This report was prepared as an account of work sponsored by the United States Government. Neither the United States nor the United States Atomic Energy Commission, nor any of their employees, nor any of their contractors, subcontractors, or their employees, makes any warranty, express or implied, or assumes any legal liability or responsibility for the accuracy, completeness or usefulness of any information, apparatus, product or process disclosed, or represents that its use would not infringe privately owned rights.

Printed in the United States of America

Available from

National Technical Information Service

U. S. Department of Commerce

5285 Port Royal Road

Springfield, Virginia 22151

Price: Printed Copy $\$ 3.00 ;$ Microfiche $\$ 0.95$ 


\title{
NEUTRON SOURCES AND APPLICATIONS
}

\author{
Proceedings of the American Nuclear Society \\ National Topical Meeting \\ April 19-21, 1971 \\ Augusta, Georgia \\ Contributed Papers \\ SESSIONS I AND II
}

April 1971

\begin{abstract}
This report was prepared as an account of work sponsored by the United States Government. Neither the United States nor the United States Atomic Energy Commission, nor any of their employees, nor any of their contractors, subcontractors, or their employees, makes any warranty, express or implied, or assumes any legal liability or responsibility for the accuracy, completeness or usefulness of any information, apparatus, product or process disclosed, or represents that its use would not infringe privately owned rights.
\end{abstract}

E. I. DU PONT DE NEMOURS \& COMPANY SAVANNAH RIVER LABORATORY

AIKEN, S. C. 29801

CONTRACT AT(07-2)-1 WITH THE

UNITED STATES ATOMIC ENERGY COMMISSION 


\section{DISCLAIMER}

This report was prepared as an account of work sponsored by an agency of the United States Government. Neither the United States Government nor any agency Thereof, nor any of their employees, makes any warranty, express or implied, or assumes any legal liability or responsibility for the accuracy, completeness, or usefulness of any information, apparatus, product, or process disclosed, or represents that its use would not infringe privately owned rights. Reference herein to any specific commercial product, process, or service by trade name, trademark, manufacturer, or otherwise does not necessarily constitute or imply its endorsement, recommendation, or favoring by the United States Government or any agency thereof. The views and opinions of authors expressed herein do not necessarily state or reflect those of the United States Government or any agency thereof. 


\section{DISCLAIMER}

Portions of this document may be illegible in electronic image products. Images are produced from the best available original document. 


\section{FOREWORD}

The speakers whose papers are published in Volume I were invited to discuss subjects in which they are acknowledged experts. To provide them with current information concerning the work of other scientists and to acknowledge the work of other persons, papers covering the many aspects of neutron sources and their applications were invited to be submitted on an international basis. Papers were accepted until March 1971. Submitted papers, which were summarized by invited speakers who acted as rapporteurs, are published here in Volumes II and III. The papers are reproduced from texts submitted by the authors and are unedited

This system of combining summary talks and submitted papers was adopted because of the widely varying and interdisciplinary nature of the rapidly growing field of neutron source application. It is hoped that the publication of this material will provide a useful reference for all who are interested in neutron sources and their applications. By being available at the meeting, Volumes II and III may assist attendees and help to stimulate discussion. Volume I will contain the invited papers, the discussions at the meeting and the remaining contributed papers. Publication will follow shortly after the meeting. 


\section{CONTENTS}

\section{SESSION I: NEUTRON SOURCES}

Experimental Capabilities and Performance of TRIGA Research and Test Reactors for Neutron Applications

G. T. Schnurer and A. T. McMain . . . . . . . . . . . . . . . . . . . .

The NBS Reactor as a Source of Neutrons

R. S. Carter.

A Versatile Nuclear Reactor Facility

A. K. Furr . . . . . . : . . . . . . . . . . . . . . . . . . . . . . .

The Control of Neutron Beams by Vibrating Crystals

A. M. Jacobs, E. S. Kenney, and J. D. E. Jeffries

The Unique Research Potential of Neutron Pulses for Nuclear Explosions

R. L. Carter ... . . . . . . . . . . . . . . . . . . . . . . .

Development of an Intense Pulsed 14-Mev Neutron Source

D. C. Gates and L. J. Demeter . . . . . . . . . . . . . . . . . . .

Using a Meson Factory as an Intense Pulsed Neutron Source

R. R. Fullwood . . . . . . . . . . . . . . . . . . . . . . . .

Gas Target Source for Fast Neutron Cancer Therapy

C. A. Kelsey, M. L. M. Boone, J. M. Hevezi,

A. L. Wiley, G. C. Spalek, H. K. Forsen and W. R. Winter. . . . . . . . . . . . . 1.56

Mobile Accelerator Facility for Neutron Interrogation and .Nondestructive Assay

B. R. Dennis, R. A. Forster, J. H. Menzel, M. M. Thorpe, and

D. B. Smith

Preparation of Industrial ${ }^{252} \mathrm{Cf}$ Neutron Sources at Savannah River Laboratory W. R. McDonell, A. R. Boulogne, J. P. Faraci, S. F. Peterson,

B. L. Dahlen, W. C. Mosley, D. J. Mahoney, and V. Whatley

Effects of Source Encapsulation Materials on the Thermal-Neutron Flux from a ${ }^{252} \mathrm{Cf}$ Source in a Water Moderator

K. D. Kok, R. Artigas, and J. W. Ray

Recent Developments in $(a n)$ Sources

K. H. Ansell and E. G. Hall . . . . . . . . . . . . . . . . . . . . . 
High Intensity $(a, n)$ Sources

K. H. Ansell and E. G. Hall

$1-100$

Evaluation of a ${ }^{244} \mathrm{Cm}$-Be Neutron Source for Activation Analysis

M. A. Wahlgren and D. C. Stewart . . . . . . .

$1-112$

Monolayer Ceramic Microsphere-Beryllium Plate Heterogeneous Isotopic Neutron Source E. D. Jordan, T. E. Carew, and B. L. Barkley

Isotopic Neutron Sources from the Los Alamos Meson Physics Facility

H. A. O'Brien, Jr. and M. E. Schillaci

Flux Enhancement with ${ }^{252} \mathrm{Cf}$ Source in a Subcritical Asseinbly

R. L. Currie, F. J. McCrosson, and P. B. Parks . . .

Comparison of Neutron Source Costs

L. A. Heinrich.

Proton Recoil Measurements of the PuBe Source Neutron Spectra

R. L. Alexander, D. F. Shook, and C. H. Ford

Absolute Measurements on Thermal Neutron Fluxes Produced in Water by $(d, d)$ and $(d, t)$ Reactions with 150 kev Deuterons

L. Holland and J. Walker . . . . . . . . . . . . . . . . . . . .

Reactor and Neutron Physics Applications of Calibrated Neutron Sources

A. DeVolpi, K. Porges, R. Karam, W. Poenitz,

A. Cox, M. Bretscher, W. C. Redman, and S. Carpenter . . . . . . . . . . .

The Neutron Spectrum From a Radioactive ${ }^{210}$ Po-Li $(a, n)$ Source

K. W. Geiger and L. van der Zwan. . . . . . . . . . . . . . . . . . . . .

Evaluation of the Neutron Source Absorption Correction in a Manganous Sulfate Bath

V. Spiegel, Jr. . . . . . . . . . . . . . . . . . . . . . . . . .

Tissue Equivalent Proportional Counters in Thin Neutron Sihield Studies

L.W. Brackenbush, G.W. R. Endres, and

L. G. Faust

Dose Rate Estimation for Neutron Source Shielding and Applications

H. E. Hootman and D. H. Stoddard.

Calculated and Measured Effectiveness of Californium-252 Source Shielding

D. H. Stoddard and R. A. Moyer

Design and Construction of a Versatile ${ }^{252} \mathrm{Cf}$ Neutron Source Shield and Experimental Facility

S. J. Gage, E. L. Draper, Jr., G. D. Bouchey, and

R. R. Day 


\section{SESSION II: MEDICAL AND FORENSIC USES OF NEUTRONS}

Neutron Radiation Therapy

M. Catterall, R. H. Thomlinson,

S. B. Field, and C. C. Rogers . . . . . . . . . . . . . . . . . . . . $\mid 1-1$

Fast Neutron Depth Doses and Spectra Obtained by Bombarding Various Targets With $16 \mathrm{MeV}$ Deuterons

C. J. Parnell.

Experience in Treatment Dose Calculations for ${ }^{252}$ Cf Patients

G. D. Oliver, Jr., P. R. Wright, and

P. R. Almond . . . . . . . . . . . . . . . . . . . . . . . . . . $11-16$

Preliminary Clinical Experience with Californium-252 Interstitial Sources

J. R. Castro . . . . . . . . . . . . . . . . . . . . . . . . . . 11.22

A Cytogenetic Determination of the OER and RBE of Californium-252

S. C. Bushong, N. Prasad, S. A. Briney, and

Introduction to Medical Neutrography *

Neutrography in Medical Research and Pathology

M. J. Flynn, G. F. Knoll, and

A. K. Poznanski

Neutron Radiography in Dental Diagnosis M. T. Weisman and M. Brown .

A ${ }^{252} \mathrm{Cf}$ Focused Neutron Gun for Activation of Skin-Tissue to Detect AboveNormal Amounts of Sodium

Practical Applications and Limitations of Forensic Activation Analysis

C. M. Hoffman and M. J. Pro

The Role of Environmental Contamination in Criminalistics: A Case History

K. K. S. Pillay, C. C. Thomas, Jr., and

G. F. Mahoney .

State-Wide Training and Service Program in Forensic Neutron Activation Analysis

J. R. Vogt, M. E. Eichor, and

R. E. Mason

Statistical Interpretation of Trace Element Patterns in Paper

H. R. Lukens and H. L. Schlesinger.

* Translated from the Journal de Radiologie d' Electrologie et de Medecine Nucleaire 51, 269 (1970). Reprinted with permission from the publisher. 


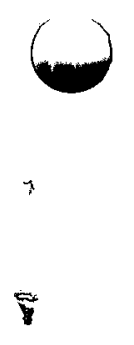

Session I

NEUTRON SOURCES 


\section{EXPERIMENTAL CAPABILITIES AND PERFORMANCE OF TRIGA RESEARCH AND TEST REACTORS FOR NEUTRON APPLICATIONS}

\section{George T. Schnurer \\ Albert T. McMain}

Gulf Energy \& Environmental Systems, Inc. San Diego, California

This paper presents an overview of the various types of TRIGA ${ }^{R}$ reactors and their experimental facilities and performance capabilities. Recently developed designs and new experimental facilities and performance capabilities are discussed in detail, and current development work in these areas is reviewed. Typical neutron research and test applications are discussed in the context of the experimental facilities and performance capabili. ties. Since the associated building and auxiliary equipment are important considerations in planning a neutron source facility, these aspects are also reviewed for the various types of TRIGA reactors. Similarly, operating considerations, including maintenance and staffing requirements, are discussed.

\section{INTRODUCTION}

TRIGA research and test reactors have found extensive application as a flexible and versatile neutron source. This acceptance is in large measure due to the experimental capabilities and performance, together with the safety, that have been exhibited by TRIGA reactors.

Historically, the first TRIGA was unveiled as part of the U.S. Government's exhibit at the Second United Nations Conference on the Peaceful Uses of Atomic Energy in 1958. The original TRIGA prototype had gone critical in San Diego just a few months earlier. It is still being used today to perform very valuable research in support of major programs, as well as to perform approximately $95 \%$ of all the work load of the Gulf Energy \& Environmental Systems Neutron Activation Analysis Service, which processes samples on a routine basis for well over 600 organizations.

The TRIGA concept was developed to fulfill the requirements for a research reactor whose safety was inherent in its intrinsic properties and not primarily dependent on mechanical or electronic safety devices. This goal was fully achieved with the development of the uranium-zirconium hydride TRIGA fuel with its large prompt negative temperature coefficient of reactivity. It is the use of this fuel that is most closely associated with the name TRIGA, a term applied to a family of reactors varying in both configuration and performance but sharing a common fuel technology.

The safety characteristics of TRIGA are exemplified by its pulsing capability, which in addition to being a useful research capability in its own right has resulted in other benefits. For example, TRIGAs are normally installed in buildings of conventional construction without pressure containment, a direct result of the TRIGA's demonstrated safety characteristics. Further, by virtue of the reactor's safety and other characteristics, TRIGA cores can be cooled by natural convection cooling for power levels up to $2000 \mathrm{~kW}$, thereby increasing their flexibility and substantially simplifying the associated cooling systems. As a result, smaller operating staffs are required and maintenance is kept to a minimum.

The history of TRIGA has been one of continuing improvement. The initial design goal of $10 \mathrm{~kW}$ steady-state has evolved into a whole class of reactors having performance levels in the megawatt range with pulsing to thousands of megawatts. At present, there are 49 TRIGA reactors in operation or under construction in 14 countries, representing more than 250 reactor years of operating experience.

As advanced power reactors are developed, there is increasing interest in the use of TRIGA research reactors to provide a test bed 
for power reactor fuel development. Compact core TRIGA reactors have already been used to perform extensive experiments on fission product diffusion in power reactor fuel matrices. The development of an annular core TRIGA reactor a few years ago for Sandia Corporation, a USAEC prime contractor, has stimulated considerable interest in the use of this concept for testing segments of power reactor fuels, including fast reactor fuels under simulated accident conditions-up to and including fuel melt-down.

\section{TYPES OF TRIGA REACTORS}

\section{GENERAL}

TRIGA reactors are available in several different configurations which use many similar parts or components to permit ease of upgrading the performance level (1). Therefore, the reactor user can acquire a reactor facility with minimum capital investment; then as the user's requirements develop, the facility can be upgraded to avoid technical obsolescence.

The performance of any given TRIGA reactor type is determined by the type of fuel and reflector configuration used. Pulsing parameters will vary slightly with the reflector material. For a given reactivity insertion, the graphite-reflected Mark I and II give a lower, broader pulse than the waterreflected Mark III. The peak power achieved during the pulse for a given reactivity insertion is a function of the total number of fuel elements, although the flux is essentially independent of the number of fuel elements for the same reactivity insertion.

The following types of uranium-zirconium hydride fuel elements (Figure la) can be employed in any of the TRIGA configurations described in succeeding sections:

1. Aluminum-clad U- $\mathrm{ZrH}_{1.0}$ (steady state), fully enriched uranium; $8.5 \mathrm{wt}-\%$ uranium for operation up to $100 \mathrm{~kW}$. This fuel was developed for use on the mini-TRIGA to permit a small compact core giving a high flux per watt.

2. Aluminum-clad U-ZrH 1.0 (steady state), $20 \%$ enriched uranium; $8.5 \mathrm{wt}-\%$ uranium. This fuel is used for steady-state operation up to $250 \mathrm{~kW}$ with natural convection cooling of the core. Higher power operation with these fuel elements is limited to fuel temperatures below $500^{\circ} \mathrm{C}$ because of a phase transition at $\sim 530^{\circ} \mathrm{C}$.
3. Stainless steel clad $\mathrm{U}-\mathrm{ZrH}_{1}$, (steady-state/pulsing). By increasing the hydrogen content by $70 \%$, the troublesome phase transition is eliminated and the fuel materlal can tolerate temperatures well in excess of $1000^{\circ} \mathrm{C}$. The stainless steel cladding also withstands higher temperatures. These fuel elements have been perated at steady-state power levels of $1500 \mathrm{~kW}$ with natural convection cooling of the core, and have been repetitively tested with core reactivity insertions up to $\$ 5.0(1)(3.5 \% \delta \mathrm{k} / \mathrm{k})$. With a $\$ 3.00$ reactivity insertion, the peak power is approximately $20 \mathrm{MW}$ per element. In the Advanced TRIGA Prototype (ATPR) at Gulf Energy \& Environmental Systems the peak power is approximately 6500 NW for a $\$ 4.60$ reactivity insertion.

4. Fuel Lifetime Improvement Program (FLIP). This fuel, dimensionally similar to standard TRIGA fuel, was specifically developed to providé core lifetimes in the range from 7 to $10 \mathrm{NW}-\mathrm{yr}$. This fuel is available with enrichments of $70 \%$ or $93 \%$. It also incorporates the use of erbium as a burnable poison ( 1.6 wt $-\%$ with the $70 \%$ enriched fue 1 ). The initial cores of TRIGA FLIP fuel will be installed early in 1971 in a TRIGA reactor at Gulf Energy \& Environmental Systems (San Diego) and at the USAEC reactor facility in Puerto Rico, which j.s now in the process of being converted to al TRIGA core.

5. Conversion Clusters. In order to facilitate the conversion of existing reactors presently designed to accommodate MTR platetype fuel, a four-rcid cluster of TRIGA fuel was developed (Figure 1b). These clusters may be formed from standard stainless steel clad $\mathrm{U}-\mathrm{ZrH}_{1}, 6$ fuel or from the FLIP fuel. End fittings ${ }^{6}$ can be adjusted to either square or circular grid plete holes.

\section{STANDARD TRIGA REACTOORS}

Mini-TRIGA. The newest and simplest of the TRIGA models, this reactor represents a budget price reactor with considerable flexibility and a high flux per watt, ${ }^{*}$ with an added capability for future upgrading. (See Figure 2.) The stardard mini-TRIGA can operate for short periods of time at steadystate power levels up to $100 \mathrm{~kW}$, providing

*In order to achieve this, the miniTRIGA utilizes a small compact core of fully enriched, aluminum-clad $\mathrm{U}-\mathrm{ZrH}_{1} .0$ fuel elements. 
a peak neutron flux of $3.5 \times 10^{12} \mathrm{n} / \mathrm{cm}^{2}-\mathrm{sec}$, which makes it an ideal. low cost neutron source for performing neutron radiography, neutron activation analysis, production of short-lived isotopes, and various research applications. With the installation of a vertical beam collimator, and a minimum of additional equipment, the mini-TRIGA becomes the basis of the simplest NEUTROVISIONTM system (2). Although the turn-key price may vary with installation circumstances, a typical mini-TRIGA costs $\$ 175,000$ in the United States, exclusive of building and pool, but including all control and operating equipment as we11 as installation and start-up.

TRIGA Mark I. The TRIGA Mark I, 1ike the mini-TRIGA, is a simple system. At first glance, it is similar in appearance to the mini-TRIGA but it uses standard TRIGA fuel, permitting operation up to $2000 \mathrm{~kW}$ with the additional capability of pulsing. Iike the mini-TRIGA and TRIGA Mark II, it employs a graphite reflector. It is located at the bottom of an open, water-filled tank in a below-ground installation. This reactor can be installed in an existing building, thus avoiding expenses for the construction of a special reactor building. Because of TRIGA's safety and proven reliability, a pressure5 type building containment is not required. For 1-MW operation and above, air confinement is recommended, but this can be accomplished with a conventional type of building construction.

All TRIGA Mark I reactors are equipped with the following types of irradiation or experimental facilities (see Table I):

1. Rotary Specimen Rack. This facility permits exposure of multiple samples. It is located in the reflector and can be rotated at a uniform rate so as to ensure that all samples are exposed to the same total integrated flux, thus reducing the number of standards required in performing neutron activation analysis.

2. Central Thimble. This facility is located in the center of the core and is equipped to be operated with a dry tube for bringing out a vertical beam.

3. Pneumatic Transfer System. The pneumatic transfer system has its terminus in an in-core position. The reactor can be equipped with multiple transfer systems and switching arrangements.

4. In-core Irradiations. All TRIGAs have the capability of performing in-core experiments in fuel element positions. A grid plate is also available with a large removable center section (Figure 3). Also with this grid plate, three fuel elements can be removed, permitting the insertion of in-core experiments which are larger than the standard fuel element grid position.

5. Bulk Irradiations. The water space above the grid and outside the reflector can also be utilized for performing the irradiation of bulk or irregularly shaped experiments. A flat face on one side of the reflector may be used to increase the flexibility in the irradiation of larger objects.

TABLE I

TRIGA Experimental Facilities

\begin{tabular}{|c|c|c|c|}
\hline $\begin{array}{l}\text { Experimental and } \\
\text { Irradiation Facilities }\end{array}$ & Mark & $\begin{array}{c}\text { Mark } \\
\text { II }\end{array}$ & $\begin{array}{r}\text { Mark } \\
\text { III }\end{array}$ \\
\hline Rotary Specimen Rack & $x$ & $x$ & $\mathrm{x}$ \\
\hline Central Thimble & $\mathrm{x}$ & $\mathrm{x}$ & $\mathrm{x}$ \\
\hline Pneumatic Transfer System & $\mathrm{x}$ & $\mathrm{x}$ & $\mathrm{x}$ \\
\hline $\begin{array}{l}\text { In-core Irradiation Facilities } \\
4,4-\text { in.-diam }\end{array}$ & $x$ & $\mathrm{x}$ & $\mathrm{x}$ \\
\hline $\begin{array}{l}\text { hexagonal space } \\
2.4 \text {-in.-diam }\end{array}$ & (a) & (a) & $\mathrm{x}$ \\
\hline $\begin{array}{l}\text { triangular spaces } \\
\text { 1.5-in.-diam }\end{array}$ & (a) & (a) & $\mathrm{x}$ \\
\hline circular spaces & $\mathrm{x}$ & $\mathrm{x}$ & $\mathrm{x}$ \\
\hline $\begin{array}{l}\text { Beam Ports, four 6-in.-diam } \\
\text { Through Ports, } \\
\text { two 8-in.-diam }\end{array}$ & & $\mathrm{x}$ & $\begin{array}{l}x \\
x\end{array}$ \\
\hline $\begin{array}{l}\text { Thermal Columns } \\
\text { Horizontal }\end{array}$ & & $x$ & $x$ \\
\hline $\begin{array}{l}\text { Vertical } \\
\text { Hohlraum }\end{array}$ & & (b) & $\begin{array}{l}\mathrm{x} \\
\mathrm{x}\end{array}$ \\
\hline Pool Irradiation Facility & $\mathrm{x}$ & $\mathrm{x}$ & $\mathrm{x}$ \\
\hline Walk-in Exposure Room & & & $\mathrm{x}$ \\
\hline
\end{tabular}

TRIGA Mark II. The TRIGA Mark II reactor (Figure 4) is similar in configuration to the TRIGA Mark I except that it is located above ground. As a consequence, a number of additional horizontally oriented experimental facilities can be incorporated. TRIGA Mark II reactors have the following types of facilities (in addition to those in the TRIGA Mark I): 
1. Beamports. A typical arrangement includes four 6-in.-ID beamports (three radial and one tangential). Other arrangements are possible, including the use of through tubes.

2. Thermal Column. All TRIGA Mark II reactors are equipped with a graphite thermal column with a cross section of $4 \mathrm{ft}$ by $4 \mathrm{ft}$. It is also possible to include a vertical, as well as horizontal, thermal column.

3. Thermalization Column and Bulk Shielding Facility. Most TRIGA Mark II reactors have a 2 ft by $2 \mathrm{ft}$ graphite thermalization column associated with a bulk shield facility.

TRIGA Mark III. The most advanced version of the compact core TRIGA reactors is the TRIGA Mark III. Unlike the Mark I and II, which have fixed cores supported from the bottom of the reactor tank, the TRIGA Mark III has a movable bridge-mounted core. It also employs a water reflector so as to maximize the leakage from the core.

Figure 5 shows a photograph of a model of this reactor together with a cross-sectional plan identifying the many beam tubes associated with the facility. A major advantage of the TRIGA Mark III arrangement is the movable core. When the reactor is operating at one end of the pool, it is possible to set up experiments at the other end without additional shielding. The TRIGA Mark III is also equipped with a large dry exposure room to permit the irradiation of large objects in a dry environment. As with the TRIGA Mark I and II, the rotary specimen rack, central thimble, pneumatic transfer system, and removable in-core sections are standard equipment.

There is also a below-ground version of the TRIGA Mark III that uses the waterreflected, movable core but is installed below ground in a manner comparable to the TRIGA Mark I. Several adaptations of this configuration are also available, permitting incorporation of inclined beams and, with the use of an experiment pit, even horizontal beam tubes.

TRIGA/ACPR (3). The TRIGA/ACPR incorporates an annular core configuration to permit the exposure of large in-core samples in a dry environment (Figure 6). The first TRIGA/ACPR in operation (since 1967) has a 9-in.-diam center test cavity which is exposed to a peak fast neutron flux during the pulse of approximately $2.5 \times 10^{17} \mathrm{n} / \mathrm{cm}^{2}-\mathrm{sec}$. (See Table II.) The reactor has 141 fuel elements plus 6 fueled follower control rods (control rods that have a fuel portion on the lower end) and 3 transient rods.

TABLE II

Status of TRIGA Compact Core

Reactivity Insertion . . \$4.60

Peak Power .. . . . . $6480 \mathrm{MW}$

Energy Release ?. . . $44.5 \mathrm{MW}-\mathrm{sec}$ (the actual energy release is a function of the number of fuel elements in the core at the time)

Reactor Period . . . . $1.68 \mathrm{msec}$

Pulse Width at one-

half peak power . . $6.2 \mathrm{msec}$

Statis of TRTGA ACPR

Peak Adiabatic Fue1

Temperature . . . . $900^{\circ} \mathrm{C}$

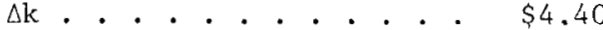

Core Energy Releàse

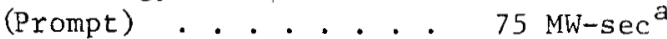

$n v t>10 \mathrm{keV}$ in experi-

mental cavity ... . $1.4 \times 10^{15^{\mathrm{b}}}$

Peak Power . . . . . . 12,000 MW

Minimum Period. . . . $1.3 \mathrm{msec}$

Pulse Width . . . . . $4.6 \mathrm{msec}$

${ }^{\text {a }}$ Sandia value is 67 based on linear $\gamma$ chamber trace.

b Includes pulse tail. Reactor shutdown is initiated $v 1 \mathrm{sec}$ after peak power; $10 \%$ to $20 \%$ of this nvt could be coming after the prompt burst.

The TRIGA/ACPR can be employed in either a simple below-ground installation such as the TRIGA Mark I or irt an above-ground configuration including the use of a movable core design. Different size test cavities are possible through suitable design modifications.

\section{CONVERSIONS}

As noted, TRITA fuel technology is being used to modernize and upgrade existing reactors presently einploying plate-type fuel elements. A four-rod cluster of either standard or FLIP TRIGA fuel elements with MTR-type end fittings is employed to permit retention of the existing grid plate. Examples of conversions using fol four-rod cluster of 
standard TRIGA fuel are the reactors at Washington State University, University of Wisconsin, and Texas A\&M. An alternative approach to conversion is to replace the grid plates and employ the standard TRIGA fuel. This was done at Pennsylvania State University (4). In the case of the reactor at the Puerto Rico Nuclear Center, the USAEC selected a four-rod cluster of TRIGA FLIP fuel. Because of the long burn-up lifetime of a core, the conversion to FLIP fuel offers some economic advantage to reactors now operating in the 1 - to 2-MW range with MTR fuel when the total cost of the reactor fuel cycle is considered.

\section{PERFORMANCE}

TRIGA reactors have been distinguished by their flexibility of operation and their unique performance capability for both steady state and pulsing operation. All TRIGAs have a high flux per watt, and there is very good; reactivity decoupling between the experiment and the reactor, thereby permitting extensive use of in-core experiments. Table III gives pertinent performance characteristics for a water-reflected TRIGA reactor using a standard stainless steel core. The fluxes indicated are per MW of operation; e.g., at $1500 \mathrm{~kW}$ steady state, the peak thermal flux is approximately $4.5 \times 10^{13} \mathrm{n} / \mathrm{cm}^{2}-\mathrm{sec}$, and the peak flux during a 2000-MW pulse is $6.4 \times 10^{16} \mathrm{n} / \mathrm{cm}^{2}-\mathrm{sec}$. Table II summarizes the present status of the pulsing character- istics of both a compact and an annular TRIGA core.

\section{SUPPORTING RESEARCH AND DEVELOPMENT PROGRAMS}

The development and growth potential of TRIGA reactors is a result of the continued research and development program which was first initiated in 1957. Gulf Energy \& Environmental Systems operates three TRIGA reactors at its laboratories in San Diego, California. One of the major uses of these reactors is the continued testing and improvement of TRIGA fuel technology and TRIGA reactor designs. For example, one of these: reactors was used for initial testing of TRIGA FLIP fuel to verify calculated nuclear parameters, both under steady-state and pulsed conditions. A complete TRIGA FLIP. core will be installed in one of these re- $\ldots$ actors and will provide round-the-clock operation at an initial power level of $2000 \mathrm{~kW}$ (still with natural convection of the cool( ing aore). The TRIGA in which it will be installed, a below-ground TRIGA Mark III, has already been modified to provide forced down-flow cooling, thereby permitting future upgrading to $3000 \mathrm{~kW}$. (Another TRIGA, the TRIGA Mark II at the University of Illinois, has already had forced down-flow cooling installed and will eventually be upgraded to $3000 \mathrm{~kW}$.).

TABLE III

Flux Data For Standard Mark III Core

Experimental Measured flux level per MW Location with clean, water-reflected core loading to give excess reactivity required for $1500-\mathrm{kW}$ steady-state and pulsing to $2000 \mathrm{MW}$

\begin{tabular}{ll} 
Peak & Peak \\
Unperturbed & Unperturbed $^{\mathrm{a}}$ Fast \\
Thermal Flux & Flux $>10 \mathrm{keV}$ \\
$\mathrm{n} / \mathrm{cm}^{2} / \mathrm{sec}$ & $\mathrm{n} / \mathrm{cm}^{2} / \mathrm{sec}$ \\
\hline
\end{tabular}

Core Center

$$
\begin{array}{cc}
\left(\mathrm{H}_{2} \mathrm{O}\right. \text {-filled } & 3.22 \times 10^{13} \quad 2.42 \times 10^{13^{\mathrm{C}}} \\
\text { thimble) } & \text { (Cd ratio }
\end{array}
$$$$
\text { (Cd ratio } 2.33 \text { ) }
$$

Water Reflec- $1.3 \times 10^{13}$

tor Peak

At Core Edge
without any

$$
1.03 \times 10^{13}
$$

$0.46 \times 10^{13}$

Reflector

Peak Effect ${ }^{b}$

$a_{\text {Where measured values are given, they }}$ are, by definition, not "unperturbed," since the method of measurement will perturb the flux. Values of unperturbed fluxes are calculated values and compare well with measured flux values.

$b_{\text {Measurements made by the University of }}$ California, Berkeley, on their TRIGA Mark III reactor, based on bare and cadmium-covered gold foil measurements. Total neutron flux was determined by $\beta-\lambda$ coincidence measurements. Gold foil measurements are accurate to within $20 \%$.

${ }^{c}$ As a result of the measurement method employed, this is an epicadmium value.

\section{TYPICAL NEUTRON RESEARCH AND TEST APPLICATIONS}

The important role that a TRIGA reactor plays in the TRIGA NEUTROVISION system for performing neutron radiography has already been noted in this paper. The unique experimental facilities, such as the rotary specimen 
rack found in most TRIGAs, make the TRIGA ideally suited for performing neutron activation analysis and producing short, half-lived radioisotopes. Such applications are well documented (5-7) and will not be reported in detail in this paper. Instead, a rather specific field of application will be discussed to illustrate how a relatively lowcost and simple neutron source, such as a TRIGA reactor facility, can be utilized in support of major power reactor development programs, particularly in the area of fuel testing. A specific field of interest at Gulf Energy \& Environmental Systems in which TRIGA reactors have played an extremely important role is the study of fission product release and diffusion in fissile matrices.

A number of different types of fission product release measurements can be carried out using TRIGA reactors in either the steadystate or the pulsing mode. The versatility of TRIGA reactors is illustrated by the examples of typical experiments which have already been performed in support of both thermal and fast power reactor programs. In addition, the ready accessibility of these reactors for the insertion of experiments and the ability to withdraw experiments from the core region at almost any time enable scientists and engineers to carry out studies on fuels and other samples at minimum cost in terms of reactor time. Finally, the inherent safety of the TRIGA reactor permits the insertion and removal of experiments by persons not highly trained in the radiation field.

\section{GASEOUS FISSION PRODUCTS}

Measurement of the release of gaseous fission products from either particulate or pellet-type fuels is carried out in the TRIGA King Furnace facility $(5,7)$. The release of gaseous fission products is normally measured under steady-state conditions by passing a sweep gas over the sample and collecting the purged fission gases in a liquid-nitrogencooled charcoal trapping system.

Samples can be studied from room temperature to approximately $2000^{\circ} \mathrm{C}$ independent of the power being generated in the sample itself. This permits studies to be carried out, for example, on fuel specimens fabricated from normal or depleted uranium, or even thorium, rather than highly enriched uranium. The cost of sample preparation and the use of highly enriched uranium are minimized.
Studies of the release of gaseous fission products can also be carried out in the pulsing mode, and a few preliminary experiments on pellet-type sj.mulated fast reactor fuels have already beer performed using a pulsing TRIGA reactor. In these experiments, the release from a fuel sample is first studied under steady-statie conditions, and immediately afterward the reactor is pulsed at a high power level to simulate transients that might be expected in operating fast reactor systems. Energy inputs up to about 2500 calories per gram of $235_{U}$ appear to be possible in 0.25 -in.-diam $\mathrm{UO}_{2}$ rods. This is well in excess of the approximately 300 calories per gram required to thelt $\mathrm{UO}_{2}$ fuel. Finally, this energy can be deposited in a pulse with a width at half maximum of about $6 \mathrm{msec}$, resulting in conditions which simulate adiabatic energy insertion.

Transient studies have been carried out with coated particle fuels of the type used in HTGRs. These fuels have been studied at energy inputs up to about 3000 calories per gram of $235 \mathrm{U}$. It has been possible to monitor the overall thermal response of these fuels during the actual time of the pulse using optical techniques.

\section{CONDENSED FISSION PRODUCTS}

The TRIGA is routinely used to produce irradiated specimens with typical fission product inventories for use in annealing experiments in which the release of condensed fission products is; studied as a function of time. A quality control program to test advanced fuels to be inserted into test capsules has been carried out using this procedure. Normally, the release of fission product strontium and barium is studied using this technique. On'e advantage of this type of postactivation annealing experiment is that several samples can be irradiated at one time and annealed individually, resulting in lower irradiation charges! per fuel sample.

\section{MISCELLANEOUS}

The TRIGA is adaptable to specialized and non-routine uses such as, for example, measurement of the release of fission product iodine from cracked fuel particles. Experiments of this type vere carried out in support of a safety analysis program for HTGRs. 


\section{CONCIUSION}

In the preceding sections, the pertinent characteristics and performance of TRIGA reactors as a neutron source have been surveyed. However, the usefulness of any device as a neutron source is viewed properly within the context of its application. For this reason, selected areas of application were discussed to underscore how certain design features and characteristics of a reactor neutron source can be used to best advantage to accomplish an experimental objective. One measure of the true versatility of a neutron source is the simplicity not only of the source itself but of the experimental apparatus that is required. The latter is frequently made unnecessarily complex because of certain performance features of the neutron source. In this paper, an attempt has been made to illustrate how the relatively simple design of TRIGA reactors together with their operating characteristics can be used to support meaningful and sophisticated work without an undue degree of complexity and expense.

\section{REFERENCES}

1. G. T. Schnurer, et al. "TRIGA - A High Performance Steady State/Pulsing Reactor." Paper presented at NUCLEX 66 Conference, Basel, Switzerland, September 8-14, 1966.

2. W. L. Whittemore, G. T. Schnurer, and A. T. McMain. "Uses of a TRIGA Neutrovision System Including Examination of Radioactive Materia1," Proceedings of 17 th Conference on Remote Systems Technology. pp. 49-57, American Nuclear Society, Hinsdale, Illinois (1969).

3. T. B. Pearson, et al. "High Pulsing in a TRIGA Annular Core Reactor - Design and Development." Transactions, 1968 Annual Meeting, American Nuclear Society and Canadian Nuclear Association, Toronto, June 10-13, 1968. pp. 283-284.

4. P. U. Fischer and G. T. Schnurer. "Conversion of Plate Type Reactors to Dual Steady State/Pulsing Reactors Through the Use of U-ZrH Fuel Elements." Paper presented at NUCLEX 66 Conference, Base1, Switzerland, September 8-14, 1966.

5. G. T. Schnurer, et al. "Uses of TRIGA Research Reactors for Engineering Research, Testing and Training," Proceedings of Panel on Engineering Programmes in Research Reactors (June 1970). Inter- national Atomic Energy Authority, Vienna (to be published).

6. G. T. Schnurer, et al. "Research Reactors - A Vital Tool for Nuclear Education and Research." Proceedings of Conference on Applications of Nuclear Energy in Lat in American Develópment, University of California, Los Angeles, 1967. Pp. 321344.

7. Federic de Hoffmann. Research Reactors and Applied Scientific Research. Gulf General Atomic Incorporated Report GA-9178 (1969). 


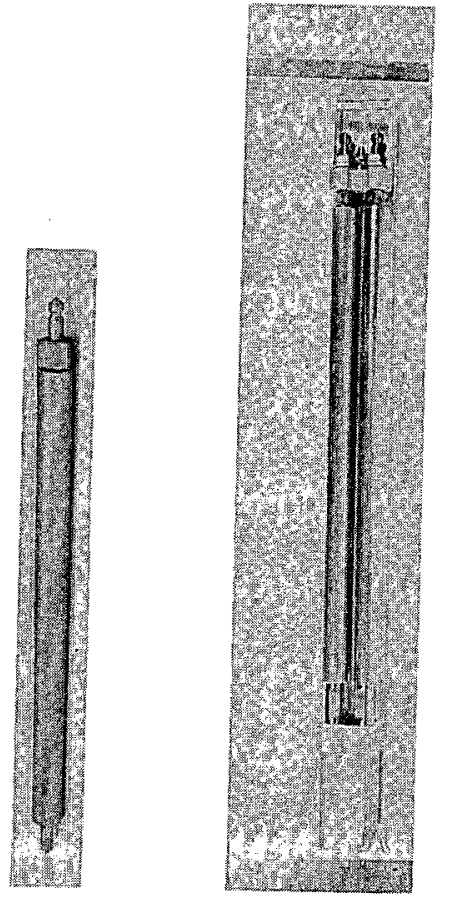

(a)

(b)

(a) Standard fuel element

(b) 4-rod fuel cluster

FIG. 1 TRIGA FUEL ELEMENTS

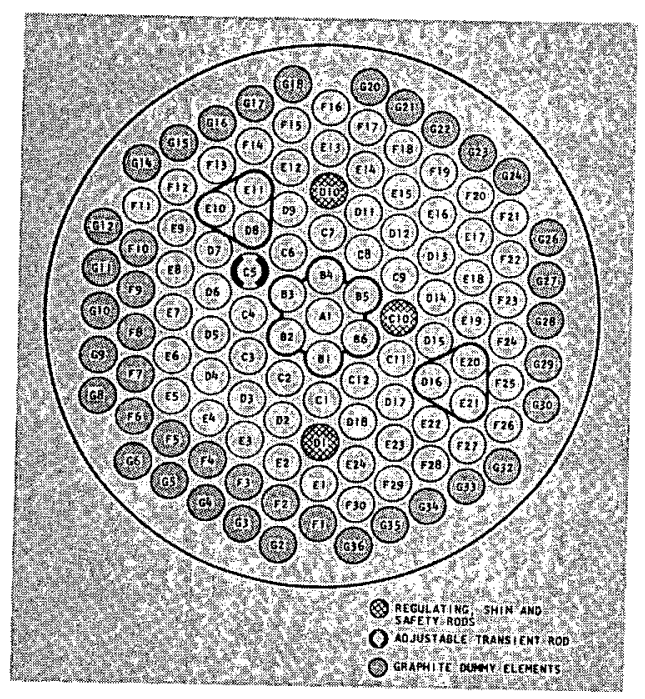

FIG. 3 TRIGA GRID PLATE WITH REMOVABLE SECTIONS

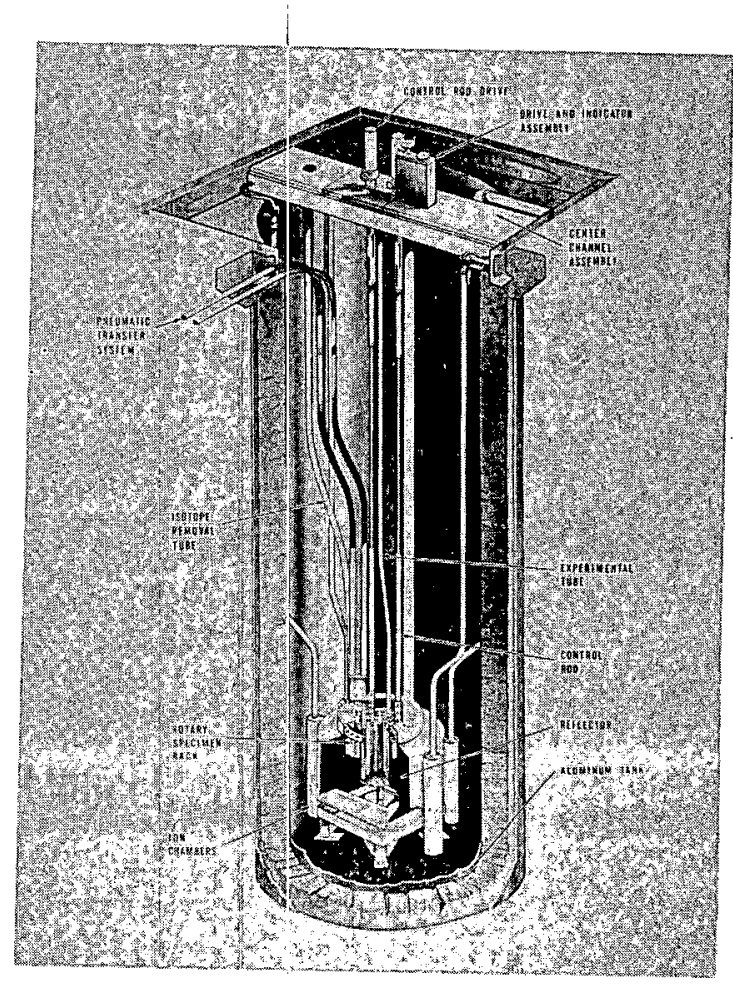

FIG. 2 MINI-TRIGA REACTOR

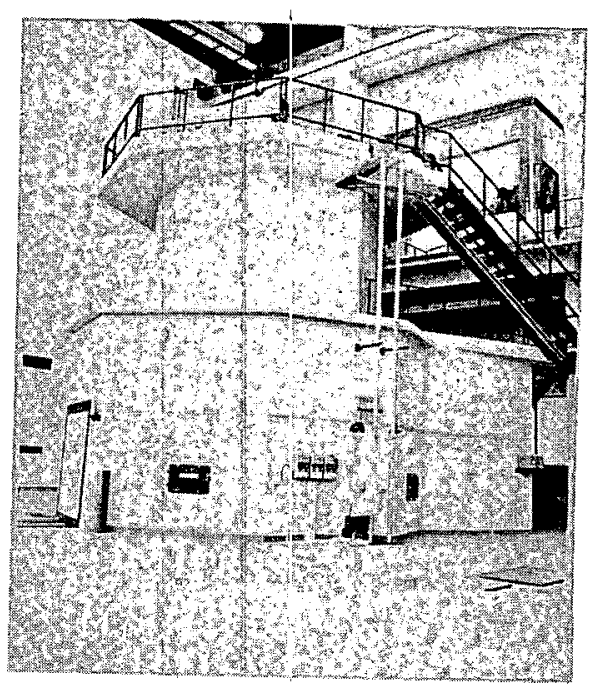

FIG. 4 TRIGA MARK II REACTOR 


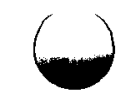

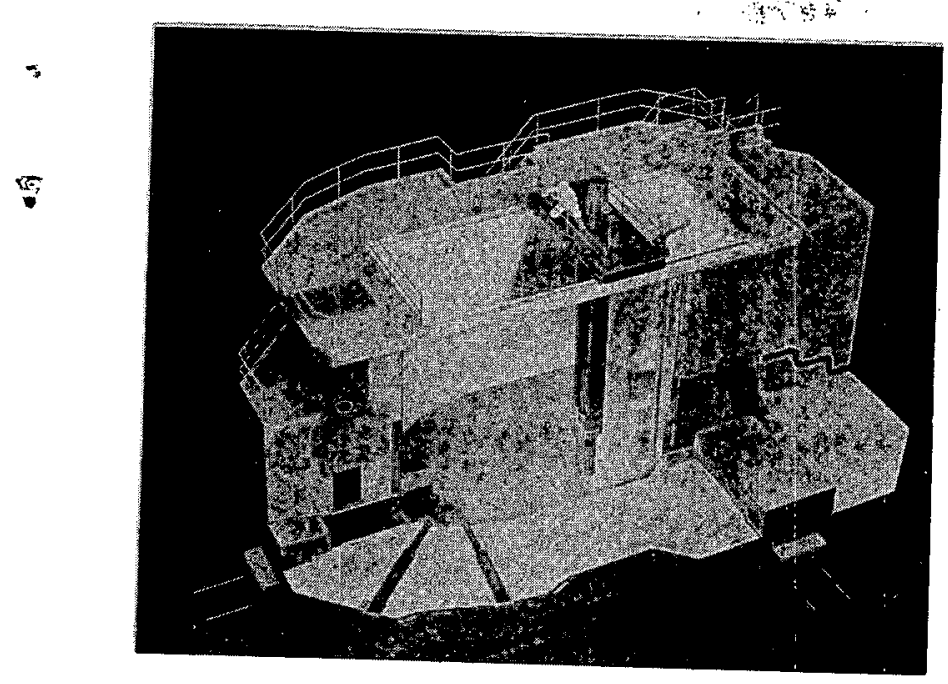

(a)

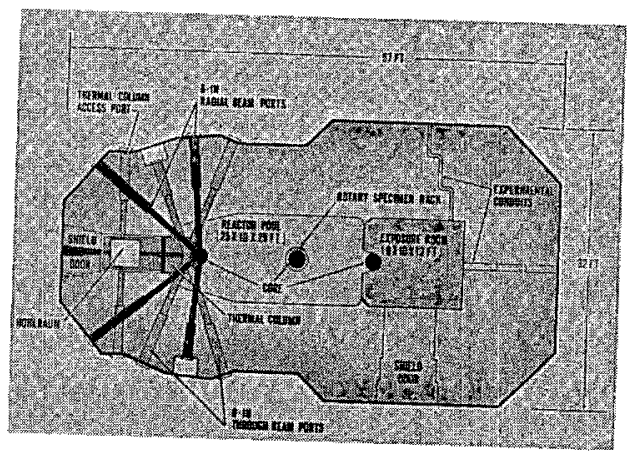

(b)

(a) Photo of model

(b) Plan view

FIG. 5 TRIGA MARK III REACTOR

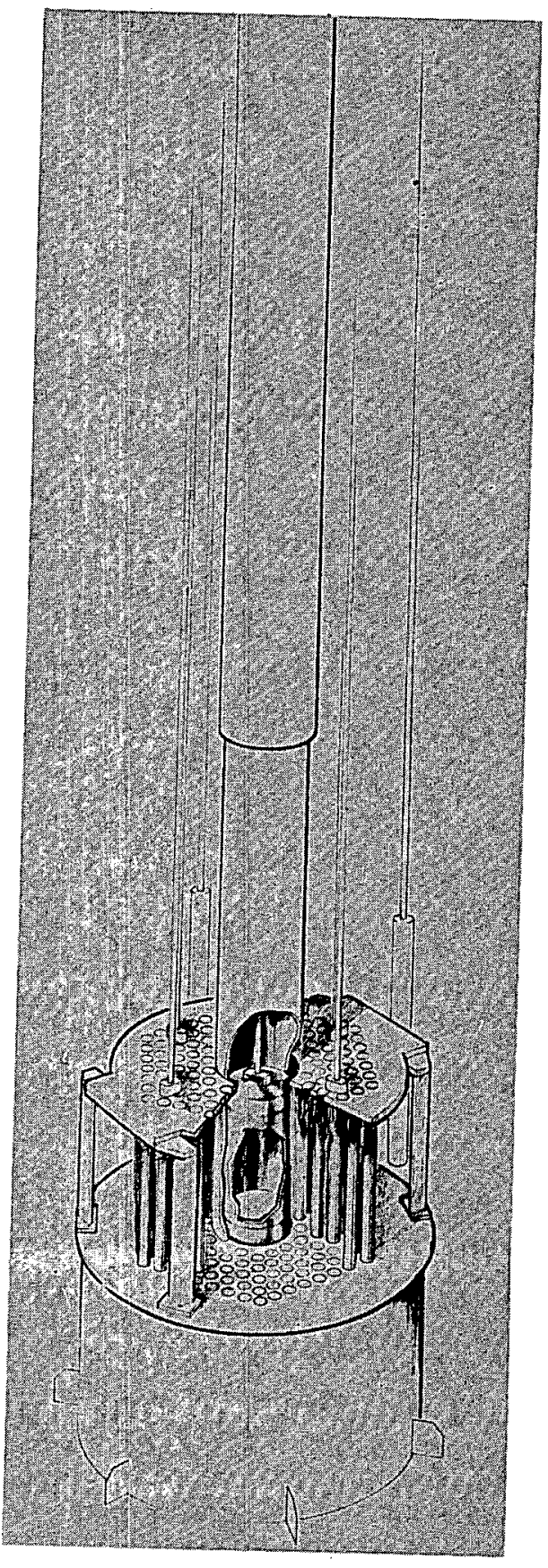

FIG. 6 TRIGA/ACPR 


\section{THE NBS REACTOR AS A SOURCE OF NEUTRONS}

\section{Robert S. Carter}

National Bureau of Standards Institute for Materials Research Washington, D. C.
The NBS Reactor (NBSR) is a $10 \mathrm{Mw} \mathrm{D}_{2} \mathrm{O}$ noderated and reflected reactor. The fuel elements are located seven inches apart in hexagonal arrays around experimental irradiation facilities. In order to support the broad range of research programs typical of a laboratory like the NBS, the reactor has extensive in-core, beam tube, and pneumatic tube facilities. Thermal neutron fluxes in the beam tubes and higher-flux irradiation facilities are about $10^{14} \mathrm{n} / \mathrm{cm}^{2} \cdot \mathrm{s}$. Several unique features of the NBSR contribute to its versatility. They include a thermal column with cadmium ratios for gold foils as high as 30,000 to 1 , a split core to minimize the intensity of very high energy neutrons in the beam tubes, and a cold neutron source to enhance the intensity of very low energy neutrons available for certain inelastic scattering experiments. Extensive, automated experimental facilities have been developed to fully utilize the neutron beams and irradiations provided by the NBSR.
INTRODUCTION

The National Bureau of Standards reactor (NBSR) was designed to meet the requirements of a broad spectrum of programs of interest to NBS and the washington scientific community. Intense neutron beams were needed for the study of the structure and dynamics of condensed materials, for a variety of nuclear physics problems, and for the development of thermal neutron standards and cross section measurements. Regions of well characterized flux, both thermal and fast, were needed for the study of radiation effects on materials and for the development of neutron flux measurement techniques. And, finally, facilities should be provided for activation analysis to supplement the Bureau's existing capability in analytical chemistry.

To fulfill these requirements, the reactor should have room for a variety of in-core irradiation facilities, pneumatic tubes, and relatively large volumes of well thermalized flux; yet, at the same time, the thermal beam intensity must be maintained. To meet these requirements a $\mathrm{D}_{2} \mathrm{O}$ moderated and reflected reactor was chosen. The large diffusion length of thermal neutrons in $D_{2} \mathrm{O}$ made it possible to place the fuel elements on seven inch centers in an hexagonal grid which allowed sufficient space for in-core irradiation thimbles. The $\mathrm{D}_{2} \mathrm{O}$ reflector also provided space for additional irradiation thimbles and assured a high thermal flux source for the beam tubes.
GENERAL DESCRIPTION OF REACTOR

The reactcr is a tank type $\mathrm{D}_{2} \mathrm{O}$ moderated and reflected reactor operating at a power of $10 \mathrm{Mw}$. Provisions for expansion to higher power are included. The plan view of the reactor is shown in figure 1 . The fuel elements are sjmilar to the conventional plate type, MTR elements and are fully enriched. The thirty elements composing the core are represented by the rectangles in figure 1. They are located about seven inches apart in hexagonal arrays around experimental irradiation failities represented in the fiqure by the larger black circles. This configuration provides seven 3-1/2" diameter irradiation thimbles, each surrounded by six fuel elements. Because each of the thimbles is located in the center of $a D_{2} \mathrm{O}$ island, the neutron flux is well thermalized and the gamma ray heating is relatively low. The smaller black circles represent 2" experimetal thimbles located between adjacent fuel elements. Their proximity to the fuel element providies a larger component of fast neutrons than is available in the larger thimbles.

The 3-1/2" thimbles are removable aluminum cylinders which fit into the bottom grid plate in the same fashion as the fuel elements. Their bottom fitting has a small hole so that a small flow of water from the plenum circulates through the thimble to provide cooling. The thimble can be removed by the fuel eliement transfer mechanism and 


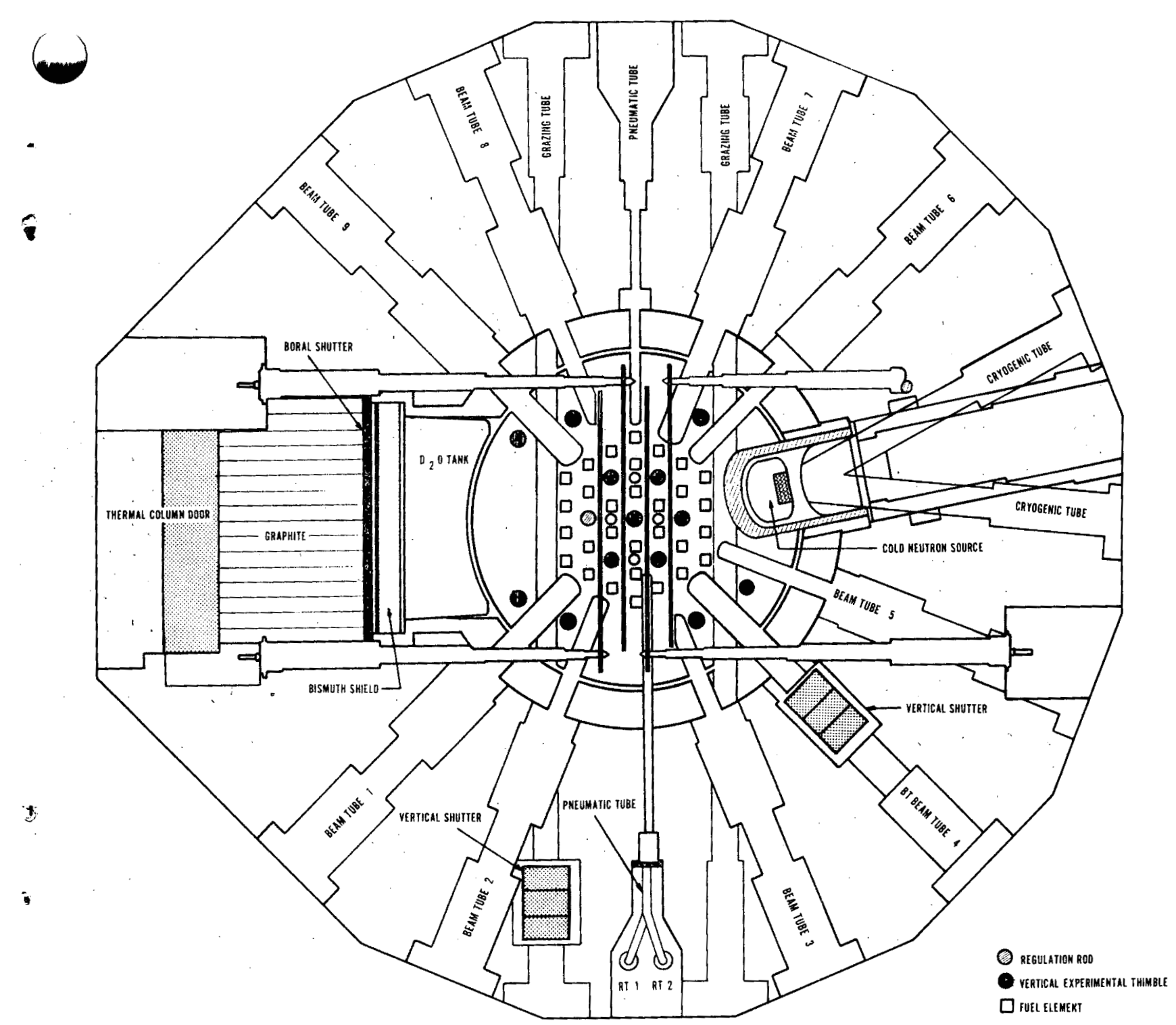

FIG. 1 PLAN VIEW OF FEACTOR

and replaced with a fuel element or a converter tube. (The converter tube will be discussed later.) Each thimble (or converter tube) is held in place by a tube penetrating the top plug. A portion of each hold down tube contains a cadmium section which provides a low flux region for temporary storage of highly radioactive samples during removal from the reactor. The 2" thimbles, on the other hand, are held in place permanently by the top grid plate. There is no tubing above them except when they are being used for irradiations. Thus each experimental position is accessible ( through the top plug without interfering in anyway with fuel transfers or other necessary reactor operations.

In order to make as much space as possible available for experiments, semaphore type shim arms are used for course reactivity control instead of the more conventional vertical control roads. The shim arms pass between the fuel elements and are rotated by horizontal shafts as indicated in figure 1 . When fully in they cut diagonally across the core from top to bottom. When fully witharawn they are in a horizontal position two feet above the fueled region of the core. The fuel elements have long top end boxes extending to a grid plate above the fully withdrawn position of the shim arms. Thus 
the coolant flow is guided past the shim arms minimizing any shim arm flutter that might result from turbulant water flow. A latching mechanism on each fuel element allows the elements to be held in place by the top grid plate leaving the region above free for access to experiments. A fuel element changing mechanism built into the top plug makes it possible to easily move fuel from one position to another or remove it entirely from the core. All this can be done without opening the shielding or disturbing the experimental thimbles. This system allows a uniform fuel changing pattern which assures essentially unchanged flux patterns from cycle to cycle of reactor operation.

The regulating rod for fine reactivity control is located in one of the $3-1 / 2$ " thimbles. But, since there are no vertical control rods the remaining 10 vertical thimbles in the core are available for experiments. In addition seven $4 "$ " ports in the top plug provide access to the reflector region. Two are used for a periscope if needed, but the remaining five can be used for experimental thimbles in the reflector.

There are nine radial beam tubes. Four are 6" in diameter and the other five are 5" in diameter. As shown in figure 1, the beam tubes penetrate up to the core surface. Two of them, however, are truncated to provide clearance for the shim arms. All the radial tubes are in the same horizontal plane 42" above the floor. Two through tubes pass on either side of the core. 24" above the floor. Each of the radial and through tubes has a vertical shutter consisting of 14" of lead and 4 " of masonite. The shutters are designed primarily to be used during shutdown when plugs or collimators are being changed.

In addition to the radial and through beam tubes, the cold neutron source and the thermal column are shown in figure 1 . These will be discussed in more detail in the next section.

Four pneumatic tube systems are built into the reactor vessel. Two are located on each side of the core, one above the other. The two upper ones are located at core midplane and terminate at the edge of the core. One of the lower ones penetrates between fuel elements into a region of high fast flux while the other lower one penetrates only a few inches into the $\mathrm{D}_{2} \mathrm{O}$ reflector in a well thermalized neutron flux region. The sendreceive terminals are located in a hood in the radiological laboratories in the basement of the confinement building. The rabbits are I" I.D. high purity polyethylene and are propelled by $\mathrm{CO}_{2}$ gas to avoid argon activation which would result if air were used.

The flux characteristics of these facilities wil be summarized in a later section after certain unique features of the NBSR are described.

\section{UNIQUE FEATURES}

Although the NBSR is in many ways very similar to the well thermalized $\mathrm{D}_{2} \mathrm{O}$ type of research reactor it has several unique features which contribute to its versatility. In addition to being the highest fliux conventional $\mathrm{D}_{2} \mathrm{O}$ research reactor in the United States, it has il specially designed thermal column, a cold reutron source, a split core, and is compatible with a cylindrical fast neutron converter assembly currently under development.

The plan view of the thermal column can be seen in figure 1 . In addition to the $\mathrm{D}_{2} \mathrm{O}$ reflector in the main reactor vessel, an additional 18" of $\mathrm{D}_{2} \mathrm{O}$ moderation is provided by a special $\mathrm{D}_{2} \mathrm{O}$ tank. Because of the increased moderating efficienty of $\mathrm{D}_{2} \mathrm{O}$ relative to graphite an unusually pure thermal neutron spectrum, is obtained.

The use of [1/20 at the core end of the thermal column also eliminates any radiation damage which might be experienced by graphite if it were used in this region. The $D_{2} O$ tank is followed by seven inches of bismuth to minimize core gamma rays. The final moderation takes place in the conventional graphite stringer configuration which allows access to substantial regions of the thermal column. To further facilitate the use of the thermal column, a boral curtain which may be raised or lowered is located between the graphite and the bismuth. The combined use of $\mathrm{D}_{2} \mathrm{O}$ and graphite results in good thermal neutron fluxes and unusually high cadmium ratios. The flux at the outer end of the thermal column is $8 \times 10^{9} \mathrm{n} / \mathrm{cm}^{2}-\mathrm{s}$ with a cadmium ratio of more than $3 \times 10^{4}$ and the flux increases to $5 \times 10^{11}$ at the inner end of the graphite with a cadmium ratio of $5 \times 10^{3}$.

A diagram of the cold source is shown in figure 2. The reactor vessel was especially constructed to provide a large volume close to the core for the cold source. The volume is sufficiently large to allow the 


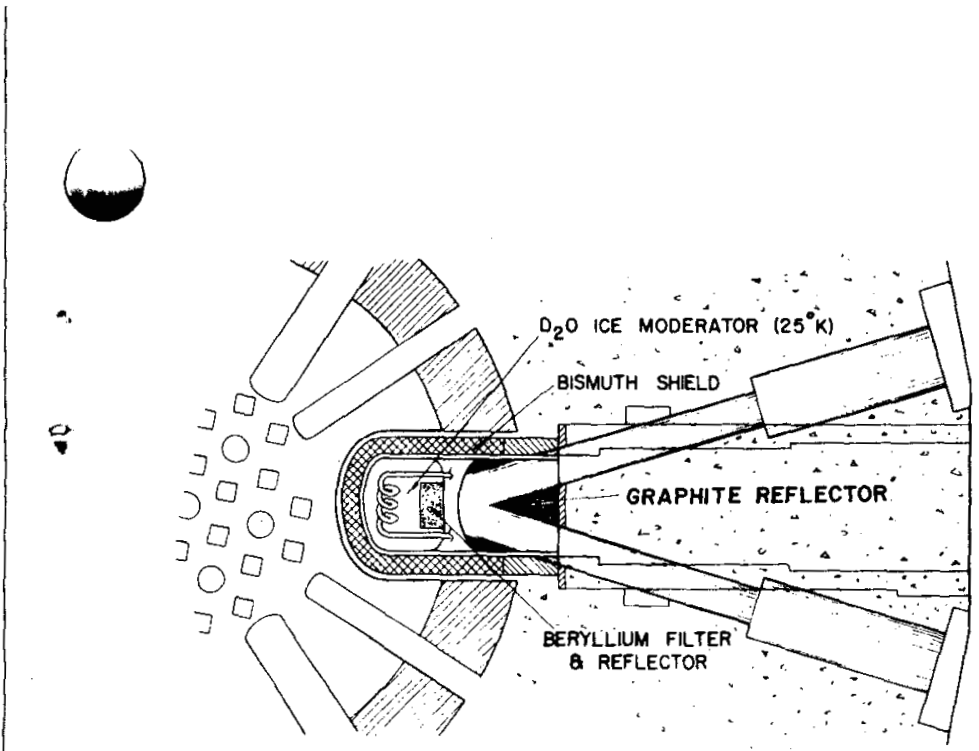

FIG. 2. COLD NEUTRON SOURCE

inches thick. It will be primarily $\mathrm{D}_{2} \mathrm{O}$ ice but the moderating capability will be increased somewhat by the addition of $\mathrm{H}_{2} \mathrm{O}$. A small percentage (about $5 \%$ ) of $\mathrm{H}_{2} \mathrm{O}$ will provide increased moderation not only because of neutron collisions with hydrogen but also by increasing the number of collisions with deuterium. The neutron absorption by this amount of hydrogen will be less than the neutron loss from neutron leakage from the moderator and so will not represent a significant increase in the overall neutron loss rate.

Experimental numbers on the effectiveness of the system are not yet available since the cryostat has not been installed. From an earlier experiment on $\mathrm{D}_{2} \mathrm{O}$ ice as a cold moderator ${ }^{*}$, an improvement factor of five to ten is estimated for this system.

use of $\mathrm{D}_{2} \mathrm{O}$ ice as the moderator. Due to the large volume of moderator, it is necessary to shield it from core and capture gamma rays fxom the surrounding structure. For this purpose, the moderator is surrounded by a bismuth shield. The shield thickness was chosen to reduce the gamma radiation to a value below that generated by neutron capture in the moderator and its cryostat. Although many kilowatts of heat are generated in the bismuth shield only about 300 watts will be generated in the source itself.

The shield is supported by a square * plug equipped with rollers. A smaller square plug rolls within the larger one and supports the cryostat containing the moderator. Two beam holes originating at the approximate center of the moderator penetrate the double plug system and the reactor shield. In addition, two smaller holes penetrate the inner plug for possible neutron guide tube use.

The moderator is vacuum insulated by an all aluminum cryostat to minimize thermal neutron capture. It is cooled to $25 \mathrm{~K}$ by helium gas circulating through cooling coils in the ice. A re-entrant hole is provided in the ice filled with a beryllium block which will pass neutrons with wavelength greater than $4 \mathrm{~A}$ but reflect shorter wavelength neutrons back into the moderator for additional moderation. The region beyond the vacuum space immediately outside the re-entrant hole is filled with graphite (except where the beam holes pass through) to enhance the neutron flux through the region of the moderator. The moderator itself is about $14^{\prime \prime}$ in diameter and twelve

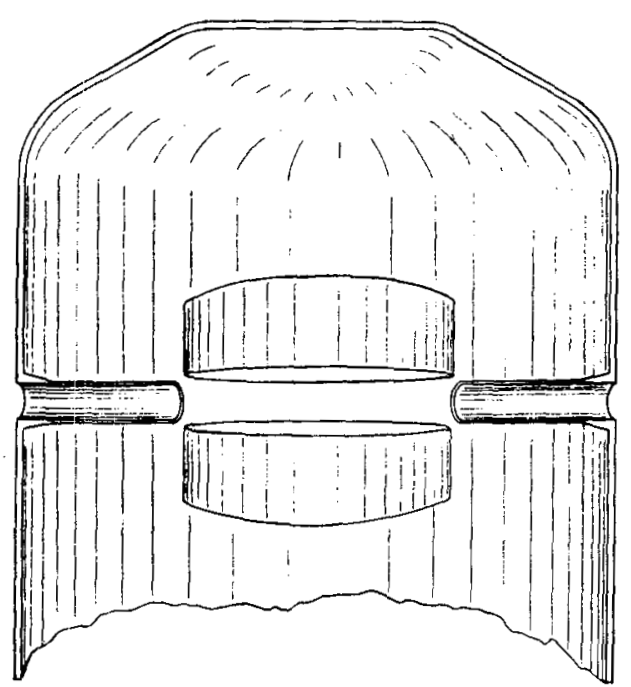

FIG. 3 SPLIT CORE

Another special feature of the NBSR is its split core illustrated in figure 3 . In order to minimize the flux of very fast neutrons in the beams, each fuel element is constructed so that there is no fuel in its center section. Thus, the core consists of two cylindrical fueled sections separated by non-fueled or gap region. The fuel sections are each ll" high and the gap is 7" high. As shown in the figure, the radial beams are located opposite the gap so no fission neutrons can get into the beams

*J.J. Rush, D. W. Connor and R. S. Carter. "Study of $\mathrm{D}_{2} \mathrm{O}$ Ice as a Cold-Neutron Source." Nuclear Science and Engineering 25, 383 (1966). 
directly. They must scatter at least once to get into the beam. It is estimated that this configuration reduces the number of very fast neutrons ( $E>2 \mathrm{Mev}$ ) in the beam by a factor of five to ten. Because of the long diffusion length of thermal neutrons in $\mathrm{D}_{2} \mathrm{O}$, the gap has no complicating effect on reactor control. This configuration has the same effect as beams tangential to the core without the geometrical complications. Furthermore, the core is easily changed to a more conventional type by simple changing fuel elements.

The final special feature is still under development. It is a cylindrical $235_{\mathrm{U}}$ converter thimble that can be placed in any one of the 3-1/2" experimental positions. It will consist of three concentric ${ }^{235} \mathrm{U}$ cylinders with cooling channels between. It will be cooled just like a fuel element and will generate about the same amount of power. Inside it will be lined with cadmium or some other thermal neutron absorbing material to create a thermal-neutron-free, fast-flux environment in a 2 " diameter void region in the center. The converter tube, although containing much more fuel than a normal element is expected to have only a very small effect on the reactivity of the core, and its lifetime should be about one year. This system will provide a region in the reactor which has a fast flux intensity and spectrum comparable to that found in testing reactors although the spectrum elsewhere in the NBSR is lower in energy that one would like for radiation effects studies. Calculations indicate that the flux of neutrons with energy greater than I Mev is about $3 \times 10^{13} \mathrm{n} / \mathrm{cm}^{2}-\mathrm{s}$ and for energies greater than $.5 \mathrm{Mev}$ it is $7 \times 10^{13}$ $\mathrm{n} / \mathrm{cm}^{2}-\mathrm{s}$. Furthermore, the absence of thermal neutrons in the region will minimize activation of the sample and greatly reduce its reactivity efect. Thus, this system will convert a sriall region of a well thermalized $D_{2} O$ reactor into a radiation environment comparable to that found in testing reactors!

\section{SUMMARY |OF FLUX INTENSITIES \\ The various features of the NBSR have} been described above. The radiation data associated with each are summarized in table I

Reactor Characteristics

\begin{tabular}{clc}
$\begin{array}{c}\text { Thermal } \\
\text { Flux } \\
\mathrm{n} / \mathrm{cm}^{2}-\mathrm{s}\end{array}$ & $\begin{array}{l}\text { Gold Foil } \\
\text { Cd Ratio }\end{array}$ & $\begin{array}{c}\text { Gamma } \\
\text { Heating } \\
\mathrm{w} / \mathrm{g}\end{array}$ \\
\hline
\end{tabular}

Radial Beam Tubes Source

$1 \times 10^{14}$

Edge of shield

$1.6 \times 10^{10}$

6.5

Through Beam Tubes

Center of tube

$6 \times 10^{13} \quad 50^{+}$

.3

In-Core Thimbles

Central 3-1/2" thimbles

Outer 3-1/2" thimbles

$1.4 \times 10^{14 *}$

$1.0 \times 10^{14}$

$1 \times 10^{14 *}$

7

7

$3 \times 10^{13 *}$

5 *

$1.5 *$

2 " thimbles

Reflector Thimbles

$8 \times 10^{9}$

Outer colum

Inner end

$5 \times 10^{11}$

$$
\begin{array}{r}
>3 \times 10^{4} \\
5 \times 10^{3}
\end{array}
$$

negligible

Pneumatic Tubes

$\mathrm{RT}-1$
$\mathrm{RT}-2$
$\mathrm{RT}-3$
$\mathrm{RT}-4$

$7 \times 10^{13}$
$7 \times 10^{13}$
$6 \times 10^{13}$

$1.4 \times 10^{13}$

$\begin{array}{rr}9 & .7 \\ 4 & 2.5 \\ 13 & .4 \\ 100 & .1\end{array}$
+Measured in an extracted beam using a black-to-thermal neutrons $\mathrm{BF}_{3}$ detector.
*Estimated. 
The thermal neutron flux values and the gold foil cadmium ratios given in table $I$ were determined experimentally except where noted otherwise. The gamma ray heating rates are estimates based on calculations and operating experience.

The high neutron fluxes achievable at the outside face of the reactor shielding relative to the internal source strengths are due to the large diameter of the beam holes and the minimum distance from the source to the reactor face. This was achieved with some loss in shielding effectiveness in the immediate vicinity of a beam hole when its shutter is up. Under these conditions, however, extensive external shielding is required to handle the beam itself so any leakage through the reactor biological shield is relatively insignificant.

The wide range of thermal fluxes and cadmium ratios available from the pneumatic tube system is evident from the table. This range will be significantly expanded in the future when pneumatic tubes in the thermal column and in an in-core thimble are added.

The table shows only the flux at the peak position in the vertical thimbles. A broader range of flux intensities and cadmium ratios, of course, can be obtained by moving the sample vertically along the axis of the thimble.

The table gives some indication of the versatility of the NBSR and the broad range of radiation environments attainable.

\section{EXPERIMENTAL FACILITIES}

The experimental facilities developed to utilize the reactor are as important as the reactor itself. Since the reactor runs continuously for several weeks at a time, it is important to have well instrumented equipment which can handle data continuously and automatically. The major experimental facilities that are of at least a semipermanent nature will be discussed briefly.

The reactor confinement building which houses the reactor and provides floor space for the experimental equipment is ninety feet on a side and contains two full floors above the basement level. The main floor which services the beam tubes is at ground level. A second full floor is flush with the top of the reactor and provides space for the reactor control room, access to the in-core facilities and ample space for auxiliary experimental equipment. This area is served by a 20 ton crane with a hook clearance of 34'. The main floor is service by an annular crane of 15 tons capacity with a 15' hook clearance. Only limited laboratory and office space is provided within the confinement building but adequate space is available for all the experiments.

The major facilities in operation as well as those under development are summarized in table II.

TABLE II

Experimental Facilities

Operational

1 3-axis neutron diffractometer

1 Single crystal neutron diffractometer

2 2-axis neutron diffractometers

I T-O-F neutron spectrometer

4 Pneumatic tubes

1 In-core irradiation facility

Under Development

1 Phased chopper $\mathrm{T}-\mathrm{O}-\mathrm{F}$ system

1 3-axis neutron diffractometer

1 Single crystal neutron diffractometer

1 Thermal column pneumatic tube

1 In-core pneumatic tube

1 In-core irradiation thimble

1 Parallel crystal neutron diffractometer

The four operational neutron diffractometers are controlled by a single live time, time shared computer system. Direct connections between the computer and each diffractometer provide for driving stepping motors to set the spectrometers and recording data. Communication between scientists and computer is provided at each station by individual teletypes which operate in a fully time shared fashion. The computer can perform a variety of sophisticated functions from receiving input from a light-pen to calculating all the reflections and diffractometer settings required to analyze a complex cirystal. One particularly advantageous feature of the computer is that all the users programs from diffractometer control instructions to data analysis can be written in FORTRAN. Although the diffractometers are all located on the ground floor, the computer itself is located on the floor above where ample space is available. The remote location causes very little 
inconvenience, because the teletypes at each diffractometer location provide direct access to the computer including all necessary input-output functions.

An example of the capability of this system is given by one of the programs written for single crystal refinement. Given the basic parameters of the crystal. the computer will direct the diffractometer to search for the exact settings of two reflections whose approximate settings had been indicated by the experimenter. Once these have been determined, the computer calculates all the settings at which reflections might be found and then proceeds to measure their intensity. At each setting it does a preliminary run over the peak to estimate its intensity. It then performs a simple statistical analysis to determine whether it is intense enough to study further and if so how much time it should spend collecting data on the peak. Thus not only is the system automatic but it also uses diffractometers efficiently.

The currently operating time-of-flight spectrometer is also controlled by the computer although the data is collected in an independent multi-channel analyzer. The spectrometer consists of a monochromating crystal before the sample and a neutron chopper and four meter flight path after the sample. The chopper, flight path, and detector bank are all mounted on a common frame. The frame is pivoted at the sample and mounted on air pads. At a signal from the computer, the air pads inflate and a driving wheel driven by a stepping motor can move the flight path to a new angle automatically. The location of the chopper after the sample provides the capability of high energy resolution work since the distance from sample to chopper has no effect on the energy resolution of the scattered neutrons. If desired, the flight path can be replaced with a diffraction arm supporting an analyzing crystal, detector and shielding to form a 3-axis spectrometer which is also run by the main computer.

The pneumatic tubes are quite straightforward and their flux characteristics were summarized in table 1 . Therefore they will not be discussed further here.

The only in-core facility fully developed so far consists of a thimble penetrating the top shield plug and located in one of the $3-1 / 2$ " in-core positions. $\mathrm{D}_{2} \mathrm{O}$ is circulated in the thimble to provide sample cooling. Samples are lowered into the thimble by nylor string or aluminum wire. Since the thimble is always full of water, adequate shielding is available to permit loading and unlcading samples even during reactor operation. A more sophisticated irradiation facility is under development.

Most of the facilities under development listed in table 2 are relatively straightforward but one, nearing completion, is of particular interest and will be described here. It is the phased chopper time-offlight system designed to use one of the neutron beams from the cold source. A schematic elevation of the system is shown in figure 4. The neutron beam from the source passes through a beryllium filter cooled with liquid nitrogen and then through the four rotor phased chopper system to the sample. The scattered neutrons travel a $240 \mathrm{~cm}$ flight path and are detected at eight angles by high pressure $\mathrm{BF}_{3}$ detectors. The detector data are then fed into a multi-channel analyzer.

The chopper system consists of two pairs of counter rotating rotors in two vacuum tanks located one meter apart. The first rotor set produce a burst of neutrons with the energy distribution of the incident beam, and the second set selects a narrow energy band from the burst. Thus a morochromatic pulse of neutrons is incident on the sample.

The rotors are two foot diameter solid aluminum discs coated with a thin layer of gadolinium which has been selectively removed to form slits along the periphery of the rotors. Each rotor has several slits so that incident energy resolution, final burst width and incident energy may all be varied simply by changing the phasing of the rotors. The rotor phasing is achieved by driving all rotors from a single stable oscillator. The signal is fed through separate channels containing amplifiers; vector resolvers are used to control the phase of each rotor. The phasing jitter of the system is less than two microseconds full width at half maximum. The burst time can be $6.6,13$ or 26 microseconds with an incident energy resolution of about $5 \%$. The energy resolution for scattered neutrons of $1.8 \mathrm{~A}$ is also about $5 \%$ for the shorter burst widths.

The detector system consists of 1 " diameter $\mathrm{BF}_{3}$ detectors filled to $2.23 \times 10^{5}$ $\mathrm{N} / \mathrm{m}^{2}(167 \mathrm{~cm}$ of $\mathrm{Hg})$. They are packaged in groups of five, and three packages are 


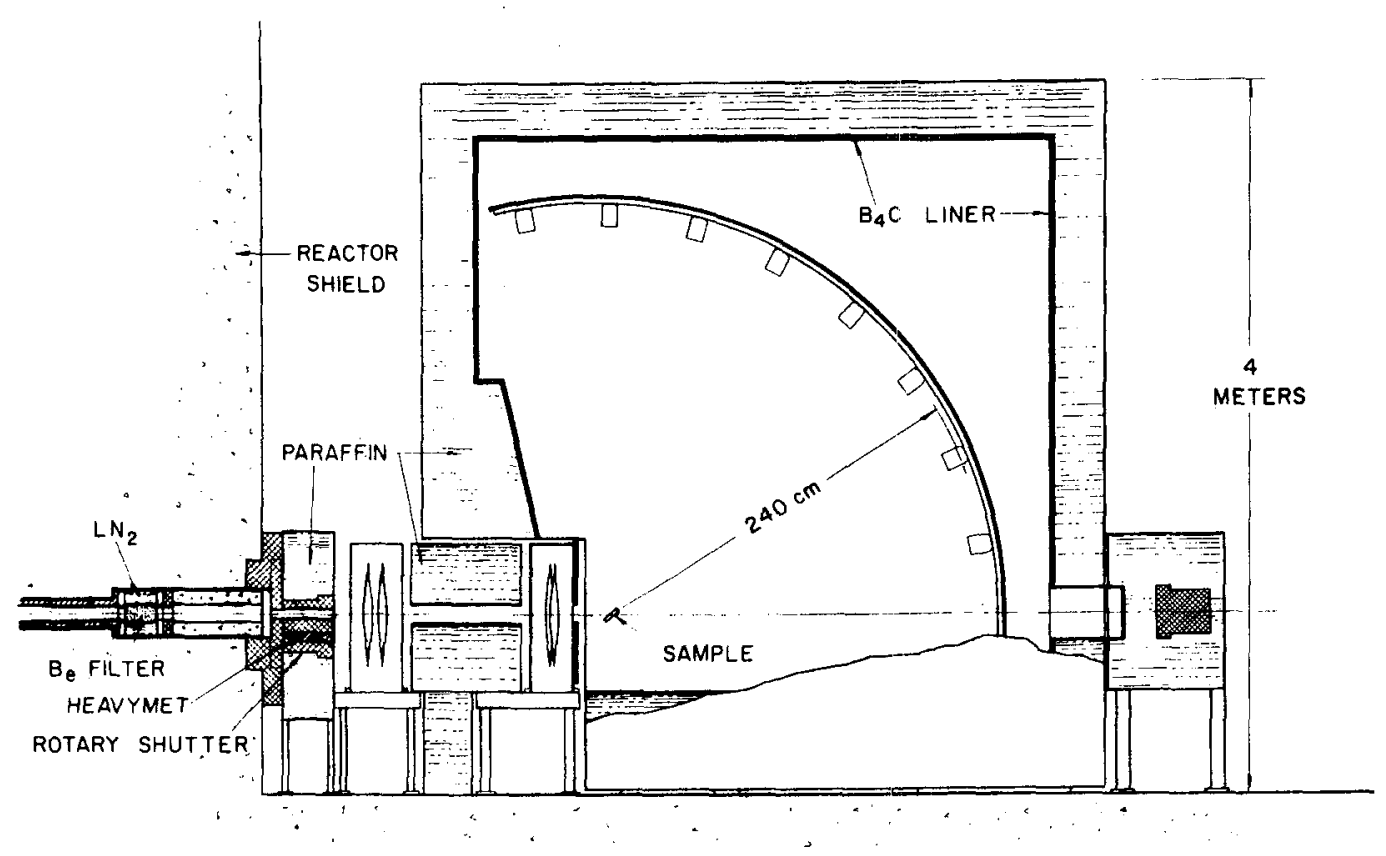

FIG. 4 PHASED CHOPPER T-O-F SYSTEM

placed along an arc about the incident beam direction at each of the eight angles. Since the active length of the detectors is 18 ", the total detector array is about $6^{\prime}$ wide by $8^{\prime}$ high. As shown in figure 4 , the sample and detector array are completely enclosed in a shielded room. The one foot thick walls are primaxily paraffin and the inside is lined with about one inch of boron carbide retained by a thin aluminum cover. With this shielding it is anticipated that most of the background will come from the incident and transmitted beams.

This system is nearing completion and should be in operation sometime in the summer of 1971.

of the other instruments being developed, two will be interfaced with the computer system already controlling the existing spectrometers. These are the single crystal and the parallel crystal neutron diffractometers. With the addition of these diffractometers, the computer will be controlling seven independent instruments.

These extensive experimental facilities were developed in cooperation with the Naval ordnance Laboratory, the Naval Research Laboratory, and Picatinny Arsenal.

SUMMARY

The NBSR is a high flux research reactor operating at $10 \mathrm{Mw}$. It has been in operation for about two years and is now almost fully utilized with only limited space left for new experimental facilities. The use of $\mathrm{D}_{2} \mathrm{O}$ as a moderator made it possible to provide adequate space within the core for experimental thimbles and at the same time maintain intense thermal beams for external experiments.. Unique features such as the split core and cold neutron facility further increase the flexibility and utility of the reactor. Thus the NBSR serves its purpose well as an intense source of neutrons for a broad range of experimental programs. 


\title{
A VERSATILE NUCLEAR REACTOR FACILITY
}

\author{
Aaron K. Furr \\ Department of Physics \\ Virginia Polytechnic Institute \\ and State University \\ Blacksburg, Virginia
}

\begin{abstract}
An unusually versatile nuclear reactor facility, has been planned for future construction at Virginia Polytechnic Institute and State University. The reactor design is basically a dual-core version of a TRIGA reactor in which one configuration is essentially a standard Mark III TRIGA with a full complement of beam ports and other irradiation facilities.

The other configuration is similar to the annular core TRIGA currently in existence at the Sandia Corporation, modified, however, to enhance the thermal component of the flux and with an evacuated central cavity. This configuration will permit an experiment of fundamental importance to nuclear physics to be carried out, viz. a direct measurement of the neutron-neutron scattering cross section.

The facility, as planned, will allow a much greater variety in the research programs than with more conventional reactors. With the interest of outside users apparent even before planning was very far advanced and with heavy utilization by the University staff, it is anticipated that the facility will be very heavily used.
\end{abstract}

\section{INTRODUCTION}

In 1969 , planning was begun on securing an unusually versatile nuclear reactor to be constructed at the Virginia Polytechnic Institute and State University in Blacksburg, Virginia. In addition to representatives of the University, scientists from several other major research institutions (Drs. C. D. Bowman and $W$. C. Dickinson from Lawrence Radiation Laboratory, Livermore; Dr. C. 0 . Muelhause, National Bureau of Standards; Dr. V. L. Sailor, Brookhaven National Laboratory and Dr. W. L. Whittemore, Gulf General Atomic, Inc.), participated in the planning for the facility. The interest of the latter group was specifically in a facility on which an experiment of fundamental importance to nuclear physics could be performed, a direct measurement of the neutron-neutron scattering length. However, the members of the $n-n$ group, as the five scientists from outside the University henceforth will be designated, recognized that a facility designed for only a single purpose would not offer as many advantages as one suitable for many programs with design features which would accommodate the $n-n$ experiment. The collaboration of the University with its broad and varied research programs and the $n-n$ group grew from a mutual recognition of the merits of such a combined facility.

The versatility of the reactor will be achieved through an unusual combination of previously emplciyed design features (see Fig.1). The unusual dual-core TRIGA configuration envisiored will provide a uniquely dense pulsed neutron gas over a large volume in a cavity within an annular core and a significant steady state power for high neutron densities and leakage fluxes in an efficient compact core configuration. A rapid repetitive pulse: mode at intermediate power levels also appears technically feasible and it is planned to develop this feature since it offers inherent advantages for beam scattering experimerts.

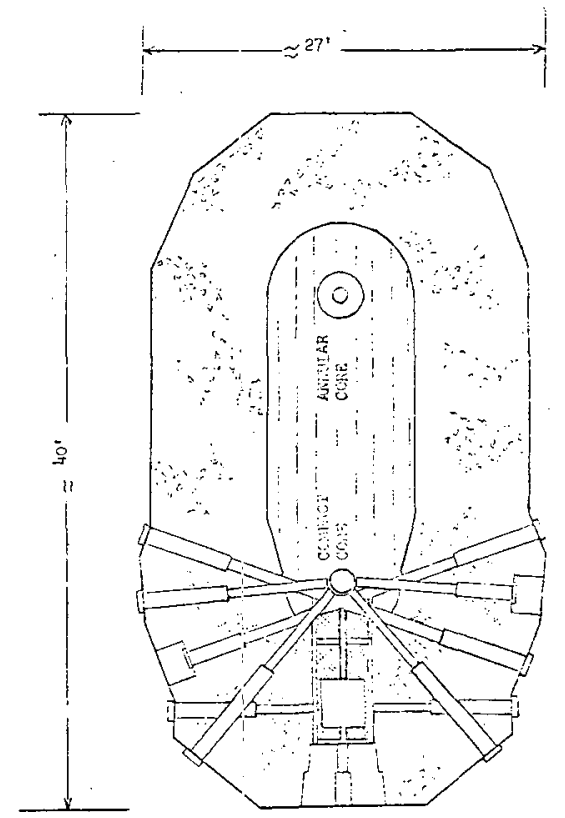


The planned facility would further enhance the already vigorous programs being carried out at the University using existing facilities and would permit exploitation of research areas where efforts are not now feastble. The unique combination of features in the proposed facility would certainly lead to programs which would take advantage of these features.

\section{THE REACTOR FACILITY}

As mentioned in the introduction, the reactor will be a multiple purpose device, designed to give good performance in steady state operation and unequaled performance in the pulsed mode. The basic concept envisions two core locations within a single pool, one for each mode of operation. Fuel elements would be available for making up either of the two configurations, but not both simultaneously. A simplified drawing of the pool design with the two core locations is shown in Fig. 1. Each of the two core configurations would be of a proven design. The "Compact Core" in the figure, with the beam port arrangement shown, is basically a "TRIGA" Mark III reactor while the "Annular Core" configuration is a straight forward adaptation of the "TRIGA" annular core pulse reactor (ACPR) at Sandia. The adaptation mentioned in the latter case requires design changes to maximize thermal neutrons rather than fast neutrons as was desired by the Sandia users and to provide the highly evacuated "through hole" needed for the $n-n$ experiment. Studies relating to the needed changes have been made (1).

An interesting mode of operation of the compact core has been suggested by Whittemore et.al. (2) which would permit repetitive pulsing at 50 to $60 \mathrm{cps}$ to power levels of 20-30 MW and it is possible that the annular core configuration might be operated in a similar manner. The average power would be much lower, however, than these peak powers. Synchronization of a Fermi chopper with this pulse rate would improve the capability over the reactor used in a steady state operation by an order of magnitude for solid state physics and other areas where chopper operation is useful. This would be due, in part, to the higher effective power and, in part, to the lower background obtaining between the pulses. This mode of operation has, as yet, not been licensed for use in any facility, but because of its obvious value, a program to incorporate it in the proposed reactor will be initiated as part of the research program of the facility.
The compact core, as shown in the figure, includes both radial and tangential beam ports. The latter ports are especially suitable for experiments which require a high leakage flux with a low fission neutron component. Now shown in the figure are two planned rabbit systems, one to be used for routine irradiations and a second high speed system which will operate on a millisecond time scale. With this type of beam port configuration, a planned "lazy susan" for sample irradiation can still be used but it will have to be in a slightly lower than normal flux region for a TRIGA reactor. However, the standard TRIGA, Mark III includes a movable bridge so that the compact core can be moved away from the beam ports which would, if necessary, permit isotope irradiations in the "lazy susan" at a slightly higher flux level. The power at which the compact core configuration can be run according to the manufacturer with normal convective cooling is $2 \mathrm{MW}$. It is planned to incorporate a capped off flange for eventual forced cooling to permit operation at even higher powers if, as expected, the demand warrants this step.

The annular core configuration is basically a copy of the Sandia Corporation annular core, pulsed reactor, with two important modiflcations. The Sandia reactor has a stainless steel liner in the cavity within the core. This stainless steel will be replaced by one or two cms of water enclosed in aluminum. This change markedly enhances the thermal component of the flux. The nine inch cavity is not evacuated in the Sandia design. and this will be necessary here in order to reduce neutron scattering by air molecules for the $n-n$ experiment. The through pipe design will extend above and below the center Iine of the reactor for about fifty feet to allow the detector for the scattered neutrons to be placed in a position where no noutrons emanating directly from the core or scattered from structural components can reach it. A simplified drawing showing the annular core configuration for the $n-n$ experiment is shown in Fig. 2 .

Although the annular core configuration is being optimized for the $n-n$ experiment, the very high flux density $\left(\simeq 10^{17} \mathrm{n} / \mathrm{cm}^{2}-\mathrm{sec}\right)$ over the large volume encompassed by the $9^{\prime \prime}$ hole makes possible other experiments which require-large neutron fluxes in similar volumes. For example, the production of free radicals in large quantities or materials testing of structural components can be carried out in this volume. Also, one possible 
way of achieving the rapid pulse mode described earlier in this section can take advantage of the cavity in the core in the annular core configuration. Thus, since there are a number of potential uses for both the compact and annular core, it will be desirable if the transfer of fuel and control rods between the two configurations can be done easily and quickly. In order that even more versatility can be had, the annular core design would permit a modest level of steady state operation at about $300 \mathrm{~kW}$ and hence a standard rabbit and a vertical beam tube will be incorporated in its design. These facilities can also be used in the pulse mode which is the primary means of operation for the annular configuration.

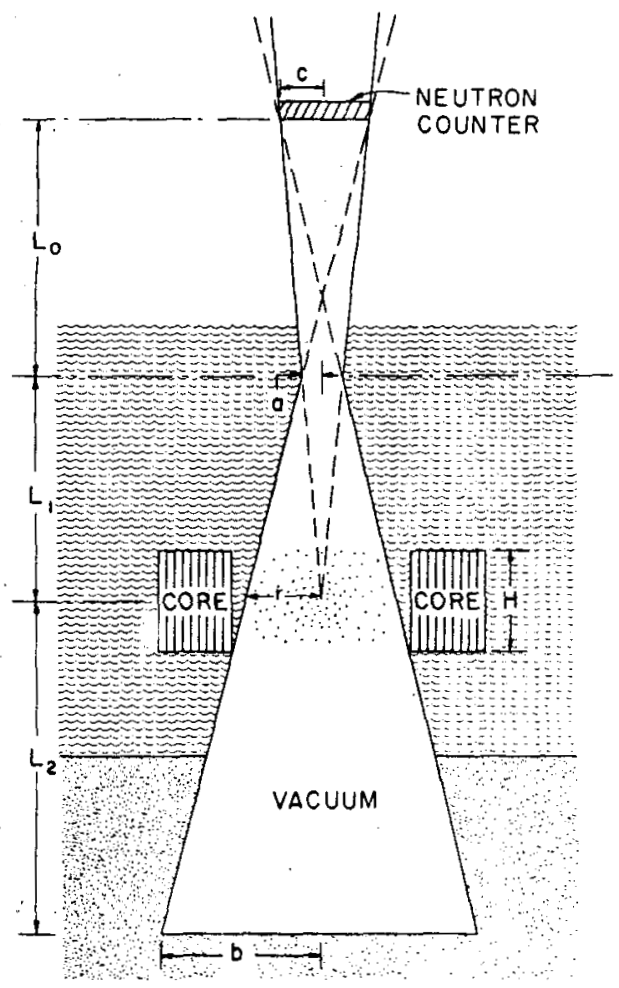

The reactor is to be housed in a separate building located at the northwest edge of the campus. The building program for areas near this site will insure a reasonable degree of isolation for some time in the future, yet the distance to departments normally expected to be users of the facility is not great. Due to the height represented by the vertical beam tube, the reactor bay will be tall which could be accommodated either in a tower, or if the site permits, below ground. Surrounding the reactor bay, adjacent to it, will be experimental 1aboratories, shops, and offices for the research and operating staff. The available floor area is planned to be approximately 20,000 square feet. This amount of space should be ample for the major users to have laboratory and office space in the building but is not intended to include any classrooms.

\section{RESEARCH PROGRAM}

The neutron-neutron scattering experiment represents an extremely significant use which will be possible with the reactor. Until now, direct measurements of neutronneutron scattering have not been practical because of the lack of adequate neutron source intensities. The only values for $a_{n n}$ have been inferced indirectly from various nuclear reactions which yield two neutrons (3), e.g., $D(n, p) 2 n, H^{3}\left(d, H e^{3}\right) 2 n$, $D\left(\pi^{-}, \gamma\right) 2 n$, etc. However, Van Oers and $I$. Slaus point out (4) that in these three particle final staie reactions, the effect of the third particle on the pair of neutrons must be removed if a scattering length is to be obtained, and that attempts to do this introduced uncertainties which are smallest except in the calse of the $D(\pi ; \gamma) 2 n$ experiment. An experiment of the latter type by

Haddock, et.a1. () gives

$$
a_{n n}=-(16.4 \pm 1.9) \times 10^{-13} \mathrm{~cm}
$$

where the quoted uncertainty represents only experimental error (this value may be revised to 18.4 with about the same error). Heller et.al. (6) calculated the range of values $-16.6<a_{n n}<-16.9 \times 10^{-13}$ by fitting various $p-p$ potential models to $p-p$ data and then "turning of $f$ " the coulomb potential to obtain $a_{n n}$. Thus, while the prediction for the scattering length from pp data, assuming charge symmetry, appears to be accurate to $\pm 1 \%$, the error in the measured value of $a_{n i}$ is at least $\pm 12 \%$. A direct measurement of $a_{n n}$, if the accuracy could be of the irder of $2 \%$, would provide a more satisfactory test of charge symmetry. 
The presently planned program for the facility includes, in addition to the neutron-neutron scattering experiment, measurements of neutron capture cross-sections by a ${ }^{10}$ B filter technique, studies of shortlived radioisotopes, preparation of Mossbauer sources and activation of solids as substrates for sputtering experiments. Reactor experiments include studles of reactor kinetics, transient boiling measurements and the previously mentioned investigation of the rapid pulse mode of operation of the reactor.

The availability of the reactor for preparation of short lived radioisotopes for use in the many programs in the life sciences will be valuable and will make possible research programs not now possible. In addition, neutron activation analysis applications in the life sciences are very numerous.

Since the originally planned program of research was formulated, a very rapid rise in interest in environmental studies has developed throughout the country. Accompanying this rise has been a correspondingly rapid rise in needs for analytical techniques for such environmentally related areas as pollution studies and quality control. Neutron activation analysis has proven to be an exceptionally powerful and competitive tool for work in this area. For example, in the recently highly publicized mercury problem, neutron activation analysis has proven to be perhaps the most selective and trouble-free method available for detection of this hazardous element. It is probable, based on extrapolation of the present demand with the existing V.P.I. reactor, that environmentally related work will occupy much of the operating time on the facility.

\section{SUMMARY}

Multiple use reactors such as the proposed Virginia Tech facility would appear to be the most appropriate reactor type for future construction. In the present financial climate, reactor facilities should not be oriented too completely toward traditional nuclear science and engineering areas and basic nuclear physics. On the other hand, preparation of radioactive sources and neutron activation analysis certainly would not represent complete utilization of the facility. The dual-core multiple purpose reactor described here thus represents an attempt to design the most efficient system which would permit the widest possible range of programs.

\section{REFERENCES}

1. G. B. West. "Study of the Adaptation of the Annular Core Pulsing Reactor for a Neutron-Neutron Scattering Experiment." GA-8:310 (1967).

2. W. L. Whittemore and G. B. West. "A Multiple Pulsed TRIGA-Type Reactor for Neuticon Beam Research." Seminar on Intense Neutron Sources, Santa Fe, New Mexico (1966).

3. Ivo Slaus, Rev. Mod. Phys. 39, 575 (1967).

4. W. T, H. van Ders and I. Slaus, Phys. Rev. 160,853 (1967).

5. R. P "Haddock, R. M. Salter, Jr., M. Zellex, J. B. Czirr, and D. R. Nygren. Phys. Rev. Ltrs. 14, 318 (1965).

6. L. Heller, P. Signell and N. R. Yoder. Phys. Rev. Ltrs. 13, 977 (1964). 


\title{
THE CONTROL OF NEUTRON BEAMS BY VIBRATING CRYSTALS
}

\author{
Alan M. Jacobs \\ Edward S. Kenney \\ Jeffrey D. E. Jeffries
}

Nuclear Engineering Department

Pennsylvania State University

University Park, Pennsylvania

\begin{abstract}
The preponderance of work on neutron diffraction has been perpetuated in the investigation of crystal structure. Recently (1965), it was suggested that diffracted neutron beams could be modulated in intensity if the diffracting crystal was mechanically vibrated. This modulation was subsequently observed along with a rather large increase in mean intensity (up to a factor of 60). This paper reviews the historical development of measurements and interpretations of the vibration-induced phenomena. An analysis, described in detail, is along conventional crystallographic lines with emphasis on the neutron effects of interest. Neutron diffraction enhancement and modulation is traced to the effects of location-dependent mechanical strain on the ranges of neutron energy and direction which satisfy the Bragg condition. Conjectures are made regarding further investigations and applications of neutron diffraction by vibrating crystals. These include areas of thermal neutron beam intensity enhancement, containment, focusing, and intensity modulation.
\end{abstract}

\section{INTRODUCTION}

About five years ago a conjecture was made regarding the expected intensity modulation of neutrons diffracted by a mechanically vibrated crystal. Since then, several investigators have studied neutron diffraction by quartz piezoelectric resonators vibrated in longitudinal wave modes. They have, indeed, found the predicted intensity modulation and, in addition, have reported mean intensity enhancements up to a factor of 60 .

A recent analytical study, based on a rather simplified model of the relevant physical phenomena, has successfully explained most of the details of reported measurements. Moreover, it appears that diffracted neutron intensities achieved to date are limited only by the magnitude of the crystal strain employed in the resonator. If this analys is is correct, then much greater enhancements can be realized using appropriate crystals which can withstand far greater strain than quartz.

Extraordinary intensification and versatile modulation of diffracted neutrons impiy the possibility of achieving control, in time and location, of thermal neutron beams of high intensity and low epithermal neutron and gamma ray contamination. This potential is manifestly intriguing.

The objectives of this paper are to initially present a historical record of the measurements of neutron diffraction by quartz piezoelectric resonators. Secondly to outline the relevant analytical considerations of the phenomena. Thirdly - to suggest further research on the diffraction of neutrons by vibrating crystals. And, finally to conjecture on various applications related to the control and manipulation of thermal neutron beams.

\section{HISTORICAL DEVELOPMENT AND MEASUREMENTS}

The first rieported efforts on the consideration of diffracted neutron beam modulation and intensification are those of Petrzilka $(1966 \mathrm{~b})$. He notes that, at the 1965 Internationial Conference on the Properties of Piezoelectric Crystals held at Liberec, it was reported that the wave amplitude associated with neutrons reflected from the lattice of a piezoelectric resonator corresponds to the amplitude of a frequency modulated wave. In later work $(1968 \mathrm{~b}, c)$, his analysis of $\mid$ this phenomenon was, indeed, along the lines of the classical radio engineering approach to the frequency modulation of radio signals. He developed a direct analogy between the Fourier spectra of the radio frequency field and the diffracted neutron field - the generated discrete spectrum of neutron energies corresponds to the side bands obseirved in radio frequency modulation. For expected vibration amplitudes, Petrzilka finds/as many as 10 pairs of side band energies which lie very close to the nominal neutron energy - roughly one part in 
$10^{7}$ displacement in frequency space. Petrzilka's arguements were addressed directly to a prediction of the modulation characteristics of the diffracted neutron beam. Mean intensity enhancement is, however, implicit in the results.

Parkinson and Moyer (1966 a) reported observation of the diffracted neutron beam modulation and mean intensity enhancement using a $2 \times 2 \times 0.25$ inch $x$-cut quartz crystal which was vibrated in the longitudinal mode at the fundamental resonance frequency of $0.5 \mathrm{MHz}$. The crystallographic $x$-axis was normal to the large surface of the crystal. Bragg reflection was accomplished from the large surface by an unspecified set of crystal planes. The incident neutron beam was collimated to an angular divergence of 13 minutes and a rather long $\mathrm{BF}_{3}$ counting tube served as the detector. Parkinson and Moyer reported:

1. A diffracted neutron beam intensity modulation at a frequency of 0.5 $\mathrm{MHz}$.

2. A mean intensity enhancement of about a factor of 2 .

3. No change in the rocking curve width with crystal vibration.

In 1ater work (1967 b), Moyer and Parkinson reported the results of a refined measurement. The diffracted neutron beam modulation was reported at a frequency of 1 $\mathrm{MHz}$ (i.e., at twice the resonator frequency), and mean intensity enhancements up to a factor of 5 were achieved. Moyer and Parkinson offer several explanations for the observed phenomena. They seem to favor the reasoning of Fox and Cork (1931) in discussing related work using $x$-rays - i.e., that cyclic lattice spacing variation reduces the effect of extinction.

Klein, et al (1967 a), reported on their studies of the diffraction of a monochromated neutron beam ( $1.09 \pm .01 \mathrm{~A}$, $1.5^{\circ}$ divergence) by slender, bar-shaped quartz crystals vibrating in the fundamental longitudinal mode. The crystal was $0.2 \times$ $0.84 \times 6.5$ centimeters in size and $-18^{\circ}$ $x$-cut. The $x$-axis was close to the long dimension direction of the crystal. The resonator frequency was $40 \mathrm{KHz}$ with a center node mounting configuration. Klein, et al, emphasized the observation of:
1. Modulation of the diffracted neutron beam intensity at twice the resonator frequency and with a shape proportional to the magnitude of an assumed harmonically varying (with location in the crystal) strain.

2. Substantial increases of the mean intensity of the diffracted beam up to 23 times that obtained with the quiescent crystal.

3. The appearance of reflections at $(0,0, h)$ reciprocal lattice points for $h \neq 3,6,9, \ldots .$. , which are space group forbidden.

4. No discernable modulation of the diffracted neutron beam when a long $\mathrm{BF}_{3}$ counting tube was employed as the detector. Modulation was observed using a thin L $i^{6} \mathrm{I}$ crystal detector in a scintillation counter.

Some of the results of Klein, et a1, are listed in Table 1. The various crystal planes used for reflection are clearly specified, and both neutron and x-ray mean intensity enhancement is reported.

TABLE 1

INTENSTTY RATIOS FOR VIBRATING AND STATIC QUARTZ BAR FOR SEVERAL REFLECTIONS

$\begin{array}{ccccc}\text { Reflection } & \begin{array}{c}\text { Intensity } \\ \text { Ratio } \\ \text { (neutrons) }\end{array} & \begin{array}{c}\text { Intensity } \\ \text { Ratio } \\ \text { (x-rays) }\end{array} \\ 1 & 0 & 0 & 10.9 & 1.1 \\ 2 & 0 & 0 & 9.8 & 1.2 \\ 3 & 0 & 0 & 14.7 & 1.7 \\ 1 & 1 & 0 & 12.1 & 1.6 \\ 2 & 2 & 0 & 16.5 & 1.8 \\ 2 & 1 & 0 & - & 4.6 \\ 0 & 1 & 2 & 23.6 & -\end{array}$

Klein, et al, attributed the increase in diffracted neutron intensity to a widening of the ranges of neutron wave length and incident angle which satisfy the Bragg condition when the crystal is vibrated. They qualitatively identified the expected harmonically distributed strain in the crystal as being responsible for the increased acceptance ranges.

In a short note (1968 a), Chalupa, et al, described measurements using thin 
crystal plates. Two crystal plates were used $-30 \times 30 \times 2.84 \mathrm{mil1}$ imeters and $30 \times$ $30 \times 5.68$ millimeters. The large surfaces of the plates; which were perpendicular to the $x$-axis, were polished and coated with silver by vacuum evaporation. Bragg reflection from the $(1,1,0)$ planes was employed and monochromated neutrons of wavelength $1.54 \mathrm{~A}$ and beam divergence of $15 \mathrm{~min}-$ utes were incident. Chalupa, et al, observed:

1. An intensification of the diffracted neutron beam up to a factor of 40. This high value was attributed to the highly perfect nature of the crystals used - i.e., the very low value of the quiescent crystal reflectivity.

2. Evidence of structure and possible saturation at high crystal drive currents.

Chalupa, et al (1969 a), reported on further efforts using a $30 \times 30 \times 5$ millimeter quartz crystal plate with a longitudinal mode resonance frequency of about 0.5 MHz. Diffraction conditions employed were as in their earlier work, however; the 'detailed effects of the mode of electrical excitation were investigated. A significant dependence of intensification on the excitation frequency was found - as little as 3 parts in 500 being sufficient to yield a factor of 2 in diffracted intensity. Parallel resonance produced a factor of about 3 increase in intensity over series resonance. A maximum diffracted neutron beam enhancement of a factor of 60 was obtained when compared to the quiescent crystal, and it was noted that saturation of the intensification was not achieved.

In another short note $(1969 \mathrm{e})$, Michalec, et al, investigated the modulation phenomenon. A monochromated neutron beam $(1.54 \mathrm{~A}, 15$ minutes) was incident upon the $(2,0,0)$ planes of two quartz crystal bars one natural and the other synthetic. The natural quartz bar had dimensions $2 \times 12.5 \times$ 70 millimeters and the synthetic bar was 2.5 $\times 16.5 \times 90$ mi11imeters. Longitudinal mode vibration resonances of $39.105 \mathrm{KHz}$ and $30.385 \mathrm{KHz}$, respectively, were achieved. Excitation current for both crystals was set at 3.6 milliamperes. The observations were completely consistent with those of Klein, et a1. In addition, Michalec, et al, observed an apparent dependence of the diffracted intensity on the direction of motion of the crystal lattice flanes. They suggested that a Doppler effect, similar to that found in the use of rotating crystals, offered a reasonable explanation.

Michalec, et al (1969 d), reported on work involving pu'lsed excitation of a quartz crystal plate $30 \times 30 \times 2.84$ millimeters with the $x$-axis perpendicular to the large surface. Diffraction of $1.54 \mathrm{~A}$ monochromated neutrons was achileved from the $(1,1,0)$ planes and detection was by means of a $\mathrm{BF}_{3}$ counter. The pulse excitation was repeated every $10 \mathrm{mi} 11$ iseconds for durations of 500 , 1000,2000 , and 4000 microseconds. During the on-time, the excitation oscillator produced a frequency of about $1 \mathrm{MHz}$ - the series resonance frequency for longitudinal mode vibrations of the crystal plate. The diffracted neutron beam intensity increased during the on-time of the excitation pulse building towards saturation. When the excitation was switched off, the diffracted intensity reduced and approached that of the quiescent crystal. Michalec, et al, conjectured on the use of pulse excited piezoelectric resonators for neutron beam modulation in neutron spectrometry and other applications.

Suggesting the application of vibrationinduced diffracted neutron beam intensification as a measure of the quality of quartz crystals, Zelenka, et al (1969 f), reported on the use of a mionochromated neutron beam (1.54A, 15 minutes) for diffraction measurements employing three natural and three synthetic crystal bars - all with dimensions 1.90 millimeters in the $x$ direction, 76.99 millimeters in the $y$-direction, and 14.04 millimeters in the $z-$ direction. Diffraction from the $(2,0,0)$ crystal planes with symmetrical transmission was studied. The crystal bars were excited in series resonance with a fundamental longitudinal mode frequency of $34.8 \mathrm{kHz}$. Agreement with earlier efforts was obtained i.e., the more pe'rfect crystals (natural) exhibited a greater diffractivity increase than the less perfect crystals (synthetic).

Englehart and Jacobs $(1969 \mathrm{c})$, reported on measurements u'sing a $2 \times 2 \times 0.22$ inch $x$-cut quartz crystal plate with a longitudinal mode resonance frequency of $0.5 \mathrm{MHz}$. Bragg reflection was utilized from the large surface perpendicular to the $x$-axis. Detection of the diffriacted neutrons was by means of a $L i^{6}$ loaded filat $\mathrm{ZnS}$ crystal mounted on a photomultiplier scintillation counter. The ZnS crystal was positioned to be parallel to 
the diffracted neutron wave front... The diffracted neutron energy spectrum was investigated using time-of-flight techniques and a Fermi chopper in the incident neutron beam. Englehart and Jacobs observed:

1. A mean intensity enhancement up to about a factor of 6 .

2. No discernable modulation in the intensity of the diffracted beam. Estimation of necessary conditions for observable modulation were developed (see equation (22) of the next section).

3. No discernable change in the spectrum of diffracted neutrons with crystal vibration.

In a short note, Englehart and Jacobs (1971), and in detai1, Englehart $(1969 \mathrm{~b})$, considered an analytical formulation of neutron diffraction by vibrating crystals which is in qualitative agreement with all the reported measurements and is in excellent quantitative agreement with those measurements for which numerical results were calculated (an outline of the analysis is included in the next section).

Summarizing the reported efforts on the measurement of neutron diffraction by vibrating crystals to date, it is clear that:

1. Only quartz piezoelectrically driven resonators have been investigated.

2. When diffracted beam intensity modulation is observed (i.e., with thin crystals and detectors), it is at twice the resonator frequency.

3. Mean intensity enhancements up to a factor of 60 are possible - varying directly as the crystal strain magnitude and perfection of the crystal mosaic structure. In Figure 1 is illustrated typical results found for mean intensity enhancements as a function of crystal drive current.

4. The shape of the modulated beam intensity is not a simple harmonic function, but rather has the form depicted in Figure 2.

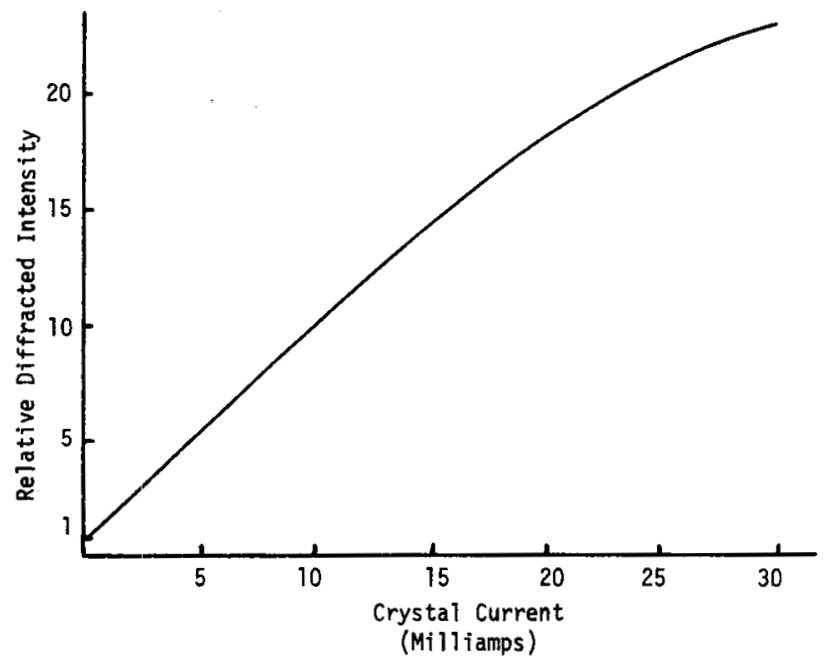

FIG. 1. ILLUSTRATIVE CURVE OF RELATIVE DIFFRACTED INTENSITY AS A FUNCTION OF CRYSTAL EXCITATION.

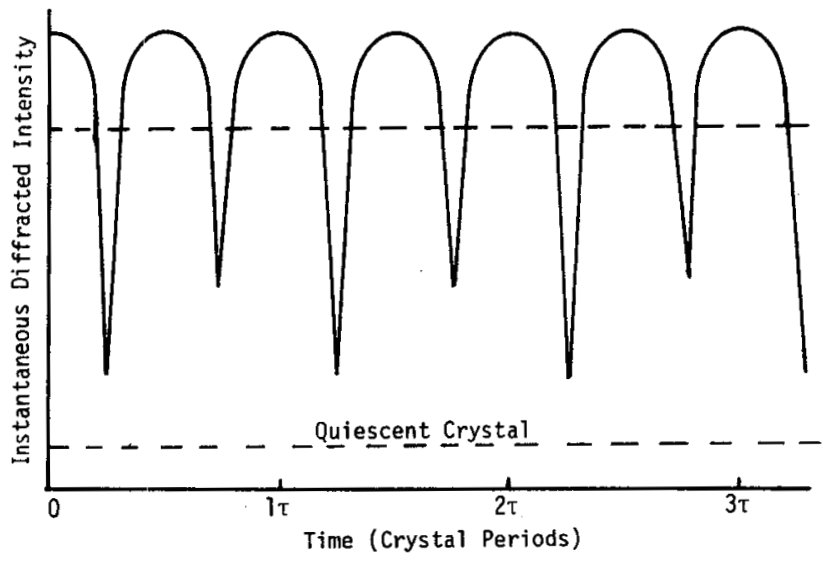

FIG. 2. ILLUSTRATIVE MODULATION CURVE FOR A VIBRATING QUARTZ CRYSTAL.

\section{REVIEW OF ANALYTICAL CONSIDERATIONS}

Several of the previously referenced investigators have commented on possible explanations of the enhancement and modulation of the diffracted neutron intensity when a crystal is mechanically vibrated. Detailed analyses of the phenomena are limited to the works of Petrzilka $(1968 \mathrm{~b}, \mathrm{c})$ and Englehart $(1969 \mathrm{~b})$. However, similar detailed considerations for the case of $x$-ray diffraction are in existence - e.g., the work of Weigle and Bleuler (1942). 
For the case of neutrons, it appears that there are at least three physical phenomena which should be considered. They are:

1. The modification of the diffracted intensity due to the mechanical strain produced by vibration. Specifically, the influence on scattering coherence of the location and time-dependent changes in the crystal lattice structure. This phenomenon is important in a perfect crystal as well as in the ideally imperfect specimens employed in the experimental efforts.

2. The change in the secondary extinction effect due to the location and time-dependent mechanical strain produced by vibration. This modification is possibly important in the reported measurements.

3. The modification in diffracted intensity and energy-directiondependence due to changes in inelastic scattering (neutronphonon energy exchange) resulting from crystal vibration. This effect is probably small but could lead to interesting possibilities as discussed later relative to applications.

Both Petrzilka and Englehart considered only the first of these phenomena. However, the methods employed to analytically express the vibration-diffraction interaction are not the same. The special case, considered in both works, is that of a piezoelectric resonator vibrating in a longitudinal (compression) mode with direction perpendicular to the crystal planes responsible for the observed diffracted neutron beam. The neutron wave elastically scattered from the quiescent crystal can be expressed as

$\Psi(\underline{r}, t)=\frac{A}{r} \exp (i \omega t-i k r) \sum_{n} \exp \left(-i \underline{k} \cdot e_{n}\right)$

where $A$ is proportional to the crystal lattice cell structure factor, $\rho_{n}$ is the location of the lattice cell indeled by $n, w$ is the neutron angular frequency, $k=k-k_{0}$, $k$ is the scattered wave vector $\bar{a} d \bar{k}_{0}$ is the incident neutron beam wave vector.

Petrzilka did not explicitly describe his assumptions regarding the crystal vibration with the exception that he did clearly express a periodic/displacement of crystal lattice plane separiation, $d$, of the form

$$
d=d_{0}(1+q \cos \Omega t)
$$

where $d_{0}$ is the latice plane separation of the quiescent crystal, $q$ is the vibration amplitude, and $\Omega$ is the angular frequency of vibration. Using the Bragg diffraction condition which rejates neutron wave length to diffraction angle, Petrzilka employed equation (2) to obtain the approximation

$$
\omega_{n} \simeq \omega_{0}-2 q \omega_{i} \cos \Omega t
$$

where $\omega_{0}$ is the neutron nominal angular frequency diffracted by the quiescent crystal, and $\omega_{n}$ is apparently the neutron angular frequency diffracted at time $t$. Without explanation, equation (3) was employed to modify equation (1) by identifying

$$
\omega=\int_{0}^{t} \omega_{n} d t
$$

The mathematical analogy between these calculations and those of frequency modulation in the field of radio engineering is clear and was noted by Petrzilka. However, the physical meaning of equation (4) with reference to the present phenomena is not clear to the present authors. Moreover, the displacement described by equation (2) is in conflict with the usual representation of the fundamental longitudinal mode in a piezoelectric resonator as a standing wave with wavelength equal to twice the crystal thickness. It should be noted that although the physical meaning of equations (3) and (4) is uncertain, the application of the results by Petrzilka yielded a modulation and, possibly, an implicit enhancement of the diffracted neutron beam. Moreover, the modulation is not symmetrical which agreed with some of the measurements. The lack of clarity in specifying the physical assumptions and mathematical model leads to difficulty in gaining understanding of the phenomenon under study.

Englehart also commenced his development with equation (1) to which he added the following assumptions:

1. There is no variation in the amplitude of vibration over the crystal plate suriface.

2. There are no parasitic vibrations. 
3. Within the limits of the incident beam collimator, the neutrons are isotropic, and within the energy interval of interest there is no variation of neutron intensity with energy.

4. The detailed relative motion of the atoms of a crystal lattice cell is negligible and thus the structure factor is not modified.

5. The crystal is perfect and thus secondary extinction is not relevant. However, the interpretation of results does include the fact that the crystals used in the measurements are ideally imperfect. Because of this it is also not relevant to include primary extinction effects as would be experienced in an actual perfect crysta 1.

6. The crystal is considered static with an arbitrary amplitude of strain in calculating the general form of the scattered neutron intensity function, $|\Psi|^{2}$.

The validity of assumptions 1-3 can be controlled by effective design of the neutron collimators and the crystal holder. It would seem that assumption 4 is not strictly valid since crystal vibration appears to yield diffraction from crystal planes which, in the quiescent state, are forbidden.

Assumptions 5 and 6 generate a great simp 1 ification in mathematical analysis, but they are not necessary.

The physical basis for Englehart's analys is is clearly presented and it appears that phenomena not included are easily identified - e.g., modified secondary extinction and neutron-phonon interaction change. Moreover, his results lead directly to a simple illustrative interpretation via an Ewald sphere construction. The details of Englehart's arguements are herein briefly reviewed.

In contrast to Petrzilka's work, the crystal strain is generated by a distortion expressed as

$$
\xi_{n_{1}}=\delta \sin \left(\pi n_{1} a_{1} / D\right) \cos (\Omega t)
$$

where $\xi_{\text {as }}$ is the displacement from the quiescent position of the crystal plane indexed by $n_{1}\left(n_{1}=0\right.$ represents the crystal center plane), $a_{1}$ and $D$ are the respective crystal lattice dimension and thickness in the direction of longitudinal vibration, and $\delta$ is the amplitude of the crystal surface vibration. Equation (5) implies a crystal lattice plane separation distribution of the form

$$
d_{n_{1}}=d_{0}\left[1+q \cos \left(\pi n_{1} a_{1} / D\right) \cos (\Omega t)\right]
$$

where $d$ is the lattice plane separation at the plahe indexed by $n_{1}$ (cf. equation (2)).

Following Englehart's assumptions, it is clear that crystal vibration modifies only the term in equation (1) which describes the relative phase shift. It is thus convenient to rewrite equation (1) for the quiescent crystal in the form

$$
\begin{aligned}
& \psi(\underline{r}, t)=\frac{A}{r} \exp (i \omega t-i k r) \psi(\underline{\underline{k}}) \\
& \psi(\underline{\underline{k}})=\sum_{n} \exp \left(i \underline{\underline{k}} \cdot \underline{\rho}_{n}\right)
\end{aligned}
$$

and realize that only $\psi(\underline{K})$ is changed when the crystal is vibrated. The crystallographic base vectors $\left(\underline{a}_{1}, \underline{a}_{2}, \underline{a}_{3}\right)$ and the recipracal space base vectors $\left(\underline{b}_{1}, \underline{b}_{2}, \underline{b}_{3}\right)$, defined by $a_{i} \cdot \underline{b}_{i}=\delta_{j i}$, provide the usual bases for the expression of $g_{n}$ and $\underline{k}$ - i.e.,

$$
\begin{aligned}
& \underline{p}_{r_{1}}=n_{1} \underline{a}_{1}+n_{2} \underline{a}_{2}+n_{3} \underline{a}_{3} \\
& \underline{\kappa}=\kappa_{1} \underline{b}_{1}+\kappa_{2} \underline{b}_{2}+\kappa_{3} \underline{b}_{3}
\end{aligned}
$$

where $n_{1}, n_{2}$ and $n_{3}$ are integers. In these terms, $\psi(\underline{\underline{k}})$ is expressed as

$$
\begin{aligned}
& \psi(\underline{\underline{K}})=\sum_{n_{1}} \exp \left(-i n_{1} \kappa_{1}\right) \sum_{n_{2}} \exp \left(-i n_{2} k_{2}\right) \\
& \cdot \sum_{n_{3}} \exp \left(-i n_{3} k_{3}\right)
\end{aligned}
$$

for the quiescent crystal. When the crystal is in the fundamental longitudinal mode vibration state, assumption 6 modifies equation (9) to the form

$$
\begin{aligned}
& \psi(\underline{k})=\sum_{n_{1}} \exp \left[-i\left(n_{1} a_{1}+\xi_{n_{1}}\right) k_{1} b_{1}\right] \\
& \cdot \sum_{n_{2}} \exp \left(-i n_{2} k_{2}\right) \sum_{n_{3}} \exp \left(-i n_{3} \kappa_{3}\right)
\end{aligned}
$$

Application of equation (5) and the identity 


$$
\exp (-i u \sin v)=\sum_{\ell=-\infty}^{+\infty} J_{\ell}(u) \exp (-i \ell v)
$$

where $J(u)$ is the $\ell$-order Bessel function of the first kind, reduces equation (10) to

$$
\begin{aligned}
& \psi(\underline{\kappa})=\sum_{\ell} J_{\ell}(w) \sum_{n_{1}} \exp \left[-i n_{1} a_{1}\left(\kappa_{1} b_{1}+\ell K\right)\right] \\
& \cdot \sum_{n_{2}} \exp \left(-i n_{2} \kappa_{2}\right) \sum_{n_{3}} \exp \left(-i n_{3} \kappa_{3}\right)
\end{aligned}
$$

where $w=\kappa_{1} b_{1} \delta \cos (\Omega t)$ and $K=\pi / D$. Identifying $N_{1}, N_{2}$ and $N_{3}$ as the respective number of illuminated lattice cells in the $a_{1}, a_{2}$ and $a_{3}$ directions, and performing the Tattice celT summation indicated in equation (11) yields

$$
\begin{aligned}
& \psi(\underline{\underline{K}})= \sum_{\ell} \mathrm{J}_{\ell}(w) G\left[N_{1}, a_{1}\left(K_{1} b_{1}+\ell K\right)\right] G\left(N_{2}, K_{2}\right) \\
& \cdot G\left(N_{3}, K_{3}\right) \\
& G(u, v)=\frac{1-\exp (-i u v)}{1-\exp (-i v)}
\end{aligned}
$$

The neutron wave intensity, $|\Psi|^{2}$, describes the flow of scattered neutrons and is proportional to $|\psi(\kappa)|^{2}$. Algebraic manipulation of equation (15) yields the result

$$
\begin{aligned}
& |\psi(\underline{K})|^{2}=\left(\sum_{\ell} J_{2 \ell}(w) \frac{\sin ^{2} \pi N_{1} a_{1}\left(K \hat{b} b_{1}+2 \ell K^{\prime}\right)}{\sin ^{2} \pi a_{1}\left(K_{1}^{\prime} b_{1}+2 \ell K^{\prime}\right)}\right. \\
& \left.+\sum_{\ell} J_{2 \ell+1}(w) \frac{\sin 2 \pi N_{1} a_{1}\left[K_{1}^{\prime} b_{1}+(2 \ell+1) K^{\prime}\right]}{\sin 2 \pi a_{1}\left[K_{1}^{\prime} b_{1}+(2 \ell+1) K^{\prime}\right]}\right) \\
& \frac{\sin ^{2} \pi N_{2} K_{2}^{\prime}}{\sin ^{2} \pi K_{2}^{\prime}} \cdot \frac{\sin ^{2} \pi N_{3} K_{3}^{\prime}}{\sin ^{2} \pi K_{3}^{\prime}}
\end{aligned}
$$

where $K_{1}^{\prime}=K_{1} / 2 \pi$ and $K^{\prime}=K / 2 \pi$. Note, in passing, that equation (13), for the case of a quiescent crystal is given by

$$
\begin{aligned}
|\psi(\underline{K})|^{2}= & \frac{\sin ^{2} \pi N_{1} K_{1}^{\prime}}{\sin ^{2} \pi K_{1}^{\prime}} \cdot \frac{\sin ^{2} \pi N_{2} K_{2}^{\prime}}{\sin ^{2} \pi K_{2}^{\prime}} \\
& \cdot \frac{\sin ^{2} \pi N_{3} K_{3}^{\prime}}{\sin ^{2} \pi K_{3}^{\prime}}
\end{aligned}
$$

It is well known that equation (14) describes a function in reciprocal space (i.e., with base $\left.\left(\underline{b}_{1}, \underline{b}_{2}, \underline{b}_{3}\right)\right)$ with sharp maxima
When $K_{1}^{\prime}, K_{2}^{\prime}$, and $\kappa_{\hat{3}}^{\prime}$ all have integer values. The set of these maxima locations defines the crystal reciprocal lattice, each point of which corresponds to a set of diffraction planes of the crystal. For the vibrating crystal, the neutron wave intensity peaks at the quiescent crystal reciprocal lattice points and at the reciprocal space locations

$$
\begin{aligned}
& \underline{k}=\left(\kappa \hat{1}-2 l K^{-} a_{1}\right) \underline{b}_{1}+\kappa \hat{b}_{2}+\underline{b}_{2} \underline{b}_{3} \\
& \underline{k}=\left[K_{1}^{1} / 2-(2 \ell+1) k^{\prime} a_{1}\right] \underline{b}_{1}+K_{2} \underline{b}_{2}+\kappa \underline{b}_{3} \underline{b}_{3}
\end{aligned}
$$

for $\kappa_{1} \hat{1}, K_{2}, K_{3}$ and $\ell$ integers. The maxima described by equation (15) are modulated by $\mathrm{J}_{2 \ell}(\mathrm{w})$ and $\mathrm{J}_{2 \ell+1}(\mathrm{wi})$.

The strength of neutron diffraction by a set of crystal planes with Miller indices ( $K_{1}^{\prime}, K_{2}, K_{3}^{\prime}$ ) is obtained by an integral of the neutron wave intensity over the portion of reciprocal space in the immediate vicinity of the lattice where the intensity has appreciable value. Thus,

$$
\mathrm{S}=\int|\psi(\underline{\underline{k}})|^{2} \mathrm{~d} \underline{\underline{k}}
$$

is a measure of the size of the region of appreciable neutron diffraction possibilities in the reciprocal lattice space about the nominal (quiescent) lattice point ( $K_{1}^{\prime}, K_{2}^{\prime}$, $\left.\kappa_{3}^{\prime}\right)$.

Englehart performs the integration indicated by equation (16) and finds that contributions from the odd-indexed reciprocal space points (i.e., the second-type listed in equation (15)) are negligible relative to the even-indexed points (first of equation (15)). He derives the approximation

$$
S \simeq \frac{\lambda^{3}}{\sin 2 \theta} \frac{N_{2} N_{2} N_{3}}{V} \sum_{\ell} J_{2 \ell}\left[W\left(1-\frac{\ell}{N_{1}}\right)\right] .
$$

where $\lambda$ and $\theta$ are the neutron wavelength and Bragg angle for the reciprocal lattice point representing the quiescent crystal, and $V$ is the crystal lattice cell volume. Moreover, Englehart points out that two conclusions can be deduced from equation (17). The first is that

$$
S \simeq \frac{\lambda^{3}}{\sin 2 \theta} \frac{N_{1} N_{2} N_{3}}{V}
$$

for all values of $\mid w=k_{1} b_{1} \delta \cos (\Omega t)$. The second is that the reciprocal lattice point spreads in only the $\underline{b}_{1}$ direction with magnitude of spread given by 


$$
\Delta b \simeq \frac{4 \kappa_{1} b_{1} \delta}{N_{1}}|\cos (\Omega t)|
$$

As illustrated in Figure 3 , the reciprocal lattice point for the quiescent crystal becomes $a$. line of length $\Delta b$ in the $\underline{b}_{1}$ direction symmetrically distributed about the point $\left(K_{1}^{\prime}, K_{2}^{\prime}, K_{3}^{\prime}\right)$. Figure 3 is a twodimensional section of the Ewald sphere construction which is familiar in the interpretation of radiation diffracted from crystals. With reference to Figure $3, k^{\prime}=$ $k / 2 \pi$ and $k_{0}^{\prime}=k_{0} / 2 \pi$ whence, $k^{\prime}=k_{0}^{\prime}=1 / \lambda$, and the conditions relating $\lambda$ and $\theta$ for neutron diffraction by the $\left(K_{\hat{1}}, \kappa_{2}^{\prime}, \kappa_{3}\right)$ planes of the quiescent crystal are illustrated.

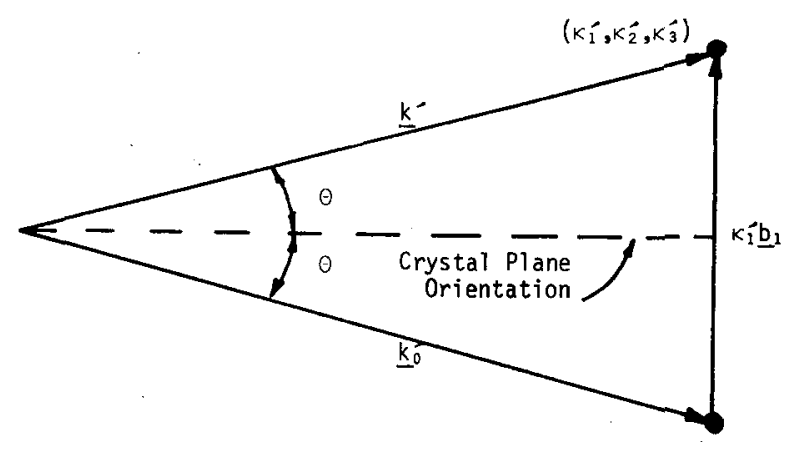

The Quiescent Crystal Case

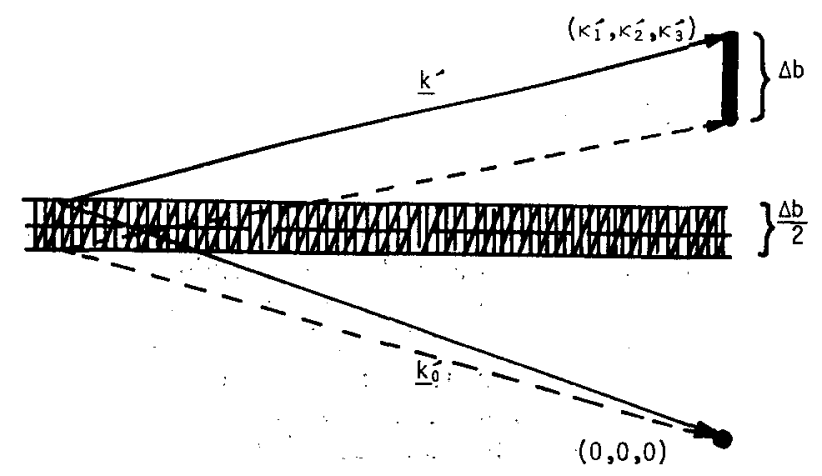

The Vibrating Crystal Case

FIG. 3. EWALD SPHERE CONSTRUCTION FOR QUIESCENT AND VIBRATING CRYSTALS

Based on assumption 3 and the result expressed in equation (18), the diffracted neutron beam intensity is proportional to

$$
\mathrm{I}=\Delta \lambda \Delta \theta
$$

where $\Delta \lambda$ and $\Delta \theta$ are the ranges of neutron wavelength and Bragg angle which can satisfy the necessary condition for diffraction by the modified ( $\left.\kappa_{1}^{\prime}, K_{2}^{\prime}, K_{3}^{\prime}\right)$ crystal planes. A series of Ewald sphere illustrations demonstrates the application and meaning of equation (20).

In Figure 4 is illustrated a twodimensional section of an Ewald sphere construction for a perfect crystal. The angles $2 \alpha$ and $2 \beta$ represent the collimation cone angles for the incident neutron beam and detector system acceptance, respectively. $P$ lane $P$ is the perpendicular bisector of $K_{i} b_{1}$ with origin at $(0,0,0)$. The intersection of the areas on plane $P$ subtended by the two collimation cones ( $2 \alpha$ and $2 \beta$ ) represents the possibilities for incident wave vector $\left(\underline{k}_{0}^{0}\right)$ origins (with terminus at $(0,0,0))$ which are diffracted by the ( $k_{i}^{\prime}$, $\left.\kappa_{2}^{\prime}, K_{3}^{\prime}\right)$ crystal planes.

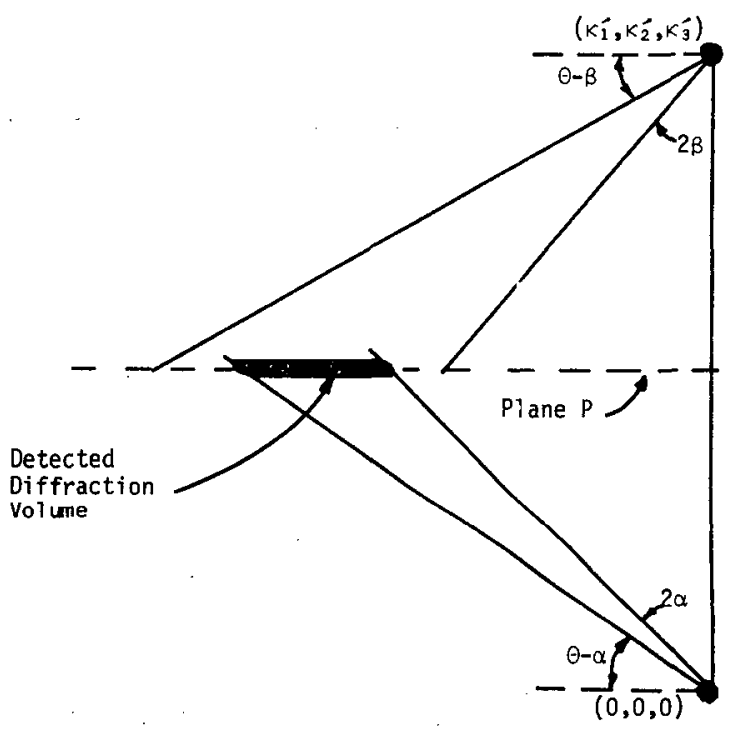

FIG. 4. EWALD SPHERE CONSTRUCTION FOR QUIESCENT PEREECT CRYSTAL

In Figure 5 is illustrated an Ewald sphere construction for a quiescent, ideally imperfect crystal with mosaic spread $2 n$. Consider two cones with common axis $b_{1}$, apexes at $K i b_{1} / 2$, and with polar angTes $\pi / 2-n$ and $\pi / \overline{2}+n$ relative to $\underline{b}_{1}$. With the volume in reciprocal space between these cones termed the mosaic spread volume, the intersection of the volume subtended by the incident beam collimation cone $(2 \alpha)$, the 
detector system collimation cone $(2 \beta)$, and the mosaic spread volume $(2 n)$ represents the possibilities for incident wave vector origins. A two-dimensional section of this volume is illustrated in Figure 5 along with the attendant spread in the reciprocal

lattice point $\left(\kappa_{1}^{\prime}, \kappa_{2}^{\prime}, \kappa_{3}^{-}\right)$. The meaning of $\Delta \lambda$ and $\Delta \theta$ specified in equation (20) can be described with reference to Figure 5 .

Specifically, $\Delta \lambda$ is the range of $\lambda$ generated by the extremes of $k_{0}$ which lie in the "detected diffraction volume." And, for each $\lambda$ (i.e., $k_{0}^{\prime}$ ), $\Delta \theta$ is the range of $\theta$ generated by extremes of $k_{0}^{\prime}$ directions which lie in the "detected diffraction volume."

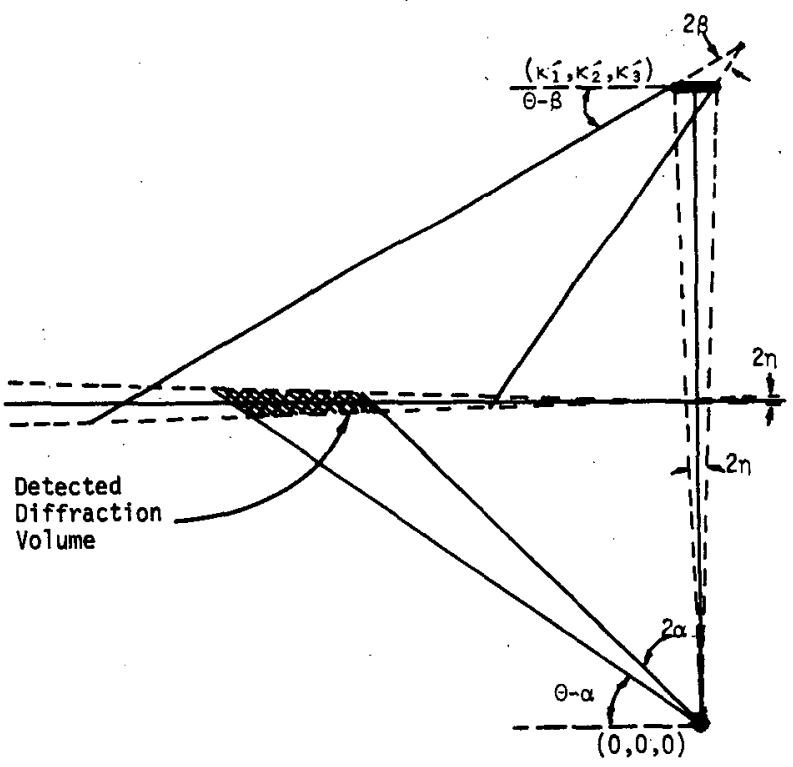

FIG. 5. EWALD SPHERE CONSTRUCTION FOR QUIESCENT IDEALLY IMPERFECT CRYSTAL

In Figure 6 is illustrated an Ewald sphere construction for a vibrating, ideally imperfect crystal at an instant when $\cos \Omega t \neq 0$. In comparison with Figure 5 , there are two differences. The lattice point ( $\left.K_{1}, K_{2} \hat{2}, K_{3}\right)$ is spread $\Delta b$ in the $\underline{b}_{1}$ direction, and thereby a separation of $\Delta b / 2$ is generated for the apexes of the mosaic cones. Clearly, the volume of possible incident wave vector origins is enlarged, and $\Delta \lambda$ and $\Delta \theta$ are increased. According to equation (20) the diffracted neutron beam intensity is thereby proportionately enhanced.

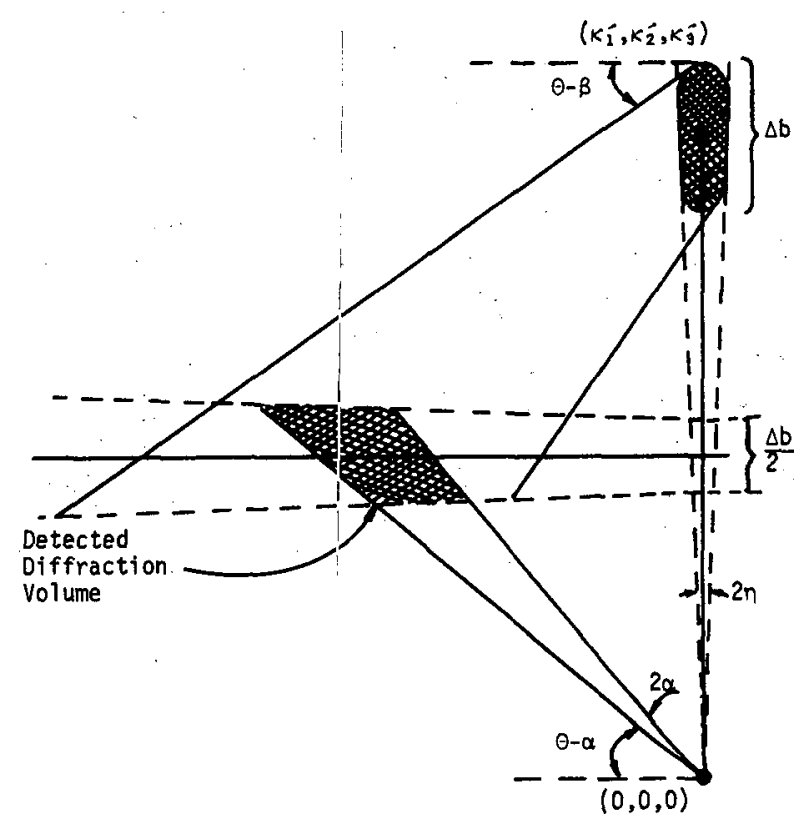

FIG. 6. EWALD SPHERE CONSTRUCTION FOR VIBRATING IDEALLY IMPERFECT CRYSTAL

Through a straight forward geometric analys is of Figure 6 (for the case of $n=0$ and $\beta>\alpha$ ), Englehart deduces the approximations

$$
\begin{aligned}
& \Delta \lambda \simeq \frac{2}{k_{1} b_{1}}\left(\frac{\sin (\theta+\alpha)}{1-\Delta b / 2}-\frac{\sin (\theta-\alpha)}{1+\Delta b / 2}\right) \\
& \Delta \theta \simeq \frac{1}{k_{1}^{1} b_{1}} \Delta b \tan \theta
\end{aligned}
$$

where $\theta$ is the nominal Bragg angle (about which collimation land vibration-induced spread is occurririg), and it should be recalled that $\Delta b$ varies directly as $\delta|\cos \Omega t|$. By the results emtiodied in equation (21), Englehart has produced analytical support of the qualitative conjectures of Klein, et al. The time-averaged (i.e., mean intensity) predictions of equation (21) and the results of Englehart's mean intensity enhancement measurements are compared in Figure 7. For the case reported the $\Delta \theta$ effect on diffracted neutron intens ty enhancement is about 650 times the $\Delta \lambda$ effect. 


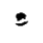

?

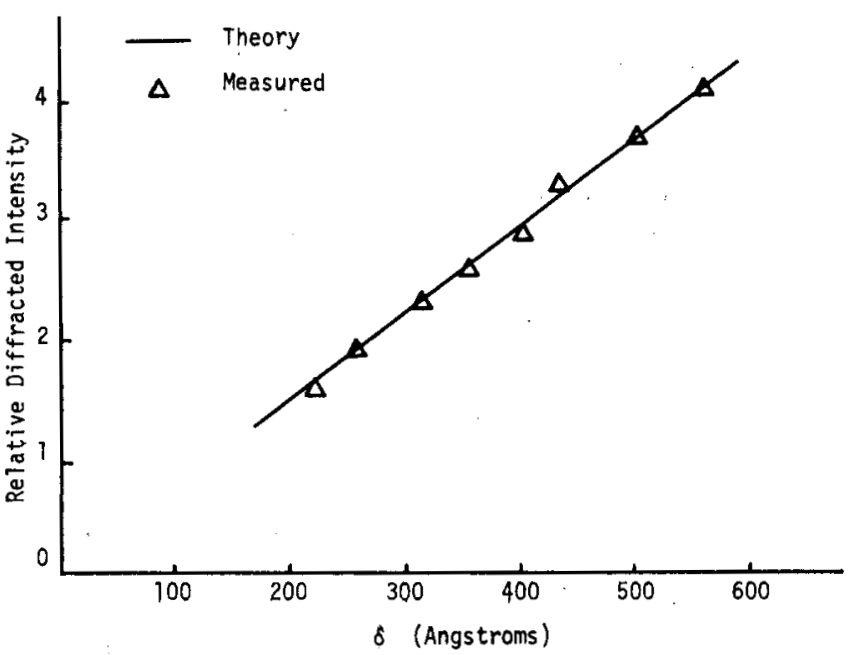

FIG. 7: RELATIVE DIFERACTED INTENSITY AS A EUNCTION OF CRYSTAL VIBRATION AMPLITUDE

Al though Englehart did not perform a dynamic calculation, he employed the interpretations gleaned from his analysis to discuss a limiting condition for finding actual modulation in the intensity of the detected diffracted neutron beam. He argues that the thickness dimension of the crystal (D) and the frequency of vibration $(\Omega)$ determine the modulation of neutron beam intensity reaching the detector. Specifically, if the crystal thickness involved in producing diffraction is such that neutrons reaching that depth are in the crystal for more than the period of crystal mechanical vibration, then there will be negligible beam modulation. Englehart derives the approximation

$$
0.009 / \mathrm{d}>\text { Dv }
$$

where $d$ is expressed in Angstroms, $D$ is in centimeters, and $v$ is the crystal vibration frequency in cycles per microsecond. Equation (22) represents the condition for observing modulation of the detected neutron beam provided that the detector has sufficient resolution. If equation (22) is satisfied then equation (21) indicates that the neutron beam will be modulated at an angular frequency of $2 \Omega$ and should have the general form depicted in Figure 2 (the modulation of the minima at frequency $\Omega$ is not predicted by Englehart).
Based on the record of measurements and interpretations presented in the previous sections, it is the present author' ' opinion that at least two important aspects of neutron diffraction by vibrating crystals deserve further investigation. Specifically, they are:

1. Methods of further increasing diffractivity, hopefully approaching total reflection. Use of crystals other than quartz (e.g., metal crystals) which can withstand far greater mechanical strains is probably indicated. These crystals need not have piezoelectric properties. They could be driven via magnetostrictive properties, or by external means.

2. Excitation of crystal mechanical vibration modes other than longitudinal compression (e.g., piezoelectric shear or surface wave modes). Such vibrational states result in location-dependent crystal strains and neutron-phonon energy exchanges which could be significant.

It is believed that such investigations will yield results that could make possible the control in time and manipulation in location and direction of thermal neutron beams. If reflection is adequate, then beams of high intensity and low epithermal neutron and gamma ray contamination may be realized. Moreover, circulation of the thermal neutron beam in a storage ring comprised of a series of vibrating crystals becomes an intriguing possibility. If neutron-phonon energy exchanges are significant, then location controlled reflected neutron velocity changes could conceivably permit focusing or phasing of the diffracted neutron beam.

It is possible to develop analytical interpretations of the relevant phenomena employing models which include the effects of secondary extinction and neutron-phonon energy exchange. This certainly should be accomplished, but it appears that some implications of Englehart's arguements will not change. The mechanical vibration of thick crystals to large strains should produce wide-band (in neutron direction and energy) diffractometers. Thus, if incoherent losses can be minimized, high intensity of the diffracted neutron beam is implied. 
Since the crystal strains are produced by time-varying phenomena, there is a natural modulation of the diffracted neutron intensity. Additional modulation can be obtained by the pulsed excitation methods discussed by Michalec, et al (1969 d) or by a clever technique suggested by Carroll and Englehart (1970) involving a tilt of the crystal diffraction axis.

In order to realize a neutron storage ring based on a series of vibrating crystals, as suggested by Jacobs and Kenney (1970) it will probably be necessary to accomplish some focusing via complex vibrational modes and neutron inelastic scattering in addition to diffracted neutron beam enhancements in excess of that previously obtained. The storage of thermal neutrons generates the capacity of producing high intensity neutron pulses - i.e., a substantial gain in duty factor by neutron storage in beam off-periods.

Recognizing the fact that the control of only low energy neutrons is considered, the potential applications of neutron beam manipulation using vibrating crystals will probably be in the areas of medical radiation therapy and diagnostics, or, in thermal neutron research. Attention has herein been confined to the effect of crystal vibration on the diffraction of neutrons and to the implications with regard to application of the phenomena. Clearly, application to the study of crystals, such as suggested by Zelenka, et al $(1969 \mathrm{f})$ or details of the mechanical vibration state are additional relevant areas of investigation.

\section{REFERENCES}

1931 Fox, G. and Cork, J., Phys. Rev., 38, 1420.

1942 Weigle, J. and Bleuler, J., Hezv. Phys. Acta., 16, 445.

1966 a Parkinson, T. and Moyer M., Nature, $211,400$.

1966 b Petrzilka, V., Czech. J. Phys., 16, 458.

1967 a Klein, A., Prager, P., Wagenfeld, H., Ellis, P., and Sabine, T., Appl. Phys. Letters, 10, 293.

1967 b Moyer, M. and Parkinson, T., Nucl. Instr. and Meth., 53, 299.

1968 a Chalupa, B., Michalec, R., Petrzilka, V., Tichy, J., and Zelenka, J., Phys. stat. Sol., 29, K51.
1968 b Petrzilka, V., Nature, 218, 80.

1968 c Petrzilka, V., Czech. J. Phys., 18, 1111.

1969 a Chalupa, B.|, Michalec, R., and Galociova, D., Nucl. Instr. and Meth., $67,357$.

1969 b Englehart, R., Neutron Diffraction by a Vibrating Crystaz, Ph.D. Thesis, The Pennsylvania State University.

1969 c Englehart, R. and Jacobs A., Amer. Nucl. Soc. Trans., 12, 2, 520.

1969 a Michalec, R., Chalupa, B., Galociova, D., and Mikula, P., Phys. Letters, $28 \mathrm{~A}, 546$.

1969 e Michalec, R., Chalupa, B., Petrzilka, V., Galociova, D., Zelenka, J., and Tichy, J., Phys. Stat. Sol., 31, K95.

$1969 \mathrm{f}$ Zelenka, J., Tichy, J., Chalupa, B., Michalec, R., and Petrzilka, V., Brit. J. Appi. Phys., 2, 1041.

1970 Carroll, R., Englehart, R., Jacobs, A. and Kenney, E., Proceedings IEEE Uttrasonics Symposium, I5, 39.

1971 Englehart, R., and Jacobs A., Nature, (in press). 


\section{THE UNIQUE RESEARCH POTENTIAL OF NEUTRON PULSES FROM NUCLEAR EXPLOSIONS}

\section{Robert L. Carter}

University of Missouri agreement to suspend all further tests using nuclear explosions. Recalling the fate of a cyclotron in Tokyo Harbor in 1945, it seems appropriate that the art of explosive sources should be examined at this time for non-weapons related applications. It is pointed out that underground explosive neutron sources offer workers in other fields of nuclear science and engineering unique approaches to certain unanswered questions through appropriately tailored experiments. The permitted extension in flux intensity by a factor of $10^{6}$ may be used to help characterize irradiation annealing mechanisms in solids. The $10^{-7}$ second duration of a derivative fission particle pulse will allow experimental examination of the immediate excitation states in fuel-bearing materials. With regard to fast breeder reactor structural materials problems, it is proposed that cross sections for $\left(n_{t}+n_{f}, a\right)$ reactions in constituent atoms of structural materials be examined for a wide variety of nuclides, using an underground explosive nuclear source of fast neutrons. The potential of explosive nuclear sources for studies of neutrino interactions should be explored further.

\section{INTRODUCTION}

Upwards of twenty underground nuclear explosions per year are set off under the aspices of the U.S. Atomic Energy Commission and other U.S. Government agencies at the Nevada Test Site northwest of Las Vegas during each calendar year. (1) On the basis of public information, only the Plowshare and Gasbuggy related events are ostensibly not associated with military interest, although many data of fundamental value to other fields have been obtained as by-products of the tests. Much of this has been reported in the open literature. The thests is herein advanced that explosive nuclear sources, tailored for the purpose, may represent the most economical approach to the procurement of information urgently needed in other areas of engineering. It appears possible that such sources may also open productive channels to important problems of nuclear physics. This approach may be technically more attractive, and in some cases, more economical than an approach through the use of high flux test reactors and particle accelerators.

Shortly after the abrupt appearance of nuclear explosive technology as a military tool in Japan in 1945, scientists and engineers within the amazingly successful Manhattan District program proposed the assuaging peaceful application of "controlled nuclear fission" for research and for domestic power. The concepts of peaceful applications have been extended further to embrace the field of "controlled nuclear fusion". Unfortunately, a suggestion that deliberately staged explosive nuclear events are somehow lacking in control is implicit in this terminology. This was undoubtedly an accurate characterization of early explosions, but it does not represent the current state of the art. By way of illustration, consider the fact that a typical nuclear fission reactor utilizes something well under 25 per cent of its fissile material before the highly radioactive fuel elements must be discharged and placed in an elaborate chemical engineering system for purification and recycle. While "controlled" fusion reactors have yet to become selfsustaining, engineering evaluations of their ultimate applications indicate that low burning efficiency, induced activity in the shell coolant, instability of structural materials are just a few of the very difficult problems remaining to be solved, even after procedures have been developed to make such reactors become "critical".(2)

In contrast, a nuclear explosive with low burning efficiency would be considered "dirty" and very wasteful. The very existence of a so-called "clean" nuclear weapon implies that highly efficlent fusion "burning" is attainable, leaving minimal residue of the initial charge of radioisotopes and of radioactive products. According to public news release(1), observable "venting" of activity occasionally is observed following underground nuclear explosive events. The writer does not have access to quantitative data on the 
amounts of activity so released, but he was permitted to walk to ground zero at the base of the crater formed following the "Physics 8 " event (pictured on the cover of the reference (3) publication) to recover items of equipment a few days after the event took place. The activity produced by such an event remains buried several hundred feet underground in the alluvial soil of the "spotted Range" test area. To place this in proper context, it should be pointed out that radioactive waste from fission reactors is slated to be buried in selected underground sites with rather similar geometry for a very long period of radioactive decay.

A neutron pulse comprised of about two moles of neutrons is produced by a ten-kiloton (TNT equivalent) yield fission explosive device, scaling from Hemendinger. (3) This corresponds to the consumption of about one mole of fissile material and the production of about two moles of radioactive fission products. A single one-million kilowatt (thermal) nuclear power reactor consumes almost $41 / 2$ moles of fissile material per day of operation; thereby producing more than four times the activity produced by a ten-kiloton explosive nuclear device. of course, the short-iived components of the activity will have decayed before burial of the waste from a reactor is undertaken.

Outside of the facts deducible from the physics of the fission and/or fusion processes, relatively little technical information on the prompt radiation emanating from explosive nuclear devices is avallable in the open literature. Glasstone's monogram (4) provides an authoritative guide to many numbers, but these are presented with a view to appraising effects upon structural and biological systems exposed to weapon explosions above ground. For the most part, Glasstone deals with radiation propagating through an atmosphere of air, although it is possible, with a little ingenuity, to use his figures to derive exposure data appropriate for a vacuum exposure to an explosive source of nuclear radiation.

Hemendinger's recent semi-popular paper (3) provides an excellent example of the research potential of pulses of neutrons from an explosive nuclear source in the production of prolific quantities of basic data on fission cross-sections of heavy nuclides as a function of energy.
THE RESEARCH POTENTIAL OF NEUTRON BURSTS FROM EXPLOSIVF NUCLEAR SOURCES

The author spent a sabbatical leave during 1968-69 with a group of workers at Los Alamos Scientific Laboratory whose primary responsibility was/the measurement of physical parameters of direct or Indirect interest to the technology of nuclear weapons. Some of the data obtained by these workers was recognized to be of fundamental interest, and selected papers have been published in the open literature. $(5,6,7)$ Most of the publications lie in the fileld of nuclear physics. In some cases, data of possible general interest are not released for publication because of classified implications of laboratory interest in the scientific area explored. With a view to pointing out the broader potential of tests which could be conducted at the Nevada Test Site, the writer presented a paper at the NASA-AEC National Symposium on Irradiation Testing Technology (18) in September 1969. Unfortunately, most of those attending the conference were concerned primarily with longterm engineering reliability tests in operating nuclear reactiors. Little interest was evinced in the profosals set forth.

Since that tinie, other areas have appeared where advances seem feasible through the application of explosive neutron sources. Great potential exists for the collection of radiation effects cata badly needed within the Civilian Reactors Frogram. Most urgent of these is a study of two-stage neutron-induced spallation processes in nuclet of moderate Znumber. Such processes may be responsible for production of helium bubbles as a consequence of reactions withir elements used as components of structural. materials within fast breeder fisston realctors. Of less urgent concern but of equal long-range importance is the possible contribution to be made by a study of the effects of high intensity fission particle pulses on materials. An elucidation of fission fragment energy degradation processes is highly desirable as a pragmatic approach to the extension of fuel element life. An esoteric by-product maly well be the suggestion of additional approaches to the practical utilization of fission particle energy short of thermalization with its environment. Exposition upon the studies mentioned above is the purpose of the presient paper. 
Hemendinger (3) characterizes a ten-kfloton device as a source of $10^{24}$ neutrons in $10^{-7}$ second. If these neutrons are assumed to radiate isotropically from a small source, the pulse flux per steradian is $10^{31 / 4 \pi}$ or about $10^{30}$ per sec. At a distance of $\mathrm{R}$ meters from the source, neglecting attenuation and scatter the pulse flux is $10^{26 / \mathrm{R}^{2}}$ per $\mathrm{cm}^{2} \mathrm{sec}$, assuming monoenergetic neutrons. The total fluence passing through a sample exposed at distance $R$ meters from a 10-kiloton equivalent device is about $1019 / \mathrm{R}^{2}$ per $\mathrm{cm}^{2}$. For samples exposed at ground level over such a nuclear device 185 meters below ground, the total fluence is about $3 \times 10^{15}$ per $\mathrm{cm}^{2}$. If a portion of the original neutron pulse is caused to pass through a moderating medium placed near the source, the initial neutron pulse will be spread in energy. The short duration of the pulse at the source serves the same role as a beam chopper in a time-of-flight neutron spectrometer, and the 185 meter drift tube serves to separate neutrons by energy in accordance with their time of arrival at the experimental area. Corrections to the source time are required as a consequence of moderator transittime delay and Doppler width. (3)

\section{APPLICATION TO STUDIES OF KNOCK-ON RADIATION DAMAGE}

Concern with the effect of recoils produced by the transit of fast neutrons through matter followed Wigner's predictions $(9)$ of crystalline changes as a consequence of neutron irradiation. Many materials show readily measurable changes in physical and chemical properties, along with significant modifications in mechanical properties and physical sinape as a direct result of fast neutron dosages often met with in the cores of existing nuclear reactors. Effects traceable to displacement phenomena are, to some degree, reversible, provided that temperatures during Irradiation are maintained at a level high enough to permit diffusion of the appropriate atomic or molecular species. Studies of the role of thermal annealing and of the kindred phenomenon of radiation annealing offer the potential for extending the useful life of reactor components formed of sensitive materials.

Neutron irradiation rate plays at least two roles in affecting the nature of the defects remaining in a given material following a period of irradiation. The higher the fast neutron irradiation rate, the higher the steady. state density of diffusing displaced atoms, and the more likely the activation of a secondorder process leading to the formation of a stable two-atom complex. Ofttimes, such a complex can serve as a condensation center for other migrant displaced atoms into larger complexes.

A countering effect may occur as a result of extremely high intensities of fast neutrons which nay increase the probability that such complezies, once formed, can fall victim to subsequent disruptive encounters. The effectiveness of such encounters in destroying complexes and permitting the return of displaced atoms to vacancies or edge positions in the crystal. would be expected to increase with the diffusion rate and hence, with the temperature. Experimental determination of parameters governing the dynamics of such competitive processes requires sources presenting a wide range of irradiation rates. (8)

The range of neutron irradiation rates which is available for such experiments (at nvt of $2 \times 10^{16}$ per $\mathrm{cm}^{2}$ is extended through the use of explosive fission sources from $2 \mathrm{x}$ $10^{23}$ per $\mathrm{cm}^{2}$ sec at a distance of 20 meters from the source down to (say) a flux of $2 \mathrm{x}$ $10^{8}$ per $\mathrm{cm}^{2} \mathrm{sec}$ for a three-year fast reactor exposure. The rate range for experimental irradiations is thus extended by a factor of about $10^{6}$ over the range of $10^{9}$ accessible through the use of laboratory fast pulse reactors.

\section{APPLICATION TO STUDIES OF SWELLING IN FAST REACTOR STRUCTURAL MATERIALS}

of serious concern to the continuing development of fast breeder power reactors is the attainment of an understanding of the processes responsible for swelling in alloy structural materials and the determination of satisfactory steps to alleviate this troublesome problem. It has been established that the process does not depend linearly upon fast f1uence. (10) Phenomenological descriptions of the progress of swelling in various alloys under specified time-irradiation-temperature histories have been developed showing thresholds or onset levels, and shoulders or saturation levels in the dilation of the material. Analyses have shown the presence of helium within the swollen material, indicating that some type of $(n, \alpha)$ process is operative. In many cases, the presence of boron impurity as the responsible agent has been clearly ruled out.

Recently reported work indicates that certain nuclides may be susceptible to a $(2 n, \alpha)$ process of appreciable crosssection. (11) The postulated process is rendered probable at high fluxes by the existence of a metastable nuclear species which is produced upon the absorption of the first 
(perhaps thermal) neutron. Such intermediate metastable species may have a high cross-section, during its lifetime, for a $(n, \alpha)$ reaction involving a fast neutron. Clearly the process has some of the requisite properties to explain the unanticipated appearance of He under conditions met with in the core of a high power-density fast reactor. Its probability will tend to rise with the square of the flux level, hence it will become much more important in high flux reactors. Furthermore, such a reaction would remain essentially unobservable in fast neutron beam cross-section measurements, if the cross-section for production of the intermediate species is appreciable only at low neutron energies, and if the metastable lifetime is of the order of milliseconds.

It would appear that data of great value to the advancement of the fast breeder development program might be obtained through arrangements to include a fairly elaborate experimental salient in one or more future underground explosive device shots. Such a salient could be used to house a sacrificial thermal reactor of a commerically available variety to provide thermal neutron flux for the production of the postulated metastable nuclides. If the metastable lifetime is $\tau *$, and the cross-section for its production is $\sigma^{*}$, the steady state metastable concentration is given by

$$
\frac{\mathrm{n}_{\mathrm{s}} *}{\mathrm{~N}}=\sigma * \phi_{\mathrm{s}} \tau *
$$

At time $t_{1}$ after cessation of saturation irradiation to thermal neutrons, the metastable concentration will have decayed to

$$
\frac{n_{1}^{*}}{N}=\sigma^{*} \phi_{t} \tau^{*} \exp \left(\frac{-t_{1}}{\tau^{*}}\right) \text {. }
$$

If, at time $t_{1}$ after cessation of thermal neutron irradiation, the material is subjected to an intense burst of fast neutron irradiation, assuming negligible delay in a helium spallation reaction, the rate of production of alpha particles is

$\left(\frac{d n}{d t}\right)_{\alpha}=N \sigma_{s p} \phi_{f}\left[\exp \left(-\int \sigma_{s p} \phi_{f} d t\right)\right] \sigma * \phi_{t} \tau * \exp \left(-\frac{t_{1}}{\tau *}\right)$

If the experiment is located at sufficient distance from the fast neutron pulse source, (slight neutron moderation being introduced near the source) the product $\sigma_{f p} \phi_{f}$ will exhibit time modulation due to itg $\mathrm{f}$ dependence upon neutron energy. This contribution will lead to an experimentally measureable variation in $\left(\frac{\mathrm{dn}}{\mathrm{dt}}\right)_{\alpha}$ during the early part of the pulse.
The production of alpha particles from various isotopic species of interest may be determined during simultaneous exposure of a variety of foils. $(3,7)$ Furthermore, the values of $\sigma^{*}$ and of $\tau^{*}$ may be determined for each by employing a timed chopper to initiate and to interrupt the thermal irradiation to several complete sets of foils, one set at each of a variety|of times, of the order of milliseconds and tnore before the fast pulse arrival time.

The energy dependence of the magnitude of $\sigma_{\mathrm{sp}}(E)$ may be letermined from the alpha particle pulse shape in time, compared with the known source function $\phi[\mathrm{E}(\mathrm{t})]$ at the exposure position! Note that

$E(t)=\frac{m D^{2}}{2 t^{2}}$ for an impulse source of neutrons of mass $m$ located at a distance $D$ from the observer. The absolute magnitude of $\sigma_{s p}$ may be determined froin the terminal portion of the alpha pulse, thich portion is governed by the depletion integral $\exp \left(-\int_{\mathrm{sp}} \phi_{f} \mathrm{dt}\right)$.

\section{APPLICATION TO FLSSION FRAGMENT DAMAGE DYNAMICS STUDIES}

The principal primary excitation imparted within fuel material exposed to high neutron flux comes from the transit of the resultant fission particles. In their rather short range these interact strongly with both conduction and valence electrons within the fuel matrix material. Remote experiments designed to observe the time required for exchange of electron energy and temperature equilibration with the electronic and vibrational states will shed light upon the processes responsible for mechanical deterioration of fuel-bearing structures, especially those containing non-metallic materials. The performance of Hall effect measurements in metals and in semiconductors will identify the presence of non-equilibrium charge carriers generated by the transient fission particles. A spatial range of magnetic field permits the simultaneous identification of charged particles with values of $\mathrm{e} / \mathrm{m}$ differing from those as sociated with conduction band electrons.

Intense fisstion particle bursts of less than a microsecond duration may be obtained by exposing plates of suitable fissile material to the high intensity fast neutron pulse. Forbidden transitions into excited states in molecular systems can be explored remotely through optical emission or transmission 
spectra for films irradiated by the fission particle bursts. Some of these metastable species may conceivably have value as alternatives to thermal transport as a means of removing energy from within cores of operating reactors.

\section{APPLICATION TO STUDIES OF NEUTRINO PULSES}

The neutrino pulse generated at any fission event accounts for about five per cent of the fission energy--about five-fold the energy of the concurrent pulse of fast neutrons. The neutrino-borne energy pulse flux density at a distance of 20 meters from a 10-kiloton equivalent explosive fission device is thus about $8.7 \times 10^{26} \mathrm{eV} / \mathrm{cm}^{2} \mathrm{sec}$. If the neutrino energy is taken to be 1.7 $\mathrm{Mev}(12)$, the neutrino flux during the pulse is $5.1 \times 10^{20} \mathrm{v} / \mathrm{cm}^{2} \mathrm{sec}$.

The cross-section for neutrino reactions with matter is notoriously. low, of the order of $6 \times 10^{-44} \mathrm{~cm}^{2}$ for the proton reaction

$$
v+\mathrm{p}^{+} \rightarrow \mathrm{n}^{\mathrm{o}}+\mathrm{e}^{+} \text {. }
$$

If a container of water is located 20 meters from the source, the most probable number of reactions per $\mathrm{cm}$ path length during transit of the neutrino pulse will be about $-\delta N_{\nu}=1.0 \mathrm{~cm}^{-1}$ per $\mathrm{cm}^{2}$ cross-section of water. For a $1 \mathrm{~m}^{3}$ tank of water exposed to the neutrino pulse (but shielded from other radiation) the yield will be approximately $10^{6}$ reactions. of course, experiments utilizing this source of neutrinos must be designed with remote instrumentation, and with expendable apparatus near the source.

\section{SUMMARY}

The purpose in presenting this paper at the National Topical Meeting on Neutron Sources and Applications is to emphatically call attention to a neutron source of unique aptitude whose availability may be abruptly terminated. At the present time efforts are being bent, at the international level, to suspend all explosive nuclear testing. This would be done, of course, in the interest of achieving a halt, on a world-wide basis, in further development of improved weapons capability--an important first step toward nuclear disarmament. The possibility remains that a formula for an internationally inspected arrangement might eventually be worked out to permit such developments as Plowshare and Gasbuggy to continue. Such an agreement, suitably safeguarded, may be some time in the making. Unless the usefulness of such can be shown soon, other peacefully-directed applications of underground nuclear tests, such as those herein outlined, would become impossible.

I.t: is proposed that all persons with an interest in the employment of neutron sources, particularly sources having high intensity and short duration, examine carefully the possibility that uniquely helpful use might be made of the underground tests while they are still occurring. If inadequate data are available to permit such appraisal, letters should be written to the Atomic Energy Commission requesting that such data be made available and, of course, stating why it is desired. It seems altogether reasonable that a facility permitting periodic detonation of a neutron source device without weapon development potential, might be agreed upon in the SALT talks, if the need for such could be set forth in the near future.

\section{REFERENCES}

1. Associated Press News Release,17 December 1970. Columbia (Mo.) Daily Tribune, P. 8, 18 December 1970 .

2. H. Postma, "Engineering and Environmental Aspects of Fusion Power Reactors" Trans. of the Amer. Nuc1. Soc., 13 No. 2 $694,(1970)$.

3. A. Hemendinger, "Neutrons from Nuclear Explosions", American Scientist, 58, No. 6, 622f, Nov.-Dec. 1970.

4. S. Glasstone, "The Effects of Nuclear Weapons", Department of the Army Pamphlet, No. 39-3, April 1962.

5. A. Hemendinger, "Cross-Section Measurements Made with Neutrons from a Nuclear Detonation", Physics Today, 18 , No. 8, 17 (1965).

6. B. Diven, Proceedings of Conference on Neutron Cross Section Technology, CONF660303 , Book 1, 105 (1966).

7. P. A. Seeger, A. Hemendinger and B. C. Diven "Fission Cross Sections of $241 \mathrm{Am}$ and $242 \mathrm{mAm}^{\prime \prime}$, Nuclear Physics A96, 605, (1.967).

8. R. L. Carter, "Use of Nuclear Explosions for the Study of Radiation Damage Processes", Proceedings of the National Symposium on Developments in Irradiation 
Testing Technology, CONF-690910, 506, (1969).

9. M. Burton, "Radiation Chemistry", J. Phys. and Colloidal Chem. 51, 618f, (1947).

10. W. N. McElroy, R. I. Simons, L. D. Blackburn, "Damage Functions for 304 Stainless Stee1", Trans. of the Amer: Nuc1. Soc., 13, No. 1, 144, (1970).
11. J. Weitman, N.. Dåverhög, S. Farvolden, "Anomalous Helium Production in Nickel", Trans. of the Amer. Nuc1. Soc., 13, No. $2,557,(1970)$.

12. D. Halliday, Introductory Nuclear Physics, p. 144, Wiley \& Sons, New York, (1955). 


\title{
DEVELOPMENT OF AN INTENSE PULSED 14-Mev NEUTRON SOURCE
}

\author{
Duane C. Gates and Laszlo J. Demeter
}

Physics International Company

San Leandro, California

Initial results are presented for a 500 kilojoule, dense plasma focus (DPF) device filled with a $50 \%-50 \%$ mixture of tritium and deuterium. The DPF gun is a coaxial plasma accelerator, which converts a fraction of the stored magnetic energy behind a moving current sheet into plasma energy. The axisymmetric current sheet is initiated on, and lifted up from a breech insulator between the electrodes and then accelerated toward the tip of the center electrode by magnetic forces. There, a violent radial ( $Z$ pinch) collapse generates a short lived, dense, high temperature plasma (DPF) wherein ${ }^{3} \mathrm{H}(\mathrm{d}, n){ }^{4} \mathrm{He}$ reactions produce the outburst of 14 Mev neutrons.

The diameters of the coaxial electrodes were 10 and 12 inches, and the operating pressure was typically 8-10 torr. The plasma gun was energized by a 160 capacitor, $20 \mathrm{kv}, 2.24 \mathrm{mF}$ bank discharged through 20 vacuum switch gaps. The current sheet reached its peak of $3 \mathrm{MA}$ at $3.2 \mu \mathrm{sec}$, then collapsed to $2.2 \mathrm{MA}$ at $4.8 \mu \mathrm{sec}$, producing a typical neutron pulse width of $100 \mathrm{nsec}$ (FWHM). Following diagnostics were used: scattering-corrected silver-activation detectors for yield; cadmium shielded sulphur tablets for fluence; and photodiode-scintillator units, enclosed in thick lead, for pulsewidth information. Maximum yield of $6 \times 10^{12}$ neutrons/pulse and fluence of $10^{11}$ neutrons $/ \mathrm{cm}^{2}$ were recorded during the initial 20-pulse operation. Neutron yields from reactions ${ }^{3} \mathrm{H}(\mathrm{d}, \mathrm{n})^{4} \mathrm{He}$ and ${ }^{2} \mathrm{H}(\mathrm{d}, \mathrm{n})^{3} \mathrm{He}$ have been compared for the device.

\section{INTRODUCTION}

Since the discovery (1) of the dense plasma focus (DPF), this mode of coaxial plasma gun operation has been studied by an increasing number of laboratories $(2, \underline{3})$. The peak neutron output from the D-D reaction was gradually increased from $1 \times 10^{8}$ to $2 \times 10^{11}$ neutrons per pulse as higher capacitor bank energies were utilized and electrode geometries were refined (4-7). In this country, the work is centered principally at Los Alamos Scientific Laboratory ( 8 ), Sandia-Albuquerque (9), Aerospace $(10,11)$, and Physics International (PI) (12). This paper describes the characteristics of a $500-\mathrm{kJ}$ DPF neutron source that has recently become operational at PI. This facility was created by doubling the capacitance of an existing system (7), reconfiguring it to be consistent with the operational needs of irradiation experiments, and adding a tritium handling capability.

This system uses a tritium-deuterium gas mixture and incorporates a sample irradiation position close to the DPF. Thus the system possesses a unique high fluence irradiation capability with pulsed $14-\mathrm{MeV}$ neutrons from the $T-D$ reaction.

\section{DPF COAXIAL GUN}

The plasma process in a coaxial gun can be considered to occur in three basic phases: (1) the initial ionization and current buildup at the breech, (2) the axial acceleration of an axisymmetric current sheet acting as a "snowplow" and (3) the violent radial collapse of the current sheet to form the DPF.

The typical sequence of current sheet propagation is shown in Figure 1. The gun is filled with a working gas to the appropriate pressure and the capacitor bank is charged to its operation voltage. The switch gaps are then triggered, applying the bank voltage across the gun electrodes. A discharge initiates at the breech insulator and current begins to flow. The resultant radial discharge propagates axially to the end of the center electrode where it rapidly collapses to the axis, forming the dense plasma focus.

The first phase is an important determinant of current sheet symmetry. The initial filamentary structure of the discharge must develop into an efficient, fully-ionizing current sheet. Gross asymmetries in current sheet structure must be suppressed to avoid instabilities and improper current sheet propagation. The current sheet, with rapid lift-off from the insulator and its canted front, produces a clean, highly ionized plasma at the end of the center electrode for the pinch phase. This behavior thus offers an advantage over the usual Z-pinch which begins with a small current and partially ionized plasma near a wall.

The last phase is a rapid pinch collapse to the axis which occurs in approximately 0.1 
usec. At the axis, a high-density plasma focus is formed by collisions of the particles that have been accelerated to high energy during the collapse. Neutrons and $\mathrm{X}$-rays are produced for about 100 nsec; thereafter, plasma cooling and dissipation occur. The DPF is short-lived because of the poor confinement geometry of the B-field, instabilities, and diffusion, however, the current continues to flow as a weak pinch for a considerable period of time.

The axial propagation phase is a result of the $j \times \underline{B}$ force on the current sheet. This phase allows the current in the system to reach a maximum value for driving the pinch collapse. Also, the magnetic energy stored in the gun during this phase is the energy source for heating the DPF.

The objective of scaling the system to higher capacitor bank energies is to store a larger amount of magnetic energy in the plasma gun and to attain higher driving currents for the pinch collapse. The resultant increase in magnetic pressure raises the energy density of the plasma focus, producing more intense neutron fluxes. When the capacitor bank energy is doubled, a coaxial gun must be developed that is matched to the bank. As the energy of a system is increased by increasing the capacitance, rather than the voltage, the system impedance is automatically reduced. For this reason, a lower impedance plasma gun is desired to optimize the energy transfer. A lower impedance load may be attained by reducing the current sheet speed or reducing the ratio of the electrode diameters. Since there is a practical lower limit to the current sheet speed for an efficient snowplow action, one is required to reduce the ratio of electrode diameters. A practical minimum electrode spacing has here been experimentally observed. Therefore, larger diameter electrodes are ultimately required as one scales up in capacitor bank energy. For this system 10- and 12-inch electrode diameters were selected as the basic sizes with which optimization studies should be performed.

\section{DPF IRRADIATION FACILITY}

A floor plan of the facility is shown in Figure 2. The major parts of the DPF system are described below.

The capacitor bank is divided, with one half on each side of the tank assembly containing the test area. The bank consists of twenty modules. Each module contains eight $14 \mu \mathrm{F}$ capacitors and is switched by a single vacuum spark gap. A master circuit is used to trigger the spark gaps simultaneously. Coaxfal cables carry the discharge current from the modules to a 6-ft. wide paralle1-plate transmission line, with the coaxial gun at the center. The center segment of the transmission line is integrated with the tank assembly as a removable unit. The capacitor bank can be operated over a wide range of stored energies, from 100 to $500 \mathrm{~kJ}$. This makes possible considerable flexibility in the output of the machine. The normal operating point is $20 \mathrm{kV}$, or $450 \mathrm{~kJ}$. The capacitance of the bank is $2240 \mu \mathrm{F}$ and its total inductance is about $7 \mathrm{nH}$, including the breech inductance of the coaxial gun. Depending on the gas pressure in the gun, the bank will deliver peak currents of 2 to $4 \mathrm{MA}$ at $20 \mathrm{kV}$.

Remote operation of the capacitor bank and tritium system is accomplished at the control console, which is located outside the screen room in order to minimize electrical noise there. Ten signal cables, shielded in conduit, connect the screen room to the test area. Considerable care has been taken to ensure clean diagnostic signals; noise levels of $2 \mathrm{mV}$ have been observed.

\section{TRITIUM SYSTEM}

The production of $14-\mathrm{MeV}$ neutrons with a high energy plasma gun introduces many complexities into the operating procedures. The kilocurie quantities of tritium gas involved impose strict standards of performance, reliability, and safety on all components of the tritium system. The approach to designing the tritium system incorporated the basic concept of double containment. The primary tritium storage and pumping apparatus, as well as the plasma gun and vacuum chamber, were enclosed in a secondary contalnment vessel. The secondary containment vessel is kept closed whenever the tritium apparatus is actuated, the plasma gun is pulsed, or the system is out of use, In this way, the chance of a catastrophic failure releasing a large quantity of tritium gas into the building or up the exhaust stack is very remote. The vessel is portable for remote decontamination if necessary. Ventilation and exhaust ducting. to a 10-foot stack on the roof of the building was also installed as was tritium monitoring equipment.

In addition to the secondary containment vesse1, another basic unit was incorporated into the system. This is a tritium scrubbing apparatus that is capable of removing tritium gas or tritiated water from an air flow. The 
purpose of this apparatus is to decontaminate the plasma gun or secondary contalnment tank in case of a system failure where air is mixed with the tritium gas. This system is also useful for some routine scrubbing operations in order to minimize the amount of contamination exhausted up the stack.

To operate the plasma generator in the 14-MeV neutron mode, a mixture of tritium and deuterium gases ( 5 to 10 torr) is required in the vacuum vessel that contains the coaxial gun. This vessel has a volume of approximately 100 liters, so that about 1000 curies of tritium must be present in a gaseous form in the operational volume. This relatively large quantity of radioactive gas represents a significant health hazard requiring special handling techniques.

A tritium-handling system was developed which delivers, recovers, stores, and purifies the tritium needed to operate the plasma generator. The components of the handling system are detailed below.

The tritium-handling facility is operated by remote control and is designed to clean and cycle the D-T mixture through the operational volume with no release of gas up the stack. Figure 3 shows two activated uranium traps $\left(U_{1}\right.$ and $\left.U_{2}\right)$, one to supply the gas mixture and one for pumping. The $U_{I}$ trap contains approximately 100 grams. Since $U_{1}$ is used for pumping, it must have a larger tubulation and a larger uranium-storage capacity to provide adequate pumping speed.

To deliver a charge of gas to the vacuum vessel, the $U_{2}$ trap is heated, causing the hydrogen to evolve from the hydride. The hydrogen is collected in the holding cylinder until a predetermined pressure is reached on the transducer readout at the control panel. The trap is then isolated from the holding cylinder, and the valves connecting the holding cylinder to the vacuum vessel are opened, allowing the gas to enter the vacuum vessel. The vacuum vessel is again isolated from the tritium system before the coaxial gun can be pulsed.

Although the coaxial gun can be pulsed many times without changing the gas in the vacuum vesse1, the tritium-storage and handling system is capable of executing this change several times during a working period. The traps contain approximately five times as much uranium as is necessary to handle the present inventory of gas. This surplus allows more rapid pumping and adequate contamination removal.
The system is equipped with a pair of vac-sorb pumps that recover the gas from the vacuum vessel. The tritium is returned from the vac-sorbs to the uranium traps and any non-reacting contaminants are processed through the scrubber system to remove residual tritium before being released to the atmosphere.

\section{SAMPLE HOLDER}

Irradiation positions were developed bearing in mind that a useful sample holder must (1) not significantly degrade the performance of the generator, (2) be readily accessible to the user, (3) have adequate electrical shielding, and (4) be safe for tritium operations.

Tests have shown that the closest approach to the plasma focus can be attained by approaching the focus along the axis. This is particularly true when a rather large size sample holder is used. The axial position of a sample holder minimizes the influence upon the collapsing current sheet.

Past experience has shown that there should be sufficient volume available beyond the end of the gun to dissipate the plasma ejected from the end of the barrel by the current sheet. The walls act as a "getter" to absorb impurities evolved from electrode surfaces and ejected by the gun operation. For this reason, the neutron sample holder necessarily took the form of a re-entrant cavity on the axis. To minimize its affect upon plasma gun operation, the diameter of the holder was kept considerably smaller than the center electrode diameter. The neutron sample holder is metallic and is bolted to the vacuum chamber wall which is at ground potential. Therefore, the re-entrant cavity provides excellent $\mathrm{RF}$ shielding for active experiments. The axial position permits quite close approach to the focus. Specifically, the target to be Irradiated can be placed approximately 2 centimeters from the source with the holder that has been developed. However, the holder does experience severe erosion which limits its useful lifetime to a relatively small number of pulses $(\approx 100)$.

Another desirable irradiation position is at 180 degrees, inside the center electrode. It should not be difficult to develop this sample position because the center electrode is hollow and large in diameter, although it would probably not be quite as close to the plasma focus as the zero degree position.

F'igure 4 shows the tank with the large, 
end door removed. The transmission lines, vacuum vesse1 and zero-degree sample holder are clearly visible. The tritium-pumping system is located on the back side of the square panel seen in the back of the tank.

Figure 5 shows a schematic of the zerodegree sample holder and its position relative to the plasma focus. The irradiation area is $3.2 \mathrm{~cm}$ in diameter and about $2 \mathrm{~cm}$ from the source. Sample holders with different areas and distances can be provided according to the user's requirements.

\section{NEUTRON INSTRUMENTATION}

This section briefly summarizes the diagnostic equipment that is used to monitor the operating characteristics and neutron output of the coaxial plasma gun.

Two silver-activation detectors of the type developed at Los Alamos Scientific Laboratory (Reference LA-3498-MS) are used as standards to measure the neutron yields of both $D-D$ and $D-T$ operations. An emitterfollower is used to match the geiger tube output to the scaler impedance. A matched set of four geiger tubes is used in each detector. The tubes are selected on the basis of similar plateau curves and response times. Only tubes are selected which have a resolution time of $125 \pm 20 \mu \mathrm{sec}$ with the $0.5-\mathrm{V}$ discrimination level of the scaler.

Correction factors for the counter readings, due to scattered neutrons, were redetermined whenever changes in the system were made and that resulted in new scattering conditions, i.e., modifications of electrodes, introduction of the tritium containment vessel and the changeover to D-T operation. The method of using two counter-scaler units was applied, where the readings of a detector, placed near the plasma focus and having known calibration characteristics, are used as standard and compared to those of the other detector located elsewhere. Low-yielding gun operation was used in order to prevent overloading the geiger tubes of the close up reference detector. Care was taken that the counting rates were maintained far below $3 \times 10^{5}$ counts per minute, above which the deadtime counting loss correction loses accuracy.

The ${ }^{32} \mathrm{~S}(\mathrm{n}, \mathrm{p}){ }^{32} \mathrm{p}$ reaction was utilized to measure the fluence of $14-\mathrm{MeV}$ neutrons. Cadmium-shielded and foam-cushioned sulphur tablets were exposed in the sample holder or on the outside wall of the vacuum chamber. After 24 hours or longer, the induced activity was counted with an RCA 6810A PM tube-scintillator-scaler unit The counts were plotted versus the scaler discrimination level and compared to corresponding background readings and counts of a uraniun glass standard. The plateau sections of the curves were used to relate the tablet activation to the uranium glass standard, representing $3 \times 10^{10} \mathrm{n} / \mathrm{cm}^{2}$.

A photodiode-scintillator detector was used to follow the higher intensity, 14-MeV neutron pulses. It consisted of an Abtronics photodiode, type PI 936, with subnanosecond response time and at 6-inch-long, 2-inch-diameter, type NE 102, plastic scintillator.

\section{DPF NE:UTRON SOURCE}

The tritium operation was performed with the Model 10x12-9 coaxial gun. The gun was first operated on cleuterium gas without the sample holder in $\mathrm{p}$ ]ace. The sample holder was then installed and comparative pulsing was performed using both cleuterium and an equal mixture of tritium and deuterium gases. The end of the sample holder was 1 inch away from the end of the center electrode for these studies.

The plasma guri was operated at a voltage of $20 \mathrm{kV}$ and a presisure of 9 torr.

An oscillograph of $\mathrm{dI} / \mathrm{dt}$ and $\mathrm{I}$ for an above average yield pinch is shown in Figure 6 . At the given operating conditions the capacitor bank delivered a peak current of $3.0 \mathrm{MA}$ at 3.2 usec: The plasma focus conditions varied, but typical values for pinch current and time were $2.2 \mathrm{MA}$ and $4.8 \mathrm{\mu sec}$. Other parameters of: interest were an initial $\mathrm{dI} / \mathrm{dt}$ of $2 \times 10^{12} \mathrm{~A} / \mathrm{sec}$ and a pinch $\mathrm{dI} / \mathrm{dt}$ value of $1.6 \times 10^{12} \mathrm{~A} / \mathrm{sec}$.

The silver activation counter was used as the primary measure of neutron yield for each pulse. These values were confirmed by counting a sulphur pellet that had been exposed at the vacuum chamber wall, $23.2 \mathrm{~cm}$ from the gun axis. A histogram of the 20 pulses above 14 $\mathrm{kV}$ is shown in Figure 7 . The average yield was $3.6 \times 10^{12} \mathrm{n} / \mathrm{pu}$ sse and the highest yield was $5.8 \times 10^{12}$.

Neutron fluence in the sample holder was determined by sulphiur activation. The forward surface of the 1/4 inch-thick sulphur pellet was set back a short distance from the maximum flux position insicle the sample holder to allow for a cadmiun shield and a polyfoam shock absorber. Many pulses were used to activate the pellet, so the single-pulse fluence results were obtained by normalizing the

$-$

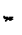


sulphur pellet reading to the total yield measurements.

It was found that because of its nearness to the plasma focus, the sulphur pellet was nonuniformly activated in the diverging flux field. The forward surface fluence determination was about 30 percent higher than the rear surface determination. For this reason, it seemed appropriate to extrapolate the measurements to the maximum flux position inside the sample holder. The maximum fluence per pulse was $1.0 \times 10^{11} \mathrm{n} / \mathrm{cm}^{2}$ and the average value was $6.2 \times 1010 \mathrm{n} / \mathrm{cm}^{2}$.

The $14 \mathrm{MeV}$ neutron pulse shape was measured with the photodiode scintillator combination. This detector, inside of a heavywalled lead shield (1.5-inch thick), was positioned at 90 degrees to the gun axis and at a distance of 35 inches from the focus.

The neutron pulse shape is generally characterized by a fast rising leading edge and a slower falling trailing edge. The full width at half maximum is approximately 100 nsec. The pulse shape varies from pulse to pulse, but exhibits some regularities that are apparent on each shot. The typical pulse exhibits a head-and-shoulders type of structure which is best exemplified by the pulse shown on the upper trace of Figure 8 . This shape is suggestive of three separate bursts of neutrons that are overlapping in time.

The occurrence of the three peaks in time relative to the leading edge of the neutron pulse is remarkably consistent from shot to shot. In the oscillograph, the pulse appears as a stepped rise to a peak and then another step on the trailing edge. The first step, or peak, occurs at $25 \pm 5 \mathrm{nsec}$, the second peak occurs at $60+10$ nsec and the third peak or step is broader and occurs at approximately $120 \pm 20 \mathrm{nsec}$. Al1 three peaks are not always apparent. When they occur, however, they occur at the previously indicated times. Generally, the second peak is of the greatest amplitude.

$4 \times 10^{19}$ The peak emission rate is approximately neutron/sec. This corresponds to a peak flux at the most advanced target position in the sample holder of approximately $0.8 \times 10^{18}$ neutron $/ \mathrm{cm}^{2}-\mathrm{sec}$.

\section{APPLICATIONS}

The DPF system provides a unique laboratory source for those applications that require a short high-fluence pulse of $14-\mathrm{MeV}$ neutrons (up to $10^{11} \mathrm{n} / \mathrm{cm}^{2}, 10^{18} \mathrm{n} / \mathrm{cm}^{2}-\mathrm{sec}$, $0.1 \mu \mathrm{sec})$. Some of the potential uses of this source are described below.

Transient and permanent neutron damage in semiconductor materials and electronic components can be studied at the high-fluence sample position near the plasma focus. Recent experiments have shown that annealing after exposure to $14-\mathrm{MeV}$ neutrons is different from that expected on the basis of investigations with reactor neutrons (Reference 13).

Material studies are performed via activation analysis of radio-isotopes produced in the capture reactions $(n, \gamma),(n, p),(n, \alpha)$, or $(n, 2 n)$. Sometimes ambiguities arise because the signal of interest is masked by the prompt gammas from inelastic scattering reactions $\left(n, n^{\prime} \gamma\right)$. The use of a short neutron pulse permits the differentiation of these reactions.

A conceptual design has been made of a slow-neutron camera using the DPF source. The space available inside the hollow center electrode would be used to hold the moderator and collimation. Optical photographs of a scintillator-produced image would be aided by an image intensifier. It is estimated that the exposure produced by high-yielding single pulses should be sufficient for stop-action photographs.

\section{SUMMARY OF SYSTEM CHARACTERISTICS}

Pulses of $14-\mathrm{MeV}$ heutrons are produced by the 500-k.J coaxial plasma gun. A tritiumdeuterium gas mixture is ionized and compressed in the coaxial gun to form a dense plasma focus within which the ${ }^{3} \mathrm{H}(\mathrm{d}, \mathrm{n}){ }^{4} \mathrm{He}$ fusion reaction occurs.

A summary of the information pertinent for potential users of the radiation source is given below.

Initial neutron radiation data

$14-\mathrm{MeV}$ neutron yield $6 \times 10^{12} \mathrm{n} / \mathrm{pulse} 3.6 \times 10^{12}$ Fluence at test area $1 \times 10^{11} \mathrm{n} / \mathrm{cm}^{2} 6.2 \times 10^{10}$ Pulse width (FWHM) $1 \times 10^{-7}$ seconds

\section{Diagnostic measurements available}

Neutron yield of pulse-by silver activation counter at remote position

Fluence at test area-by sulphur activation at test position

Pulse shape-by fluor-photodiode pulse monitor 


\section{Pulse repetition rate}

Working rate 20 pulse/day Minimum pulse interval 5 minutes

(The minimum pulse interval is inherently short. However, experience has led us to project the above working rate for an instrumented test program.)

\section{Access to test area}

The test area is located at the the top of a vertical 12-inch by 1.25 -inch ID sample ho1der.

The sample holder is located inside of a 13foot by 8-foot diameter steel tank (at atmospheric pressure).

\section{REFERENCES}

1. J. W. Mather, "Investigation of the HighEnergy Acceleration Mode in the Coaxial Gun," Phys. Fluids (Supp1.) 7, 11, S28 (1964)

2. Proceedings of the APS Topical Conference on Pulsed High-Density Plasmas, LA-3770, Los Alamos Scientific Laboratory (September 1967).

3. "Program of the 1968 Annual Meeting of the Division of Plasma Physics," Bull. Am. Phys. Soc. (II), 13, No. 11 (1968). See Session 5C and Abstract No. 6C6, 7B5, $3 \mathrm{C} 10,1 \mathrm{~A} 14$.

4. J. W. Mather, "Dense High-Temperature Deuterium-Plasma Focus," Bu11. Am. Phys. Soc. 10, 228 (1965).

5. J. W. Mather, "Formation of a High-Density Deuterium Plasma Focus," Phys. Fluids, $\underline{8}$, 366 (1965).

6. H. F. Rugge, D. E. Maxwe11, and S. A. Zwick, Plasma Focus Device Study, SAMSo TR-67-107 (October 1967).

7. D. C. Gates, C. K. Hinricks, and D. L. Lafferty, Development of a Laboratory Coaxial Plasma Radiation Source, AFWLTR-68-88 (1968).

8. J. W. Mather and P. J: Bottoms, "Characteristics of the Dense Plasma Focus Discharge," Phys. Fluids, 11 (1968).
9. E. H. Beckner! "Production and Diagnostic Measurements of Kilovolt High-Density Deuterium, Helium, and Neon Plasmas," J. App1. Phys. 37, 4944 (1966); "Pulsed, High-Intensity Source of Soft X-rays," Rev. Sci. Insitrum. 507 (1967); "Detailed Measurement of the $\dot{X}$-ray Emission Spectra of High-Densily Kilovolt Plasmas," Proc. APS Topical Conf. on Pulsed High-Density Plasma," LA-3770, Los Alamos Scientific Laboratory (September 1967).

10. D. A. Meskan, H. L. van Paassen, and G. G. Comisar, "Neutron and $\mathrm{X}$-ray Production in a Forward Z-P nch," Proc. APS Topical Conference on Puised High-Density Plasma, LA-3770, Los Alamos Scientific Laboratory, (September 1967).

11. H. L. van Paasisen, X-ray Measurements on the Mark 1-A Three Dimensional Plasma Pinch, Aerospace Report No. TR-1001 (2550-10)-1 (ig67).

12. D. Gates, L. Iemeter, and A. Noeth, Deve1opment of a Coaxial Plasma Gun, AFWL-TR70-159, Kirtliand Air Force Base, New Mexico, 87117, February 1971.

13. J. R. Srour, 0. L. Curtis, Jr., J. App1. Phys., 40, 40132 (1969). 


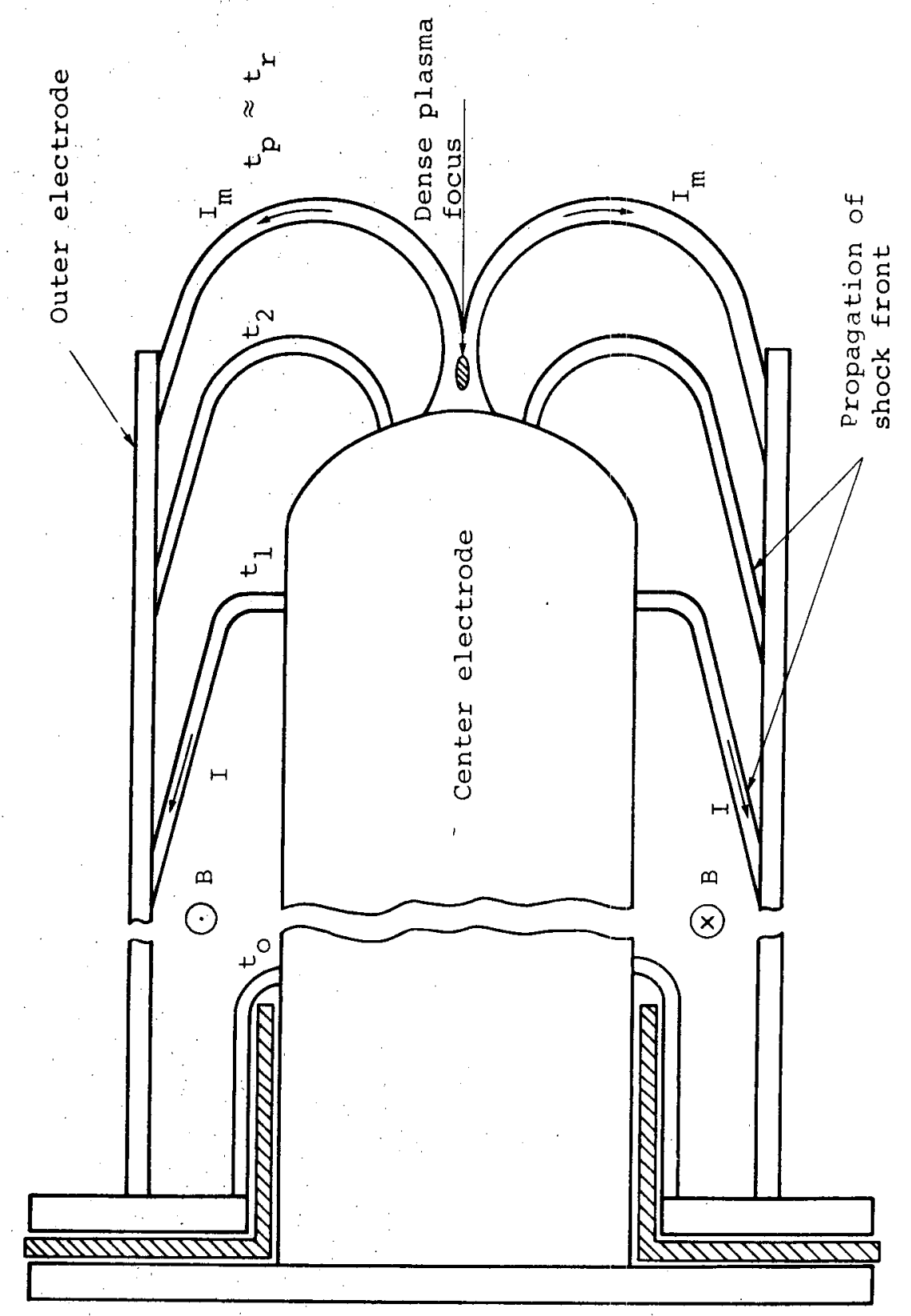

FIGURE 1. CURRENT SHEET PROPAGATION IN COAXIAL PLASMA GUN 


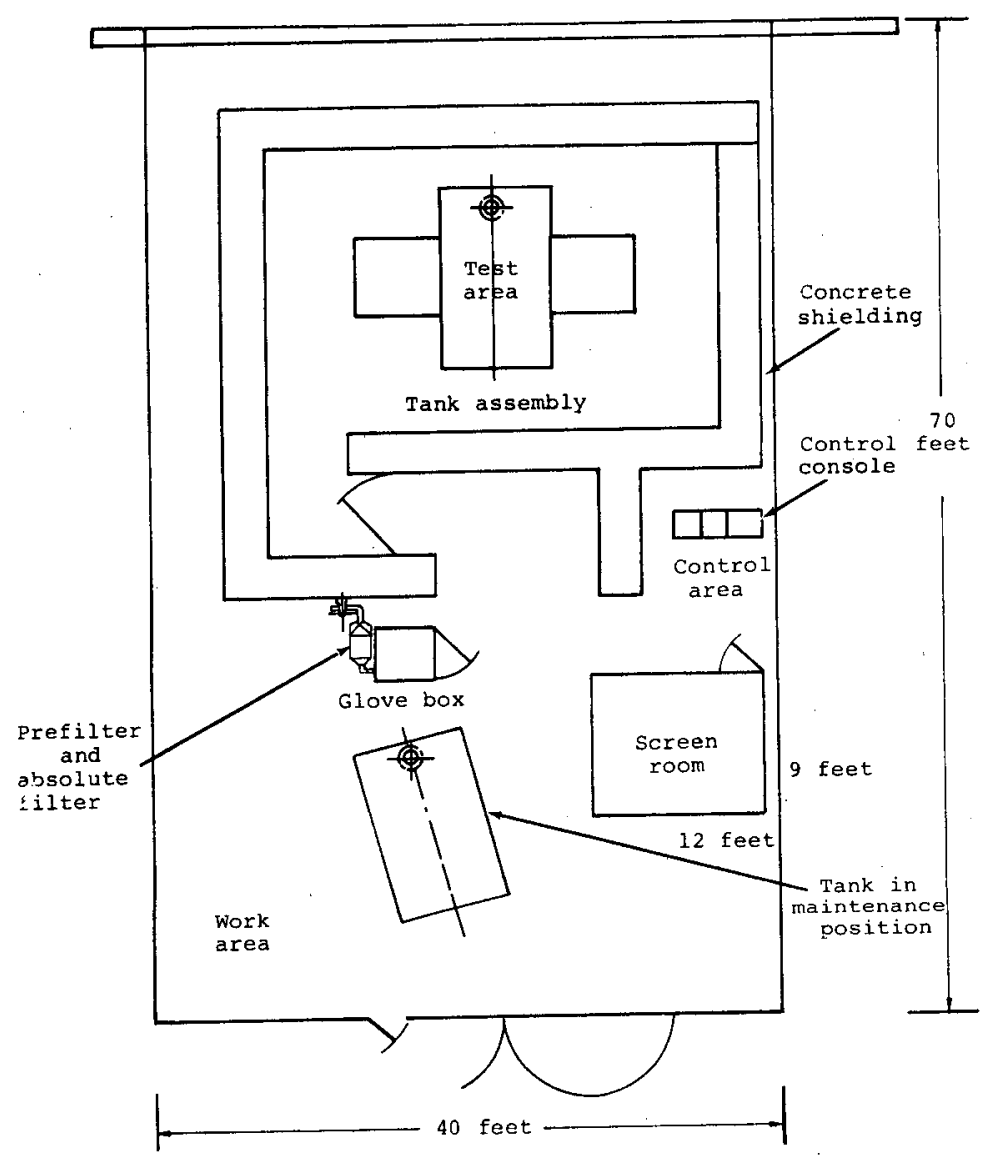

FIGURE 2. PLASMA GENERATOR LAYOUT

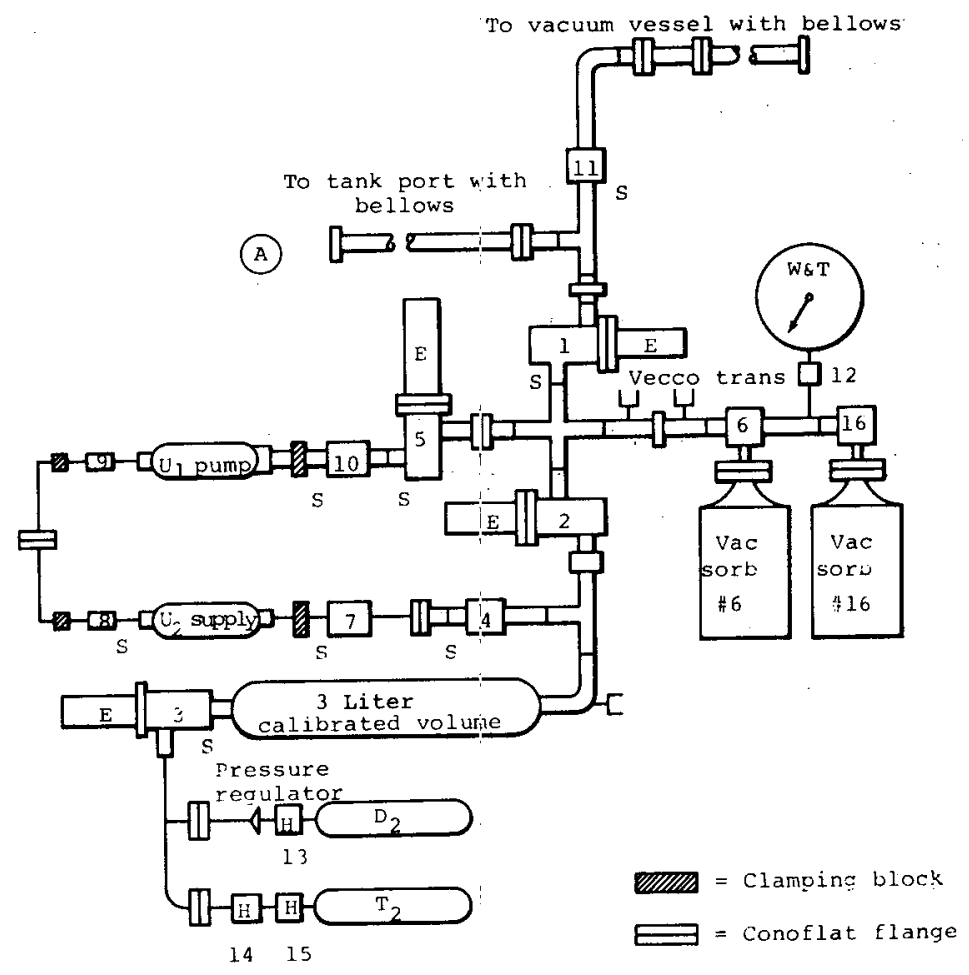




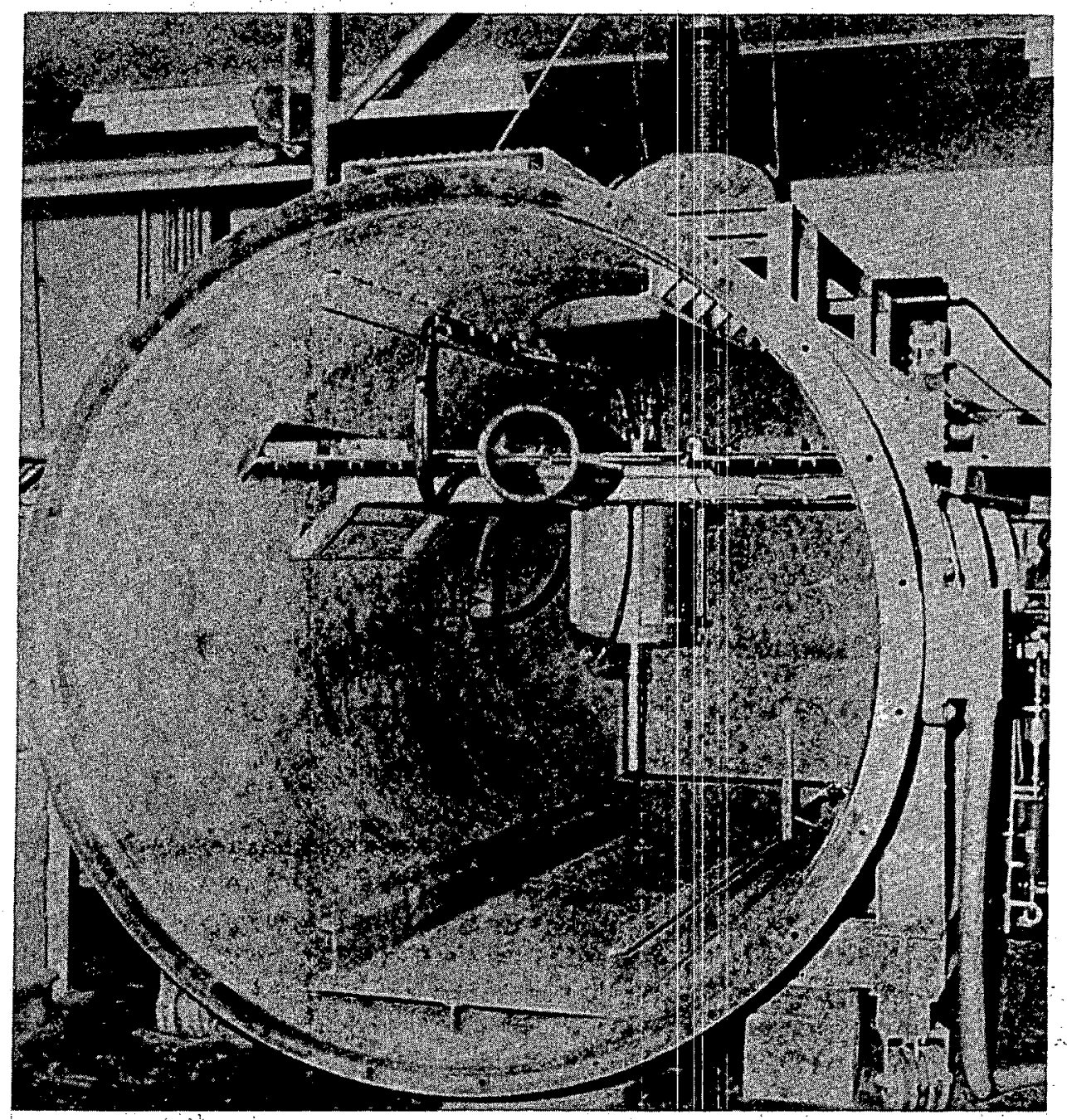

FIGURE 4. PLASMA GENERATOR-NEUTRON MODE : 


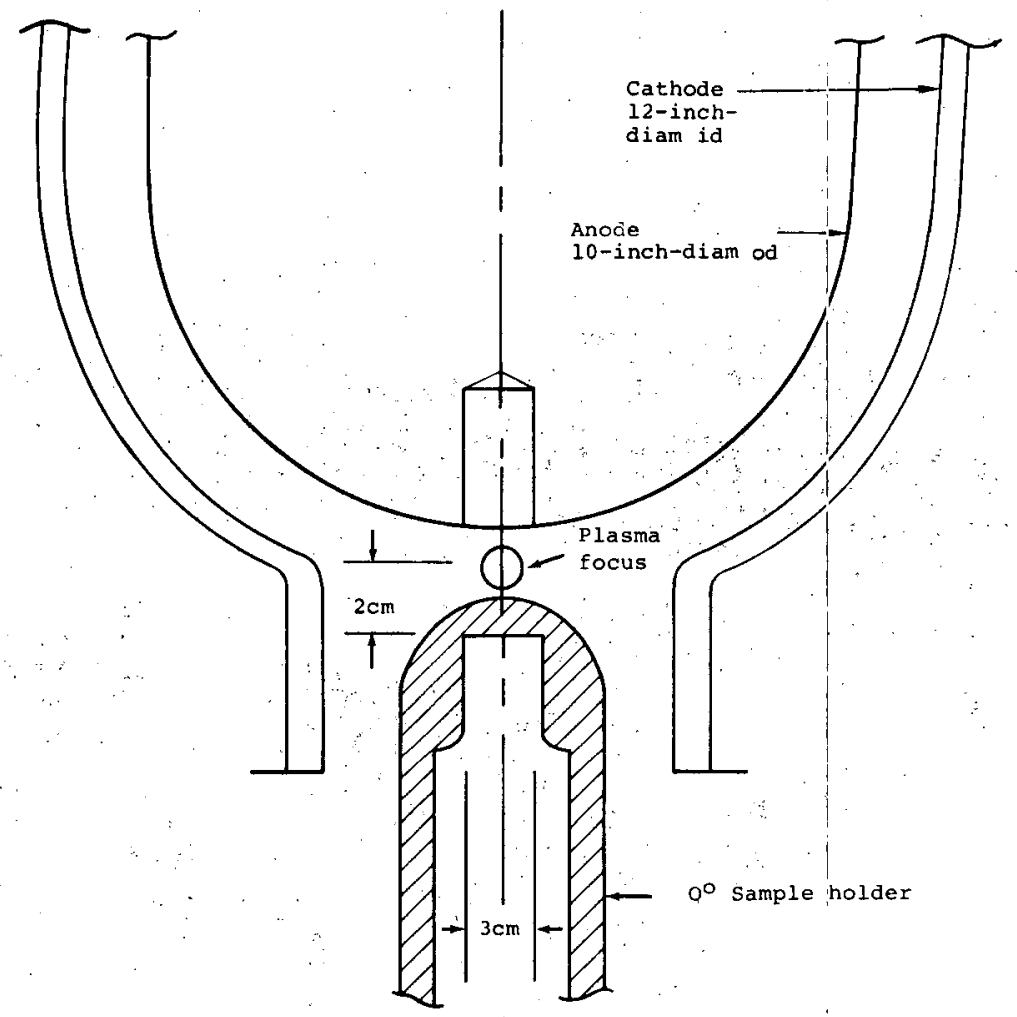

FIGURE 5. ZERO-DEGREE SAMPLE HOLDER AND NEUTRON PLASMA GUN ELECTRODES
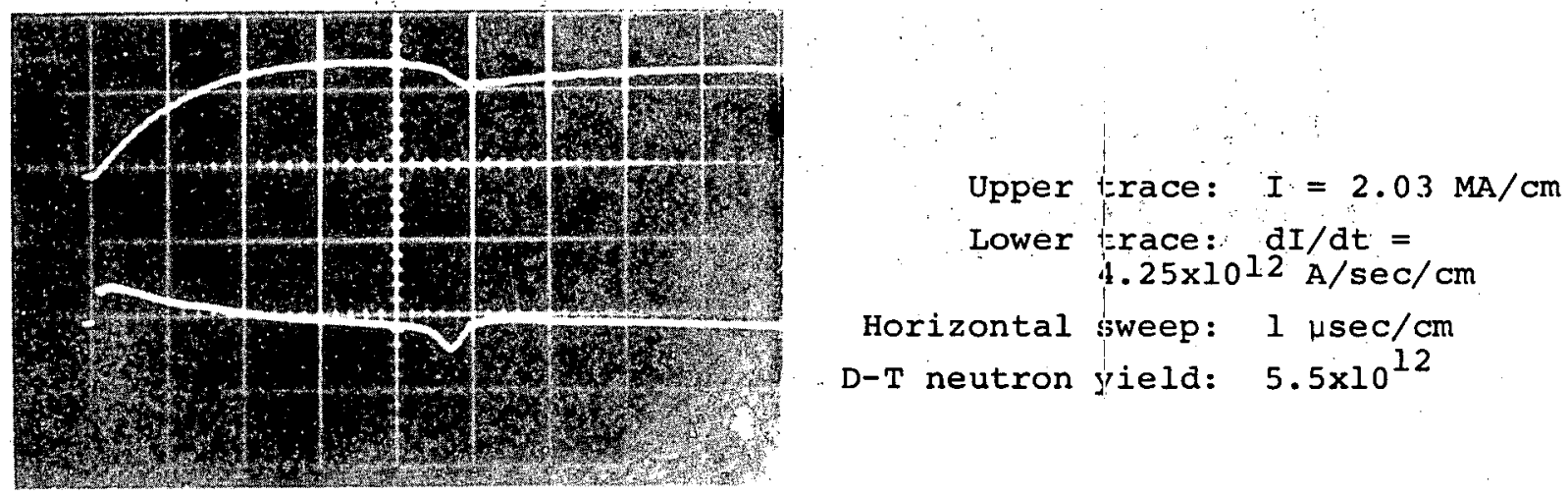

Horizontal sweep: $1 \mathrm{\mu sec} / \mathrm{cm}$ D-T neutron yield: $5.5 \times 10^{12}$

FIGURE 6. OSCILLOGRAPH OF 10x12-9 PLASMA GUN PULSE AT $20 \mathrm{kV}$ AND 9 TORR D-T GAS MIXTURE 


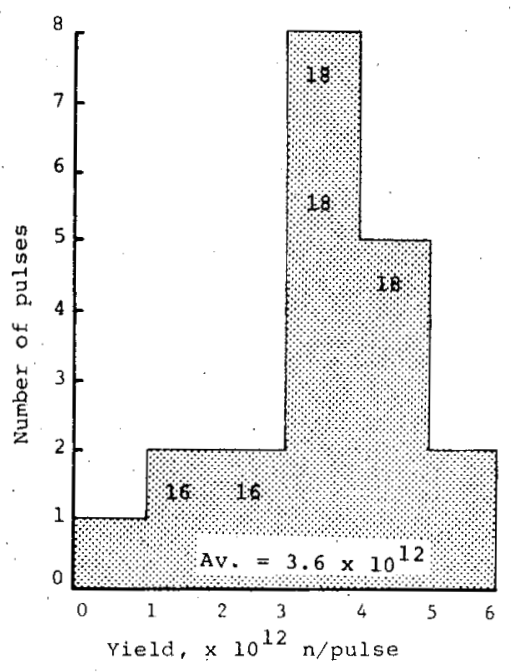

FIGURE 7. HISTOGRAM OF 14-MeV NEUTRON PULSES FOR COAXIAL PLASMA GUN 10x-12-9. NUMBERS WRITTEN INSIDE SHADED AREA SHOW POSITION OF YIELDS FOR PULSES BELOW $20 \mathrm{kV}$

FIGURE 8, OSCILLOGRAPHS ILLUSTRATING VARIATIONS IN D-T NEUTRON PULSE SHAPES OF $10 \times 12-9$ PLASMA GUN

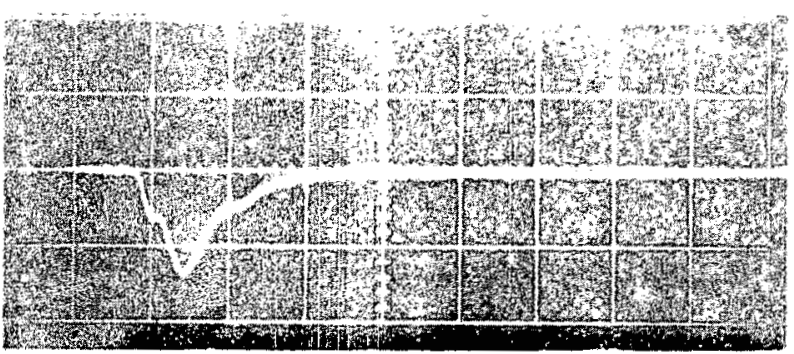

a.

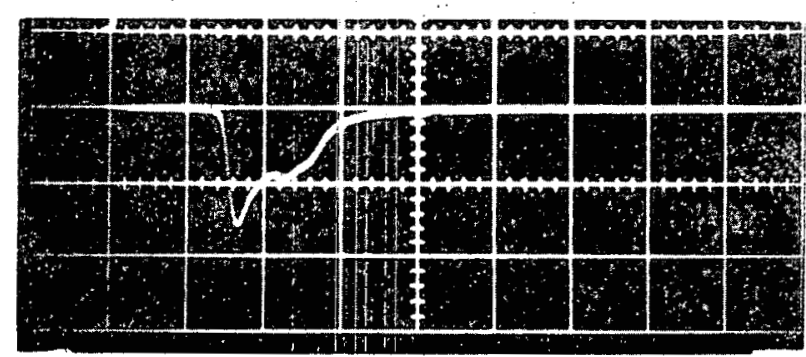

b.

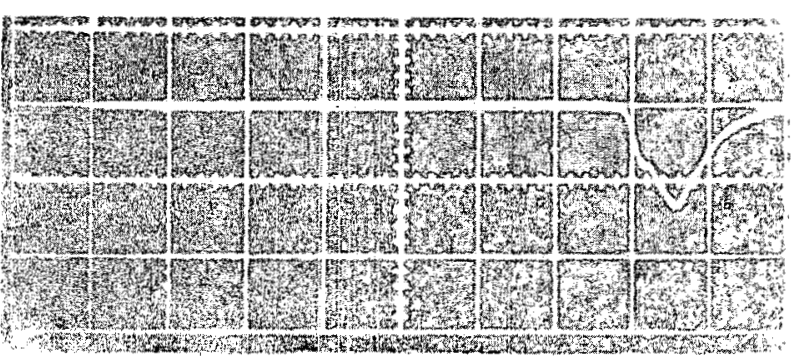

c.

Sweep: $0.1 \mu \mathrm{sec} / \mathrm{cm}$ 


\section{USING A MESON FACTORY AS AN INTENSE PULSED NEUTRON SOUNRE}

\section{Ralph R. Fullwood}

Los Alamos Scientific Laboratory

Los Alamos, New Mexico
The Weapons Neutron Research (WNR) facility is proposed for adapting the Los Alamos Meson Physics Facility (LAMPF) to pulsed neutron production in the range 1-20 Mev. Intensity estimates are given and associated problems are discussed.

\section{INTRODUCTION}

What will be the characteristics of neutron sources five or more years in the future, as seen from todays experience? If present trends continue, these sources will likely be used for programmatic experiments. The source that is the most versatile and would most probably fit this need is the pulsed "white" source, as exemplified by the high-powered electron linacs. The general trend of these accelerators has been working up the neutron energy and intensity scale to the point that they cover the range from thermal energy to a few $\mathrm{MeV}$, or slightly above the $\gamma-n$ production spectrum maximum. An extrapolation that predicts future white sources will be covering a considerably higher energy range is not hard to believe.

The following are characteristics based largely on experiences at this Laboratory as to the desirable characteristics for future neutron sources:

(1) It should be a copious source of neutrons in the 1-20 MeV range.

(2) The pulse duration should be variable from a few nsec to a few usec. Pulse duration below one nsec does not seem particularly desirable because of dedector and target problems.

(3) The source should produce only neutrons with minimal other penetrating radia- tion. The gamma flash associated with electron linacs has been a compromising element in extending the neutron energy range. Many of the techniques for "riding through" the gamma flash do so at a sacrifice in source intensity. Many neutron detectors are actually more gamma than neutron sensitive.

(4) The charged-particle beam should be of good quality to facilitate low-loss transport to the experiment as required. This would seem to be the limitation of circular machines such as those at Karlsruhe or Columbia that use an internal beam of protons for neutron production.

(5) The source should produce a maximum number of neutrons for a minimum amount of target heating to allow intensities to rise above the present levels.

(6) The source should be in a we11-shielded enclosure with a wide variety of flight paths, including several very short ones. At least one spacious room for the suppression of neutron room return should be available. Facilities for plutonium experiments should be provided. The target:s and enclosures should be designed to facilitate repair in spite of high resiclual activity.

Before investigating how well LAMPF can be adapted to fill these requirements, the 
characteristics of neutron production by high energy protons incident on heavy nuclei targets must be examined. This has been studied both theoretically $(1,2)$ and experimentally $(3,4)$. If $800-\mathrm{MeV}$ protons interact in a $238_{\mathrm{U}}$ target, from 17 to (perhaps) 30 neutrons are produced, depending upon whether it is a thin or a very thick target. In general, thick targets are desired because of higher efficiency, softer spectrum, and suppressed gamma output. In this case, the gamma output is from deexitation of nucle $i$ and is soft, compared with typical electron linac bremstrahlung.

The relative efficiency of several neutron sources is presented in Table I. Some

\section{TABLE I}

BEAM ENERGY TO PRODUCE ONE NEUTRON FOR VARIOUS SOURCES

\section{Source}

Hermes II (15-MeV electrons on ${ }^{238} \mathrm{U}$ )

Energy $(\mathrm{MeV})$

ORELLA (140-MeV electrons on $\mathrm{Ta}$ )

8800

Harwe11 (40-MeV electrons

3200 and 10X booster)

WNR ( $800-\mathrm{MeV}$ protons on ${ }^{238} \mathrm{U}$ )

caution should be used with Table I in that this is the beam power-it does not reflect the target heating from nuclear reactions occurring within the target; however, fission in a target should produce the same heating regardless of the accelerator source.

Another requirement is that the source be copious in 1-20 MeV neutrons. Figure 1 shows a comparison between $B(e, \gamma, n), P B(e, \gamma, n)$, and $\mathrm{U}(\mathrm{p}, \mathrm{xn})$ spectra. The $\mathrm{U}(\mathrm{p}, \mathrm{xn})$. spectra is normalized to the $\mathrm{Pb}(e, \gamma, n)$ spectra at $1 \mathrm{MeV}$. An inspection of Fig. I shows that the spectra from a $U(p, x n)$ target resembles the $B_{e}(e-\gamma-n)$ target, but in employing $\mathrm{B}_{e}$ instead of $\mathrm{Pb}$ the $(e-\gamma-n)$ source pays a penalty in intensity.

The $(p, x n)$ source, while being low in gamma-flash relative to the $(e-\gamma-n)$ source, has two disadvantages. It has a high-energy neutron component. Figure 2 shows the neutron angular distribution from this source. It is believed that problems from this component can be avoided by working at back angles, by neutron time-of-flight, and by the fact that they are very few and the efficiency for detection is very low. Another disadvantage of the $(p, x n)$ source for high-energy protons is the targets must be about $30 \mathrm{~cm}$ long to be thick for this proton energy. This results in spatial uncertainty which can be minimized by viewing the source at right angles to the beam and by a reduction in target fluence $\left(\mathrm{n} / \mathrm{cm}^{2}\right)$ which must be considered in the design of an experiment.

To see how these ideas may be incorporated into LAMPF, certain characteristics are needed which are given in Table II.

TABLE II

\section{MAXIMUM CHARACTERISTICS OF LAMPF}

Beam energy

$800 \mathrm{MeV}$

Average proton current during

$17 \mathrm{~mA}$

the pulse

Average $\mathrm{H}^{-}$current during the pulse $1.7 \mathrm{~mA}$

Extraction efficiency

$100 \%$

Pulse duration

Pulse repetition rate

$500 \mu \mathrm{sec}$

Average proton beam power

120 pps

Energy spread

$800 \mathrm{~kW}$

Beam emittance

$\pm 0.4 \%$

Beam microstructure

$\pi / 3 \mathrm{mrad}-\mathrm{cm}$

0.25 nsec pulses at 5 nsec period

Duty factor

$6 \%$

Anticipated utilization

$120 \mathrm{hr} / \mathrm{wk}$

Using the optimistic number, 30 neutrons/ proton arid the beam current of Table II, the instantaneous neutron production rate is $3 \mathrm{x}$ $10^{18} \mathrm{n} / \mathrm{sec}$. which is nearly the maximum rate of ORELLA with a Ta target. (If ORELLA used a $238 \mathrm{U}$ target, a rate of $8 \times 10^{18} \mathrm{n} / \mathrm{sec}$ would be expected.). Thus, by simply chopping the beam, the production rate compares we 11 with the best of present accelerators at their maximum production rate. An important consideration is that the number of neutrons per pulse will be proportional to the pulse width for LAMPF, while for ORELLA it is practically independent of pulse width. (ORELLA operates on stored energy.)

Next are two related problems. The pulse is too long for most applications and, with a utilization of $120 \mathrm{hr} / \mathrm{wk}$, there is essentially no time left for the neutron experiments being considered. The solution to 


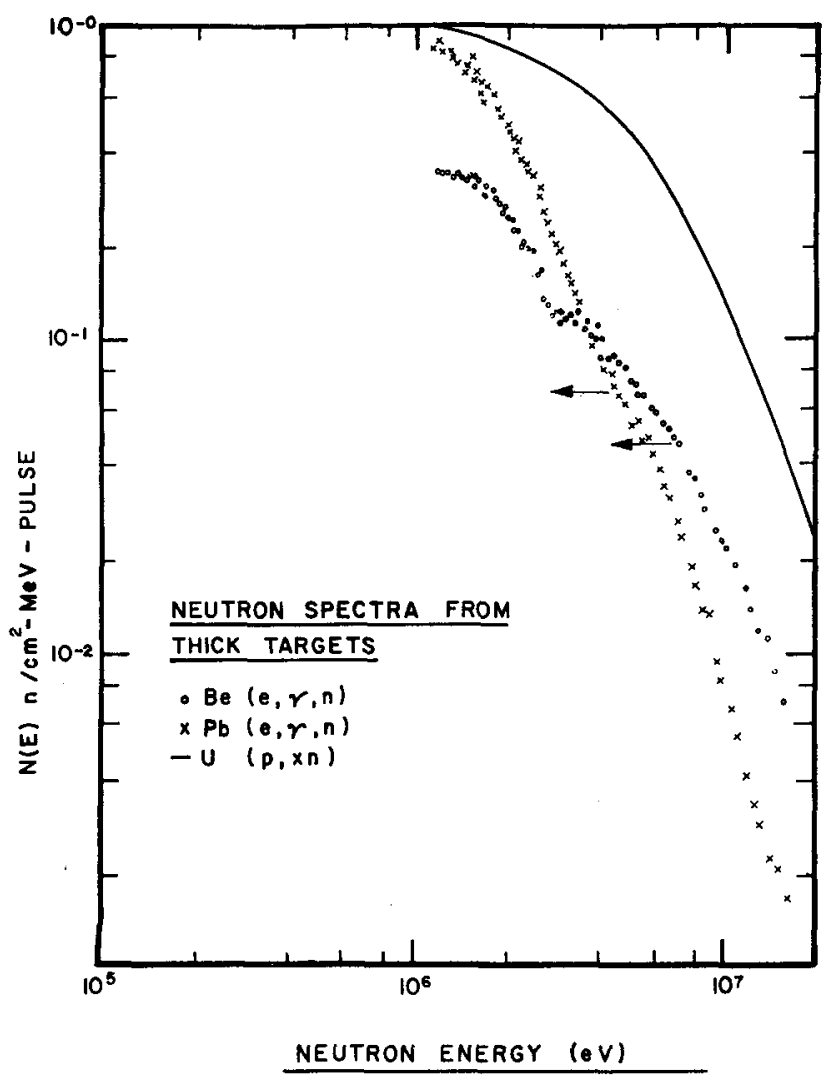

Fig. 1. Neutron spectrum from an electronneutron source (Ref. 6) compared with a proton-neutron source (Ref. 7).

both problems is to chop the beam into the desired pulse duration and, since the duty is low, to multiplex between the neutron experimental facility and the medium energy experimental area so that both types of experiments may run concurrently.

WNR

These ideas culminated in the proposed WNR facility. The major planners of this facility are A. Hemmendinger, J. R. Beyster, L. E. Agnew, and R. R. Fullwood.

Figure 3 shows, schematically, the processes involved in chopping and time-sharing the beam. The chopping would be done while the protons are at $750 \mathrm{keV}$. It is considered too difficult to try to chop at lower energy in the ion column; however, at this location the beam is at ground potential and there is room for the necessary chopping. The proposed technique for intensity control is to use a pulsed electrostatic quadrupole to defocus the beam during the WNR pulse. This defocus-

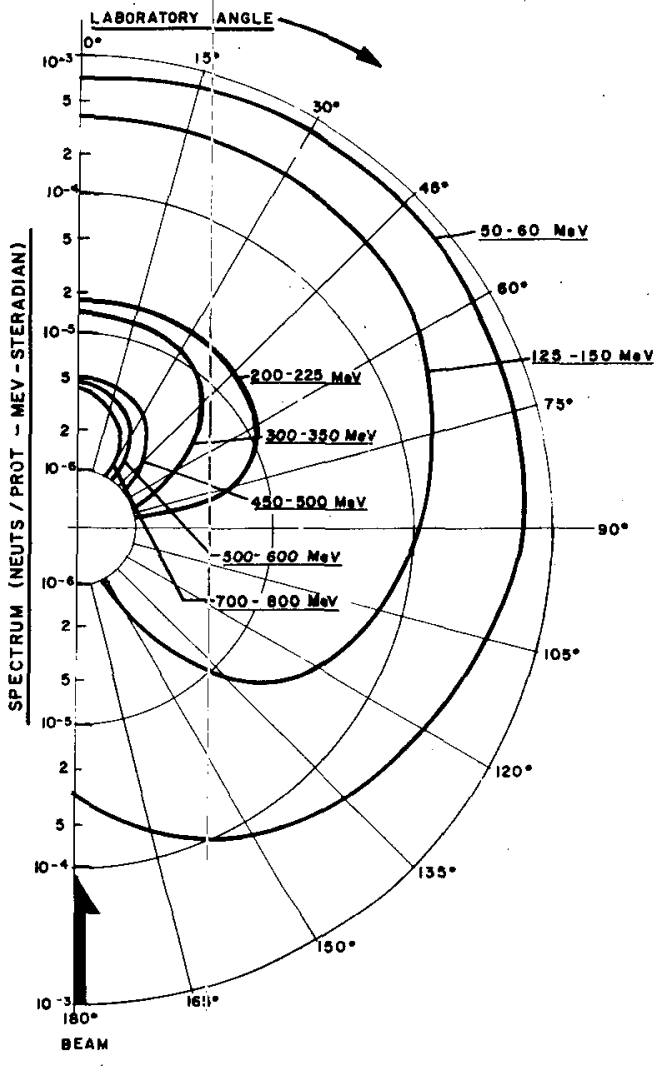

Fig. 2. Ángula neutron distribution from a 10-cm-diam $\times 30-\mathrm{cm}-1$ ong $U$ target, $800-\mathrm{Mel}$ protons incident on axis, as calculated with the ORNL code NMTC with the LASL code GMC treating the neutron transport below $25 \mathrm{MeV}$.

ing must be completely removed at the time for the meson-producing pulse. Four usec are allowed for this mode switch-over. It is considered that 20 -nsec pulses are about as short as can be produced by electrostatic chopping. Also, higher intensities are desired at the very short pulse lengths. A subharmonic chopper (5) is under study to provide several nsec pulses of perhaps $4-6$ times the $5 \times 10^{8}$ protons that are ordinarily in a microstructure piacket. In addition subharmonic bunching would allow a blank space to facilitate the chopping. Further advantages result from the fact that there are some neutron timi-of-flight experiments and detector calibration problems that can profitably use a burst repetition rate of $20 \mathrm{MHz}$.

When the WNiR pulse reaches the ferrite core magnet, labsled $\mu$-pulser, this magnet is pulsed to $2 \mathrm{kG}$ and this pulse is deflected $3^{\circ}$ into a dc magnet channel where it is bent through $87^{\circ}$ in the horizontal plane and $17.5^{\circ}$ 


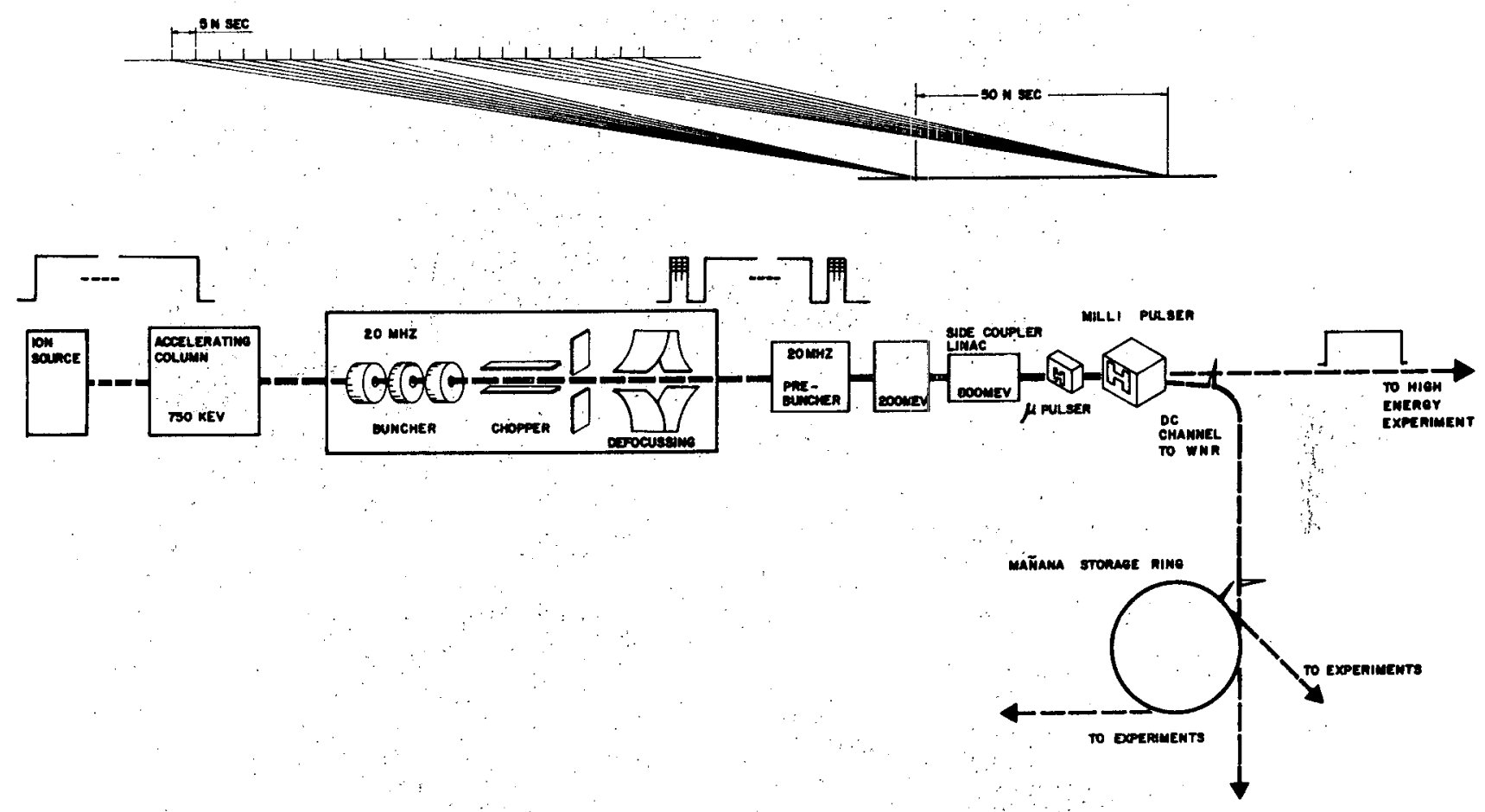

Fig. 3. Block diagram of the beam multiplexing arrangement to provide parallel operation between neutron and medium-energy experiments. The Applegate diagram (top of figure) shows the velocity modulation necessary for subharmonic bunching.

downward in the vertical plane. After. $90 \mathrm{ft}$, it is deflected upward $17.5^{\circ}$ so that it is traveling horizontally south for $490 \mathrm{ft}$ to reach WNR.

The magnet labeled milli-pulser in Fig. 3 is a laminated-core magnet that operates on a millisecond time scale so that whole $500 \mu \mathrm{sec}$ or less pulses from LAMPF can be diverted to WNR. Of course, when this is done, no pulse is available for meson production.

The storage ring shown in Fig. 3 is a design study to store the charge from many $\mu-$ pulses or a milli-pulse--or possibly an $\mathrm{H}^{-}$ pulse (the design is not determined). The ring of magnets would have a period of about $100 \mathrm{nsec}$ so that if all the current could be extracted in one revolution, the resulting proton pulse would be $100 \mathrm{nsec}$. It may be possible, by making the frequency of the ring $10 \mathrm{MHz}$ which is a subharmonic of $200 \mathrm{MHz}$, to have axially asymmetric current distributions in the ring and, thus, be able to store a shorter pulse than the period of the ring.
Figure 4 shows a simplified concept of the WNR proposa1. The beam coming from LAMPF emerges from the horizontal beam channel just described. It may be deflected by a bending magnet into Target Room 1 , which has a thin grating floor to give a minimum distance of $15 \mathrm{ft}$ from the experiment to the walls, thereby reducing the neutron room-return problem. Target Room 1 is intended for neutron transport problems that require long setup time and fairly short running time. It is intended to keep the average radiation level: low in this room to facilitate personnel entry.

When personnel are working in Target Room 1 , the beam pipes would be plugged and the beam would enter the storage ring or travei straight to Target Room 2 (the high average radiation level room). Here it is anticipated that high residual activities will exist inhibiting access; hence, experiments would not be performed in this room but the room would house the neutron production targets used for various experiments. The distance from the target to the wall is $12 \mathrm{ft}$, as shown in Fig. 


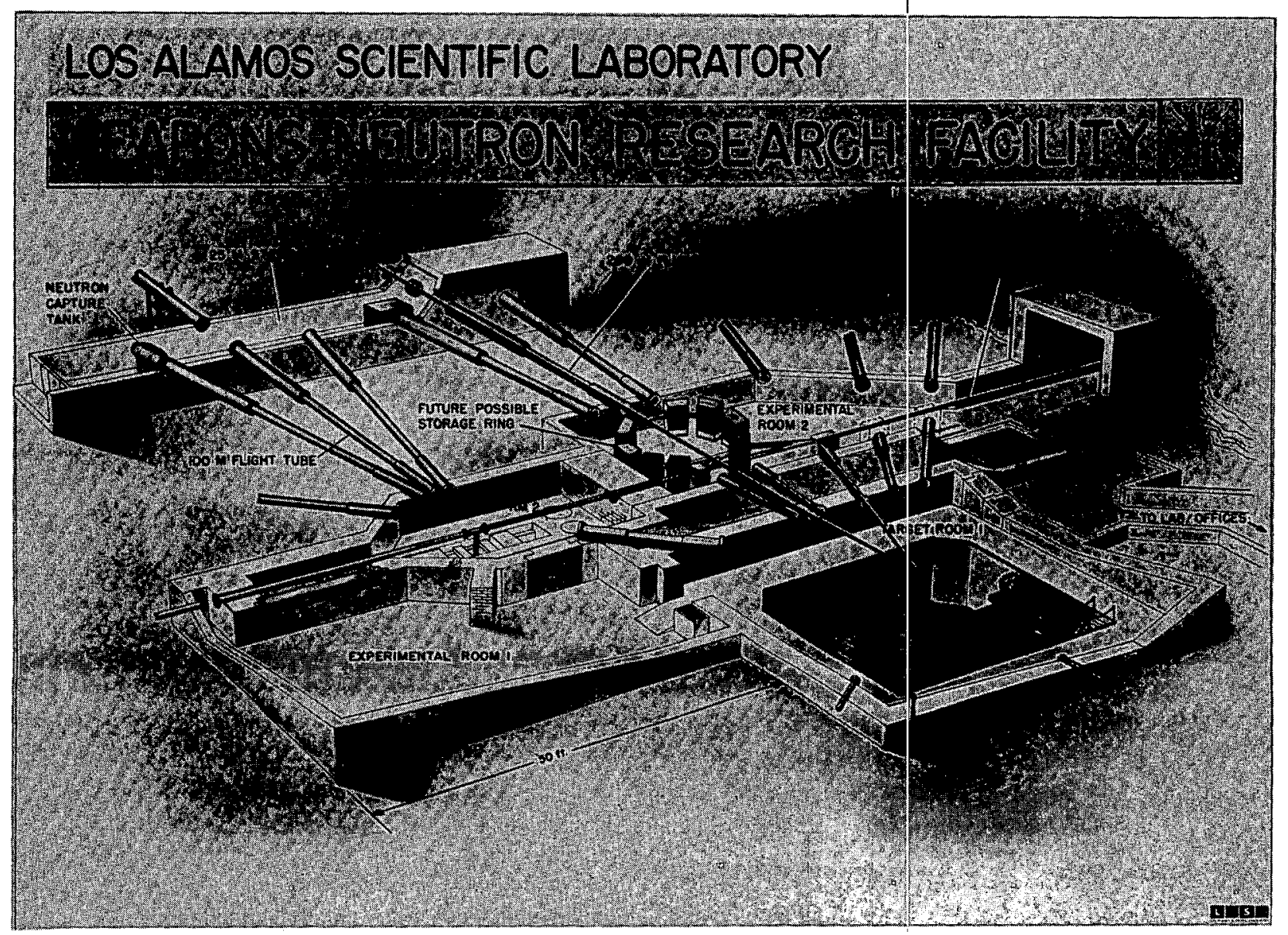

Fig. 4. Artist's concept of WNR facility. The beam enters from the upper right. The storage ring is not in the proposal, but is under study.

4 , in order to minimize the room scatter that would constitute a second source and compromise time-of-flight experiments.

The target trench extends to the end of the narrow part of Target Room 2. The targets are mounted in integral shields, called crypts, and are raised from the crypt by a compound hydraulic lift. It is planned to mount severa1 targets, one above the other, and by remotely controlling the height, select any one of several targets--depending upon the neutron spectrum desired. By rolling the crypt into the narrow part of Target Room 2, a shielded flight path as short as $6 \mathrm{ft}$ is possible. To obtain the maximum flexibility on these short flight paths, pipes are not cast into the wall but a large-stepped, special plug containing the pipes required for the experiment is fitted into the window (shown in Fig. 4). The break in the pipe at the end of Target Room 2 is for possible installation of a quadrupole lens for transporting the proton beam out of WNR for some possible future application. This beam pipe can also serve for highenergy neutron time-of-f1ight experiments.

The entire facility would be buried under a minimum of $20 \mathrm{ft}$ of dirt with the thickness being considerably increased over Target Room 2. This berm would extend to the retaining wall at the $25-\mathrm{m}$ station. The reason for the large diameter pipes at this location is to facilitate resonance neutron radiography. Neutron radiography is also planned for the 6-ft station.

The diagonal pipe shown connecting Target Rooms 1 and 2 might also be used for radiography, but is primarily for allowing neutrons 
produced by a target in Target Room 2 to be incident on an assembly whose neutron transport properties are being studied in Target Room 1. This will allow accurate normalization of the experiments.

Objectives of the WNR facility are presented in Table III.

TABLE III

OBJECTIVES OF THE PROPOSED WNR FACILITY

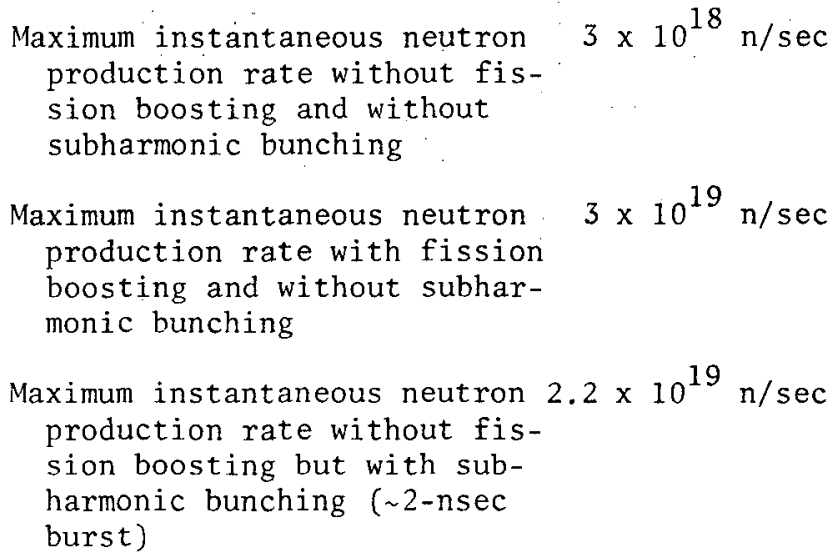

Maximum instantaneous neutron $2.2 \times 10^{19} \mathrm{n} / \mathrm{sec}$ production rate without fission boosting but with subharmonic bunching ( $\sim 2-\mathrm{nsec}$ burst)

Maximum average beam power $4 \mathrm{~kW}$

Maximum average neutron pro- $.8 \times 10^{15} \mathrm{n} / \mathrm{sec}$ duction rate

Repetition rates

Manual to. 240 pps

Pulse Widths

2 nsec $-1 \mu \mathrm{sec}$ and $500 \mu \mathrm{sec}$ at $<1$ pps

SUMMARY

To extend neutron sources to higher intensities and higher energies than the present level would seem to require a different production mechanism than the presently used (e, $\gamma, n)$. The reaction $(p, x n)$ would seem to provide some desirable qualities for future sources. It would seem that a meson factory can be reasonably adapted for use as a pulsed neutron source with negligible interference with its medium energy role.

\section{REFERENCES}

1. J. C. D. Milton and J. S. Fraser, A Monte Carlo Calculation of Neutron Production in Heavy Element Targets by Protons in the Range 0.3-1 BeV. Atomic Energy of Canada Ltd. AECL-2259, Chalk River Laboratories, Chalk River, Ontario (1965).

2. H. W. Bertini, "Calculation of Nuclear Reactions for Incident Nucleous and $\pi$ Meson in the Energy Range 30-2700 MeV," Intemational Conference on Radiation Shielding and Dosemetry, Stanford, California (Nov. 1970). To be submitted to the Physical Review.

3. J. S. Fraser, J. S. Hewett, and J. Wa1ker, Neutron Spectra and Angular Distribution Produced by $1 \mathrm{GeV}$ Protons on a Thick Lead Target. Canadian Association of Physicists, Sherbrooke, Quebeck (1969).

4. L. R. Veeser, J. F. Morgan, and R. R. Fullwood, experiment in progress and private communication (December 1970).

5. N. J. Norris and R. K. Hanst, "Velocity Modulation System for Enhancement of 50 Picosecond Radiation Pulse," IEEE Trans. Nuc. Sci. 16, 323 (1969).

6. D. Groce, Feasibility Study of an Accelerator-Booster, Fast Pulsed Reactor, USAEC Report GA-8087, General Atomics, San Diego, California (1967).

7. C. A. Bartholomew, Proceedings of the Internationa 1. Conference on the Study of Nuclear Structure with Neutrons, Antwerp Belgium, North Holland Publishing Co., Amsterdam, Holland (1966).

\section{ACKNOWLEDGMENTS}

Work was performed under the auspices of the United States Atomic Energy Commission. 


\section{GAS TARGET SOURCE FOR FAST NEUTRON CANCER THERAPY}

C. A. Kelsey, M. L. M. Boone, J. M. Hevezi, A. L. Wiley,

G. C. Spalek, H. K. Forsen, and W. R. Winter

University of Wisconsin

Madison, Wisconsin

Experimental and theoretical studies are undernay to investigate the use of a differentially pumped gas target as a source of fast netutrons for cancer therapy. A target containing tritium gas at a pressure of $10 \mathrm{~mm} \mathrm{Hg}$ bornbarded by $16 \mathrm{ma}$ of $250 \mathrm{kev}$ deuterons should produce $10^{13} \mathrm{n} / \mathrm{sec}$. Gas flowing out through the target entrance aperture will be returned to the target through a pumping recirculation system. Current efforts are utilizing proton and deuteron beams with hydrogen ind deuterium targets to study the focusing and cooling requirements of the system. Priesent results indicate a gas target producing $10^{13} \mathrm{n} / \mathrm{sec}$ for clinical use is technically feasible.

\section{NTRODUCTION}

Fast neutrons may be more effective for cancer therapy because they deposit their energy through secondary particles having a high linear energy transfer (LET). Such particles are expected to be more effective in destroying cells in the hypoxic or anoxic regions of tumor systems (1). These regions are believed to be the source of some local tumor recurrences. Fast neutron radiation therapy from a cyclotron is currently under study at the Hammersmith Hospital (2). Cyclotrons, however, a re not ideal radiotherapy machines because of their expense, size and lack of mobility. All of these factors are important in the development of clinically useful machines. The $d, T$ reaction is an attractive alternate neutron source because $150 \mathrm{keV}$ deuterons incident on a tritium target can produce $15 \mathrm{MeV}$ neutrons. Thus a relatively inexpensive low energy accelerator could be used to produce a beam of high energy neutrons. Accelerators having large beam outputs are presently available.

Supported in part by Grant No. CA12079-01 from the National Cancer Institute.
The only limitation on the use of the $d, T$ neutrons for cancer therapy appears to be the lack of a target capable of withstanding the required beam current and having a satisfactorily long lifetime. It is the purpose of this paper to describe the efforts underway at the University of Wisconsin to construct a gas target neutron source. The goal of the effort is a neutron source producing $10^{13} \mathrm{n} / \mathrm{sec}$ having a lifetime of greater than 500 hrs. and capable of withstanding the incident beam current of $16 \mathrm{milliamps}$ required to produce the desired neutron flux.

The goal of the source strength $10^{13} \mathrm{n} / \mathrm{sec}$ will provide a dose rate of $20 \mathrm{rads} / \mathrm{min}$ at a source-to-skin distance (SSD) of $125 \mathrm{~cm}$. Goodman has made measurements showing the depth at which the $\mathrm{d}, \mathrm{T}$ neutron dose equals $50 \%$ of the dose maximum is at a depth of $12 \mathrm{~cm}$ (for a $15 \times 15 \mathrm{~cm}$ field) (3). Moving the source closer to the patient (decreasing the SSD) increases the dose rate but also results in a smaller $50 \%$ dose maximum depth. In our opinion the $125 \mathrm{~cm}$ SSD provides a reasonable compromise between the two conflicting requirements of high dose rate and high percentage depth dose. An additional benefit of the $125 \mathrm{~cm}$ SSD is that sufficient collimation 
material can be placed between the source and the patient to reduce the neutron dose outside the radiation field.

\section{RATIONALE}

The rationale for employing a gas target for production of $d, T$ neutrons is that the power dissipation requirements of such a system are calculated to be considerably less than those of other systems which employ a solid substrate such as copper or zirconium to support the target material. This is because al1 interactions of the incident deuteron beam within the gas target contribute to the neutron flux. Figure 1 presents a comparison of the yield from gaseous and solid targets as calculated by Coon (4).

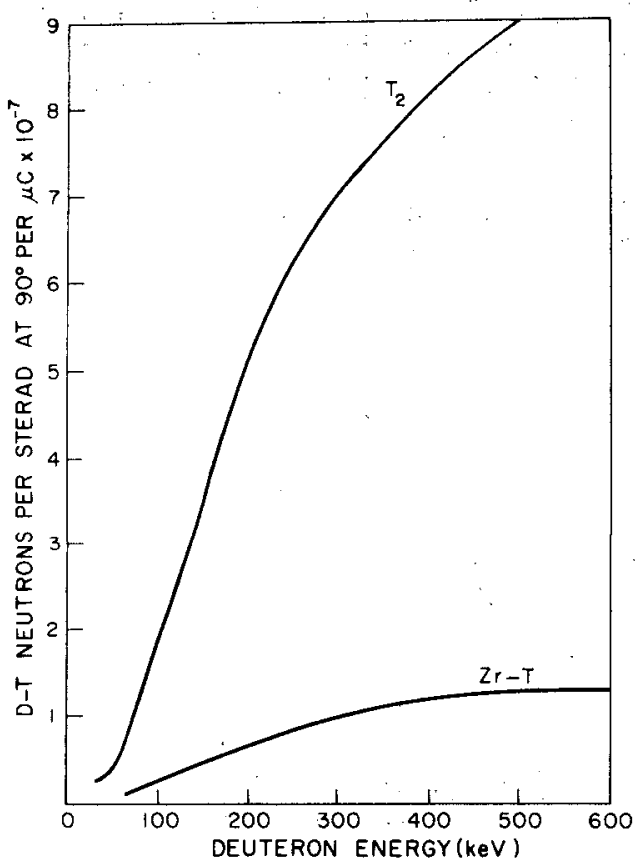

FIGURE 1

Neutron yield as a function of deuteron bombarding energy for solidbacked and gaseous tritium targets.
Calculations were made to determine the beam current required to produce $10^{13} \mathrm{n} / \mathrm{sec}$ as a function of deuteron bombarding energy. The results of these calculations are shown in Figure 2. We have chosen a deuteron bombarding energy of $250 \mathrm{KV}$ as a reasonable operating point and since the insulation requirements are not prohibitive, a $250 \mathrm{KV}$ accelerator can be operated in air.. The reduction in beam current resulting from further increases in accelerating voltage were not judged to be sufficiently advantageous to outweigh the disadvantages of working with higher voltages and more difficult insulation techniques.

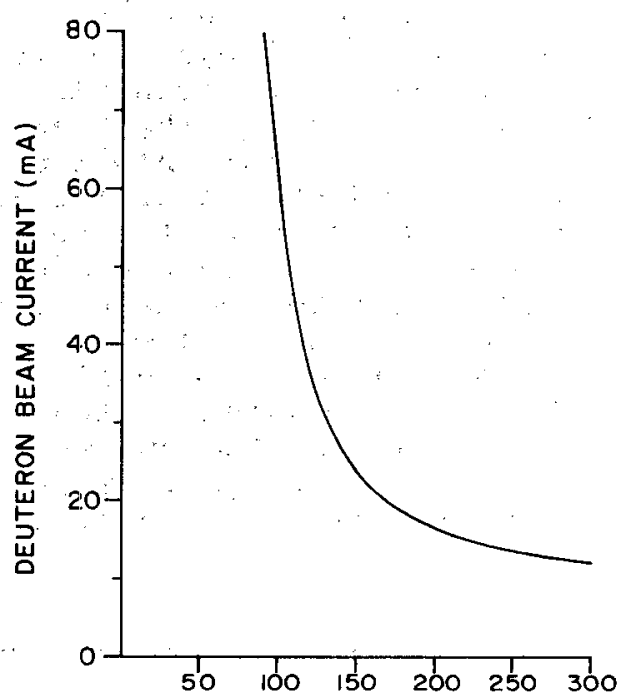

ENERGY OF ACCELERATED DEUTERONS ( $\mathrm{keV}$ )

\section{FIGURE 2}

Beam current required to produce $10^{13} \mathrm{n} / \mathrm{sec}$ as a function of deuteron bombarding energy.

\section{GAS TARGET SYSTEM}

Figure 3 presents a schematic view of the system currently under investigation to test the feasibility of using a gas target for high flux neutron production. A duo-plasmatron ion source provides a 16. $\mathrm{mA}$ deuteron beam which passes 
through a quadrapole lens after acceleration to $250 \mathrm{KV}$. The beam then passes through an intermediate aperture and comes to a focus at the final aperture which is also the entrance to the gas target. The gas target containing tritium at a pressure of 10 torr $(\mathrm{mm} \mathrm{Hg})$ serves as the source of $d, T$ neutrons. Because of the high beam currents required to produce $10^{13} \mathrm{n} / \mathrm{sec}$ it is not possible to cover the entrance aperture with a foil capable of withstanding both the pressure of the gas within the target and the beam current passing into the gas target. Thus we are designing a differentially pumped system to maintain the gas in the target and eliminate it from the beam tube. The target gas leaking out through the entrance aperture is removed by the target pumping system and returned to the gas target through a recirculator. The various apertures in the beam line reduce the gas flow into the beam pipe. To reduce gas scattering of the beam, the pressure. in the beam tube near the ion source and the accelerrating tube are maintained below $10^{-5}$ torr by the ion source pump. The patient will be positioned along an extension of the beam axis $160 \mathrm{~cm}$ from the entrance aperture of the gas target. Thus the irradiated area "sees" only a $2 \mathrm{~cm}$ diameter source.

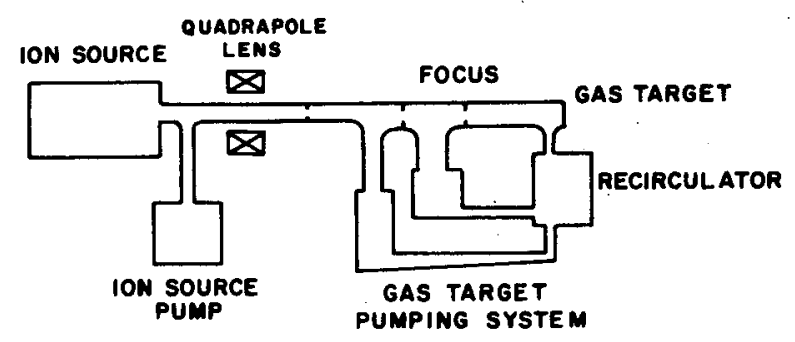

FIGURE 3

Schematic view of differentially pumped gas target system.
Neutrons are produced all along the length of the gas target. Thus the irradiated field on the patient's skin will receive neutrons from an extended source having a diameter of $2 \mathrm{~cm}$. Calculations have been performed to determine the number of neutrons reaching the patient surface from the gas target length. These calculations include both the effect of d-T cross section variation with deuteron energy and the inverse square law variation due to the length of the gas target. The results of these calculations are shown in Figure 4 for an incident deuteron energy of $250 \mathrm{KV}$, a distance between the entrance aperture and the patient sulrface of $160 \mathrm{~cm}$ and a gas target pressure of 10 torr. Notice that more than $90 \%$ of the neutrons are produced in the first $38 \mathrm{~cm}$ of the target. The length of the gas target could be reduced by a factor of 2 by increasing the pressure to 20 torr. This would however increase the gas outflow by the same factor of 2 requiring a larger pumping system.

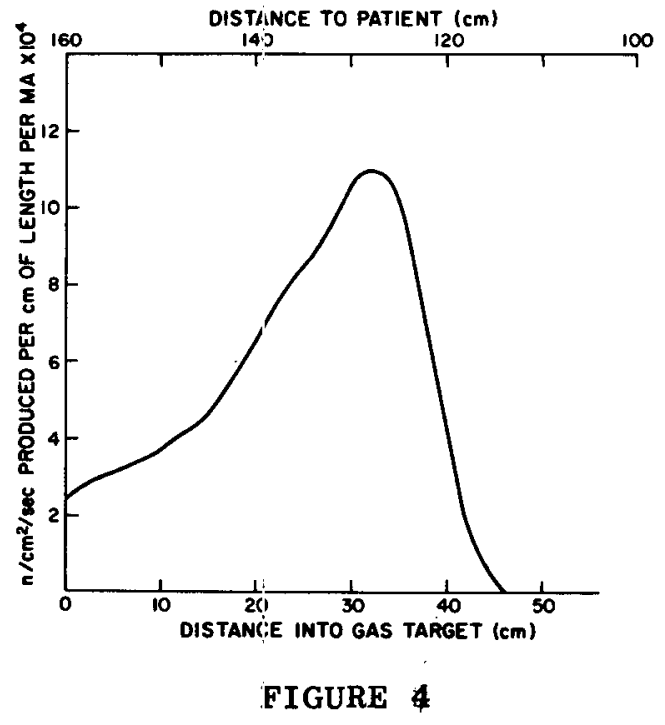

Number of neutrons produced per $\mathrm{cm}$ of target length.

Calculations were aiso performed to investigate how an extended gas target source affects the depth dose within the patient. Such calculations demonstrate that 
the depth at which the dose equals $50 \%$ of the maximum dose is the same $(+1 \mathrm{~mm})$ for the extended gas target as it is for a point source located at $125 \mathrm{~cm}$ SSD. As the irradiation field width is increased, the apparent width of the source also increases. Geometrical calculations were made to determine the apparent width of a $2 \mathrm{~cm}$ diameter gas target as a function of field size. Figure 5 presents the apparent width of the source versus field size for $160 \mathrm{~cm}$ entrance aperture to patient distance. Note that the larger apparent width occurs for the larger field sizes where the increased penumbra is relatively less important. Studies on the effect of the collimating system parameters on the dose distribution within the patient are currentiy underway at the University of Wisconsin using a commercial low flux d, $\mathrm{T}$ generator.

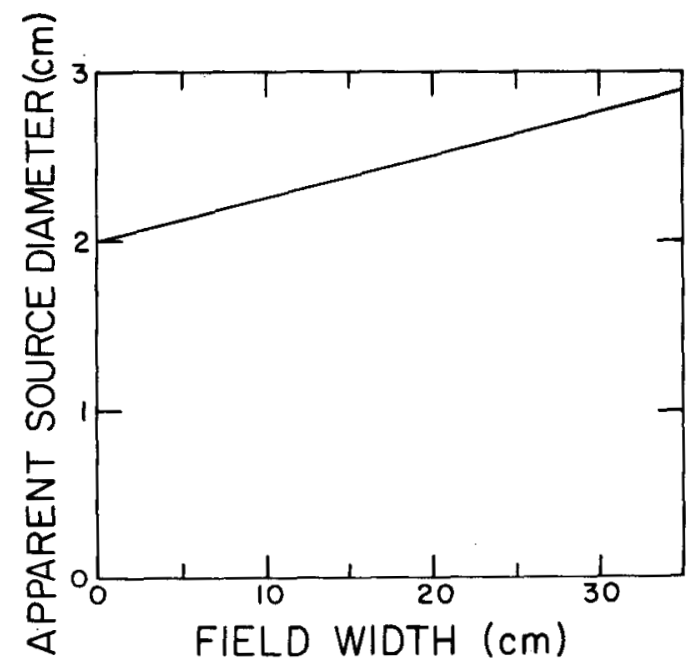

FIGURE 5

Apparent width of $2 \mathrm{~cm}$ diameter gas target as a function of field size.

In the design of any target system, provisions must be made to remove the heat deposited in the target by the incident deuteron beam. Water cooling around the gas target can easily remove the heat load of approximately $4 \mathrm{~kW}$ which will be present in our gas target system. water flow rate of 4 gallons per minute will remove this heat load with only a $4^{\circ} \mathrm{C}$ temperature rise.
Table I presents the calculated parameters for the gas target source under investigation. All of these parameters are technically possible and no advances in the state of the art are required.

TABLE I

\section{Calculated Gas Target Source parameters}

Deuteron Energy
$\begin{aligned} & \text { Deuteron Beam } \\ & \text { Current }\end{aligned}$
$\begin{aligned} & \text { Heat deposited } \\ & \text { by beam }\end{aligned}$

by beam

Gas Target - 10 torr

Tritium Pressure

Length of Gas - $40 \mathrm{~cm}$

Target

Diameter of Gas - $2 \mathrm{~cm}$

Target

Distance between - $160 \mathrm{~cm}$

entrance aperture

and patient surface

Neutron flux at $-4 \times 10^{7} \mathrm{n} / \mathrm{cm}^{2} / \mathrm{sec}$ patient surface

Dose rate at - $20 \mathrm{rad} / \mathrm{min}$

patient surface

The most significant disadvantage of the gas target system is probably the necessity for handling large amounts of gaseous tritium in a hospital environment. The type and volume of the pumping system has not been finally determined so the total tritium inventory is not yet accurately known. Current estimates indicate approximately $100 \mathrm{~atm}$ cc of gaseous tritium may be required. Such an amount of radioactive gas (more than $200 \mathrm{Ci}$ ) will obviously require a very reliable pumping system contained within a second outer leakproof vessel. We are investigating both mercury diffusion pumps and turbo molecular pumps for use in the pumping system. It is not possible to use oil diffusion pumps because tritium exchange with the hydrogen in the oil results in a contamination of the pump oil and reduction of the tritium available for the gas target system. 
The incident deuteron beam will deposit approximately $0.1 \mathrm{~atm} \mathrm{cc} / \mathrm{min}$ of deuterium in the tritium gas target. Assuming a treatment time ("beam on" time) of $5 \mathrm{~min}$. and a tritium inventory of $100 \mathrm{~atm} \mathrm{cc,} \mathrm{the}$ decrease in neutron output due to the dilution of tritium by deuterium can be calculated to be about $0.5 \%$ per patient. To prevent this decrease from becoming excessive, the target gas will be periodically passed through a fractional distillation system. An alternative approach also being considered is to use a mixed beam of deuterium and tritium and a gas target containing a mixture of deuterium and tritium. Such a mixed beam system would eliminate the need for an isotope separation system but would require twice the beam current.

We plan to store the tritium in the form of $\mathrm{UH}_{3}$ contained in a stainless steel bottle. At room temperature the $\mathrm{UH}_{3}$ has a vapor pressure of 1 micron and is very stable. Gaseous tritium at a pressure of 10 torr can be obtained by heating the bottle to $270^{\circ} \mathrm{C}$ and decomposing the $\mathrm{UH}_{3}$ (5). This cycle can be repeated indefinitely with no loss of efficiency.

\section{CONCLUSION}

We have described the current efforts at the University of Wisconsin to develop a $10^{13} \mathrm{n} / \mathrm{sec}$ source for clinical cancer applications by using a gas target $d, T$ source. The tests on the pumping requirements and ion optics using a proton beam currently underway will be followed by initial studies of neutron production using $d, D$ reaction. The lower yield of this reaction requires significantly less shielding than would be required if the $d, T$ reaction were used. Our studies appear to show that the use of an extended gas target neutron source is technically feasible, will not be a significant disadvantage in clinical applications and can provide the $1013 \mathrm{n} / \mathrm{sec}$ source required for fast neutron radiation therapy.

\section{REFERENCES}

1) Bewley, D, K. and Hornsey, S., In, Biolopical Effects of

Neutron and Proton Irradiations, Proceedings of the Symposium on Biological Effects of Neutron Irradiations, Nat. Agency for Int. Publ! Vol. II (1964).

2) Fowler, J,F. and Morgan, R.L., Brit. J. Radiology 36: 115-121 (1963).

3) Goodman, $\mathrm{L} . \mathrm{J}$. and Koch, U. AEC Report NYO 2740-6: 32-95 (1969).

4) Coon, J.H., In; Fast Neutron Physics, ad. by J.B. Marion and J.F. Fowler, Interscience Publ., New York (1960).

5) Spedding, F.H., Newton, A.S., Warf, J.C., Johnson, O., Nottorf, R.W., Johns, I.B. and Dáane, A.H. Nucleonic's 4: 4-15 (1949). 


\title{
MOBILE ACCELERATOR FACILITY FOR NEUTRON INTERROGATION AND NONDESTRUCTIVE ASSAY
}

\author{
Bert R. Dennis, R. A. Forster, J. H. Menzel,
}

$M$. M. Thorpe, and D. B. Smith

Los Alamos Scientific Laboratory

Los Alamos, New Mexico

\begin{abstract}
The Mobile Nondestructive Assay Laboratory (MONAL) employed by the Nuclear Safeguards Research Group at Los Alamos utilizes a 200 kv positive ion accelerator for active neutron interrogation and assay of fissionable materials. The accelerator produces neutrons by the $\mathrm{D}+\mathrm{T} \rightarrow \mathrm{n}+{ }^{4} \mathrm{He}$ reaction and is operated in a pulsed or modulated mode. Addition of a post-acceleration beam deflection system ensures a large neutron source "on-to-off" ratio. The accelerator has been designed for operation specifically in the MONAL and procedures have been developed for fast efficient transshipment of the entire mobile laboratory, including accelerator, between deployment locations.

An integral part of the active neutron interrogation system in the MONAL is the necessary personnel shielding surrounding the interrogation chamber; the main neutron shield consists of refillable water tanks which are drained prior to transit. Shield design characteristics and neutron flux distributions in the interrogation chamber were investigated using three-dimensional Monte Carlo calculations. Similar calculations were made on spectrum tailoring assemblies for appropriately moderating and reflecting the $14 \mathrm{Mev}$ source neutrons. These calculations, plus supporting measurements, provide guidance in the development of active interrogation methods for nondestructive assay of fissionable materials using the MONAL facility.
\end{abstract}

\section{INTRODUCTION}

Plutonium and enriched uranium are nuclear materials of high economic and strategic value. As these materials flow through the nuclear fuel cycle from the enrichment plant or plutonium production reactor to the fuel fabricator, to the power reactor, and finally to the fuel reprocessor, repeated material transfers occur between government contractors and private firms and there arise numerous opportunities for inventory and ship-per-receiver differences and hence a very real possibility of diversion of such material to unlawful uses, viz the nuclear materials safeguards problem. In addition, the fis sionable material scrap and so-called discardable waste generated by each facility and containing widely varying amounts of uranium and plutonium in a variety of matrix materials and container configurations as well as material held up in dissolver tanks, process lines etc. present an almost insurmountable problem of material accountability and safeguards. Consequently, the Los Alamos

Scientific Laboratory is engaged in an extensive $R \& D$ program to develop techniques and instrumentation for nondestructive assay of fissionable materials in whatever form they may occur, e.g., solutions, inventory samples, fuel pins and subassemblies, irradiated fuel, scrap and waste (1). The nonde- structive assay techniques employed range from passive methods (2) based on the measurement of characteristic gamma rays with $\mathrm{Ge}(\mathrm{Li})$ and $\mathrm{NaI}(\mathrm{Tl})$ detectors and spontaneous fission neutrons with neutron coincidence counters to active interrogation methods based on neutron-induced fission via a neutron generator or ${ }^{252} \mathrm{Cf}$ source combined with the observation of prompt and/or delayed neutrons (2).

At Los Alamos development of these techniques is carried through to complete nondestructive assay systems which will perform reliably not only under controlled laboratory conditions but also under the demanding conditions of in-plant or field deployment. Techniques and instrumentation must be fully tested in the field in order to define their capabilities and limitations. Therefore, LASL has developed a Mobile Nondestructive Assay Laboratory, known by the acronym MONAL, to test, demonstrate and further improve these new assay techniques in the "real world" of the nuclear industry.

Field tests of the MONAL were initiated early in 1970 at the Los Alamos plutonium facility (DP-Site). During May through July 1970, the MONAL was used to measure plutonium scrap at the Dow 
Chemical Company, Rocky Flats Division, in Golden, Colorado. Fuel rods and incinerator ash containing depleted, normal, and low enrichment ( $\leq 10 \%, 235 \mathrm{U}) \mathrm{UO}_{2}$ and $\mathrm{U}_{3} \mathrm{O}_{8}$ were assayed at the Feed Materials Production Center, National Lead Company of Ohio, Cincinnati, during the period November 1970 to February 1971. Following this assignment the MONAL will be reassigned to the Nuclear Materials and Equipment Company of Apollo, Pennsylvania, where various highly-enriched materials will be assayed largely by neutron interrogation methods.

The MONAL is an extremely versatile laboratory (3) employing the complete range of on-board passive and active nondestructive assay. systems. However, in view of the main theme of this conference, Neutron Sources and Applications, the present paper is restricted to the design and ope rational characteristics of only one of these systems, namely the $14-\mathrm{MeV}$ neutron generator and associated shielding used for active neutron interrogation and assay of fissionable materials.

The Mobile Nondestructive Assay Laboratory's $200 \mathrm{keV}$ accelerator plus necessary support equipment and the shielded experimental cavity where neutron interrogation is carried out, have been described (3). Figure 1 shows the exterior of the $10 \times 50 \mathrm{ft}$. cargo container housing the laboratory. A general layout of the facility, shown in Fig. 2, describes the arrangement of the control room, data acquisition and analysis facilities, sample preparation room, electronic and instrument shop, the shielded cavity and the rear equipment room where the accelerator is housed. The accelerator target (14-MeV neutron source) is reentrant into the experimental cavity through one of the shield penetrations. Large samples, up to 55 -gallon drums, are handled by a remotely operated conveyor system (Fig. 3). An elevator (at left in Fig. 3), transports these samples into the experimental cavity where they may be "scanned" (i.e., rotated while being elevated) for interrogation.

\section{4-MeV NEIUTRON GENERATOR}

The neut:con source employed in the MONAL utilizes a pumped-tube type accelerator as shown in Fig. 4. This consists of a Penning ion gauge source, $200 \mathrm{kV}$ accelerating column, a vac-ion pump, postacceleration beam deflection plates, isolation valve, drift tube, and target assembly. The accelerator is cooled by a closed-loop refrigerator system employing freon gas as the heat-transfer medium. The $200 \mathrm{kV}$ power supply is housed in a steel tank and insulated with pressurized $\mathrm{SF}_{6}$. This unit provides the ion beam accelerating voltage as well as the $5 \mathrm{kV}$ pulse-controlled ion source voltage. The accelerating column (also insulated with pressurized SF ) is housed in a stainless steel dome thus 6 allowing the exterior of the entire accelerator to be at ground potential. To improve the accelerator "on-to-off" performance an external $5 \mathrm{kV}$ pulsed power supply is provided to control the post-acceleration beam deflection plates. $\mathrm{A}_{2}$ manifold provides deuterium gas to a palladium leak which: controls the flow of gas supplied to the ion source.

A commercially available accelerator (Kaman Nuclear A-1254) was adapted for use in the MONAL. Existing state-of-the-art pulsing capabilities necessary for certain delayed neutron assay applications were further developed to meet Los Alamos specifications (4). Both the ion source and the deflection plate power supplies are controlled from a single pulse control unit designed and fabricated at Los Alamos. The $T+D \rightarrow n+$ ${ }^{4} \mathrm{He}$ reaction produces $14-\mathrm{MeV}$ neutrons with a yield of approximately $2 \times 10^{11}$ neutrons / sec. Target life depends on the quality of the targets as well as the vac-ion system, target cooling and accelerator voltages. Since the accelerator employed in the MONAL is normally operated in the pulsed or modulated mode for dielayed neutron assay applications, operating target life is greatly extended. A typicall duration of 80 millis econds on and 120 milliseconds off is used in normal 
assay work. Typical target life operating time has been approximately 22 hours. A simple, fast target changing procedure has been developed which takes less than 20 minutes for the complete target change operation.

An important performance characteristic of the accelerator is its "on-to-off" ratio, which is defined as the neutron production rate when the beam is on compared to the "source background" when the beam is off. An "on-to-off" ratio of $\sim 10^{8}$ is desirable for active interrogation and delayedneutron assay. To measure this ratio it is necessary to monitor neutron production rate at two levels which differ by a factor of $\sim 10^{8}$. This ratio was measured in the MONAL experimental cavity, using three detectors. A " $Q$ " monitor, normally used in the cavity to measure neutron yield, recorded total neutron production. This detector is a small $\mathrm{BF}_{3}$ counter imbedded in a boron loaded poly sleeve. A thin slab detector (5) optimized for delayed-neutron counting, recorded the "off" background. The "on-tooff " ratio is then determined by the ratio of relative efficiency and count rate of the $\mathrm{BF}_{3}$ detector as compared to the ratio of relative efficiency and count rate of the thin slab detector. A bare ${ }^{3} \mathrm{He}$ counter was used to determine the relative efficiencies of the two primary detectors at intermediate yields. The "on-to-off" ratio determined in this manner was measured as $2.06 \times 10^{7}$, which is quite acceptable; it should be noted, however, that a value of $2 \times 10^{7}$ does not necessarily represent the maximum achievable on-to-off ratio for the A-1254 neutron generator itself since the contribution of $17 \mathrm{~N}$ delayed neutron emitters produced from the ${ }^{17} \mathrm{O}(\mathrm{n}, \mathrm{p}){ }^{17} \mathrm{~N}$ reaction was not separately determined; viz this reaction could contribute significantly to the "off" or "source background" counting rate.

Figure 5 shows the accelerator assembled in its operating configuration. The entire accelerator is mounted on a dolly at its approximate center of gravity, (i.e., the vac-ion pump). The dolly allows withdrawal of the accelerator into the rear equipment room to facilitate target changes. Commercially available accelerators have not been designed for transshipment in their operating configurations. Thin-walled stainless steel flanges are incorporated in various sections of the accelerator as vacuum seals and are subject to fatigue from prolonged vibrations. Such vibrations occur when MONAL is transported from one field location to another. Experience has shown the necessity of separately packing the accelerator column in a specially designed padded container for transshipment from one field location to another.

Recent developments in the technology of sealed tube neutron sources having the required neutron yield, on-to-off ratio, and pulsing characteristics should extend the scope of portable accelerators in the near future. The sealed-tube modular design (i. e. , Accelerators, Inc. Model 801-102 type ST$1000 \mathrm{P}$ ) would bring several advantages, including greater operational simplicity and transportability as well as easier, faster maintenance in the field.

\section{SHIELD CHARACTERISTICS}

Active interrogation employing accelerator-produced neutrons requires shielding of operating personnel in the MONAL. Consideration of the size of the interrogation chamber vs. the shield size, weight and composition resulted in the choice of water as the shielding material. The water shield has several primary advantages, the first being high neutron shielding effectiveness per unit weight. The low cost and general availability of water allows draining of the shieid prior to transit and therefore a sizable ( 15 ton) reduction in gross MONAL weight during transit. A layout of the shield construction is included in Fig. 2. The overall dimensions of the shield are $13 \mathrm{ft}$. 5 in. by $9 \mathrm{ft} .4 \mathrm{in}$. by $8 \mathrm{ft} .1$ in. high. The inner tank, six inches thick, surrounding the cavity is filled with $\mathrm{O}_{2}$-free oil. The experimental cavity measures $4 \mathrm{ft}$. 3 in. by $5 \mathrm{ft}$. $11 \mathrm{in}$. by

$7 \mathrm{ft}$. 0 in. high. The cavity walls and ceiling are covered with $1 / 2$ in. thick sheets of borated $\mathrm{PVC}$ to aid in hardening the neutron spec- 
trum. Personnel access into the cavity is provided by a rotating shield door shown in Figure 6 with a passageway 20 in. wide by $6 \mathrm{ft}$. high. A removable plug in the overhead was included for fuel element insertion. Two large penetrations provide access into the cavity for the accelerator and various auxiliary equipment. The gamma port provides a penetration that may be used for prompt and delayed gamma measurements. It may also be used for monitoring the $17 \mathrm{O}(\mathrm{n}, \mathrm{p}){ }^{17} \mathrm{~N}$ reaction. It provides a convenient location to investigate the use of radioactive neutron sources such as ${ }^{252} \mathrm{Cf}$ and to facilitate evaluation of a sealed tube accelerator at some future time. Separate smaller penetrations provide access for power and signal cables, air conditioning and a pneumatic transfer system. A 36 in. by $54 \mathrm{in}$. opening in the cavity floor serves as a port for the large sample handling elevator. Figure 7 shows the cavity interior with the thin slab detector in the for eground, and 55-gallon scrap barrel elevated into position in front of the accelerator for delayed neutron assay.

The design of the water shield was investigated using the three dimensional Monte Carlo code TDMC 1G. A prediction of dose rate at various distances was obtained by calculating the transmission of an isotropic point source of $14 \mathrm{MeV}$ neutrons through an infinite slab of water 30 inches thick (6). Thermal neutron dose outside the water shield was shown to be negligible. The DTF-IV code was used to determine the gamma dose of the $2.2 \mathrm{MeV}$ gamma ray produced by thermal neutron capture in hydrogen. The calculated $2.2 \mathrm{MeV}$ gamma dose at a distance of five feet from the shield was $0.43 \mathrm{mrem} / \mathrm{hr}$ for a neutron source strength of $5 \times 10^{10}$ neutrons per second.

A second consideration of the shield characteristics was the contribution of delayed neutrons from the ${ }^{17} \mathrm{O}(\mathrm{n}, \mathrm{p})^{17} \mathrm{~N}$ reaction on nondestructive assay results. This reaction was investigated by approximating the experimental cavity and surrounding shield by spherical shells. For a $14-\mathrm{MeV}$ neutron source strength of $5 \mathrm{x}$ $10^{10}$ neutrons per second, the number of delayed neutrons in the cavity due to the ${ }^{17} \mathrm{O}$ in the shield was computed to be $5.3 \times 10^{4}$ neutrons/sec. This number was reduced to $9.6 \times 10^{1}$ neutrons/sec by replacing the first six inches of shield water with oxygen-free oil.

The above calculations did not include neutrons leaving the $\epsilon$ xperimental cavity through the elevator port To guide in the design of external water tainks to shield against ground scattered neutrohis, calculations were performed again using the three dimensional Monte Carlo code (7). The neutron flux distribution at a position six feet from the front of the neutron shield and three feet above the trailer floor was computed. This position is the distance of closest approach to the shield during accelerator operation because of an interlocked guard rail. The neutron flux distribution at a position corresponding to an exclusion area of approximately 50 feet from the side of the van was also computed. A spherical neutron dosimeter system consisting of a 9 in. diameter cadmium loaded polyethylene sphere with a $\mathrm{BF}_{3}$ tube in the center and an energy range of thermal to about $10 \mathrm{MeV}$ neutrons was used to measure the actual dose rate for comparison with the above cited calculations. These data are listed in Table I.

The good agreement between the calculated and measured dose rate verifies the Monte Carlo result. The close agreement is partially due to the fact that the effect of thermal neutrons|was negligible. Neutr ons were computed in four energy bins ranging from $10 \mathrm{eV}$, to $14 \mathrm{MeV}$, energies below $10 \mathrm{eV}$ being discarded.

The $14 \mathrm{MeV}$ neutron generator has proved quite adaptable for mobile assay applications. It offers a hard spectrum for neutron penetrability to induce fission throughout the sample materials under study. The energy of the incident neutrons can be varied by utilizing "beam-tailoring" or moderator assemiblies to take advantage of increased isotopic discrimination afforded by sub-threshold and super-threshold fission characteristics of various fissionable 
TABLE I

Shielding Parameters

LASL Mobile Nondestructive Assay Laboratory

Computed Dose $\mathrm{mr} / \mathrm{hr}$

Shield Configuration

No ground shield

Ground shield $1.5 \times 6 \times 4 \mathrm{ft}$

Ground shield $2 \times 9 \times 4 \mathrm{ft}$

况

$15 \mathrm{ft}$. from source

象棌

$47 \mathrm{ft}$. from source
Measured Dose $\mathrm{mr} / \mathrm{hr}$

$\begin{array}{ccc}\text { Front }^{*} & & \text { Side } \\ 21 & & 0.82 \\ 5.6 & 0.88 \\ 1.7 & 0.72\end{array}$

Front $^{*} \quad$ Side $^{* ; * k}$

15

7.8

0.83

0.73

$2.9 \quad 0.50$ isotopes. Monte Carlo computer calculations were performed to specify guide lines for use in the design of spectrum-tailoring assemblies to be used in the MONAL (8). These calculations contain some modifications to previous assemblies not designed for the MONAL's experimental cavity (9).

To determine the effectiveness of beam-tailoring, it was necessary to consider the neutron spectrum anticipated in the experimental cavity. Ideally the spectrum would remain hard ( $14 \mathrm{MeV}$ ) throughout the experimental area. However, room return neutrons soften the spectrum to some degree depending on the location with respect to the source. The neutron flux (neutrons $/ \mathrm{cm}^{2} /$ unit source) in various energy bins at several points in the cavity were computed using a detailed Monte Carlo calculation (10). Parameters considered in the computation included a lead tailoring assembly surrounding the $14 \mathrm{MeV}$ source, and all walls except the floor were covered with borated PVC. A low-energy cutoff at $0.25 \mathrm{eV}$ was chosen to expedite the calculations. The ${ }^{235} \mathrm{U} /{ }^{238} \mathrm{U}$ ratio is of special interest and was chosen as a figure of merit. Estimates of cadmium-covered ${ }^{235} \mathrm{U} /{ }^{23} \dot{8}_{\mathrm{U}}$ ratios were determined at three distances from the tailoring assembly, namely, 6 in., 15 in. and 60 in. To confirm the calculations, several measurements were made in the cavity using a ${ }^{235} \mathrm{U}$ fission chamber "back-to-back" with a ${ }^{238} \mathrm{U}$ fission chamber and covered with a Cd sleeve.

The results of the calculations and measurements are listed in Table II. The measured data have been corrected for the mass ratios of the back-to-back fission chambers. Again there is good agreement between the calculations and the measured values. To study thermalization of neutrons in the cavity a $235 \mathrm{U}$ cadmium ratio was measured at various distances from the source. These data are listed in Table III.

As apparent from the se data, the water shield provides adequate personnel shielding but also contributes significantly to a softer spectrum in the experimental

TABLE II

Cavity Neutron Spectral Indices $235 \mathrm{U} /{ }^{238} \mathrm{U} \mathrm{Cd}$-Covered Ratios in LASL Mobile Nondestructive Assay Laboratory

\begin{tabular}{|c|c|c|}
\hline Distance & ${ }^{235_{U} / 238_{U}}$ & $235_{U / 238} U$ \\
\hline Source & Computed & Measured \\
\hline $6 \mathrm{in.}$ & 4.7 & 4.8 \\
\hline $15 \mathrm{in.}$ & 8.0 & 6.6 \\
\hline $60 \mathrm{in.}$ & 19.0 & 23.0 \\
\hline
\end{tabular}




\section{TABLE III}

Cavity ${ }^{235} \mathrm{U}$ Cadmium Ratio

Los Alamos Scientific Laboratory Mobile Nondestructive Assay Laboratory

Distance from Source

Cadmium Ratio

7
12
19
24
36
48

1.094

1.26

1. 32

1.46

1. 56

1. 61

cavity. The cadmium ratio could probably be improved by lining the cavity walls with cadmium, should a specific application warrant it. For the majority of applications encountered in the MONAL thus far, the softer spectrum is of little consequence. Small to intermediate samples may be isolated from room return by simply surrounding them with a cadmium-covered borated sleeve. The interrogation of 55-gallon drums may be classified into two categories: drums containing non-hydrogenous scrap which are not affected by the cavity spectrum and drums containing hydrogenous materials which then become the major contributor to the neutron spectrum.

Since the above calculations were made, a remotely operated sliding shield floor has been fabricated to cover the cavity port. The movable floor adds two inches of borated PVC when in place. Its dual purpose is, first, to shield the experimental cavity from background contributed by adjacent samples loaded on the sample handling conveyor and, second, to aid in hardening the neutron spectrum from ground-scattered neutrons.

In addition to providing a suitable environment for active interrogation, it should be noted that the shielded cavity provides an area in the mobile laboratory that is decoupled from plant or other exterior backgrounds; this feature has proved especially advantageous when performing passive assay at plutonium facilities.

\section{REFERENCES}

1. G. R. Keepin, H. O. Menlove, M. M. Thorpe, R. H. Augustson, C. N. Henry, D. B. Smith, and T. D. Reilly. Application Areas and Results of Nondestructive Assay Measurements. Paper No. SM-133/49, IA EA Symposium on Progress in Safeguards Techniques, Karlsruhe, Germany (6-10 July 1970).

2. R. H. Augustson, H. O. Menlove, R. B. Walton, L. V. East, A. E. Evans, and M. S. Krick. Development of Techniques for Active and Passive Assay of Fissionable Miaterials. Paper No. SM-133/51, IAEA Symposium on Progress in Safeguards Techniques, Karlsruhe, Germany (6-10 July 1970).

3. J. H. Menzel, B. R. Dennis, M. M. Thorpe, R. B. Walton, D. B. Smith, and G. R. Keepin. A Mobile Laboratory for Nuclear Safeguards. Paper No. SM-133/s7, IAEA Symposium on Progress in Safeguards Techniques, Karlsruhe, Germany (July 6-10, 1970).

4. C. N. Henry, Darryl B. Smith, and H. O. Menlove. Mobile Neutron Sources and Their Applications. Paper presented at the American Chemical Society meeting, Houston, Texas (Feb. $24-26,1970)$.

5. Nuclear Safeguards Research and Development Program Status Report ILA 4368MS, p. 13-14, Los Alamos Scientific Laboratory, Los Alamos, N. M. (1969).

6. Nuclear Safegguards Research and Development Quarterly Progress Report N-6-1016, p. 23-24, Los Alamos Scientific Laboratory, Los Alamos, N. M. (1969).

7. Nuclear Safeguards Research and Development Program Status Report N-61035 , p. 24, 25, 26, Los Alamos Scientific Laboratory, Los Alamos, N. M. (1969). 
8. Nuclear Safeguards Research and Development Program Status Report N-61035, p. 23-24, Los Alamos"Scientific Laboratory, Los Alamos, N. M. (1969).

9. Nuclear Safeguards Research and Development Program Status Reports LA$3921-\mathrm{MS}$, p. 11, and LA-4070-MS, p. 13. Los Alamos Scientific Laboratory, Los Alamos, N. M. (1968).

10. Nuclear Safeguards Research and Development Program Status Report LA4523-MS, Los Alamos Scientific Laboratory, Los Alamos, N. M. (1970). 


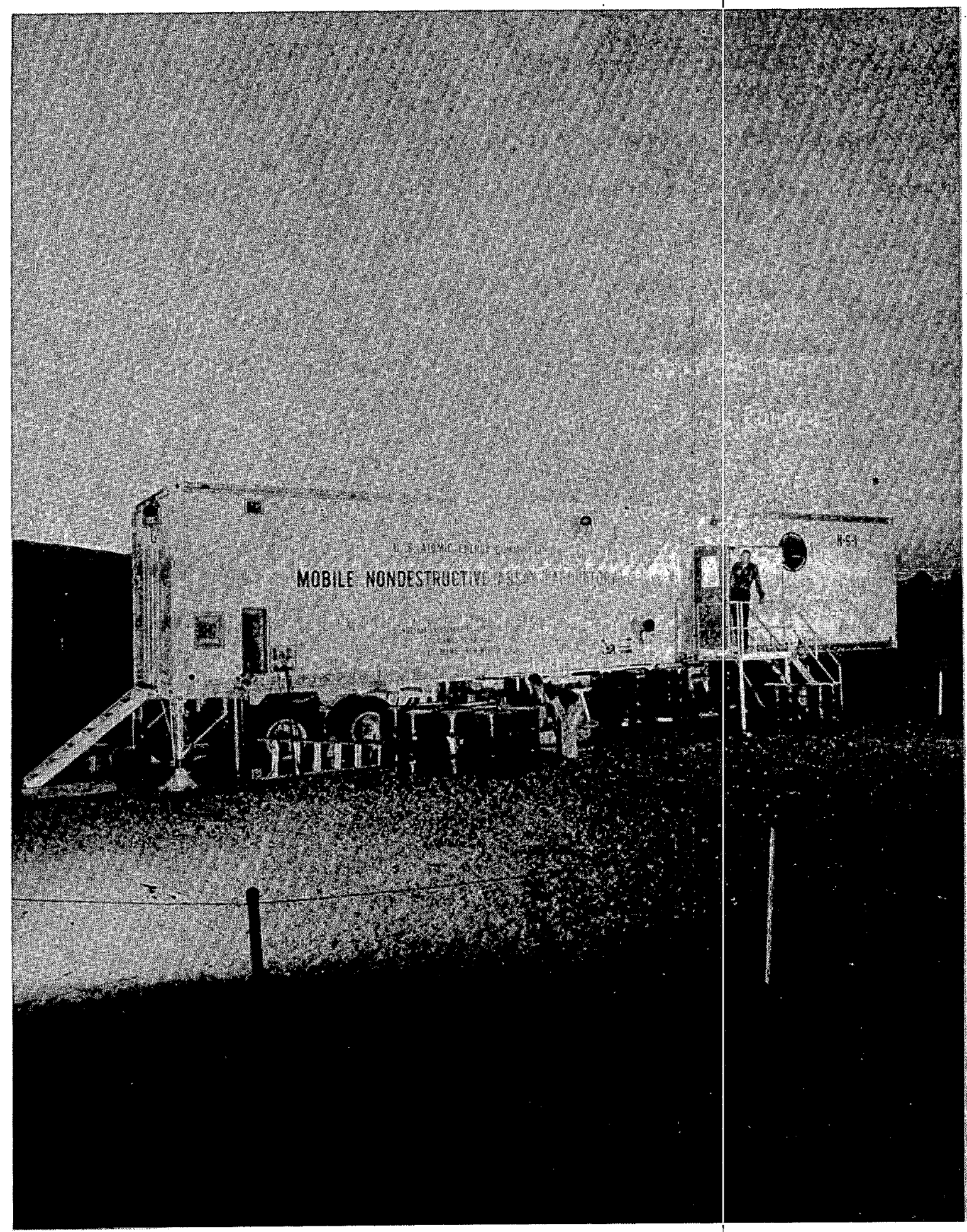

Figure 1. The Mobile Nondestructive Assay Laboratory developed by the Los Alamos Scientific Laboratory. 

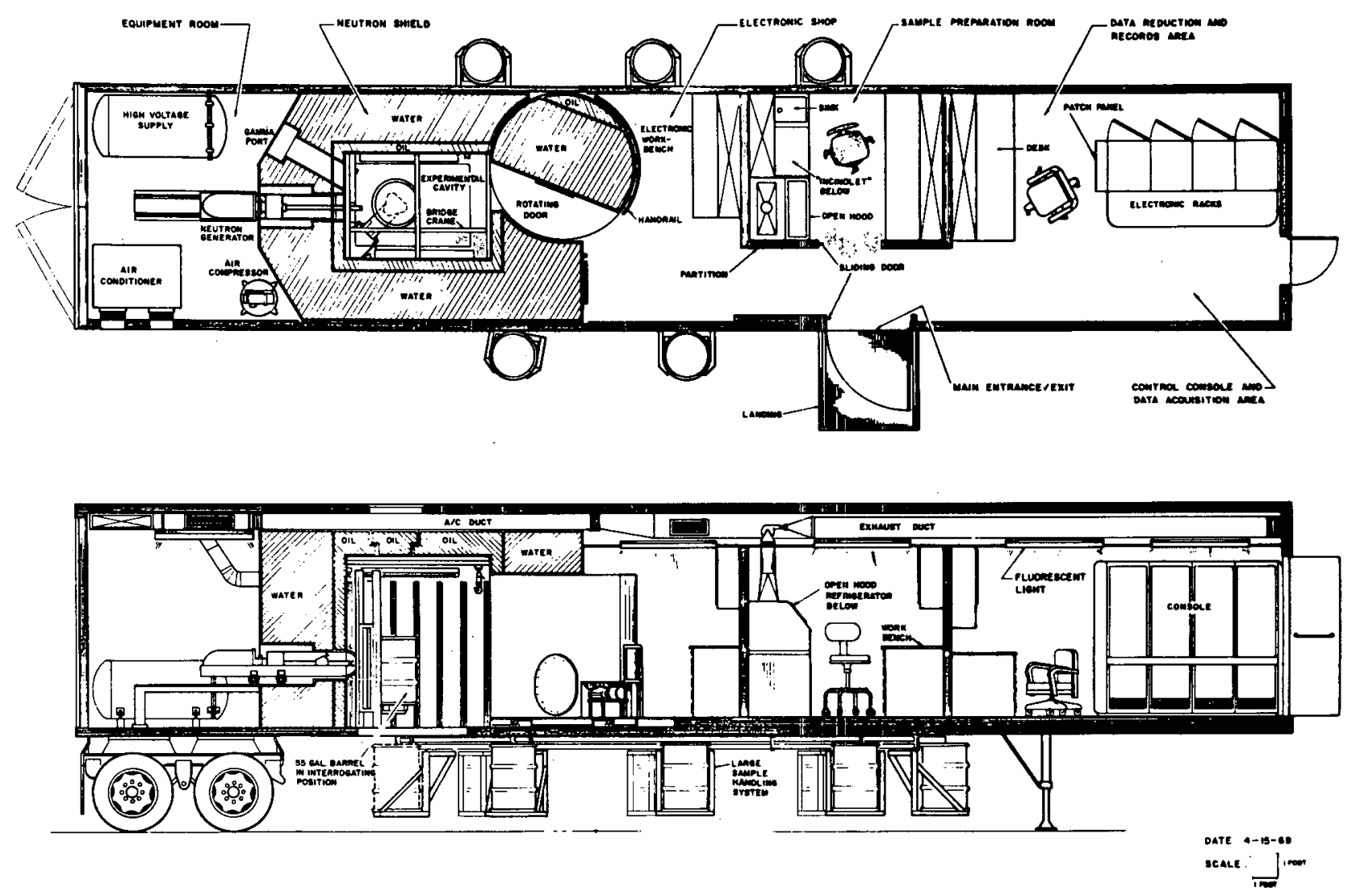

Figure 2. General layout of the MONAL facility.

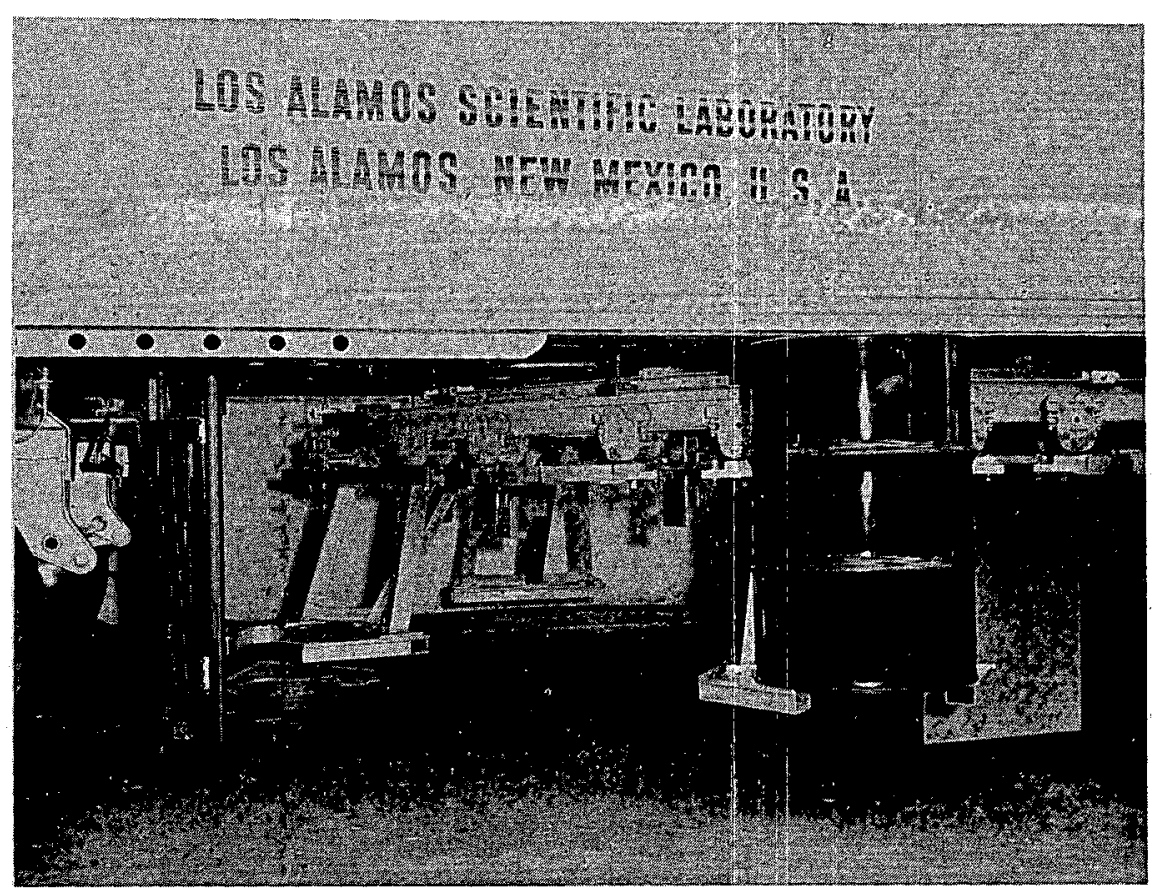

Figure 3. Portion of the large-sample handling system. 


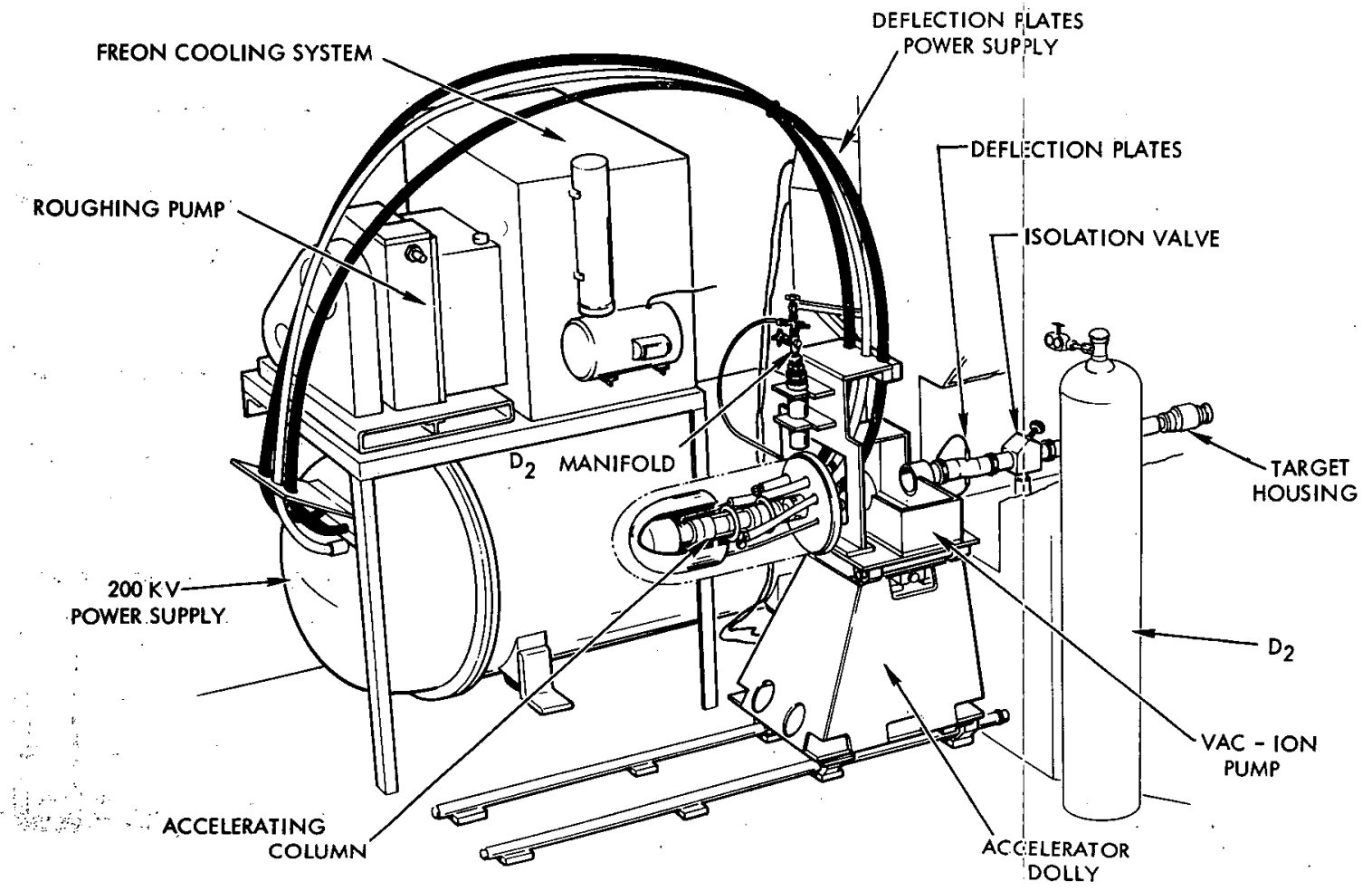

Figure 4. Diagram of the accelerator and auxiliary units located in the rear equipment room.

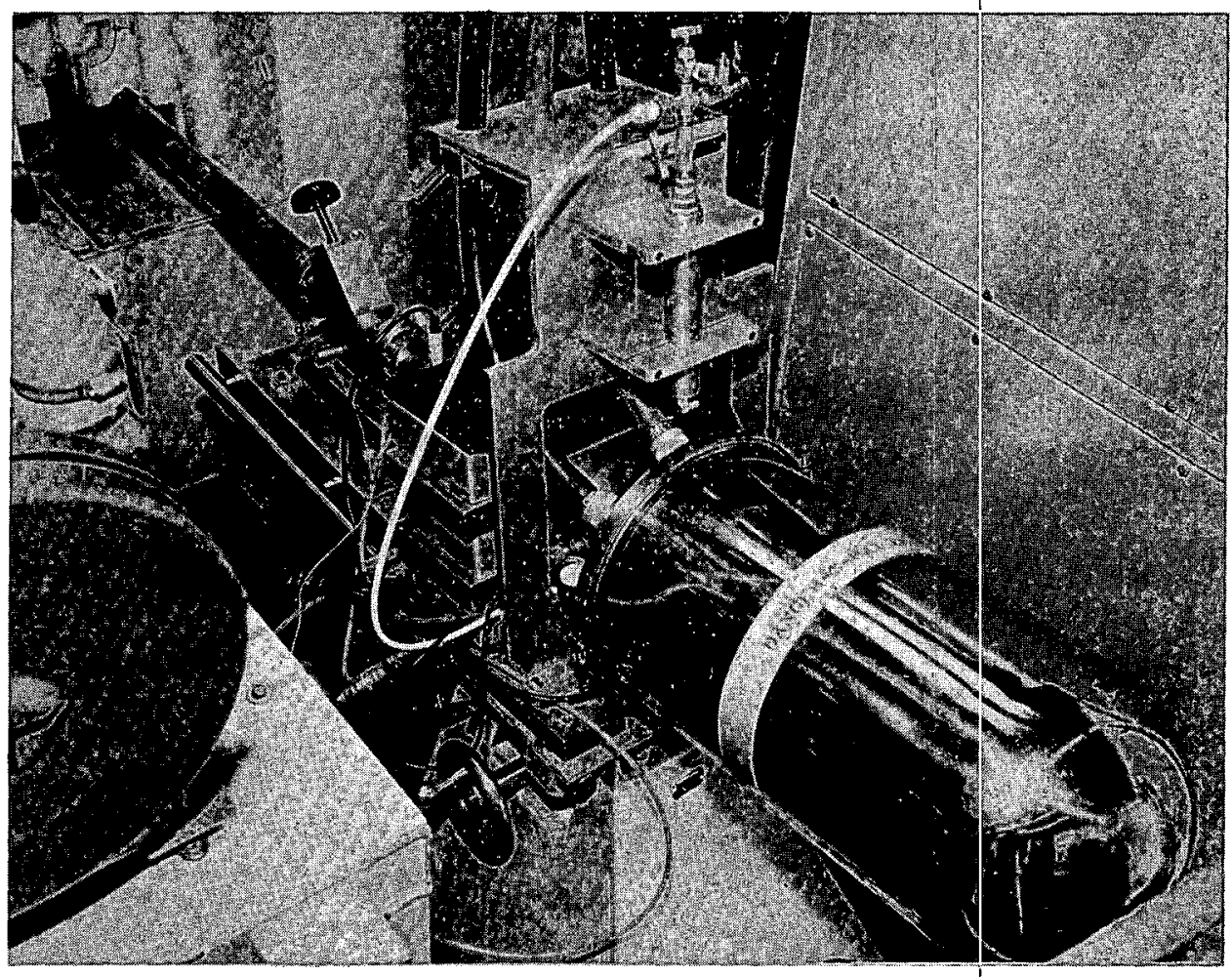

Figure 5. Accelerator in its operating configuration. 


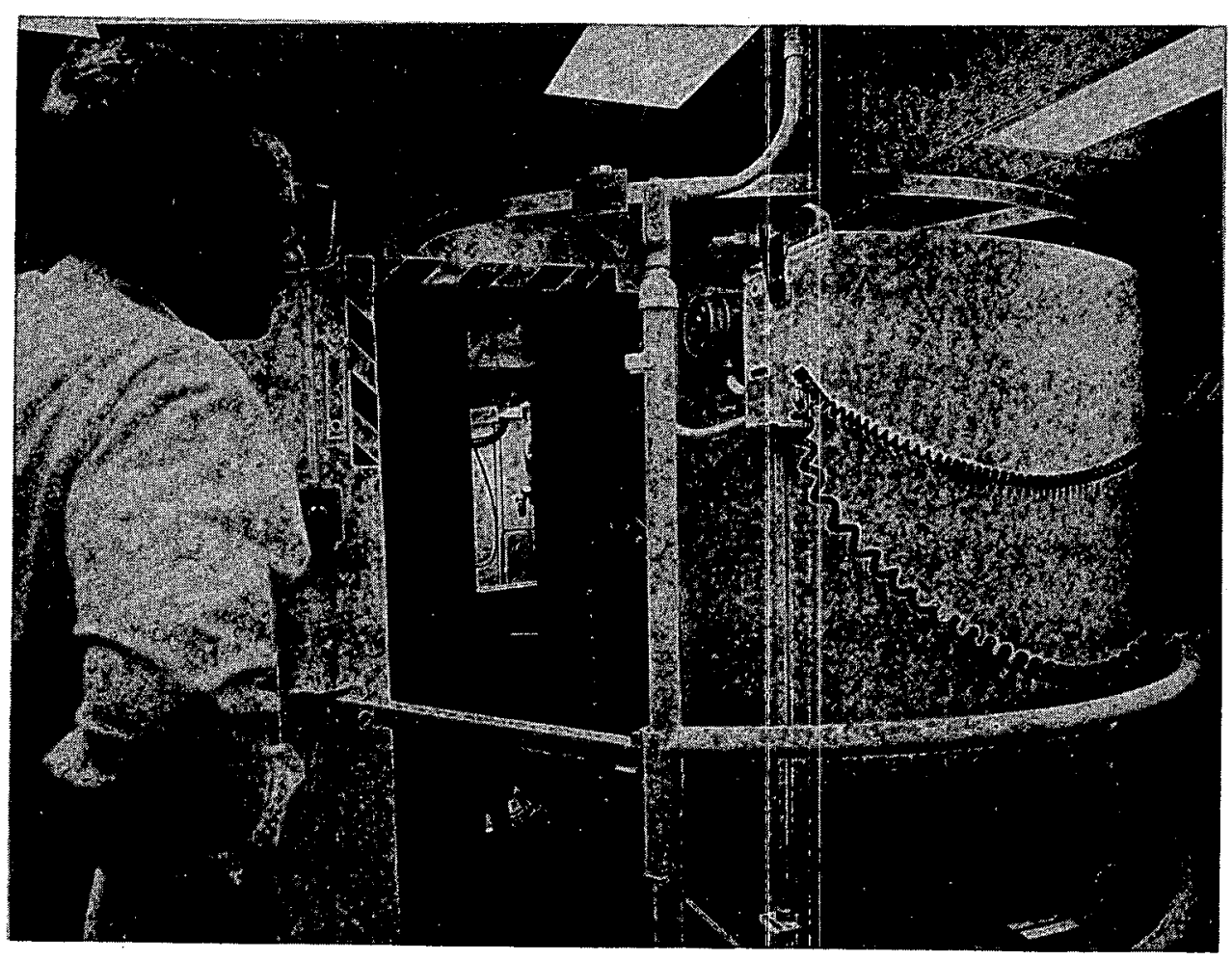

Figure 6. Rotating door and passageway into the cavity.

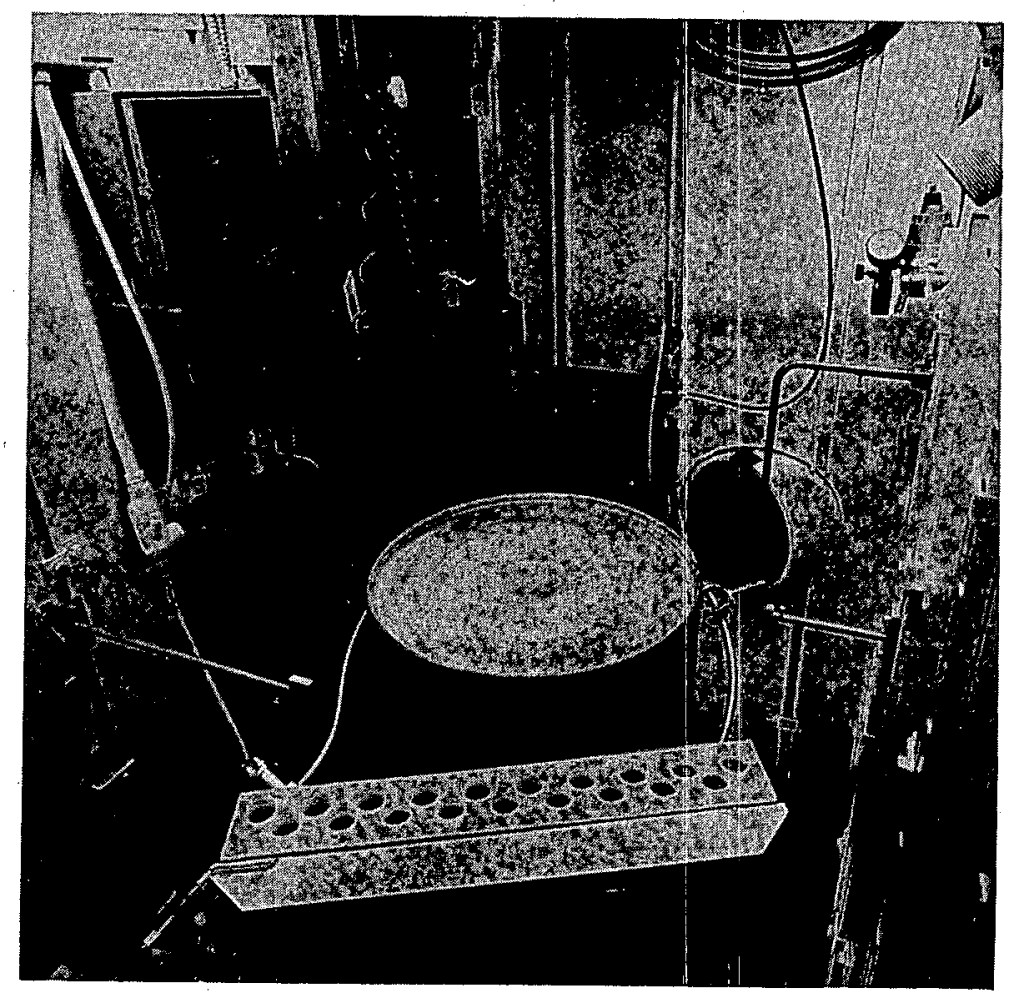

Figure 7. Shielded cavity--55-gallon barrel positioned for assay. 


\section{PREPARATION OF INDUSTRIAL $252 \mathrm{CF}$ NEUTRON SOURCES AT SAVANNAH RIVER LABORATORY}

W. R. McDonell, A. R. Boulogne, J. P. Faraci, S. F. Peterson, B. L. Dahlen, W. C. Mosley,

D. J. Mahoney, and $\mathrm{V}$. Whatley

Savannah River Laböratory

E. I. du Pont de Nemours and Company

Aiken, South Carolina

About 50 neutron sources containing up to $5 \mathrm{mg}$ of ${ }^{252} \mathrm{Cf}$ have been prepared at $S R L$ for industrial applications in the ${ }^{252} \mathrm{Cf}$ market evaluation program. Most of the sources were prepared by oxalate precipitation and calcination to form californium oxide. The oxide was contained within $\mathrm{Pt}-10 \% \mathrm{Rh}$ inner capsules and Type 304L stainless steel or Zircaloy-2 outer capsules. Feed received recently from ORNL as the product of the calcination of ${ }^{25}{ }^{2} \mathrm{C}$-loaded cation exchange resin, and from SRL separations operations as purified ${ }^{252} \mathrm{C}$ solution, had present 455 parts cationic impurities perpart ${ }^{252} \mathrm{Cf}$. This feed was used for source preparation without further purification. Techniques and facilitjes were developed to minimize radiation exposures incurred during source fabrication and handling operations.

Interfering gamma radiation from the stainless steel capsules was substantially reduced by substituting Zircaloy- 2 capsules for sources to be used for prompt gamma activation analyses; several other suitable capsule materials were also defined.

Assessments of the chemical stability of the californium oxide, the potential for deleterious interactions between source components, and the heat transfer and strength capabilities of the capsules indicated that high integrity containment of the ${ }^{252} \mathrm{Cf}$ in the sources should be maintained under many adverse conditions.

\section{INTRODUCTION}

Californium-252 is a potentially useful radioisotopic neutron source. The nuclide, which decays with a 2.65-year half-1ife predominantly by $\alpha$-emission, emits also $2.31 \times 10^{9} \mathrm{n} / \mathrm{sec}-\mathrm{mg}$ due to a significant spontaneous fission mode of decay.

Potential industrial applications include oil and mineral exploration, materials analyses and process control, neutron radiography, nuclear materials assay, and shortlived radioisotope production (i). $252 \mathrm{Cf}$ neutron sources for these applications may range from $5 \mathrm{\mu g}$ to $5 \mathrm{mg}$ or more.

The AEC is currently conducting a market evaluation program to estimate the future requirements for ${ }^{252} \mathrm{Cf}$, so that production can be planned accordingly. Under this program, ${ }^{252} \mathrm{Cf}$ sources are being loaned to a limited number of organizations interested in evaluating the nuclide in promising applications.

By the beginning of 1971 , about $50252 \mathrm{Cf}$ sources had been prepared at the Savannah River Laboratory for distribution under the

The information contained in this article was developed during the course of work under Contract AT $(07-2)-1$ with the U. S. Atomic Energy Commission. loan program. A list of the users and applications to be evaluated is given in Table I.

The purpose of this report is to describe the sources, their method of preparation, and materials-related radiation characteristics. Results of source design and materials evaluations to assure safe use under extreme conditions are discussed, and the facilities employed to prepare and handle the highly radioactive sources are described.

\section{PREPARATION OF SOURCES}

\section{SOURCE DESIGN}

Most of the industrial $252 \mathrm{Cf}$ sources prepared at SRL were of the SR-Cf-100 series design, shown in Figure 1. In this design, purified califorrium oxide is contained within a $90 \%$ platinum - $10 \%$ rhodium alloy inner capsule, which is enclosed within a Type $304 \mathrm{~L}$ stainless steel or Zircaloy-2 outer capsule." The Zircaloy-2 outer capsule is employed to minimize interfering gamma emissions in pronpt gamma activation analyses.

\section{SOURCE PREPARATION}

Sources of the SR-Cf-100 series are prepared using an oxalate precipitation-calcination technique developed by Boulogne and 
Faraci (2) In the technique as currently employed, $252 \mathrm{Cf}$ oxalate is precipitated from a $0.1 \mathrm{~N}$ nitric acid feed solution by addition of excess oxalic acid, and then is filtered onto a porous Type 316 stainless steel or platinum alloy disk in the inner capsule of the source. For sources containing under $500 \mu \mathrm{g}$ of $252 \mathrm{Cf}$, an additional $500 \mu \mathrm{g}$ of terbium is added in nitrate form as a carrier to ensure efficient precipitation of the ${ }^{252} \mathrm{Cf}$ oxalate. The precipitation is complete after about $\mathrm{l}_{\frac{1}{2}} \mathrm{hr}$. The precipitation-filtration operations are carried out in a completely enclosed appratus which minimizes $252 \mathrm{Cf} 1$ losses during transfer operations. After another porous metal disk is inserted over the filtered precipitate in the inner capsule, the ${ }^{252} \mathrm{Cf}$ oxalate is dried for 1 hr at $200^{\circ} \mathrm{C}$ and calcined for $30 \mathrm{~min}$ at $700^{\circ} \mathrm{C}$ in an air furnace, to convert the oxalate to an oxide. The inner capsule is then sealed with TIG-welded end plugs, heated for $15 \mathrm{~min}$ at $1000^{\circ} \mathrm{C}$, pressurized in $300 \mathrm{psi}$ He for $60 \mathrm{~min}$, and leak tested to ensure the inner capsule closure. The sealed capsule is decontaminated ultrasonically using alternate flushes of $1 \mathrm{M} \mathrm{HNO}_{3}$ and distilled water unti 1 a $1 \mathrm{~m} 1$ aliquot contains less than $50 \mathrm{dis} / \mathrm{min}$ alpha and 200 $\mathrm{c} / \mathrm{min}$ beta-gamma contamination. Large closure leaks that might not be detected by the helium leak test are revealed during the decontamination procedure.

The decontaminated inner capsule is then assembled into the stainless steel or Zircaloy-2 outer capsule, which is similarly sealed with a welded end plug, pressurized in helium and leak tested, and ultrasonically decontaminated to a level of less than 9 $\mathrm{dis} / \mathrm{min}$ alpha and $10 \mathrm{c} / \mathrm{min}$ beta-gamma transferable radioactivity as determined by a wipe test.

The equipment used for source fabrication operations has been previously described (2). A major improvement in the rate at which sources can be processed through the final calibration operations was achieved by development by $T$. R. Herold of a fission counter technique for measuring the rate of emission of source neutrons to replace the $5 \mathrm{MnSO}_{4}$ bath method previously used (3): Source calibration measurements using the fission counter technique require only about $15 \mathrm{~min}$, compared to $16 \mathrm{hr}$ using the $55_{\mathrm{MnSO}_{4}}$ bath technique. Neutron emission rates of sources containing up to $5 \mathrm{mg}$ of $252 \mathrm{Cf}$ are within $1 \%$ of the values obtained by ${ }^{5} \mathrm{MnSO}_{4}$ bath measurements.

Yields of ${ }^{252} \mathrm{Cf}$ retained in the source capsules using the oxalate precipitation procedure have generally exceeded $90 \%$, depending upon the source size and whether or not terbium carrier was used. In a typical series of five $100 \mu \mathrm{g}{ }^{252} \mathrm{Cf}$ sources prepared using $500 \mathrm{Hg}$ terbium carrier, source yields ranged from 94.0 to $99.7 \%$, averaging $95.8 \%$. Without terbium carrier, lesser yields would have been realized in these small sources. For a $1000-\mu g$ 252 Cf source prepared without terbium carrier, typically $92 \%$ of the original $252 \mathrm{Cf}$ was retained in the capsule. For the two $5-\mathrm{mg}$ ${ }^{252} \mathrm{Cf}$ sources that have been prepared, however, greater than about $98 \%$ of the $252 \mathrm{Cf}$ introduced as feed was retained in the capsule. In each case, the $252 \mathrm{Cf}$ not retained in the capsule appeared in the filtrate and wash solutions and was concentrated and recycled through the encapsulation operations for other sources. Better than $99 \%$ overall utilization of the feed $252 \mathrm{Cf}$ can be achieved using these procedures.

\section{FEED CHARACTERISTICS}

Feed for the ${ }^{252} \mathrm{Cf}$ sources has been obtained from the Savannah River Plant and Oak Ridge National Laboratory. The isotopic content of the californium feed varied considerably, as shown in Table II, depending on the time elapsed since reactor production. The SRP product, produced several years earlier, contained less than half $252 \mathrm{Cf}$. The ORNL product, which is also typical of recent SRP production, contained about $85 \% 252 \mathrm{Cf}$.

Chemical impurities in the feed have depended most notably on the method of packaging for transfer to the SRL source preparation facilities. The SRP feed was transferred as a nitric acid solution in polyethylene bottles. The ORNL feed, on the other hand, was shipped in solid form, originally as the dry nitrate and recently as the product of the calcination of a $252 \mathrm{Cf}$-loaded ion exchange resin contained initially in stainless steel and recently in platinum-plated stainless steel or Pt-Rh alloy columns. Although the 252 Cf was readily recovered from the dry nitrate product, shipments in large quantities were thought to pose problems that could be avoided by preparation of the more stable form resulting from resin calcination. Recent ORNL shipping packages with platinum alloy or platinum-plated columns were prepared by calcining the $252 \mathrm{Cf}$-loaded "Dowex $50^{\text {"1* }}$

* Registered tradename of Dow Chemical Co. 
cation resin at $550-650^{\circ} \mathrm{C}$, with air passing through the column to promote complete combustion of the resin. The $252 \mathrm{Cf}$ product was recovered essentially quantitatively by eluting with $0.1 \mathrm{~N} \mathrm{HNO}_{3}$, and this solution used to prepare sources without further purification. Recovery of $252 \mathrm{Cf}$ from earlier stainless steel columns calcined at $400-500^{\circ} \mathrm{C}$ without air flow required prolonged treatment with strong $\mathrm{HNO}_{3}$, and, as a consequence, at least part of the recovered product needed extensive repurification to remove organic and container material contaminants before source preparation.

Studies at SRL (4) have demonstrated that the product of the resin calcination is probably the oxysulfate, (CfO) ${ }_{2} \mathrm{SO}_{4}$. Typical recent analyses of the recovered ORNL product is compared with similar analyses of the SRP product in Table III. Cationic impurities are in both cases 4-5 times the 252Cf content of the feed solutions. The major impurity constituents ( $>20 \% 252$ Cf content) are the lanthanides (fission products), iron and chromium (from stainless steel containers), silicon, aluminum, sodium, potassium, and calcium (probably from glass containers), zinc (from separations processing reagents), sulfur (from the ion exchange resin), and lead (source unknown). Of these, only the lanthanides, calcium, and possibly lead and zinc are expected to be co-precipitated as oxalates from acid solution and retained along with californium and other actinides in the capsules.

Other actinides $(244 \mathrm{Cm}, 243 \mathrm{Am})$ in the $\mathrm{Cf}$ feed solutions have varied considerably depending on previous processing. Most of the ORNL feed was specially processed before shipment to recover the long-lived $248 \mathrm{~cm}$ produced by ${ }^{252} \mathrm{Cf}$ decay and thus contained almost no actinide impurities. The SRL feed contained actinides $243_{\mathrm{Am}}$ and $244 \mathrm{Cm}$ in quantities up to $10 \%$ of the californium content; larger scale processing methods being developed for SRP separation operations will reduce these quantities by several orders of magnitude.

Sources containing up to $1 \mathrm{mg}$ of ${ }^{252} \mathrm{Cf}$ were successfully prepared from the earlier, less pure ORNL feed solutions containing up to 18 parts cationic impurities per part of $252 \mathrm{Cf}$. Larger sources (5-10 mg) in the Sr-Cf-100 series, however, require the lower impurity levels typical of the current feed, to avoid an excessive quantity of inert materials in the capsule. Goal specifications for the $252 \mathrm{Cf}$ feed have been tentatively set as follows: none

Organic degradation products: essentially

Total solids (except organics): maximum 4 times Cf concentration

Actinides-Lanthanides (other than $\mathrm{Cf}$ ): Maximum equal to $\mathrm{Cf}$ concentration

\section{SOURCE FABRICATION FACILITIES AND HANDLING EXPERIENCE}

The ${ }^{252}$ Cf sources prepared to date have been fabricated in one cell of the High Level Caves (HLC) facility or in two cells of the Target Fabrication Facility (TFF) at SRL. The strength of sources that can be fabricated in these facilities is limited to about $10 \mathrm{mg}$ and $2 \mathrm{mg}$ of ${ }^{252} \mathrm{Cf}$, respectively. Sources and shipping packages containing up to $100 \mathrm{mg}$ 252 Cf will be prepared in a new installation, the Californium Packaging Facility, presently under construction.

Operations in the HLC cell shielded with 3-ft. of high density concrete have been previously described (2). An open top stainless steel box is proviled inside the shielded cell to minimize contamination of the shield walls and assist in disposal of contaminated equipment. The ceill operates with a oncethrough air atmosphere exhausted at 500-750 $\mathrm{ft}^{3} / \mathrm{min}$ through one bank of absolute filters in the cell and two banks of absolute filters outside the cell.

During preparation of $5-\mathrm{mg}{ }^{252} \mathrm{Cf}$ sources in the cell, combined neutron and gamma exposures at the operating location in front of the high-density glass window of the cell were accumulated at a rate of about $3 \mathrm{mrem} / \mathrm{hr}$. Inventories of $>5 \mathrm{mg} 252 \mathrm{Cf}$ could be accomodated by providing local shielding within the cell.

The TFF consists of three interconnected stainless steel containment boxes shielded at front and back by steel water tanks (36 inches total thickness), at each side by $3 \mathrm{ft}$ of concrete block constructed against a steel plate, and at the top by $24 \mathrm{in}$. thick concrete slabs. An inert gas (argon) atmosphere. is maintained in the TFF, although it is not required for ${ }^{252} \mathrm{Cf}$ source preparations. Process operations are viewed through water-filled glass windows in each cell. A pair of masterslave manipulators is provided for each box, and a periscope is available in one box for close-up viewing. 
Radiation intensities during fabrication of $1-\mathrm{mg}{ }^{252} \mathrm{Cf}$ sources in the TFF cells were typically less than $3.5 \mathrm{mrem} / \mathrm{hr}$ in operating areas in front of the cell window and less than $10 \mathrm{mrem} / \mathrm{hr}$ in intermittently occupied areas. Localized shielding within the cells could be used to maintain inventories of $252 \mathrm{Cf}$ $>1 \mathrm{mg}$ or to produce larger sources.

Sources prepared in the HLC or TFF were transferred via concrete or "Benelex"* casks to a Shielded Transfer Station (STS) for calibration of neutron emission from the decontaminated sources and assembly, as necessary, into special source holders required by the user. The STS is a $6 \times 12$-ft-enclosure shielded on all sides by 24-in. thickness of ordinary concrete block, and on the top by 8-in.-thick "Benelex" slabs. The station is provided with a 3-ft-thick water-filled glass window (with additional "Benelex" shielding below the window to protect the operator) and a pair of master-slave manipulators for process operations.

The station was designed for short time handling of decontaminated sources; no containment boxes are provided. Exposures from 1 -mg ${ }^{252} \mathrm{Cf}$ sources in the station totalled about $6.5 \mathrm{mrem} / \mathrm{hr}$ at the manipulator operating station.

Handling of $5-\mathrm{mg}{ }^{252} \mathrm{Cf}$ sources presented greater problems. After fabrication in the HLC ce11, the decontaminated 5-mg sources, suspended from the midpoint of a cord held at each end by operators $20 \mathrm{ft}$ away, were drawn through an access port in the cell roof slab and deposited in a "Benelex" cask for transfer to the STS. Radiation intensities from the bare source in air ranged up to about 1000 $\mathrm{mrem} / \mathrm{hr}$, principally neutron radiation, at 20 ft. Shielded by $22 \frac{1}{2}$ in. of "Benelex" and $1 \frac{1}{2}$ in. of lead in a "Benelex" cask, the $5-\mathrm{mg}$ source produced a radiation field of $100-250$ $\mathrm{mrem} / \mathrm{hr}$ at 5 in. from the cask surfaces. During remote transfer from the cask to the source calibration unit within the Shieided Transfer Station, radiation intensities were less than about $25 \mathrm{mrem} / \mathrm{hr}$ at 5 -in. from the outer surfaces of the station walls. During storage in the "Benelex". cask in the STS, radiation levels were $<1 \mathrm{mrem} / \mathrm{hr}$ at all. positions around the station.

* Registered tradename of Masonite Corp.

\section{ALTERNATIVE CAPSULE \\ MATERIALS FOR REDUCED GAMMA EMISSIONS}

Activation analysis techniques are being developed for terrestrial and underseas mineral explorations and process control applications, in which the prompt gamma radiation from capture of $252 \mathrm{Cf}$ source neutrons serve to identify the elements present (5). Utilization of the prompt $\gamma$-rays in the high energy region (3-10 Mev) avoids major interference from other source and sample emissions and allows a large sample volume to be assayed. Lithium-drifted germanium detectors permit highly selective detection of such metals as titanium, vanadium, chromium, manganese, iron, cobalt, nickel, copper, zinc, gold, and mercury, using the prompt gamma activation analysis techniques.

The presence of such metals in the source components, however, constitutes a major interference in the technique (6). The prompt gamma emissions resulting from capture of moderated neutrons in the stainless steel outer capsule of ${ }^{252} \mathrm{Cf}$ sources show peaks characteristic of the iron, nickel, and chromium present (Figure 2). Alternative capsule materials with improved radiation characteristics, as well as suitable strength, corrosion resistance, and fabricability, were sought.

A survey using neutron absorption and capture gamma data for the elements revealed several promising alternative capsule materials (6). A listing of Interference Parameters $\mathrm{CS}_{\mathrm{T}}$ ), which are summations of the magnitudes of potentially interfering gamma rays in the 3-10-Mev range, is given in Table IV for several elements of interest. Zircaloy2 ( $\mathrm{Zr}-1.5 \% \mathrm{Sn}-0.1 \% \mathrm{Fe}-0.1 \% \mathrm{Cr})$ represented an evident improvement over Type $304 \mathrm{~L}$ stainless steel (Fe-18 to $20 \% \mathrm{Cr}-8$ to $12 \% \mathrm{Ni}-2 \%$ $\max \mathrm{Mn}-1 \% \max \mathrm{Si}$ ) as an outer capsule material. Other high melting materials with improved radiation characteristics included niobium and tantalum. Low melting magnesium and aluminum might be useful in moderate temperature environments.

Platinum used for the inner capsule appeared acceptable, although substitution of ruthenium for the high interference parameter, rhodium in the inner capsule alloy might be beneficial. Among the lanthanide elements, only cerium had a lower interference parameter than terbium, for use as a carrier material in small sources. 
Experimental measurements of gamma emissions from $252 \mathrm{Cf}$ sources with alternative outer capsule materials at the U. S. Geological Survey and SRL verified the predicted reduction in high-energy gamma emissions, to those resulting only from $252 \mathrm{Cf}$ spontaneous fission. USGS measurements showed that gamma radiation intensities from an approximately $100 \mu \mathrm{g}{ }^{252} \mathrm{Cf}$. source with a Zircaloy-2 outer capsule were significantly decreased above about $5 \mathrm{Mev}$, as compared to the radiation from a similar source in a stainless steel capsule ( (7). Below 5. Mev, the ${ }^{252} \mathrm{Cf}$ gamma radiation was sufficiently intense that differences due to the alternative capsule material had little significance.' ANISN computer code calculations by H. E. Hootman at SRL of the gamma spectrum. from ${ }^{252} \mathrm{Cf}$ encapsulated in stainless steel and Zircaloy- 2 capsules supported these results.

Analogous SRL measurements showed similar results for other outer capsule materials (Figure 3). For these measurements; $4.4 \mathrm{\mu g}$ of $252 \mathrm{Cf}$ in a $\mathrm{Pt}-10 \% \mathrm{Rh}$ inner capsule was inserted successively into outer capsules of Type 304L stainless steel, Zircaloy-2, niobium, and tantalum and suspended in a light-waterfilled plastic bottle.. :High-resolution gamma ray spectra were measured, using a $15 \mathrm{~cm}^{3}$. $\mathrm{Ge}(\mathrm{Li})$ detector positioned $50 \mathrm{~cm}$ from the source and a 4096 channel pulse height analyzer. The detector was shielded from the source with 1/4-in. $\mathrm{Pb}$ to reduce low-energy gamma intensities and with 1/32-in. Cd to minimize thermal neutron damage to the detector.

The data in Figure 3 show the gamma intensities recorded for the various outer capsule materials, as summed over $0.5-\mathrm{Mev}$. intervals and normalized to 1000 min counting time. Tabulation of specific peaks in the spectra revealed characteristic radiations. from the iron, chromium, and nickel of the stainless steel encapsulated sources, but no evidence of specific radiations from other source materials (except $252 \mathrm{Cf}$ ). It was concluded that the alternative capsule materials did not contribute significantly to the gamma emissions from the source, and moreover, that the $\mathrm{Pt}-10 \% \mathrm{Rh}$ inner capsule, the Type 316 stainless steel porous disks in the capsule, and the terbium carrier used to prepare the source also did not contribute significant interfering gamma radiation.
SOURCE SAFETY EVALUATIONS

STABILITY OF 252 CF OXIDE

The chemical and structural stability of ${ }^{252} \mathrm{Cf}$ oxide was assessed by analogy with lanthanide and other actinide oxides to as sure safe handling and use of the sources under adverse conditions!

Calcination of the ${ }^{252} \mathrm{Cf}$ oxalate at $700^{\circ} \mathrm{C}$ in air during source preparation should produce a bcc (C-type) $\mathrm{Cf}_{2}\left(\mathrm{l}_{3}\right.$, possibly containing excess oxygen in sources with terbium carrier or actinide impurities (including $248 \mathrm{Cm}$ decay product). Only the $+3 \mathrm{Cf}$ oxides have been observed to date, al though variations in lattice parameter of the bcc $\mathrm{Cf}_{2} \mathrm{O}_{3}$ indicate possible deviations from +3 stoichiometry $(8,9)$. Terbium adcled as carrier to sources containing less than $500 \mu \mathrm{g}$ of $252 \mathrm{Cf}$ would be present after calcination in a form equivalent to $\mathrm{TbO}_{1.71}$, which would further oxidize on cooling in air : $\mathrm{TbO}_{1.83}(10)$. Impurity curium would be present as $\mathrm{CmO} 1.63$, which would oxidize to $\mathrm{CmO}_{2}$ on cooling in air (11). Other impurity oxides such as $\mathrm{AmO}_{2}, \mathrm{CaO}, \overline{\mathrm{PbO}}$, or $\mathrm{ZnO}$ might also be present.

Europium, samarium, and gadolinium sesquioxides were selected as the best structural homologs for $\mathrm{Cf}_{2} \mathrm{O}_{3}$ on the basis that actinide sesquioxides exist in forms structurally analogous to the three basic types of lanthanide sesquioxides. Stability ranges of the C-type (bcc), B-type (monoclinic), and A-type (hexagonal) 1 anthanide sesquioxides, and of other high temperature forms (12) are shown in Figure 4 , with the actinide sesquioxides of equivalent cationilc radii superimposed.

Based on the behavior of these lanthanide oxides, the $\mathrm{C}$-type $\mathrm{Cf}_{2} \mathrm{O}_{3}$ formed on calcination of the oxalate should transform irreversibly on heating to a stoichiometric B-type (monoclinic) $\mathrm{Cf}_{2} \mathrm{O}_{3}$ at $970-1100^{\circ} \mathrm{C}$, and possibly to $\mathrm{A}$ or $\mathrm{H}$-type (hexagonal) $\mathrm{Cf}_{2} \mathrm{O}_{3}$ near $2000^{\circ} \mathrm{C}$, before melting at $22300^{\circ} \mathrm{C}$. The $\mathrm{B}$-type (monoclinic) $\mathrm{Cf}_{2} \mathrm{O}_{3}$ and the $\mathrm{C}$-type (bcc) $\mathrm{Cf}_{2} \mathrm{O}_{3}$ have been experimentally observed $(8,9)$.

By analogy wilth ${ }^{244} \mathrm{Cm}_{2} \mathrm{O}_{3}$, a self-radiationinduced transformation of the C-type ${ }^{25}{ }^{2} \mathrm{Cf}_{2} \mathrm{O}_{3}$ to $\mathrm{B}$ or A-type oxildes may also occur at low temperatures. Wallman (13) observed C-type ${ }^{244} \mathrm{Cm}_{2} \mathrm{O}_{3}(244 \mathrm{Cm}$ half-1ife $=18.1$ years $)$ to transform to A-type oxide in two to four weeks at $700^{\circ} \mathrm{C}$; a similar transformation in $252 \mathrm{Cf}_{2} \mathrm{O}_{3}$ (252 Cf half-1ife $=2.65$ years) should occur much more rapidly. It is likely that $\mathrm{Cf}_{2} \mathrm{O}_{3}$ in a finished source capsule exists, if pure, 
as B-type or A-type sesquioxide, formed either during the $1000^{\circ} \mathrm{C}$ heating test or by selfradiation-induced transformation of the C-type oxide at a lower temperature.

Carrier $\mathrm{TbO}_{1} .83$ or impurity $\mathrm{CmO}_{2}$ present in the source oxide after calcination would lose oxygen during the $1000^{\circ} \mathrm{C}$ heating test to form the corresponding sesquioxides. These components of the source oxide would increase or decrease, respectively, the temperature for thermal transformation of the C-type to $\mathrm{B}-$ type $\mathrm{Cf}_{2} \mathrm{O}_{3}$, in accord with Figure $4.248 \mathrm{Cm}$ generated in the source by alpha decay of the ${ }^{252} \mathrm{Cf}$, for example, would lower the C-B transformation temperature of the product oxide by $150^{\circ} \mathrm{C}$ over two half-1ives $(5.3 \mathrm{yr})$. If, however the radiation-induced transformation at low temperatures predominated as expected, the 1 anthanide-actinide impurities present should not greatly change the final form of the $\mathrm{Cf}_{2} \mathrm{O}_{3}$ oxide in the source capsule.

Melting points of the lanthanide and actinide elements, shown in Figure 5 as a function of cationic radius, indicate $\mathrm{Cf}_{2} \mathrm{O}_{3}$ oxide should melt between 2300 and $2400^{\circ} \mathrm{C}$. High melting 1 anthanide and actinide impurity oxides should not markedly lower this; other impurities could have a greater effect.

\section{MATERIALS COMPATIBILITY}

No deleterious incompatibility of californium sesquioxide with the $\mathrm{Pt}-10 \% \mathrm{Rh}$ inner capsule of the sources is expected at temperatures up to $1000^{\circ} \mathrm{C}$, although impurities present in the oxide may promote minor reactions. In nonoxidizing atmospheres at high temperatures, the lanthanide and higher actinide sesquioxides $\left(\mathrm{Cm}_{2} \mathrm{O}_{3}\right)$ generally are non-reactive in noble metal containers. In air atmosphere at high temperatures, however, the noble metals may oxidize to some extent, and the resulting noble metal oxide may react with sesquioxides present, though no direct reaction of the sesquioxides with the unoxidized noble metal occurs (14). Thus, ${ }^{244} \mathrm{Cm}_{2} \mathrm{O}_{3}$ has been observed to change structure when heated in air in contact with Pt above $850^{\circ} \mathrm{C}$; no reaction was observed in inert or reducing atmospheres.

Reaction of ${ }^{25}{ }^{2} \mathrm{Cf}_{2} \mathrm{O}_{3}$ with noble metal oxides in the Pt-Rh inner capsule analogous to the similar reaction of $\mathrm{Cm}_{2} \mathrm{O}_{3}$ could occur to only the very limited extent that oxygen is available in the capsule atmosphere. Since only minor amounts of oxygen, derived possibly from higher oxide impurities, could be present in the sealed capsules, formation of $\mathrm{Pt}-\mathrm{Rh}$ oxides capable of reaction with the sesquioxides in the capsule would be of negligible significance.

Chemical reaction of the source oxides with the stainless steel sintered disk in the capsule would be similarly restricted.

No incompatibility of practical significance has been observed between the other material components of the sources. Metallographic examinations by $\mathrm{C}$. L. Angerman of Pt$10 \% \mathrm{Rh}$ inner capsule specimens heated in contact with the porous Type 316 stainless steel disks and Type 304L stainless steel, Zircaloy2 , niobium, and tantalum outer capsule specimens at $1000^{\circ} \mathrm{C}$ for $1 \mathrm{hr}$ in vacuum showed only minor reaction layers $(<1 \mathrm{mi} 1)$. No significant degradation of capsule material properties due to such interactions during capsule preparation and testing is expected.

More extensive compatibility evaluations would be required if long duration use of the sources at high temperature were anticipated.

\section{HEAT TRANSFER AND STRENGTH ANALYSES}

Evaluation of heat transfer and capsule strength parameters indicated the ${ }^{252} \mathrm{Cf}$ source design is capable of maintaining integrity under severe operating and accident conditions.

Sources with assumed ${ }^{252} \mathrm{Cf}$ content of up to $10 \mathrm{mg}$ were evaluated; capsule materials considered were $\mathrm{Pt}-10 \% \mathrm{Rh}$ for the inner capsule and Type 304L stainless stee1, Zircaloy-2, niobium, and tantalum for the outer capsule.

The heat from alpha decay and fission of 252 Cf, approximately 40 watts/g, was assumed to be generated entirely within the source and transfered to the capsule. For heat removal from the capsule by natural convection to the surrounding air, the bare inner capsule contair ing $10 \mathrm{mg}$ of $252 \mathrm{Cf}$ would be $50^{\circ} \mathrm{C}$ higher than the average air temperature; an outer capsule enclosing the inner capsule would be $34^{\circ} \mathrm{C}$ higher than ambient air (Table V). These capsule temperatures could be reduced by heat sinks attached to the capsule surfaces.

Sources containing up to $10 \mathrm{mg}$ of ${ }^{252} \mathrm{Cf}$ would not overheat severely in contact with any amount of insulating material ( $\mathrm{K}=0.02$ $\left.\mathrm{pcu} / \mathrm{hr}-\mathrm{ft} \mathrm{t}^{2}{ }^{\circ} \mathrm{C}-\mathrm{ft} \mathrm{t}^{-1}\right)$. The adiabatic temperature rise of the inner capsule, if perfectly insulated, would be about $19^{\circ} \mathrm{C} / \mathrm{min}$, and of the inner and outer capsules combined, $4^{\circ} / \mathrm{min}$. Enclosed in a 1-in. thickness of insulation, the outer capsule surface would not rise more 
than $57^{\circ} \mathrm{C}$ higher than the ambient air temperature; in one foot of insulation the maximum temperature rise would be $73^{\circ} \mathrm{C}$. Additional insulation thickness would increase this temperature rise by only small amounts.

Helium produced by $\alpha$-decay of the ${ }^{252} \mathrm{Cf}$ is the principal gas generated in the source during service; fission gases are less than $1 \%$ of the helium generated. Complete $\alpha$-decay would produce about $88 \mathrm{st} \mathrm{cc}$ He per gram of 252Cf, as indicated in Figure 6. After 10 years decay, internal gas pressure in an inner capsule containing an initial $10 \mathrm{mg}$ of $252 \mathrm{Cf}$ (1-atm. pressure originally present) would be $89 \mathrm{psi}$ at $0^{\circ} \mathrm{C}$ and 413 psi at $1000^{\circ} \mathrm{C}$ (assumed accidental fire condition), as shown in Table VI. If released to the outer capsule (by weld leak for example), the corresponding gas pressures would be $59 \mathrm{psi}$ at $0^{\circ} \mathrm{C}$ and $275 \mathrm{psi}$ at $1000^{\circ} \mathrm{C}$.

Calculation of the capsule wall strengths, assuming thick-walled cylindrical geometry, indicates these pressures could be contained with ample safety factors. The Pt-10\% Rh inner capsule could safely contain internal pressures up to $14,600 \mathrm{psi}$ at $25^{\circ} \mathrm{C}$ and up to 6500 psi for short times at $1000^{\circ} \mathrm{C}$. The Type $304 \mathrm{~L}$ stainless steel outer capsule could contain internal pressures up to $24,000 \mathrm{psi}$ at $25^{\circ} \mathrm{C}$ and $2400 \mathrm{psi}$ at $1000^{\circ} \mathrm{C}$, and the Zircaloy2 outer capsule $18,000 \mathrm{psi}$ at $25^{\circ} \mathrm{C}$ and 5,800 psi at $1000^{\circ} \mathrm{C}$. Niobium and tantalum outer capsule materials would prove equally satisfactory.

\section{PROTOTYPE TESTS}

The integrity of prototype source designs was demonstrated experimentally by subjecting outer capsules with circumferential seal welds to internal and external pressures far in excess of the pressures expected under adverse service and accident conditions. Hydrostatic burst tests at $25^{\circ} \mathrm{C}$ showed that the internal pressures required to burst the outer capsule welds were 47,500 psi for Type $304 \mathrm{~L}$ stainless steel and 41,000 psi for Zircaloy -2 .

Outer capsules of Type $304 \mathrm{~L}$ stainless steel and Zircaloy-2 subjected to 25,000 psi external He pressure did not deform and showed no leaks after pressurization, within a detection limit of $1.0 \times 10^{-8} \mathrm{std}$ cc He per $\sec$.

Capsules pressed between stainless steel anvils to loads up to 10 tons deformed, but remained leak free.

\section{ACKNOWLEDGMENT}

Many people at Savannah River and Oak Ridge contributed to the technology developed for the preparation of $252 \mathrm{Cf}$ sources at SRL. A major contribution was made by G. A. Burney in the separation and purification of SRL $252 \mathrm{Cf}$ feed solutions. The diligence and cooperation of J. E. Bigelow and associates at ORNL in development and preparation of suitable packages for shipment of $252 \mathrm{Cf}$ to SRL is also greatly appreciated.

REF,ERENCES

1. Californium-252: Its Use and Market Potential. Savannah River Operations Office, Aiken, S. C. (1969); Californium252 Progress (Numbers 1-5). Savannah River Operations Office, Aiken, S.'C. (1969-1970).

2. A. R. Boulogne and J. P. Faraci. "Californium-252 Neution Sources for Industrial Applications.' to be published in Nucl. App 2. Tech.

3. A. R. Boulogne and A. G. Evans. "Californium-252 Neution Sources for Medical Applications." Int. J. App Z. Rad. and Isotop. 20,453 (1969).

4. W. H. Hale. "Thermal Decomposition of Neodynium-Loaded Cation Exchange Resin." to be published in $J$. Inorg Mucl. Chem.

5. F. E. Senftle, D. Duffey, and P. F. Wiggins. "Mineral Exploration of the Ocean Floor by In-situ Nelitron Absorption Using a Californium-252 (252Cf) Source." Marine Tech. Soc. Journ. 3 (5), 9 (1969).

6. A. G. Evans, I. E. Senftle, P. F. Wiggins, and D. Duffey! "Construction Materials for Neutron Capture Gamma-Ray Spectroscopy with 252Cf Sources." Trans. Am. Mucl. Soc. 13, 488 (1970).

7. P. Philbin, Fl. Senftle, D. Duffy, P. Wiggins, and A. G. Evans. "Comparison of Stainless Steel and Zircaloy-2 as Encapsulation Materials for Californium-252." to be published.

8. J. L. Green. "The Absorption Spectrum of ${ }^{+}{ }^{3} \mathrm{Cf}$ and Crystallography of Californium Sesquioxide and Californium Trichloride." UCRL-16516 (1965). 
9. J. C. Copeland and B. B. Cunningham. "Crystallography of the Compounds of Californium-II. Crystal Structure and Lattice Parameters of Californium Oxychloride and Californium Sesquioxide." J. Inorg. Nucl. Chem. 31, 733 (1969).

10. J. Kordis and L. Eyring. "A Tensimetric Study of Terbia and Praseodymia Systems and the Mixed Praeseodymia-Terbia System." J. Phys. Chem. 72, 2044 (1968); D. A. Burnham, L. Eyring, and J. Kordis. "HighTemperature X-Ray Diffraction Studies of the Terbuim Oxide-Oxygen and Mixed Cerium Terbuim Oxide-Oxygen Systems." J. Phys. Chem. 72, 4424 (1968).

11. W. C. Mosley. "B-Type ${ }^{244} \mathrm{Cm}_{2} \mathrm{O}_{3}$ - A Candidate Isotopic Power Fuel." Abstracts of Papers, American Chemical Society Meeting, San Francisco, March 31 - Aprit 5, 1968.
12. M. Föex and J.P. Traverse. "Remarques sur les différentes transformations cristállines présentées à haute température par les sesquioxydes des terres rares." C. R. Acad. Sc. Paris t. 262 (Série C), 636 (1966).

13. J. C. Wallman. "A Structura1 Transformation of Curium Sesquioxide." J. Inorg. Mucl. Chem. 26, 2053 (1964)。

14. S. J. Schneider, J. L. Waring, and R. E. Tressler. "Phase Relations Between Iridium and the Sesquioxides in Air." J. Research NBS 69A, 245 (1965).

TABLE I

Californium Sources Fabricated at SRL $(9 / 18 / 68-12 / 31 / 70)$

\begin{tabular}{|c|c|}
\hline $\begin{array}{c}\text { Source } \\
\text { No. }\end{array}$ & $\begin{array}{c}252 \text { cf Equivalents, } \\
\text { U日 }\end{array}$ \\
\hline \multicolumn{2}{|l|}{ sk-t:f- } \\
\hline 1 & 78 \\
\hline 2 & 3 \\
\hline 3 & 17 \\
\hline 4 & 135 \\
\hline 5 & 141 \\
\hline 0 & $1 \mathrm{t}$ \\
\hline \multicolumn{2}{|l|}{ SR-cif- } \\
\hline 101 & 49 \\
\hline 102 & 703 \\
\hline 10.3 & 50 \\
\hline 10.4 & 122 \\
\hline 105 & 100 \\
\hline 108 & 917 \\
\hline 167 & 81 \\
\hline 108 & $8 \tilde{3}$ \\
\hline 109 & 82 \\
\hline 116 & 81 \\
\hline 111 & 81 \\
\hline 112 & 52 \\
\hline 115 & 1121 \\
\hline 114 & 197 \\
\hline 115 & 57 \\
\hline 110 & $36 !$ \\
\hline 117 & 519 \\
\hline 118 & 24 \\
\hline 119 & 24 \\
\hline 120 & 25 \\
\hline 121 & 24. \\
\hline 122 & 4 \\
\hline 123 & 12 \\
\hline $124-z^{a}$. & 23 \\
\hline $125-z$ & 112 \\
\hline 126 & 1006 \\
\hline 127 & 0.1 \\
\hline 128 & 9,3 \\
\hline 129) & 93 \\
\hline 130 & 86 \\
\hline 131 & 10.3 \\
\hline 132 & 97 \\
\hline 133 & 105 \\
\hline 134 & 80 \\
\hline 135 & (1) 46 \\
\hline 136 & 119 \\
\hline 137 & 45 \\
\hline 138 & 5212 \\
\hline 139 & 6 \\
\hline 140 & 105 \\
\hline 141 & 220 \\
\hline 142 & 352 \\
\hline 145 & 31 \\
\hline 144 & 152 \\
\hline & \\
\hline
\end{tabular}

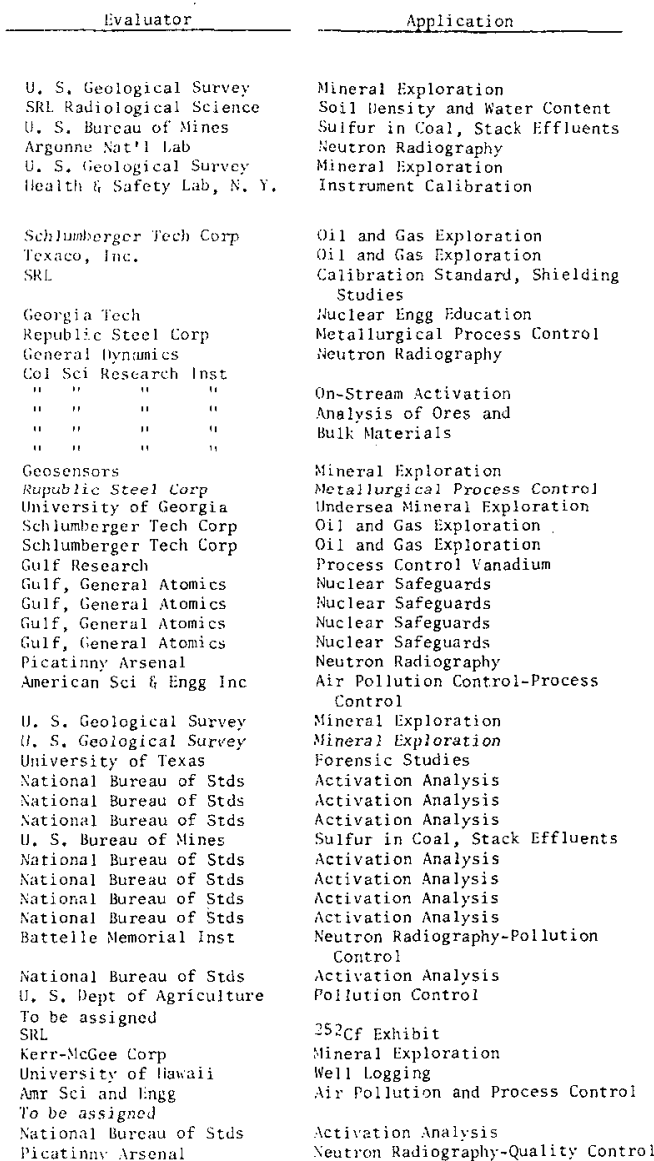

$\bar{a}$ a designates outer capsule in realoy-2; at 11 others type $30+1$ stainless steel 
TABLE II

Isotopic Purity of ${ }^{252} \mathrm{Cf}$ Feed Solutions

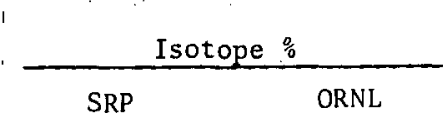

$\begin{array}{lcc} & 3 / 19 / 69 * & \underline{7 / 13 / 70 *} \\ { }^{249} \mathrm{Cf} & 23.92 & 1.05 \\ { }^{250} \mathrm{Cf} & 24.88 & 9.69 \\ { }^{251_{\mathrm{Cf}}} & 5.95 & 2.77 \\ { }^{252} \mathrm{Cf} & 45.22 & 86.43 \\ { }^{253} \mathrm{Cf} & <0.3 & 0.045 \\ { }^{254} \mathrm{Cf} & -\ldots .- & 0.019\end{array}$

Assay date

TABLE III

Typical Analyses of ${ }^{252} \mathrm{Cf}$ Feed Solutions

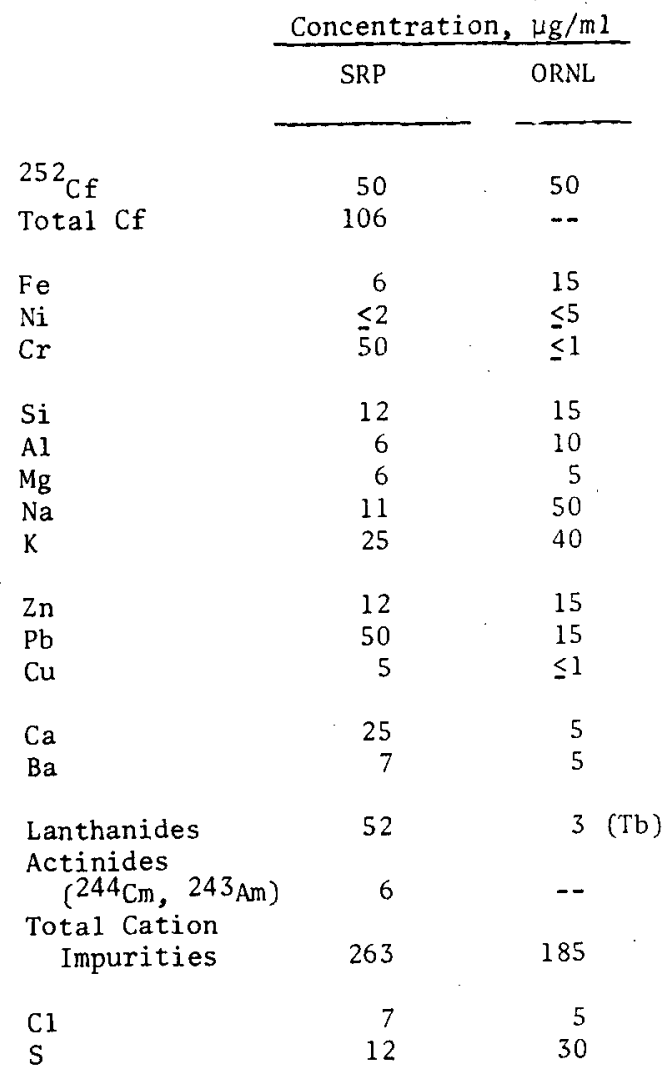

TABLE: IV

Gamma Radiation Interference Parameters

for Selected Elements (ㅁ)

Element

$\mathrm{C}$

$\mathrm{Sn}$

$\mathrm{Zr}$

$\mathrm{Be}$

$\mathrm{Ce}$

$\mathrm{Mg}$

$\mathrm{Nb}$

$\mathrm{Al}$

$\mathrm{Ru}$

Mo

. Ta

$\mathrm{Pd}$

$\mathrm{Pt}$

$\mathrm{Tb}$

W

$\mathrm{Cr}$

$\mathrm{Cu}$

$\mathrm{Ni}$

$\mathrm{Ti}$

$\mathrm{Mn}$

$\mathrm{Rh}$

Co

Ir
Interference

Parameter (ST)

0.028

0.085

0.106

0.116

0.230

0.291

0.310

0.633

0.659

0.724

1.15

1.48

1.63

2.04

3.55

4.66

5.76

5.94

9.02

16.5

23.4

24.0

31.6

52.2

54.5

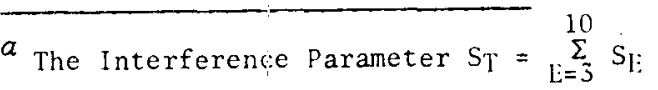
represents the summation of Interference Indices $S_{E}$ for $g$ anma lines from 3 to 10 Mev. The Interference Index $S_{E}=I_{E} \sigma_{c} / A$, where $\mathrm{I}_{\mathrm{E}}=$ intensity of gamma of energy $\mathrm{E}$ per 100 captured neutrons, $\sigma_{C}=$ thermal neutron capture cross section in barns, and $A=$ atomic weight of element.

T'ABLE V

Capsule Surface Temperatures in Air at $25^{\circ} \mathrm{C}$

Bare Inner Capsule

Outer Capsule Containing Inner Capsule

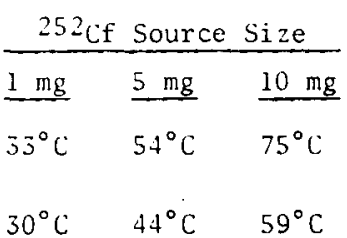


TABLE VI

Internal Gas Pressures in ${ }^{252} \mathrm{Cf}$ Capsules

Pressure in Inner Capsule after 10 Years Decay of ${ }^{252} \mathrm{Cf}$
Source, $\mathrm{mg}{ }^{252 \mathrm{Cf}}$

1

5

10
Internal Gas Pressure, psia

Pressure in Outer Capsule if Inner Capsule Ruptured
Source, mg ${ }^{252} \mathrm{Cf}$

\section{1}

5 $0^{\circ} \mathrm{C} \quad 1000^{\circ} \mathrm{C}$

$22 \cdot 101$

56260

$89-413$

Internal Gas Pressure, psia

\begin{tabular}{|c|c|}
\hline $0^{\circ} \mathrm{C}$ & $1000^{\circ} \mathrm{C}$ \\
\hline 18 & 82 \\
\hline 36 & 166 \\
\hline 59 & 275 \\
\hline
\end{tabular}
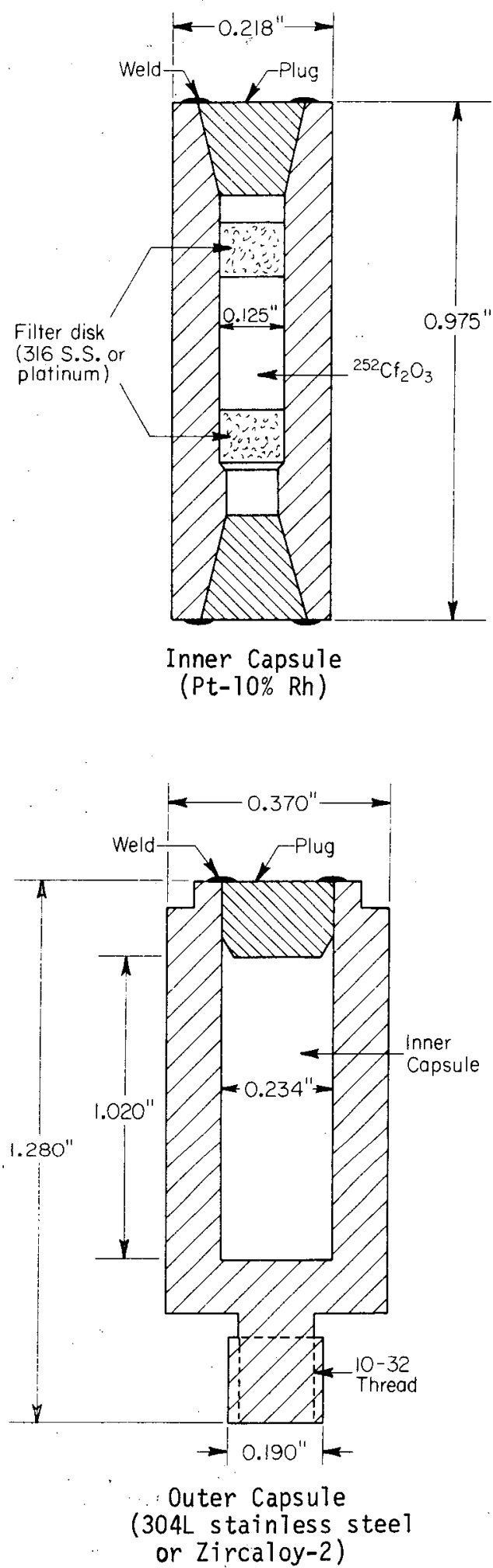

FIG. 1 CALIFORNIUM-252 SOURCE CAPSULES (Sr-Cf-100 Series) 


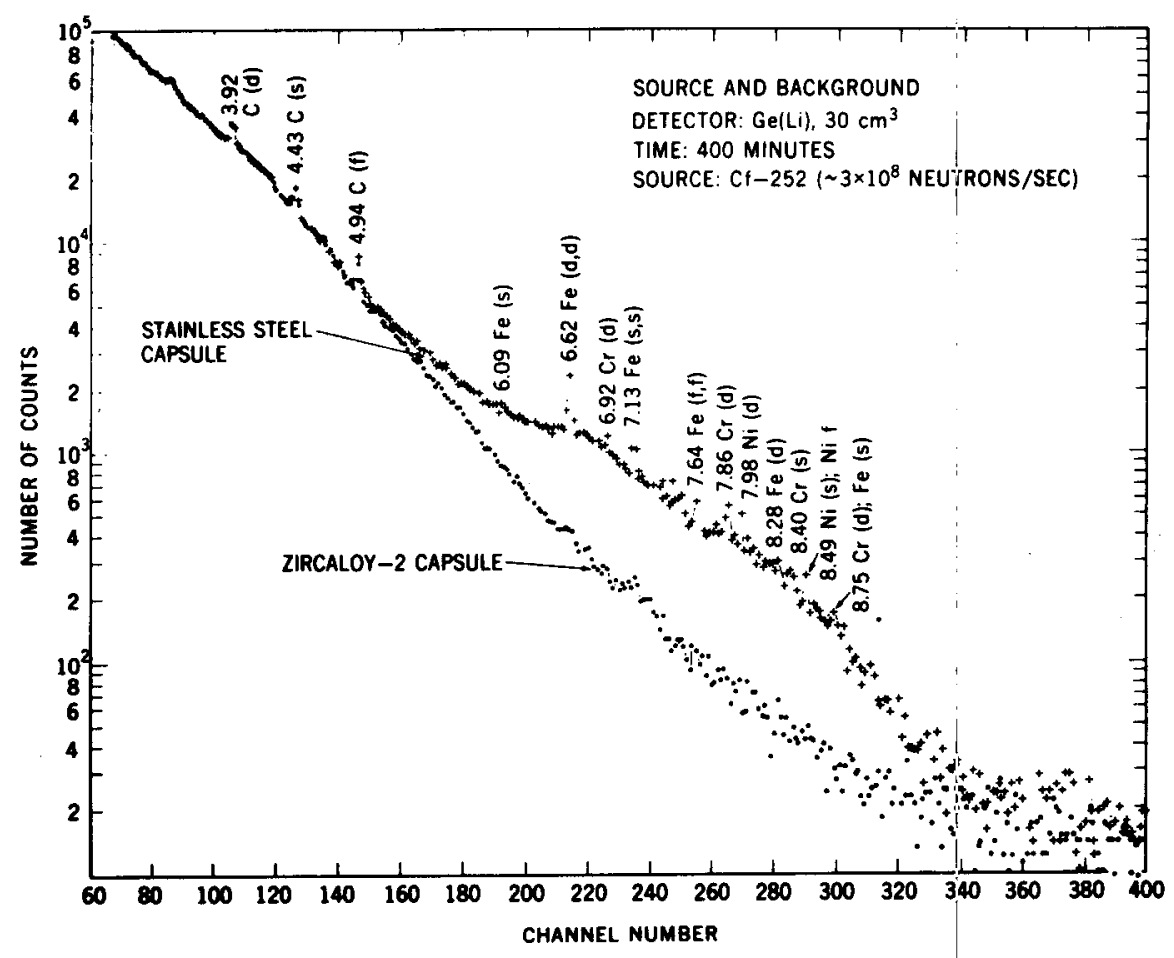

FIG. 2 COMPARISON OF GAMMA EMISSIONS FROM

STAINLESS STEEL AND ZIRCALOY-2

ENCAPSULATED 252Cf SOURCES (Ref. 7)

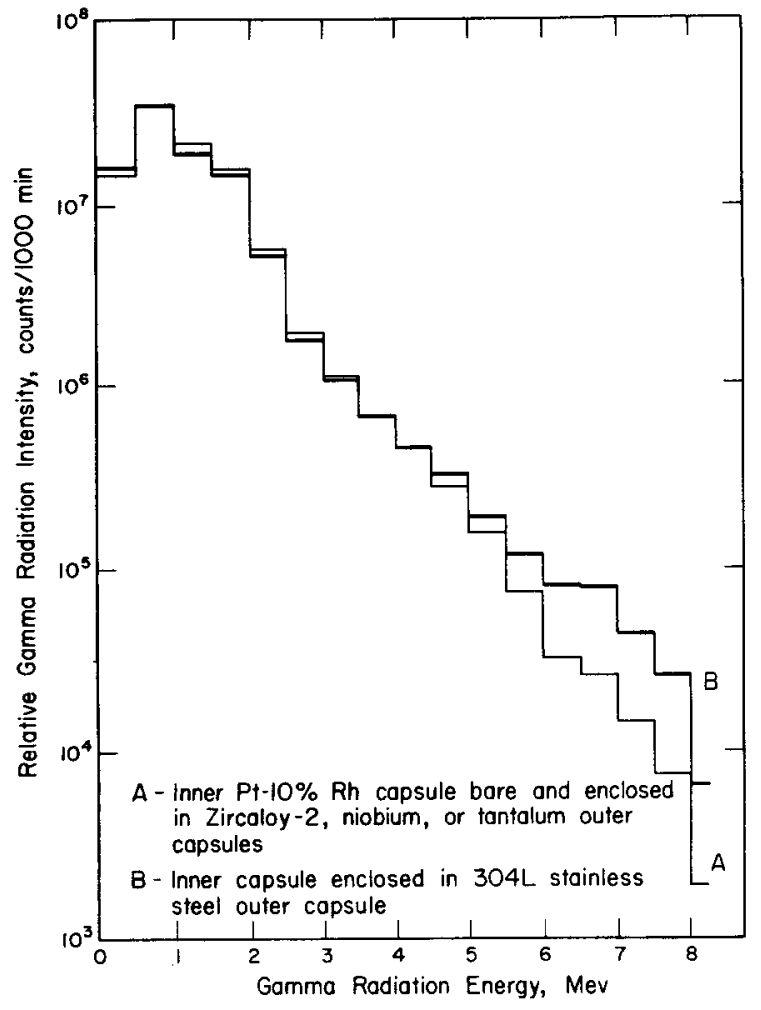

FIG. 3 EFFECT OF OUTER CAPSULE MATERIAL ON GAMMA RADIATIONS FROM A $252 \mathrm{Cf}$ SOURCE

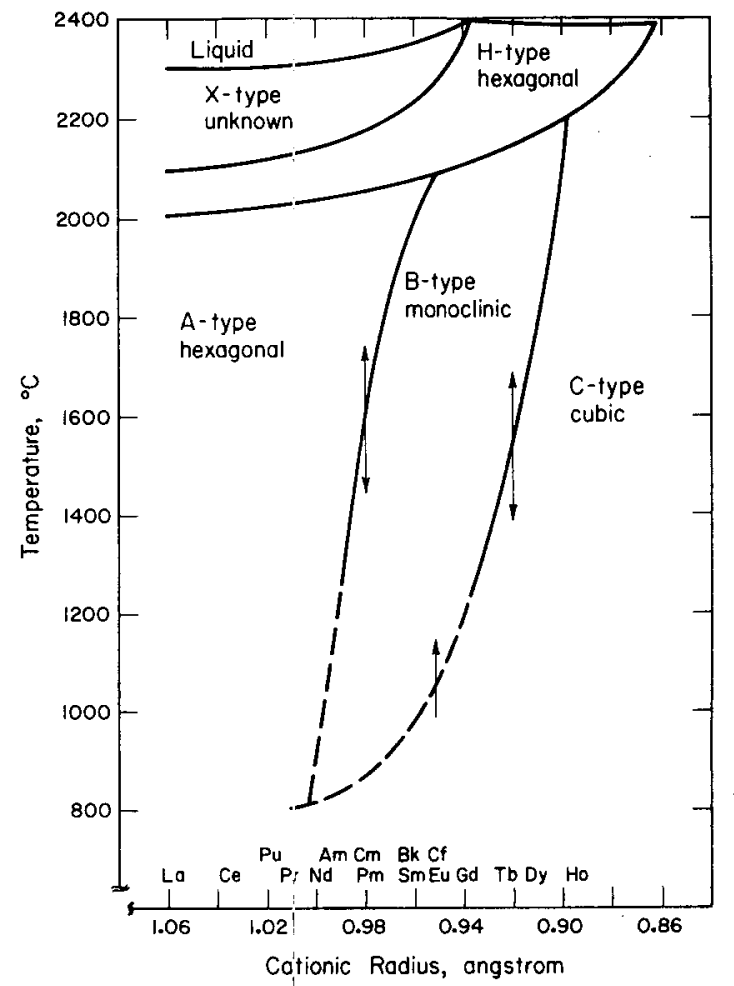

FIG. 4 STABILITIES OF SESQUIOXIDE POLYMORPHS 


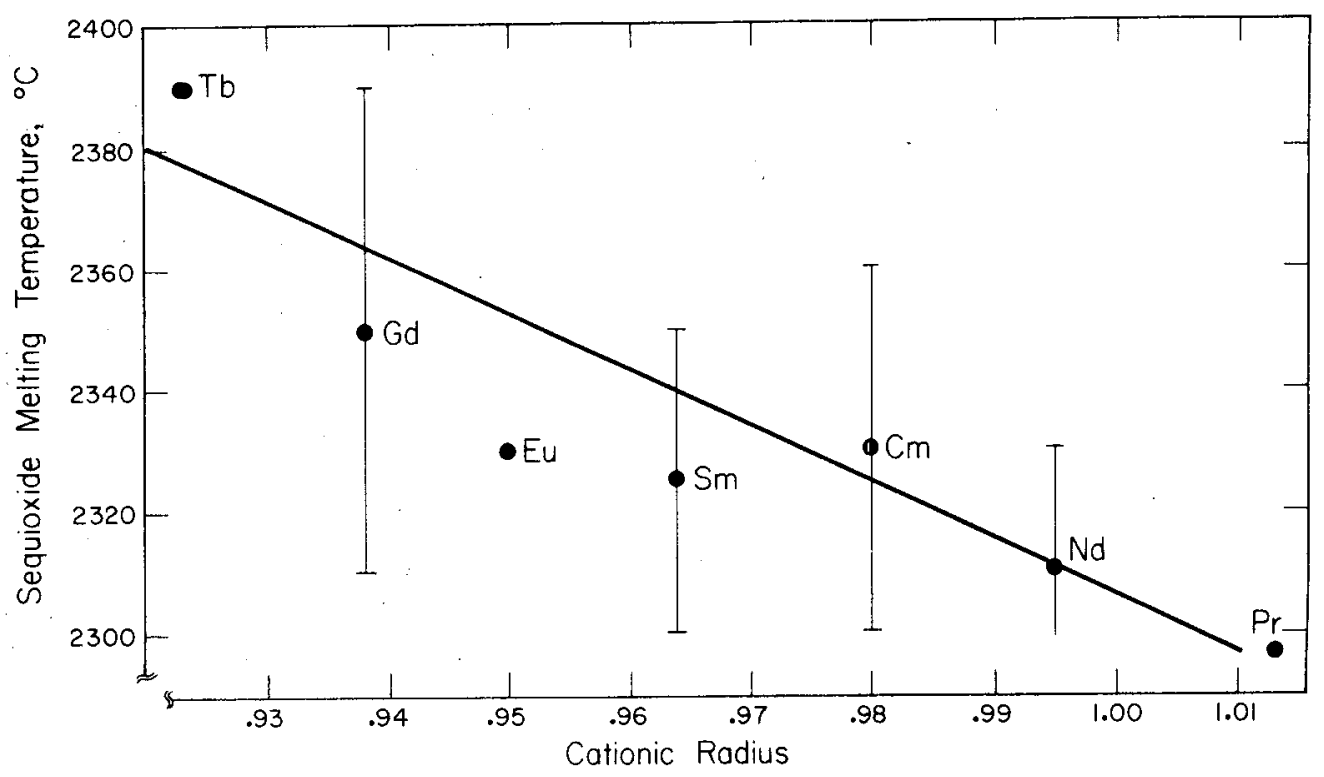

FIG. 5 SESQUIOXIDE MELTING POINTS AS A

FUNCTION OF CATIONIC RADIUS

References: Tb, Eu, Pr (no error limits

given): Georg Brauer in Progress in the

Science and Technology of the Rare Earths,

III, p. 444, ed. by L. Eyring, 1968; Cm:

B. L. Dahlen, unpublished work at SRL,

1969; Gd, Sm, Nd: M. Föex, COMPTES RENDU,

Vol. 260, p. $6389,1965$.

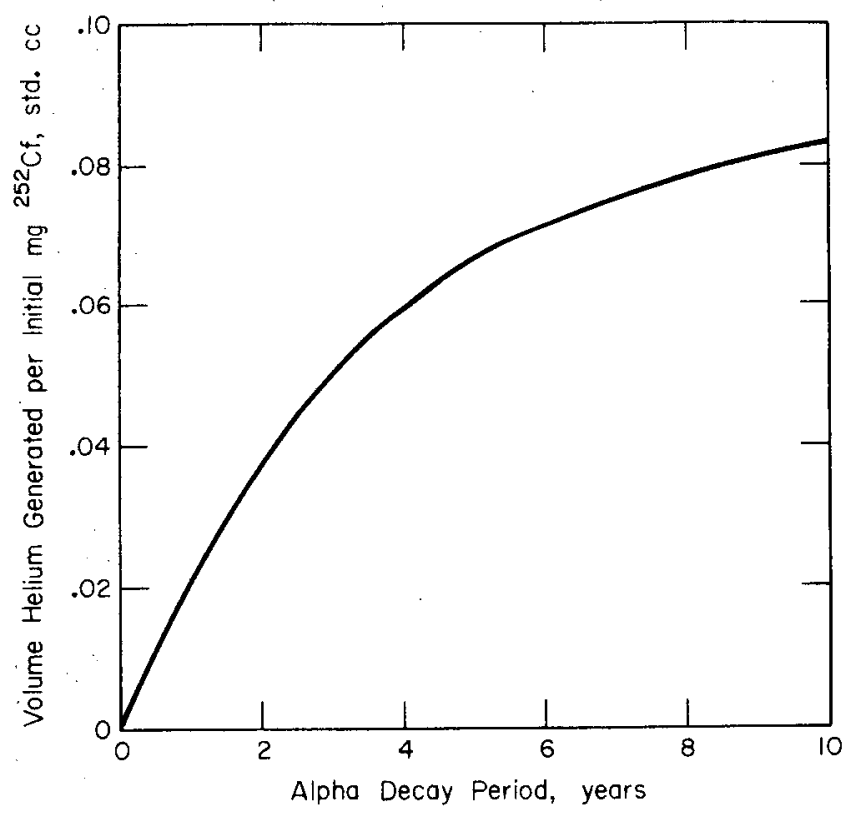

FIG. 6 HELIUM GENERATION FROM ALPHA DECAY

OF 252Cf

(Half-Life $=2.65 \mathrm{yr}$ ) 


\title{
EFFECTS OF SOURCE ENCAPSULATION MATERIALS ON THE THERMAL-NEUTRON FLUX FROM A ${ }^{252}$ Cf SOURCE IN A WATER MODERATOR
}

\author{
Kenneth D. Kok, Ricardo Artigas, \\ and Joseph W. Ray
}

Battelle Memorial institute Columbus Laboratories

Columbus, Ohio

\begin{abstract}
Many possible applications of small neutron sources require the optimization of the thermal flux from the source. Since these are fast-neutron sources, the peak thermal flux available from the source occurs in the moderator surrounding the source. The material in which the source is encapsulated can have an effect by perturbing the thermal flux around the source and decrease the peak flux available?

Experimental measurements were carried out using a ${ }^{252} \mathrm{Cf}$ source encapsulated in a Pt-Rh inner capsule and a Type 304 stainless steel outer capsule. The outer capsule dimensions were 0.375 inch $O D$ and 1.25 inches long. The calibrated source strength was $2.385 \times 10^{9}$ neutrons/second. Flux measurements made using gold-foil activation techniques gave a peak flux of $2.4 \times 10^{7}$ neutrons $/ \mathrm{cm}^{2}$-seclapproximately 0.5 inch from the source.

Calculations were made using the two-dimensional transport code DOT to determine the effect of encapsulation material on thermal-neutron flux and flux distribution in a water moderator. Calculations were made for the actulal encapsulation material (Pt-Rh inner, stainless steel outer) and for capsules made entirely of aluminum. These calculations indicate a $\mathbf{2 0}$ percent higher thermal-neutron flux with the aluminum capsule than with the Pt-Rh/stainless steel capsule.
\end{abstract}

\section{INTRODUCTION}

A major consideration in the use of ${ }^{252} \mathrm{Cf}$ neutron sources for many applications is that the thermal-neutron flux in the moderator surrounding the source must be as high as possible. This requires careful selection of both the source-encapsulation material and the moderator material. Considerable attention has been given to the evaluation of moderator materials. This paper reports the results of research aimed at evaluating the effect of candidate sourceencapsulation materials on peak thermalneutron flux in a water moderator surrounding a $252 \mathrm{Cf}$ source. Two material combinations were considered: a Pt-10 percent Rh inner capsule surrounded by a Type 304 stainless steel outer capsule, and a pure aluminum capsule. The former combination is the standard Sr-Cf-100 series capsule.

Transport calculations were made to determine the peak thermal-neutron flux for the two capsules. These results were compared with experimental data from BattelleColumbus (BCL) on the Sr-Cf-100 capsule and from Oak Ridge National Laboratory (ORNL) on the aluminum-encapsulated source.

\section{ANALYTICAL METHOD}

The thermal flux in water, from two different californium- 252 sources, was

Work performed under AEC Contract AT $(38-1)-593$. computed by the use of the two-dimensional transport code, DOT-IIW (1)*. The code solves the two-dimensional, Boltzmann transport equation with general anisotropic scattering by using a diamond difference solution technique'. The method of discrete ordinates or Carlson's Sn method has been implemented in the code (2).

Figure 1 shows the $\mathrm{Sr}-\mathrm{Cf}-100$ and the aluminum-encapsulated $252 \mathrm{Cf}$ source configurations as used for computational purposes. A water region of approximately 7 inches in radius and 14 inches in height was considered. Reflective boundary conditions $(d \varphi / d \bar{X}=0)$ were used at the left and bottom boundaries, and vacuum (no reflection) boundary conditions were used at the top and right boundaries. This set of boundary conditions was used to take advantage of the problem's symmetry. The thermal fluxes of interest are the one at the midplane along a radial axis (see Figure 1).

The radial and axial mesh intervals were picked using the criteria given below to assure accurate solutions, within the limitations of BCL's computer system. These criteria, used near boundaries between regions or in regions with rapidly changing fluxes, are given by

*The two-dimensional $\mathrm{D} \emptyset \mathrm{T}$ code was used in perference to the one-dimensional ANISN code because of the difficulty of representing the Sr-Cf-100 source in a one-dimensional model. 


$$
\Delta \mathrm{R} \leq 1.0+\sum_{i \rightarrow i}^{\mathrm{S}_{1} \mathrm{t}} / \Sigma \text { and } \Delta \mathrm{Z} \leq 2.0 / \Sigma_{i}^{\mathrm{t}}
$$
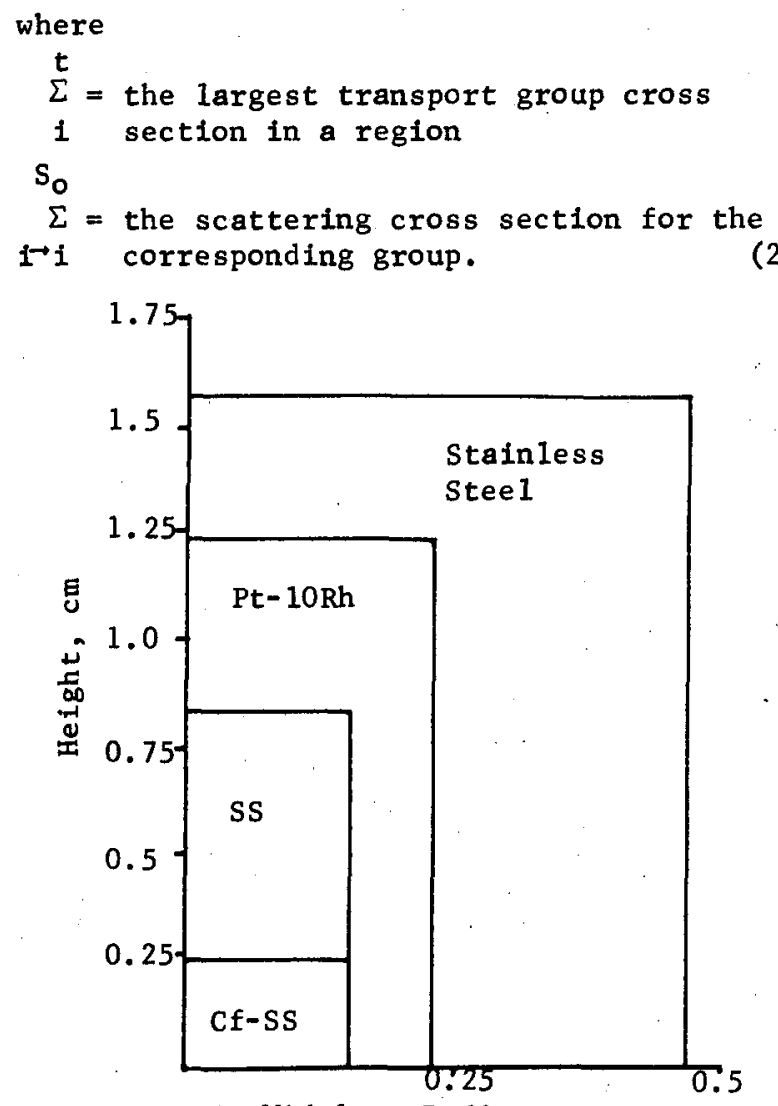

Midplane Radius, $\mathrm{cm}$

(a) Battelle Source

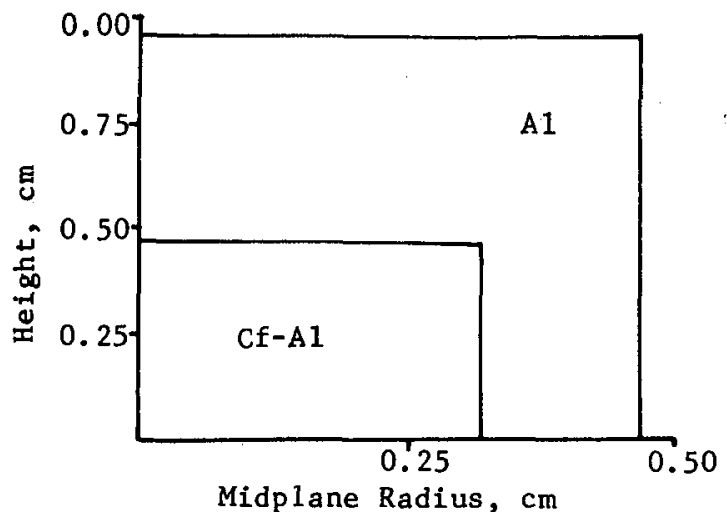

(b) ORNL Source

FIG. 1 DESCRIPTION OF ${ }^{252}$ Cf SOURCES USED IN DØT
These criteria were relaxed for mesh spacings away from boundaries where the thermal fluxes resulted essentially from scattering-down processes (1).

The energy range was divided into 15 energy groups, the fifteenth group corresponding to the thermal-energy range. A $\mathrm{P}_{2}$ multigroup set of cross sections was obtained by $1 / \mathrm{E}$ weighting of the 99 -group DLC- 2 set of cross sections with ANISN (3). The Pt-Rh alloy cross sections were approximated.

Sixteen space angles (discrete directions) were used in the calculation, $S_{4}$, with the symmetric quadrature sets (direction cosine and angular quadrature weight data) given in the $\mathrm{D} \emptyset \mathrm{T}-\mathrm{II}-\mathrm{W}$ code.

A11 runs were normalized to a total source strength of 1 neutron/second.

\section{EXPERIMENTAL TECHNIQUE}

The spatial neutron-flux distribution radially outward from the $\mathrm{Sr}-\mathrm{Cf}-100$ source was measured by dysprosium-foil activation techniques. Dysprosium foils, 0.25 inch in diameter and 0.001 inch thick, were mounted on $0.05-$ inch-long polystyrene rods. These rods were stacked in a polystyrene tube and the tube placed in contact with the ${ }^{252} \mathrm{Cf}$ source as shown in Figure 2. Polystyrene was selected since its cross sections closely approximate those of water, and no significant activation of the polystyrene occurs.

The foils were counted using the gamma scintillation system at the Battelle Research Reactor. The system consists of a $3 \times 3$-inch NaI(T1) we11 crysta1 and a 512-channe1 analyzer. The $0.0948-\mathrm{MeV}$ decay gamma ray from dysprosium-165 was used for analysis. At least 60 minutes was allowed for the complete decay of dysprosim-165 m before counting.

The absolute flux was determined by gold-foll activation. Bare and cadmium (0.040 inch) covered gold folls were positioned 0.563 inch from the surface of the source capsule. The $0.4118-\mathrm{MeV}$ gamma from the decay of ${ }^{198} \mathrm{Au}$ was used for analysis. Gold foils were used to obtain an absolute measurement; since the counting system was calibrated using gold foils activated at the National-Bureau of Standards. The absolute flux was calculated and the flux distribution normalized to the absolute flux value. 
The experimental data for the aluminumencapsulated source were taken from the work of Magnuson (4). The experimental techniques used for collecting these data are described in Magnuson's paper.

\section{RESULTS AND CONCLUSIONS}

The thermal-neutron flux distribution obtained for the Sr-Cf-100 source at BCL is shown in Figure 3 . The flux is normalized to a source of 1 neutron per second for comparison purposes. The peak flux per source neutron of $1.06 \times 10^{-2} \mathrm{n} / \mathrm{cm}^{2} /$ source $\mathrm{n}$ corresponds to an absolute flux of $2.53 \times 10^{7}$ $\mathrm{n} / \mathrm{cm}^{2} / \mathrm{sec}, 0.75$ inch from the center of the source.

Figure 4 shows the $B C L$ data for the SrCf-100 source and the ORNL (4) data for the aluminum-encapsulated source. The peak normalized flux obtained at ORNL is 1.37 $x 10^{-2} \mathrm{n} / \mathrm{cm}^{2} /$ source $\mathrm{n}$, which is 25 percent greater than the peak obtained with the SrCf -100 source at BCL. The two sets of data begin to converge as the distance from the source increases, and are nearly the same at a distance of 3 inches or greater.

The primary difference between the two sources is the encapsulation materials. The absorption cross sections for thermal neutrons is greater for the stainless steel, platinum, and rhodium in the Sr-Cf-100 source than for the aluminum capsule in the ORNL source. It is apparent that the thermal flux in the region of the source is perturbed by the materials used for the ${ }^{252} \mathrm{Cf}$ encapsulation.

Figure 5 shows the calculated therma1flux distributions obtained for the two sources using the $D \emptyset \mathrm{T}-I I-W$ code. The magnitude and shape of the calculated distributions, Figure 5, is the same as for the experimental data, Figure 4, up to about 1-1/2 inches fron the source. At distances from the source greater than $1-1 / 2$ inches the calculated filuxes decrease more rapidly than the experimental; however, the calculated fluxes for both sources become essentially identical beyond 3 inches, as was true for thelexperimental data.

The rapid decrease in the thermal flux more than 3 inches from the source is due to the increased mesh spacing at greater distances. This is/caused by computer size limitations and the desire to obtain an extremely fine mesh near the source, which was the area of prime interest. . This decrease has very little effect on the accuracy of the solution near the source since the distribution is calculated from the source outward. If the mock-up size had been decreased to allow a finer mesh, the effects of increased leakage at the boundary could have caused a greater error in flux near the source.

These results show that the material of which a ${ }^{252} \mathrm{Cf}$ capsule is constructed can have a significant effect on the peak thermal flux obtainable from the source. This suggests that a high-strength, low-cross-section

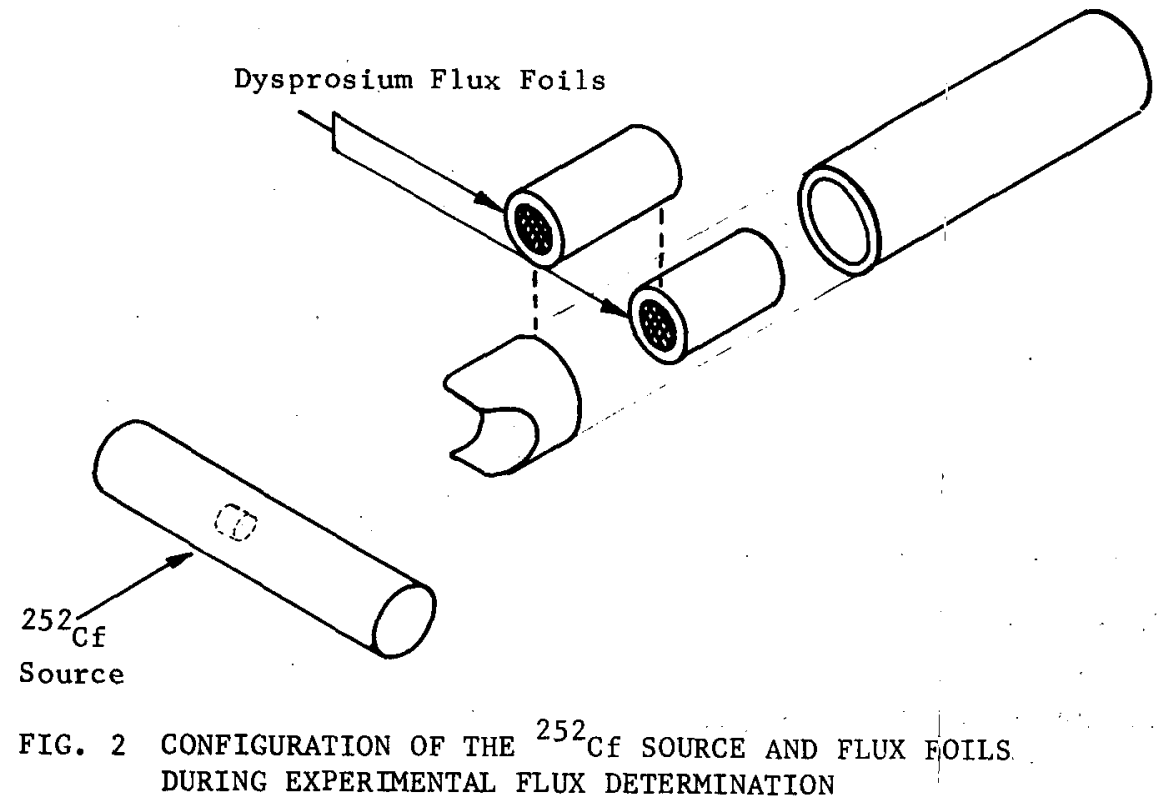


material such as zirconium should be studied for possible use as a ${ }^{252} \mathrm{Cf}$ encapsulation material.

\section{REFERENCES}

1. R. G. Soltesz and R. K. Disney, "User's Manua1 for the DOT-IIW Discrete Ordinates Transport Computer Code", WANL-TME-1982 (December 1969).

2. C. E. Lee, "The Discrete Sn Approximation to Transport Theory", LA-295 (March 1962)
3. W. W. Engle, Jr., "A User's Manual for ANISN", ORNL-K-1693 (March 1967).

4. D. W. Magnuson, "Therma1 Neutron F1ux Distribution from a Cf-252 Source", Californium-252, Proceedings of a Symposium Sponsored by the New York Metropolitan Section of the American Nuclear Soclety, New York City (October 22, 1968), USAEC Report CONF681032 (1969).

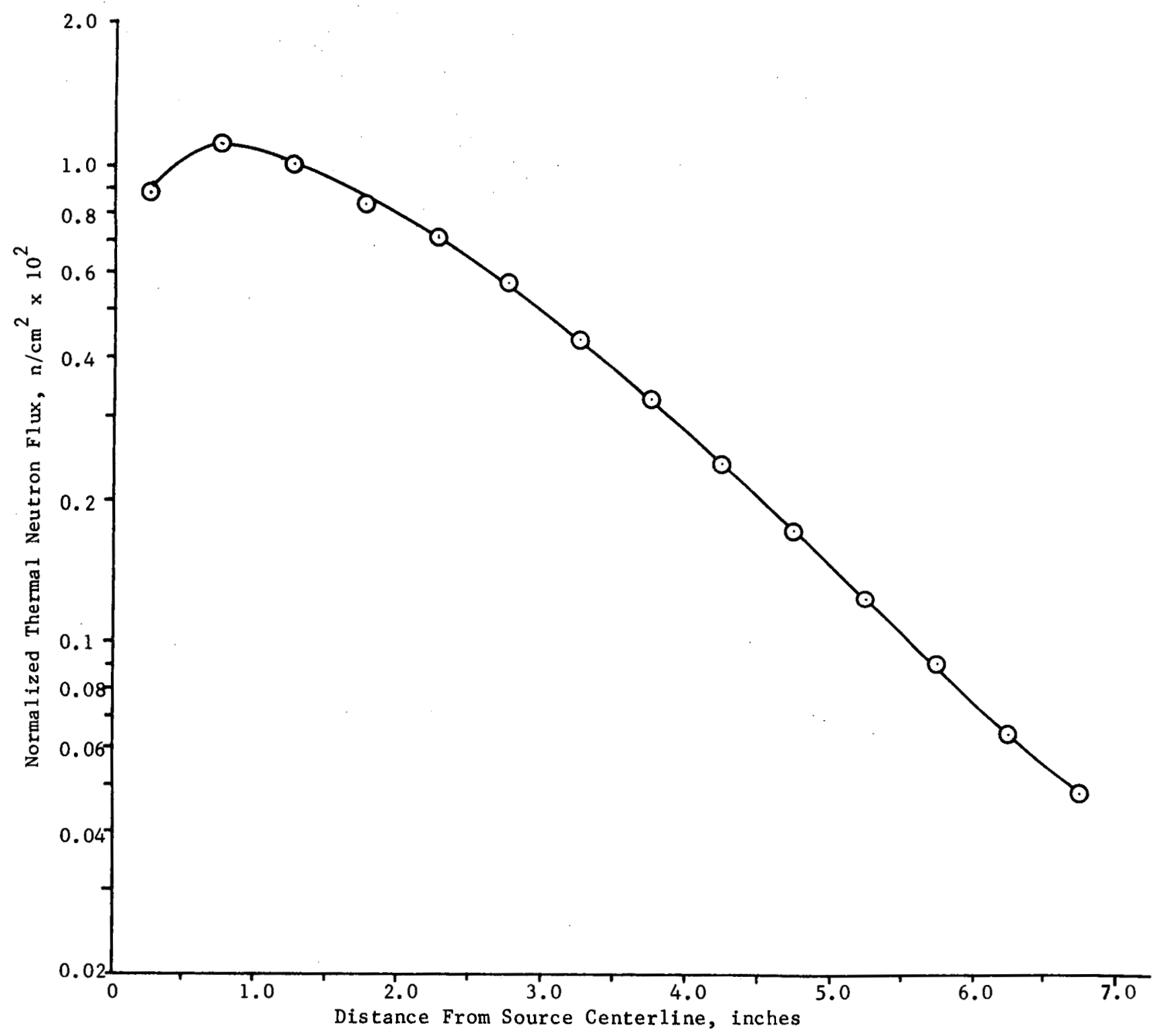

FIG. 3 THERMAL NEUTRON FLUX DISTRIBUTION FROM A ${ }^{252}$ Cf SOURCE 

RECENT DEVELOPMENTS IN $(a, n)$ SOURCES

\author{
Kenneth H. Ansell and Edward G. Hall
}

The Radiochemical Centre

Amersham, Buckinghamshire, England

For many applications the conventional type of $(a, n)$ neutron source is perfectly satisfactory. However, with the development of new techniques, sources with alternative constructions are required: This paper describes recent developments in neutron source design which have been carried out in the UK to meet this new situation.

As an alternative to the simple cylindrical ource, there is a growing demand for annular and line sources for industrial and research applications. For medical use a curium-242-beryllium needle may have some advantages and a source similar to'a conventional radium needle has been produced.

Sources with different physical properties have been developed in addition to sources with different external shapes. The incorporation of the active material in ceramic form can give increased source safety, where the source is used in high risk environmental conditions. The development of some sources is discussed and the results of integrity tests are presented. For instrument calibration, the ability to switch the neutron output may be an advantage The construction of such a source presents an interesting problem and one possible: solution to this problem is discussed.

It is possible that in the future californium-252 may meet many of the demands for high activity neutron sources, but the long half-lives of nuclides like americium-241 and plutonium-238 make them very suitable for use in instrumentation sources; and: the demand for $(a, n)$ neutron sources is likely to continue for many years

\section{INTRODUCTION}

Isotopic neutron sources have been available for more than three decades and over these years there has been a substantial and steady increase in the numbers in use.

The main development over this period has been the rapid increase in the number of nuclides available. Before the advent of the nuclear reactor, neutron sources had to be based on radium-226 or radium-228 and their decay products. Currently a choice may be made from about 20 nuclides. This has resulted in the development of a wide range of sources, based on $(\alpha, n)$ and $(\gamma, n)$ reactions.

Although $(\gamma, n)$ sources (1) and irradiated $(\alpha, n)$ sources $(\underline{2}, \underline{3})$ have been used for many applications, such as neutron activation analysis, the simple $(\alpha, n)$ source forms by far the most important group.

As new types of $(\alpha, n)$ sources have become available they have been used with advantage in existing applications and have also led to new developments in

instrumentation. For some applications the process of change has been continuous for many years. In the Western World oil well logging sources present a good example of this process. The initial use of radium-226-beryllium was superseded by polonium-210-beryliium which had the advantage of higher output, small size and lower gamma emission. The advantages of the longer half lives of plutonium-239beryllium and amiericium-241-beryllium were utilised as soon as these sources became. available although it was not possible to produce sources of equivalent physical size. In recent years it has become obvious that this disadvantacie would be overcome by the use of plutoniun-238-beryllium because of its higher specific activity and hence greater neutron output for a given volume. It now seems probable that the use of $(\alpha, n)$ sources for this application will diminish with the advent of californium-252.

Other applications have not shown the same rate of change. Moisture meters were developed when radium-226-beryllium sources were the only $(\alpha, n)$ sources available and some current moilels still use them because the gamma radiation may be utilised for density measureinents. "The advantages of americium-241-beryllium for this application have been recoghised and many instruments now use this type of source or combined americium-241-beryllium/caesium-137 sources for moisture plis derisity measurements. There is little doubt that $(\alpha, n)$ sources will continue to be ised for this application for many years and further dramatic changes are unlikely.

The other main area of development has been in source design and construction, and until recently the rate of development has been rather slow, the basic design of $(\alpha, n)$

-

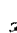


neutron sources remaining virtually unchanged. They normally consist of a mixture of the alpha emitting nuclide and beryllium, the active material being in the form of a powder, pressed pellet or alloy. The main developments in source construction have been improvements in encapsulation materials, sealing techniques and testing. A cross section of a typical source of current design is shown in Figure 1.

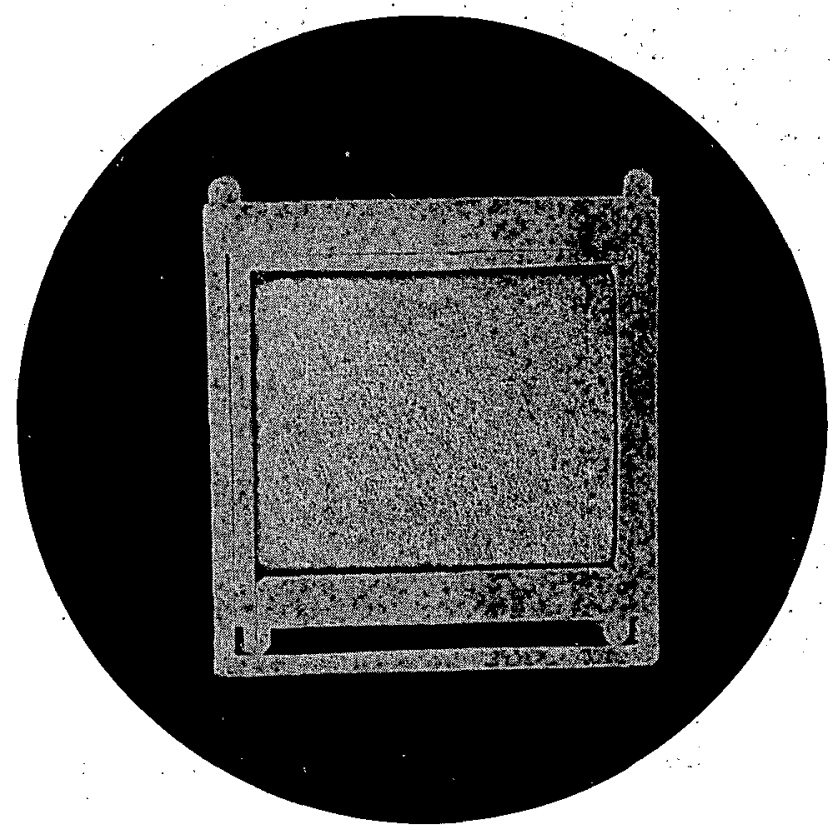

Fig.1 CROSS SECTION OF TYPICAL SOURCE

In this example a pressed pellet is doubly encapsulated in argon arc welded stainless steel.

The capsule design and material can be modifed for particular environments and it seems probable that this type will continue to be completely satisfactory for many applications.

However, new applications and increased emphasis on source safety in some countries have now resulted in the need for more sophisticated source designs and annular, line, high temperature and variable output sources have been developed. 'Work in the UK with high intensity $(\alpha, n)$ sources (> $108 \mathrm{n} / \mathrm{sec}$ ) has been described elsewhere (4) but some of the recent developments carried out in the United Kingdom on the design and construction of low interisity $(\alpha, n)$ sources are outlined in the following sections.

\section{ANNULAR SOURCES}

Hollow cylindrical sources have been developed for two distinct applications soil moisture determination and neutron activation analysis. Of these the former is currently the more important in terms of numbers of sources required.

\section{SOURCES FOR SOIL MOISTURE METERS}

The use of small neutron sources for soil moisture determination has been described on many occasions and was discussed by an IAEA Panel in 1967 (5). . The source strength required varies from instrument to instrument but is usually in the region of $10^{5}-10^{6} \mathrm{n} / \mathrm{sec}$.

Some designs of soil moisture meter require a hollow cylindrical source to fit around the $\mathrm{BF}_{3}$ counter in the probe unit. In order to keep this unit to a reasonable size the external dimensions of the annular source must be kept to a minimum. The internal diameter of the source is of course limited by the dimensions of commercially available $\mathrm{BF}_{3}$ tubes. These restrictions lead to a source design with a very small active volume. A typical example is shown in Figure 2.

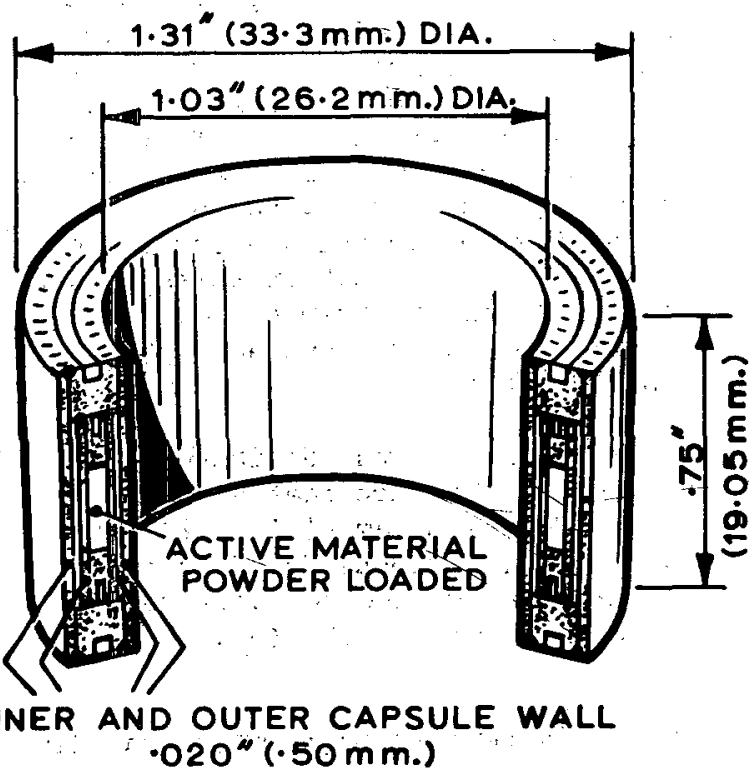

FIG.2 ANNULAR SOURCE FOR MOISTURE METER 
The problem facing the source manufacturer is that of producing a robust source with the active material homogenously distributed round the annulus and with a stable neutron emission.

Americium-241 is a suitable: nuclide but the conventional method for the production of americium-beryllium neutron sources by pellet loading. is not feasible because of the small active volume. In this case the powder mixture is loaded into the inner stainless steel capsule using an annular loading funnel designed to keep external contamination to a minimum. After sealing, decontamination and testing, a second encapsulation in stainless steel is carried out.

\section{TABLE I}

Design Data for Source in Fig.1

\begin{tabular}{|c|c|c|c|}
\hline Active Length & $(\operatorname{Max})$ & - & 0.23 inch \\
\hline Beryllium & $(\operatorname{Max})$ & - & $0.72 \mathrm{~g}$ \\
\hline *Activity & $(\operatorname{Max})$ & - & $200 \mathrm{mCi}$ \\
\hline
\end{tabular}

* Neutron output (Max) - $4.4 \times 10^{5} \mathrm{n} / \mathrm{sec}$

- At normal target/nuclide ratio.

In order to test the constancy of the neutron emission from this type of source under normal working conditions, a sample source was vibrated at $100^{\circ}$ cycles/sec at 0.02 inch amplitude peak to peak for 24 hours. It was also subjected to free fall impact tests through a distance of 1 " at a rate of 100 impacts per hour for 24 hours. The source was measured before and after. the test and no change in neutron emission was detected. The radial symmetry of the neutron emission was checked and no anistropy was found.

The integrity of the design has been demonistrated by the fact that a source containing $100^{\circ} \mathrm{mCi}$ of americium-241 has passed all the IAEA tests for "special form" material which include very severe percussion and impact tests (6).

\section{SOURCES FOR NEUTRON ACTIVATION ANALYSIS}

There has only been a limited use of $(\alpha, n)$ sources for activation analysis in the UK but a number of groups have been studying the determination of fluorine

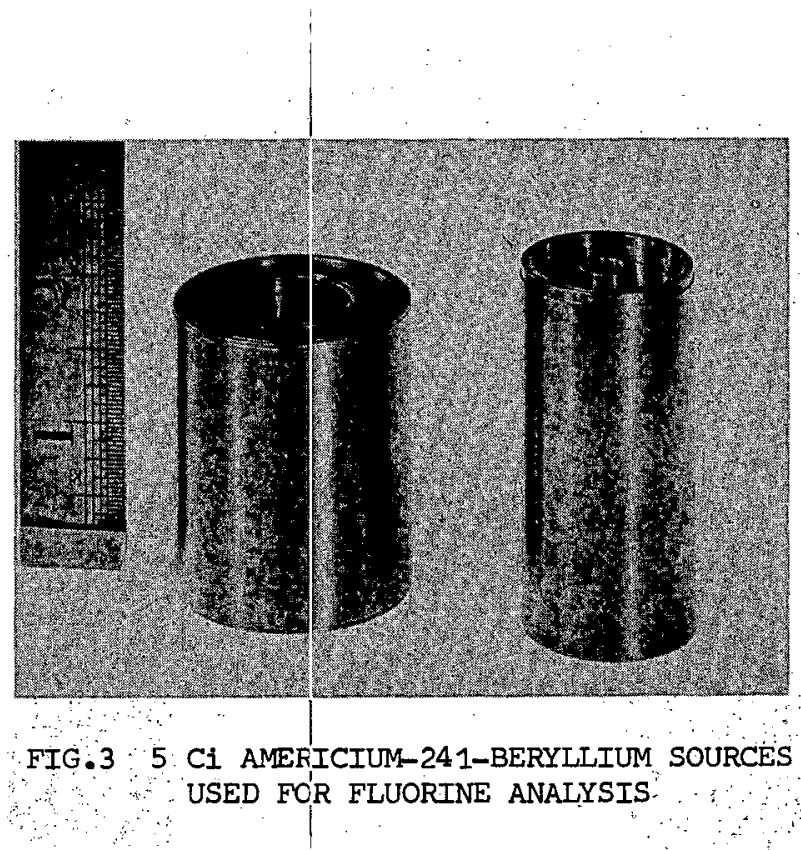

using americium-241-beryllium and plutonium238-beryllium neutron sources, and in one case a source is being used for routine industrial measurements on a semi-automatic basis $(\underline{7}, \underline{8}, \underline{9})$.

The reactions of interest are:${ }_{9}^{19} \mathrm{~F} \stackrel{(n, p)}{\longrightarrow}{ }_{8}^{19} \stackrel{29, \mathrm{pec}}{\longrightarrow}{ }_{\mathrm{F}}^{19} \gamma^{\prime} \mathrm{s}$ at $0.197+\underset{\mathrm{MeV}}{1.37}$ ${ }_{9}^{19} \stackrel{(n, 2 n)}{\longrightarrow}{ }_{9}^{18}{ }_{F}^{1} \frac{\beta^{+}}{109 \mathrm{~min}}{ }^{18} \circ \gamma^{\prime} s$ at $0.511 \mathrm{MeV}$ ${ }_{9}^{19} \stackrel{(n, \alpha)}{\longrightarrow}{ }_{7}^{16} \underset{7 \mathrm{sec}^{1}}{\stackrel{\beta}{\longrightarrow}}{ }^{16} \gamma^{\prime} \mathrm{s}$ at $6.13+7.11 \mathrm{MeV}$

For the determination of fluorine, sources with outputs in the range $10^{7}$ $10^{8} \mathrm{n} / \mathrm{sec}$ have been successfully used, the fluorine content of the sample being measured by counting the 6.1 - $7.1 \mathrm{MeV}$ gamma rays which are easily resolved from other gamma rays present. Two geometrical arrangements have been used, a cylindrical source with an annular sample and a cylindrical sample with an annular source. $5 \mathrm{Ci}$ americium-241-berylitium sources of both geometries have been developed for fluorine analysis and are shown in Figure 3 .

In this case the physical dimensions were such that the manufacture of pressed annular pellets was a practical proposition and was considered to be preferable to the powder loading technique. 
The use of similar techniques is bound to grow in the future and already there is interest in the hospitals for the determination of calcium in bone. This may require hollow cylindrical sources large enough to fit round a human hand or foot.

\section{LINE SOURCES}

The production of long cylindrical sources has usually in the past resulted from the need for a specified neutron output from a source of fixed diameter using a low specific activity nuclide such as plutonium-239 or americium-241.

Recently however, new applications have arisen which require the active material to be uniformly distributed along the length of source capsules with very small active diameters. Two such applications are the industrial measurement of hydrogenous materials in tanks and the production of clinical sources for interstitial and intracavitary neutron therapy. For this type of source, pellet loading has a number of disadvantages and alternative production techniques must be considered.

\section{PRODUCTION OF LINE SOURCES}

For most industrial applications of line sources the neutron emission must be uniform along the active length of the source, and the source capsule must be physically robust. In addition the production technique must be capable of producing large numbers of sources with similar and constant neutron emissions, at a reasonable cost. The conventional method of incorporation of the active material in the form of pressed pellets is very tedious as large numbers of small diameter peliets have to be pressed and loaded into the same tube. Such a design is far from ideal from the point of view of source safety because if the capsule is ruptured in an accident, material from the active pellets could escape. Vibration compaction of powder is a practical technique which has been used for similar sources but suffers from the same objections on the grounds of source safety. In addition if very long sources are required there may be some segregation of the active mix during loading which would lead to nonlinearity of the neutron emission.

A rotary swaging technique has been developed at the Radiochemical Centre,
Amersham, for the production of sealed radiation sources. The manufacture of gamma sources utilising this technique has been reported previously $(10,11)$.

The use of a similar technique has been investigated in Japan for the fabrication of fuel elements (12).

This process consolidates the active matrix inside a ductile metal cladding. The production procedure developed for manufacture of neutron sources is as follows:-

i) Intimate mixing of ${ }^{241} \mathrm{AmO}_{2}$ and beryllium metal powder in appropriate proportions.

ii) Loading of the powder mixture into an aluminium tube and sealing with end plugs.

iii) Extension of the sealed compact in a rotary swaging machine to form a wire of the required diameter.

iv) Cutting of the swaged wire to the correct length.

v) Double encapsulation in stainless steel tubing sealed by argon arc welding.

One design of source produced by this technique is shown in Figure 4.

This gives a neutron emission of $1.2 \times 10^{5} \mathrm{n} / \mathrm{sec}$ from a source containing $50 \mathrm{mCi}$ of americium-241. The Be/AmO mixture was consolidated within the swaged rod to a density of approximately $1.7 \mathrm{~g} / \mathrm{cc}$ which compares very favourably with the densities of pressed pellets $(\sim 1.3 \mathrm{~g} / \mathrm{cc})$ and vibration compacts $(\sim 1.1 \mathrm{~g} / \mathrm{cc})$. The tools used to cut the swaged wire to length are designed to form cold sheared ends which are virtually leak free.

Tests on the unencapsulated swaged wire show that although some release of active material occurs when the outer wall is fractured because of the brittle nature of the active core, it is extremely smali compared with the release of active material in the form of pressed pellets or powder enclosed in a stainless steel capsule fractured in a similar manner. It is clear that the active component of the source is already in a basically safe form even before the final double encapsulation in stainless steel. 


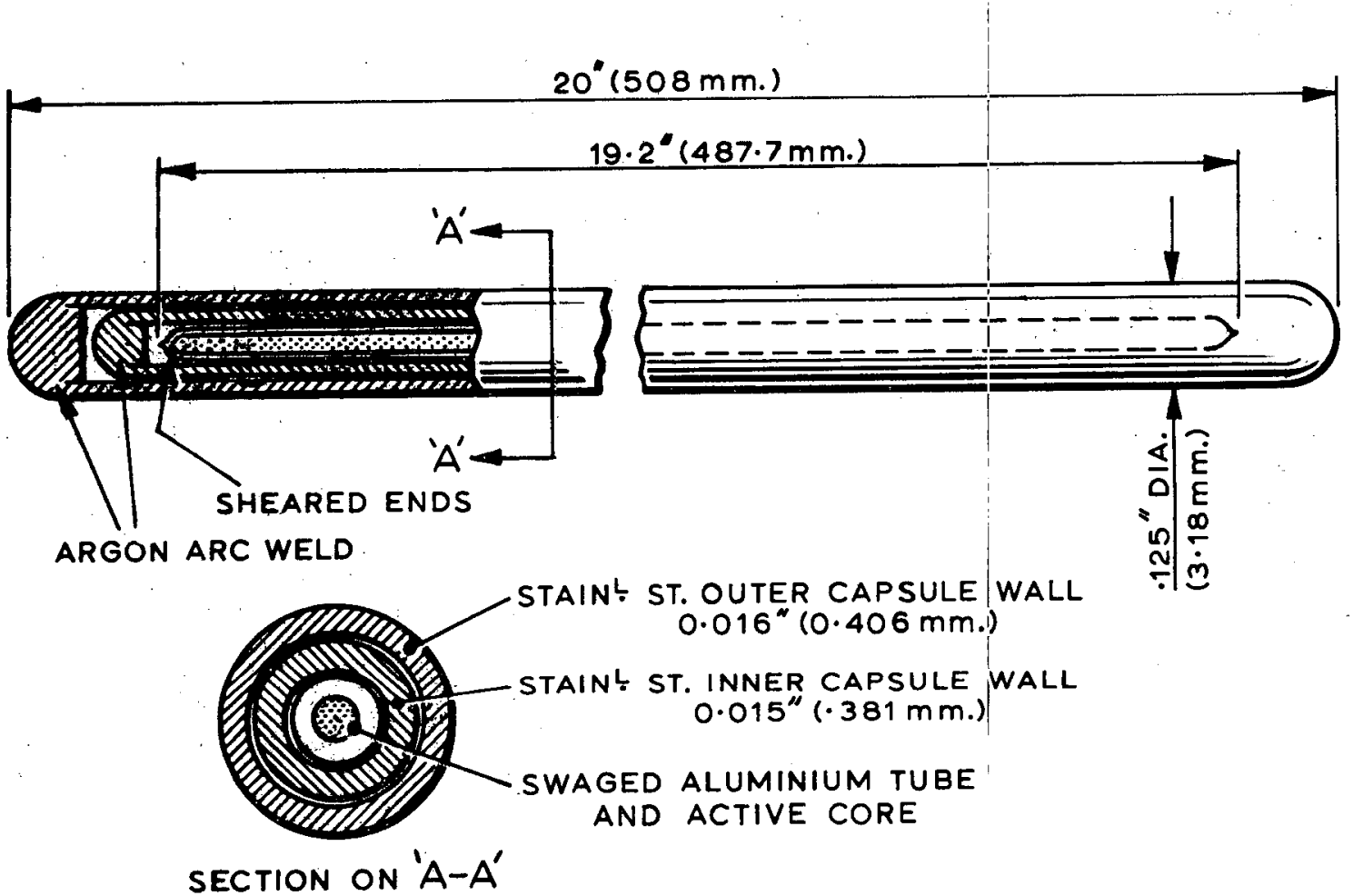

FIG.4 AMERICIUM-241-BERYLLIUM LINE NEUTRON SOURCE

An active source of this design containing $50 \mathrm{mCi}$ has been tested and approved as "special form" material. In addition to the impact, percussion, temperature and corrosion tests required by the IAEA regulations, an additional $90^{\circ}$ bend test was carried out (Figure 5).

The uniformity of neutron output along the length of the source has been checked by measuring the $4.4 \mathrm{MeV} \gamma$ from the excited carbon-12 using a gamma spectrometer in a collimated system. The variation per inch along the length is less than $\pm 10 \%$.

The technique is extremely versatile and examples of other possible rods are given in Table II.

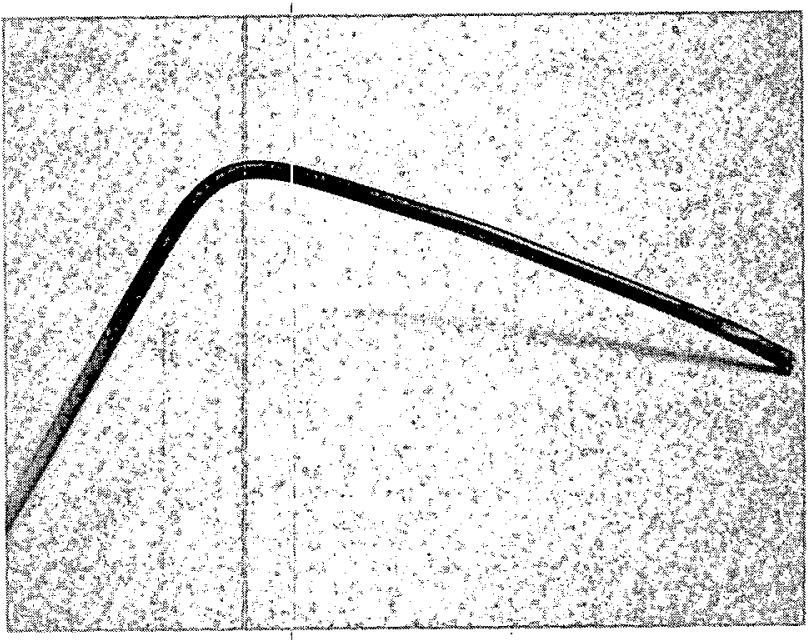

FIG.5: BEND TEST ON

$50 \mathrm{mCi}$ AM RICIUM-241-BERYLLIUM LINE SOURCE 
TABLE II

Americium-241-Beryllium Line Neutron Sources

\begin{tabular}{|c|c|c|}
\hline $\begin{array}{c}\text { External dia. } \\
\text { of swaged rod } \\
\text { in. }\end{array}$ & $\begin{array}{c}\text { Activity/foot } \\
\text { at 10:1 } \\
\text { Be/Amo ratio }\end{array}$ & $\begin{array}{c}\text { Neutron } \\
\text { output/ } \\
\text { foot }\end{array}$ \\
\hline 0.050 & 30 & $7 \times 10^{4}$ \\
0.075 & 100 & $2.2 \times 10^{5}$ \\
0.100 & 150 & $3.3 \times 10^{5}$ \\
0.150 & 340 & $7.5 \times 10^{5}$ \\
\hline
\end{tabular}

Swaging is already established for the production of industrial line sources and can obviously be used for the manufacture of clinical needles and tubes.

\section{CLINICAL NEUT'RON SOURCES}

The use of neutrons for clinical therapy has been discussed from time to time ( 13 $14,15)$ but it is only recently with the availability of californium-252 that detailed experimental brachytherapy has been undertaken. Sources similar in design to conventional radium-226 needles and tubes have been manufactured in USA (16), and have been loaned to interested institutions for evaluation.

Although californium-252 appears to be the most suitable neutron enitting material for this typerof clinical source, we felt that it would be a worthwhile exercise to see if alternative clinical sources could be produced which would be independent of the US californium-252 programme.

The problem is to achieve a useful neutron output from the very small active volumes available in the well established designs of conventional needles and tubes. A typical radium needle (Figure 6) has a volume of approximately $0.0085 \mathrm{cc}$ available for active material. Beryllium is the best target material for an $(\alpha, n)$ source and by using vibration loading techniques a density of $\sim 1.1 \mathrm{~g} / \mathrm{cc}$ can be achieved.

The weight of target which can be loaded is therefore only $\sim 10 \mathrm{mg}$ and this leads to the conclusion that only $\alpha$ emitters with high specific activities are suitable.

Both polonium-210 and curium-242 have high specific activities and the additional possible advantage of low gamma emission. For preliminary studies curium-242 was selected.

In order to achieve the optimum neutron yield/Ci a ratio of beryllium/nuclide in excess of 10:1 is necessary. A source containing $1 \mathrm{Ci}(0.30 \mathrm{mg})$ curium-242 has been made to establish manufacturing technique and for preliminary measurements.

A powder mixture of beryllium and curium-242 as oxide was loaded into a platinum inner capsule $0.85 \mathrm{~mm}$ ID, $1.25 \mathrm{~mm}$ OD, $15 \mathrm{~mm}$ long, and sealed by gold brazing. After decontamination and testing

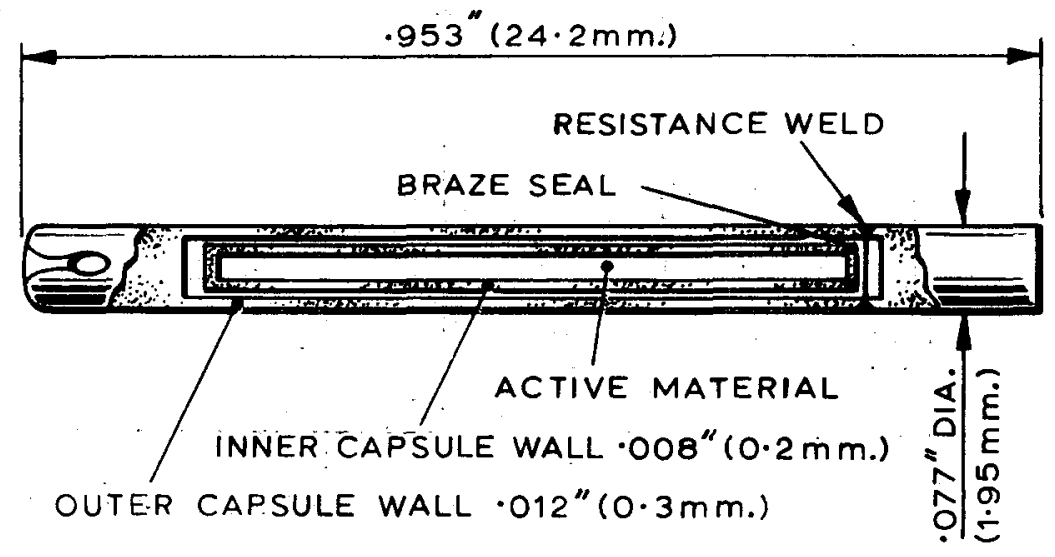

FIG.6 CURIUM-242-BERYLIIUM NEUTRON EMITTING NEEDLE 
for leakage the inner capsule was inserted in a platinum/iridium sheath and sealed by resistance welding.

A neutron output of $3 \times 10^{6} \mathrm{n} / \mathrm{sec} / \mathrm{Ci}$ was achieved with this trial needle and our experience with other sources indicates that neutron emissions in excess of $10^{7} \mathrm{n} / \mathrm{sec}$ will be practicable for the needle illustrated.

One possible extension of this work is into the field of neutron emitting seeds based on short lived nuclides like radium-223. Should beryllium be unacceptable for clinical use, oxygen-18 is a suitable alternative target.

\section{CERAMIC NEUTRON SOURCES}

The standard design of $(\alpha, n)$ source shown in Figure 1 gives extremely robust sources which may be adapted for use under particular operating conditions by careful design and selection of encapsulation materials.

There are however a number of disadvantages inherent in the use of beryllium metal powder in its construction. At temperatures in excess of $600^{\circ} \mathrm{C}$ beryllium will attack stainless steel (17) and this will lead to weakening and possible rupture of the source capsule. If the capsule is ruptured through overheating or severe mechanical damage, the active material will be in a form which is readily attacked chemically and may, because of its powder form become dispersed over a wide area. Although source capsules are designed to withstand foreseeable damage, for some high risk applications additional safety precautions are required. With such applications in mind we have developed neutron sources where the active material is incorporated in a ceramic form.

Two possible routes were considered initially:-

1. The manufacture of: a glass containing various combinations of beryllium, boron and fluorine as target material.

2. The manufacture of high temperature sintered compacts with beryllium oxide as the target.
The second method was selected for a development study/using $241_{\mathrm{AmO}_{2}}$.

Equilibrium diagrams of some oxide systems were avaitable eg $\mathrm{ThO}_{2} / \mathrm{BeO}, \mathrm{UO}_{2} / \mathrm{BeO}$ which showed eutectics in the $2000-2500^{\circ} \mathrm{C}$ region. No data for $\mathrm{AmO}_{2} / \mathrm{BeO}$ was found.

Experience with manufacture of americium-241-berflilum neutron sources has shown that the specific neutron emission increases with $\mathrm{Be} / \mathrm{AmO}_{2}$ ratio. A ratio of approximately bet;er than 10:1 is normally used in practice to achieve an emission of $2.2 \times 10^{6} \mathrm{n} / \mathrm{sec} / \mathrm{C}$. It has been shown that a similar ef:Eect applies when beryllium oxide is used as the target but that the emission achieved with a target/AmO ratio of $10: 1$ is $1.1 \times 10^{6} \mathrm{n} / \mathrm{sec} / \mathrm{Ci}$ (see Figure 7). 
Experimental work has shown that the density of sintered compacts increases with increasing temperature up to $1600-1700^{\circ} \mathrm{C}$. The rate of heating to this temperature has been shown to be critical but the optimum cycle has not yet been determined. Current practice involves a heating cycle of 6 hours. The density achieved is typically $2.6 \mathrm{~g} / \mathrm{cc}$ compared with $1.3 \mathrm{~g} / \mathrm{cc}$ for pressed pellets of $\mathrm{Be} / \mathrm{AmO}_{2}$. It is therefore possible to achieve comparable neutron yields for a given volume with BeO and Be targets (Figure 8) although double the activity is required with beryllium oxide. The active content could be reduced by the use of $\mathrm{Be}^{18} \mathrm{O}$.

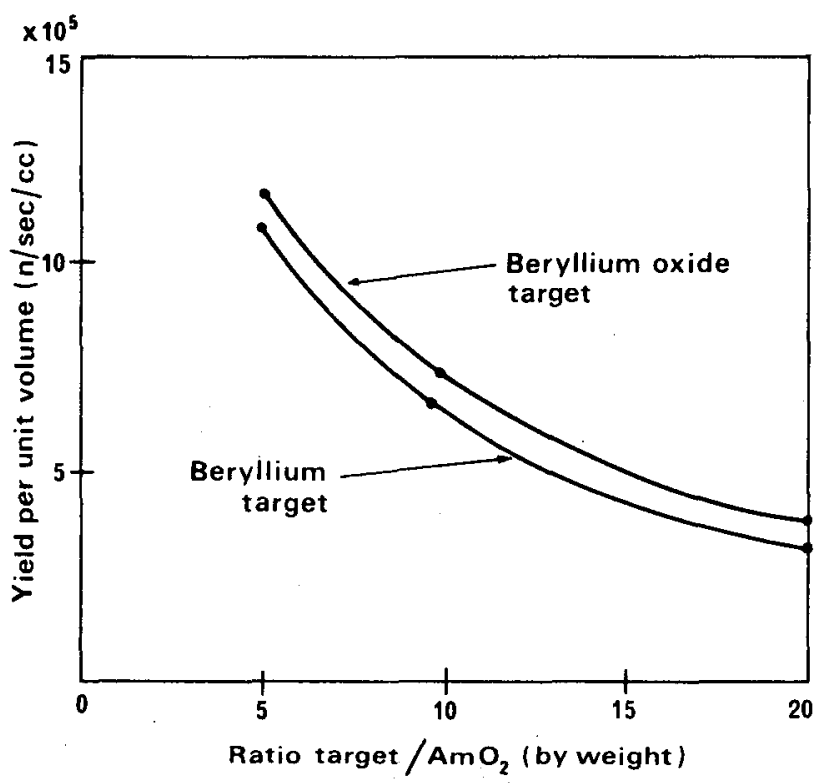

FIG.8 COMPARISON OF NEUTRON YIELD PER UNIT VOLUME

Sintered pellets are extremely hard and when subjected to pressure tests do not powder but ultimately break into discrete pieces.

After preliminary decontamination to remove loose active material arising in the processing plant, trial pellets containing $10 \mathrm{mC} i$ of americium-241 have been subjected to 8 hour immersion in water at $50^{\circ} \mathrm{C}$ and the amount leached out was $<0.05 \mu \mathrm{Ci}$.
Further tests on the integrity of the sintered ceramic pellets are continuing but it has already been established that they have considerable safety advantages compared with the pressed pellet sources.

\section{SWITCHABLE NEUTRON SOURCE}

$(\alpha, n)$ sources consisting of an intimate mixture of alpha emitter and target emit neutrons continuously and for many applications this is acceptable but there are potential uses where it can be a disadvantage.

The development of a source where the neutron output could be switched on and off was prompted by a requirement for a permanently installed standard in a neutron dosimeter.

The specification called for an output of $10^{4} \mathrm{n} / \mathrm{sec}$ when operational and a reduction of at least tenfold when switched off. A compact source with long half life and low gamma emission was required. Various designs of switchable sources have been described previously but none of them met our particular specification $(18,19,20,21)$. All these are based on the use of an alpha emitter and a suitable target arranged in such a way that it is possible to prevent the alphas reaching the target.

In order to achieve the maximum neutron emission, the alpha emitting source should be as close as possible to the beryllium target, and the alpha energy should be as high as possible. In order to achieve this, our alpha emitting source consisted of an electrodeposit on a thin metal backing.

Preliminary experiments indicated that emissions of approximately $10 \%$ of conventional sources could be achieved by bringing an alpha emitting source in close proximity to beryllium. We selected americium-241 as the nuclide and in order to achieve an emission of $10^{4} \mathrm{n} / \mathrm{sec}$ an activity of approximately $5 \mathrm{mCi}$ was indicated.

The design of the assembled source is shown in Figure 9. It consists of two half shells of alpha emitting foil and beryllium attached to a small button magnet. The magnet equipped with spindles is mounted and sealed in an argon arc welded stainless steel capsule. A second magnet is mounted external to the sealed capsule and is equipped with an operating spindle, rotation 
of which enables the alpha source and beryllium shells to be brought into close proximity.

A prototype source containing $\sim 5 \mathrm{mCi}$ americium-241 gave an emission of $10^{4} \mathrm{n} / \mathrm{sec}$ in the ' $O N$ ' position and $1.5 \times 10^{2} \mathrm{n} / \mathrm{sec}$ in the 'OFF' position. The neutron emission in the 'OFF' position is due to:-

i) Neutrons from the alpha source itself due to $(\alpha, n)$ reaction with light element constituents eg oxygen.

ii) Neutrons from $(\alpha, n)$ on beryllium due to scattered alphas and close proximity at the edges of the shells even when in the 'OFF' position.

We consider that this background neutron emission could be considerably reduced.

The source has been in use for over one year and the ON/OFF system is functioning satisfactorily.
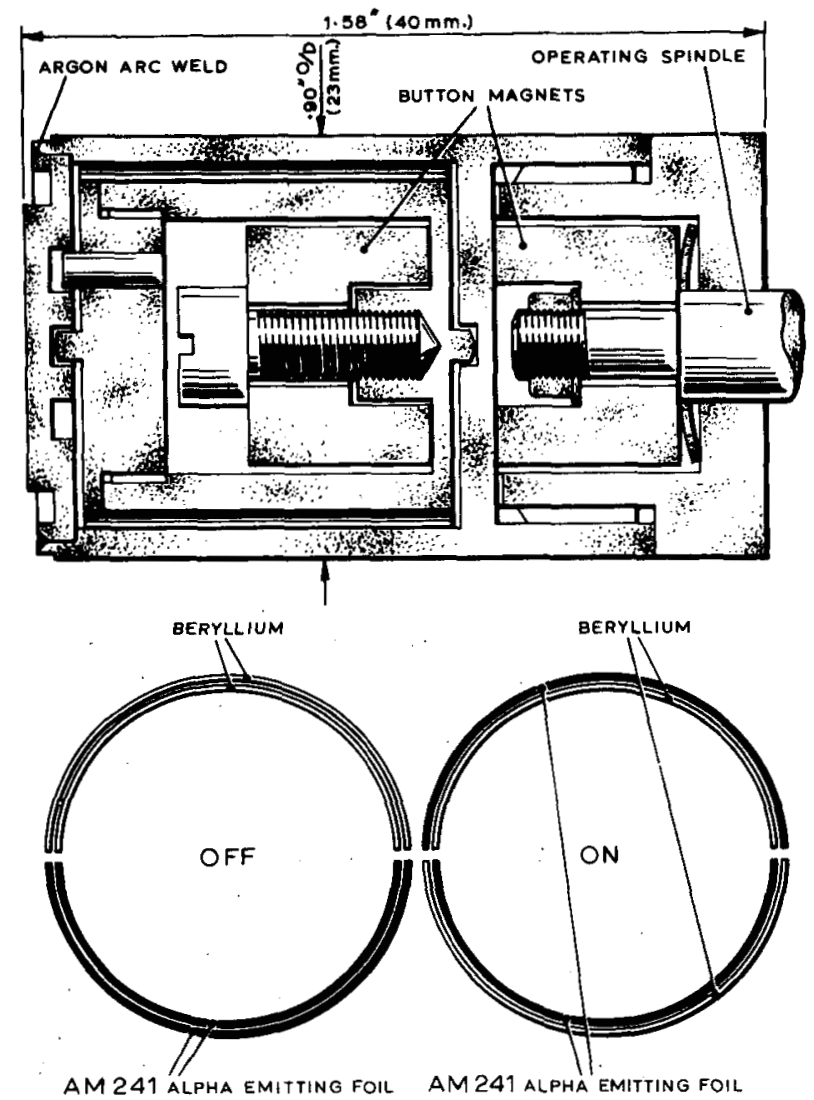

FIG.9 VARIABLE OUTPUT NEUTRON SOURCE
THE FUTURE FOR $(\alpha, n)$. SOURCES

The neutron output per unit volume from californiun-252 is so much higher than that from any $(\alpha, n)$ source that it is virtually the only possibility where outputs in excess of $10^{10} \mathrm{n} / \mathrm{sec}$ are required from radioisotopic sources. For reactor start up califo-nium-252 again has advantages becailse of the Iow mass of active material|and the absence of target material such as beryllium which raises problems of compatibility and helium production under irradiation.

The future use of neutron emitting needles and tubes in brachytherapy is dependent on the current programme with californium-25\%. If clinical trials show advantages over the conventional $\gamma$ emitting sourices then californium-252 will probably kie the source of choice. However, there lare alternatives and the ability to vary the neutron and gamma ratio which is possible with $(\alpha, n)$ sources may be an advaritage. Short lived $\alpha$ emitters in $(\alpha, n)$ sources might be worth considering as permanent implants.

For many of the applications of neutron sources in instrumentation, high neutron output is not hecessary, and constancy of output may be imuch more important. Half lives of less than 15 - 20 years are inconvenient, and in these cases curium-244, plutonium-238 and americium-241 are likely to be preferred. At this time curium-244 is not generally available, and plutonium-238 is subject to many restrictions for general commercial use because it is technically a fissile material. Thus in the short term at least, americium-241 iwill continue to be an important nuclide for neutron sources.

\section{REFERENCES}

1. R. W. Tolmile and C. J. Thompson. "Field Equipment for Neutron Activation Analysis." IAEA Publication SM-112/31. (Nuclear Techniques and Mineral Resources) 489 (1969).

2. J. W. Mander and C. W. Terrell. "Utilization of High Yield AmericiumBeryllium-Gurium Sources for in situ Elemental Analyses: Trans.Amer. Nucl.SoC. $10,31(196 \overline{7)}$. 
3. J. Wing and M. A. Wahlgren. "Detection Sensitivities in Nuclear Activation with an Isotopic Fast Neutron Source." Anal. Chem. 39, No.1, 85 (1967).

4. K. H. Ansell and E. G. Hall. "High Intensity $(\alpha, n)$ Sources." Proceedings of ANS Topical Meeting on Neutron Sources and Applications, Atlanta, Georgia. April (1971).

5. (International Atomic Energy Agency Tech.Report Series No.112). "Neutron Moisture Gauges." IAEA, Vienna, 1970.

6. International Atomic Energy Agency. "Regulations for the Safe Transport of Radioactive Materials." 1967 edition. Vienna, 1967. (IAEA Safety Series No.6)

7. P. E. Starnes. "Improvements in Activation Analysis." British Patent $1,124,992$ (1965).

8. P. G. Jeffrey and J. M. Bakes. "The Determination of Fluorine in Fluorite Ores and Concentrates by Isotope Source, Fast Neutron Activation Analysis." The Analyst. Vol.92, 151 (1967).

9. M. T. Gundy. "Use of ${ }^{241}$ Am-Be Source for On-line Activation Analysis." Private Communication. 1970.

10.A. F. C. Horsler, J. C. Jones and A. J. Stacey. "Caesium-137 Sources for Use in Intracavitary and Interstitial Radiotherapy." Brit.J.Radiol. 37, 385 (1964).

11.K. H. Ansell and E. G. Hall. "Recent Developments in Low Energy Photon Sources." Proceedings of $3 \mathrm{rd}$ Conf. on Low Energy $\mathrm{X}$-Ray and Gamma Sources and Applications, Boston (1970). :
12.K. Naruki. "Vib-Swaging Process for Fuel Rod." J.Nucl.Sci. and Tech. 7, 327 (1970).

13.J. F. Fowler. "Oxygen Enhancement Ratios and Depth Doses as Opposing Factors in Fast Neutron Therapy." Proc.Roy.Soc.Med. 58, 1601965 .

14.R. L. Morgan, D. K. Bewley, D. D. Von Berg, R. Morrison. "Neutrons against Cancer." Brit.Med.J. 1, 241 (1967).

15.L. E. Farr and L. Y. Yamamoto. "Neutron Capture Therapy of a Cerebellar Hemangiosarcoma after Surgical and Radiation Treatment." J.Nuclear Med. 2, 252 (1961)

16. Californium-252: Its Use and Market Potential. USAEC, Savannah River (1969).

17.J. D. Baird, G. A. Geach, A. G. Knapton and K. B. C. West. "Compatibility of Beryllium with Other Metals used in Reactors." Proc.of the 2nd UN Int.Conf. on Peaceful Uses of Atomic Energy, Geneva. Vol. 5.328 (1958).

18.V. A. Emel'yanov and I. I. Beskins. Russian Patent 154623 (1963).

19.S. Amiel. "Neutron Source." Canadian Patent 730778 (1966).

20.W. M. Holm. "Variable Intensity Neutron Generator." United States Patent 3,400,269 (1968).

21.S. Suzuki. "Neutron Source with Variable Yield using a Radioisotope." Japanese Patent.20159 (1969). 


\title{
HIGH INTENSITY $(a, n)$ SOURCES
}

\author{
Kenneth H. Ansell \\ Edward G. Hall \\ The Radiochemiçal Centre \\ Amersham, Buckingsnamshire, England
}

Radioisotope neutron sources have been used extensively in the field of power reactors for neutron flux measurements during start-up, reactor design studies and in teaching reactor technology. All $(a, n)$ neutron sources consist of a mixture of an alpha emitter and a light element target, usually beryllium. Experience in the UK is that for $(a, n)$ sources with outputs above $10^{8} \mathrm{n} / \mathrm{sec}$ the nuclides selected are polonium-210, thorium-228 and curium-242.

Polonium-210 and thorium-228 have been used in high intensity neutron sources for more than a decade but curium- 242 has only recently become commercially available. The use of polonium- 210 is likely to decline and this paper concentrates on sources containing thiorium-228 and curium-242. These nuclides are produced by the reactor irradiation of radium-226 and americium-241 respectively. The problems associated with these irradiations and the subsequent chemical processing to yield multicurie quantities of pure nuclide are discussed.

The design, manufacture and testing of thorium-228-beryllium and curium-242-beryllium neutron sources with outputs up to $1.2 \times 10^{9} \mathrm{n} / \mathrm{sec}$ which are safe for reactor use over a number of years are outlined. The properties of thorium-228-beryllium and curium-242-beryllium including neutron spectra are given.

\section{INTRODUCTION}

$(\alpha, n)$ neutron sources have been prepared during the past few decades with outputs ranging from a few neutrons/second up to $10^{10} \mathrm{n} / \mathrm{sec}(1)$. For the purposes of this paper we regard sources with outputs in excess of $10^{8} \mathrm{n} / \mathrm{sec}$ as "high intensity".

Such sources are of interest for neutron activation, neutron radiography and for reactor instrumentation (2). Before the USAEC launched its programme for the production of californium-252 the choice of a neutron source for these applications lay between nuclear reactors, accelerators, $(\alpha, n)$ and $(\gamma, n)$ sources and in the majority of cases the cost per neutron for sources over $10^{8} \mathrm{n} / \mathrm{sec}$ is higher for the $(\alpha, n)$ source than for the other three alternatives. Mainly because of this cost factor the use of $(\alpha, n)$ sources manufactured in the United Kingdom has been restricted to the one case where the alternatives were ruled out either because of physical size or short half life, namely reactor instrumentation.

$(\alpha, n)$ sources have been used in exponential stacks and sub-critical assemblies during reactor design studies, for zero energy reactors and power reactors to provide an adequate neutron count rate so that accurate measurements can be made of neutron flux and doubling time during start up and for safety control during core changes in complete reactor systems.
For large power reactors a short lived source is usually acceptable as they subsequently rely on $(\gamma, n)$ sources such as antimony-124-berylium activated within the reactor.

It is appropritate at this moment in time when it is 1ijely that californium-252 will become a suitable alternative to the $(\alpha, n)$ source for reactor instrumentation, to review some of the progress made in this field in the UK during the past decade.

\section{AVAILABLE $(\alpha, n !)$ NEUTRON SOURCES}

Most of the chemical elements with atomic number below 20 give useful yields of fast neutrons when irradiated with alpha particles in the $5 \mathrm{MeV}$ region. These neutrons have a wide spread of energy covering the range $1-12 \mathrm{MeV}$, the exact shape of the energy spectrum depending upon the energies of the alpha particles and the nuclear reactions involved. Since for most reactor applications the energy of the neutrons emerging from the source is not critical, the target usually chosen is beryllium-9 as this has the highest $(\alpha, n)$ yield of all the light elements.

The theoretical neutron emission from an $(\alpha, n)$ beryllium neutron source is governed by the energy of the alpha particles and the number of alpha particles in the decay chain. 
TABLE I

Properties of $(\alpha, n)$ Neutron Sources

\begin{tabular}{|c|c|c|c|c|c|c|}
\hline Source & $\begin{array}{l}\text { Half- } \\
\text { life }\end{array}$ & $\begin{array}{c}\text { Number } \\
\text { of } \\
\text { alphas }\end{array}$ & $\begin{array}{r}\text { Average } \\
\alpha \text { energy }\end{array}$ & $\begin{array}{c}\text { Practical } \\
\text { emission } \\
\text { n/sec/Ci }\end{array}$ & $\begin{array}{c}\text { Weight of } \\
\text { carrier-free } \\
\text { nuclide per Ci }\end{array}$ & $\begin{array}{l}\gamma \text { exposure rate } \\
\mathrm{mR} / \mathrm{hr} / \mathrm{m} \text { per } \\
10^{6} \text { neutrons }\end{array}$ \\
\hline${ }^{210} \mathrm{Po}-\mathrm{Be}$ & $138.4 d$ & 1 & 5.3 & $2.5 \times 10^{6}$ & $0.22 \mathrm{mg}$ & $<0.1$ \\
\hline${ }^{242} \mathrm{Cm}-\mathrm{Be}$ & $162.5 d$ & 1 & 6.1 & $3.0 \times 10^{6}$ & $0.30 \mathrm{mg}$ & $<1$ \\
\hline${ }^{228} \mathrm{Th}-\mathrm{Be}$ & $1.91 y$ & 5 & 6.4 & $2.0 \times 10^{7}$ & $1.2 \mathrm{mg}$ & 30 \\
\hline${ }^{244} \mathrm{Cm}-\mathrm{Be}$ & $18.1 y$ & 1 & 5.8 & $2.5 \times 10^{6}$ & $12 \mathrm{mg}$ & $<1$ \\
\hline${ }^{210} \mathrm{~Pb}-\mathrm{Be}$ & $22 y$ & 1 & 5.3 & $2.3 \times 10^{6}$ & $13 \mathrm{mg}$ & 9 \\
\hline $227 \mathrm{AC}-\mathrm{Be}$ & $21.8 y$ & 5 & 6.5 & $2.0 \times 10^{7}$ & $14 \mathrm{mg}$ & 8 \\
\hline${ }^{232} \mathrm{U}-\mathrm{Be}$ & $72 y$ & 6 & 6.2 & $2.2 \times 10^{7}$ & $48 \mathrm{mg}$ & 30 \\
\hline${ }^{238}{ }_{\mathrm{Pu}-\mathrm{Be}}$ & $86.4 y$ & 1 & 5.5 & $2.2 \times 10^{6}$ & $60 \mathrm{mg}$ & $<1$ \\
\hline $241_{\mathrm{Am}-\mathrm{Be}}$ & $458 y^{(a)}$ & 1 & 5.4 & $2.2 \times 10^{6}$ & $309 \mathrm{mg}$ & 1 \\
\hline${ }^{226} \mathrm{Ra}-\mathrm{Be}$ & $1620 y$ & 4 & 5.6 & $1.3 \times 10^{7}$ & $1 \mathrm{~g}$ & 60 \\
\hline${ }^{239}$ Pu-Be & $24400 y$ & 1 & 5.1 & $1.5 \times 10^{6}$ & $16.4 \mathrm{~g}$ & $<1$ \\
\hline
\end{tabular}

(a) $433 y$ is an accepted alternative value.

The practical emission will depend upon the ratio of alpha emitting nuclide to target element and the efficiency with which they are mixed. Practical results are rarely above $85 \%$ of the theoretical values, and are much lower for longer-lived nuclides like plutonium-239 because of self absorption of the alpha particles. Although there are over 300 known alpha emitting nuclides, consideration of half life, availability and cost reduces those worthy of practical consideration to eleven. The main properties of these are given in Table $I$.

The choice of nuclide for a particular reactor source will depend on a number of factors including half life, the physical size of the finished. source, and cost.

Frequently in reactor work there are restrictions on the physical dimensions of the source. The maximum external diameter may be governed by fuel element channel size, and the maximum length over which the active material is distributed is often limited for instrumentation reasons. The properties of the source under reactor irradiation must also be considered.

For start up sources half lives as short as that of polonium-210 can often be tolerated but longer half lives are desirable for reactor design studies. For sources with outputs greater than $10^{8} \mathrm{n} / \mathrm{sec}$ polonium-210, curium-242 and thorium-228 are considered to be the only suitable nuclides. In the UK the others have been rejected on the grounds of cost, availability or space considerations. These three nuclides have the further advantage for many applications that the change of neutron output under reactor conditions follows a predictable decay scheme.

. The weight of a curie of a radionuclide is related to its half life and as a general principle the longer the half life of the radionuclide the larger the neutron source will have to be for a given neutron output. However, very compact sources can be made 
using thorium-228 because the five high energy alpha particles emitted by thorium-228 and its decay products result in an unusually high specific emission of $2 \times 10^{7} \mathrm{n} / \mathrm{sec} / \mathrm{Ci}$. Sources containing thorium-228 have been selected for the following applications:-

i) For reactor study work extended over periods of up to several years;

ii) For reactor start up where extended commissioning trials were required;

iii) For reactor work where there has been difficulty in forecasting start up schedules.

Curium-242-beryllium and polonium-210beryllium are both acceptable where the short half lives can be tolerated and have the advantage over thorium-228 of lower gamma emission and lower cost per neutron.
Curium-242-bieryllium has been preferred to polonium-210 for high output sources manufactured in the UK. This is because the time scale for the production of curium-242 from ainericium-241 is very much shorter than that of polonium-210 from bismuth-209. There is no major production of polonium-210 for heat sources in the UK and therefore the nuclide production time is critical if the source manufacturer is to have any flexibility in allowing for the inevitable changes in reactor commissioning schedules.

The propertiles of thorium-228 and curium-242 are gitven in Table II.

TABLE II

Properties of Nuclides and Decay Product:s

\begin{tabular}{|c|c|c|c|c|c|}
\hline Isotope & Half life & $\begin{array}{c}\text { Main } \\
\text { energies } \\
\mathrm{MeV}\end{array}$ & $\begin{array}{c}\text { Main } \\
\gamma \text { energies } \\
\text { MeV }\end{array}$ & $\begin{array}{r}\text { Relati } \\
n / s \\
\text { pa }\end{array}$ & $\begin{array}{l}\text { Ye Yield } \\
\text { c/Ci } \\
\text { jent) }\end{array}$ \\
\hline $228 \mathrm{Th}$ & $1.91 \mathrm{y}$ & $\begin{array}{l}5.2-1 \% \\
5.3-28 \% \\
5.4-71 \%\end{array}$ & $0.084-2 \%$ & 2.6 & $\times 10^{6}$ \\
\hline${ }^{224} \mathrm{Ra}$ & $3.6 \mathrm{~d}$ & $\begin{array}{l}5.5-5 \% \\
5.7-95 \%\end{array}$ & $0.24-4.2 \%$ & 3.2 & $\times 10^{6}$ \\
\hline $220_{\mathrm{Rn}}$ & $55 s$ & $6.3-100 \%$ & & 4.6 & $\times 10^{6}$ \\
\hline${ }^{216} \mathrm{Po}$ & $0.15 \mathrm{~s}$ & $6.8-100 \%$ & & 6.1 & $\times 10^{6}$ \\
\hline${ }^{212} \mathrm{~Pb}$ & $10.6 \mathrm{~h}$ & & $\begin{array}{l}0.24-47 \% \\
0.30-4 \%\end{array}$ & & \\
\hline${ }^{212} \mathrm{Bi}$ & $60.6 \mathrm{~m}$ & $6.1-35 \%$ & $\begin{array}{l}0.5-4 \% \\
0.73)-9 \% \\
1.08)-9 \\
1.62-2 \%\end{array}$ & $\begin{array}{l}\text { ) } \\
\text { ) } \\
\text { ) } \\
\text { ) } \\
\text { j }\end{array}$ & $\times 10^{6}$ \\
\hline${ }^{212}$ Po & $3 \times 10^{-7} \mathrm{~s}$ & $8.8-64 \%$ & & ) & \\
\hline${ }^{208} \mathrm{TI}$ & $3.1 \mathrm{~m}$ & & $\begin{array}{l}0.58-31 \% \\
2.62-36 \%\end{array}$ & ' & \\
\hline${ }^{208} \mathrm{~Pb}$ & Stable & & & & \\
\hline
\end{tabular}

\begin{tabular}{|l|r|r|r|r|r|}
\hline${ }^{242} \mathrm{Cm}$ & $162.5 \mathrm{~d}$ & $\begin{array}{c}6.12-74 \% \\
6.07-26 \%\end{array}$ & $\begin{array}{l}\text { Lxrays } 12 \% \\
0.0440 .04 \%\end{array}$ & $3 \times 10^{6}$ \\
${ }^{238} \mathrm{Pu}$ & $86.4 \mathrm{y}$ & $\begin{array}{c}5.5-72 \% \\
5.46-28 \%\end{array}$ & L xrays $13 \%$ & $1.2 \times$ & $10^{4}(\max )$ \\
$234 \mathrm{U}$ (plus $4 \mathrm{n}+2$ sefies) & & \\
\hline
\end{tabular}


The energy spectra of the neutrons emitted from these two types of source have been measured at The Radiochemical Centre using a stilbene crystal and pulse shape discrimination ( 3 ). In common with other $(\alpha, n)$ sources they show a range of energies up to $12 \mathrm{MeV}$ (Figures 1 and 2).

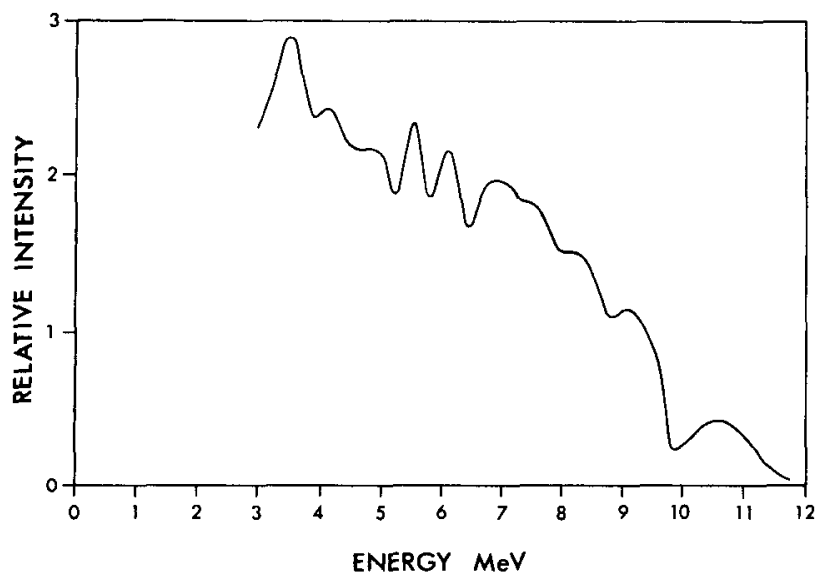

FIG. I ${ }^{228}$ Th-Be NEUTRON SPECTRA

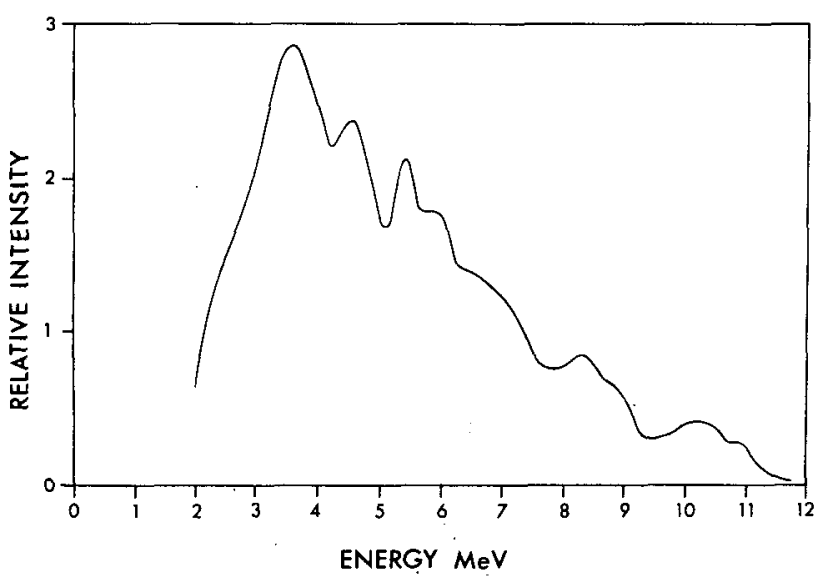

FIG. $2^{242}$ Cm-Be NEUTRON SPECTRA

Two obvious alternatives to thorium-228beryliium and curium-242-beryllium are the mixed radium/actinium/thorium/beryllium (4) and americium/curium/beryilium sources (5) made by the irradiation of radium-226beryllium and americium-beryllium sources respectively. The applications for these mixed sources are limited by their inherent disadvantages of high gamma emission, high internal pressure due to helium and fission gas production, lower specific activity and variable neutron output under in-pile conditions.
PRODUCTION OF CURIUM-242 AND THORIUM-228

For neutron sources containing curium-242 and thorium-228 the production of the nuclide is a major part of the problem. This is in marked contrast to many other $(\alpha, n)$ sources such as radium-226 and americium-241 where the nuclide is available from a number of commercial suppliers. Both nuclides are made by the reactor irradiation of other alpha emitting radionuclides and the problems associated with the design and production of the irradiation targets are similar to those encountered during the manufacture of reactor neutron sources.

To comply with reactor safety regulations the design of the irradiation target has to take into account heating due to alpha decay, nuclear reactions, and gamma heating and pressure build-up due to the production of helium and fission product gases. The approved design for UKAEA heavy water reactors like DIDO and PLUTO is shown in Figure 3. It is designed to withstand a pressure of $500 \mathrm{psi}$ at $500^{\circ} \mathrm{C}$ and specimens have been internally hydraulically pressure tested to 10,000 psi at room temperature.

\section{CURIUM-242}

Curium-242 is produced by the irradiation of americium-241; the possible nuclear reactions are set out in Table III.

TABLE III

Irradiation of Americium-241

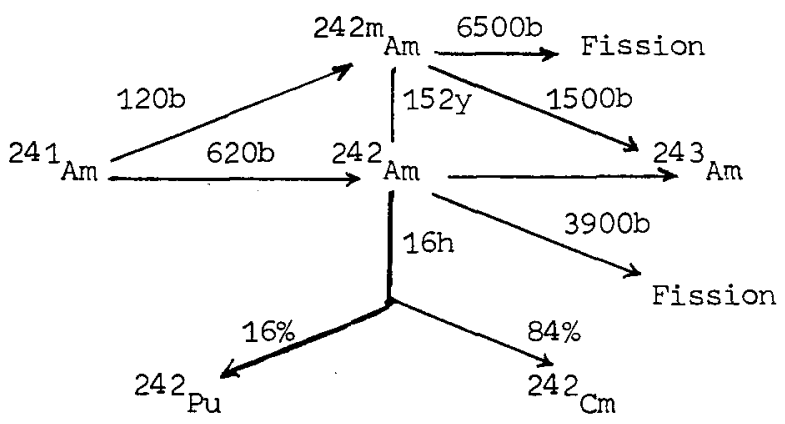




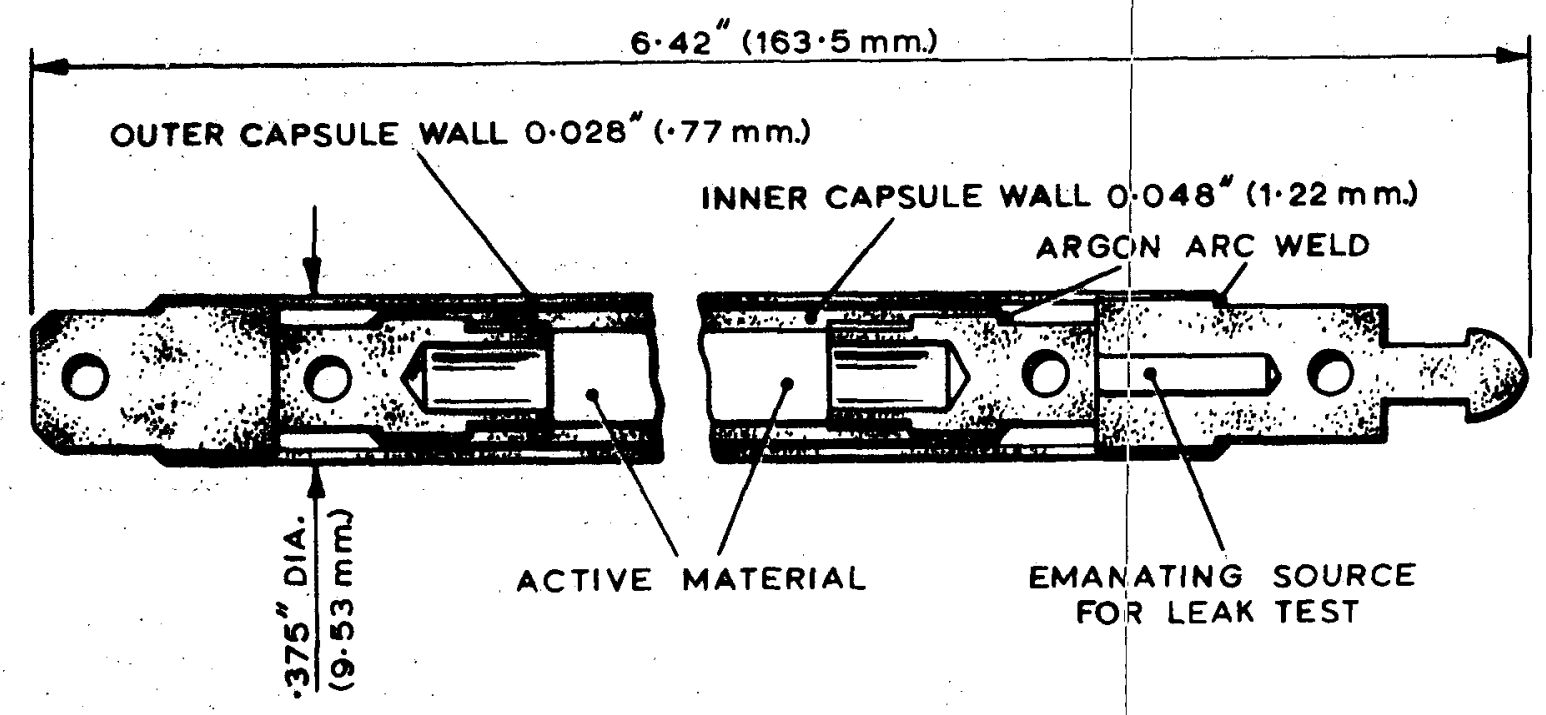

FIG.3 IRRADIATION TARGET CAPSULE

A typical target consists of $400 \mathrm{mg}$ americium-241 as oxide homogenously dispersed in nickel powder and cold compacted into pellets. The pellets are a push fit in the inner capsule which is sealed by argon arc welding after introduction of a helium atmosphere. The integrity of the seal is checked by helium mass spectrometry.

The primary capsule is loaded in the outer capsule which is sealed by argon arc welding after filling with helium. The integrity of the complete target is tested by helium leak test, wipe test and emanation test. For the purposes of this test a radon emitting (radium-226) source is enclosed in one of the end caps prior to sealing and this emanation source is also used as a control for leakage during irradiation.

\section{TABLE IV}

Data for Curium-242 Production

\begin{tabular}{|c|c|c|}
\hline Target loading & - & $\begin{array}{l}400 \mathrm{mg}{ }^{241} \mathrm{Am} \text { as } \\
\text { americium oxide } \\
\text { dispersed in } 8.6 \mathrm{~g} \text { nickel } \\
10^{14} \mathrm{n} / \mathrm{sec} / \mathrm{cm}^{2}\end{array}$ \\
\hline Irradiation time & - & 3 weeks \\
\hline Typical yield & - & $150 \mathrm{Ci}$ \\
\hline
\end{tabular}

In order for the curium-242 to be suitable for neutrion source production, material of high specific activity (at least $1 \mathrm{Ci} / \mathrm{mg}$ ) and low gamma emission (less than $1 \mathrm{mR} / \mathrm{hr}$ at $1 \mathrm{~m}$ ) isequired.

The irradiated targets are opened mechanically, and the pellets removed and dissolved in nitric acid. The actinide lanthanide fraction is separated from the bulk of the nickel and the soluble fission products by classical chemical methods.

Americium and curium are separated from the other actinides (mainly plutonium) and lanthanides (fission products) present by ion exchange and the americium is finally removed from the furium by oxidation of the americium to the Five valent state with persulphate.

The final material usually contains traces of iron, nickel and cerium-144 but is satisfactory for heutron source production.

\section{THORIUM-228}

The nuclide is produced by reactor irradiation of radium-226 and the production route is shown in Table, $V$. 
Irradiation of Radium-226

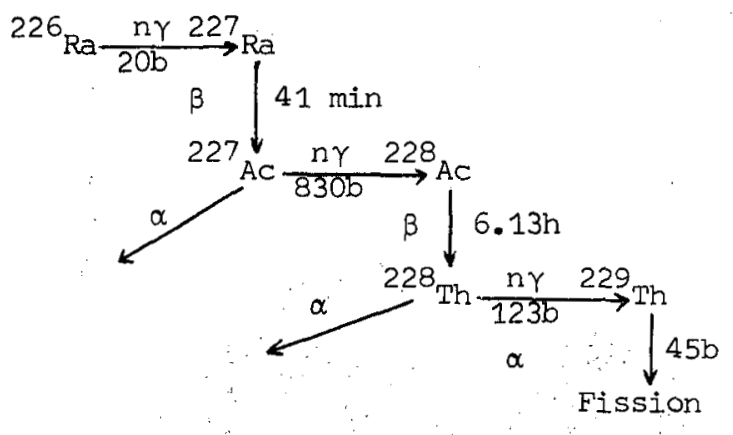

In this case the space in the inner capsule is completely filled with radium carbonate by a vibration loading technique. The testing procedures are as specified for the curium-242 production target"

\section{TABLE VI}

Data for Thorium-228 Production

Target loading - $\quad 3 \mathrm{~g}_{\text {carbonate }}^{226} \mathrm{Ra}$ as radium
Irradiation flux - $10^{14} \mathrm{n} / \mathrm{sec} / \mathrm{cm}^{4}$
Irradiation time -9 months
Typical yield $-40 \mathrm{Ci}$
Dose rate from
irradiated target $-40 \mathrm{R} / \mathrm{hr}$ at $1 \mathrm{~m}$

The target is mechanically opened and the radium carbonate removed and dissolved in nitric acid. The thorium-228 is separated from the radium and actinium by ion exchange. When the thorium-228 is eluted from the ion exchange column it is free from its daughter. product radium-224 and consequently neutron sources made from this thorium-228 will not reach maximum. neutron emission until several weeks after the final chemical purification. This fact can be used to advantage to reduce the radiological dose to the operators during source manufacture.

Both the curium-242 and thorium-228 separations are carried out in suites of lead-shielded tong boxes (Figure 4) and a similar plant is used for source manufacture.

\section{DESIGN}

Although curium-242 and thorium-228beryllium neutron sources are available in standard capsules, each reactor system usually requires a specially designed source. Critical to source design are the required neutron output and the end use to which the source is going to be put. Within this framework the source designer must consider the following points:-

(a) Operating conditions such as temperature, pressure and environment because these will determine the material and strength of the outer encapsulation.

(b) Internal pressure due to build-up of helium from the alpha decay of the radionuclide and from reactor irradiation of the beryllium because the source. capsule must be designed to withstand this pressure in the unlikely event of the helium being released from the lattice.

(c) Any restrictions on external dimensions and the need for handling attachments as this will fix the overall design of the source capsule.

(d) When (a), (b) and (c) have been considered, the most important point is the volume left available for the radionuclide and beryllium mixture as this fact taken in conjunction with the required neutron output will determine the quartity of radionuclide to be used, the ratio of active material to target and the possible method of loading.

(e) For applications where a number of sources with closely similar neutron emissions are needed, this requirement must be allowed for at the design stage. One technique is to produce a series of small inner capsules so that the neutron emissions from each can be measured and these capsules arranged in sets to give the required neutron emission, prior to final encapsulation.

We have designed and manufactured many different high intensity neutron sources during: the past ten years. The designs for two such sources with outputs in excess of $10^{9} \mathrm{n} / \mathrm{sec}$ are shown in Figures 5 and 6 . 


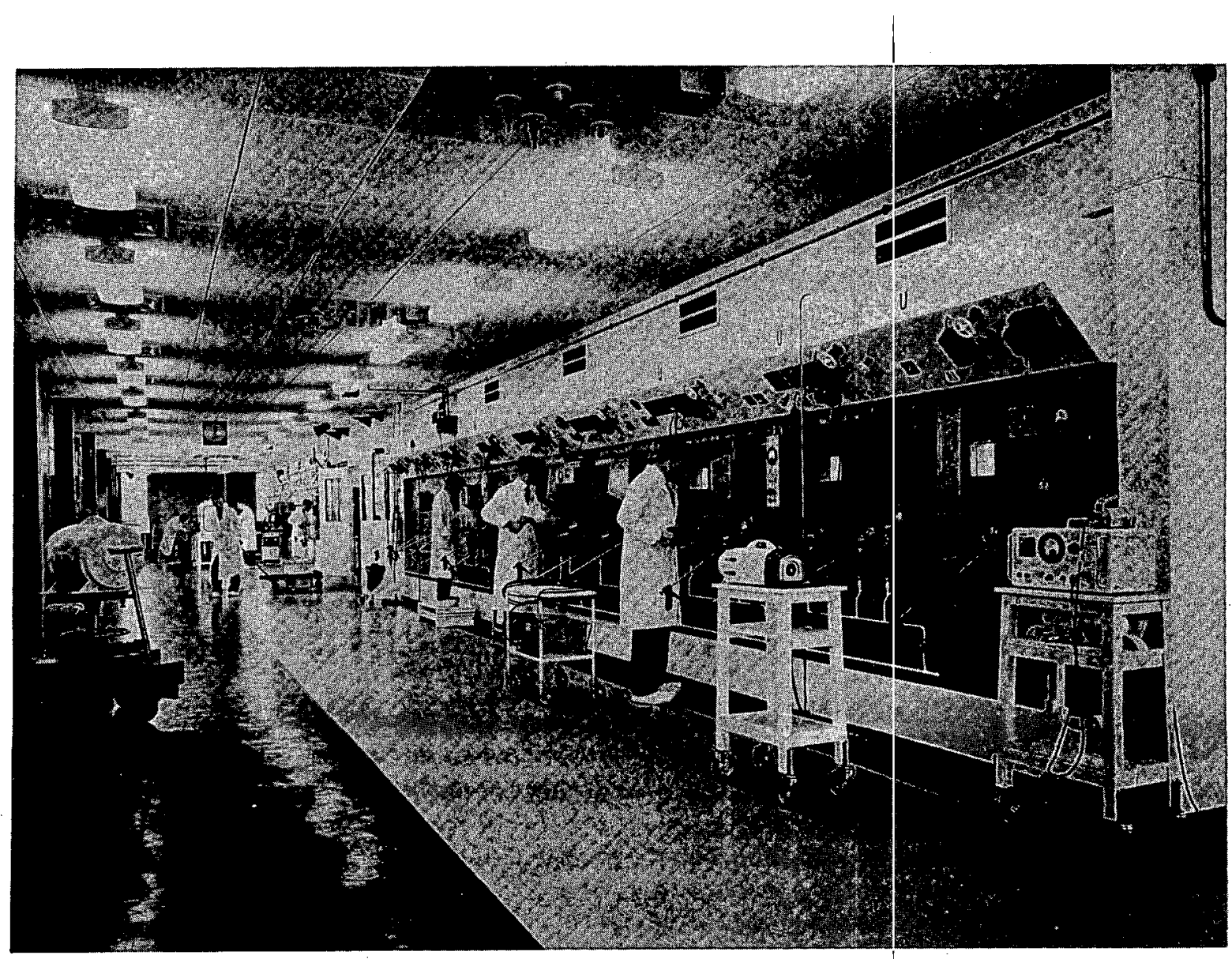

FIG.4 SUITE OF LEAD-SHIELDED TONG BOXES

The outer diameter of the thorium-228beryllium source (Figure 5 ) was restricted to $9 \mathrm{~mm}(0.354 ")$ and the capsule was designed to enable attachment of handling equipment for remote withdrawal from the transport container. The curium-242beryllium neutron source (Figure 6) was designed to fit into a fuel element channel. A further handling rod was attached to it for removal from the transport container and loading into the reactor.

\section{TABLE VII}

Design Data for Thorium-228-Beryllium Source

Active volume

- $2.14 \mathrm{cc}$

Active dimensions

- $3.4 \mathrm{~mm} \varnothing 23.6 \mathrm{~cm}$ long

Activity

- $60 \mathrm{Ci}$

Weight of beryllium - $2.35 \mathrm{~g}$

Density of active mixture $-1.1 \mathrm{~g} / \mathrm{cc}$

Neutron output

$-1.2 \times 10^{9} \mathrm{n} / \mathrm{sec}$
TABLE VIII

Design Data for!Curium-242-Beryllium Source Active volume $-6.2 \mathrm{~mm} \varnothing \times 65 \mathrm{~mm}$

Number of inner capsules - 2

Activity per inner capsule - $200 \mathrm{Ci}$ Weight of Be per capsule - $2.4 \mathrm{~g}$ Density of activé mixture - $1.25 \mathrm{~g} / \mathrm{cc}$ Neutron output $\quad->10^{9} \mathrm{n} / \mathrm{sec}$

The basis used for calculating build-up of pressure due to helium production is shown in Appendiz $I$. 


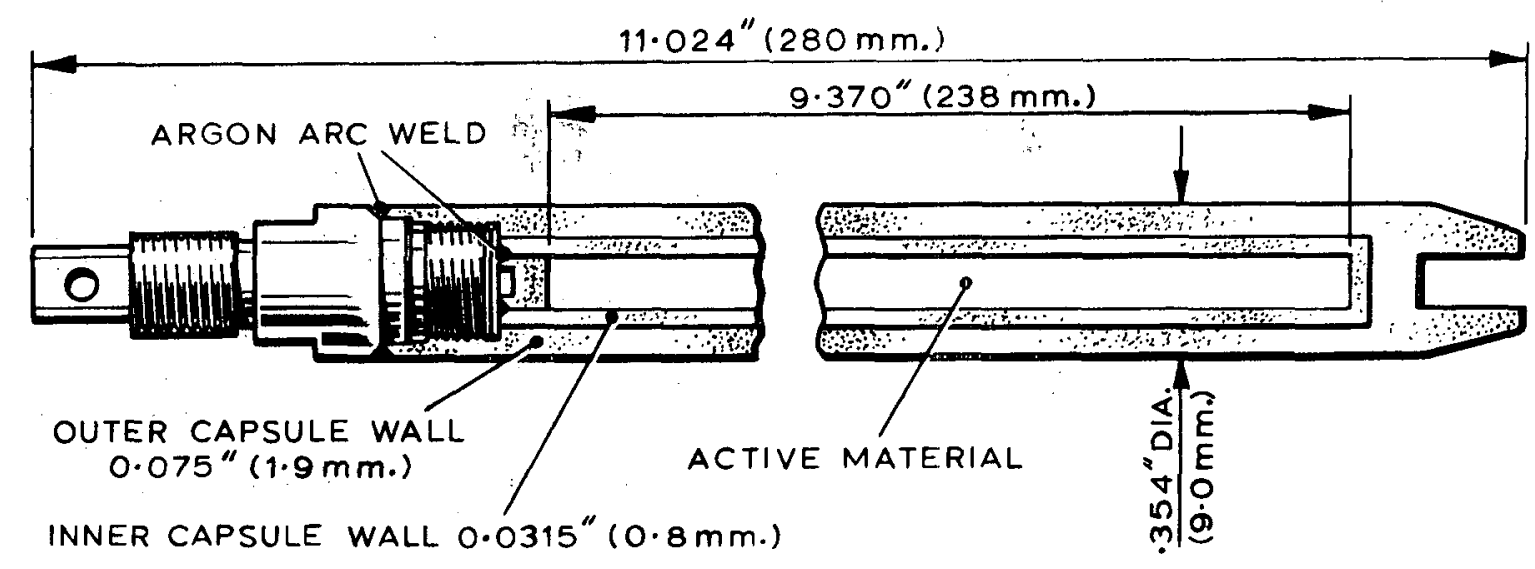

FIG.5 THORIUM-228-BERYLLIUM SOURCE

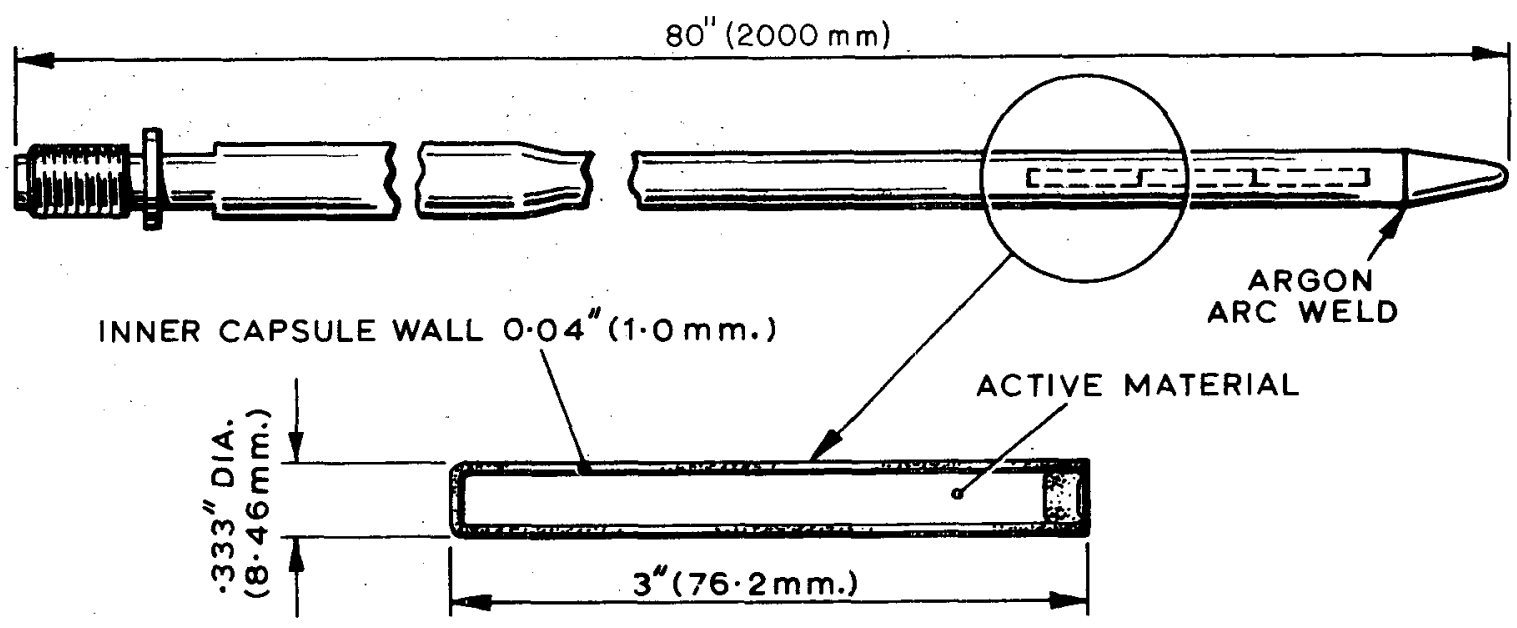

FIG.6 CURIUM-242-BERYLLIUM SOURCE

FABRICATION AND TESTING

Source fabrication, testing and measurement techniques obviously vary from source to source but a brief outline of these techniques is set out below.

The first stage in the fabrication of the neutron source is to produce an intimate mixture of the nuclide and target. This is normally accomplished by a combination of wet chemical and dry mechanical methods.

In the case of thorium-228 the nuclide is normally separated from its decay products immediately prior to mixing to reduce to a minimum the exposure dose rate due to neutron and gamma. When thoroughly dried the powder mixture is either loaded as a powder by vibration compacting or in the form of pressed pellets.

\author{
Density of vibration loaded \\ compact - approx. $1.1 \mathrm{~g} / \mathrm{cc}$ \\ Density of pressed pellets \\ - approx. $1.3 \mathrm{~g} / \mathrm{cc}$
}

After loading, the inner capsule is sealed by argon arc welding and decontaminated by chemical and electropolishing techniques. The tests used to check for leakage and freedom from surface contamination include bubble, wipe and emanation tests and are described in detail in Reference 3.

The inner capsules, after these tests have been successfully carried out, are loaded into outer capsules, filled with helium and sealed by argon arc welding.

In addition to the tests previously described, the outer capsules are tested 
by a helium mass spectrograph method, a leak rate of less than $10^{-8}$ standard $\mathrm{cc} / \mathrm{sec}$ being the normally accepted level.

The welding parameters for all capsules are established by trials on inactive samples, metallographic examination being carried out to check weld penetration and weld structure. Control samples are then made under exactly similar conditions to those used for the active specimens immediately before and after the active specimen.

To ensure that physical dimensions of completed sources conform with specification, full checks are carried out. Jigs and thread gauges are designed for remote handling operation.

The most normal additional request is for external pressure testing of completed sources. As a typical example, the thorium-228-beryllium source shown in Figure 5 was hydraulically tested to 10,000 psi external pressure and the tests for leakage and surface contamination repeated.

\section{MEASUREMENT OF NEUTRON EMISSION}

Neutron emissions are measured by comparison with standard sources calibrated at the National Physical Laboratory using an enriched $\mathrm{BF}_{3}$ counter moderated with either wax or polyethylene. In the case of the thorium-228-beryllium sources, several measurements are made over a period of about ten days after sealing in order that an extrapolated equilibrium value can be obtained. Final measurements are made after the full growth period (approximately one month).

\section{MEASUREMENT OF ACTIVITY CONTENT}

Thorium-228 The gamma emission from each source is measured using an ionisation chamber, several measurements being made in the first ten days to obtain an extrapolated value and a final measurement after full growth.

Thorium-228 and Curium-242 The heat output from sources is measured (subject to limitations of dimensions) using an adiabatic calorimeter.
TRANSPORT

The transport of "high intensity" sources is usually expensive as very large shielded containers are needed. A typical container used for sources similar to the curium-242 sources described on page 7 is shown in Figure 7. One adilitional problem with transport is that the limit for an alpha emitting nuclide in a "B" type container is normally $20 \mathrm{Ci}$, and this figure will be exceeded for many high intensity sources. It is therefore imbortant to show that the neutron source cap'sules meet the "special form" requirements of the IAEA.

"SPECIAL FORM" TESTIING

The IAEA Regulations for the Safe Transport of Radioactive Materials (1967) (6) gave limits for the maximum quantities of various nuclides wich can be transported in Type 'A' or ' $B$ ' containers.' If the radioactive material itself or its capsule can be shown to conform to the conditions laid down for "special form" materials, the maximum quantity allowed for transport may be considerably increased. The maximum activities for Toxicity Group I which includes curium-24.2 and thorium-228 are as follows:-

T'ABLE VIII

Transport Container Activity Limits

\begin{tabular}{|r|c|}
\hline Class A transport container \\
\hline Usual form & Special form \\
\hline $1 \mathrm{mCi}$ & $20 \mathrm{Ci}$ \\
\hline Class B transport container \\
\hline Usual form & Special form \\
\hline $20 \mathrm{Ci}$ & 5000 Ci \\
\hline
\end{tabular}




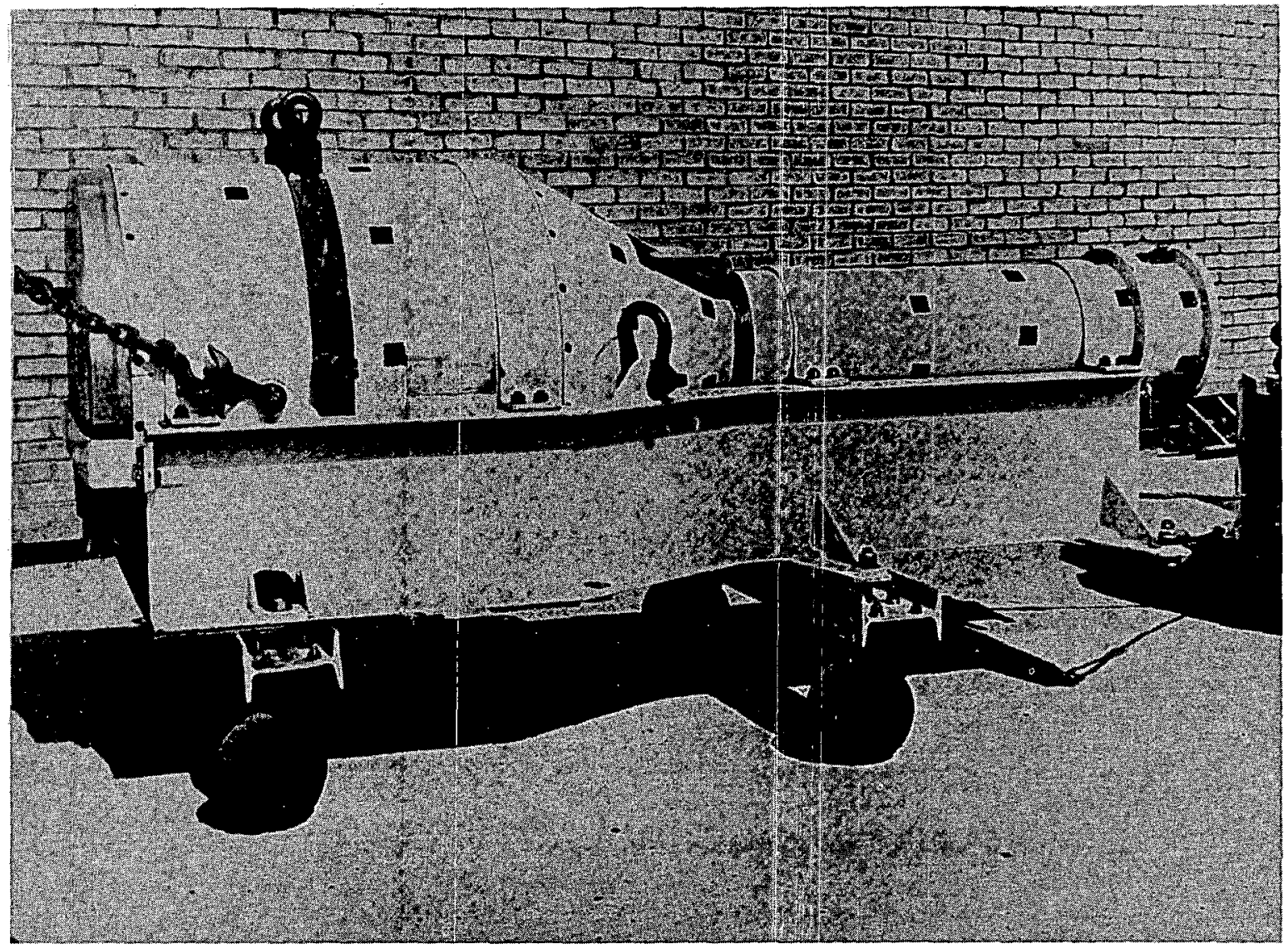

FIG. 7 TRANSPORT CONTAINER

In the UK in order to obtain a certificate of approval of radioactive material as being in "special form", it is necessary to demonstrate that the material or capsule will pass the IAEA tests, and then make an application to the Ministry of Transport.

The active component of curium-242beryllium and thorium-228-beryllium clearly cannot be classed as "special form" material, tests on the encapsulated source are therefore necessary.

The tests required by the IAEA include impact, percussion, heating and corrosion and are given in reference (6).

After each of the specified tests a leakage assessment is performed using an approved method (7).

The sample capsule tested should be constructed as for transport and the contents of the sample capsule should duplicate as closely as practicable the radioactive material which it is to contain, in particular with regard to the activities and specific activity.

In the case of high activity sources it is impractical and unnecessary to carry out tests on fully active sources. Tests are either carried out on low activity sources or on capsules where the alpha active nuclide is replaced with $10-20 \mu \mathrm{Ci}$ of a soluble caesium-137 salt.

The thorium-228-beryllium source illustrated in. Figure 5 passed all the tests and can be classed as "special form".

Due to the length of the curium-242beryllium source shown in Figure 6, it was impractical to test the complete source. In cases like this it has been our practice to submit the inner capsule to all the tests and this has been acceptable to the UK Competent Authority. 


\section{CONCLUSIONS}

In the UK we have found the two nuclides curium-242 and thorium-228 to have appropriate properties for the production of high intensity sources and have produced a large number of such sources for reactor instrumentation during the past ten years. It now seems certain that californium-252 will] supplant these $(\alpha, n)$ sources and the demand for curium-242-beryllium and thorium-228beryllium must diminish. We do not think that this means that high intensity $(\alpha, n)$ sources have no future. The half lives of curium-242 and thorium-228 have always been rather short for some applications and californium-252 with its half life of 2.6 years is not much better. It seems likely that as californium-252 stimulates new markets for high intensity neutron sources, there will be an increasing demand for longer half lives and this may well lead to the development of sources based on curium-244, actinium-227 or uranium-232.

\section{REFERENCES}

1. J. Wing and M. A. Wahlgren. "Detection Sensitivities in Nuclear Activation with an Isotopic Fast Neutron Source." Anal.Chem. 39, No. 1, 85. (1967).

2. G. Olive, J. F. Cameron, C. G. Clayton. "A Review of High Intensity Neutron Sources and their Applications in Industry." UKAEA Report AERE-R-3920. HM Stationery Office (1962).

3. "Radiation Sources for Industry and Research." RS-13. 56 (1971). The Radiochemical Centre, Amersham, UK.

4. D. J. Dudziak and I. B. Freeman. "Irradiated Ra:Be Neutron Sources." Nucl.Sci. and Eng. 11. 324 (1961).

5. E. H. Acree. "Americium-BerylilumCurium Neutron Sources." Trans.Am. Nucl.Soc. 7. 348 (1964).

6. International Atomic Energy Agency. "Regulations for the Safe Transport of Radioactive Materials." 1967 edition, Vienna IAEA 1967 (IAEA Safety Seriés No. 6).
7. K. H. Ansell. "Leakage Assessment Methods used at The Radiochemical Centre, Amersham, for testing Source Capsules for Approval as 'Special Form'" UKAEA Report RCC M-223. HM Stationery Office. 1969.

Design of sources takes into account the pressure built-up due to helium: and assumes that all helium is released from the lattice. Adequate safety margins are allowed when desichning source capsules.

Helium will be produced by:-

(a) radioactive decay of the isotope;

(b) reactions on beryllium under neutron irradiation.

(i.) the $n, 2$ n reaction;

(ii) the $n, \alpha$ reaction;

(iii) the $n, \gamma$ réaction.

The effect of (iii) on the production of helium is negligikle compared to the effects of fast neutron reaction and is not normally taken into account.

The relative importance of the two other routes for helium production, by decay and by fast neutron reactions on beryllium depends on the reactor flux to which sources are exposed and the time of exposure.

The following calculations for a typical source containing, $10 \mathrm{Ci}$ thorium-228 and $0.5 \mathrm{~g}$ beryllium ililustrate this point.

(a) Radioactive decay of thorium-228

$228 \mathrm{~g}$ of thorium-ri28 gives $20 \mathrm{~g}$ of helium when decay is complete.

$11.8 \mathrm{mg}(10 \mathrm{Ci})$ of ${ }^{228} \mathrm{Th}$ gives $1.035 \mathrm{mg} \mathrm{He}$.

$4 \mathrm{mg}$ He occupies $22.4 \mathrm{cc}$ at STP.

Volume of helium froduced at full decay $=\frac{1.035}{4} \times 22.4=5.8 \mathrm{cc}$

Helium production is calculated on the half life of 1.91 years neglecting any conversion of thorium-228. 
(b) Helium produced under irradiation

Helium is produced by neutron irradiation of a target according to the equation:-

Volume of $\mathrm{He} / \mathrm{g}$ of target $=$

$\frac{22.4 \times 10^{3}}{M} \cdot t . f . \sigma \cdot x \cdot \quad$ CC at STP

where $M$ is atomic weight $(\mathrm{Be}=9)$;

$f$ is neutron flux $\left(\mathrm{n} / \mathrm{sec} / \mathrm{cm}^{2}\right)$

$t$ is time ( $\mathrm{sec}$ )

$\sigma$ is reaction cross section $\left(\mathrm{cm}^{2}\right)$

$x$ is the number of helium atoms produced per incident particle

(i) $n, 2 n$ reaction

${ }^{9} \mathrm{Be}+\mathrm{n} \rightarrow 2^{4} \mathrm{He}+2 \mathrm{n}-1.85 \mathrm{MeV}$

This reaction may be taken to have an effective threshold of $3 \mathrm{MeV}$ and a constant cross section of 530 millibarns above this energy.

The volume of helium produced from $0.5 \mathrm{~g}$ beryllium per year in a typical flux of $10^{13} \mathrm{n} / \mathrm{sec} / \mathrm{cm}^{2}$ is $0.42 \mathrm{cc}$.

(ii) $n, \alpha$ reaction

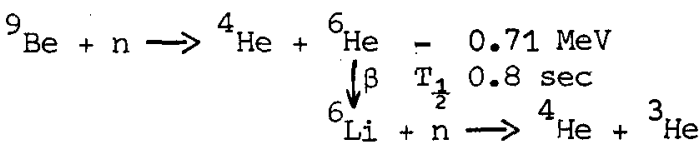

This reaction may be taken as having an effective threshold of $2.0 \mathrm{MeV}$ and a constant cross section of 83 millibarns above this energy.

The volume of helium produced from $0.5 \mathrm{~g}$ beryllium per year in a typical flux of $10^{13} \mathrm{n} / \mathrm{sec} / \mathrm{cm}^{4}$ is $0.05 \mathrm{cc}$.

The total volumes of helium produced by fast neutron reactions on beryllium and decay of thorium-228 for the example given above are as follows:-

\begin{tabular}{|c|c|c|c|}
\hline \multirow{2}{*}{ Time } & \multicolumn{3}{|c|}{ Helium produced cc } \\
\cline { 2 - 4 } & Decay & $\mathrm{n}, 2 \mathrm{n}$ & $\mathrm{n}, \alpha$ \\
\hline 1 & 1.8 & 0.42 & 0.05 \\
2 & 2.96 & 0.84 & 0.1 \\
5 & 4.88 & 2.10 & 0.25 \\
10 & 5.63 & 4.20 & 0.5 \\
\hline
\end{tabular}




\section{EVALUATION OF A ${ }^{244} \mathrm{Cm}$-Be NEUTRON SOURCE FOR ACTIVATION ANALYSIS}

\section{Morris A. Wahlgren \\ Donald C. Stewart}

Argonne National Laboratory

Argonne, Illinois
INTRODUCTION
A ${ }^{244} \mathrm{Cm}-\mathrm{Be}$ neutron source has recently been fabricated at ANL by Stewart, Horwitz, Youngquist and Wahlgren. A 0.64 gram quantity of 99 atom \% pure ${ }^{244} \mathrm{Cm}$ was mixed with -325 mesh Be metal in a 1:100, (i:m:Be ratio and compressed at 10,000 psi to produce the source. This source has a half-life of 17.6 years and has a low gamma ray background. The neutron emission rate was measured to be $1.2 \times 10^{8}$ neutrons/second.

The gamma radiation from the bare source and from the water or paraffinmoderated source have been measured with a $20 \mathrm{cc}$ GeLi detector. Experimental detection sensitivities in a rabbit irradiation facility have been obtained for the light elements where $(n, p)$ and $(n, a)$ reactions occur. Some comparative data taken with a ${ }^{252} \mathrm{Cf}$ source of nearly equivalent intensity in the same assembly are also presented.

When ${ }^{244} \mathrm{Cm}$ becomes readily available as a byproduct of power reactor fuel reprocessing, these sources will be very useful for certain applications because of the neutron spectral characteristics, the convenient half-life, and the relatively low cost where long-term utilization is possible.
Isotopic neutron sources are useful in a variety of laboratory as well as field applications in analytical problems because of the simplicity of operation and neutron flux reproducibility in day-to-day use. High-intensity ( $10^{10} \mathrm{n} \mathrm{sec}^{-1}$ ) $124 \mathrm{Sb}-\mathrm{Be}$ neutron sources were produced for on-site studies at the Savannah River Laboratory by Hennelly (1) in 1961 and offered commercially by the Atomic Energy of Canada Limited in 1968. A source assembly fabricated of $12242 \mathrm{Cm}-\mathrm{Be}$ capsules was produced at Argonne (2) in 1965 which yielded $7.5 \times 109 \mathrm{n} \mathrm{sec}-1$. The half-lives of $124_{\mathrm{Sb}}\left(60\right.$ days) and of $242_{\mathrm{Cm}}$ ( 163 days) are inconveniently short for many purposes which necessitates frequent source replenishment. The recent commercial availability (3) of milligram amounts of $252 \mathrm{Cf}(2.5$ years $)$ and recent evaluation studies (4) indicate a considerable potential for neutron sources in the range of $10^{9}-10^{10} \mathrm{n} \mathrm{sec}^{-1}$. Since ${ }^{244} \mathrm{Cm}(17.6$ years) will become a readily available byproduct of nuclear fuel reprocessing within a defade, we have constructed a prototype ${ }^{244} \mathrm{Cm}-\mathrm{Be}$ neutron source. The characteristics of this source and a number of experimental measurements taken with this source are described below.

Work performed under the auspices of the U. S. Atomic Energy Commission.

\section{SOURCE PREPARATION}

The physical details of the neutron source are shown in Fig. 1. Due to the limited amount of $244 \mathrm{Cm}$ available the capsule was only partially filled. The capsule volume is sufficient to hold about 1 gram of $244 \mathrm{~cm}$ in the 100:1 atom ratio of $\mathrm{Be}$ to $\mathrm{Cm}$ used for this source. Two features of the source will be noted. Helium gas built up in the source during alpha decay is leaked through a powdered aluminum plug (5). Aluminum was chosen as capsule material for the prototype source because of the low neutron capture cross section as well as ease of fabrication, since the neutron source is maintained in a controlled area. The helium leak technique could very likely be used with other capsule materials but no further work along this line has been done. Carrying of the Be powder by a curium hydroxide precipitation was utilized to avoid the losses incurred in mechanical grinding and mixing operations. The neutron yield of 56 neutrons per $10^{6}$ alphas indicates that a homogeneous mixture was obtained. Gamma ray spectral measurements on the purified $24+\mathrm{Cm}$ showed on $1 \mathrm{y}_{4}$ the lines of 239 Np formed in the decay of ${ }^{43} \mathrm{~cm}$. present in about $0.05 \%$ abundance. A detailed description of the chemical purification and inechanical assembly of the neutron source are given in a recent publication ( $\underline{6})$. 
ANL ${ }^{244} \mathrm{Cm}$ NEUTRON SOURCE

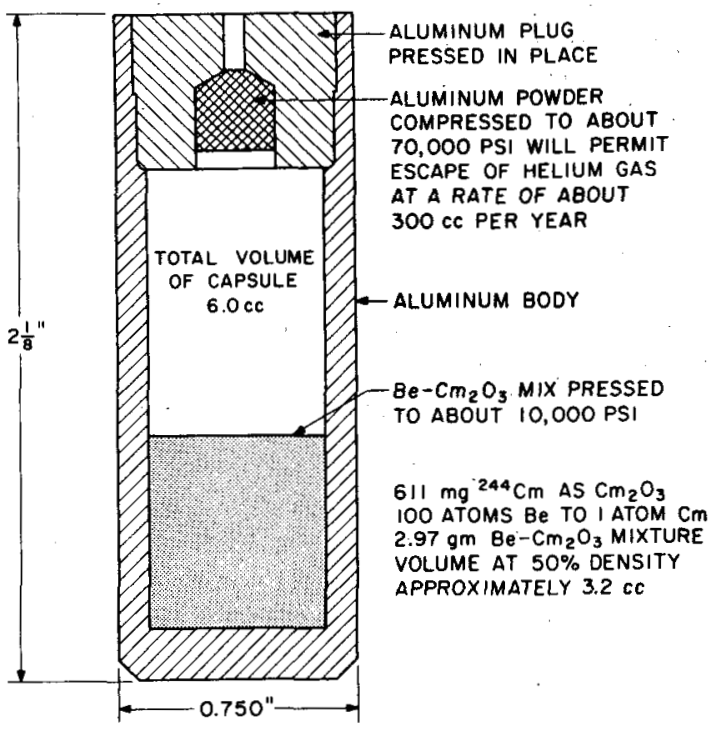

FIG. 1 DETAILS OF PROTOTYPE CAPSULE

\section{EXPERTMENTAL STUDIES}

Much of the evaluation study was carried out with the experimental arrangement indicated schematically in Fig. 2. Most of the elements of this arrangement could be removed from the system or varied in thickness to determine whether a significant effect on the gamma ray spectra was observed. The goals in this portion of the study were to establish (1) conditions for analysis of solutions or slurries, and (2) whether the $252 \mathrm{Cf}$ data now appearing in the Iiterature can be extrapolated to designs for $(x, n)$ facilities and vice versa. The moderator consisted of two concentric right circular cylinders, the inner of $15 \mathrm{~cm}$ diameter and the outer of $27 \mathrm{~cm}$ diameter. The inner cylinder was filled with distilled water, solutions, or paraffin, and the outer cylinder left empty or filled with distilled water to double the moderator thickness. A number of comparison measurements have been made in which a $2.2 \times 10^{8} \mathrm{n} \mathrm{sec}-1$ ${ }^{252} \mathrm{Cf}$ source was substituted for the $1: 2 \mathrm{x}$ $10^{8} \mathrm{n} \mathrm{sec}-1244 \mathrm{Cm}-\mathrm{Be}$ source. It was hoped initially that studying the variations in the shielding assembly with only the inner. cylinder filled would be useful in estimating the contributions of fast and slow neutron reactions to the "background" gamma ray spectrum.

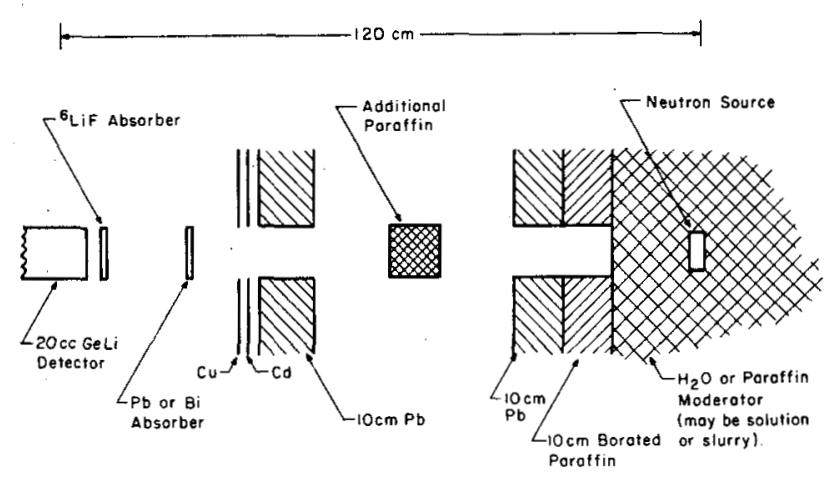

FIG. 2 EXPERIMENTAL ARRANGEMENTT FOR CAPIURE GAMMA RAY STUDIES

THERMAL NEUTRON MFASUREMENTS

The results of gold foil activations in the $15 \mathrm{~cm}$ diameter water moderator are shown in Fig. 3. For comparative purposes these radial measurements have been normalized to source strengths of $1 \times 10^{8}$ $\mathrm{n} \mathrm{sec}^{-1}$. The higher thermal neutron yield from $25 \dot{2}_{\mathrm{Cf}}$ is consistent with the comparisons reported by Barton (7) for $252 \mathrm{Cf}$ and $241_{\mathrm{Am}-\mathrm{Be}}$. The shape of the curves shows the effect of the lower average neutron energy in the fission spectrum ( $\sim 1 \mathrm{MeV})$ than that of the $(\mathcal{X}, n)$ spectrum $(4-5 \mathrm{MeV})$. The thermal flux distribution in this region is improved as expected when the outer moderator cylinder is also filled.

\section{CAPTURE GAMMA RAY SPECTRA}

Typical gamma ray spectra are shown in Fig. 4 The spectra of the bare sources are (a) ${ }^{244} \mathrm{Cm}-\mathrm{Be}$ and (b) ${ }^{252} \mathrm{Cf}$. The sourcedetector distance was $180 \mathrm{~cm}$, and a $10 \mathrm{~g} \mathrm{~Pb}$ $\mathrm{cm}^{-2}$ absorber was used. The dominant feature of spectra (a) is the Dopplerbroadened line at $4.43 \mathrm{MeV}$ due to recoil of the excited ${ }^{12} \mathrm{C}$ nucleus from the ${ }^{9} \mathrm{Be}(\alpha, \mathrm{n})$ reaction. The complex set of low energy lines in all spectra arise from neutron reactions in the GeLi detector $(\underline{8}, \underline{9})$. Reaction lines were observed at energies of $511,567,598,610,650,695,802$, and 1013 $\mathrm{KeV}$. Spectra (c) and (d) for the same sources were obtained with the components of Fig. 1, the moderator cylinder being moved sideways to utilize the lead as a shadow shield for the neutron source gamma rays. The slit in the lead shielding defined a vertical section of the moderator $3 \mathrm{~cm}$ wide by $10 \mathrm{~cm}$ high, parallel to the axis of the 


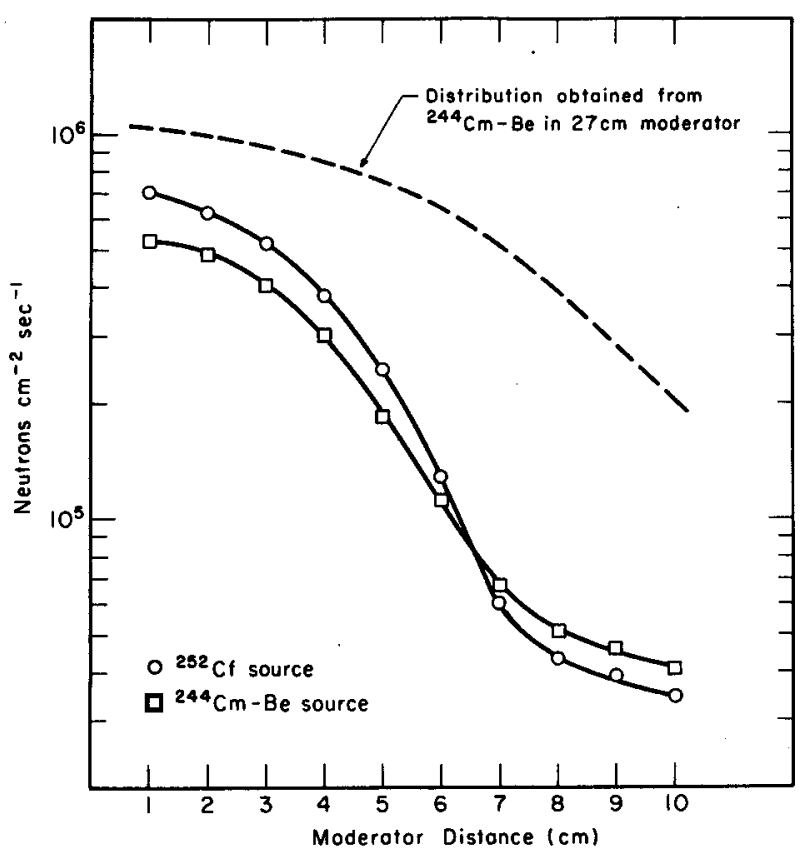

FIG. 3 THERMAL NEUTRON FLUX PLOT

neutron source. The borated paraffin slit was closed and only the inner moderator cylinder was filled. The spectra show that the "boekground" gamma ray spectra of the two sources are almost equivalent. Some comparative data are given in Table $I$. The peak area of the $695 \mathrm{KeV}$ line is representative of the fast neutron leakage, the $2223 \mathrm{KeV}$ line is a measure of thermal neutron yield, and the integral counts in the 5.8-6.2 MeV region indicate the high energy background. Absorption of hydrogen garma rays in the near-source moderator region accounts for the small difference in the $2223 \mathrm{KeV}$ peak areas. For higher energy lines this effect would be considerably less.

TABLE I

"Background" Spectra Comparison *

\begin{tabular}{|c|c|c|c|}
\hline Source & $695 \mathrm{KeV}$ & $2223 \mathrm{KeV}$ & $5.8-6.2 \mathrm{MeV}$ \\
\hline${ }^{252} \mathrm{Cf}$ & 3870 & 1830 & 760 \\
\hline${ }^{244} \mathrm{Cm}-\mathrm{Be}$ & 7000 & 1600 & 1330 \\
\hline
\end{tabular}

Further studies with the ${ }^{244} \mathrm{Cm}-\mathrm{Be}$ source showed lititle effect on the "background" spectra of removal of the ${ }^{6} \mathrm{LiF}$ thermal neutron absorber, the $\mathrm{Cd}-\mathrm{Cu}$ lining of the detector shield, or the additional $10 \mathrm{~cm}$ paraffin block. Closing the slits in the borated paraffin and the adjacent lead shield, or doubling the borated paraffin thickness also had little effect. Filling the outer moderator cylinder reduced the fast neutron background both at $695 \mathrm{KeV}$ and at 5.8-6.2 MeV by approximately a factor of two, and doubled the intensity of the $2223 \mathrm{KeV}$ line. To reduce counting times the detector could be moved about $50 \mathrm{~cm}$ closer to the moderator assembly without an excessively high background count. The minimum detectable concentration for chloride in solution was found to be about $0.1 \mathrm{M}$.

\section{PAST NEUTRON ACTIJATIONS}

Samples of pixre compounds of ca 8 $\mathrm{cm}^{3}$ volume were irradiated with unmoderated neutrons for $5 \mathrm{~min}$ and counted for 5 min with a $7.5 \mathrm{~cm} \times 7.5 \mathrm{~cm}$ NaI(TI) crystal. The plastic sample capsules (18mm diameter, $35 \mathrm{~mm}$ high) were irradiated end-to-end with the neutron source capsule, separated by $7 \mathrm{~mm}$ by a mechanical capsule stop. Comparative results are summarized in Table II. For $244 \mathrm{Cm}-\mathrm{Be}$, irradiations of a sample 20 times larger and surrounding the neutron capsule gave nearly the same specific activity as the arrangement above. A complete list of relative sensitivities obtained with a similar $(\chi, n)$ source is given in an earlier publication (10).

\section{TABEE II}

Fast Neutron Activation Sensitivities (a)

\section{Reaction}

$27 \mathrm{Al}(\mathrm{n} ; \mathrm{p}) 27 \mathrm{Mg}$

$28 \mathrm{Si}(\mathrm{n}, \mathrm{p}){ }^{28 \mathrm{Al}}$

$31_{\mathrm{P}}(\mathrm{n}, \alpha) 28_{\mathrm{A} I}$

${ }^{5} l_{V}(n, p) \quad 5 l_{\mathrm{T} i}$

${ }^{52} \mathrm{Cr}(\mathrm{n}, \mathrm{p}){ }^{52} \mathrm{~V}$ (b)

$19 \mathrm{~F}(\mathrm{n}, \alpha) 16_{\mathrm{N}}(\mathrm{c})$

(a)

a) results normalized to neutron outputs of

$1 \times 10^{8} \mathrm{n} \mathrm{sec}-1$

(b) irradiation tine 17 hours, count time

(c) 5 minutes irradiation time 30 seconds, count time
Counts per Counts per gram using gram using $24{ }^{\mathrm{Cm}-\mathrm{Be}} \quad{ }^{252} \mathrm{Cf}$

110

620

360

290

100

170

740
110

70

40

30

10

low

low 


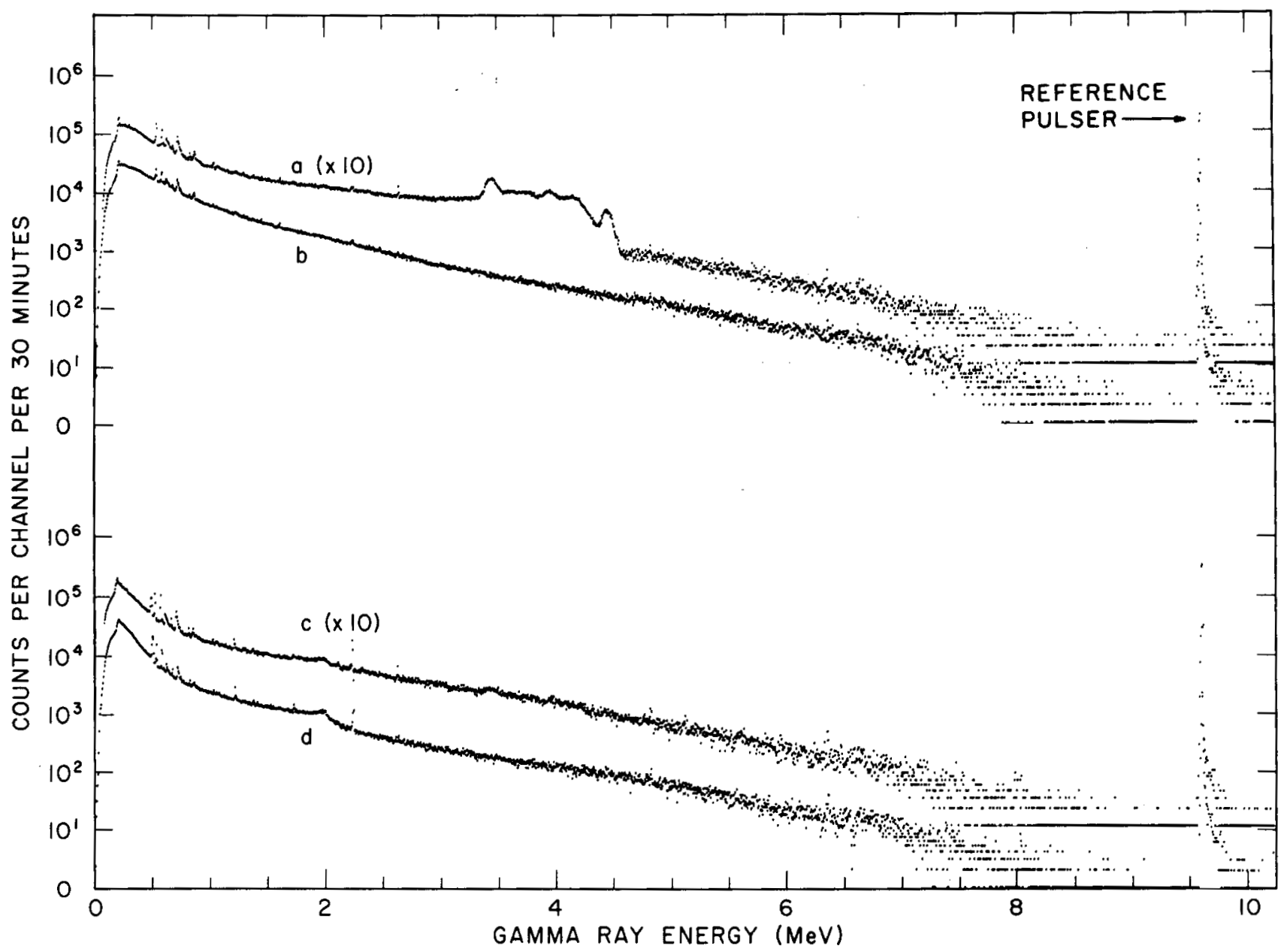

FIG. 4 COMPARISON GAMMA RAY SPECTRA

\section{CONCLUSSIONS}

The results of this work confirm that ${ }^{252}$ Cf offers a higher thermal neutron yield for radioactivation of small solid samples. The difference appears to be less significant in the application to solution or slurry samples utilizing capture gamma radiation. For fast neutron radioactivation of most light elements which undergo $(n, p)$ or $(n, \alpha)$ reactions, the ${ }^{244} \mathrm{Cm}-\mathrm{Be}$ neutron source is approximately 10 times as efficient as the ${ }^{25} \mathrm{C} \mathrm{Cf}$ source.

A definitive comparison of relative costs per neutron is not possible in view of the uncertainties in future pricing and availability. However, when $244 \mathrm{~cm}$ becomes available as a byproduct, a $2 \times 10^{8} \mathrm{n} \mathrm{sec-1}$ source would cost about $\$ 1800$ (assumes $\$ 200$ per gram of $244 \mathrm{~cm}$ and packaging-handiing costs of $\$ 1600)$. For long-term applications this source would yield $8 \times 10^{16}$ neutrons in 17.6 years for $\$ 1800$, which appears to be competitive with currently available neutron sources. $252 \mathrm{Cf}$ sources offer the greatest advantage for very intense sources ( $\sim 5 \mathrm{x}$ $10^{9} \mathrm{n} \mathrm{sec}^{-1}$ ) because of the compact size and low heat output.

\section{ACKNOWLEDGEMENTS}

The assistance of John Buzzell in handling neutron sources, and of John Barton for the loan of the $252 \mathrm{Cf}$ source is gratefully acknowledged. 
1. E. J. Hennelly. "Intense Sb-Be Sources Make $10^{10}$ Neutrons/Second." Nucleonics 19, 124 (1961).

2. J. A. Wing and M. A. Wahlgren. Detection Sensitivities in Nuclear Activation with an Isotopic Neutron Source. USAEC Report ANI-7242, Argonne National Laboratory, Argonne, Illinois (1966).

3. W. B. McCool. Californium-252 Price Decrease. U. S. Govermment Federal Register 35, (August 29, 1970).

4. Anonymous. Califormium-252 Progress, Vols. 1-5. U. S. Atomic Energy Commission, Savannah River Laboratory, Aiken, S.C. (1969-70).

5. C. H. Youngquist. A Controlled Leak Capsule to Contain Intense Alpha Emitters. Proc. 18th Conf. Remote Systems Technology, American Nuclear Society, Hinsdale, Illinois (1970).

6. D. C. Stewart, E. P. Horwitz, C. H. Youngquist and M. A. Wahlgren. "A ${ }^{244} \mathrm{Cm}-\mathrm{Be}$ Isotopic Neutron Source." Nucl. Appl. Tech. 2, 875 (1970).

7. J. P. Barton. Neutron Extraction and Collimation. Califormium-252 Progress 1, U. S. Atomic Energy Commission, Savannah River Laboratory, Aiken, S.C. (1969).

8. C. Chasman, $K_{0}$ W. Jones and R. A. Ristinen. "Fast Neutron Bombardment of a Iithium-Drifted Germanium Gamma Ray Detector." Nucl. Instr. Meth. 37, 1 (1965).

9. J. L. Rodda, R. I. Macklin and J. H. Gibbons. "Response of a $25 \mathrm{~cm}^{3}$ $\mathrm{Ge}$ (Li) Detector to NeutronsShielding Factors." Nucl. Instr. Meth. 74, 224 (1969).

10. J. Wing and M. A. Wahlgren. "Optimal Detection Sensitivities in Activation with Fast Neutrons from an $241_{\mathrm{Am}-} 24 \mathrm{C}_{\mathrm{Cm}-\mathrm{Be}}$ Source." Appl. Spectroscopy 23, 5 (1969). 


\title{
MONOLAYER CERAMIC MICROSPHERE-BERYLLIUM PLATE HETEROGENEOUS ISOTOPIC NEUTRON SOURCE
}

\author{
Edward D. Jordan, Thomas E. Carew, \\ and Barry L. Barkley
}

Catholic University of America Washington, D. C.

A heterogeneous isotopic neutron source consisting of an alternating array of plates containing an alpha emitter such as polonium-210 and beryllium would have the advantage of a controlled variable neutron output when the plates are moved in apposition to each other. A $6: 25 \mathrm{~cm}^{2}$ plane alpha source of 3 millicurie activity, consisting of a monolayer of polonium bearing ceramic microspheres of $50 \mu$ diameter, affixed to the surface of an aluminum plate by an epoxy film, was tested to evaluate its effectiveness in producing neutrons in a beryllium plate through the $(a, n)$ reaction. The alpha yield and energy spectra as a function of angle of incidence from the source was measured using a silicon surface barrier detector. Fifty percent of the alpha particles emitted in the half space above the source surface emerged from the source with energies equal to or greater than $1 \mathrm{Mev}$, and were thus effective in neutron production in beryllium.

A neutron production rate of 110 neutrons per second per $\mathrm{cm}^{2}$ of source surface was calculated and measured when a 10 mil thick beryllium plate was placed over the plate alpha source. Neutron measurements were made with a NE 213 liquid scintillation detector. It is calculated that a microsphere-plate source of optimum specific alpha activity could enhance neutron production by a factor of 30 . Thus, neutron yields in excess of 1000 neutrons-sec- $\mathrm{cm}^{2}$ are believed to be achievable using this source design concept.

\section{INTRODUCTION}

Previous work on heterogeneous isotopic neutron source development $(1,2)$ indicates that ceramic microspheres containing entrapped polonium-210 form an efficient contained source of alpha radiation. This suggests their possible use in a disk plate array heterogeneous neutron source in lieu of the window covered bare polonium metal. Thus, microspheres containing polonium-210 affixed in a monolayer to the surface of a metal plate might result in an effective neutron source when brought into close proximity to a beryllium plate. This concept has the advantage that there would be no need for a containment window between the alpha source and the beryllium plate. Hence, window failure would not be a limitation on useful source lifetime, and source fabrication would be facilitated. A possible disadvantage of this concept is that there may be a significant reduction in the available effective alpha radiation per unit area of source plate, resulting in larger dimensions for the source.

Aluminum plates coated with poloniumbearing ceramic microspheres fixed in position by a thin epoxy film are manufactured. for industrial use as static eliminators: A small plate source of this type was obtained for experimental evaluation of its effectiveness as an alpha source in a heterogeneous isotopic neutron source.
The purpose of this investigation was to measure the alpha output effectiveness and energy spectrum from a commercially produced static eliminator, polonium-microsphere plate source. These results were then applied to predict the maximum neutron output from a heterogeneous plate array source of optimum design. Neutron output predictions were also experimentally verified.

\section{ALPHA SPECTRUM AND YIELD FROM POLONIUM MICROSPHERE PIATE SOURCES}

\section{DESCRIPTION AND SPECIFICATIONS OF A STATIC ELIMINATOR ALPHA SOURCE.}

Plate sources of ceramic microspheres containing polonium-210 are used as static eliminators. The static eliminator sources consist of a thin layer of alpha emitting ceramic microspheres affixed by epoxy cement to the surface of a small aluminum plate. The dimensions of the microsphere alpha source aluminum strip used in this study are shown in Fig. 1. The alpha-active source area was $6.25 \mathrm{~cm}^{2}$. The polonium-210 alpha source activity on the date of manufacture (April 17, 1967) was approximately 3 millicuries. Table I lists various specifications of the polonium-210 static eliminator sources. 


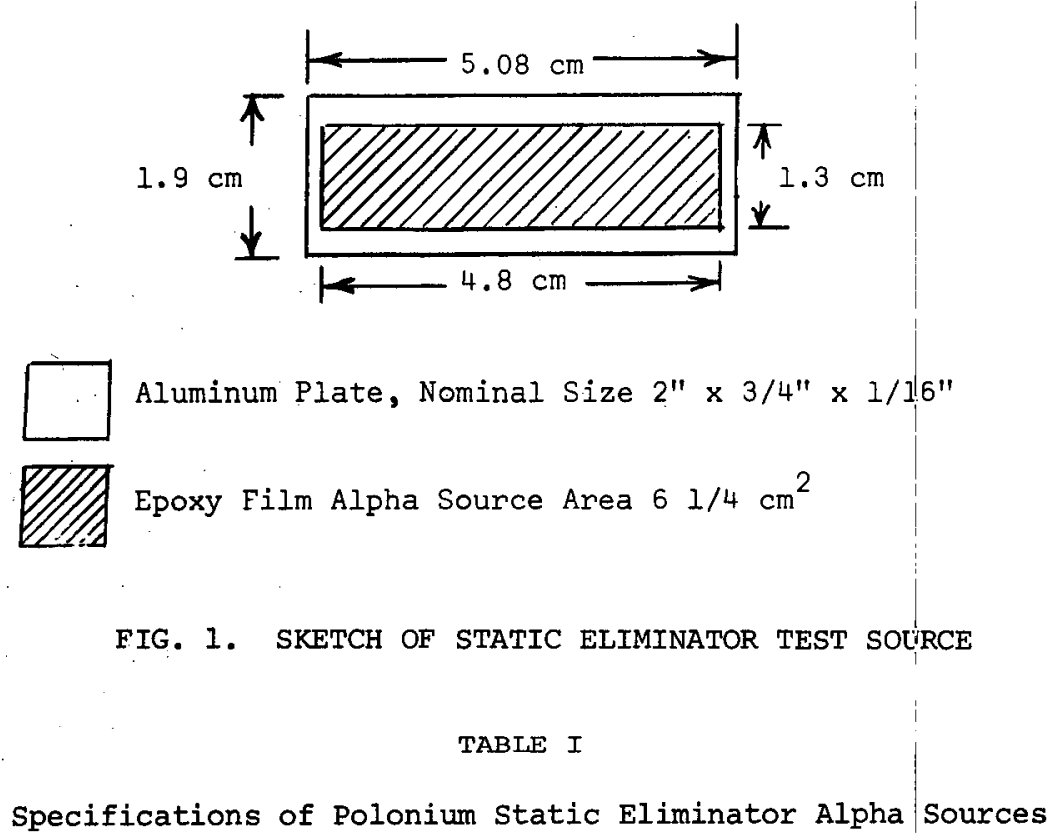

Static Eliminator Test Source

Static Eliminator A].pha Sources Now Available

Total Source Activity

Source Surface Area

Activity per Unit Area

Microsphere Activity

Microsphere Diameter

Microsphere Density

Microsphere Bulk Density

Number of Microspheres per Unit Area (Perfect Packing)

ALPHA YIELD AND SPECTRA FROM TEST SOURCE.

Neutron generation due to the alphaneutron reaction in beryllium is a sensitive function of alpha energy (1). To predict neutron production from a $\bar{d}$ isc-array source, it is necessary to know the alpha flux impinging on the beryllium target as $\simeq 3 \mathrm{mc} \quad$ Vairiable

$6.24 \mathrm{~cm}^{2} \quad$ Vàiriable

$\simeq 480 \mu \mathrm{Ci} / \mathrm{cm}^{2} \quad$ Up to $5 \mathrm{mCi} / \mathrm{cm}^{2}$.

$1 \mathrm{Ci} / \mathrm{g} \quad 1$ to $3 \mathrm{ci} / \mathrm{g}$

$\simeq 50$ microns $\quad \simeq 10 \mu$ to over $100 \mu$
$3 \mathrm{~g} / \mathrm{cm}^{3}$
$3 \cdot \mathrm{g} / \mathrm{cm}^{3}$
$2 \mathrm{~g} / \mathrm{cm}^{3}$
$2 \mathrm{~g} / \mathrm{cm}^{3}$
$46,188 / \mathrm{cm}^{2}$
Dépends on Micro- sphere Diameter

a function of alpha energy. Alpha particles emitted from the test source are degraded in energy by passage through the ceramic material of the microspheres and the epoxy film. The alpha yield and energy spectrum from the surface of the test source will, in general, not be isotropic since alpha particles enitted at small angles 
from the plane of the plate surface, on the average, will have traveled a greater track length through the ceramic and epoxy material than those emitted in a direction perpendicular (normal) to the plate. Thus, the needed experimental information is alpha output rate per unit area of the source surface as a function of both alpha energy and angle to the plate normal.

Alpha yields and energy spectra from the polonium-210 static eliminator test source were measured using a silicon surface barrier detector whose amplified and shaped pulse signals were passed to a multichannel pulse height analyzer. Measurements were made with the source and detector mounted in a vacuum chamber assembly specially designed to reduce the alpha intensity at the detector to acceptable levels and to permit the angle from the source plane to the detector to be changed. Experimental details are given in Appendix $A$.

Multichannel analyzer-detector system calibration with standard alpha sources permitted the identification of analyzer channel numbers with alpha energy groups. The alpha energy groups were selected with an energy spacing to facilitate neutron yield prediction ( 1 ). The observed alpha countrate for each selected energy group at angles of $0^{\circ}, 45^{\circ}$, and $85^{\circ}$ from the normal to the source plane is given in Table II and plotted in Fig. 2. Measurements were also made at $90^{\circ}$ and at intermediate angles from $0^{\circ}$ to $85^{\circ}$. The countrate at $90^{\circ}$ was essentially zero. The countrates at intermediate angles showed the same energy distribution as those plotted in Fig. 2. The resulting integrated countrates as a function of angle are presented in Table III. These values, normalized to the countrate at $0^{\circ}$ are plotted in Fig. 3 .

As may be seen in the semi-log plot in Fig. 2, the alpha energy spectrum over most of the energy range may be represented as a simple exponential of the form

$f(E)=A e^{E / E^{\prime}}$ where $E$ is the energy in Mev and $1 / E^{\prime}$ is empirically derived from the slope of the semi-log plot. The empirical equation is

$$
f(E)=1,920 e^{.455 E} 0 \leq E \leq 5 \mathrm{MeV}
$$

for $0^{\circ}$ to the normal where the constant is given in alpha counts per minute and $\mathrm{E}$ is in Mev. of significance is the fact that the slopes of all three curves plotted in Fig. 2 are approximately the same. Thus, the shape of the alpha energy spectrum is not a sensitive function of angle of incidence from the plate.

\section{ALPHA SOURCE ANISOTROPY}

A truly isotropic plane source would have a constant alpha output and energy spectrum at all angles rather than showing the angular dependence seen in Fig. 2 and Fig. 3 .

To simplify neutron yield calculations, neutron production can be calculated for the alpha yield and spectrum measured in a direction perpendicular to the source face (zero degrees in Fig. 3). The calculated yield can then be corrected by an anisotropic correction factor $F$ to account for the change in alpha an output with angle in the half space above the source face. only a single correction factor need be used, as the angular dependence curves shown in Fig. 2 all have approximately the same shape when normalized to equal areas.

The anisotropic correction factor, $F$ an' for the half space is obtained by integration under the curve in Fig. 3, rotated $2 \pi$ around its zero axis, and divided by the volume of a right circular cylinder of equal base and of height equal to the maximum ordinate value in Fig. 3. This calculation leads to a value of $\mathrm{F}_{\text {an }}=0.60$. That is, the average yield of alpha particles with energies greater than $1 \mathrm{Mev}$ in the half space above the source surface is $60 \%$ of the yield measured in a direction normal to the source surface.

\section{ALPHA PRODUCTION EFFICIENCY OF TEST SOURCE}

The anisotropic correction factor does not account for the fractional loss of a1pha particles in the perpendicular direction. The fractional loss in the perpendicular direction is implied, however, in the experimental data plotted as the $0^{\circ}$ curve in Fig. 2. The ratio of the integrated measured to total areas under this curve extrapolated to $0 \mathrm{Mev}$ and to, $5.3 \mathrm{Mev}$ suggests that $83 \%$ of the total number of alpha particles emitted in this direction have energies equal to or greater than 1 Mev. The net 
TABLE II

Alpha Spectrum Measurements

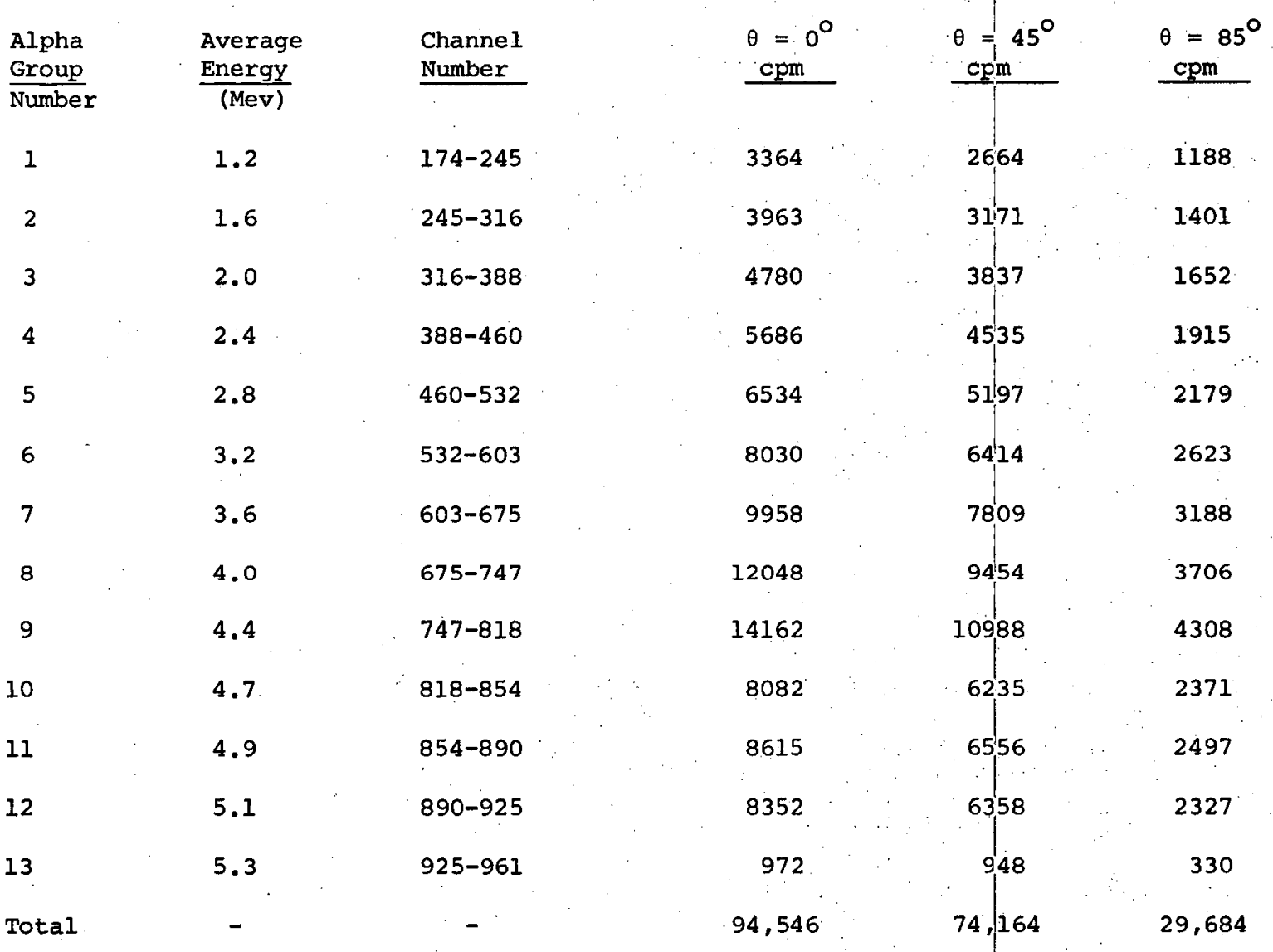

\section{TABLE III}

Total Alpha Countrate vs. Angle to Source Plate Normal

\begin{tabular}{|c|c|c|c|c|c|c|c|}
\hline Angle & $0^{\circ}$ & $10^{\circ}$ & $30^{\circ}$ & $45^{\circ}$ & $60^{\circ}$ & $80^{\circ}$ & $85^{\circ}$ \\
\hline cpm & 94,546 & 94,162 & 86,283 & 74,164 & 58,381 & 38,381 & 29,684 \\
\hline
\end{tabular}




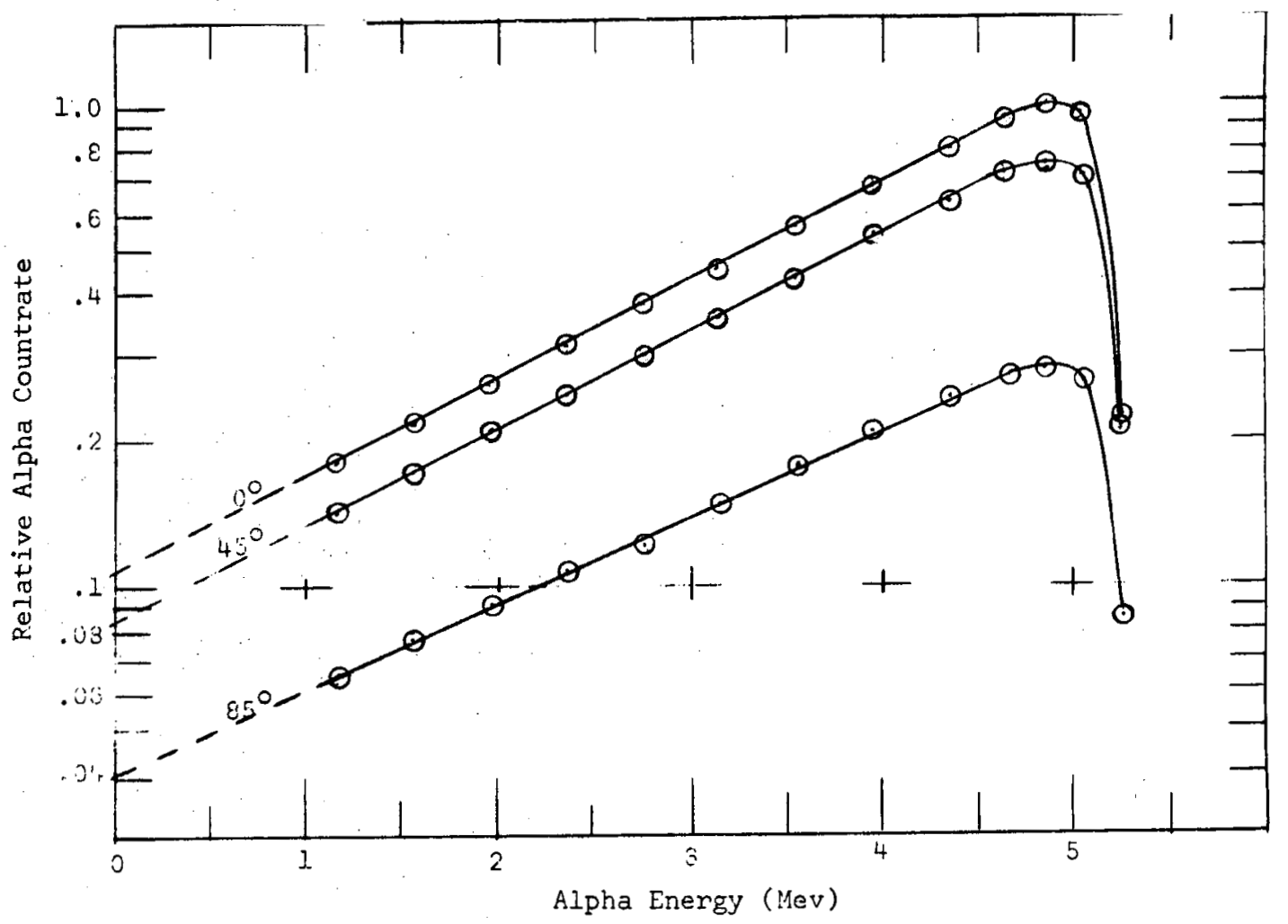

FIG. 2 RELATIVE ALPHA COUNTRATE AS A FUNCTION OF ALPHA ENERGY

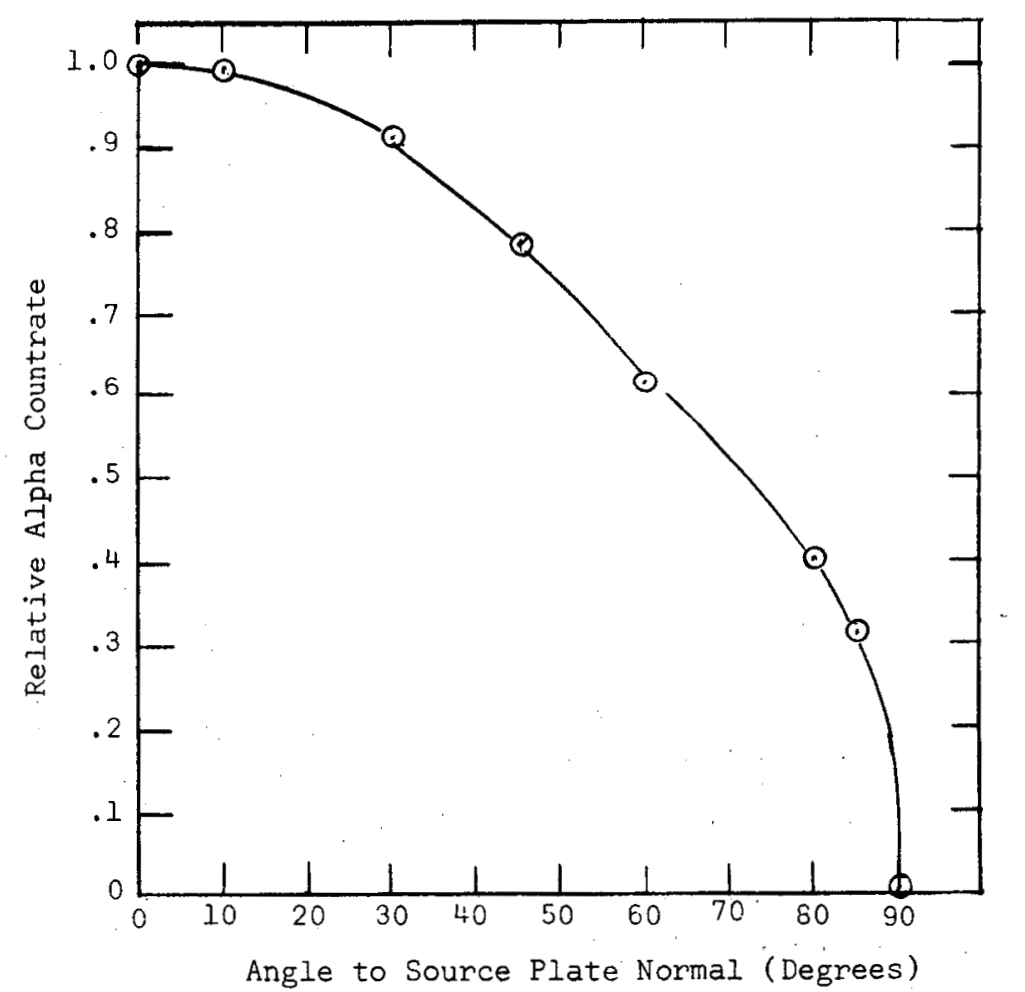

FIG. 3 RELATIVE ALPHA COUNTRATE AS A FUNCTION OF ANGLE TO SOURCE NORMAL 
alpha production efficiency ( $\geq \geq 1 \mathrm{Mev}$ ) in the half space for the source is then $0.83 \times 0.60 \simeq 508$.

\section{ALPHA DATA TREATMENT}

The surface barrier detector efficiency is essentially unity for alpha particles with energies greater than a few tens of Kev. That is, each alpha particle of non-negligible energy impinging on the detector will give rise to a voltage pulse whose amplitude is proportional to the kinetic energy of the incident alpha. As given in Appendix A, the geometrical efficiency factor for the source-detector geometry employed was $2.01 \times 10^{-4}$. The data may then be corrected for the effects of the detector system by dividing the observed countrate by $2.01 \times 10^{-4}$.

Due to the half life of polonium-2lo, a radioactive decay correction factor must be applied to the data to transpose relative countrates observed on different dates to a common date. The date selected was the date of test source manufacture (4-1767).

As an example of the data treatment, $94,546 \mathrm{cpm}$ were recorded on October 24 , 1967 at an angle of $0^{\circ}$ from the source normal, cf. Table II. The polonium decay correction results in $\simeq 245,000 \mathrm{cpm}(4-17-67)$. For an effective source area of $3.16 \mathrm{~cm}^{2}$ (App. A) this is $\simeq 77,550 \mathrm{cpm} / \mathrm{cm}^{2}$. The anisotropic correction factor, and the geometric efficiency factor of $2.01 \times 10^{-4}$ results in a calculated net alpha production rate per unit area of $2.32 \times 10^{8}$ alphas/min$\mathrm{cm}^{2}$. This is $3.87 \times 10^{6}$ alphas $/ \mathrm{sec}-\mathrm{cm}^{2}$ of energies equal to or greater than $1 \mathrm{Mev}$. The total alpha output rate in the half space of $E \geq 1 \mathrm{Mev}$ from the test source of $6.25 \mathrm{~cm}^{2}$ is thus $2.42 \times 10^{7}$ alphas $/ \mathrm{sec}$. Taking the net alpha production efficiency of $\mathrm{E} \geq 1 \mathrm{Mev}$ in the half space, this becomes $4.84 \times 10^{7}$ alphas/sec in the half space or $\simeq 10^{8}$ alphas/sec, $4 \pi$. This total production rate is equivalent to $2.7 \mathrm{milli}$ curies. Thus, the experimental results are in agreement with the nominal polonium-210 source strength value of $\simeq 3 \mathrm{mCi}$ on the date of manufacture.

NEUTRON YIELD FROM A MICROSPHERE-BERYLIIUM PLATE SOURCE

ALPHA-NEUTRON EXPERIMENTAL PLATE SOURCE
A rectangular beryllium foil, $10 \mathrm{mil}$, (greater than $\alpha$ stopping thickness) was placed on the surface of the static eliminator test source, resulting in $(\alpha-n)$ neutron production over a source area of $4.95 \mathrm{~cm}^{2}$. Neutron production was measured using an NE 213 liquid scintillation detector. The phototube output pulse was fed to a scaler through a pulse shape discrimination network (2) to discriminate against the gamma response of the scintillator (see App. A). Using a conventional poloniumberyllium source of known neutron output as a calibration standard, the neutron production per unit area of the test source was determined. As given in Appendix A, the measured neutron output from the test source was 111 neutrons/sec- $\mathrm{cm}^{2}$ of source area.

\section{CALCULATED TEST SOIJRCE NEUTRON YIELDS}

The measured alpha spectrum data in Table II was used to calculate a predicted neutron output (1). The experimental data for each alpha energy group at $\theta=0^{\circ}$ as given in Table II was converted as described above to the equivalent yield of alphas per second-m $\mathrm{m}^{2}$ of source surface on the date of source manufacture. The calculations are given in Table IV. The resultant prediction is 114 neutrons $/ \mathrm{sec}-\mathrm{cm}^{2}$.

PREDICTED NEUTRON YIELD FROM OPTIMUM PLATE SOURCE

The static eliminator source used for these measurements 'was not designed for optimum alpha actirity per unit area. The manufacturer advises that a factor of three increase can be obtained by increasing the specific activity of the microspheres and that ar additional factor of three can be achieved by the elimination of dilution of the microspheres by inert material (3). A denser packing of the spheres on the source surface may result in a further increase by a factor of 3-4. Thus, an optimized source manufactured under existing technclogy could be expected to result in a neutron yield higher by a factor of 30 than that of the test source, or $\simeq 13 \mathrm{mCi} / \mathrm{cm}^{2}$ of polonium-210.

The predicted optimized source yield is $3 \times 10^{3} \mathrm{n} / \mathrm{sec}-\mathrm{cm}^{2}$. The resulting predicted yield of $2.3 \times 10^{5}$ neutrons/sec-curie with a surface area of $77 \mathrm{~cm}^{2}$ per curie compares favorable with the yield and source area requirements. 
TABLE IV

Calculated Neutron Yield

\begin{tabular}{|c|c|c|c|c|}
\hline $\begin{array}{l}\text { Alpha } \\
\text { Energy } \\
\text { (Mev) } \\
\end{array}$ & $\begin{array}{c}\text { Exper. } \\
\begin{array}{c}\theta=0^{\circ} \\
(\mathrm{cpm})\end{array}\end{array}$ & $\begin{array}{l}\alpha / \mathrm{sec}^{-\mathrm{cm}^{2}} \\
(4-17-67) \\
\left(\mathrm{x} 10^{6}\right)\end{array}$ & $\begin{array}{l}\text { Neuts/* } \\
10^{6} \alpha\end{array}$ & $\begin{array}{l}\text { Neutron } \\
\text { Yield } \\
\text { n/sec-cm }\end{array}$ \\
\hline 1.2 & 3364 & 0.1377 & 1.6 & 0.22 \\
\hline 1.6 & 3963 & 0.1622 & 2.9 & 0.47 \\
\hline 2.0 & 4780 & 0.1957 & 4.7 & 0.92 \\
\hline 2.4 & 5686 & 0.2327 & 7.0 & 1.63 \\
\hline 2.8 & 6534 & 0.2675 & 9.99 & 2.67 \\
\hline 3.2 & 8030 & 0.3287 & 13.8 & 4.54 \\
\hline 3.6 & 9958 & 0.4076 & 19.1 & 7.79 \\
\hline 4.0 & 12048 & 0.4932 & 26.6 & 13.12 \\
\hline 4.4 & 14162 & 0.5797 & 36.9 & 21.39 \\
\hline 4.7 & 8082 & 0.3308 & 47.3 & 15.65 \\
\hline 4.9 & 8615 & 0.3526 & 55.9 & 19.71 \\
\hline 5.1 & 8352 & 0.3419 & 66.0 & 22.56 \\
\hline 5.3 & 972 & 0.0398 & 76.0 & 3.02 \\
\hline
\end{tabular}

${ }^{*}$ cf. Ref. (4)

\section{CONCLUSIONS}

It is concluded that a ceramic microsphere-beryllium plate heterogeneous neutron source can be made with a neutron output per unit area equal to that predicted for a polonium metal-beryllium plate source separated by a thin window to contain the polonium (1). The microsphere plate array source can be made using existing technology. Its design and manufacture is basically simpler than that of the window disc source, and window failure would not be a controlling factor on useful source lifetimes. Fifty percent (50\%) of the alpha particles from the epoxy coated ceramic microspheres in the half space above the source plate emerge with energies equal to or greater than $1 \mathrm{Mev}$, and are thus effective in neutron production.

A microsphere-beryllium plate source can be made to yield approximately 3,000 neutrons per second per $\mathrm{cm}^{2}$ of source area, at an efficiency of $2.3 \times 10^{5}$ neutrons per second per curie of polonium-210.

It is desirable, but not essential that the microsphere plates and beryllium plates be contained inside of an encapsulation housing. With proper care, the plates could be stacked to assemble a small neutron source, of the order of $10^{6}$ neutrons/second. Such a source could consist of an array of 33 discs $2^{\prime \prime}$ in diameter coated on both sides with an epoxy film polonium microsphere surface. The discs 
would be separated by $10 \mathrm{mil}$ sheets of beryllium. The total source dimensions would be approximately 2 " in diameter $x$ " high.

\section{APPENDIX A}

\section{EXPERIMENTAL MEASUREMENTS}

\section{ALPHA MEASUREMENTS}

Alpha yield and spectra measurements were made using a silicon surface barrier detector. The large (13/16 in. diameter) solid state alpha detector having an alpha energy resolution of $30 \mathrm{Kev}$ FWHM, was mounted in a specially devised vacuum chamber assembly to permit rotation of the source at a source to detector separation distance of $201 / 4$ inches. A sketch of the vacuum chamber and a block diagram of the electronic circuit is given in Fig. A-I.

The separation distance of $201 / 4$ inches $(\simeq 51.4 \mathrm{~cm})$ was selected to reduce the alpha flux impinging on the detector in order to prolong detector life. At close distances, the surface barrier detector could receive its threshold level of significant alpha damage within a few seconds of exposure to the high intensity alpha radiation from the test source.

Four spacers, each having a 1 inch center aperature, were arranged in the vacuum cylinder as shown in Fig. $\mathrm{A}-1$, to reduce the effect of scattering. This system resulted in a 1 inch diameter diaphragm opening near the source, producing an effective source area of $3.16 \mathrm{~cm}^{2}$.

The absolute pressure of the vacuum chamber during measurements was kept at $0.5 \mathrm{~mm} \mathrm{Hg}$. The source to detector distance of $201 / 4$ inches represents less than $1 \%$ of the alpha range in air at $0.5 \mathrm{~mm} \mathrm{Hg}$. Thus, the alpha particles undergo a negligible energy degradation in traversing the distance from source to detector.

Due to the relatively large source to detector separation distance $(51.4 \mathrm{~cm})$ the $3.16 \mathrm{~cm}^{2}$ source could be treated as an approximate geometrical point relative to the distance to the detector. A simple geometrical efficiency factor can then be calculated (detector area to area of half sphere).

The detector diameter was $13 / 16$ inch, resulting in a geometric efficiency factor of 0.000201 ; that is, $0.0201 \%$ of the alpha particles emitted from an isotropic point source a distance of $51.4 \mathrm{~cm}$ away would arrive at the detector. The alpha yield from the test source is not isotropic; however, this fact can be accounted for in the data treatment by a separate correction factor (anisotropic correction factor, $\mathrm{F}_{\text {an }}$ ).

\section{NEUTRON MEASUREMENTS}

Neutron output was measured using an NE 213 liquid scintillator with a pulse shape discriminator network. Neutrons were produced from the $(\alpha-n)$ reaction by placing a $10 \mathrm{mil}$ beryllium sheet over the alpha emitting surface of the static eliminator test source, as described above.

Neutron yield from the beryllium sheet was found to be anisotropic. The measured neutron countrate in the direction of the alpha particles was consistently found to be higher than the countrate in the opposite direction by a factor of two. The greater neutron emission in the direction of the incident alpha particle can be qualitatively explained by considering the energetics of the $(\alpha, n)$ reaction on beryllium. The $Q$ of the reaction to the ground state of ${ }^{12} \mathrm{C}$, for example, is $5.71 \mathrm{Mev}$. In the compound nucleus model of the reaction

$$
{ }_{4}^{9} \mathrm{Be}+{ }_{2}^{4} \alpha \rightarrow\left({ }_{6}^{13} \mathrm{C}\right)+{ }_{6}^{*} \rightarrow{ }^{1} \mathrm{~N}+{ }_{6}^{12} \mathrm{C}+5.71 \mathrm{Mev},
$$

neutron emission from ${ }^{13} \mathrm{C}$ is isotropic in the center of mass system. From momentum conservation, however, the compound nucleus will have momentum in the laboratory system equal to the momentum of the incident alpha particle. The momentum carried by a 5 Mev alpha particle is significant compared to the 5.71 Mev released in the reaction. Thus, the neutrons will be given off with a momentum vector component in the direction of the incident alpha particle. The anistropic nature of the $(\alpha, n)$ process from a plate source may prove useful for some source applications, for example in radiography, since it provides a means of enhancing the neutron flux in a preferred direction.

Counting both a Po-Be homogeneous source of known rieutron output and the heterogeneous test source at the same distance from the detector enables a direct 


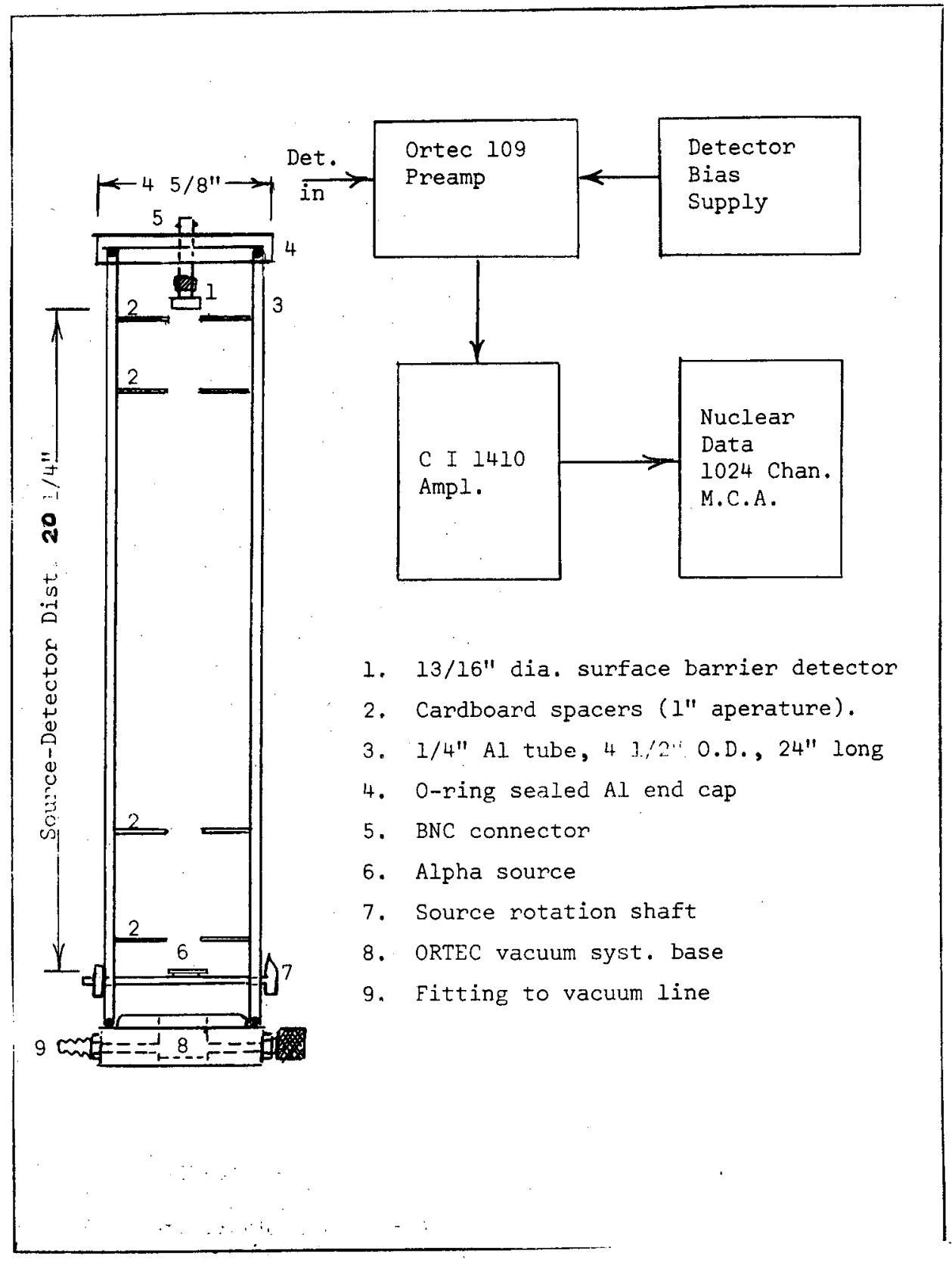

FIG. A-1. ALPHA MEASUREMENT EXPERIMENT

calibration of the test source, since the observed countrates will be directly proportional to the neutron output of the two sources. The counting should be done with sufficient source to detector distance so that the source and the detector geometry is essentially the same for both sources. The relatively low neutron production from$$
\text { . }
$$

the test source; however, prevented direct calibration at large source-to-detector distances. The high neutron output, plus the great difference in source geometry (a flat plate vs. a small cylinder) prevented direct calibration at very small distances. Calibration was made at a source-to-detector distance of 3 in. Data is given in Table $A-I$. 
TABLE A-I

Neutron Output Measurements

NE 213 liquid scintillator - PSD probe at discriminator dial setting of 25.0

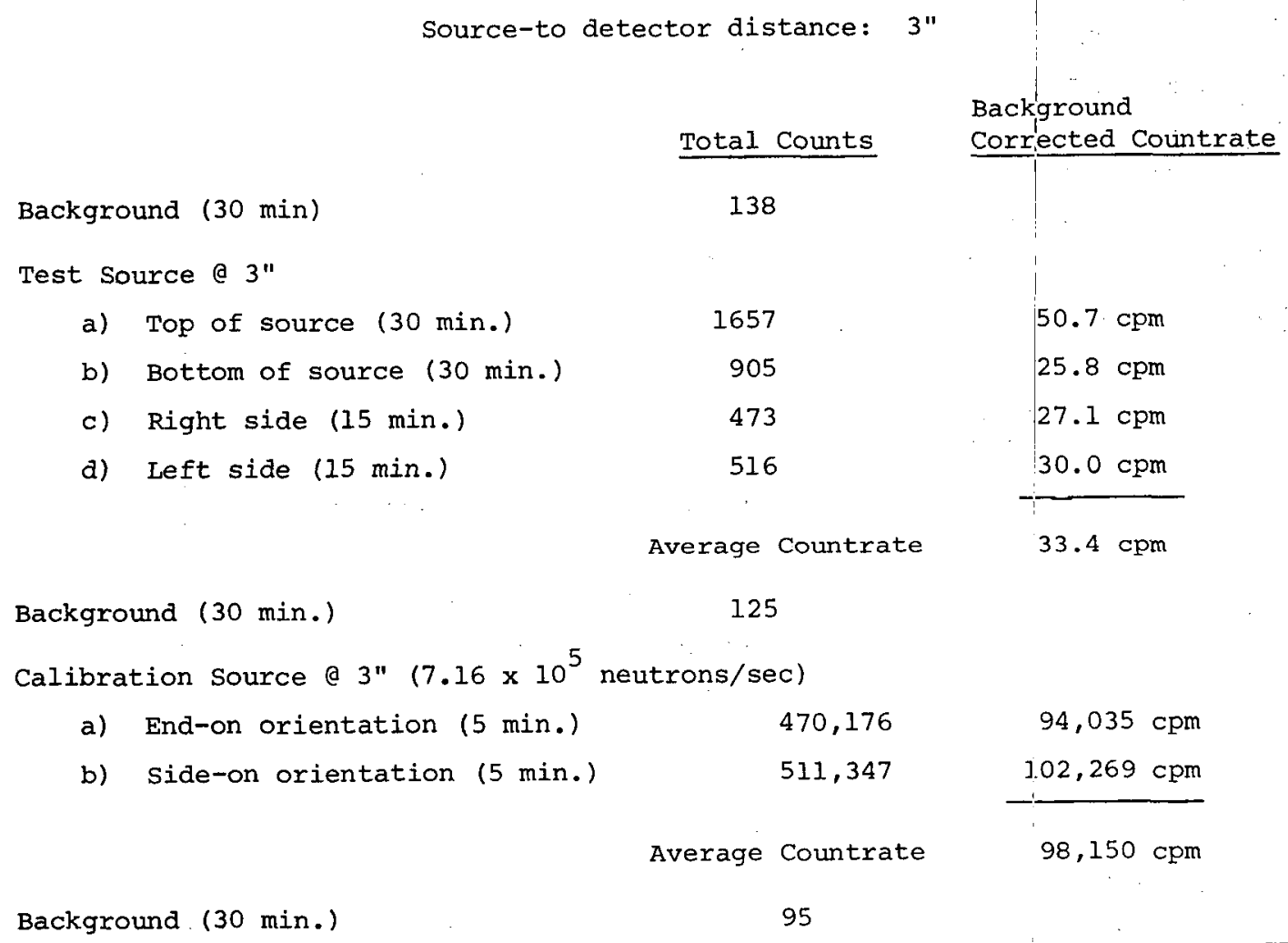
Source-to detector distance: 3"

516

Average Countrate

$50.7 \mathrm{cpm}$

$25.8 \mathrm{cpm}$

$27.1 \mathrm{cpm}$

$30.0 \mathrm{cpm}$

$$
33.4 \mathrm{cpm}
$$

Calibration Source e 3" (7.16 x $10^{5}$ neutrons/sec)
a) End-on orientation $(5 \mathrm{~min}$.)
470,176
$94,035 \mathrm{cpm}$
b) Side-on orientation (5 min.)
511,347
$1.02,269 \mathrm{cpm}$

Average Countrate

$98,150 \mathrm{cpm}$

Background ( $(30 \mathrm{~min}$.

95

Neglecting the small effect of different source geometries, the above data permits a direct neutron output calibration of the test source by

$$
\frac{\mathrm{N}_{1} \text { (Neut./sec.) }}{\mathrm{N}_{2} \text { (Neut./sec.) }}=\mathrm{k} \frac{\mathrm{C}_{1}(\mathrm{c} / \mathrm{m})}{\mathrm{C}_{2}(\mathrm{c} / \mathrm{m})}
$$

where the proportionality constant, $k$, has a value of unity for NE 213-PSD probe neutron detection system counting rates less than $40,000 \mathrm{cpm}$. $\mathrm{k}$ has an experimentally determined value of 0.75 due to PSD system deadtime losses at a discriminator setting of 25.0 for counting rates in the order of $100,000 \mathrm{cpm}$. Thus:

$\mathrm{N}_{1}=(.75) \frac{33.4}{98,150}\left(7.16 \times 10^{5}\right)=$

$183 \mathrm{neut} / \mathrm{sec}$ for $4.95 \mathrm{~cm}^{2}$ source area

$$
\begin{aligned}
\mathrm{N}_{1} & =37 \text { neut } / \mathrm{sec}-\mathrm{cm}^{2}(11-25-67) \\
\mathrm{N}_{1} & =\frac{37}{.3309}(\mathrm{jo}-210 \text { decay correction }) \\
& =111 \text { neut } / \mathrm{sec}-\mathrm{cm}^{2}(4-17-67)
\end{aligned}
$$

\section{REFERENCES}

1. E.D. Jordan and D.F. Knuth, "Neutron Production by the $(x, n)$ Reaction from Heterogeneous Isotopic Sources," Trans. ANS, Vol. 8, No. 2, 317, (1965)

2. E.D. Jordan and T.E. Carew, "Neutron Yields from Mixtures of Beryllium Powder and 
Polonium Impregnated Microspheres,"

Trans. ANS, Vol. 10, No.: 1, 84, (1967).

3. T. Lahr, Minnesota Mining and Manufacturing Co., St. Paul, Minnesota. Private communication.

4. E. Segré and C. Wiegand, "Thick Target Excitation Functions for Alpha Particles," MDDC-185, September 15, 1944. 


\title{
ISOTOPIC NEUTRON SOURCES FROM THE LOS ALAMOS MESON PHYSICS FACILITY*
}

\author{
Harold A. O'Brien, Jr. and Mario E. Schillaci
}

Los Alamos Scientific Laboratory

Los Alamos, New Mexico
After passing through several principal target areas, the main beam of the LAMPF accelerator will reach the main beam-stop with approximately $1 / 2$ ma of $700 \mathrm{Mev}$ protons. This excess beam of high-energy protons will be utilized for the production of radionuclides, which, at this energy, takes place primarily by spallation reactions. The principal features of the LAMPF accelerator and the conceptual design of the Isotope Production Facility are described. The systematics used to estimate the spallation reaction cross sections are discussed together with thick-target considerations. Several examples of yields of radionuclides that are potentially useful for photoneutron sources are given. One of the more promising examples is that of 107 day ${ }^{88} \mathrm{Y}$, of $/$ which approximately $75 \mathrm{Ci} / \mathrm{month}$ can be produced with exceptionally high specific activity from a one-inch-thick zirconium target. An ${ }^{88} \mathrm{Y}$-Be source will emit $150 \mathrm{kev}$ neutron:s with an intensity of about $3 \times 10^{6}$ $\mathrm{n} / \mathrm{sec} / \mathrm{Ci}$.

The LAMPF machine, which is scheduled to achieve full beam in July 1972, will make available, in commercially useful quantities, several photoneutron sources with a variety of neutron energies. This will represent a significant expansion in the available supply of such sources and will, hopefully, stimulate further afplications.

- Work performed under the auspices of the U. S. Atomic Energy Commission.

\section{INTRODUCTION}

The Los Alamos Meson Physics Facility (LAMPF) is a linear accelerator that will accelerate protons to an energy of $800 \mathrm{MeV}$ and have an average beam current of one milliampere. The projected date for the full-energy beam is July, 1972, and it is expected that operations will commence about January, 1973. At the peak usage of this machine, it is anticipated that the residual beam reaching the main beam-stop will have approximately $1 / 2 \mathrm{~mA}$ of $700 \mathrm{MeV}$ protons. P1ans are now being made to utilize this excess beam of high-energy protons for the production of radionuclides. Since the cost of prime beam time that is normally associated with accelerator-produced isotopes is not present here, a substantial economy can be realized. Furthermore, the combination of high energy and high intensity of the LAMPF beam will allow the production, in copius quantities, of many radionuclides currently not available.

The purpose of this communication is to point out the utility of LAMPF for producing selected radionuclides which can be used as the active components in low energy $(<1 \mathrm{MeV})$ photo-neutron sources. Many such sources have been described in the literature (1) for several years; however, because the radionuclides used in these sources cannot be economically produced with sufficiently high activities or because of their very short half-lives, almost all of these sources are not commercially useful. One source, ${ }^{124} \mathrm{Sb}-$
Be, which is readily produced in reactors at relatively little cost and has a half-life of $60 \mathrm{~d}$, has proved useful in several applications. We herein present a list of several gamma-emitters, with at least several-day half-lives, which can perhaps be economically produced with sufficient activity to provide additional usefu]. isotopic neutron sources with a variety of neutron energies. One in particular - vizi, ${ }^{8}{ }^{8} \mathrm{Y}(107 \mathrm{~d})$ - would seem to compete favorably with ${ }^{124} \mathrm{Sb}$.

\section{THE LAMPF ACCE'LERATOR AND THE ISOTOPE PROIUCTION FACILITY}

For the past five years a group of scientists at the Los Alamos Scientific Laboratory in New Mexico has been engaged in the development of a high-flux meson physics facility. The $800-\mathrm{MeV}$ linear accelerator will have an average proton beam current of one milliampere, |whereas the largest proton linear acceleratir now in existence with a comparable current has an energy of only 68 $\mathrm{MeV}$. It was proved feasible to increase this energy more than tenfold and, further, to increase the external beam intensity by approximately 10,000 times that which is available from any accelerator in the energy region under consideration.

The external beam power from this accelerator is at least as great as that available from any accelerator now operating or under construction, energy notwithstanding. The only accelerators which approach LAMPF in 
operating characteristics are the machines being built in Zurich and in Vancouver (TRIUMF) and the soon-to-be-completed, modified synchrocyclotron of Columbia University. However, these projects are designed for peak energies several hundred $\mathrm{MeV}$ lower than that of LAMPF. Also, external beam intensities are 10 times less in the case of the Zurich accelerator and TRIUMF and 30 times less for the Columbia machine.

The LAMPF accelerator is approximately one-half mile in length and is composed of three separate accelerators, each optimized for a particular energy range. First is a conventional Cockcroft-Walton injector by means of which protons will be accelerated to $0.75 \mathrm{MeV}$. These protons will be injected Into a section of the machine known as the Alvarez structure, so named after its inventor, Professor Luis Alvarez of Berkeley. This section will accelerate protons to $100 \mathrm{MeV}$. From the Alvarez section the beam will proceed into a waveguide section. This structure, a side-coupled cavity system, was developed at Los Alamos and is characterized by good stability and high efficiency for transforming the $800-\mathrm{MHz}$ rf power into beam power. A schematic representation of LAMPF showing the principal components of the 800$\mathrm{MeV}$ proton accelerator is shown in Fig. 1 . Present plans call for the first beam in July, 1972 .

\section{LOS alamos meson phrsics facility}

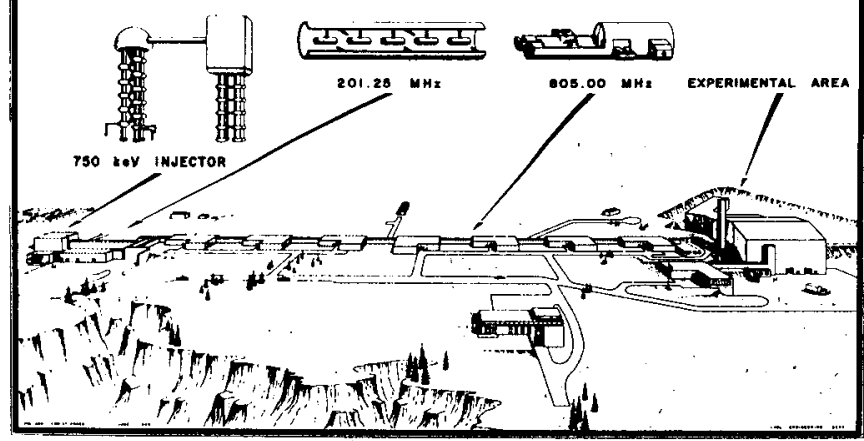

Fig. 1. Schematic representation of the three major components of the LAMPF accelerator.

The main purpose of this facility is to provide sufficient intensities of nucleons, pions, muons, and neutrinos to make possible their use as probes to explore the structure of the atomic nucleus and the forces by which this structure is governed. Consequently, several experimental areas are planned, each requiring particular beam energies and intensities. A complex system of bending magnets and quadrupole lenses will be available to transport the proton beam to various experimental locations. In addition, several innovations will allow portions of the 500- $\mu$ sec proton beam to be deflected to experimental areas requiring only a small amount of beam. A schematic diagram of the experimental area is shown in Fig. 2.

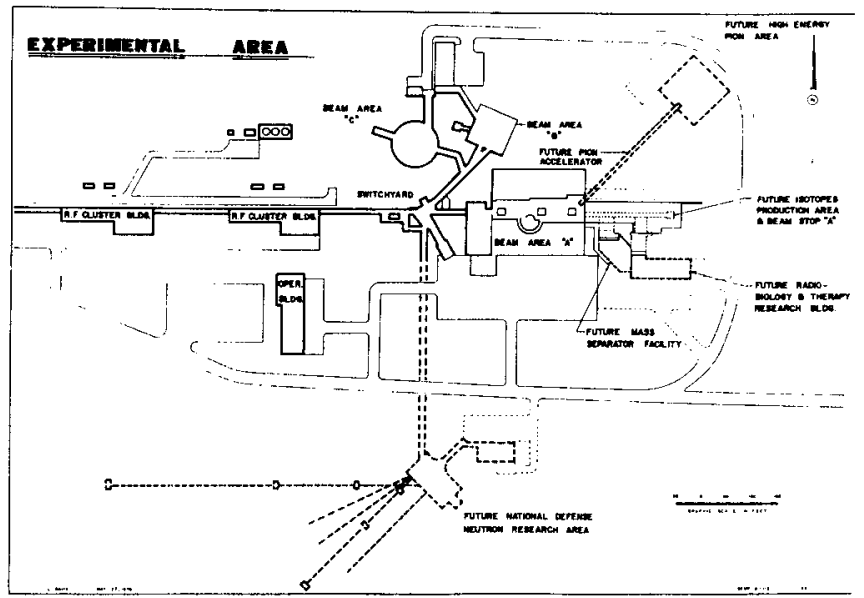

Fig. 2. LAMPF Experimental Area showing the location of the Isotope Production Facility.

Along the main high-intensity proton beam path, at least six experiments will use the same beam. After each target, the beam will be refocused and transported to the next target. Even so, a major portion of the proton beam will remain and must, of necessity, be disposed of. As a result, it is proposed to locate an Isotope Production Facility at the end of the main proton channel for the purpose of using the excess proton beam to prepare a wide variety of both neutron-deficient and neutron-excess radionucldies. $\mathrm{Re}-$ cent estimates show that, if all of the target stations up-stream of this facility were occupied, the beam reaching this facility will contain protons of $700 \pm 20 \mathrm{MeV}$ at a current of $400 \pm 100 \mu \mathrm{A}$.

The target assembly of the Isotope Production Facility will provide for six target stations, each of which is independently operated. A conceptual design of this assembly is presented in Fig. 3. Each target station will accommodate a target or combination of targets up to about 1 in. thickness; and will contain provisions for target cooling, instrumentation, and retraction mechanism to remove the target station from the 


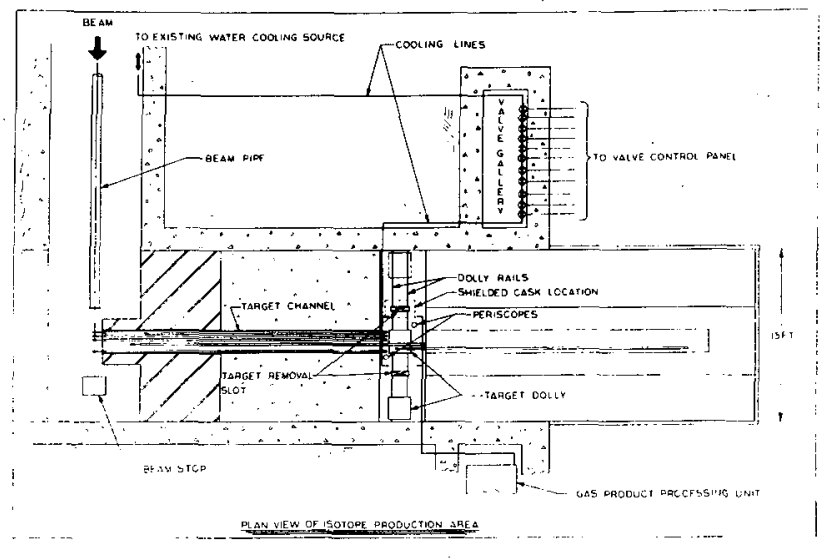

Fig. 3. Proposed target assembly for the Isotope Production Facility.

proton beam when not in use. In addition, it is proposed that one or more of the target stations be equipped to provide for the continuous removal of gaseous products during bombardment.

The independent-target-station concept allows the necessary flexibility for choosing the optimum irradiation time for each targetproduct combination, as we 11 as the insertion and removal of targets from the beam without interrupting the irradiations in the other stations.

Taking into account both beam attenuation and scattering, our calculations indicate that if $700 \mathrm{MeV}$ protons enter the first target station, approximately $500 \mathrm{MeV}$ protons will exit from the sixth target. Also the sixth target will see about $20 \%$ of the beam that impinges on the first station.

\section{YIELD PREDICTIONS}

The principal mechanism involved in the production of radionuclides at LAMPF is the high-energy proton-induced spallation reaction. In this reaction, many nucleons are knocked out of the nucleus, both singly and in clusters, resulting in a variety of possible nuclei. Some idea of the distribution of products that is obtained is provided by the example shown in Fig. 4. Here are shown the mass-yield curves from a $\mathrm{Bi}$ target at several energies. The $800 \mathrm{MeV}$ curve has been sketched in to provide a qualitative comparison with the other curves which represent data (2). The general features to be noted are: 1) a peak corresponding to spallation

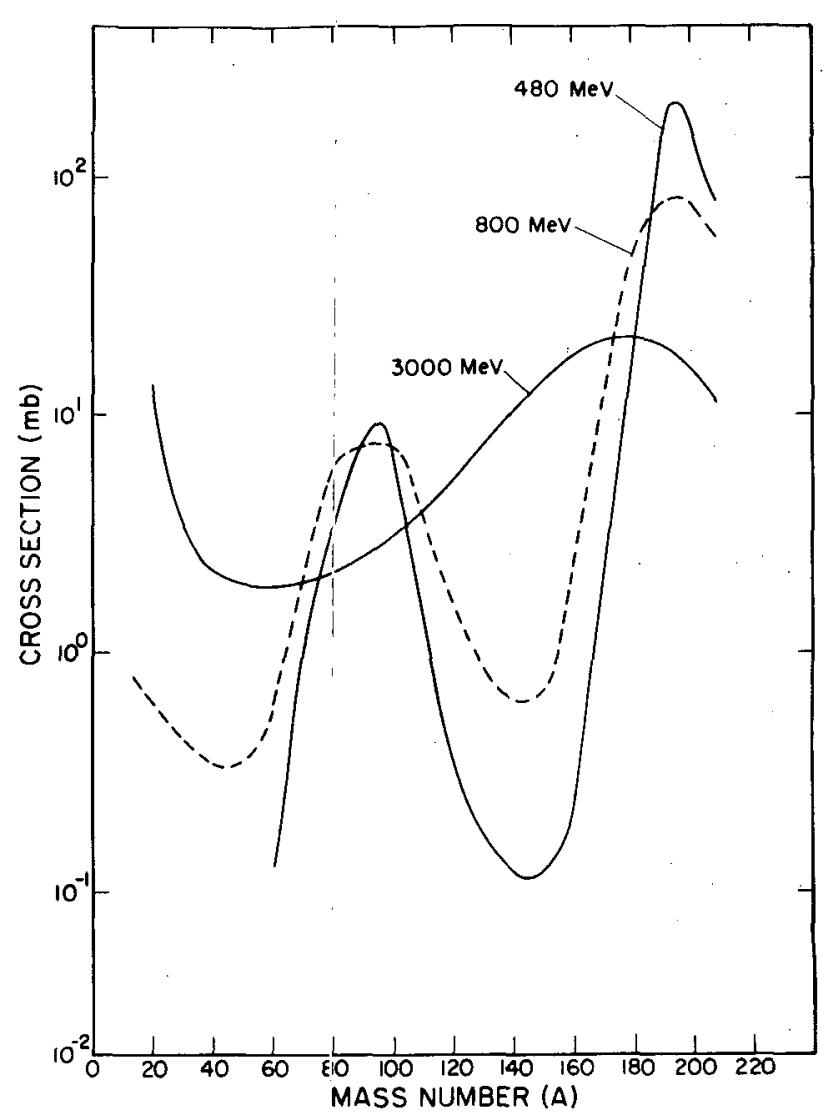

Fig. 4. Mass-yield curves for the proton bombardment of bismuth (2).

products near the target mass; 2) another peak (for higher-nass targets) corresponding to fission products at intermediate masses; and 3) a rise at the low-mass end corresponding to fragmentation products. It is the region of the first peak - viz., spallation products - that is of interest to us here.

Since the available cross section data at or near $700 \mathrm{MeV}$ are insufficient for most of the target-product combinations that are of interest, we have relied on a calculational approach in order to make rapid, approximate yield predictions. We refer to the empirical systematics developed by G. Rudstam (3) in 1966. Rudstam's empirical formula represents approximately 1200 data points available at the time and was shown to be accurate to within a factor of two or three for almost all of the data. The accuracy tends to degenerate for low-mass targets because of the contribution of secondary particles, and for high-mass targets because of the importance of fission. In addition, this formula is meant to be used only for products that are three or more mass units removed from the target. Within these restrictions, 
it is felt that Rudstam's systematics are useful for estimating spallation cross sections for most target-product combinations. The mathematical details of the development of this formula can be found in Rudstam's paper and, so, will not be treated here. Suffice it here to say that this formula was programmed so that by providing the incident energy, target mass, and the product mass and charge, the cross section is then calculated. An additional refinement to Rudstam's formula, later made by Chackett and Chackett (4), which takes into account the charge of the target, was also included in our program. This program has been used to calculate the cross sections of products lying within 40 mass units of each of about three dozen targets.

The calculations described above refer to thin-target cross sections. Since the production targets to be used at LAMPF will be relatively thick ( $\sim 1$ inch), some account must be made of the effective cross section as a function of depth. An example of thicktarget data, taken by Shedlovsky and Rayudu (5), is illustrated in Fig. 5. What is shown here is the effective cross section for producing various nuclides from a thick iron target with incident protons of $1 \mathrm{GeV}$ energy. The solid curves represent an attempt to fit the data with an empirical formula (6). An important feature to note is that for products with relatively small $\triangle \mathrm{A}$ ( $\equiv \mathrm{A}_{\text {target }}{ }^{-\mathrm{A}}$ product
there is a buildup in the effective cross section, peaking at about 60 $\mathrm{gm} / \mathrm{cm}^{2}$ for the $1 \mathrm{GeV}$ case. This effect is even more pronounced at higher energies (5) and is believed to be caused by secondary particles producing such nuclides in addition to the primary protons. Since almost all of the products of interest to us will have relatively small $\Delta \mathrm{A}$, an obviously conservative way of handling the thick-target problem is to assume a constant cross section as a function of depth. In addition, let us assume that this constant is given by the thin-target cross section calculated using the Rudstam systematics. This too is a conservative estimate, since the secondary particles produced in a thick target surely contribute something to the effective cross section. Thus, the most naive approximation for our thick-target cross sections is clearly justified.

The activity produced ( $C i$ ) of a specific nuclide is given by

$Y=\frac{1}{c}\left(\frac{N}{A_{T}} \rho_{T} x\right) I\left(\sigma_{0} \cdot 10^{-2} 7\right)\left[1-\exp \left(-0.693 t_{i} / \tau_{\frac{1}{2}}\right)\right]$

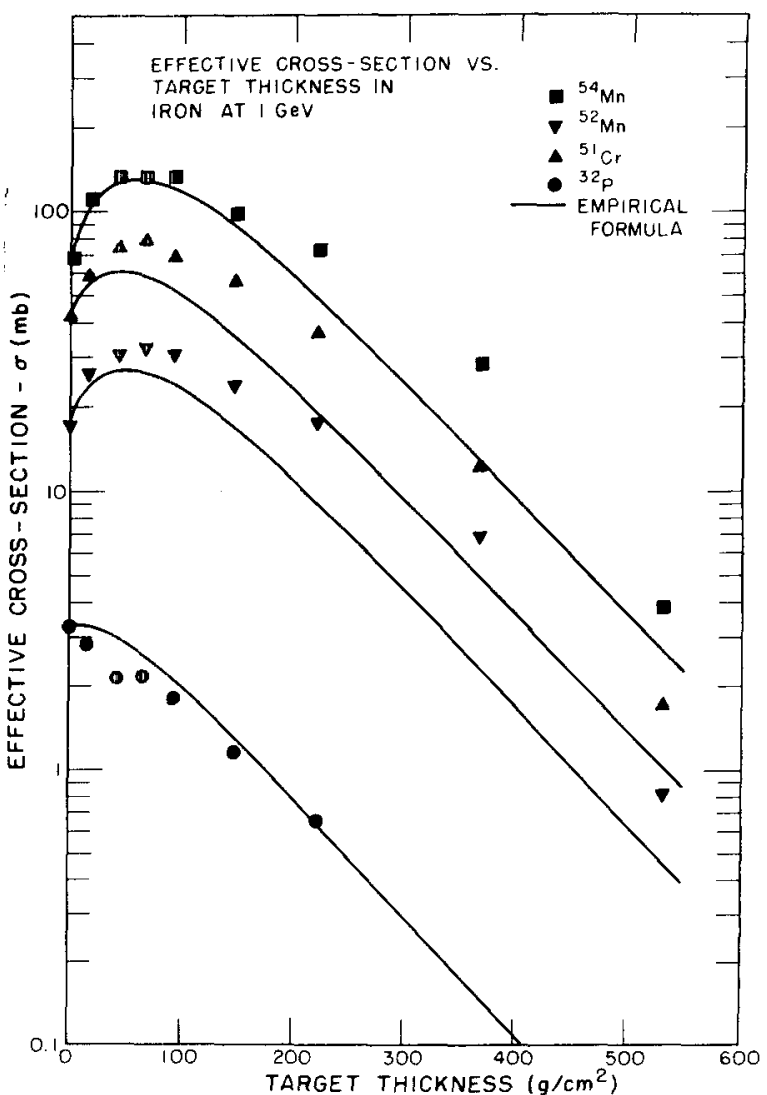

Fig. 5. Thick-target yields in iron at $1 \mathrm{GeV}$ (5).

where the constant $c$ coverts disintegrations/ sec to $\mathrm{Ci}\left(\mathrm{c}=3.7 \times 10^{10}\right), \mathrm{N}$ is Avagodro's number, $A_{T}$ is the target mass number, $P_{T}$ is the target density $\left(\mathrm{gm} / \mathrm{cm}^{3}\right), x$ is the target thickness $(\mathrm{cm})$, I is the beam intensity (protons/sec), $\sigma$ is the thin-target cross section (mb), $t_{i}^{o}$ is the irradiation time, and $\tau_{\frac{1}{2}}$ is the product half-life.

\section{PHOTO-NEUTRON SOURCES OF INTEREST FROM LAMPF}

In selecting a list of suitable gamma emitters, two criteria are used: 1) a halflife of at least a few days; and 2) a gamma energy greater than the threshold for neutron production in ${ }^{9} \mathrm{Be}-\mathrm{viz}, \mathrm{E}>1.666 \mathrm{MeV}$ (1). Another commonly used materlal is $\mathrm{D}_{2} \mathrm{O}$; however, since the binding energy of the deuteron is $2.226 \mathrm{MeV}$, considerably more energetic photons are required to produce neutrons. Such a list fulfilling these criteria can be culled from a standard table of isotopes (7) and is presented in Table $I$. The targets to be irradiated, the spallation cross sections and the yields for each specific product are also listed in Table $I$. In addition, we list the energies of the relevant gamma-rays emitted from each product, together with the 
TABLE I

GAMMA EMITTERS OF INTEREST FROM LAMPF

(700 MeV, 1/2 mA, 1-inch targets)

Product $\left(\tau_{1 / 2}\right)$

$\sigma_{0}(\mathrm{mb})$

$\mathrm{E}_{\gamma}(\mathrm{MeV})$

Yield (Ci) ${ }^{a}$

${ }^{88} \mathrm{Y}(107 \mathrm{~d})$

Target

45

$1.836-99.5$

210

${ }^{206} \mathrm{Bi}(6.24 \mathrm{~d})$

$\mathrm{Pb}$

$77^{b}$

$1.720-36$

$450^{b, c}$

${ }^{205} \mathrm{Bi} \quad(15.3 d)$

$\mathrm{Pb}$

$37^{\mathrm{b}}$

$1.766-27$

$1.864-6$

1.906 -

${ }^{56}$ Co $(77.3 d)$

$\mathrm{Cu}$

8.8

$1.76-15$

$2.02-11$.

$2.60-17$

$3.26-13$

${ }^{124}$ I (4.2d)

La

32

$1.69-14$

$2.09-2$

$2.26-1.5$

92

79

\section{$130^{\mathrm{b}}$}

$$
2.26-1.5
$$

${ }^{156} \mathrm{Eu}(15.2 \mathrm{~d})$

$\mathrm{Tb}$

39

$$
\begin{aligned}
& 2.187-3.5 \\
& 2.181-2.2 \\
& 2.098-3.9 \\
& 2.026-4.9 \\
& 1.966-4.0 \\
& 1.937-3.1 \\
& 1.877-1.6
\end{aligned}
$$

130

$156_{\mathrm{Tb}}(5.4 \mathrm{~d})$

Ho

100

$1.85-3$

340

${ }^{119} \mathrm{Te}(4.7 \mathrm{~d})$

$\mathrm{Sb}$

100

$2.09-4$

$360^{d}$

${ }^{48} \mathrm{~V}(16 \mathrm{~d})$

$\mathrm{Cr}$

32

$2.24-3$

290

${ }^{106} \mathrm{Ag}(8.3 \mathrm{~d})$

$\mathrm{Sb}$

17

$1.83-3$

$60^{\mathrm{d}}$

Irradiation time $\left(t_{1}\right)=$ Half-life $\left(\tau_{1 / 2}\right)$.

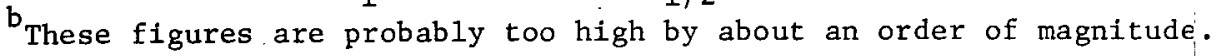

$c_{t_{i}}=15.3 \mathrm{~d}$.

$\mathrm{d}_{\text {These }}$ yields should be multiplied by the fraction of the excited state produced relative to the ground state. 
percentage of disintegrations that result in each gamma-ray of interest. The cross sections and yields contained in Table $I$ are based on the assumptions of a $700 \mathrm{MeV}, 1 / 2$ $\mathrm{mA}$ proton beam, a target thickness of one inch and an irradiation time equal to the product half-life. This choice of irradiation time simplifies the presentation of the results and gives a yield which is one-half the saturation yield.

It should be noted that the cross sections used for the production of $20{ }^{5} \mathrm{BI}$ and ${ }^{206} \mathrm{Bi}$ from a lead target are surely too high. This is to be expected since the Rudstam formula tends to overestimate the cross section when $\Delta \mathrm{A}$ is less than three. In addition, no account is made of the increased importance of fission for heavier targets, resulting in decreased spallation cross sections. Indeed, the results of an intranuclear cascade calculation (8) indicate that the cross sections in question are approximately an order of magnitude lower than indicated by the Rudstam systematics. Nevertheless, we shall use the yields as stated in Table $I$, but with a note of caution in the $\mathrm{Bi}$ cases.

The material most commonly used for the production of neutrons by the emitted $\gamma$-rays is beryllium. Let us assume, for the purpose of calculation, a simplified model of the photo-neutron source in which the radioactive component is enclosed in a spherical volume of beryllium of thickness $x$. In such a model, the intensity of the source per unit activity of the radioactive component (neutrons/sec/Ci) is given simply by

$$
n=\operatorname{cf}\left(\frac{N}{A_{T}} \rho_{T} x\right)\left(\sigma \cdot 10^{-2} 7\right) \text {, }
$$

where $\mathrm{c}=3.7 \times 10^{10}$ as before, $\mathrm{N}$ is Avagodro's number, $A_{T}$ is the mass number, $P_{T}$ is the target density $\left(\mathrm{gm} / \mathrm{cm}^{3}\right), x$ is the target thickness $(1 \mathrm{~cm}), \sigma$ is the $(\gamma, n)$ reaction cross section at the particular energy of the $\gamma$-ray (mb), and $f$ is the fraction of the disintegrations resulting in the emission of the particular $\gamma$-ray. In all cases but one, the assumed target material in which the production reaction takes place is beryllium $\left(A_{T}=9\right)$; and for the case when the radioactive component is ${ }^{56} \mathrm{Co}, \mathrm{D}_{2} \mathrm{O}\left(\mathrm{A}_{\mathrm{T}}=2\right)$ is assumed. The $(\gamma, n)$ reaction cross sections for ${ }^{9} \mathrm{Be}$ and deuterium are given in Fig. 6 as a function of the $\gamma$-ray energy.

The energy, $\mathrm{E}$ ( $\mathrm{MeV}$ ), of a neutron produced by a $(\gamma, n)$ reaction in a nucleus of mass $A_{T}$ is given by $(\underline{1})$

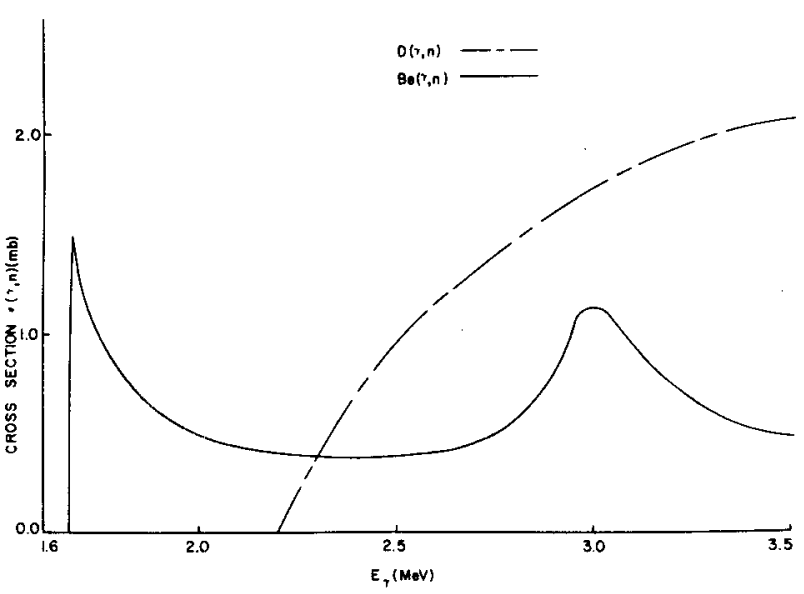

Fig. 6. $(\gamma, n)$ cross sections for beryllium and deuterium vs. $\gamma$-ray energy.

$$
\begin{aligned}
E_{\mathfrak{n}} & =\frac{A_{T}-1}{A_{T}}\left[E_{\gamma}-E_{0}-\frac{E_{\gamma}^{2}}{1862\left(A_{T}-1\right)}\right] \\
& +\left[\frac{2\left(A_{T}-1\right)\left(E_{\gamma}-E_{0}\right)}{931 A_{T}^{3}}\right] E_{\gamma} \cos \theta,
\end{aligned}
$$

where $E_{\gamma}$ is the $\gamma$-ray energy $(\mathrm{MeV}), \mathrm{E}_{0}$ is the threshold energy for the reaction ( $\mathrm{MeV}$ ), and $\theta$ is the production angle. The second term, which corresponds to the kinematic spread in the energy, is very small compared to the first term and will, therefore, be neglected.

In Table II we list the neutron intensity per unit activity, $n$, for each of the sources together with the corresponding neutron energies. Also, we have reproduced the yields listed in Table $I$, and, in the last column, have indicated the total neutron intensities resulting from such yields. The neutron energy and intensity for the $12{ }^{4} \mathrm{Sb}-\mathrm{Be}$ source is also listed for comparison. A most striking feature illustrated in Table II is the great varlety of neutron energies that will be available with such sources. It should also be noted that $\gamma$-rays of a given energy produce a very sharply spiked distribution of neutrons -- i.e., essentially monoenergetic. Thus, it would seem that this expanded arsenal of isotopic neutron sources would prove very useful for energy-dependent applications.

In addition to the neutron energy spectrum, other factors which are important in considering such isotopic neutron sources are: 1) half-1ife; 2) total neutron intensity: and 3) specific activity. Regarding 
TABLE II

BERYLLIUM- $(\gamma, n)$ SOURCES USING LAMPF-PRODUCED IS(OTOPES

\begin{tabular}{|c|c|c|c|c|c|c|}
\hline$\gamma$-Source $\left(\tau_{1 / 2}\right)$ & Yield (Ci) & a & $\mathrm{E}_{\mathrm{n}}(\mathrm{keV})$ & $\mathrm{n} / \mathrm{sec} / \mathrm{C} 1 \cdot 10^{-6}$ & \multicolumn{2}{|c|}{$\mathrm{n} / \mathrm{sec} \cdot 10^{-6}$} \\
\hline${ }^{124} \mathrm{Sb}(60 \mathrm{~d})$ & & & 24 & 2.8 & & \\
\hline${ }^{88} \mathrm{Y} \quad(107 d)$ & 210 & & 151 & 2.9 & 610 & \\
\hline${ }^{206} \mathrm{Bi}(6.24 \mathrm{~d})$ & $450^{b}$ & & 48 & 1.8 & $810^{b}$ & 1000 \\
\hline${ }^{205}{ }_{\mathrm{Bi}}(15.3 \mathrm{~d})$ & $130^{b}$ & & 89 (peak) & 1.5 & $190^{\mathrm{b}}$ & \\
\hline${ }^{56}$ Co $(77.3 d)$ & 79 & & $185,514\left(\mathrm{D}_{2} \mathrm{O}\right)$ & 1.2 & 91 & \\
\hline${ }^{124} \mathrm{I}(4.2 \mathrm{~d})$ & 92 & & 21 (peak) & 0.73 & 68 & \\
\hline${ }^{156} \mathrm{Eu}(15.2 \mathrm{~d})$ & 130 & (7) & $188-463$ & 0.38 & 50 & \\
\hline $156_{\mathrm{Tb}}(5.4 \mathrm{~d})$ & 340 & & 164 & 0.080 & 28 & \\
\hline $119 \mathrm{~m}_{\mathrm{Te}}(4.7 \mathrm{~d})$ & $360^{c}$ & & 377 & 0.053 & 19 & \\
\hline${ }^{48} \mathrm{v}(16 \mathrm{~d})$ & 290 & & 511 & 0.040 & 12 & \\
\hline $106 \mathrm{~m}_{\mathrm{Ag}}(8.3 \mathrm{~d})$ & $60^{c}$ & & 146 & 0.087 & 5 & c \\
\hline
\end{tabular}

$a_{t_{1}}=\tau_{1 / 2}$

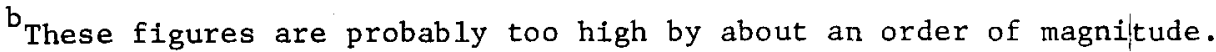

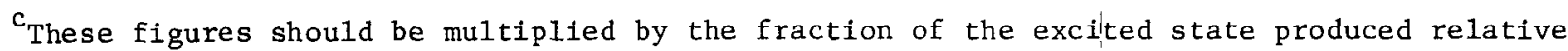
to the ground state.

half-life alone, only two of the sources listed are comparable or better than the current1y used $60 \mathrm{~d}^{124} \mathrm{Sb}-\mathrm{viz},{ }^{107 \mathrm{~d}^{88} \mathrm{Y} \text { and }}$ $77 \mathrm{~d}{ }^{56} \mathrm{Co}$. The remaining sources are, perhaps, too short-lived to be useful for widescale commercial applications; however, if these sources can be produced economically, they may prove feasible for more specialized. applications requiring the variety of neutron energies that they offer.

The specific activity of the radionuclide being used in the neutron source is an important quantity as it bears directly on the size of the source. We estimate that the yttrium source consists of about $70 \%{ }^{88} \mathrm{Y}$ nuclei, while the cobalt source consists of only about $6 \%{ }^{56}$ co nuclei. Another possibly interesting case is the bismuth source. Ca1culations indicate that this source consists of about $20 \%{ }^{20}{ }^{5} \mathrm{Bi}$ and ${ }^{206} \mathrm{Bi}$. However, since the cross sections for ${ }^{2}{ }^{07} \mathrm{Bi},{ }^{2}{ }^{8} \mathrm{Bi}$, and ${ }^{209} \mathrm{Bi}$ are probably more greatly exaggerated than those for ${ }^{2}{ }^{5} \mathrm{Bi}$ and ${ }^{206} \mathrm{Bi}$, the specific activity of this source is likely to be much higher. All of the sources listed can probably be made much smaller than the $12{ }^{4} \mathrm{Sb}$ source, as the latter is produced by the $(n, \gamma)$ reacticn in ${ }^{123} \mathrm{Sb}$ (a sealed unit is irradiated in a nuclear reactor).

Perhaps the most obviously important characteristic of any neutron source for most applications is the total neutron intensity. 
On this basis alone, the bismuth source would seem to be the best choice; however, it should be remembered that, as noted earlier, the total neutron intensity associated with this source is probably about an order of magnitude too high. Nevertheless, the more realistic figure is still quite significant. The yttrium source has the best total neutron intensity of all the isotopic sources listed, and, on a "per Curie" basis, is equal to the ${ }^{124} \mathrm{Sb}$ source. The cobalt source is comparable in strength to the bismuth source, assuming about $10^{8} \mathrm{n} / \mathrm{sec}$ for the latter case.

Thus, considering the three factors stated above, the ${ }^{8}{ }^{8} \mathrm{Y}-\mathrm{Be}$ source is far superior to all of the others listed. In comparison with the currently used ${ }^{124} \mathrm{Sb}-\mathrm{Be}$ source, the yttrium source has almost a factor of two advantage in half-life, can probably be made much smaller, and is equivalent in strength, on a "per Curie" basis. In addition, the $150 \mathrm{keV}$ neutrons provided by the yttrium source are more penetrating than the $24 \mathrm{keV}$ neutrons provided by the ${ }^{12}{ }^{4} \mathrm{Sb}$ source. Although this characteristic may be a disadvantage for some applications - e.g., thermal neutron radiography, it may provide a distinct advantage for others, such as in assay systems (9). For applications which require very high intensities $\left(\sim 10^{10}\right.$ $\mathrm{n} / \mathrm{sec}),{ }^{252} \mathrm{Cf}$ will undoubtedly remain the best choice among isotopic neutron sources; however, for some applications requiring an intermediate intensity $\left(\sim 10^{7}-10^{8} \mathrm{n} / \mathrm{sec}\right)$, the ${ }^{88} \mathrm{Y}-\mathrm{Be}$ source may indeed prove superior to any other isotopic neutron source. Recall for example, that ${ }^{252} \mathrm{Cf}$ neutrons are characterized by a fission spectrum. A cost analysis of the production of an ${ }^{88} \mathrm{Y}-\mathrm{Be}$ source, which includes target material, chemical processing, and fabrication costs, is described elsewhere in these proceedings (9). In this analysis, the ${ }^{8}{ }^{8} \mathrm{Y}-\mathrm{Be}$ source is found to be economically competitive with the ${ }^{124} \mathrm{Sb}-\mathrm{Be}$ source. We should point out that this result is due in no small measure to the fact that, at LAMPF, there will be no prime beam time cost for producing radioisotopes. In addition, further economies will be realized by the extraction of several desired radionuclides from a single target.

The yield of ${ }^{88} \mathrm{Y}$ presented in Table I is based on an irradiation time equal to the half-life of ${ }^{88} \mathrm{Y}-\mathrm{viz}$., 107 days. This would not seem to be the optimum production schedule for making this nuclide; rather, an irradiation time of about one month would be preferred. In this case, about $75 \mathrm{Ci}$ of
${ }^{8}{ }^{8} \mathrm{Y}$ can be made each month, utilizing just one of the six target stations. Accounting for the beam attenuation in passing through each of the targets, the total facility capability of the production of ${ }^{88} \mathrm{Y}$ is about $240 \mathrm{Ci} /$ month; however, this upper limit is unrealistic, as there will certainly be demands for other radionuclides.

\section{References}

1. E. Amaldi, Handbuch der Physik 38/2, p. 1 ; edited by S. Flugge, SpringerVerlag, Berlin (1959).

2. J. M. Miller and J. Hudis, Ann. Rev. Nuc1. Sci. 9, 159 (1959).

3. G. Rudstam, Z. Naturforschg. 21a, 1027 (1966).

4. K. F. Chackett and G. A. Chackett, Nuc1. Phys. A100, 633 (1967).

5. J. P. Shedlovsky and G. V. S. Rayudu, J. Geophys. Research 69, 2231 (1964).

6. M. E. Schillaci, "Radioisotope Production at LAMPF", LASL MP-Division report MPDOT/MES-1 (February 1970).

7. C. M. Lederer, J. M. Hollander, and I. Perlman, Table of Isotopes - Sixth Edition, Wiley (1968).

8. H. Bertini, "Preliminary Data from Intranuclear-Cascade Calculations of $0.75-, 1-$, and $2-\mathrm{GeV}$ Protons on Oxygen, Aluminum, and Lead, and $1-\mathrm{GeV}$ Neutrons on the Same Elements", ORNL report ORNLTM-1996 (December 1967).

9. L. A. Kull, M. E. Schillaci, and J. R. Beyster, ${ }^{88} \mathrm{Y}$ - A New Replacement for ${ }^{124} \mathrm{Sb}$ in a Nuclear Materials Assay System", these proceedings. 


\title{
FLUX ENHANCEMENT WITH ${ }^{252}$ Cf SOURCE IN A SUBCRITICAL ASSEIMBLY
}

\author{
R. L. Currie, F. J. McCrosson, and P. B. Parks \\ Savannah River Laboratory \\ E. I. du Pont de Nemours and Company \\ Aiken, South Carolina
}

The spontaneous fission neutrons emitted by ${ }^{252} \mathrm{Cf}$ can be thermialized by surrounding the source with a neutron moderating material. If a subcritical multiplying system is substituted for the simple moderator, the thermal flux can be enhanced.

To determine the extent of this enhancement, discrete ordinates codes were used to calculate neutron fluxes in ${ }^{233} \mathrm{U}_{-} \mathrm{H}_{2} \mathrm{O}$ and ${ }^{235} \mathrm{U}-\mathrm{H}_{2} \mathrm{O}$ solutions containing a central ${ }^{252} \mathrm{Cf}$ neutron source. Compared to a nonmultiplying medium of $\mathrm{H}_{2} \mathrm{O}$, spherical ${ }^{233} \mathrm{U}-\mathrm{H}_{2} \mathrm{O}$ solutions with $\mathrm{k}_{\mathrm{eff}}=0.98$ can enhance the thermal flux at the source by a maximum factor of 6.4 ; similarly ${ }^{235} \mathrm{U}-\mathrm{H}_{2} \mathrm{O}$ solutions can increase the flux by a maximum factor of 4.8 .

The dependence of the enhanced thermal flux on nuclear parameters was estimated with two-group diffusion theory to be $\mathrm{M} / \nu \sigma_{\mathrm{f}} \mathrm{m}_{\mathrm{f}}$ where $\nu$ is the average number of neutrons per fission, $\sigma_{\mathrm{f}}$ is the microscopic fission cross section for the thermal group, $m_{f}$ is the mass of fissile material, and $M$ is the integral source multiplication.

The $S_{n}$ calculations were extended to cylindrical multiplying systems to examine the effects of reentrant ports and thus to be more directly applicable to activation analysis and neutron radiography.

\section{INTRODUCTION}

Several investigators have proposed that the useful thermal flux from a moderating system with ${ }^{252} \mathrm{Cf}$ as the neutron source could be enhanced using subcritical multiplication $(1,2)$. The enhancing system would provide a higher flux level over a larger useful volume thereby reducing the requirements for the $252 \mathrm{Cf}$.

Significant flux enhancement is realized only if the subcritical system is quite close to criticality. In this paper, systems with multiplication constants (keff) of 0.98 and 0.99 have been considered. It is the opinion of the authors that systems of this multiplication, or higher, require a reasonably complete calculational program to verify that anticipated benefits balance the potential criticality risks before development of experimental facilities is undertaken.

This paper reports initial calculations of flux enhancing possibilities. Multigroup discrete ordinates calculations were performed in one dimension to determine the effect of varying certain parameters. A simple twogroup diffusion theory solution to the problem was obtained which gives physical insight into the results of the more sophisticated

The information contained in this article was developed during the course of work under Contract AT (07-2)-I with the U. S. Atomic Energy Commission. calculations. Extension of the calculations to two dimensions examined more realistic systems that might be used in activation analysis or neutron radiography. However, the calculational program is not complete, and additional study is needed before prototype development is warranted.

\section{CALCULATION OF FLUX ENHANCEMENT WITH TRANSPORT METHODS}

The multigrcup discrete ordinates code ANISN (3) was used with the 16-group HansenRoach cross section sets (4) to calculate fluxes in the $\mathrm{S}_{4} \mathrm{P}_{1}$ approximation. The subcritical systems investigated were fully reflected multiplying spheres of homogeneous $235 \mathrm{U}-\mathrm{H}_{2} \mathrm{O}$ and $233 \mathrm{U}-\mathrm{H}_{2} \mathrm{O}$ solutions with ${ }^{252} \mathrm{Cf}$ sources in a $1 \mathrm{~cm}^{3}$ central Al sphere. The $233 \mathrm{U}-\mathrm{H}_{2} \mathrm{O}$ systems had calculated eigenvalues of $k_{\text {eff }}=0.98$ and 0.99 , and concentrations ranged from 20 to $200 \mathrm{~g} / \mathrm{l}$. A more limited range of fuel concentrations was studied for the $235 \mathrm{U}-\mathrm{H}_{2} \mathrm{O}$ systems, and only the cases for $k_{\text {eff }}=0.98$ were considered.

The calculational program consisted of using the zone search option of ANISN to compute the radius of the multiplying core for input $k_{\text {eff }}$ of 0.98 or 0.99 and then using these radii to set core size for the fixed source calculations. The results of the calculations are shown in Table I. 
The ratio of the thermal flux at the center of the subcritical sphere to that at the center of an essentially infinite $\mathrm{H}_{2} \mathrm{O}$ sphere is a measure of the effective enhancement of the system. The maximum enhancement with $\mathrm{k}_{\text {eff }}=0.98$ is 6.4 for $233 \mathrm{U}-\mathrm{H}_{2} \mathrm{O}$ and 4.8 for $235 \mathrm{U}-\mathrm{H}_{2} \mathrm{O}$.

Figure 1 shows the radial shape of the thermal flux and Figure 2 the radial dependence of the ratio of the flux above $0.4 \mathrm{eV}$ to that below for the $40 \mathrm{~g} / 1, \mathrm{k}_{\mathrm{eff}}=0.98233 \mathrm{U}-$ $\mathrm{H}_{2} \mathrm{O}$ system and for the non-multiplying system. The thermal flux in the multiplying system is greater and remains relatively more flat than that in the non-multiplying system indicating that the anticipated increase in useful flux and flux volume is achieved. Furthermore, the fast-to-slow ratio is constant over a fairly large distance making the corrections for fast neutron reactions in activation analysis less dependent on sample geometry.

No consideration has been made in these calculations for the effects of non-fissionable impurities in $233 \mathrm{U}$ or $235 \mathrm{U}$. Moreover, practical systems may be cylindrical in shape and have re-entrant or beam ports. However, these one dimensional calculations do give some understanding of the degree of flux enhancement available through the use of practical subcritical systems.

\section{TWO-GROUP DIFFUSION THEORY}

Some qualitative features of the ANISN results can be established using two-group diffusion theory. In spherical geometry, the appropriate equations are:

$$
\begin{aligned}
& -\mathrm{D}_{1} \nabla^{2} \phi_{1}(\mathrm{r})+\left(\Sigma_{\mathrm{R}}+\Sigma_{\mathrm{a}_{1}}\right) \phi_{1}(\mathrm{r})-\nu \Sigma_{\mathrm{f}} \phi_{2}(\mathrm{r})= \\
& \mathrm{S}(\mathrm{r})
\end{aligned}
$$

and

$$
-\mathrm{D}_{2} \nabla^{2} \phi_{2}(\mathrm{r})+\Sigma_{\mathrm{a}_{2}} \phi_{2}(\mathrm{r})-\Sigma_{\mathrm{R}} \phi_{1}(\mathrm{r})=0
$$

Here $D_{i}$ and $\Sigma_{a i}$ denote the diffusion coefficient and macroscopic absorption cross section associated with group $i(i=1,2)$, and $\Sigma_{R}$ is the removal cross section from group 1 . Fissions induced by fast neutrons have been neglected. It will be assumed that neutrons are emitted by a simple point source of radius $\mathrm{R}_{\mathrm{o}}$ located at the origin, viz.,

$$
S(r)=\left\{\begin{array}{lll}
Q, & r \leqslant R_{O} \\
0, & R_{O}<r \leqslant R
\end{array}\right.
$$

and that

$$
\phi_{i}(R)=0 \quad(i=1,2)
$$

when $R$ denotes the extrapolated radius of the sphere.

It is convenient to analyze the above problem in terms of the eigenfunctions of the corresponding critical problem. In this approach the group fluxes and source distribution are represented by the following expansions :

$$
\phi_{i}(r)=\frac{1}{r} \sum_{n=1}^{\infty} a_{i n} \sin B_{n} r
$$

and

$$
S(r)=\frac{1}{r} \sum_{n=1}^{\infty} q_{n} \sin B_{n} r
$$

where

$$
B_{n}=\frac{n \pi}{R}
$$

The coefficients $a_{i n}$ can be related to $q_{n}$ by substituting $\mathrm{Eq} .3$ into $\mathrm{Eq}$. 1. In this manner it can be shown that

$$
\phi_{1}(r)=\frac{1}{r} \sum_{n=1}^{\infty} q_{n} \frac{\sin _{n} r}{\left(D_{1} B_{n}^{2}+\Sigma_{a_{1}}+\Sigma_{R}\right)\left(1-k_{n}\right)}
$$

and

$$
\begin{aligned}
\phi_{2}(r)= & \frac{1}{r} \sum_{n=1}^{\infty} q_{n} \Sigma_{R} \sin B_{n} r /\left[\left(D_{2} B_{n}^{2}\right.\right. \\
& \left.\left.+\Sigma_{a_{2}}\right)\left(D_{1} B_{n}^{2}+\Sigma_{a_{1}}+\Sigma_{R}\right)\left(1-k_{n}\right)\right]
\end{aligned}
$$

where 


$$
k_{n}=\frac{\Sigma_{R}}{D_{1} B_{n}^{2}+\Sigma_{a_{1}}+\Sigma_{R}} \cdot \frac{v \Sigma_{F}}{D_{2} B_{n}^{2}+\Sigma_{a_{2}}}
$$

If fast fission is neglected, $k_{1}$ is the usual two-group multiplication constant. The orthogonality of the sine terms can be used to show that

$$
q_{n}=\frac{2 Q}{R B_{n}^{2}}\left(\sin B_{n} R_{o}-B_{n} R_{o} \cos B_{n} R_{o}\right) .
$$

To simplify the analysis it will be assumed that the system is nearly critical and that only the fundamental mode in Eq. 5 is significant. Using the explicit expression for $\mathrm{ql}, \mathrm{Eq}$. $5 \mathrm{~b}$ then takes the form

$$
\phi_{2}(r)=\frac{2 Q R k}{\pi^{2}(1-k)} \frac{\sin B R_{0}-B R_{0} \cos B R_{0}}{\nu \Sigma_{f}} \frac{\sin B r}{r},
$$

when the subscript 1 has been dropped for convenience. Since the thermal flux peaks at the center of the sphere, this region is of special interest:

$$
\phi_{2}(0)=\frac{2 Q k}{\pi(1-k)} \frac{\sin B R_{0}-B R_{0} \cos B R}{\nu \Sigma_{f}}
$$

This expression for the peak flux can be further simplified by noting that $B R_{O}$ is generally small enough to make the identification

$$
\sin B R_{O}-B_{O} \cos B R_{O}=\frac{1}{3}\left(B R_{O}\right)^{3}=\frac{\pi^{3} V_{O}}{3 V},
$$

where $V$ and $V_{o}$ are the volumes of the system and source respectively. Eq. 9 can then be rewritten to obtain the central result

$$
\phi_{2}(0)=\frac{2 \pi^{2} Q V_{0}}{3 v \sigma_{f} m_{f}} \frac{k}{1-k}
$$

That is, $\sigma_{f}$ and $m_{f}$ denote the microscopic fission cross section and mass of the fissile material in the moderating medium. Eq. 11 states that the flux increases as $k$ approaches unity and that, for a given $k$, the flux can be amplified best by minimizing the product $\sigma_{f m f}$ of the three common fuels, $233 \mathrm{U}, 235 \mathrm{U}$ and $239 \mathrm{Pu}, 233 \mathrm{U}-\mathrm{H}_{2} \mathrm{O}$ systems can yield the smallest value of 'ofmf and hence, according to Eq. 11, the highest flux enhancement for a given $\mathrm{k}$; $239 \mathrm{Pu}$ systems should yield the lowest enhancement for a given $k$.

To obtain quantitative agreement of the twogroup treatment wilth ANISN results, higher order harmonics would be necessary. However, Eq. 11 does provide useful physical insight into enhancements from multiplying systems.

\section{EXTENSION OF/ CALCULATIONS TO THE} RE-ENTRANT OR BEAM PORT PROBLEM

The one dimensional code ANISN is unable to treat the problem of re-entrant or beam ports in moderating or multiplying systems. The $\mathrm{S}_{\mathrm{n}}$ code DOT (5) can be used to solve two dimensional problems, and it was used to determine the flux distributions within ports in cylindrical systems. These results, while preliminary, are of more direct application to neutron radiography and activation analysis.

DOT incorporates a fixed source option; however, very long computer running times were encountered when using this option at the keff values of this study. Therefore, an alternative approach to fixed source problems has been employed. The technique involves creating a "fake" additional material for the source zone whose only non-zero cross section entry is $U \Sigma_{f}$ in the $P_{0}$ thermal group. A search is made for, the concentration of this fake material that will lead to $\mathrm{keff}=1.0$. The ratio of the total fission source in the system to the fission source in this material should be the integral source multiplication which would be obtained from a normal fixed source calculation on the subcritical system.

Since regular fixed source solutions could not be obtained wilh DOT for the high $\mathrm{k}_{\mathrm{eff}}$ systems, a direct comparison of the fake and regular solutions was not possible. Instead the DOT fake solutions for a keff $=0.98 \mathrm{re}-$ flected cylinder (lequal height and dianeter) of $40 \mathrm{~g} / 1,233 \mathrm{U}-\mathrm{H}_{2} \mathrm{O}$, was compared to ANISN solutions for a reflected sphere of the same mixture at $k_{\text {eff }}=0.98$ where both the fake source routine and the regular fixed source option have been employed. These results are given in Table II and show that the fake 
source technique is adequate; the integral source multiplications agree within $1 \%$.

DOT calculations have been performed on $40 \mathrm{~g} / 1,233 \mathrm{U}-\mathrm{H}_{2} \mathrm{O}$ multiplying systems at $\mathrm{k}_{\mathrm{eff}}=$ 0.98 in a 4 group, $\mathrm{S}_{6}, \mathrm{P}_{1}$ approximation. Two basic geometries were employed and are shown in Figure 3. For the first, an air filled port was $0.0,2.0$, and $4.0 \mathrm{~cm}$ from the core boundary in the three problems run, the intervening space being filled with $\mathrm{H}_{2} \mathrm{O}$. The core radii, as found from zone thickness searches in the absence of the fixed source, decreased slightly from $15.468 \mathrm{~cm}$ as the port-core boundary distance increased.

The thermal group axial flux distributions are shown in Figure 4 . The flux at the inner face of the ports is not altered by providing the intervening $\mathrm{H}_{2} \mathrm{O}$ moderator; however, the flux spectrum is thermalized considerably.

Other calculations were performed with the source at other positions along the axis toward the core boundary. No significant change in either the thermal flux level or the flux spectrum was noted for source-core boundary distances $\geqslant 5 \mathrm{~cm}$. This is to be expected at such high source multiplication levels in these small homogeneous cores.

In the second basic core geometry (see Figure 3) the re-entry port was extended into the core in the hope of realizing higher thermal neutron flux levels. Only one multiplying system calculation was made for this geometry, the $5 \mathrm{~cm}$ radius port extending 4.5 $\mathrm{cm}$ into the core with an additional $3 \mathrm{~cm}$ of $\mathrm{H}_{2} \mathrm{O}$ moderator between the core boundary and the inner port face. The axial thermal flux distribution and the flux ratio are shown in Figure 5. Note that the increased fast flux feed from the core regions at the side of the port has greatly increased the flux ratio.

Calculations were also performed for nonmultiplying, purely $\mathrm{H}_{2} \mathrm{O}$ systems with the same geometries as above. Presumably the point of most interest for activation analysis would be the region of highest accessible thermal flux, the port inner face. However, for neutron radiography purposes, the point of interest is the radiographic detector position.

The flux levels, flux ratios, and enhancements given in Table III are for two axial positions within the port, at the inner and outer faces. The thermal flux enhancement is not particularly sensitive to the position within the port. This indicates that the angular flux distributions at the inner port face must be quite similar in the multiplying and non-multiplying systems. Thus we could reasonably expect the enhancement at an external radiography station to be similar to that within the port.

Although the apparent enhancements in Table III are about 20 , the $\mathrm{H}_{2} \mathrm{O}$ and multiplying system results are not directly comparable because the flux ratios in the port are different. One way to increase the flux ratio of the $\mathrm{H}_{2} \mathrm{O}$ system to that of the multiplying system would be to move the source nearer to the re-entry port. This would raise the $\mathrm{H}_{2} \mathrm{O}$ system fluxes but decrease the enhancement. Calculations are in progress to produce directly comparable systems.

\section{CONCLUSIONS}

From an economic point of view, enhancing the flux with a subcritical system is attractive only if the costs of the system do not exceed the cost of additional $252 \mathrm{Cf}$ that would yield the equivalent flux. Moreover, an enhancing system must be demonstrably safe against all criticality hazards. Otherwise, it will have to be treated as a potential critical system, entailing licensing difficulties and extra added costs for criticality monitoring and maintenance. Indeed, if the system is labeled as a potential critical system, many, if not all, of the advantages seen for simple $252 \mathrm{Cf}$ source enhancement systems may disappear.

An important distinction must be made between integral flux multiplication and flux enhancement. It is the latter quantity which is of interest to most potential users, and we have shown it to be a complex function of

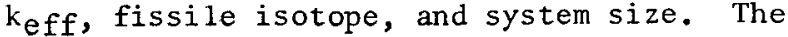
implication is that potential users must carefully examine their planned systems in light of many more factors than simple keff.

Our calculations show that if enhancement is defined as the ratio of thermal fluxe: in multiplying and non-multiplying systems at points with the same flux spectrum, then factors between 6 and 20 are the maximum to $b_{1}$ expected for systems with $\mathrm{k}_{\mathrm{eff}}=0.98$. Ultimately, however, source enhancement must be defined in the context of the specific tasks to be accomplished with the system. No universally applicable definition appears to exist and thus each case must be examined separately. 


\section{REFERENCES}

1. F. J. McCrosson. Subcritical Multiplication of $252 \mathrm{Cf}$ Sources. USAEC Report DP-1250, Savannah River Laboratory, E. I. du Pont de Nemours and Co., Aiken, S. C. (to be issued).

2. Califomium-252 Progress No. 2, 40 (Jan 1970), No. 3, 26 (April 1970), and No. 4, 25 (August 1970), reporting work done by. Idaho Nuclear Corp.

3. W. W. Engle, Jr. ANISN Users Manual: A One-Dimensional Discrete Ordinates Transport Code With Anisotropic Seattering. USAEC Report K-1693, Union Carbide Corporation, Nuclear Division, Gaseous Diffusion Plant, Oak Ridge, Tenn. (1967).

4. G. E. Hansen and W. H. Roach. Six and Sixteen Group Cross Sections for Fast and Intermediate Critical Assembiies. USAEC Report LAMS-2543, Los Alamos Scientific Laboratory, Albuquerque, New Mexico (1961).

5. F. R. Mynatt. A Users Manual for DOT. USAEC Report K-1694, Union Carbide Corporation, Nuclear Division, Computer Technology Center, Oak Ridge National Laboratory, Oak Ridge, Tenn. (1969).

TABLE I

Detailed Comparison of Flux Enhancing Sys tems

\begin{tabular}{|c|c|c|c|c|c|}
\hline $\begin{array}{c}k_{\text {eff }} \\
\text { (no source) } \\
\end{array}$ & $\begin{array}{l}\text { Conc., } \\
g / 1\end{array}$ & $\begin{array}{c}\text { Core } \\
\text { Radius, } \\
\mathrm{cm}\end{array}$ & $\begin{array}{l}\text { Mass, } \\
\mathrm{kg}\end{array}$ & $\begin{array}{c}\text { Multipli- } \\
\text { cation } \\
\text { (with source) } \\
\end{array}$ & $\begin{array}{c}\text { Enhancement } \\
(\mathrm{r}=0)\end{array}$ \\
\hline \multicolumn{6}{|c|}{${ }^{33}{ }_{\mathrm{U}-\mathrm{H}_{2} \mathrm{O}}$ systems } \\
\hline \multirow[t]{8}{*}{0.98} & 20 & 23.76 & 1.12 & 100.1 & 5.015 \\
\hline & 30 & 17.47 & 0.670 & 94.8 & 6.38 \\
\hline & 40. & 15.06 & 0.572 & 91.3 & 6.35 \\
\hline & 50 & 13.74 & 0.543 & 86.0 & 5.76 \\
\hline & 60 & 12.90 & 0.539 & 84.7 & 5.31 \\
\hline & 80 & 11.88 & 0.562 & 82.8 & 4.43 \\
\hline & 100 & 11.27 & 0.599 & 81.3 & 3.70 \\
\hline & 200 & 10.00 & 0.837 & 75.5 & 1.73 \\
\hline \multirow[t]{8}{*}{0.99} & 20 & 24.33 & 1.21 & 240.6 & 10.64 \\
\hline & 30 & 17.76 & 0.704 & 216.1 & 13.45 \\
\hline & 40 & 15.28 & 0.598 & 203.0 & 13.24 \\
\hline & 50 & 13,93 & 0.566 & 186.8 & 11,86 \\
\hline & 60 & 13.07 & 0.561 & 174.4 & 10.42 \\
\hline & 80 & 12.03 & 0.583 & 171.1 & 8.77 \\
\hline & 100 & 11.41 & 0.622 & 165.0 & 7.25 \\
\hline & 200 & 10.12 & 0.869 & $146 . ?$ & 3.24 \\
\hline
\end{tabular}

${ }^{235} \mathrm{U}_{-} \mathrm{H}_{2} \mathrm{O}$ Systems

0.98

$\begin{array}{lllll}13.3 & 55.37 & 9.46 & 98.5 & 1.50 \\ 35.1 & 17.75 & 0.822 & 88.2 & 4.77 \\ 50.7 & 15.24 & 0.752 & 84.8 & 4.41 \\ 85.8 & 13.15 & 0.817 & 82.7 & 3.3 .3\end{array}$

TABLE II

Comparison of Results Obtained with Fake and Regula Fixed Source Routines in DOT and ANISN

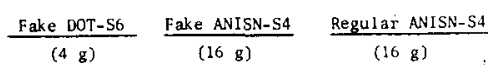

\begin{tabular}{|c|c|c|c|}
\hline Fixed Source & $1.106 \times 10^{-2}$ & $1.099 \times 10^{-2}$ & 1.0 \\
\hline Total Source & 1.0 & 1.0 & 91.26 \\
\hline $\begin{array}{l}\text { Integral Multiplication } \\
\text { (Total Source/Fixed Source) }\end{array}$ & 90.42 & 90.97 & 91.26 \\
\hline Running Time ${ }^{b}$ & $49.1 \min$ & $1.5 \mathrm{~min}$ & $20.9 \mathrm{~min}$ \\
\hline
\end{tabular}

.

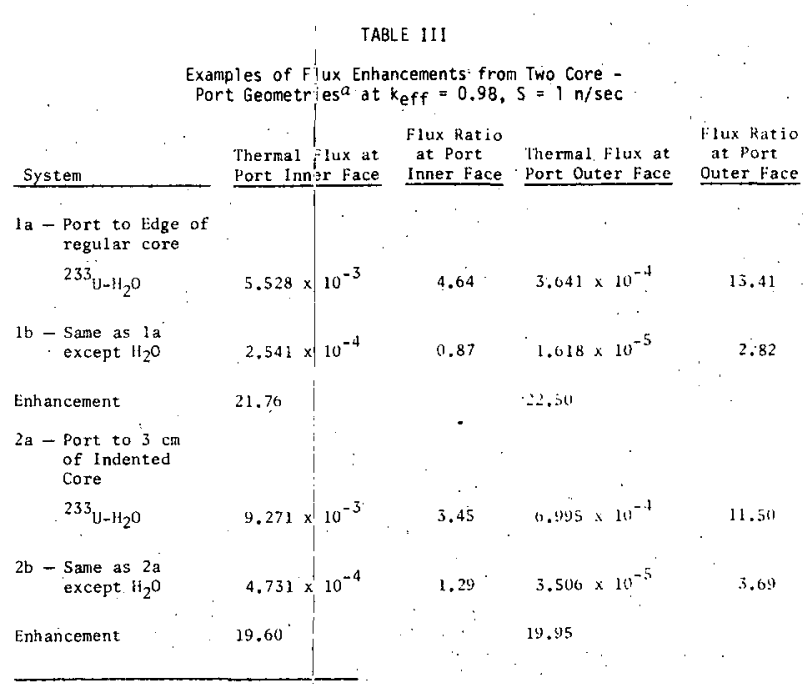

${ }^{a}$ See figure 3 for geometry definitions.

Enhancement

of Indented

Core

${ }^{233} \mathrm{U}-\mathrm{H}_{2} \mathrm{O}$

$\therefore$

19.45

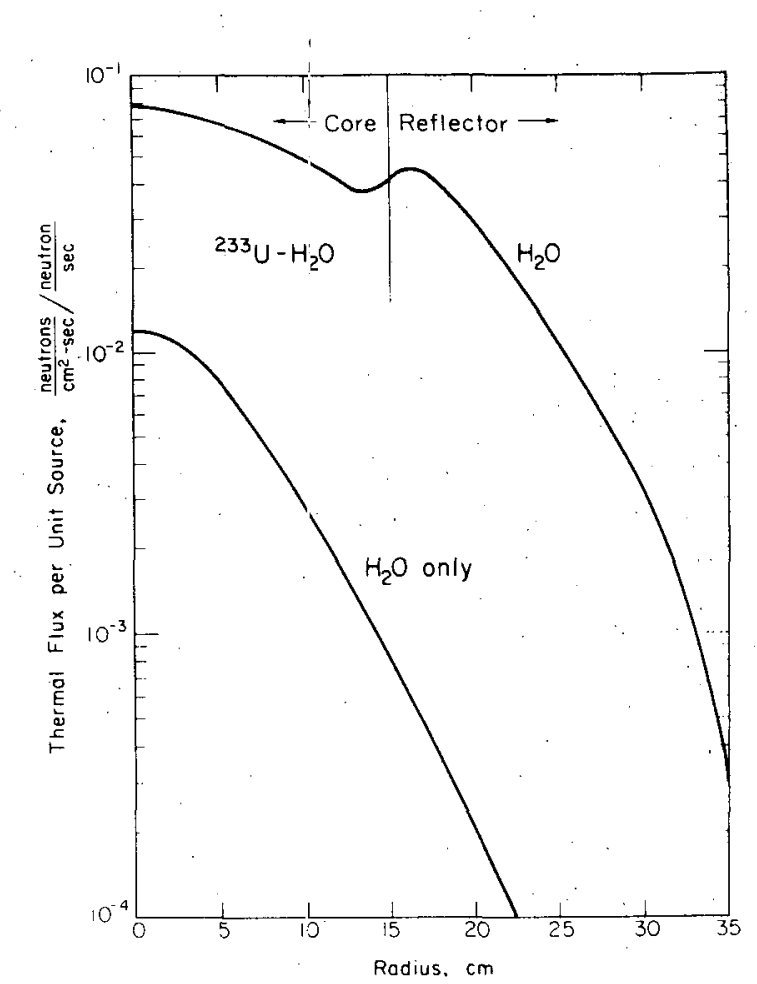

FIG. 1 THERMAL NEUTTRON FLUX (GROUP 16) AS A FUNCTION OF RAOIAL POSI ION IN H2 OND $233 \mathrm{U}-\mathrm{H}_{2} \mathrm{O}$ SYSTEMS AT $40 \mathrm{~g} / 1, \mathrm{k}=10.98$ 

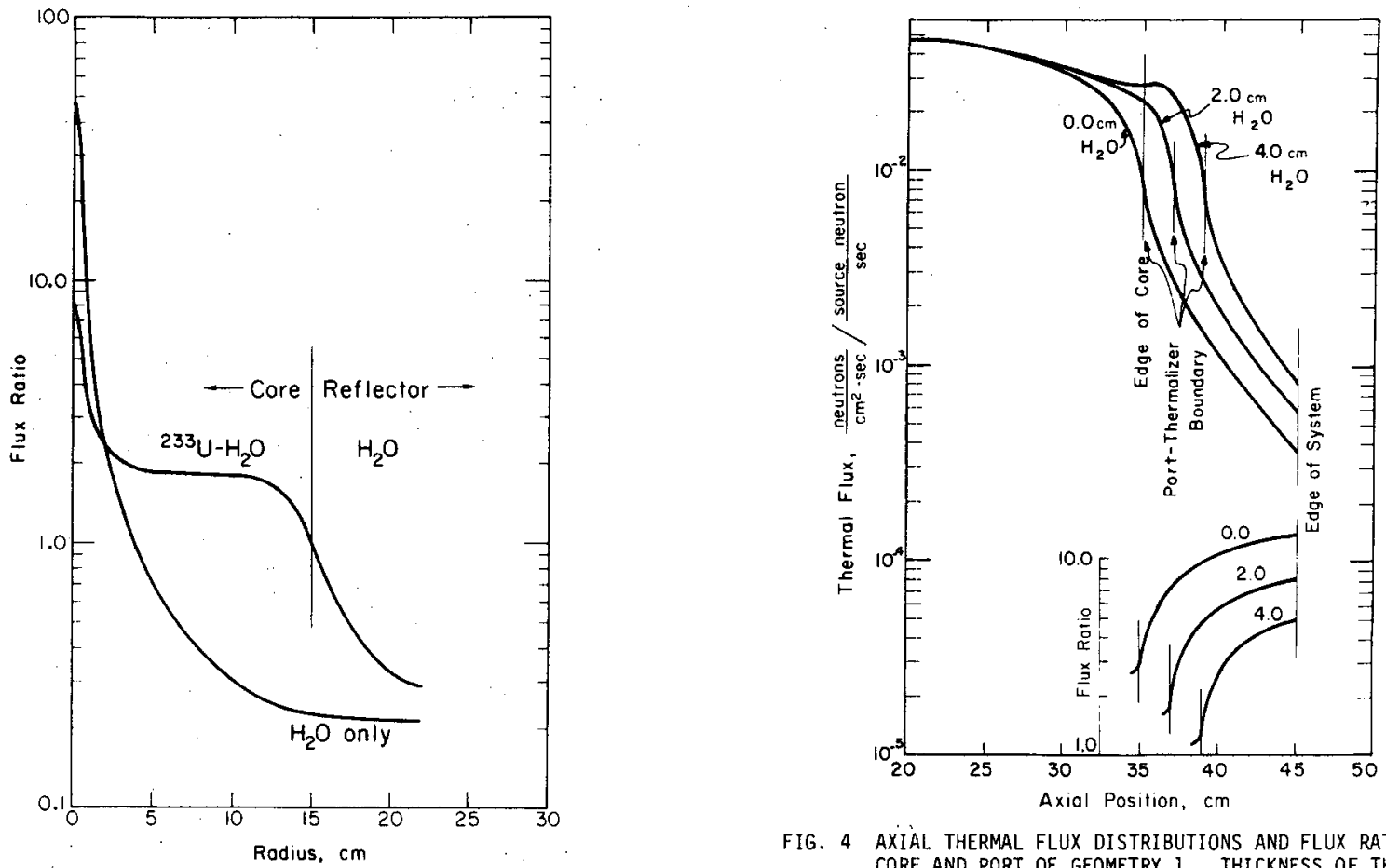

FIG. 2 FAST TO SLOW FLUX RATIO FOR $\mathrm{H}_{2} \mathrm{O}$ AND $233 \mathrm{U}-\mathrm{H}_{2} \mathrm{O}$ SYSTEMS AT $40 \mathrm{~g} / \mathrm{l}, k=0.98$

Flux ratio defined as the ratio of the flux above $0.4 \mathrm{eV}$ to that be $70 w 0.4 \mathrm{eV}$
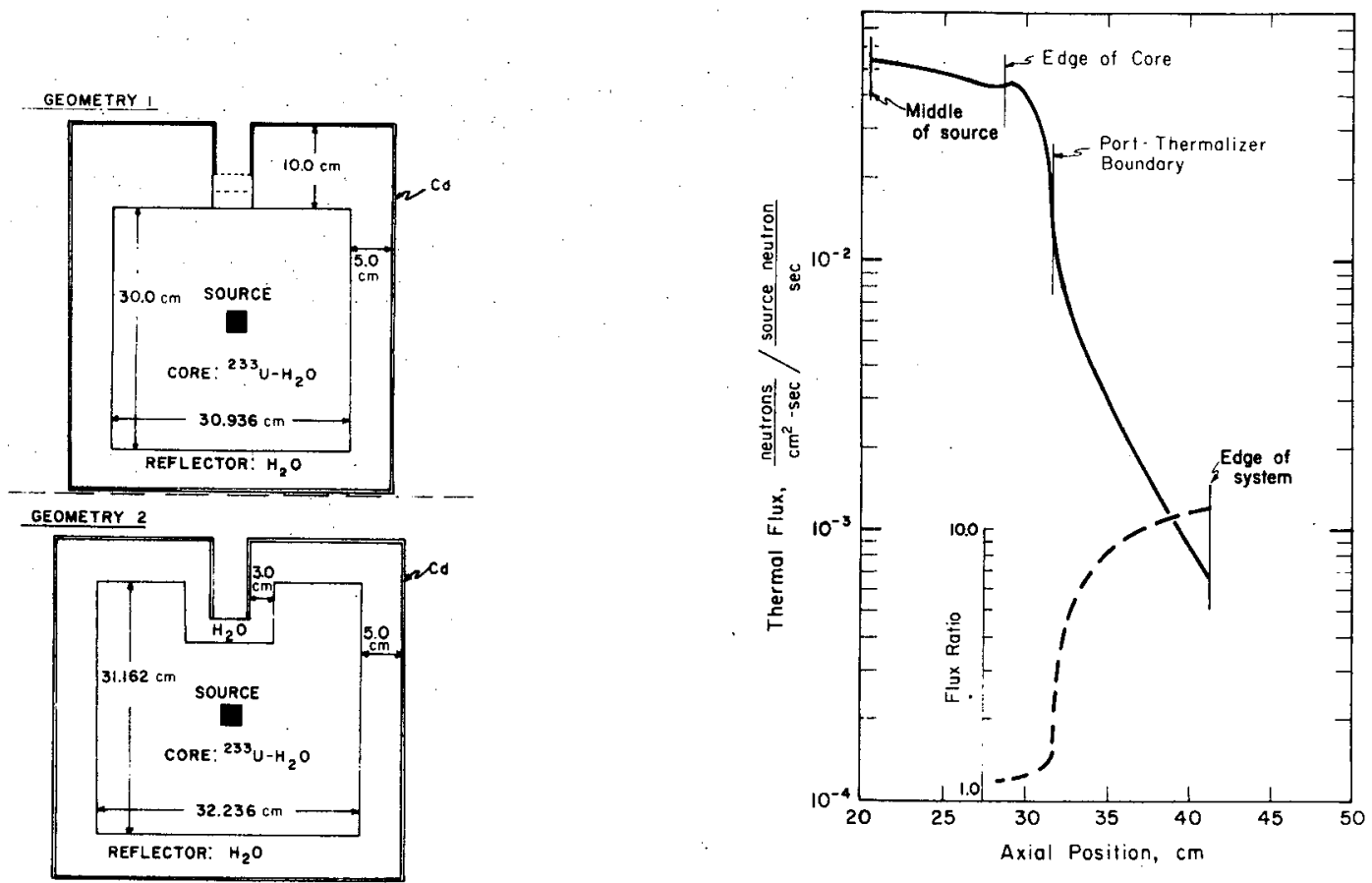

FIG. 3 SOURCE, CORE, REFLECTOR, AND PORT GEOMETRIES USED IN DOT CALCULATIONS (SOURCE IN MIDDLE OF CORE WITH

$0.64 \mathrm{~cm}$ RADIUS BY $1.28 \mathrm{~cm}$ HEIGHT)

FIG. 5 AXIAL THERMAL FLUX DISTRIBUTION AND SPECTRUM RATIO IN CORE AND PORT OF GEOMETRY 2. THICKNESS OF THERMAL. IZER ( $\mathrm{H}_{20}$ BETWEEN CORE AND PORT) $=3.0 \mathrm{~cm}$ ON BOTTOM AND SIDES CORE AND PORT OF GEOMETRY 1 . THICKNESS OF THERMALIZER ( $\mathrm{H}_{2} \mathrm{O}$ BETWEEN CORE AND PORT) $=0.0,2.0$, AND 


\section{COMPARISON OF NEUTRON SOURCE COSTS}

\section{A. Heinrich}

Advanced Operational Planning

Savannah River Laboratory

E. I. du Pont de Nemours and Company

Aiken, South Carolina

A general method for calculating the cost of maintaining isotopic neutron sources has been developed. The method is oriented toward industrial applications and includes costs incurred by source decay, encapsulation, and shipping, as well as interest on investment. Direct comparison between sources with different nuclealr characteristics is facilitated by expressing source costs as the average yearly cost over a ten-year period, optimizing individual source use times to minimize costs, and expressing costs as a function of source strength. Source characteristics pertinent to specific appilications, such as physical size and heat generation, must also be considered in addition to the maintenance costs.

Under pricing assumptions that were thought to be representative of conditions over the next ten years, an application of the method to the current complement of isotopic neutron sources indicates that ${ }^{238} \mathrm{Pu}-\mathrm{Be}$ sources are the most economical below a source strength of about $7 \times 10^{8} \mathrm{n} / \mathrm{sec}$ and that ${ }^{252} \mathrm{Cf}$ sources are the most economical above that level.

\section{INTRODUCTION}

While technical factors such as source dimensions or the neutron energy spectrum can sometimes be controlling, in most cases the principal factor governing the choice of a neutron source for industrial use is cost. This paper provides a method for evaluating the costs of radionuciide neutron sources on a commercial basis in terms of the average yearly costs to be assigned to the source over a ten year period. The analysis includes the radionuclide decay charges, the source fabrication charges, the source shipping charge, and the interest-on-investment charges. Under both present and assumed future charges, $238 \mathrm{Pu}-\mathrm{Be}$ and $252 \mathrm{Cf}$ have the lowest annual costs with the $238 \mathrm{Pu}-\mathrm{Be}$ having the lowest costs at sizes below an emission of about $7 \times 10^{8} \mathrm{n} / \mathrm{sec}(14.3 \mathrm{~g}$ of $238 \mathrm{Pu}$ ) and $252 \mathrm{Cf}$ an increasing advantage at larger sizes (above $300 \mu \mathrm{g}$ of ${ }^{252} \mathrm{Cf}$ ).

\section{LOGIC}

The basis for the source comparisons chosen for this study was "average annual cost over a ten-year period." The "average annual" concept is compatible with most industrial accounting practices; the "ten-year period" represents an assumed "technological useful-

The information contained in this article was developed during the course of work under Contract AT (07-2)-1 with the U. S. Atomic Energy Commission. ness" lifetime that is limited by the advent of new technology or by the enforced replacement of a long-lived source because of real or anticipated physical deterioration.

Four types of costs were included in the analyses: source encapsulation, shipping charges, decay of the radionuclide, and interest on the average source value. Each of these costs is discussed separately below. Figure 1 illustrates a time-sequenced representation of source decay and the cost allotment that parallels the cost descriptions.

\section{ENCAPSULATION}

The cost of source encapsulation was taken to be $\$ 2000$ per source at the present time with a linear decrease to $\$ 500$ per source in ten years. The present value is based on cost experience in using Savannah River Laboratory cave facilities to encapsulate sources up tio $1010 \mathrm{n} / \mathrm{sec}$; the linear decrease attempts to foresee the growth of an encapsulation industry. These costs apply to larger sources $\left(10^{9} \mathrm{n} / \mathrm{sec}\right.$ or better $)$. For smaller sources $\left(10^{7}-108 \mathrm{n} / \mathrm{sec}\right)$ the current commercial price for encapsulation is about $\$ 750$ per source (1). The effect of encapsulation in a glove box is evaluated later in this report. In cases where little or no encapsulation work is required ( $\mathrm{Sb}-\mathrm{Be}$ sources, for example) no charge is made. 


\section{SHIPP ING}

Shipping costs for the large sources were assumed to be $\$ 250$ per source in each direction for a total of $\$ 500$ per source. They are based on a study of cask requirements and construction costs that was made at the Savannah River Laboratory. About half of this cost represents overhead invested in the handling facilities required. This cost was assumed to be constant over the ten year period. In cases where the value of the source at the end of its use period was less than $\$ 250$, it was assumed that the source was not returned, and the return shipping charge not included. This usually corresponds to a source size of $\leq 2 \times 10^{7} \mathrm{n} / \mathrm{sec}$.

\section{DECAY}

Decay costs are charged to the user for the time the source is in use plus the shipping time each way. They are represented by

Decay Costs $=N_{E} A-N_{R}[A-B(y+1)]$, where the nomenclature is as follows:

Symbo1

NE

NS

N

$\mathrm{N}_{\mathrm{R}} \quad$ Source weight when returned

$y$

$y+\frac{1}{2}$,

$y+1$

A

B

C

D

$\lambda$

V

$t$

i for resale 0.5 months after end of use.

Source weight at encapsulation

Source weight at start of use, 0.5 months later.

Source weight at end of use; the weight used to characterize source size.

Source use time in months

Use time plus 1 shipment, 2 shipment times.

Unit cost of source at start of 10-year period

Rate of change of source unit cost

Encapsulation charge at start of 10-year period

Rate of decrease of encapsulation charge

Source decay constant, month-1

Value

Time, month

Interest rate, $15 \%$ in this study
If $\mathrm{N}_{\mathrm{E}}$ and $\mathrm{N}_{\mathrm{R}}$ are expressed in terms of $\mathrm{N}$, the above equation becomes, after rearrangement

$$
\begin{aligned}
\text { Decay Costs } & =\mathrm{N}\left\{\mathrm{A}\left[\mathrm{e}^{\lambda\left(y+\frac{1}{2}\right)}-\mathrm{e}^{-\lambda / 2}\right]\right. \\
& \left.+\mathrm{B}\left[\mathrm{e}^{-\lambda / 2}(1+\mathrm{y})\right]\right\}
\end{aligned}
$$

This equation makes provision for a change in the unit cost of a radioisotope over the use period. In this study a linear change was assumed in the unit cost of the principal isotope and byproducts produced within the source were assumed to have no value. Thus in the case of $252 \mathrm{Cf}$, for example, any returned 252 cf was valued at the current unit price, but $248 \mathrm{Cm}$ accruing from $\alpha$ decay of $252 \mathrm{Cf}$ during the use period was assumed to have no value.

\section{INTEREST CHARGES}

Interest charges were calculated as a percentage of the average value of the source over the use plus shipping $(y+1)$ period. The average value can be expressed as

$$
\bar{V}=\frac{\int N E e^{-\lambda t}(A-B t) d t}{\int d t}
$$

Integrating over the period 0 to $y+1$ months and expressing $\mathrm{NE}$ in terms of $\mathrm{N}$, the interest charge, $I$, is

$$
\begin{gathered}
I=\frac{\bar{V} \dot{i}}{100}=\frac{N i}{1.2 \times 10^{3} \lambda}\left\{A\left[e^{\lambda\left(y+\frac{1}{2}\right)}-e^{-\lambda / 2}\right]+\right. \\
\left.\frac{B}{\lambda}\left[(\lambda y+\lambda+1) e^{-\lambda / 2}-e^{\lambda\left(y+\frac{1}{2}\right)}\right]\right\}
\end{gathered}
$$

In the above derivations $y$ is treated as an independent variable. In the calculations for specific sources the costs were optimized with respect to $y$; that is, the average annual costs for each source were set to a minimum with respect to use time as encapsulation and shipping charges were spread out over longer periods and balanced by the decay and interest charges which increase with use time. This procedure requires the assumption that the initial size of a source is inconsequential (that only the final size, $N$, is important) and that sources larger than $\mathrm{N}$ can be accommodated by reducing the exposures. 


\section{APPLICATION}

The cost formulas described above were applied to a group of nine radionuclide neutron sources. The sources, plus pertinent cost and decay constants, are listed in Table I.

The results of the calculations are shown in Figure 2. These calculations show that $238 \mathrm{Pu}-\mathrm{Be}$ and $252 \mathrm{Cf}$ are the most economical neutron sources with $238 \mathrm{Pu}-\mathrm{Be}$ having the lowest average annual cost up to a source size of $7 \times 108 \mathrm{n} / \mathrm{sec}$ and $252 \mathrm{Cf}$ having the lowest cost above that size. The 1970 price schedules used in the calculations are, at the time of writing, the most recent commercial prices available to the author. However they are subject to possible major changes.

\section{SENSITIVITY STUDIES}

Major postulates included in this study are that any price variation is linear with respect to time and that a reasonable interest rate to apply to source investment is $15 \%$. Both of these postulates affect the absolute magnitude of source costs but have little effect on the relationship between different sources.

An exponential relationship between source price and time, (price $\sim c / t+c^{\prime}$ ), might well be one result of a rapidly growing neutron source industry. A price-time relationship that reduces the 10-year price differential by half in two years instead of five years reduces the 10-year average annual cost approximately $40-50 \%$ in the $10^{9} \mathrm{n} / \mathrm{sec}$ source size range, and, if applied to either $238 \mathrm{Pu}-\mathrm{Be}$ or $252 \mathrm{Cf}$ and not to the other, changes the indifference source size by $\pm 2.5 \times 10^{8} \mathrm{n} / \mathrm{sec}$.

Interest charges account for about half of the $238 \mathrm{Pu}-\mathrm{Be}$ and one quarter of the $252 \mathrm{Cf}$ annual cost at a source size of $10^{9} \mathrm{n} / \mathrm{sec}$. A change of $\pm 5 \%$ (absolute) in the interest rate thus changes the indifference source size by a factor of about two: to $1.5 \times 10^{9}$ $\mathrm{n} / \mathrm{sec}$ at $10 \%$ interest and $4 \times 10^{8} \mathrm{n} / \mathrm{sec}$ at $20 \%$ interest. Similar changes occur in the average annual costs of other sources.

The use of cave facilities to encapsulate small sources (approximately $2 \times 10^{7}$ $\mathrm{n} / \mathrm{sec}$ ) is probably not required, and encapsulation can be accomplished in a less expensive glove box facility. The estimated cost for encapsulation under these conditions is $\$ 750$ or less per source compared with $\$ 2000$ per source for the larger sources. The average annual costs of $10^{7} \mathrm{n} / \mathrm{sec} 238 \mathrm{Pu}-\mathrm{Be}$ and $252 \mathrm{Cf}$ sources encapsulated in glove boxes are $\$ 120 /$ yr and $\$ 180 / y r$, respectively, about half the cost if encapsulated at $\$ 2000 /$ source.

Radionuclide costs that are constant with respect to time can be used in the formulas derived for this study to calculate source costs representative of equilibrium conditions between the supply and demand for neutron sources. A comparison of the cost of three neutron sources, $238 \mathrm{Pu}-\mathrm{Be}$ at $\$ 100 / \mathrm{g}$, $244 \mathrm{Cm}-\mathrm{Be}$ at $\$ 200 / \mathrm{g}$, and $252 \mathrm{Cf}$ at $10^{6} \$ / \mathrm{g}$, is shown in Figure 3 . The unit costs assigned to these isotopes aje estimated to be typical of equilibrium production in the 1980-1990 period (2). The significant point of this comparison is that, / while the absolute costs in equilibrium cond tions are expected to be lower than current costs, the relative economic positions between different radionuclides is about the same. $238 \mathrm{Pu}-\mathrm{Be}$ sources are expected to be the cheapest up to about $10^{8} \mathrm{n} / \mathrm{sec}$ with $252 \mathrm{Cf}$ having a substantial economic advantage for larger sources.

\section{REF=ERENCES}

1. Radioactive Sourice Handbook. Monsanto Research Corporation, p 36 (1968).

2. J. L. Cranda11. "Tons of Curium and Pounds of Californium." Proceedings of The Internationd 7 Conference on Constructive Uses of Atcmic Energy. American Nuclear Society (1968).

3. F. P. Baranowskj. "Development and Production of $252 \mathrm{Cf}$." Californium-252. CONF. 681032, USAEC Division of Technical Information (1969).

4. C. A. Rohman, Values in Spent Fuel from Power Reactors." Isotop. and Radiat. Tech. 6, $19(196.8)$. 
TABLE I

Source Input Data

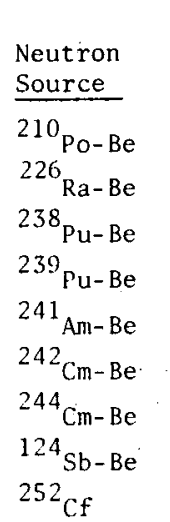

\begin{tabular}{|c|c|}
\hline 1970 & $1980(2,4)$ \\
\hline $360,000^{a}$ & 10,000 \\
\hline $20,000^{b}$ & 20,000 \\
\hline $750^{a}$ & 250 \\
\hline $50^{b}$ & 10 \\
\hline $150^{a}$ & 100 \\
\hline $10,000^{b}$ & 500 \\
\hline $4,000^{c}$ & 400 \\
\hline $87,400^{b}$ & 1,200 \\
\hline $10^{7 a}$ & $2 \times 10^{6}$ \\
\hline
\end{tabular}

\begin{tabular}{c}
$\begin{array}{c}\text { Half-Life, } \\
\text { years }\end{array}$ \\
\hline 0.378 \\
1620 \\
89 \\
24,360 \\
458 \\
0.448 \\
17.6 \\
0.167 \\
2.646
\end{tabular}

gram-sec
$1.13 \times 10^{10}$
$1.3 \times 10^{7}$
$4.75 \times 10^{7}$
$1.35 \times 10^{5}$
$6.98 \times 10^{6}$
$9.2 \times 10^{9}$
$2.05 \times 10^{8}$
$2.78 \times 10^{10}$
$2.34 \times 10^{12}$

Neutrons Per

watt-sec

a particle

$7.8 \times 10^{7}$

0.000067

$8.5 \times 10^{7}$

0.00042

$9.1 \times 10^{7}$

0.000077

$6.2 \times 10^{7}$

0.000050

$6.7 \times 10^{7}$

0.000053

$8.3 \times 10^{7}$

0.00011

$8.3 \times 10^{7}$

0.000067

$2.5 \times 10^{9}$

(no $\alpha$ )

2.646

$6.3 \times 10^{10}$

0.12

$a_{\text {Federal Register. }}$

${ }^{b}$ Current commercial price.

${ }^{c}$ Assumed price. See Reference 3.

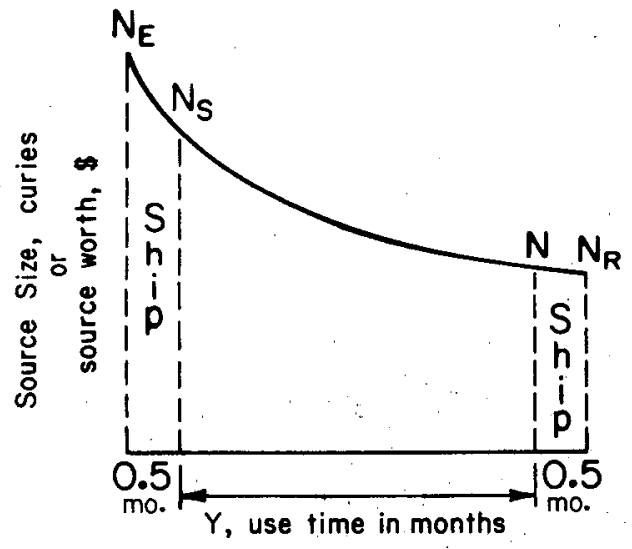

FIG. 1 USE NOMENCLATURE. DIAGRAM

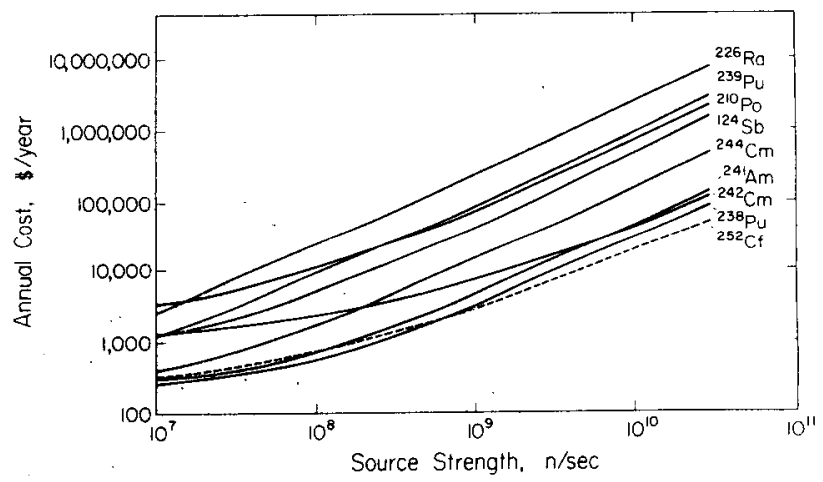

FIG. 2 ANNUAL SOURCE COST COMPARISON 


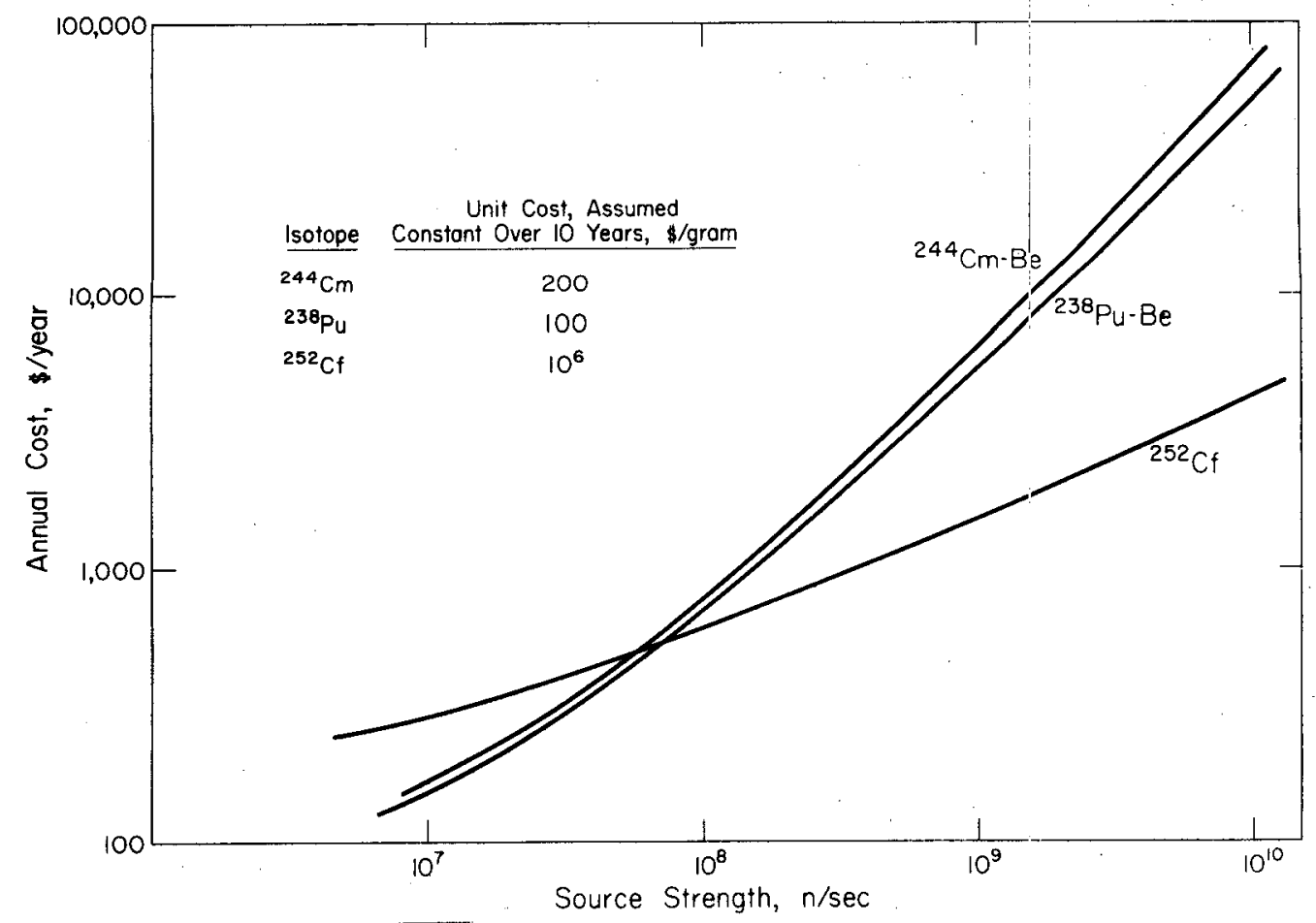

FIG. 3 ANNUAL SOURCE COST COMPARISON AT EQUILIBRIIJM CONDITIONS

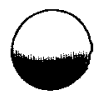




\section{PROTON RECOIL MEASUREMENTS OF THE PUBE SOURCE NEUTRON SPECTRA}

Roger L. Alexander, Donald F. Shook, and C. Hubbard Ford

NASA - Lewis Research Center

Cleveland, Ohio

The spectrum of a $5 \mathrm{Ci} \mathrm{PuBe}$ neutron source has been measured from 40 to $800 \mathrm{kev}$. The measurement was made using a spherical proportional counter containing 4 atmospheres of hydrogen. Pulses due to gamma-ray interactions in the chamber were distinguished by rise time analysis using a two-parameter pulse-height analyzer. The proton recoil spectrum was analyzed using a code written by Benjamin which accounts for contributions to the proton recoil spectrum from neutrons of energy greater than that to be analyzed.

The proportional counter measurement is combined with a recent liquid scintillator measurement to give a complete spectrum. Good agreement exists between the two measurements in the region of overlap from 500 to $800 \mathrm{kev}$. The proportional counter data show that the flux is fairly smooth and flat in the region from 40 to $800 \mathrm{kev}$.

The present measurements indicate a neutron yield below 1 Mev for this source of $20.2 \pm 3.9$ percent.

\section{INTRODUCTION}

For many applications of radioactive neutron sources it is necessary to have an accurate knowledge of the neutron spectrum as well as the source strength: The use of large water bath techniques has made it possible to determine source strengths to an accuracy of 3 percent or better but the neutron spectrum is not nearly so well known. Considerable past work with emulsions and organic scintillators has resulted in fairly well established spectra at energies greater than about $1 \mathrm{MeV}$ but below this energy the spectra of many radioactive sources have not beèn measured.

In the present work a 5 Curie (Ci) PuBe source spectrum is measured from $800 \mathrm{keV}$ down to $40 \mathrm{keV}$ using a spherical proportional counter (1). This measurement is combined with a recent liquid scintillator measurement (2) to give a complete spectrum. The primary difficulties in making a proportional counter measurement of the low energy part of the $\operatorname{Be}(\alpha, n)$ spectrum are caused by the large number of high energy neutrons emitted by these sources, and by the gamma-ray background.

\section{EXPERIMENIT}

A spherical proportional counter designed by Benjamin et.al., (1) was used in the measurements. The counter was filled with 4 atmospheres of hydrogen and a trace of helium-3 and had an inside diameter of $3.94 \mathrm{~cm}$. The counter wall was $.051 \mathrm{~cm}$ thick stainless steel. A lead shield about $.64 \mathrm{~cm}$ thick, and an outer cadmium cover about $.075 \mathrm{~cm}$ thick surrounded the counter.

The measurements were made with the counter mounted on a light frame 1.8 meters above the floor of a room 4 meters high, and 6 meters square. The PuBe source was supported with its axis vertical at $15 \mathrm{~cm}$ from the counter by a. $62 \mathrm{~cm}$ diameter threaded iron rod.

A block diagram of the electronics is shown in figure 1 . To separate protons from electron recoils, a two-parameter analyzer was driven by an identification signal as well as by a linear signal. To preserve the linear response for large pulses in hydrogen, which may take up to $6 \mu$ sec to rise, the preamplifier decay time was made $130 \mu \mathrm{sec}$, and a single $7 \mu$ sec $R C$ integration and differentiation time constant was chosen in the linear amplifier. To obtain an identification pulse separating fast-rising proton recolls from electrons, the preamplifier output was R-C clipped with a short time constant in the second linear amplifier. This pulse was used to drive a fast stretcher and a slow stretcher of about $5 \mu \mathrm{sec}$ duration. The slow stretcher pulse was adjusted to reach 1ts peak at its end. Finally, the identification pulse was delayed 4. $75 \mu$ sec. These precautions ensured that the two-parameter analyzer would always 
convert the full voltage of the linear signal.

Careful measurements were made of the analyzer linearity and pulse height zero. Amplifier gains were measured and absolute gas gains were obtained using the $764 \mathrm{keV}$ hellum-3 peak. A plot of $\ln \mathrm{A} / \mathrm{V}$ versus in $\mathrm{V}$ gave a straight line over a range of gas gain from $A=8$ to $A=250$.

Measurements were made with gas gains of from 45 to 180 . The resolution of the helium-3 peak was about 10 percent full width at half maximum (FWHM).

\section{THE 5 CURIE PUBe SOURCE}

This source is a right cylinder, outer diameter $3.33 \mathrm{~cm}$, and length $6.91 \mathrm{~cm}$. It contains 79.88 gms of plutonium and $39.30 \mathrm{gms}$ of beryllium intimately mixed and melted in a tantalum cup and doubly encapsulated in stainless steel.

The source emission rate was $9.50+.29$ neutrons per second in september of 1962. The isotopic fraction of plutonium-241 was - 74 percent (3). This corresponds to an initial rate of increase of source strength due to the formation of americium-241 of 2.1 percent per year.

The source strength in September 1970 is estimated to be 13.6 percent greater than in 1962. The relative emission rate of this source as a function of direction was measured with a long counter, the center of the front face of which was at $100 \mathrm{~cm}$ from the center of the source. The emission rate in the direction perpendicular to the source axis was measured to be $1.067 \pm .020$ times as great as the emission rate averaged over the total solid angle. The calculated neutron flux at one meter from the source perpendicular to the axis is then

$$
\phi=\frac{9.50 \times 1.067 \times 1.136 \times 10^{6}}{4 \pi(100)^{2}}=91.6 \mathrm{n} / \mathrm{cm}^{2} \mathrm{sec}
$$

\section{DATA ANALYSIS}

The maximum pulse size that can be produced by a gamma-ray in this chamber corresponds to that of about a $100 \mathrm{keV}$ proton, so that there is no gamma-ray interference above this energy. Consequently, the proton recoil data analysis is straightforward. The Spec 4 code of Benjamin (4) was used. This code requires a neutron spectrum shape above the highest lenergy to be analyzed. The required shape was obtained from the data of reference 2. The code generates a proton recoll response shape for neutrons. above the energy to be analyzed, using analytic response functions. It then normalizes this shape to the data, and strips the differentiated remainder.

Below about $100 \mathrm{keV}$ proton energy, gamme events occur in the chamber. A twodimensional plot of counts from a cokalt- 60 source is shown in flgure 2. The pulses from a $40 \mathrm{keV}$ electron travel a total distance equivalent to the diameter of this chamber, and may require as long as $6 \mu \mathrm{sec}$ to rise. Consequently, at a given energy the clipping circuit places most of the pulses near the eriergy axis.

Figure 3 show's data from the PuBe source. A $100 \mathrm{keV}$ proton in the counter travels only about a millimeter, so that all of the $100 \mathrm{keV}$ proton pulses should rise within the clipping time of the identification circuit. These pulses appear as a peak in the identification channel. Since the identification pulse size for these pulses is proportional to the enerby, this peak occurs in a diagonal line on the plot (5).

Unfortunatelj, not all the pulses on this plot can be cilearly ascribed to the proton-recoil peak, or to gamma-rays. Above $100 \mathrm{keV}$, where the gamma-ray induced pulses are gone, there is a flat continuum of pulses which heive smaller identification pulses than those in the peak. It is believed that these pulses are due to higher energy protons theit lose only a fraction of their energy in the gas before colliding with the wall. At $2.5 \mathrm{MeV}$ for example, a proton will lose $1.00 \mathrm{keV}$ while travelling nearly $2 \mathrm{~cm}$. in the gas.

Figure 4 shows the spectrum of pulses from the source surrounded by a spherical shell of tungsten; which sharply reduces the fraction of high-energy neutrons in the spectrum. The ca]culated fraction of the flux above $2 \mathrm{MeV}$ in the tungsten leakage spectrum is only 1.7 percent compared with To percent for the bare source. The number of counts not on the diagonal have been much reduced. This nor-peak fraction is 35 percent at $100 \mathrm{keV}$ for the bare source, and only 4 percent for: the tungsten leakage spectrum. 
It is possible to subtract an arbitrary fraction of a gamma-ray spectrum from a mixed spectrum so as to obtain a smooth continuation below $100 \mathrm{keV}$ of the distribution above $40 \mathrm{keV}$, so that the subtraction is from the non-peak counts.

Figure 5 shows the total counts measured for the $5 \mathrm{Ci}$ PuBe source. Two slightly different gamma fractions are subtracted below $100 \mathrm{keV}$. The non-peak contribution to the spectrum for these subtractions is shown also. A modified version of Spec 4 was used to calculate the fraction of recoils from neutrons of energy greater than $1.5 \mathrm{MeV}$. This is also shown in figure 5. Figure 6 shows similar results for the tungsten leakage spectrum, where the nonpeak fraction is much lower.

In both fluxes, the fraction of nonpeak counts is not a constant with energy. For a fixed clipping time, there will be some energy of proton-recoil below which all the recoils will be in the group along the diagonal. The rise time for these very small pulses will be within the clipping time. Since the absolute number of recoils from high energy events increases only about 10 percent from $100 \mathrm{keV}$ to $10 \mathrm{keV}$, the number of counts occurring off the diagonal should, at most, rise no more than this.

The uncertainty in the gamma-ray subtraction above $40 \mathrm{keV}$ is of the order of the difference between the two subtractions shown in figures 5 and 6 . The approximate upper limit of the counts in the non-peak fraction is the upper curve shown. If one subtracts more than enough to give the lower curve, portions of the identification channel region become negative.

\section{RESULTS AND DISCUSSION}

The solid line of figure 7 shows the neutron flux spectrum reduced to 1 meter distance for the $5 \mathrm{Ci}$ PuBe source as presently measured by the 4 atmosphere hydrogen proportional counter. The curve drawn through the data is the weighted averages of two runs each above and below about 100 $\mathrm{keV}$. The hatched area represents the estimated error. The analysis is not inconsistent with a nearly featureless spectrum. But the analyses of runs taken on different days show the source spectra features reproduced here. Below $70 \mathrm{keV}$, the large uncertainty in the spectrum is caused by the uncertainty in the gamma fraction subtracted from the non-peak data. Because the non- peak proton recoil contribution is expected to decrease with decreasing neutron energy, the upper limit of the spectrum shown is believed to be near the upper limit for the spectrum in this region. The lower limit is not so clearly defined.

The integral of the flux from 0 to 800 $\mathrm{keV}$ with a flat $30 \mathrm{n} / \mathrm{cm}^{2}$ sec $\mathrm{MeV}$ below 40 $\mathrm{keV}$ is $15.6 \pm 2.7 \mathrm{n} / \mathrm{cm}^{2} \mathrm{sec}$. The total flux at one meter is calculated to be $91.6 \pm 3.7$ $\mathrm{n} / \mathrm{cm}^{2} \mathrm{sec}$. The data of reference 2 , therefore, have been normalized to include $76.0 \mathrm{n} / \mathrm{cm}^{2} \mathrm{sec}$ between $800 \mathrm{keV}$ and $11 \mathrm{MeV}$. The fraction of the neutron spectrum below $1 \mathrm{MeV}$ is then $20.2 \pm 3.9$ percent.

The spectrum of reference 2 is very similar to that of Anderson and Bond (6) who measured the spectrum of a similar PuBe source with nuclear emulsions. Their measured spectrum extrapolated smoothly to zero neutron energy indicated about 18 percent below I MeV. St. Romain, et.al., (7) using Bonner spheres to estimate neutron yields found about 17 percent of the spectrum of a $1 \mathrm{Ci}$ PuBe source below $1 \mathrm{MeV}$. They inferred a peak in the neutron spectrum at $0.3 \mathrm{MeV}$ due to inelastic scattering of alpha particles from beryllium. The excited beryllium nucleus was postulated to decay by neutron emission. Shook, et.al., (8) measured the age of neutrons in various media and using a similar $5 \mathrm{Ci}$ PuBe source, estimated that 21 percent of the neutrons were emitted below $1 \mathrm{MeV}$. They used the Anderson and Bond spectrum above $1 \mathrm{MeV}$.

In conclusion, the neutron spectrum of a $5 \mathrm{Cl}$ PuBe source measured from $40 \mathrm{keV}$ to $800 \mathrm{keV}$ is generally flat and smooth. The measurement indicates there are about $20.2+3.9$ percent of the neutrons emitted below $1 \mathrm{MeV}$, which is consistent with other measurements in magnitude but not in shape. The method of estimation of the contribution of gamma-rays to the recoil spectrum below neutron energies of $100 \mathrm{keV}$ is the principal source of uncertainty in the present protonrecoil measurements which have large fractions of the proton recoils caused by high-energy neutrons.

\section{REFERENCES}

1. Benjamin, P.W. , Kemshall, C. D., Redfearn, J. A. : High Resolution Spherical Proportional Counter; AWRE No. NR 1/64, June 1964. 
2. Shook, D. F.:A Small Differentiable

Liquid Scintillator Neutron Spectrometer;

NASA TM X-52828, June 1970.

3. NUMEC Corporation, private communication.

4. Benjamin, P.W., Kemshall, C. D.,

Brickstock, R.: The Analysis of Recoil

Proton Spectra; AWRE Report No. 09/68, March 1968.

5. Bennett, E. F. : Fast Neutron Spectroscopy by Proton-Recoil Proportional Counting; Nucl. Sci. \& Eng., Vol. 27, 1967, pp. 16-27.

6. Anderson, M. E., and Bond, W. H., Jr.: Neutron Spectrum of a Plutonium Beryllium Source; Nucl. Phys., Vol. 43, 1963, pp. $330-338$.

7. St. Romain, F. A., Bonner, T. W., Bramblett, R. I., and Hanna, J.: LowEnergy Neufrons from the Reaction ${ }^{9} \mathrm{Be}(\alpha, \mathrm{n}){ }^{12} \mathrm{C}$; Phys. Rev., Vol. 126, 1959, pp. 1794-1797.

8. Shook, D. F., Alexander, R. L., Bogart, D. and Krueger, M. : Ages of PlutoniumBeryllium Neutrons in Tungsten-Water Media; NASA TN D-3695, Nov. 1966. 
ISI - I

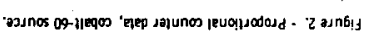
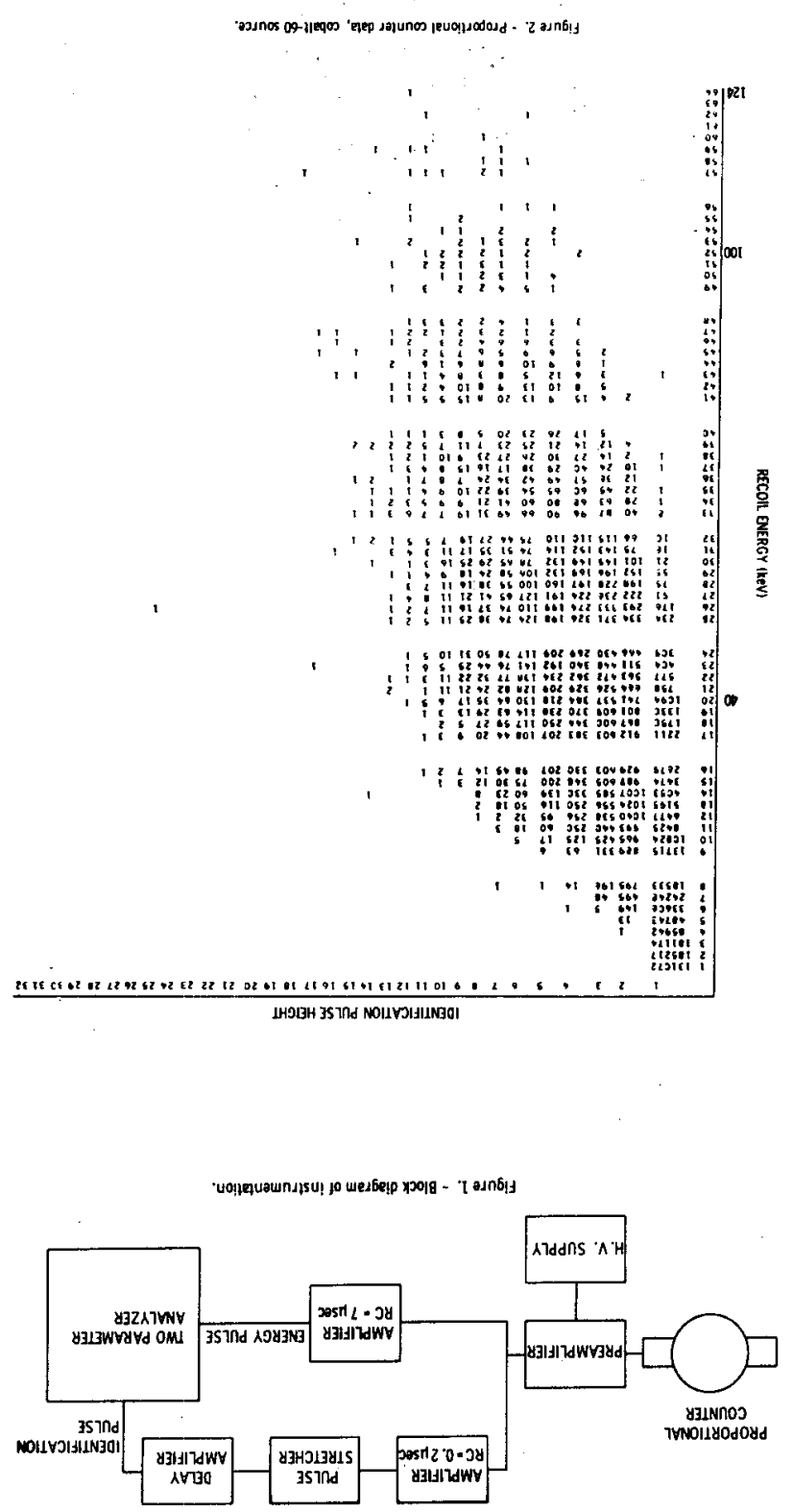


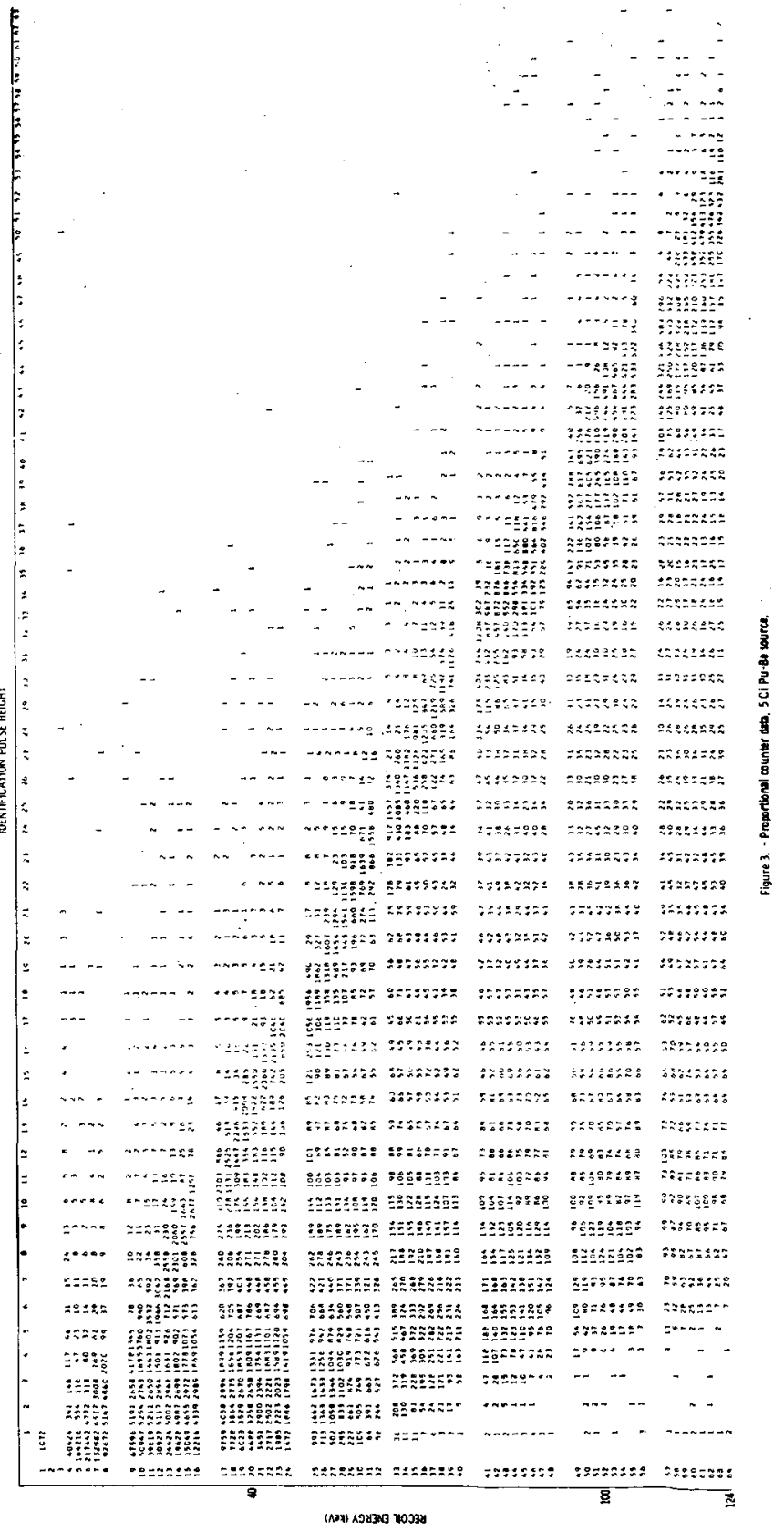



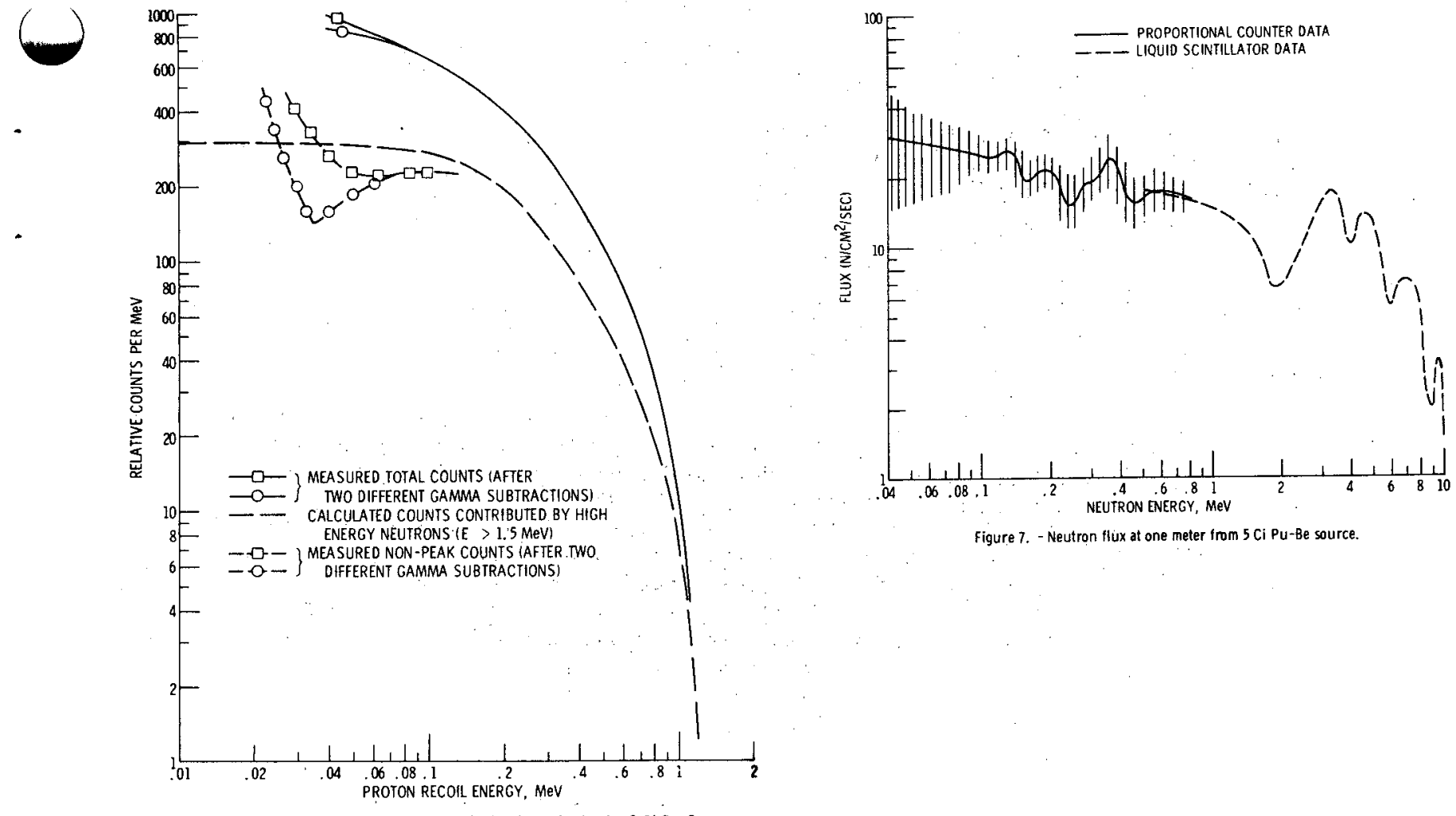

Figure 5. - Comparison of measured with calculated count rates for 5-Ci Pu-Be source.

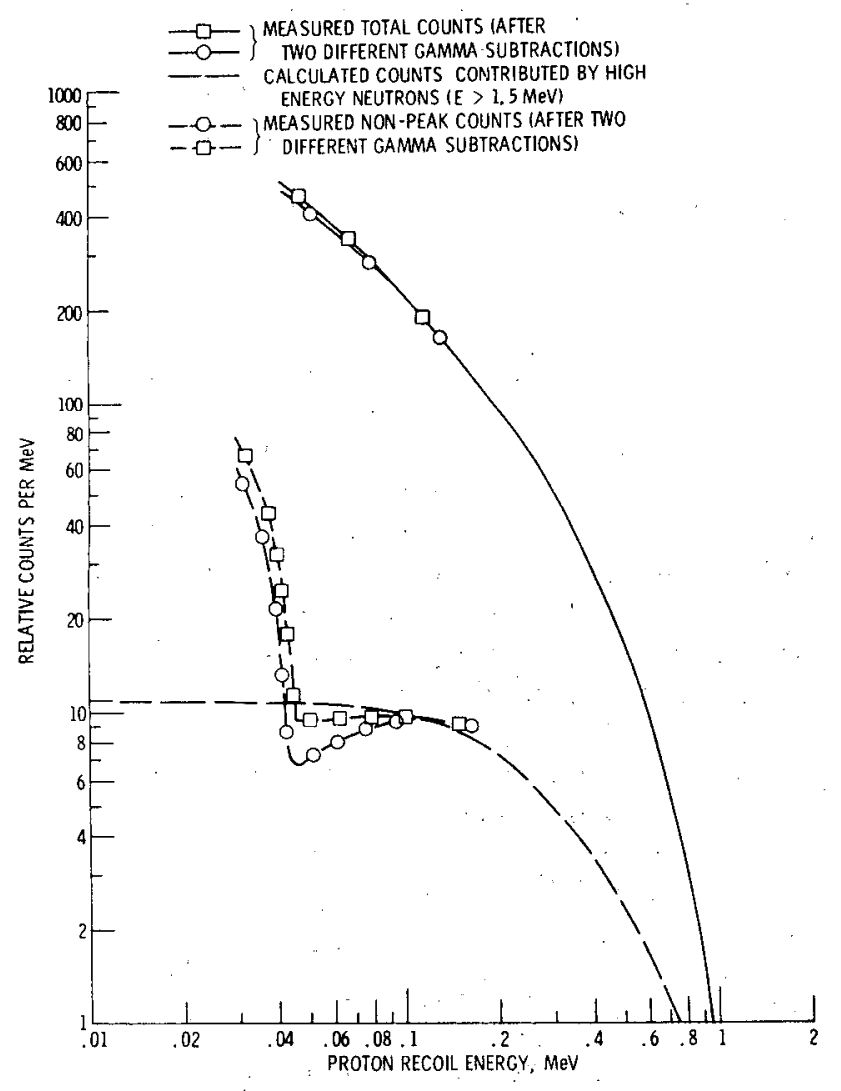

Figure 6. - Comparison of measured and calcuilated count rates for tungsten leakage flux. 


\title{
ABSOLUTE MEASUREMENTS ON THERMAL NEUTRON FLUXES PRODUCED IN WATER BY (d,d) AND (d,t) REACTIONS WITH 150 kev DEUTERONS
}

\section{Holland}

J. Walker

Department of Physics

University of Birmingham

Birmingham, England

\begin{abstract}
A small accelerator producing $150 \mathrm{kev}$ deuterons has been used with conventional adsorbed deuterium and tritium targets to produce neutrons which were moderated in ordinary water. The thermal neutron flux distributions were measured with a small scintillation detector which was calibrated against the activation of thin foils of gold; in turn, the gold foils were calibrated in a standard flux of thermal neutrons. Absolute values of the fluxes per fast neutron injected were obtained by an associated-particle measurement of the actual target yield. Simple age-diffusion calculations have been made for both cases and gave flux distributions in reasonable agreement with the experimental results for the $(d, d)$ neutrons but more refined computational methods are required for the $(d, t)$ case; results of an S-N computation are included for the latter.
\end{abstract}

\section{INTRODUCTION}

Absolute measurements of the thermal neutron fluxes produced by $(d, d)$ and $(d, t)$ reactions with $150 \mathrm{keV}$ deuter ons were undertaken as part of a general study of the suitability of non-reactor sources for thermal neutron radiography; the as sociated-particle method has been used. The results have been used to assess the usefulness of age-diffusion theory in estimating the ratio of peak thermal fluxes from these sources in water.

A systematic review of the method and errors involved in the associated-particle technique has been given by Fewell (1). For the $\mathrm{T}(\mathrm{d}, \mathrm{n})^{4} \mathrm{He}$ reaction the neutron yield is isotropic in the centre-of-mass system at the deuteron energies involved here, and when the associated-particle detector is positioned at $90^{\circ}$ to the incident deuteron beam, the neutron yield per detected $a$-particle is given by

$$
N=\frac{\int_{0}^{E^{\prime}} \frac{\sigma(E)}{d E / d x} d E}{\left(\frac{d \omega^{\prime}}{d \omega}\right)_{\alpha} \Delta \Omega \int_{0}^{E^{\prime}} \frac{\sigma(E)}{d E / d x} d E}
$$

where $\sigma(E)$ is the reaction cross-section $\Delta \Omega$ is the solid angle subtended at the target by the detector

$d \omega^{\prime}$ is the solid angle conversion $d \omega$ factor from the centre-ofmas is to the laboratory system for the detected $\alpha$ particle

and $d E / d x$ is the rate of energy loss of deuterons in the target material.

Total neutron yield measurements from the $\mathrm{D}(\mathrm{d}, \mathrm{n})^{3} \mathrm{He}$ reaction are more difficult because the reaction is not isotropic in the centre of mass system for incident deuterons of $150 \mathrm{keV}$ and the ${ }^{3} \mathrm{He}$ particles are not easily resiolved in the presence of deuterons scattered from the target and of tritons from the competing $\mathrm{D}(\mathrm{d}, \mathrm{p}) \mathrm{T}$ reaction. However, by measuring the easily resolved pi:otons from the last reaction the neutron yield per detected particle can be calculated from the relationship

$N=\frac{4 \pi}{\Delta \Omega} \frac{\int_{0}^{E} \frac{\sigma(d, p)}{d E / d x} d E}{\left.4 \pi \int_{0}^{E^{\prime}} \frac{\frac{d \sigma(d, p)}{d \omega^{\prime}}\left(\frac{d \omega^{\prime}}{d \omega / d x}\right)}{d \omega}\right)_{p}^{E^{\prime}} \frac{\int_{0}^{E^{\prime}} \frac{\sigma(d, n)}{d E / d x} d E}{d E / d x} d E}$ 


$$
=\frac{4 \pi}{\Delta \Omega} R^{\prime}\left(\frac{Y(d, n)}{Y(d, p)}\right)_{\text {Thick }}
$$

Values of $R^{\prime}$ and $[Y(d, n) / Y(d, p)]$ have been tabulated by Ruby and Crawford (2) .

\section{APPARATUS AND MEASUREMENTS}

An unanalysed deuteron beam from a conventional Cockroft-Walton $(0-200 \mathrm{kV})$ accelerator bombarded the neutron producing target (fig. 1) which was mounted in a cubical tank of water ( $3 \mathrm{ft}$ side) as shown in figure 2 . The charged particles associated with either the $(d, d)$ or $(d, t)$ reaction were detected with a $3000 \Omega-\mathrm{cm}$ silicon surface-barrier detector mounted in a $1^{\prime \prime} \mathrm{O}$. D. dural tube at $90^{\circ}$ to the deuteron beam (fig. 3). Accurate alignment and support of the target-detector assembly were simplified by enclosing the accelerator drift tube (AISI $32.1 \mathrm{grade}$ stainless steel, l"O.D., 0.010" thick) and detector a ssembly in a 3 " O.D. perspex (lucite) tube joined at right angles and mounted in two adjacent ports of the moderator tank. Both the drift tube and detector assembly were centrally located in the perspex by $1 / 4^{\prime \prime}$ thick perspex discs and the annular space was flooded with water.

Aluminium stops positioned at $3.6 \mathrm{~cm}$. $12.6 \mathrm{~cm}$ and $22.1 \mathrm{~cm}$ from the target . defined the solid angle between the active target area and the detector. Care was taken in the construction of the apparatus to minimise the importance of scattered $a$-particles and ther efore no allowance.was made for them in the analysis. For the surface near the beam, the stops were tapered to $0.25 \mathrm{~mm}$ thickness and they were positioned so that particles not. travelling directly to the detector could only be detected after at least three scatters. Careful alignment ensured that the a-particle detector viewed the whole active target area.

For the $(d, t)$ measurements, a conventional tritiated titanium target was used with the active target defined by a $3.2 \mathrm{~mm}$ dia. beam stop made from a $0.010 "$ thick disc of tantalum. For the (d, d) target, stops (6.7 mm dia.) were placed at $1.5 \mathrm{~cm}, 51.5 \mathrm{~cm}$ and $89 \mathrm{~cm}$ from the target; the two additional stops were necessary to $r$ educe the importance of secondary sources of neutrons.

Thermal neutron flux distributions were measured in water with a LiF-ZnS scintillation detector coupled by a perspex light guide $61 \mathrm{~cm}$ long to an E.M.I. $9524 \mathrm{~S}$ photomultiplier. To minimise flux perturbations the detector was kept thin and used $5.62 \mathrm{mg} \mathrm{cm} \mathrm{cm}^{-2}$ of lithium fluoride enriched to $99.6 \%$ in $6 \mathrm{Li}$. The detector was calibrated with the thermal flux from a $1 \mathrm{Ci}$ Am-Be source in water. This flux had been measured previously by activating bare and cadmium-covered (0.040" thick Cd) gold foils (0.020" thick, $0.6 "$ diameter). In turn, the foil activities were compared with those produced by activation in the standard flux reactor GLEEP.

Thermal neutron flux distributions per neutron per second from the $(d, t)$ source were measured at $0^{\circ}, 90^{\circ}$ and $135^{\circ}$ to the incident deuteron beam; for the $(d, d)$ source the maximum distribution was obtained for the $0^{\circ}$ direction only. For the $(d, t)$ source the $90^{\circ}$ direction was actually vertical and in this case the measurements were repeated with different depths of water in the moderator tank; these measurements gave information on the minimum moderator size necessary for the maximum peak flux.

Additional absolute flux measurements were made using bare and cadmiumcovered manganese foils (0.003" thick, $1 "$ dia., $12 \% \mathrm{Ni}$ and $88 \% \mathrm{Mn})$; the foil activities, as before, were compared with those produced by activation in the thermal neutron flux from a $1 \mathrm{Ci} \mathrm{Am}-\mathrm{Be}$ source in water. For this group of measurements, 
a small fission chamber in the moderator was used to monitor the neutron output from the accelerator source to avoid radiation damage to the surface-barrier detector; the counting rates of the fission and surface-barrier detectors were recorded simultaneously at both the beginning and end of an activation run and no changes in the condition of the fission chamber were made during a run.

In addition to the absolute flux measurements, relative ones were also made for the $90^{\circ}$ direction with a $(\mathrm{d}, \mathrm{t})$ source and for the $0^{\circ}$ direction with a (d,d) source; the scintillation counter recorded the fluxes and was normalised against a fission monitor. A simpler and more compact target assembly was used; it had no beam stops and, in consequence, there was an uncertainty of $\pm 1.1 \mathrm{~cm}$ in the position of the beam on the target.

\section{RESULTS AND DISCUSSION}

Figure 4 shows the measured thermal neutron flux distributions obtained for the two accelerator sources in the full water tank. Absolute flux distributions obtained for the $(d, t)$ source at $0^{\circ}, 90^{\circ}$ and $135^{\circ}$ to the incident deuterium beam were found to be identical to within the uncertainty of the measurements. Although the relative flux measurements were normalised to the absolute flux distributions for 8 sourcedetector separations in the range 6 to 42 $\mathrm{cm}$, to avoid confusion only the two points closest to the source are shown. Figure 5 gives the $(d, t)$ thermal neutron flux distributions (at $90^{\circ}$ ) obtained for different depths of water.

As already stated the large uncertainties in the $(d, d)$ measurements are a direct consequence of the reaction anisotropy and the variation of $d \omega^{\prime} / d \omega$ (eqn. 2) with deuter on energy. The main causes of uncertainty are target loading, cross section and slowing down data $( \pm 5 \%)$ and the beam composition $( \pm 7 \%)$. Of less importance are uncertainties produced by counting statistics and the detector efficiency $( \pm 2 \%)$, the position of the deuter on beam on the target $( \pm 7 \%)$, the accelerating voltage $( \pm 0.6 \%)$ and multiple scattering in the tirget $( \pm 0.5 \%)$. The overall R.M.S. uricertainty in the absolute flux per emitted neutron for the $(d, d)$ reaction is $\pm 9.0 \%$.

An overall uncertainty of $\pm 2.2 \%$ is ascribed to the (d,t) flux measurements; the main factors are the uncertainty produced by counting statistics and the detector efficiency $( \pm 2 \%)$, small uncertainties arising from the position of the beam on the targe $( \pm 0.7 \%)$ and other causes such as target loading (approximately $\pm 0.5 \%$ ).

A comparison has been made between the present absolute flux measurements and earlier relative flux distributions obtained for the $(d, t)[3]$ and $(d, d)[4]$ sources in water. These relative flux distributions were normalised to the absolute measurements by using an average multiplying factor and the deviation of the measured points from the normalised curve was obtained for each source at 7 radial points. For distances from the target in the range 6.6 to $35 \mathrm{~cm}$ the R.M.S. deviation is $\pm 4.8 \%$ for the $(d, t)$ case and $\pm 5.4 \%$ for the $\overline{(d, d)}$ one. The (d, d) relative distribution [4] was obtained with a $250 \mathrm{keV}$ deiteron beam and was measured in the $0^{\circ}$ direction.

As earlier work by Walker (5) had shown that age-diffusion theory gives a realistic prediction of the thermal neutron flux produced by a $\mathrm{Ra}-\mathrm{Be}$ source in water, it was considered useful to apply the theory to the present measurements. Using the age theory expressions of Wallace and Le Caine (ㅁ) , the absolute neutron flux per emitted source neutron was calculated for point fast sources of $(d, d)$ and $(d, t)$ neutrons. For the calculations, the $(d, t)$ neutrons were ascribed a single energy of $14 \mathrm{MeV}$; the $(d, d)$ source spectrum, taken from Marion and Fowler (7) and Beckurts and Wirtz (ㅇ) , covered the range 2.12 to $3.1 \mathrm{MeV}$. The ages to thermal energies for neutrons from the two sources were taken from Goldstein et al. (9). Figure 4 shows that while age 
theory is useful for predicting the thermal neutron fluxes from a $(d, d)$ source, it is quite inapplicable to the $(d, t)$ case.

As seen in figure 4 a Carlson 58 calculation gives a good fit to the $(d, t)$ thermal flux distribution. This calculation was made in the U.K.A.E.A. as part of a separate study and actually gives the thermal flux distribution for a point 14 $\mathrm{MeV}$ neutron source in oil (radius $75 \mathrm{~cm}$, composition $\mathrm{C}_{7} \mathrm{H}_{1} 3$ and density 0.865 $\mathrm{g} \mathrm{cm}^{-3}$ ) of hydrogen density $4.2 \%$ higher than that in water. Work by Cutforth (10) suggests that the peak thermal flux produced by a $14 \mathrm{MeV}$ source in water may be a little lower than in oil, but by no more than $5 \%$.

\section{ACKNOW LEDGMENTS}

\section{$:$}

We are grateful to the U.K.A.E.A., Harwell for financial support for one of us (L.H.) and to the Science Research Council, London for other support.

\section{REFERENCES}

1. T.R. Fewell. "An Evaluation of the Alpha Counting Technique for Deter mining 14-MeV Neutron Yields". Nucl. Inst. and Meth. 61, 61 (1968).

2. L. Ruby and R.B. Crawford. "Anisotropy Factors for the Determination of Total Neutron Yields from the $\mathrm{D}(\mathrm{d}, \mathrm{n})^{3} \mathrm{He}$ and $\mathrm{T}(\mathrm{d}, \mathrm{n})^{4} \mathrm{He}$ reaction ${ }^{\prime \prime}$. UCRL-10752 suppl. Lawrence Radiation Laboratory, Berkeley, California. (1963).

3. R.S. Caswell; R.F. Gabbard; D. W. Padgett; W. P. Doering. "Attenuation of 14.1 MeV Neutrons in Wat er". Nucl. Sci. and Eng. 2 , 143 (1957).

4. V. Spiegel Jr., D. W. Oliver and R.S. Caswell. "Age to Indium Resonance for D-D Neutrons in Water". Nucl. Sci. and Eng. $\underline{4} 546$ (1958).
5. J. Walker "Some Neutron Measurements with simulated ING targets"

AECL 2635, Chalk River Laboratory, Ontario, Canada (1966).

6. P.R. Wallace and J. Le Caine "Elementary Approximations in the Theory of Neutron Diffusion" AECL 336, Chalk River Laboratory, Ontario, Canada (1957).

7. J.B. Marion and J. L. Fowler. (Eds). "Fast Neutron Physics Part 1:

Techniques" P. 80, Int erscience

Publishers Inc.; New York (1960).

8. K.H. Beckurts and K. Wirtz. "Neutron Physics" 2nd Edn., P. 39, Springer-Varlag, Berlin (1964).

9. H. Goldstein; J.G. Sullivan, Jr.; R.R. Coveyou; W.E. Kinney and R.R. Bate. "Calculations of Neutron Age in $\mathrm{H}_{2} \mathrm{O}$ and Other Materials". ORNL 2639, Oak Ridge National Laboratory, Oak Ridge, Tennessee. (1961).

10. D.C. Cutforth "Neutron sources for Neutron Radiography" Ph.D. Thesis, Utah State Univer sity, Logan, Utah. (1969). 


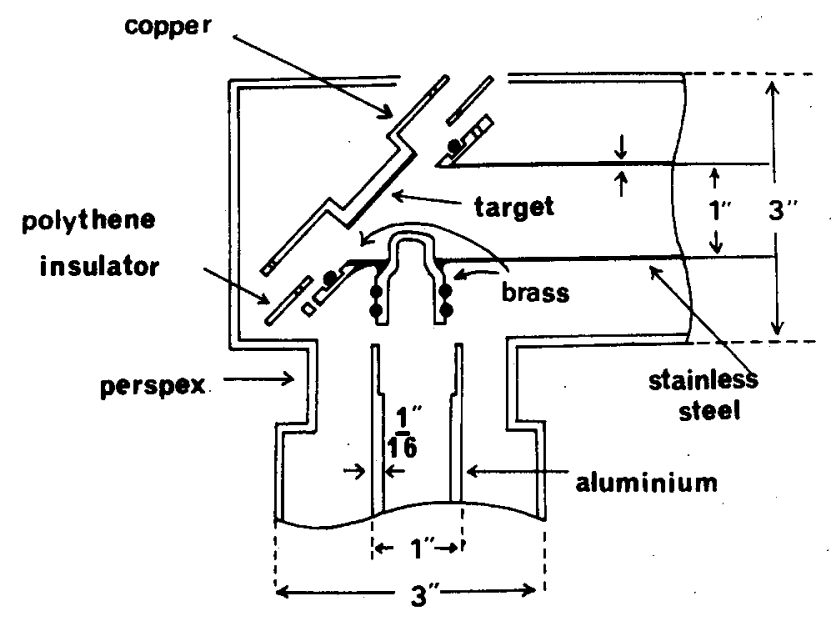

FIG.1. NEUTRON PRODUCING TARGET.

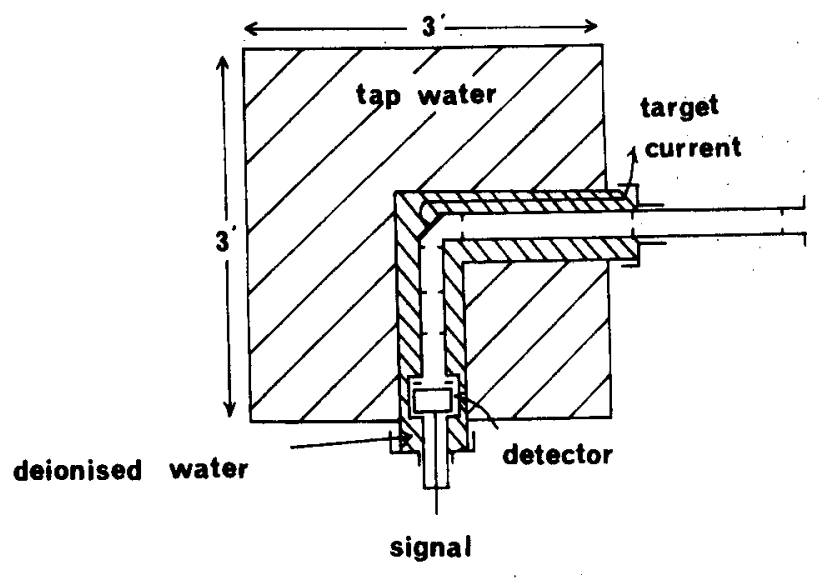

FIG. 2. ACCELERATOR TARGET ASSEMBLY IN MODERATOR TANK.

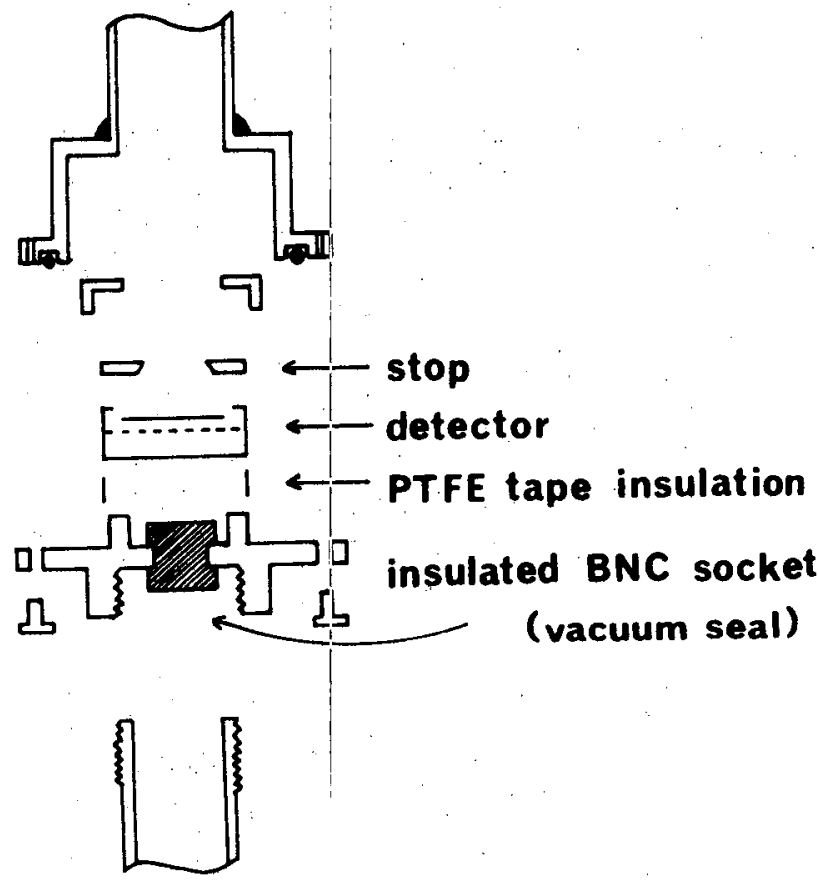

FIG 3. RECOIL : PARTICLE DETECTOR. 


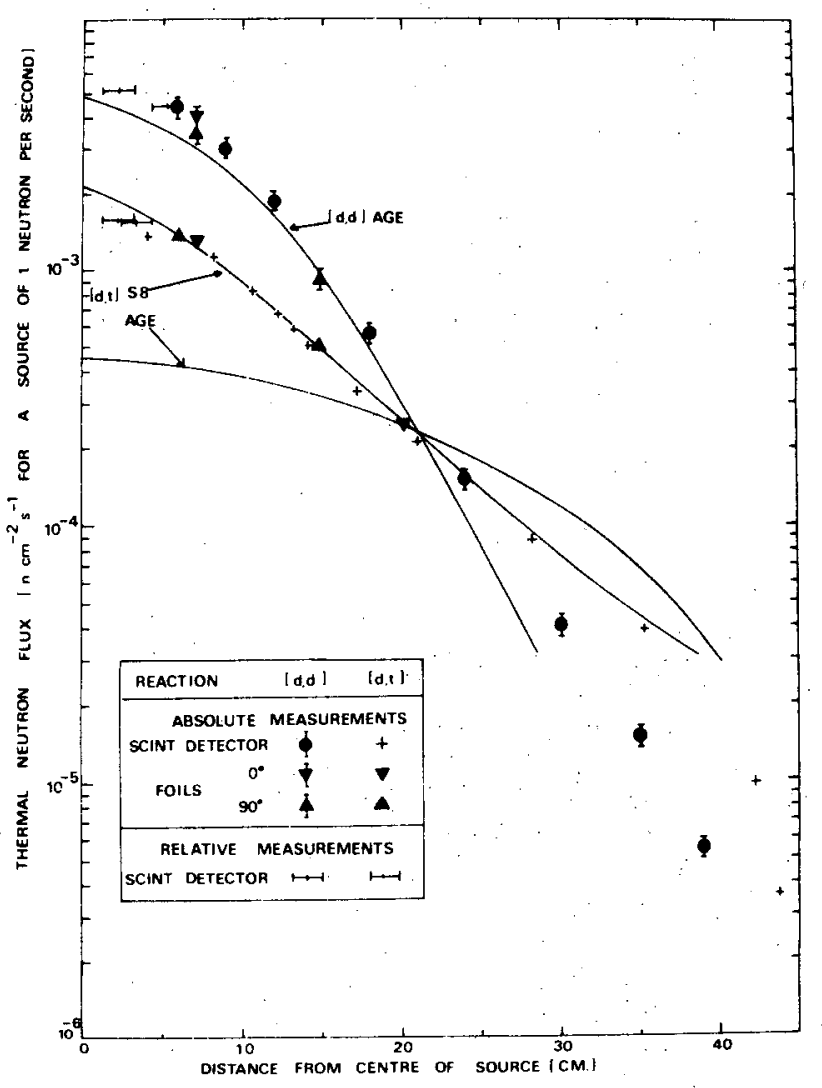

FIG 4 MEASURED AND CALCULATED tHERMAL NEUTRON FLUX DISTRIBUTIONS FOR OId,n]- AND $T(d, n)$ SOURCES.

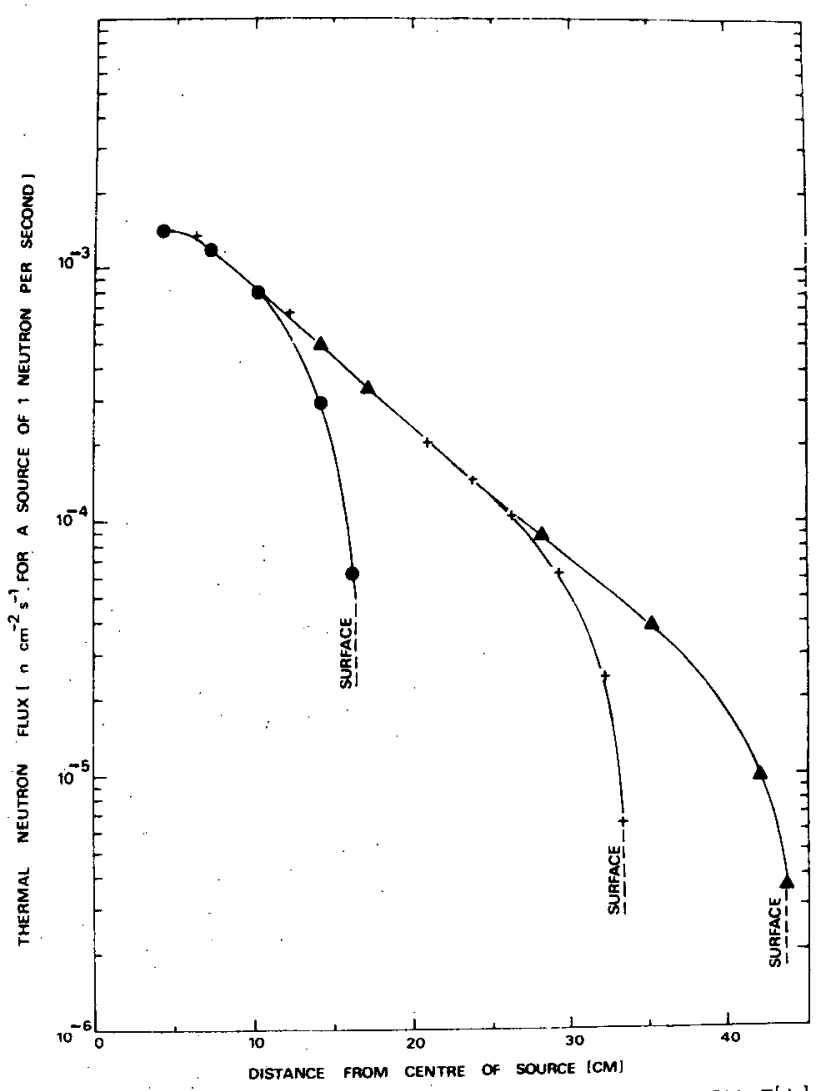

FIG 5. MEASURED THERMAL NEUTRON FLUX DISTRIBUTIONS FROM T[d, SOURCE WITH DIFFERENT DEPTHS OF WATER. 


\section{REACTOR AND NEUTRON PHYSICS APPLICATIONS OF CALIBRATED NEUTRON SOURCES}

A. De Volpi, K. Porges, R. Karam, W. Poenitz, A. Cox, M. Bretscher, W. C. Redman, and S. Carpenter

Argonne National Laboratory

Argonne, Illinois

Neutron sources calibrated to high accuracy have been employed in a number of reactor and neutron physics applications. Three categories of use can be distinguished: for integral measurements in critical facilities (such as $\bar{a}, \beta$ eff, and the normalization integral); directly for differential cross-section measurements ( $\nu$ and $\eta)$; and indirectly in other neutron physics experiments (branching ratios and flux calibration). Although the initial need for independent absolute neutron cápability arose from requirements of high accuracy in measurements of the neutron yield from fission $(\nu)$, both the standardized sources and the calibration facility have benefited other users.

Extensive efforts in verification of the various procedures leading to absolute neutron emission rates permit this Laboratory to claim calibration to $\pm 0.4 \%$. Among those independent efforts going into reducing the quoted error have been studies of parasitic absorption of high energy neutrons in sulfur and oxygen, thermal absorption in sulfur and hydrogen, neutron escape, source and cavity self-absorption, and absolute detection of manganese activity --- all carried out at differing concentrations and for sources having a wide range of neutron emission spectra.

\section{INTRODUCTION}

The availability of neutron sources calibrated to high accuracy has given rise to new or improved applications in reactor and neutron physics. For some of these uses, an accuracy of about $1 \%$ is adequate; for other applications, an accuracy near $1 / 2 \%$ is needed. We can distinguish, somewhat arbitrarily, three categories of use at Argonne: (1) integral measurements in zero-power reactors; (2) direct employment in differential cross-section measurements; and (3) indirect utilization in other neutron physics experiments.

The initial need at this Laboratory for absolute measurement capability arose from requirements of high accuracy in determination of the neutron yield from fission ( $v$ ) for fissile isotopes. With the availability of ${ }^{252} \mathrm{Cf}$ it became evident that the neutron yield from spontaneous fission would make an excellent universal reference. To detect fission neutrons on an absolute scale, a manganese bath calibration facility was set up at ANL at a time when worldwide confidence in neutron source standardization was about 2 or $3 \%$. Extensive efforts here and at other laboratories have now reduced this uncertainty to less than $1 \%$. With the aid of verification of all absolute steps in the calibration procedure, it has been possible

Work performed under the auspices of the U. S. Atomic Energy Commission. for this Laboratory to justify a total error of $\pm 0.4 \%$, representative of the state-ofthe-art at the moment (I).

Sources which emit neutrons in different energy ranges are Idesignated for different applications. Sone of the best calibrated sources are the International Standard NRC 200-1 $\operatorname{Ra}-B e(\alpha, n)$ and the U. S. National Bureau of Standards $\operatorname{Ra}-\operatorname{Be}(\gamma, n)$. These neutron standards have been readily available for intercomparison on an international basis. The photoneutron source produces a soft spectrum with average energy of about $100 \mathrm{keV}$ (2), and the alpha-reaction source has a hard spectrum with many neutrons in the MeV region. The fission spectrum falls somewhat in between.

When accuracies better than a few percent are desired, the manganese bath facility requires special attention to energy-dependent corrections. Neutron escape, source self-capture, parisitic capture in components of the solution, and other effects require compensation which can vary by one or two percent, depending on the neutron spectrum. For this reason, inany of these energy-dependent factors havel been independently investigated at Argonn' for several sources with differing emission spectra.

${ }^{252} \mathrm{Cf}$ has soine special advantages over other sources, Because of its high specific emission rate, it can be considered a true point source. Its low gamma radiation 
fraction allows relatively safe handing and minimal encapsulation. The combination of minimum encapsulation and mean energy of about $2.0 \mathrm{MeV}$ cause little self-absorption. The most energetic neutrons from spontaneous fission do not lead to excessive leakage from the bath, and the neutrons are generally below thresholds for parasitic capture in sulfur and oxygen. As a result, despite the relatively short-life (which we have measured to be $2.621 \pm 0.006 \mathrm{y}(3)),{ }^{252} \mathrm{Cf}$ is the most convenient source susceptible to the best attainable accuracy.

\section{MANGANESE BATH CALIBRATION}

Calibration of the various neutron sources at the Argonne Reactor Physics Laboratory has been fully described elsewhere (I). Thus only an outline of the calibration procedure will be given here with the addition of some recent neutron metrology developments.

The primary system for calibration consists of a nominal $100-\mathrm{cm}$ diameter sphere filled with $\mathrm{MnSO}_{4}$ dissolyed in distilled water -- the so-called manganese bath. A beam tube runs through the center of the sphere, allowing convenient insertion of sources. Mechanical and electronic features of the system were described at the 2nd Conference on Neutron Cross Sections and Technology $(\underline{4})$. When a source is introduced to the center of sphere, neutrons are moderated by the aqueous solution and captured predominantly in manganese and hydrogen. The solution is continuously circulated by pumping through an annular sodium iodide crystal, where detection of the ${ }^{56} \mathrm{Mn}$ decay products takes place. The crystal is usually located in a well-shielded container at least 10 or 20 feet from the bath.

Automatic and continuous collection of data allow full coverage of the two-day irradiation to saturation (half-life of ${ }^{56} \mathrm{Mn}$ is just over 2.5 hours) and subsequent decay. A computer program takes care of all decay and pumping corrections.

The detection efficiency of the sodium iodide counter can be calibrated by comparing aliquots of reactor-irradiated manganese in the counter and in an absolute coincidence system. A limited international comparison of six laboratories, including Argonne, resulted in a range of $0.6 \%$ and an $\mathrm{rms}$ error of $\pm 0.1 \%$ for absolute calibration of a ${ }^{56} \mathrm{Mn}$ sample sent out by the National Physical Laboratory of England (5).
Correction for thermal neutron losses to hydrogen and sulfur, for resonance absorption in manganese, and for high energy neutron capture in sulfur and oxygen are based on experimental results obtained at Argonne and els ewhere (I).

Two additional correction categories recently studied take into account neutron source self-absorption and cavity-wall absorption, as well as neutron escape from the bath. Inconsistencies in published data and in calculations of these effects led to the necessity of direct experimental attack.

Even nearly massless sources require some encapsulation. In addition, to measure the emission rate these capsules are generally placed in an air-filled cavity at the center of the bath. All of these materials -m- source and cavity walls --- absorb thermal neutrons which pass through the center. The relative number of neutrons intercepted near the origin is strongly dependent upon the initial energy distribution of the source. Thus, we made a series of measurements in a variety of cavity and source geometries to directly determine the absorption effects for neutron emitters ranging from $24 \mathrm{keV}$ up to several $\mathrm{MeV}$ (2).

The number of neutrons which escape from the bath is strongly dependent upon the uncollided flux. Such numbers are difficult to calculate from theory with any accuracy; so again a direct experimental approach was ut1lized (6). The leakage from a small sphere ( $60 \mathrm{~cm}$ diameter) was compared with the measured rate from the $100-\mathrm{cm}$ bath. By iteration, the escape fraction was determined for energetic neutron emitters in both dilute and concentrated solutions of manganous sulfate.

As a result of these supplementary efforts, the neutron rate measurements can be considered to be of increased reliability with an attendant improved estimate of the systematic error component.

With the manganese bath apparatus we have calibrated the following types of sources: $\operatorname{Sb}-B e(\gamma, n), \operatorname{Na}-B e(\gamma, n), \operatorname{Ra}-B e(\gamma, n)$, ${ }^{252} \mathrm{Cf}, \operatorname{Ra}-\operatorname{Be}(\alpha, n)$, and $\mathrm{Am}-\operatorname{Be}(\alpha, n)$. The californium has been in the form of small sealed capsules and large fission fragment detectors. In addition, the manganese bath and vanadium bath (about l-hour irradiation cycle) (4) have been applied to measurement of accelerator and reactor produced neutron 
beams.

\section{APPLICATIONS}

In view of the variety offered in neutron energies, emission rates, and source configurations, a broad range of applications has developed. Once the absolute calibration capability was established, some of the applications became easier to accomplish to the required accuracy. Examples of calibrated source use will be divided into two categories: (I) integral measurements in zero-power reactors and (2) neutron physics.

\section{INTEGRAL MEASUREMENTS IN ZERO-POWER REACTORS}

\section{Capture-to-Fission Ratio Measurements} The use of a calibrated ${ }^{252} \mathrm{Cf}$ spontaneous fission neutron source for integral measurements of capture-to-fission ratios $(\bar{\alpha})$ in low flux reactors was first proposed by Redman (7). Derived from first order perturbation theory, the equation which forms the basis of this reactivity-reaction rate method for $\bar{\alpha}$ determinations is

$$
\begin{aligned}
& (I+\bar{\alpha})=\left[\mathrm{R}_{a}(A)\left[\rho / R_{f}\right)\left(1-\rho_{s} / \rho\right)\right. \\
& \left.\left.-(\bar{\nu} / S) \rho^{\prime}\left(C_{f}\right) \bar{\phi}_{f}^{*} / \bar{\phi}_{f}^{*}(C f)\right] \bar{\phi}_{a}^{*}(A) / \bar{\phi}_{a}^{*}\right] \\
& /\left(\rho(A)\left[1-\rho_{s}(A) / \rho(A)\right]\right)
\end{aligned}
$$

The measured quantities are the relative reactivities of the fissile material $\rho$ and the reference absorber (Mn for thermal, ${ }^{6} \mathrm{Li}$ for fast criticals) $\rho(A)$; the relative apparent reactivity of a californium source $\rho^{\prime}(\mathrm{Cf})$; the absolute fission and absorption rates $R_{f}$ and $R_{a}(A)$; and the neutron emission rate $S$ from the californium source. The remaining factors in this equation must be calculated from fundamental nuclear data. $\bar{v}$ is the average number of neutrons emitted per fission in the sample. The term $\left(1-p_{s} / p\right)$ corrects for the scattering contribution to the total reactivity; $\bar{\phi}_{\mathrm{a}}^{*}(\mathrm{~A}) / \bar{\phi}_{\mathrm{a}}^{*}$ accounts for the small difference in neutron importance between neutrons absorbed in the reference sample and those absorbed in fissi.le material; while $\bar{\phi}_{f}^{*} / \bar{\phi}_{f}^{*}(\mathrm{Cf})$ corrects for the difference in importance between neutrons from induced fission in the sample and from spontaneous fission in the source. These correction factors are generally small, but do depend somewhat on the neutron spectrum used in the calculation.

Equation (1) applies to measurements made at the center of the reactor where flux gradients are essentially zero. For the equation to be valid, the measured values of $R_{f}, R_{a}(A)$ and $\rho^{\prime}\left(C f_{f}^{\prime}\right)$ must be normalized to a common power level. This method has been successfully applied to the measurement of $25^{3} \mathrm{U} \bar{\alpha}$ in thermal $(8)$ and epicadmium ( 9 ) spectra and of ${ }^{239} \mathrm{Pu}, 23{ }^{5} \mathrm{U}$ and ${ }^{238_{\mathrm{U}}}$ in $\mathrm{a}$ variety of fast reactor spectra (10,11).

Beff Measurements in Two Fast Reactor Crit cal Assemblies As part of a continuing effort to study the experimental accuracy of small sample reactivity measurements; $\beta$ eff has been measured in two cores. One core had primarily U-235 fissions and the other Pu-239 fissions.

The error in the calculated value of Beff in any one isotope relative to another is not large and does not affect reactivity calibrations to an important extent. The reactivity reported in cents or inhours should therefore not be in exror more than 1 or $2 \%$ and nowhere near the $30 \%$ difference found in certain cores when compared with calculations.

Since the experimental results for the small sample reactivities are in cents or inhours and the calculated value is in $\Delta \mathrm{k}_{e}$, an incorrect value of $\beta_{\text {eff }}$ will produce $a$ difference in the comparison of the same percentage as the error in $\beta_{\text {eff. }}$. The purpose of these experiments was to measure Beff in two cores which were particularly simple in that most of the fissions occurred in only one isotope. Insofar that $B_{\text {eff }}$ is not greatly different from $B$ it is expected that any major uncertainty is not particularly sensitive to core but only to isotope.

An experiment is performed to measure the neutron production rate relative to any instrument reading used to measure the power level. This is done with absolute fission counters for each isotope in the core producing significant fissions.

When $\phi^{+}$is the average importance of a source neutron at the source position (center), $\phi_{f}^{+}$is the average importance of a fission neutron in the reactor and $S$ is the absolute source strength, a relationship to find Beff may be derived. The ratio of importance is determined from the measured fission distribution, with a calculated correction since the energy distribution of source (Cf-252) neutron is not exactly the same as those from U-235 or Pu-239.

The results are then used to find 


$$
\beta_{\text {eff }}=\frac{S \phi^{+}}{V \vee F \phi_{f}^{+}} \text {, }
$$

where $\mathrm{V}$ is the apparent reactivity of the calibrated neutron source $S$ and the reactor fission rate is $F$.

The absolute source strength, S, for the smalier source was measured using the manganese-sulfate vath solution. A larger source strength was compared to the smaller by both a manganese sulfate bath and neutron counter using a liquid proton recoil detector.

Measurements of the Normalization Integral and the Spatial Distribution of the Importance of Fission Neutrons (12) The denominator of the perturbation expression, which is the importance-weighted production of neutrons throughout a reactor, is commonly referred to as the normalization integral (N.I.). If the calculated value of N.I. is in error because of configurational effects such as irregular boundaries, then the calculated reactivity effects such as sodium void, Doppler, and danger coefficients would also be in error regardless of whether or not the basic cross sections are at fault.

Core zoning is a very useful technique whereby the total fuel inventory required to study a large fast reactor is substantially reduced. The success of the use of zoned cores to establish characteristics of fullsize systems depends to a large extent on the accuracy with which N.I. can be determined.

Measurements of N.I. and the importance of fission neutrons provide additional and valuable indices with which basic data and analytical tools are checked.

If a constant point neutron source that does not absorb any neutrons is introduced at position $r$ into a reactor that is exactly critical, the power level increases linearly with time. The increase in power level may be equated to an apparent reactivity whose value depends on the power level at which the source was introduced.

The normalization integral was measured in Argonne's ZPR-6 and ZPR-9. Additionally, the spatial distribution of the importance of fission neutrons was measured in ZPR-6.
The neutron source used in the measurements was a microgram quantity of ${ }^{252} \mathrm{Cf}$ calibrated by the manganese bath technique. The container was driven with a stepping motor attached to a screw lead with practically no backlash. Positioning was reproducible to within $0.002 \mathrm{~cm}$. The reactivity effect of the container without the source but with a glass ampule similar to the source ampule was measured with a calibrated fine autorod, by determining the timeaveraged position of the autorod which maintained constant power level, for each radial (or axial) position of the sample holder. The dummy ampule was then replaced with the ${ }^{252} \mathrm{Cf}$ ampule. The apparent reactivity associated with the ${ }^{252} \mathrm{Cf}$ source at a particular position was then measured with a calibrated control rod while maintaining the same control conditions previously arrived at, i.e., the same power level and the corresponding time-averaged position of the autorod now held constant by manual operation.

The measurement of the importance of fission neutrons was accomplished by monitoring the linear rise of the power level due to only the spontaneous fission of the ${ }^{252} \mathrm{Cf}$ source at a particular point within the system. A chi-squared goodness-of-fit test of the data showed that a straight line approximates very well the distribution of the data. Perturbation denominators measured with a calibrated ${ }^{252} \mathrm{Cf}$ neutron source have been found to be in favorable agreement with those obtained by other techniques (13).

\section{NEUTRON PHYSICS}

Number of Neutrons Per Fission from $252 \mathrm{Cf}$ (14) In order to determine the neutron yields for various fissile isotopes, one of the most direct methods developed has been to make separate measurements of both the neutron rates and the fission rates for a fissile source in which fission is induced by an external beam. The manganese bath was used to measure the neutron emission rate, and the fission rate was found from calibrated fission counters. Because of background problems and difficulties in absolute fission fragment detection, these experiments have not achieved better than $1-1 / 2 \%$ accuracy.

The accumulation of adequate quantities of spontaneous fission. ${ }^{252} \mathrm{Cf}$ led to a twostep procedure of better accuracy. Some experimenters were well equipped to measure neutron yields from fissile isotopes relative 
to the yield from ${ }^{252} \mathrm{Cf}$. And other experimenters through a variety of techniques have been able to contribute absolute values of the yield from ${ }^{252} \mathrm{Cf}$.

The technique at Argonne made use of a manganese bath for the neutron assay, and this was the primary reason why the equipment was originally set up. Measurement of neutrons for this experiment differs little from calibration of other sources. The main. difference is that the californium is contained in a fission counter with a supporting tube.

The fission rate for the yield experiment was determined by absolute promptcoincidence detection of fission fragments and neutrons coming from the spontaneous activity. It was possible to find the fission rate with an uncertainty in the order of $0.1 \%$; thus the final error on the total neutron yield ( $3.729 \pm 0.015$ neutrons/ fission) is dominated by the $0.4 \%$ uncertainty in the neutron assay.

Neutron Flux Measurements. The manganese bath which has been used to calibrate neutron sources is also adaptable to measuring external beams of neutrons with corresponding accuracy. The procedure consists of collimating the beam (which may come either from a reactor or an accelerator) and introducing the beam to the center of the manganese bath via a re-entrant hole. Beams ranging in energy from thermal to the fission spectrum may be evaluated in this manner. Calibration of the bath need not occur on each use; instead, neutron sources previously calibrated under better conditions are brought to the site of the beam experment, and the bath is thus standardized. Frequently, special corrections must be applied for beam effects in the re-entrant hole.

Two experiments conducted at the Argonne CP -5 reactor thermal column have been the measurements of neutrons/fission ( $v$ ) and neutrons/absorption $(n)$ for ${ }^{235} \mathrm{U}$ and ${ }^{239} \mathrm{Pu}$.

The branching ratios for ${ }^{7} \mathrm{Be},{ }^{51} \mathrm{Cr}$, and $65 \mathrm{Zn}$ may also be determined with an indirect procedure using the manganese bath. Protons on beryllium, vanadium, and copper targets produce neutrons which can be detected by a bath surrounding the target in nearly 4-pi geometry. The decay of the associated activity from each of these reactions is measurable by absolute gamma detection, which provides the other property needed to determine the branching ratio. In practice, the vanadium bath .... which has a quicker response (4) -.. has been used instead of the manganese bath. Again, calibration was carried out by transporting the standard neutron sources to the experimental area.

Another recent measurement with the vanadium bath has been to determine the yield of thick targets of $\mathrm{Be}$ and $\mathrm{Li}$ subjected to beams of proton's and deuterons.

Finally, one must include the calibration -... with standardized neutron sources -- of various detectors which are used for neutron flux measurement. Long counters, for example, which may be calibrated as to shape by other means, have been absolutely calibrated by making use of the range of neutrons available from $\mathrm{Sb}-\mathrm{Be}(\gamma, n),{ }^{252} \mathrm{Cf}$, and other sources.

\section{REFERENCES}

1. A. De Volpi and K. G. Porges, "Absolute Calibration of Neutron Sources Having a Wide Range cf Emission Spectra." Metrologia 5, 128 (1969).

2. A. De Volpi, "Thermal Neutron Absorption in Neutron Sources and Cavity Walls Centered in Mciderating Solutions." (Submitted for Publication).

3. A. De Volpi ard K. G. Porges, " $252 \mathrm{Cf}$ Half-Life by Neutron Counting:

Revision." Incirg. Nucl. Chem. Letters 5, 699 (1969).

4. A. De Volpi arid K. G. Porges, "Advances in Accurate Fe.st Neutron Detection." Second Conf. on Neutron Cross Sections and Technology, NBS Spec. Publ. 299, Vol. I, p. 312? (1968).

5. A. De Volpi (compilor), "International Comparison of $56 \mathrm{Mn}$ Activity in 1968." Metrologia 6, 65 (1970).

6. A De Volpi, "Neutron Escape from WaterModerated Tanks." (J. Nucl. Energy, in press).

7. W. C. Redman, "Pile Oscillator Determination of Capture-to-Fission Ratio." TID-17738, USHEC (1962).

8. W. C. Redman and M. M. Bretscher, "Direct Determination of $235 \mathrm{U}$ Capture-to-Fission Ratio in a Zero-Power Reactor." Nucl. Sci. Eng. 27, 34 (1967). 


\section{REFERENCES (Cont'd.)}

9. W. C. Redman and M. M. Bretscher, "Lowm Flux Measurement of $235 \mathrm{U}$ Epi Cadmium Capture-to-Fission Ratio." Nucl. Sci. Eng. 31, 247 (1968).

10. M. M. Bretscher and W. C. Redman, "Iow Flux Measurements of $23{ }^{2} \mathrm{Pu}$ and ${ }^{235} \mathrm{U}$ Capture-to-Fission Ratios in a Fast Reactor Spectrum." Nucl. Sci. Eng. 39, 368 (1970).

11. M. M. Bretscher, J. M. Gasidlo and W. C. Redman, "Comparison of Measured and Calculated Capture-to-Fission Ratios in a Soft Spectrum Fast Critical." Trans. Am. Nucl. Soc. 13, 89 (1970).

12. R. A. Karam, "Measurements of the Normalization Integral and the Spatial Distribution of the Importance of Fission Neutrons." Nucl. Sci. Eng. 37, 192 (1969).

13. W. C. Redman and M. M. Bretscher, "Experimental Determination of the Perturbation Denominator in Fast Critical Assemblies." Nucl. Sci. Eng. (to be published).

14. A. De Volpi and K. G. Porges, "Neutron Yield of ${ }^{252} \mathrm{Cf}$ Based on Absolute Measurements of the Neutron Rate Fission Rate." Phys. Rev. 틀. 683 (1970). 
THE NEUTRON SPECTRUM FROM A RADIOACTIVE 210 Po-Li $(a, n)$ SOURCE

\author{
Klaus W. Geiger \\ Len van der Zwan \\ Division of Physics \\ National Research Council \\ Ottawa, Canada
}

\begin{abstract}
The neutron spectrum of a ${ }^{210} \mathrm{Po}-\mathrm{Li}(a, n)$ sciurce was determined by $(i)$ a ${ }^{3} \mathrm{He}$ proportional counter, (ii) a stilbene scintillation detector, (iii) a calculation using the ${ }^{7} \mathrm{Li}(a, n){ }^{10} \mathrm{~B}$ differential cross section as measurecl with an accelerator beam. The spectrum rises to a maximum at $0.1 \mathrm{MeV}$ and then smocithly decreases to an endpoint at $1.32 \mathrm{MeV}$. It is believed that the calculated spectrum is the most accurate one but on the other hand it did not include the effect from the sceittering of neutrons within the source capsule. The spectrum taken with the ${ }^{3} \mathrm{He}$ counter needed rather large corrections for geometric effects; the stilbene detector did not pernit to establish the spectrum for neutron energies below $0.5 \mathrm{MeV}$.
\end{abstract}

\section{INTRODUCTION}

The neutron spectra of commercially available radioactive neutron sources are by now quite well known except for the spectrum from $\operatorname{Li}(\alpha, \mathrm{n})$ sources. Compared to other $(\alpha, n)$ type sources, this spectrum is of considerably lower energy, apparently peaking in the $100 \mathrm{keV}$ region and extending to 1.3 $\mathrm{MeV}(1,2)$. Although the neutron yield of a Li $(\alpha, \bar{n})$ source is lower by a factor of about 80 than that of a $B e(\alpha, n)$ source, the different spectrum makes a $\operatorname{Li}(\alpha, n)$ source a good candidate for applications in this energy range. The spectrum is difficult to determine because few practical neutron spectrometers exist for the energy range in question. A ${ }^{3} \mathrm{He}$ proportional counter as well as a stilbene scintillation detector were evaluated, using a commercial neutron source* which contained $4.6 \mathrm{Ci}$ of $210_{\mathrm{Po}}$ together with $\mathrm{LiH}$ as target material. In addition, a calculation of the spectrum was carried out, based on differential $\mathrm{Li}(\alpha, \mathrm{n})$ cross sections determined experimentally with the $\alpha$-beam from an accelerator.

\section{THE ${ }^{3}$ HE PROPORTIONAL COUNTER}

The proportional counter uses the reaction ${ }^{3} \mathrm{He}(\mathrm{n}, \mathrm{p}){ }^{3} \mathrm{H}, \mathrm{Q}=0.764 \mathrm{MeV}$. The neutron energy is simply obtained by subtracting

\footnotetext{
The Radiochemical Centre, Amersham, X-3
} capsule. the reaction Q-value from the total energy released. In conjunction with the ${ }^{3} \mathrm{He}(\mathrm{n}, \alpha)$ cross section which varies smoothly within the energy range of interest (3), the source spectrum can be obtained. A limitation arises from competing elastic neutron scattering on ${ }^{3} \mathrm{He}$. The ${ }^{3} \mathrm{He}$ recoils have a continuous energy distribution from $E=0$ to $E=0.75 E_{n}$. Neutrons of more than $1 \mathrm{MeV}$ will therefore produce recoils with energies above the Q-valiue of the reaction. Fortunately, this effiect does not lead to a major correction here since the source spectrum is of low intensity above $1 \mathrm{MeV}$, adding relatively few events to the low energy component. A commercial 3 He proportional counter ${ }^{*}$ of $15.5 \mathrm{~cm}$ active length and $2.4 \mathrm{~cm}$ diameter was selected. To keep wall and end effects within acceptable limits a gas filling of $4 \mathrm{~atm}$ of ${ }^{3} \mathrm{He}$ with an admixture of 2 atm of $\mathrm{Kr}$ was chosen so as to provide increased stopping power for protons. For the thermal neutron peak a resolution at FWHM of $5 \%$ was obtained which compares favorably with an intrinsic resolution of about $2 \%$ (4). When making source measurements the counter was covered with cadmium so as to reduce the thermal peak priduced by neutrons scattered from the surrouidings.

Figure 1 shows the measured source spectrum together with the spectrum corrected for ${ }^{3} \mathrm{He}$ recoils and for geometric effects (5). The geometric correction is

* Texas Nuclear Corporation 
the major one; it is seen in the figure that it nearly doubles the relative number of neutrons detected at high energies. The errors in the corrected spectrum are believed to be about $+5 \%$. For comparison, the spectrum measured by Vialettes and Le Thanh (1) using a similar counter, is also shown.

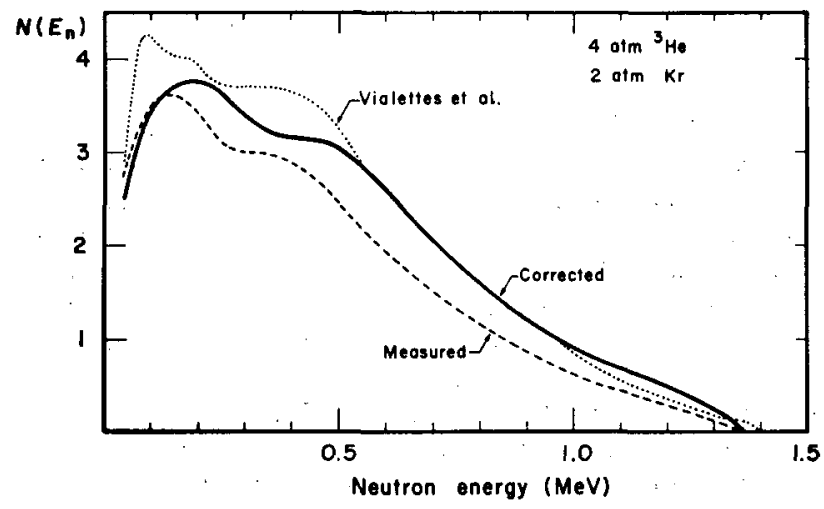

FIG. 1 The measured and correçted spectra of a Po-LiH source using a ${ }_{\mathrm{He}}^{\mathrm{He}}$ proportional counter.

\section{THE STILBENE SPECTROMETER}

Principally these measurements were carried out to demonstrate the low energy limit for this type of detector. A stilbene crystal of $2.5 \mathrm{~cm}$ diameter and $1.2 \mathrm{~cm}$ thickness was used, mounted on a RCA 8575 photomultiplier. The techniques for the unfolding of the proton recoil spectra and for the discrimination against $\gamma$-rays by pulse shape selection have been described elsewhere $(\underline{6}, 7)$. The method requires calibration with monoenergetic neutrons which were obtained from the ${ }^{7} \mathrm{Li}(\alpha, \mathrm{n}) 10 \mathrm{~B}$ reaction on a thin target.

The spectrum of the source as well as the calibration line are shown in Fig. 2 . Below $0.45 \mathrm{MeV}$ the intensity increases rapidly; this is caused by failure of the pulse shape discriminator to reject all. pulses caused by $\gamma$-radiation". The cathode sensitivity of the photomuitipiier-stilbene system was found to be 1.5 photoectrons $/ \mathrm{keV}$ electron energy. Since a $500 \mathrm{keV}$ proton produces a light pulse corresponding to an electron energy of only $57 \mathrm{keV}$, it is evident $\therefore$ : that the rise time of the integrated pulse ceases to be well defined. Quantitative measurements on the rise time spectrum are difficult, but it was estimated that the error in the measured spectrum is about $+15 \%$ at $500 \mathrm{keV}$, decreasing to the width of the drawn curve at $1 \mathrm{MeV}$. It is worth noting that Goryachev et al. (8) succeeded to measure spectra by this method down to $0.2-0.3$ $\mathrm{MeV}$, although substantial lead shielding was needed to reduce $\gamma$-radiation to acceptable levels. At this low energy, an average of only 10 photoelectrons are available in our system to define the pulse shape. The authors in (8) used a FEU-13 photomultiplier but its photocathode efficiency is not quoted.

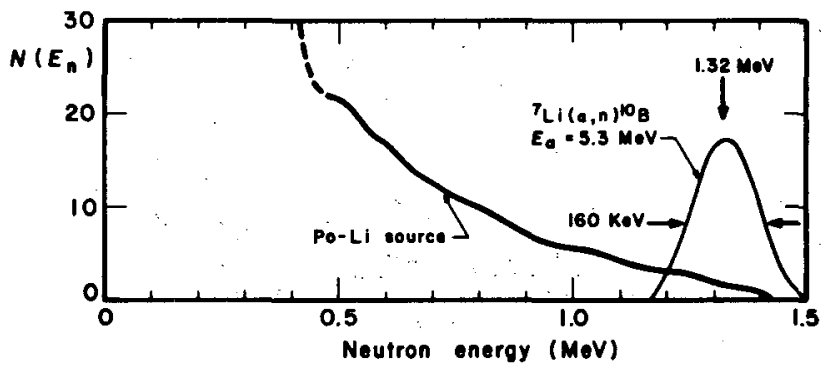

FIG. 2 The source spectrum as measured with a stilbene detector. The spectrum obtained with $1.32 \mathrm{MeV}$ neutrons, needed for calibration, is also shown.

\section{CALCULATION OF THE SPECTRUM}

When the differential cross sections, $\sigma(\theta)$, for the $\alpha$ energy range encountered within the source are known, it is possible to calculate the neutron spectrum. For the ${ }^{7} \mathrm{Li}(\alpha, n){ }^{10}{ }_{\mathrm{B}}$ reaction, only the neutron production cross sections were known $(9,10)$; these had to be complemented by neutron angular distribution measurements on a thin Li target using the N.R.C. Van de Graaff. The kinematics of the reaction is shown in Fig. 3. For the energy range of the $\alpha-$ particles occuring within the source, only neutrons to the "ground state of $10_{\mathrm{B}}$ are produced. The neutron' energy is therefore uniquely defined and the angular distribution could be measured with a flat response detector, in the present case a "Precision Long Counter" (11). The results after normalization to (9.,10) are shown in Fig. 4 . Corrections for neutron scattering in the target assembly causes the large errors at back angles. The curves drawn in Fig. 4 were obtained by a least-squares fit with Legendre polynomials up to the second degree. For the lowest $\alpha$-energy used the fit is compatible with an isotropic distribution in the center of mass system. The neutron energies become double valued below $E_{\alpha}=4.65$ $\mathrm{MeV}$ which precluded measurements by. the 
present method. An isotropic distribution has been assumed from $E_{\alpha}=4.78 \mathrm{MeV}$ down to the reaction threshold.

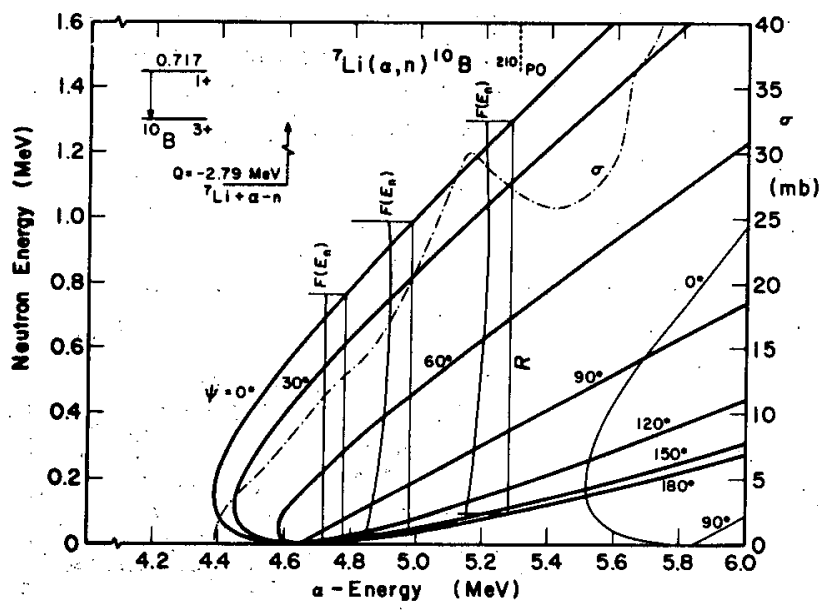

FIG
$7_{\mathrm{LI}(\alpha, n)}^{3}$ Energy diagram for the
$\mathrm{B}$ reaction. $\psi$ is the laboratory angle for the outgoing neutrons. $R$ is the range of neutron energies permitted at energy $E_{\alpha}$. Neutron spectra for three $\alpha$-energies are also shown. $\sigma$ is the neutron production cross section of $(\underline{9}, \underline{10})$.

Because of the slowing down of the 5.30 $\mathrm{MeV}$ ofparticles from the ${ }^{210} \mathrm{Po}$ in the target

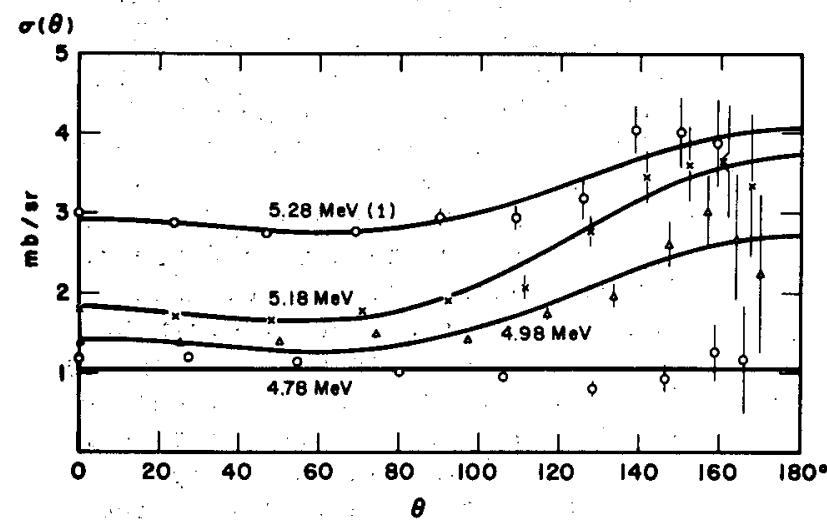

FIG. 4 The experimental differential cross sections, normalized to $(\underline{9}, \underline{10})$ as functions of the center-of-mass angle $\theta$. For clarity, the curve for $\mathrm{E}_{\alpha}=5.28 \mathrm{MeV}$ has been raised by $1 \mathrm{mb} / \mathrm{sr}$.

material of the source, $\alpha$-particles of all energies up the maximum are present. Also there is no preferred direction of the $\alpha$ particles. These effects tend to smear out structure in a source neutron spectrum which would otherwise be found with thin targets. For $\alpha$-particles of fixed energy $E_{\alpha}$ the number of neutrons produced within the source of energy between $E_{n}$ and $E_{n}+d E_{n}$ is (12):

$$
F\left(E_{n}\right) d E_{n}=A 4 \pi \frac{\sigma\left(E_{n}\right)}{\varepsilon R} d E_{n}
$$

The factor A is a constant which depends on the composition of the source material and $\varepsilon$ is the stopping cross section of this material at $E_{\alpha}$. The cross section $\sigma\left(E_{n}\right)$ is equivalent to $\sigma(\theta)$ pf Fig. 4 but expressed as a function of $E_{n}$ instead of $\theta ; R$ is the range of the possible neutron energies at $E_{\alpha}$ as shown in Fig. 3! The figure also includes examples of neutron spectra for three energies $\mathrm{E}_{\alpha}$. An isptropic neutron distribution in the center-o -mass system results in a neutron spectrun of rectangular shape. By interpolation of the differential cross sections of Fig. 4 in steps of $\Delta E_{\alpha}=10 \mathrm{keV}$, a family of spectra was obtained, which after summation resulis in the source neutron spectrum of Fig 5. If the angular distributions were assumed to be isotropic throughout the a-energy range, the dashed curve is obtained.

\section{DISCUSSION}

The three spectra are compared in Fig. 5. Except for ninor variations, the two directly measured spectra agree fairly well with each other. The somewhat lower resolution of the stilbene spectrometer is evident from the fact that the neutron energy extends to $1.42 \mathrm{MeV}$; the kinetics of the reaction allows a maximum energy of 1.32 $\mathrm{MeV}$. The calculated spectrum decreases smoothly with ircreasing energy from its rather sharp peak at $100 \mathrm{keV}$. In particular it shows some bilging in the neighborhood of $1 \mathrm{MeV}$, a region where the directly measured spectra exhibit a slight dip. Clustered polonium with consequent self-absorption of $\alpha$-particles could produce such dip, this however is rather unlikely for the two following reasons:

(a) The polonium metal is usually evaporated into the target material and would therefore not cluster to a great extent.

(b) Clustering affects predominantly 
the neutron yield and not so much the spectral shape (12). The yield of our Po-LiH source (in neutrons per $10^{6} \alpha$-particles) was 0.91 , compared to a calculated yield of 1.09 . The source we had used was therefore a rather efficient one.

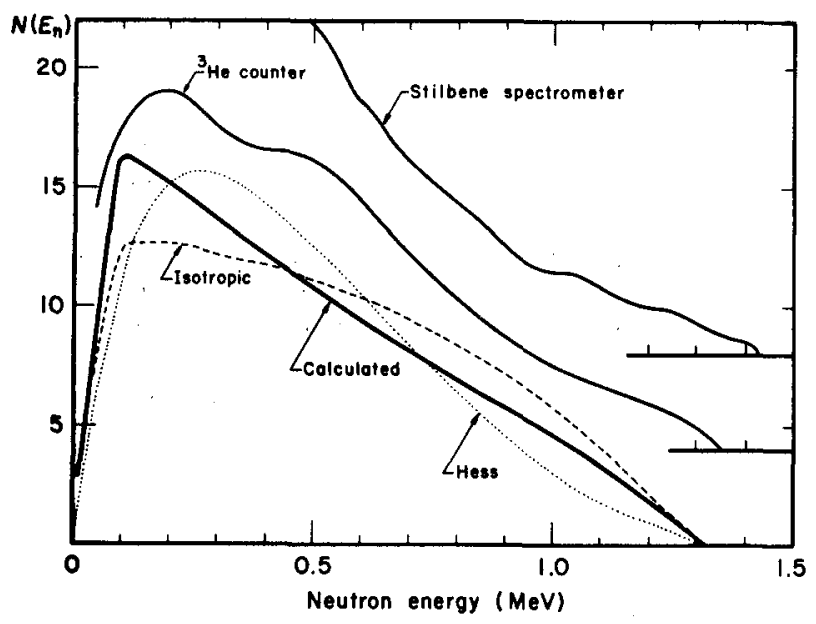

spectra.

It is quite possible that the stainless steel source capsule affects the neutron spectrum to some extent. Many resonances appear in the neutron cross section curves of the elements which make up the components of stainless stee1; this may explain some of the differences between the calculated and the directly measured spectra.

The frequently quoted Po-Li source spectrum calculated by Hess. (2) using an isotropic angular distribution is also shown in Fig. 5. His spectrum i's quite different from ours, principally because Hess erroneously. used a distribution proportional to $\sin \theta$ instead of the rectangular neutron energy distribution produced by monoenergetic $\alpha-$ particles. Also we have used the newer and more accurate neutron production cross sections of $(9,10)$. It is however interesting to note that the experimental spectrum of (1) and the spectra measured here fit the Hess. curve somewhat better than our calculated. spectrum. This fact is presumably quite fortuitous and mainly caused by the source encapsulation as mentioned above.

\section{REFERENCES}

1. H. Vialettes and P. Le Thanh, Neutron Monitoring, p. 375, Proceedings Series,
International Atomic Energy Agency, Vienna (1967).

2. W.N. Hess. "Neutrons from $(\alpha, n)$ Sources." Annals of Physics. 6, 115 (1959).

3. J.R. Stehn, M.D. Goldberg, B.A. Magurno and $R$. Wiener-Chasman. Neutron Cross Sections, Vol. I, $Z=1$ to 20 . USAEC Report BNL-325, 2nd ed., sup1. 2, Sigma Center, Brookhaven National Laboratory (1964).

4. R. Batchelor and G.C. Morrison. Fast Neutron Physics, Part I. Edited by J.B. Marion and J.L. Fowler, p. 413, Interscience Publishers, Inc., New York (1960).

5. R. Batchelor, R. Aves, and T.H.R. Skyrme. "Helium-3 Filled Proportional Counter for Neutron Spectroscopy." Rev. Sci. Inst. 26, 1037 (1955).

6. L.H. Heistek and L. van der Zwan. "Pulse Shape Discrimination with a Comparator Circuit." Nuc1. Instr. and Meth. 80, 213 (1970).

7. L. van der Zwan and K.W. Geiger." "The ${ }^{9} \mathrm{Be}(\alpha, \mathrm{n})^{12} \mathrm{C}$ Cross Section between 1.5 and $7.8 \mathrm{MeV}$." "Nuc1. Phys. A152, 481 (1970).

8. I.V. Goryachev, V.I. Kukhtevich, L.A. Trykov, and O.A. Trykov. Neutron Monitoring, p. 187, Proceedings Series, International Atomic Energy Agency, Vienna (1967).

9. J.H. Gibbons and R.L. Macklin. "Total Neutron Yields from Light Elements under Proton and Alpha Bombardment." Phys. Rev. 114, 571 (1959).

10. R.L. Macklin and J.H. Gibbons." "Study of ${ }^{10} \mathrm{~B}(\mathrm{n}, \alpha){ }^{7} \mathrm{Li},{ }^{7} \mathrm{Li}^{*}$, for $30<\mathrm{E}_{\mathrm{n}} \mathrm{keV}<500$." Phys. Rev. 165, 1147 (1968).

11.. J. DePangher and L.L. Nichols. A Precision Long Counter for Measuring Fast Neutron Flux Density. USAEC Report BNWL-260, Batel1e Memorial Instituté, Pacific North West Laboratory, Richland, Washingt on (1966).

12. L. van der Zwann. "Calculated Neutron Spectra from $\mathrm{Be}(\alpha, n)$ Sources." Can. J. Phys. 46, 1527 (1968). 
EVALUATION OF THE NEUTRON SOURCE ABSORPTION CORRECTION IN A MANGANOUS. SULFATE BATH

\author{
Valentine Spiegel, Jr. \\ Nuclear Radiation Division \\ Center for Radiation Research \\ National Bureau of Standards \\ Washington, D. C.
}

\begin{abstract}
A program has been written and experimentally verified for the correction to the Manganous Bath Technique for thermal neutron self-absorption in spherical and cylindrical neutron sources. The correction is generally small for most absolutely calibrated neutron sources, but may be as large as four or fiye percent for some commercially available sources with large thermal-neutron cross sections. Neutron multiplication in Pu-Be sources may partially or even overcompensate for neutron absorption. The thermalneutron flux at the source location, the source dimensions, and the macroscopic fission and absorption cross sections for all materials in the source are required as program input.
\end{abstract}

\section{INTRODUCTION}

A recent review article by Gelger (1) describes the manganous bath method of neutron source calibration as the most convenient to use and capable of the best accuracy. The correction for thermal neutron absorption within the source is sald to be typically below about 1\%. This is certalnly true for the absolute cal1bration of $\operatorname{Ra}-\mathrm{Be}(\alpha, n)$ and $\operatorname{Ra}-\operatorname{Be}(\gamma, n)$ sources with total nuclear thermalneutron absorption cross sections of about $1 \mathrm{~cm}^{2}$, which have been absolutely callbrated and adopted as standards for several laboratories. Most secondary or working sources, however, are thickly encapsulated radium-, plutonium-, polonium-, or americiumberyllium sources. The total nuclear neutron absorption cross section for these sources varies from about 15 $\mathrm{cm}^{2}$ to several hundred $\mathrm{cm}^{2}$. They are no longer "thin" absorbers and multiplication of neutrons in plutonium in the core material may also be present.

\section{DESCRIPTION OF THE CORRECTION}

A recent calculation and experimental verification of neutron ab-

CONTRIBUTION OF THE NATIONAL BUREAU OF STANDARDS NOT SUBJECT TO COPYRIGHT sorption in the source 1tself $(2,3)$ removes the Limitation of this correction to thin sources only. Brief$1 y$, the calcilation is applicable to sources of aill ranges of absorption or fission cross section and encapsulations of any 3 materlals. The calculation is carried out in a single interaction approximation, 1.e., the effects of elastic and inelastic scattering of thermal neutrons are neglected.

The absiorption programs were written for spherical and cylindrical neutron sources. The relative fraction of neutrons captured in the core and each of the cladding materlals is tabulated in the spherical source program, and the ratio of neutrons captured in the core to those captured In all cladding materials is tabulated in the cylindrical source program.

Calculations were performed for spherlcal sciurces with volumes of source and cladding materials equal to those in cylindrical sources. The net absorption, particularly for sources of $\epsilon$ qual length and outside dlameter, wáls so close that use of the cylindrical program with its factor of about; 60 in running time is warranted only for exceptional reasons. 
RESULTS

The results of the spherical and cylindrical source program are shown in Table I for a cylindrical Pu-Be and Am-Be source together with spherical sources of equal volumes of core and cladding materials. Our bath uses a tetrafluoroethylene spherical cavity of $10 \mathrm{~cm}$ outside diameter surrounding the source to reduce the thermal flux. The loss of neutrons would be about a factor of 3 higher if the cavity were not used.

\section{TABLE I}

Neutron Source Absorption Correction in a Manganous Sulfate Bath

$\begin{array}{lcccc} & \text { Pu-Be } & \begin{array}{c}\text { Pu-Be } \\ \text { Equiv. Sphere }\end{array} & \text { Am-Be } & \begin{array}{c}\text { Am-Be } \\ \text { Equiv. Sphere }\end{array} \\ \text { Interaction } & 2.007 \% & 1.955 \% & 0.966 \% & 0.960 \% \\ \text { Absorption } & 1.341 \% & 1.352 \% & 0.965 \% & 0.959 \% \\ \text { Fission } & 1.864 \% & 1.688 \% & 0.003 \% & 0.003 \% \\ \text { Loss } & 0.143 \% & 0.267 \% & 0.963 \% & 0.957 \%\end{array}$

The pertinent data for the calculations in the above table were: Cylindrlcal Pu-Be source encapsulated in tantalum and stainless steel-- Dimenslons in inches, $L=2.72, O D=1.31$, $B$ wall $=0.07, B$ ends $=0.25$ and 0.1 , $\mathrm{C}$ wall $=0.03, \mathrm{C}$ ends $=0.1$ and 0.25 . Pu-Be equivalent sphere dimensions-$\mathrm{OD}=1.913, \mathrm{~B}$ shell $=0.11, \mathrm{C}$ shell = 0.071 . Macroscop1c cross sections per $\mathrm{cm}--$ Siga $=1.865$, Sigaf $=4.305$, Sigb $=1.16, \mathrm{SIgc}=0.2811$. Neutrons per fission $=2.80$. Thermal flux $=0.12 \%$ of source neutrons per $\mathrm{cm}^{2}$. Cylindrical Am-Be source encapsulated in tantalum and stainless steel-- Dimensions in inches, $\mathrm{L}=1.355, \mathrm{OD}=1.355, \mathrm{~B}$ wall $=0.07, \mathrm{~B}$ ends $=0.07, \mathrm{C}$ wall $=$ $0.03, C$ ends $=0.05$. Am-Be equivalent sphere dimensions-- OD $=1.551, B$ shell $=0.080, C$ shell $=0.042$. Macroscopic cross sections per cm-$\mathrm{S} 1 \mathrm{ga}=0.1704, \mathrm{~S} 1 \mathrm{gaf}=0.00082, \mathrm{~S} 1 \mathrm{gb}$ $=1.16, \mathrm{~S} 1 \mathrm{gc}=0.281$. Neutrons per flssion $=2.89$. Thermal flux $=0.121 \%$ of source neutrons per $\mathrm{cm}^{2}$. Source materials are labeled A for the core
$B, C$, and $D$ for the Ist, 2nd, and 3rd encapsulations. S1ga and Sigaf are the absorption and fission macroscopic cross sections of material. A. Sigb, Sigc, and Sigd are the macroscopic absorption cross sections of the $B, C$, and D encapsulations.

\section{REFERENCES}

I. K. W. Gelger. "Recent Improvements in the Absolute Calibration of Neutron Sources." Metrologia 4, 8 (1968).

2. V. Splegel, Jr. and W. M. Murphey. Computer Code for the Calculation of Thermal Neutron Absorption in Spherical and CyIindrical Neutron Sources. Nat. Bur. Stand.(U.S.), Tech. Note (In preparation).

3. V. Spiegel, Jr. and W.M. Murphey. "Calculation of Thermal Neutron Absorption in Cylindrical and Spherical Neutron Sources." Metrologia (I971) (In press). 


\section{TISSUE EQUIVALENT PROPORTIONAL COUNTERS IN THIN NEUTRON SHIELD STUDIES}

\author{
Larry W. Brackenbush \\ G. W. R. Endres \\ Leo G. Faust
}

Pacific Northwest Laboratories

Battelle Memorial Institute

Richland, Washington

\section{INTRODUCTION}

In general there are two methods of determining absorbed neutron dose in tissue in a mixed (gamma and neutron) field. The first is to measure the neutron fluence and the neutron energy spectrum and multiply by the appropriate fluence to dose conversion factors. The second method of determining the absorbed dose in tissue is to use cavity techniques employing the Bragg-Gray relation $(\underline{1}, \underline{2})$.

Tissue equivalent ion chambers were used by Failla and Rossi (3) to determine the total absorbed dose in tissue. The main disadvantage of this technique is that other instruments must be used to determine the relative proportions of dose from electromagnetic radiation, beta particles, etc., which make up the total dose. Rossi and Failla used ion chambers lined with carbon or conducting Teflon and filled with carbon dioxide gas to measure the dose to tissue from electromagnetic radiations.

An alternate method is to use proportional counters to separate the gamma from the neutron everts. This method was first used by Hurst ( 4 ), who used a proportional counter lined with polyethylene and filled with ethylene gas, which satisfied the israggGray principle. This counter vas almost tissue equivalent, since the energy deposited by neutrons per gram of ethylene is almost the same as that deposited in soft tissue. This differs from the ion chamber technique in that the number of ion pairs produced in the gas is determinec by summing pulse heights rather than by measurinc currents. The pulse neizht is proportional to the nurater of ion pairs created if: (1) there is no electron attachment to the ions and (2) the pulse height from the anplifier is indeperient of the track orientation in the proportional counter. ire first concition can be thet $\mathrm{by}$ exclucinin water vapor, oxygen, halogens, anc other nighly electrone;ative ases from the counter, the second condition car be met by using amplifiers with time constants greater than the collection time of the electrons in the proportional counter (5). In the riurst counter the gamme everts are separated from tine neutron events by applying a wias and recordiris only the total enerby deposition from neutron inuluced events. Solite reutron eneroy is lost in applying the vias, wich ceperis upon the energy of the cianna rays.

Fossi $(\underline{6}, \underline{7})$ has developed a spherical proportional counter of Sionka (3) tissue equivalent jastic sinilar to the courter shown in $\mathrm{Fi}_{\tilde{\mathrm{C}}}$. 1. The recoil particle spectrum set up at the $1 / 8$ inch tilick wall of such a counter will ie essentially the sane as that occuring in tissue for neutrons with energies of under $10 \% \mathrm{eV}$, assuming brabe-Gray conuitions are met with chareed particle equilinfium. Fihe counter is filled with high purity tissue equivalent ges

\begin{abstract}
ingly important. Tissue equivalent proportional counters (TEPC) have been developed to from fluence and difficult neutron spectra measurements. Exposures made with monokerma values calculated from fluence measurements. The ability of the TEPC to separate
I model for analysis of linear energy transfer distributions from TEPC data is shown to be
mode adequate for determining quality factors from fast neutrons with energies above about
$0.4 \mathrm{Mev}$. Neutron dose reduction measurements and quality factor measurements are presented for various hydrogeneous shields with neutrons from californium fission sources Transport computer code calculations for the dose reduction with polyethylene shields Transport computer code calculations for the

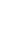




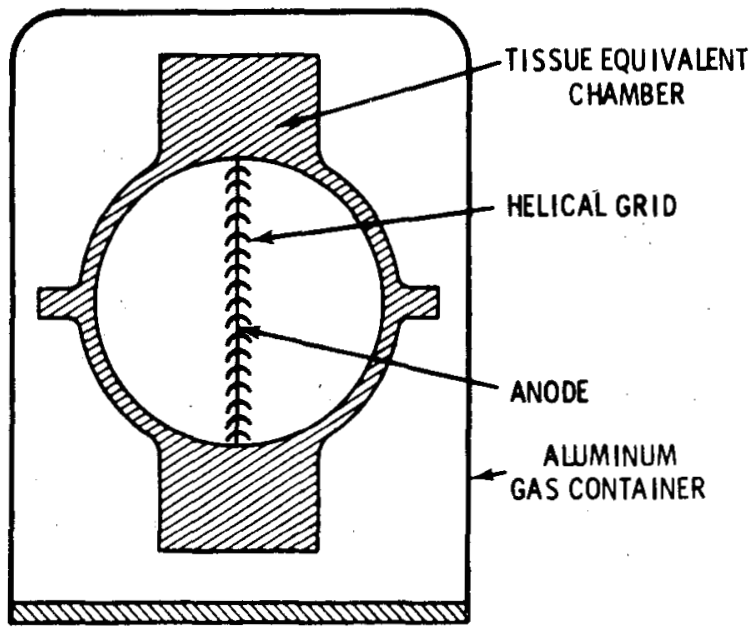

Fig. 1 - Cross Sectional View of the Tissue Equiva?ent Proportional Counter

containing $64.4 \%$ methane, $32.4 \%$ carbon dioxide, and $3.2 \%$ nitrogen. Table I gives the compositions of the TE plastic, counter gas, and soft tissue. The energy absorbed in the counter gas is essentially that absorbed in soft tissue, except that the oxygen and carbon fractions are reversed. This introduces little error, since most of the recoils come from hydrogen. It is necessary to use very high purity dry gas to avoid electron attachment to the ions. The Rossi counter has a helical grid operated at about $20 \%$ of the anode voltage for more uniform gas multiplication, so that the pulse height does not depend upon track orientation.

\section{TABLE I}

Composition of Tissue, Tissue Equivalent Plastic, and Tissue Equivaient Gas

$\begin{array}{cccc}\text { MUSCLE } & \text { STANDARD } & \text { TE } & \text { TE } \\ \text { TISSUE } & \text { MAN } & \text { PLASTIC } & \text { GAS } \\ \text { (WEIGHT } & \text { (WEIGHT } & \text { (WEIGHT } & \text { (WEIGHT } \\ \text { ELEMENT } & \text { PERCENT) } & \text { PERCENT) } & \text { PERCENT) } \\ \text { PEERCENT) }\end{array}$

$\begin{array}{rrrrr}\mathrm{H} & 10.2 & 10 & 10 & 16.2 \\ \mathrm{C} & 12.3 & 18 & 76 & 57.0 \\ \mathrm{~N} & 3.5 & 3 & 3.2 & 3.2 \\ \mathrm{O} & 72.9 & 65 & 5.2 & 23.6\end{array}$

The main difference between the Hurst counter and the Rossi counter is the operating pressure of the gas. In the Hurst counter almost all of the energy of the recoils is measured in the counter, and the gamma events, along with a predictable fraction of the neutron recolls, are eliminated by applying a bias. On the other hand, in the Rossi counter the gas pressure is so low that most of the secondaries completely traverse the counter, and gamma and neutron events are separated on the basis of differences in the stopping power or linear energy transfer of the secondary electrons from gamma rays and secondary proton and heavy ion recoils from neutrons.

If the cavity is of very low density, a typical charged secondary will lose only a small fraction of its energy in crossing the cavity, and energy loss spectrum is indicative of the linear energy transfer (LET) of the secondaries. The energy deposited in the cavity for particles crossing the cavity is approximately the product of the LET times the track length, so that there is some overlap of neutron and gamma events. The spectrum of the energy deposited in a cavity equivalent to a sphere of tissue slightly over 2 microns in diameter is shown in Fig. $6 \mathrm{~A}$ and is labeled the $\mathrm{D}(\mathrm{Y})$ distribution. Note the separation between neutron events and gamma events. The dotted line in Fig. $6 \mathrm{~A}$ is the result obtained by exposing $a$ 2-1/4 inch diameter tissue equivalent proportional counter to only gamma rays from a ${ }^{60} \mathrm{Co}$ source. In most instances $97 \%$ of the neutron events can be separated from the gamma events, which occur below about 10 $\mathrm{keV} / \mathrm{micron}$. Unfortunately, at high. neutron energies the neutron and gamma events become more difficult to separate because the LET of the recoil protons becomes lower. (This will be explained in greater detail later in the section on LET analysis.)

The ability of the tissue equivalent proportional counter (TEPC) to measure the neutron dose while rejecting almost all of the gamma dose is shown in Table II. In this experiment a constant neutron dose was delivered to the tissue equivalent proportional counter using a long counter (2) as a fluence monitor. A $6{ }^{\circ} \mathrm{Co}$ source was used to give gamma exposure rates as. high as 1400 $\mathrm{mR} / \mathrm{hr}$ before the proportional counter was unable to completely resolve neutron and gamma events.

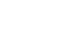


TABLE II

Mixed Gamma -Neutron Dose Measurements

\begin{tabular}{|c|c|c|c|}
\hline $\begin{array}{l}\text { LONG COUNTER } \\
\text { KERMA } \\
\text { (erglg } \times 10^{-1} \text { ) }\end{array}$ & $\begin{array}{c}\text { TEPC } \\
\text { DOSE (mrads) }\end{array}$ & $\begin{array}{c}\text { GAMMA } \\
\text { EXPOSURE } \\
\text { RATE (mR/hr) }\end{array}$ & $\underline{L C / T E P C}$ \\
\hline 62.2 & 57.3 & 0 & 1.09 \\
\hline 62.2 & 58.4 & 7 & 1.06 \\
\hline 62.2 & 57.7 & 14 & 1.08 \\
\hline 62.2 & 57.7 & 56 & 1.08 \\
\hline 62.2 & 56.9 & 224 & 1.09 \\
\hline 62.2 & 56.2 & 662 & 1.11 \\
\hline 62.2 & 55.4 & 1400 & 1.12 \\
\hline
\end{tabular}

The Rossi proportional counter has several disadvantages - (1) the electrons. can be backscattered from the tissue equivalent plastic wall, and the energy deposited will be twice as much as it should be and (2) two or more electrons can cross the cavity within the resolving time of the counter, which is about 1 microsecond. Such events do happen, but with very low frequency if the gamma exposure rates are restricted to low values. Very high gas purity must be maintained, and outgassing from the TE plastic walls can be a serious problem. One great advantage of Rossi's counter is that it is self calibrating - the so called proton drop point, which corresponds to the maximum energy loss event occuring when a proton of very high LET traverses the diameter of the sphere, occurs at a value of about $98 \mathrm{keV} /$ micron for a 2 micron equivalent diameter chamber (10). In Fig. 6A this point is recognized as the point of inflection or the point of the largest negative derivative.

In summary the Rossi counter is (I) able to distinguish between gamma and neutron events over a wide range of exposure rates, (2) self calibrating, and (3) able to make an absolute measurement of the dose imparted to a tissue equivalent system.

\section{APPARATUS}

The proportional counter used in the experimental measurements is a $2-1 / 4$ inch diameter sphere with $1 / 8$ inch thick walls of tissue equivalent plastic as shown in Fig. I. This proportional counter is operatea at 650 volts and is filled to about 28 torr pressure, which gives an equivalent diameter of slightly over 2 microns in tissue of unit density. To differentiate the gamma events from electronic noise, it is necessary to use a very low noise charge sensitive preamplifier. The signal from the preamplifier is sent through a linear amplifier with 1.6 microsecond time constants and analyzed by a 1024 channel pulse height analyzer with a 100 megahertz analog to digital converter.

There are many experimental difficulties encountered in using this counter. The principal problem is that of gas contamination - thie presence of minute traces of water vapor oxygen, or carbon monoxide which diffuse from the tissue equivalent plastic cause rapid deterioration of the gas multiplication. The Shonka tissue equivalent plastic which has been exposed to air often outgasses to such an extent that the counter has a useful life-time of only a few hours. It is often necessary to evacuate the chambers for weeks to eliminate the outgassing problem. Another serious problem is that of large gain changes with temperature, which interferes with long neutron exposures. As mentioned before, the tissue equivalent gas composition is not exactly the same as sort tissue, and this introduces a small error in the measurements.

Absorbed dose measurements were made with sealed neutron sources with $30 \mathrm{~cm}$ separation between the centers of the neutron source and the tissue equivalent proportional counter. The slab shielas used in the dose reduction measurements were 12 inches by 24 inches. It was also necessary to carefully control the temperature of the roon in which the measurements were made and a!lso to remove all large neutron scattering materials from this room.

Some neutrion exposures were made with a positive ion Van de Graaff accelerator in a special low neutron scatter room, and the fluences were measured with a long counter. Some errors were made in the fluence measurements since it s necessary to have a nearly uniform lilux over the face of the long counter for accurate measurements. Many of the nuciear reactions employed to produce monoenergetic neutrons have large changes in the reutron yield and energy at different angles. Small errors were also introduced into the fluence measurements by neutrons scattered into the long counter by the neutron shields placed in front of the tissue equivalert proportional counter. This error was found to be less than about 
5\% from measurements made with a second proportional counter placed close to the accelerator target.

\section{EXPERIMEITAL RESULTS}

A series of tissue equivalent proportional counter (TEPC) measurements were made with nearly monoenergetic neutrons produced by a Van de Graaff accelerator using a long counter to measure the neutron fluence. For these irradiations the kermas were calculated using the fluence to kerma conversion factors published by Pitts et al. (11). The calculated kermas were some $4 \%$ to $12 \%$ higher than the absorbed doses measurea by the TEPC for neutrons with energies from 0.5 to $5 \mathrm{MeV}$. Data were also taker at $7.5 \mathrm{MeV}$ and $5.25 \mathrm{MeV}$ using a fission chamber to measure fluence; at tinese energies the locally ebsorbed dose neasured by the tissue equivalent proportional counter agrees within $3 \%$ of the calculated kermas. It is suspected that the lon counter messured fluences are slightly in error due to nonuniform fluxes over the front face of the long counter.

Some of the results of the Van ce Graaff irradiations are shown in Fig. 2 for various neutron shielding materials which are commercially available. Fig. 2 shows the relative absorbed dose reduction which results from various slab shields measured by a TEPC exposed to neutrons from a thick tritium target bombarded with protons. The thick target produces neutrons from a few hundred $\mathrm{keV}$ to $733 \mathrm{keV}$ with an average neutron energy of about $500 \mathrm{keV}$. In this case boron locdec polyethylene is the best shield material. Since the mean free path of these low energy neutrons is under about $3 \mathrm{~cm}$, it is suspected that many of these relatively low energy reutrons are slowea cown, and the addition of boron to the shields removes the slow reutrons. The cose rates behind polyethylend shields are probably hifiner because of the protons produced from $\left.14_{i j}(n, p)\right)^{14} \mathrm{C}$ reactions incuced by slow neutrons in the tissue equivalent plastic walls.

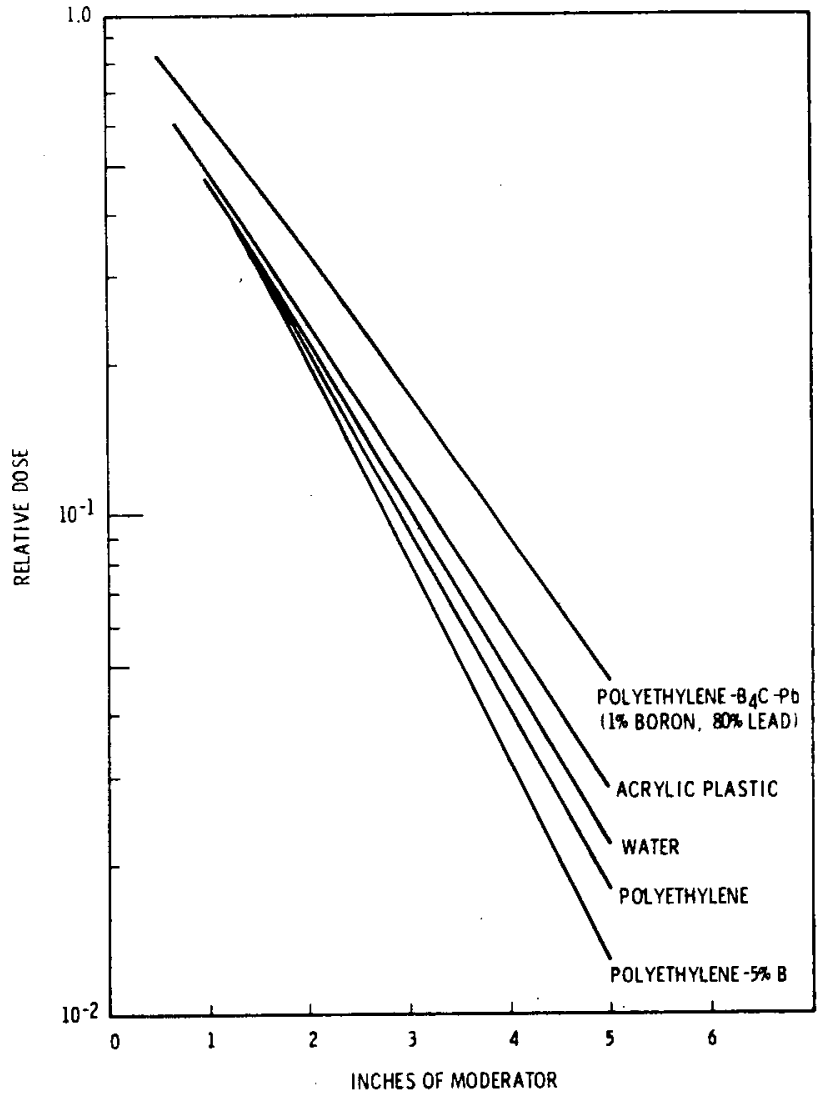

Fig. 2 - Neutron Dose Reduction Jith lydrogeneous Slab Shields and About $1 / 2$ YeV Veutrons

Tifure 3 shows the results of neutron shielaing measuremerts using polyethylene and a ${ }^{252} \mathrm{Cf}$ spontaneous fission source (with an average neutron energy of $2.3 \mathrm{MeV}$ (12)) and a high exposure plutonium dioxide* neutron source. The average energy of this plutonium cicxide source was calculated (13) to be about $2.1 \% \mathrm{eV}$ assuming that $68 \%$ of the neutrons came from spontaneous fission with an average energy of about $2 \mathrm{MeV}$ and $32 \%$ of the neutrons came from $O(\alpha, n)$ reactions viti an average energy of about 2.3 :EV. The plutonium aioxide measurements required very long exposure tiries, and considerable difficulty ras encountered with gain sints ciue to temperature fluxuations.

\footnotetext{
* Tizh exposure plutonium has a $239 \mathrm{Pu}$ content of less than $80 \%$.
} 
Dose Raduction with Polyethylene Slab Shields

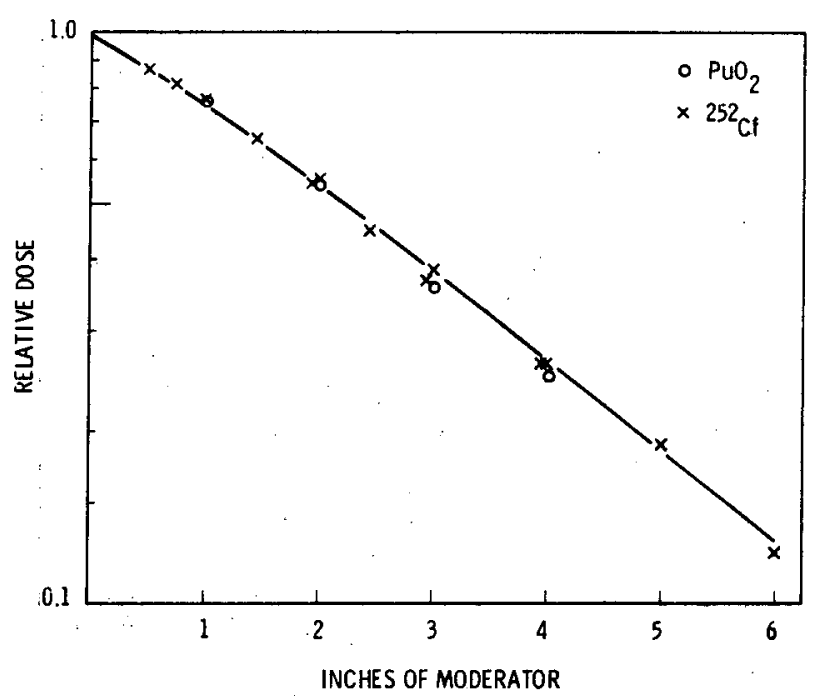

Fig. 3 - Neutron Dose Reduction With Polyethylene Slab Shields and Neutrons From $\mathrm{PuO}_{2}$ and ${ }^{252} \mathrm{Cf}$.

Fig. 4 shows a set of shield effectiveness measurements for a plutonium tetrafluoride neutron source with an average neutron energy of $1.4 \mathrm{MeV}$ and the same set of slab shields as in Fig. 2. The data points in this graph give an idea of the precision which can be achieved with the tissue equivalent proportional counter. In this case polyethylene is the most effective shield material. The addition of boron to the shields does not reduce the dose rate, because only a small fraction of the absorbed dose comes from slow neutrons. In general polyethylene seems to be the best shield material for plutonium compounds.

Fig. 5 shows the dose reduction for a Californium-252 spontaneous fission neutron source (with an average neutron energy of $2.3 \mathrm{MeV}$ ) shielded by polyethylene. The triangles indicate TEPC measurements made with spherical polyethylene shields, and the circles indicate measurements made with the 12 inch by 24 inch slab shields. In addition the crosses indicate the relative reduction in kerma calculated from fluence values calculated by the computer code ANISN (14) and the fluence to kerma factors of Ritts et al. (op. cit.). ANISN-is a one dimensional discrete ordinates transport code which gives results agreeing within $1 \%$ to $2 \%$ of the values measured by the tissue equivalent proportional counter.

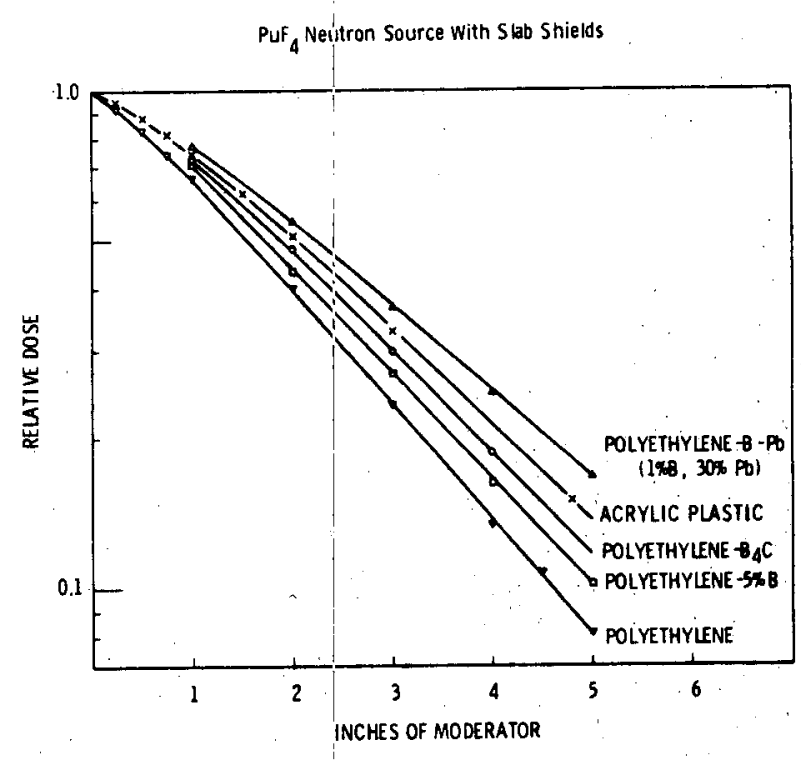

Fig. 4 - leutron Dose Reduction With hydrogeneous SIab Shields and Neutrons Fror: $\mathrm{PuF}_{4}$

In general the results verify the maxim that the best fast neutron shield is the one with the greatest hydrogen density. The addition of boron or other thermal neutron absorbing materials seems to have little effect in shielding fast neutrons from plutonium corpounds and may, in fact, reduce the effectiveness of thin shields by reducing the hydrogen density.

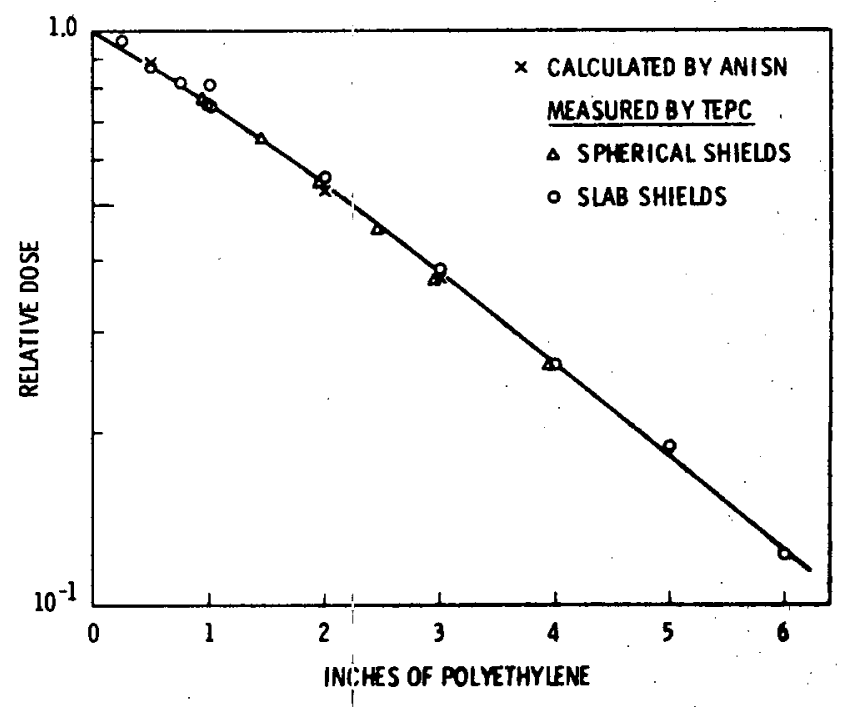

Fig. 5 - Measured and Calculated Dose Reduction With Polyethylene Shields and Neutrons From ${ }^{252} \mathrm{Cf}$. 


\section{LINEAR ENERGY TRANSFER AND QUALITY FACTOR ANALYSIS}

It is possible to derive linear energy transfer (LET) values for the secondary particles in tissue equivalent proportional counters. In investigating the various methods and mathematical nodels available, it was decided to use the simplest system possible - a spherical cavity. To obtain IET data it is necessary for the charged particle secondaries to lose as little energy as possible in crossing the cavity, which can be acnieved only at very low gas pressures. For a cavity of a few centimeters in diameter filled with tissue equivalent gas at pressures of 10 to 100 torr, the equivalent diameter in tissue of unit density is only a few microns. This cavity simulates the dose delivered to a single sell, or even to a single chromosone, which is of great interest in microdosimetry and radiation biology.* Since there are several different methods of interpreting TEPC data, it was decided to investigate the simplest model available - the model first proposed by h. H. Rossi $(17,10,19,20)$. This model considers a spherical counter with neutron secondaries which have a con-m stant, uniform energy loss alors straight line paths which completely cross the cavity. It is also assumed there is no backscatter from the walls. This simple model obviously has many disadvantages and is not valid for heavy ion recoils or proton recoils from low energy neutrons (below several hundred $\mathrm{keV}$ ) which lose a large fraction of their energy in crossing the cavity or stop inside the cavity. These problems and others are discussed in greater detail by Caswell (2l), Turner (22), and Dvorak (23). In a 2 micron equivalent diameter cavity the Rossi model is valid for most proton recoils from neutrons with energies of about $700 \mathrm{keV}$ to about $3 \mathrm{MeV}$. It is a simple analytic model which does not involve complicated computer programs. For a complete description of the model see Chapter 2 of Vol. 1 of Radiation Dosimetry (reference 15 ).

Before discussing the Rossi model in detail, it is necessary to define several quantities. The equivalent diameter of the proportional counter cavity is the diameter

* For more information the reader is referred to Chapter 2 of Vol. I of Radiation Dosimetry (15) or the proceedings of tine Ispra conference on microdosimetry (16). of a small sphere of tissue of unit. density ( $1 \mathrm{gm} / \mathrm{cm}^{3}$ ) which has the same stopping power, or causes the same energy loss, as the sphere of low pressure tissue equivalent gas. Consider a small sphere of tissue of unit density within a uniformly irradiated mass of uniform composition. Neutron or gamma radiation interact with the mass and create cinarged particles. A charged particle, together with its secondaries, may deposit a certain amount of energy $E_{y}$ in the sphere. The energy deposition is called an event and the event size, $Y$, is defined as the energy deposited within the sphere divided by the diameter of the sphere. In the case of particles traversing the sphere in straight lines with a uniform linear energy transfer (LET), $Y$ has a constant value regardless of the size of the small sphere. Note that event size has the same dimensions as LET - energy/length. Multiplying the event distribution observed by the event size, $Y$, one obtains the distribution for the energy deposited as a function of the event size - this is the dose distribution in event size, $D(Y)$. Ideally, under the conditions given above the dose distribution is independent of the sphere size. However, because the charged particles do have curved tracks (or even branched tracks), energy loss variations, and finite track diameters, the dose distribution, $D(Y)$, does depend upon the sphere size. This is a measure of the inability of the LET concept to properly characterize local energy deposition. These problems are discussed in detail in the ICRU Report on Linear Energy Transfer (24).

If one considers only the case where charged particles cross the sphere along straight lines with a continuous, uniform energy loss, and statistical effects are neglected, then it is possible to relate the dose distribution in event size to the dose distribution as a function of LET. In Radiation Dosimetry (op. cit.) Rossi gives the following formula for determining the (unnormalized) absorbed dose distribution, $A(L)$, in terms of the linear energy transfer, $\mathrm{L}$ :

$$
A(L)=\frac{I}{2 \pi r^{2}}\left[Y N(Y)-Y^{2} \frac{d i}{d Y}\right] Y=L
$$

where

$\mathrm{Y}$ is the event size (units of $\mathrm{keV} / \mathrm{micron}$ )

$Y(Y)$ is the event distribution measured by the IEPC 
$r$ is the radius of a sphere of tissue of equivalent size

$\frac{d V}{d y}$ is the derivative of the event distribution evaluated at $\mathrm{Y}=\mathrm{L}$.

Fig. 6A shows the absorbed dose distribution in terms of the event size $Y$ as defined by Rossi. Again note that the neutron and gemma events can be separated, and that only a few neutron events occur below $10 \mathrm{keV} /$ micron. The above formula was used to determine the rad absorbed dose distribution as a function of LET, and the resulting aistribution is shown in Fig. $6 \mathrm{~B}$. Wote the number of low energy events which do not appear on the LET distributions calculated by ìoag (25). It is suspected these events may be due, in part, to secondary particles which start or stop in the gas cavity. Boag predicts that the number of Low LET events increases at higher neutron energies, which is indeed observed to happen.

In Fig. $6 B$ the dotted line represents a function for quality fector, $Q F(I)$, as a function of linear energy transfer, $L_{\infty}$, with units of $\mathrm{keV} / \mathrm{micron}$. The formula for this curve,

$$
Q \dot{Q}(L)=0.2 \mathrm{~L}-0.0005 \cdot \mathrm{L}^{2}
$$

was taken from Haffner (26) and is a reasonably good approximation of the quality factor data presented in NBS Hanabook 59 (27). The data in liandbook 59 are based on a quality factor* equal to one for $x$-rays and electrons, and consiciers the effects of radiations of different LET's to critical organs (such as cancer in skin, leukemia in blood-forming organs, loss of fertility in gonads, and cataract formation in eyes). The product of the quality factor, $Q F(L)$, times the fractional absorbed dose, $D(L)$, gives the dose equivalent distribution, $\mathrm{DE}(\mathrm{L})$, as a function of LET as shown in Fig. 6C. The average quality factor is found by dividing the dose equivalent by the absorbed dose.

There are many different definitions of quality factor used by various authors (28),

* Note that prior to 1963 the term relative biological effectiveness (RBE) was used. This was changed to quality factor for radiation protection purposes to avoid confusion with biological experiments. See "Report on the RBE Committee to the International Commissions on Radiological Protection and on Radiological Units and Measurements." Health Physics 9, 357 (1963).

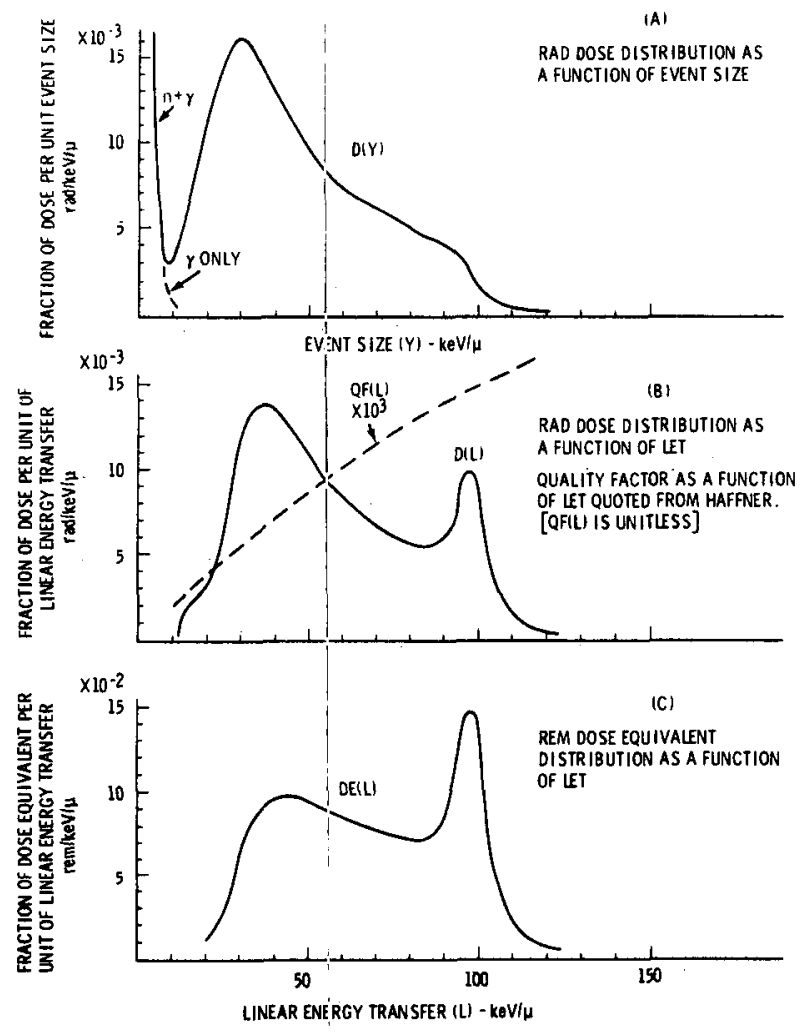

FiE. 6 - Dosel and Dose Equivalent Distributions From Tissue Equivalent Proportional Dounter Data With Two Micron Equivalent Diameter and $1.5 \mathrm{MeV}$ Neutrons

which causes somelconfusion. Quality factor now is generally eccepted to be an administrative guideline in radiation protection. The values of quality factor recommended by the NCRP (in Nis Handbook 59) as a function of the linear energy transfer, $L_{\infty}$, increase with increasing LET. However, plots of the relative biologicall effectiveness ( $R B E$ ) verses the average linear energy transfer show that peaks occur. The value of LET at which the maxinum occurs appear to differ depending unon the biological material studied and the conditions of the irradiation. For mammalian cells the curve of REE verses LET usually has a maximum at roughly 100 $\mathrm{keV} / \mathrm{micron}$. (See ICRU Report IG (op. cit.) for more information.)

Yudir (29) gives a table of "effective" quality factors for monoenergetic neutrons; these data are showr as the solid line in Fig. 7. The quality factor** is also given in NBS landiook 63 (30) for monoenergetic reutrons, and these date are show dis tite 


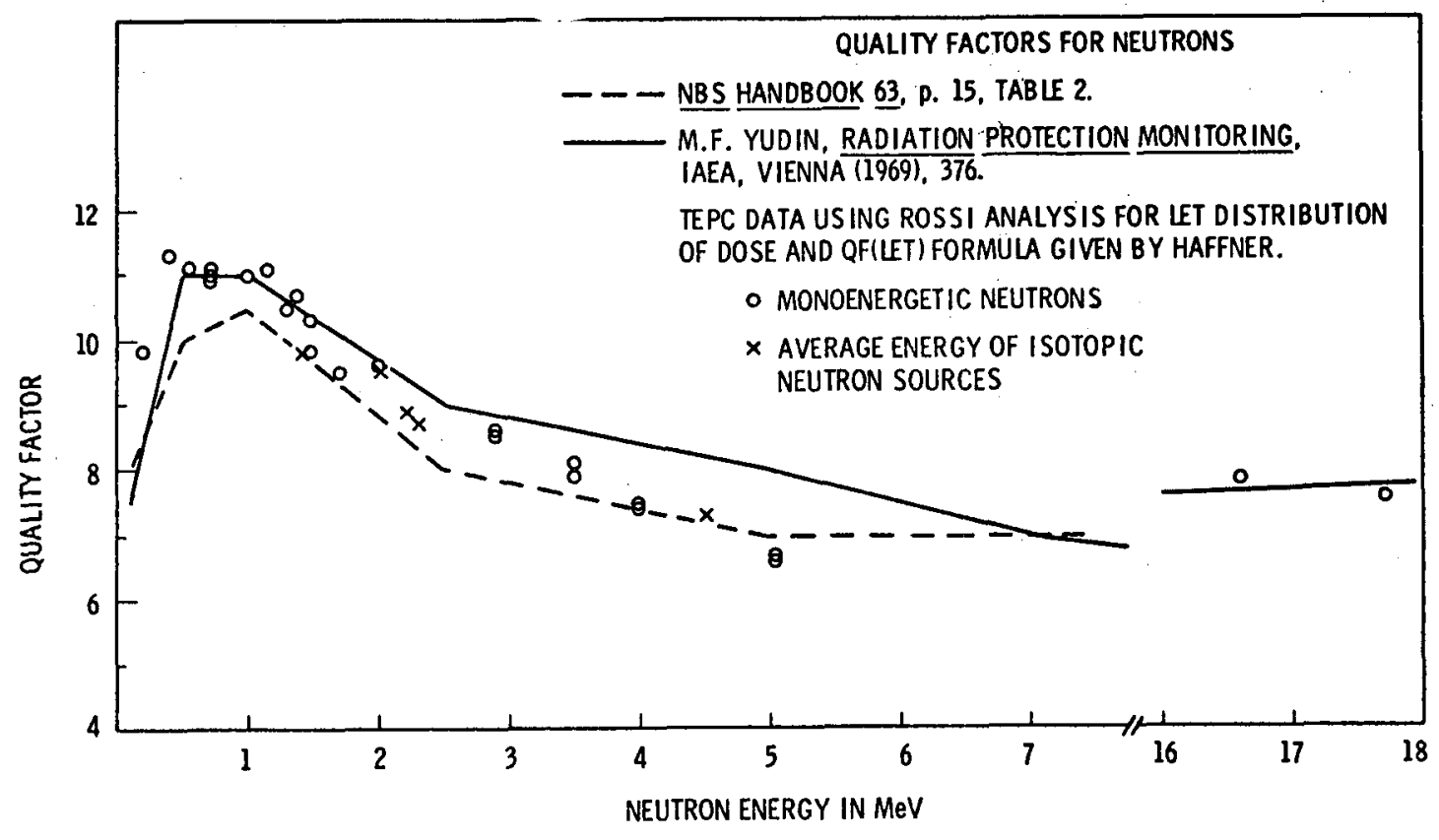

Fig. 7 - Quality Factors for Neutrons of Various Energies

dashed line. The points in Fig. 7 are average quality factors calculated from tissue equivalent proportional counter data by the computer program TISSUE, which uses the Rossi model for the dose distribution in LET and the formula quoted from Haffner (op. cit.) for all recoils with energies above the minimum separating gamma and neutron events at about $10 \mathrm{keV} / \mathrm{micron}$. Note these quality factors are calculated for a small mass of tissue equivalent material and are not exactly the same as the quality factors in NBS handbook 63, which were calculated for an infinite slab of tissue $30 \mathrm{~cm}$ thick. In TISSUE a low bandpass digital filtering technique is necessary to smooth the data and find its derivative. The proton drop point - the point of the largest negative derivative - is assumed to occur at $98 \mathrm{keV} /$ micron for the 2 micron equivalent

** These quality factors are called RBE values in NBS Handbook 63. They are the results of computer calculations for monoenergetic neutrons normally incident on an infinite slab of tissue $30 \mathrm{~cm}$ thick. The recoils from the primary neutrons and scattered neutrons were calculated, and the quality factor as a function of LET data from NBS llandbook 59 were used to calculate diameter TEPC measurements. Note the good correlation between quality factors which are derived from different bases. The simple model predicts quality factors with reasonable agreement, even at high neutron energies $(16-17 \mathrm{MeV})$. The Rossi model obviously is not valid at high neutron energies; the heavy recoils do not have a constant LET along their path and may even stop inside the cavity. It is suspected that the agreement is fortuitous - a compensating error occurs in the formula for the quality factor as a function of $L E T$, and the quality factor is too large at high LET values.

Tissue equivalent proportional counter measurements show the simple Rossi model works reasonably well for monoenergetic neutrons with energies between 0.4 and $5 \mathrm{MeV}$ and even as high as $17 \mathrm{MeV}$. From this one can conclude that this model should work

the dose equivalent as a function of depth in the phantom and initial neutron energy. The quality factors given in NBS Handbook 63 are the maximum values of the ratio of the dose equivalent (called RBE dose) divided by the absorbed dose at various depths in the phantom. 
reasonably well for many isotopic neutron sources. The crosses in Fig. 7 are the results of TEPC measurements of various neutron sources $-\mathrm{PuF}_{4}$ (mean energy, $\overline{\mathrm{E}}=1.4$ $\mathrm{MeV}), \mathrm{PuO}_{2}(\overline{\mathrm{E}}=2.1 \mathrm{MeV}),{ }^{252} \mathrm{Cf}(\mathrm{E}=2.3 \mathrm{MeV})$, and PuBe $(\bar{E}=4.5 \mathrm{MeV})$. The data from plutonium isotopic sources indicate that thin shields (under 6 inches) do not significantly change the average quality factor.

There are some problems involved in the quality factor analysis of experimental data. Since the derivative is involved in the formula for finding the absorbed dose distribution in LET, the quality factor derived from this distribution is dependent somewhat upon the resolution of the proportional counter, for a counter with poor resolution seems to give lower quality factors.

\section{CONCLUSIONS}

In general the ability of the tissue equivalent proportional counter to make absolute neutron dose measurements in a tissue equivalent system has been demonstrated. One must be careful in interpreting this measured dose, however. The absorbed dose measured is the dose delivered to a small tissue site and is not always indicative of the dose delivered to critical organs involved in a whole body irradiation. Deep within a phantom there may be a significant component of absorbed dose caused by the build-up of $2.2 \mathrm{MeV}$ gamma rays from $1_{H}(n, \gamma)^{2}$ reactions induced by slow neutrons; the free space TEPC measurement does not include this effect. The problems of charged particle equilibrium, comparison of kerma and absorbed dose in a phantom or body, and dosedepth distributions are beyond the scope of this paper, and the reader is referred to authoratative works such as Attix and Roesch, Radiation Dosimetry, Vol. 1, Chapters 1 and 6 . The ability of the TEPC to measure the neutron dose in gamma fields as. high as $1 \mathrm{R} / \mathrm{hr}$ has been demonstrated experimentally. The main problems involved in higher exposure rates are analyzer dead time and pulse pile-up.

The simple Rossi model seems to be adequate for predicting quality factors for monoenergetic neutrons with energies between $0.4 \mathrm{MeV}$ to $5 \mathrm{MeV}$ for tissue equivalent proportional counters with a 2 micron equivalent diameter in tissue. It also seems to be adequate for measuring average quality factors for many isotopic neutron sources. One must remember that quality factor may be considered to be an administrative guideline for radiation protection purposes, and that the biological effectiveness of a neutron irradiation may depend upon many other factors, such as dose rate, dose level, time fractionization of the dose, the biological system considered, etc. The quality factors computed from the TEPC data were for free air exposures and may not be exactly the same as those measured at various points in a phantom or body. However, quality factor analysis of TEPC date taken behind hydrogeneous shields indicate there is little change in the quality factor for thin shields (under ' inches) for neutrons with energies above about $1 \mathrm{MeV}$. In any case it is possible to make TEPC measurements inside a phantom to resolve any ambiguities.

One great advantage of the tissue equivalent proportional counter over other types of "rem meters" is that even if the dose equivalent (or quality factor) is not measured correctily, the absorbed dose in a small tissue site is always measured. It is also usually possible to obtain information about the neutron energy spectrum from the TEPC recoil spectrum, so that one has ar idea of the ralidity of the quality factor value.

One of the purposes of these TEPC measurements wais to verify the use of the one dimensional computer code ANISN for slab shielding problems. Using current fluence to kermi conversion factors, the calculated redultion in kerma with polyethylene shieldis and fission sources agree within $2 \%$ of the TEPC measurements for thin shields. 


\section{BIBLIOGRAPHY}

1. I. H. Gray. "An Ionization Method for the Absolute Measurement of Gamma Ray Energy." Proc. Roy. Soc. (London) A156, 578 , (1936).

2. L. H. Gray. "The Ionization Method of Measuring Energy." Proc. Cambriage Phil. Soc. $40,72(1944)$.

3. H. H. Rossi and G. Failla. "Tissue equivalent Ionization Charbers." Nucleonics 14, Ho. 2, 32 (1956).

4. G. S. Hurst. "An Absolute Tissue Dosimeter for Tast Neutrons." Brit. J. Radiology 27, 353 (1954).

5. R. F. Healey and J. W. Reed. "The Behavior of Slow Electrons in Gases. Amalgamated wireless Valve Co., Sydney, Australia (1941).

6. H. H. Rossi and W. Rosenzweig. "A Device for the Measurement of Dose as a Function of Specific Ionization." Radiology 64, 404 (1955).

7. H. H. Rossi and W. Rosenzweig. "Measurement of Neutron Dose as a Function of Linear Energy Transfer." Radiation Research 2, 417 (1955).

8. F. R. Shonka, J. E. Rose, and G. Failla. "Conducting Plastic Equivalent to Tissue, Air, and Polystyrene." Proc. of the Soc. United Nations International Conf. on the Peaceful Uses of Atomic Energy (1958).

9. L. L. Nichols and J. DePangher. A Precision Long Counter for Measuring Fast Neutron Flux Density. USAEC Report BNWL-260, Battelle-Northwest, Richland, Wash. (1966).

10. W. A. Glass and D. N. Samsky. "Ionization in Thin Tissue-like Gas Layers by Monoenergetic Protons." Radiation Res. 32, 138 (1967).

11. J. J. Ritts, et al. "Calculation of Neutron Fluence to Kerma Factors for the Human Body." Nucl. Applications and Technology 7, 89 (1969).

12. J.W. Meadows, "252Cf Fission Neutron Spectrum from 0.003 to 15.0 MeV." Phys. Rev. 157, 157 (1967).

13. L. W. Brackenbush and L. G. Faust. Neutron Spectra of Plutonium Compounds. USAEC Report BNWL-1262, Battelle-Northwest, Richland, Wash. (1970).

14. W. W. Engle, Jr. A User's Manuel for ANISN, A One Dimensional Discrete Ordinates Transport Code with Anisotropic Scattering. USAEC Report K-1693, Union Carbide Corp., Oak Ridge, Tenn. (March, 1967).

15. F. H. Attix and W. C. Roesch, ed. Radiation Dosimetry. Vol. 1, Academic Press, New York (1968).
16. H. G. Ebert, ed. Proc. of Symp. on Microdosimetry at Ispra, Italy, Nov. 13, 1967. European Atomic Energy Community, Brussels (1968).

17. H. H. Rossi. "Specification of Radiation Quality." Radiation Research 10, 522 (1959).

18. W. Rosenzweig and H. H. Rossi. "Determination of the Quality of the Absorbed Dose Delivered by Monoenergetic Neutrons.' Radiation Res. 10, 532 (1959).

19. H. Biavati et $a \bar{l}$. "The Dependence of RBE on the Energy of Fast Neutrons." Radiation Res. 19, 512 (1963).

20. I. F. Phillips et a.l. "Dose Distributions as a Function of LET and Measurements, of QF around the BNL Medical Research Reactor." Health Phys. 13, 1175 (1967).

2l. R. S. Caswell. "Deposition of Energy by Neutrons in Spherical Cavities." Radiation Res. 27, 92 (1966).

22. J. E. Turner. "The Determination of LET Spectra From Energy-Proportional Pülse-Height Measurements." Fealth Phys. 18, 15 (1970).

23. R. F. Dvorak. Calculation of $d E / d x$ and Energy Loss Distributions in Spherical Cavities for Monoenergetic Neutron Fields. USAEC Report ANL-7454, Argonne Nat. Lab., Argonne, Ill. (1968).

24. Linear Energy Transfer. International Commission on Radiation Units and Measurements Report 16, Wash. D. C. (1970)

25. J. W. Boag. "The Distribution of Linear Energy Transfer or 'Ion Density' for Fast Neutrons in Water." Radiation Research 6, 323 (1954).

25. J. W. Haffner. "RBE of Protons and Alpha Particles." Proc. Sec. Symp. on Protection Against Radiation in Space, Gatlinburg, Tenn. (1964).

27. Permissible Dose From External Sources of Ionizing Radiation. Recommendations of the National Committee on Radiation Protection, Nat. Bureau of Standards Handbook 59. U. S. Govt. Printing Office, Wash. D. C. (1954).

23. J. Neufeld. "Comments on Quality Factor." Health Phys. 17, No. 4, 625 (1969).

29. M. F. Yudin. "Periodic Checking of Dosimetric Instruments," Radiation Protection Monitoring p. 373, IAEA, Vienna (1969).

30. Protection Against Neutron Radiation up to 30 Million Electron Volts. National Bureau of Stardards Handbook 63, U. S. Govt. Printing Office, Wash. D. C. (1957). 


\section{DOSE RATE ESTIMATION FOR NEUTRON SOURCE SHIELDING AND APPLICATIONS}

\section{H. E. Hootman \\ D. H. Stoddard}

Savannah River Laboratory

E. I. du Pont de Nemours \& Company

Aiken, South Carolina

The ANISN discrete ordinates code which solves the energy-dependent transport equation from coupled neutron-gamma cross section sets was used to generate dose attenuation curves for common shielding materials. Calculated data are furnished for a ${ }^{252} \mathrm{Cf}$ fission source, a ${ }^{124} \mathrm{Sb}-\mathrm{Be}$ photoneutron source, and a ${ }^{239} \mathrm{Pu}-\mathrm{Be}(a, n)$ source.

\section{INTRODUCTION}

Neutron emitting radioisotopes pose unique shielding problems both with respect to the nature of the radiations to be shielded and to portability requirements. Source neutrons react with the shield to produce secondary radiation sources distributed throughout the shield. The ANISN (1) discrete ordinates code which solves the energydependent transport equation from coupled neutron-gamma cross section: sets was used to generate dose attenuation curves for common shielding materials. Calculated data are furnished for a $252 \mathrm{Cf}$ fission source, a $124 \mathrm{Sb}-\mathrm{Be}$ photoneutron source, and a $239 \mathrm{Pu}-\mathrm{Be}$ $(\alpha, n)$ source.

\section{RADIOISOTOPIC NEUTRON SOURCE CHARACTERISTICS}

Isotopic neutron sources do not require maintenance, power supplies, or elaborate control systems. Their portability depends only on shielding requirements: Thus, the importance of the neutron shield for specific applications requires that a means for estimating shield dose rates be available.

The emitted neutron energy distribution has a marked effect upon dose rate. Three neutron sources with different neutron

The information contained in this article was developed during the course of work under Contract AT (07-2)-1 with the U. S. Atomic Energy Commission. spectra were used in calculating dose rate curves in common materials. The thirteen neutron energy groups chosen to describe their respective spectra are shown in Table 1 .

The ${ }^{252} \mathrm{Cf}$ fission source neutron energy distribution is intermediate between the ${ }^{239} \mathrm{Pu}-\mathrm{Be}(\alpha ; \mathrm{n})$ source and the ${ }^{124} \mathrm{Sb}-\mathrm{Be}$ photoneutron source. A $239 \mathrm{Pu}-\mathrm{Be}$ source emits more energetic neutrons and an $124 \mathrm{Sb}-\mathrm{Be}$ source emits relatively low energy neutrons.

Calculated results shown in Figures 1 to 6 reflect the influence of these differing neutron spectra on relative neutron and capture gamma contributions to the dose rate at various thicknesses of shielding.

\section{METHOD OF CALCULATION}

Dose rate attenuation curves were calculated with the ANISN code. ANISN embodies a discrete ordinates $\left(\mathrm{S}_{\mathrm{n}}\right)$ method of approximating the solution of the energy-dependent linear Boltzmann transport equation with anisotropic scatiering.

The angular flux was approximated by a sixteenth order quadrature $\left(\mathrm{S}_{16}\right)$, and the anisotropy of elastically scattered neutrons and Compton-scatiered photons was approximated by a third order Legendre expansion $\left(\mathrm{P}_{3}\right)$. 
The spectrum has been divided into 13 neutron energy groups and 9 gamma ray energy groups as shown in Table I. The neutron groups have been coupled with the gamma ray groups to calculate the inelastic and capture gamma rays that arise on neutron scatter or absorption.

Cross sections for neutron-induced reactions and energy group-to-group transitions were taken from a 100 group ENDF/B library. These cross sections were compressed from 100 to 13 neutron energy groups with ANISN using a $\mathrm{H}_{2} \mathrm{O}$ medium. Gamma ray group-to-group transfer cross sections were generated by the MUG code (2) which includes photoelectric and pair production absorptions as well as Compton scattering. Cross sections for neutron-induced secondary and inelastic scattering gamma rays were coupled between the neutron and photon matrices. Capture gamma ray yields were taken from thermal neutron data and were assumed to be invariant to neutron energy.

Conversion factors used to convert neutron flux to biological dose rate (Table I) were based on the Snyder-Neufeld data with relative biological effectiveness factors based on NBS handbook values (3). Gamma ray flux to dose conversion factors were based on the maximum exposure dose proposed by Claiborne (4).

In every case, the source was assumed to be in an aluminum matrix, spherical in shape, with a volume of $1 \mathrm{~cm}^{3}$. Shield geometry was also spherical.

\section{ATTENUATION DATA}

Figures 1 to 6 showing the results of dose attenuation calculations provide a means for estimating neutron dose rate and neutroninduced gamma ray dose rates associated with several common neutron sources. To facilitate comparison, dose rates are normalized to one source neutron per second multiplied by $4 \pi$ times the shield thickness squared to reduce the effect of. spherical divergence.

Dose rates listed are for the surface of tissue surrounding the material of interest. The data can be used to compare shield materials and to estimate required: shield thickness.

Neutron and neutron-induced dose rates at the surface of a shield material may be estimated by adding the neutron dose rate to the gamma dose rate at a common shield thickness as shown

$D, m r e m / h r=\left[\left(D_{n}+D_{\gamma}\right)\right]\left[\frac{\text { Source Strength, Neutrons/sec }}{4 \pi(\text { Shield Thickness, cm })^{2}}\right]$

The source strength in neutrons/sec may be calculated from the following data:

\begin{tabular}{|c|c|c|}
\hline Source & Type & Neutron Yield \\
\hline${ }^{252} \mathrm{Cf}$ & Fission. & $2.3\left(10^{12}\right)$ neutrons/sec/gram \\
\hline${ }^{239} \mathrm{Pu}-\mathrm{Be}$ & $\alpha, n$ & $2.2\left(10^{6}\right) *$ neutrons/sec/curie \\
\hline${ }^{124} \mathrm{Sb}-\mathrm{Be}$ & $\gamma, n$ & $\begin{array}{l}5.1^{*} \text { neutrons per } 10^{6} \\
\text { disintegrations for } 1 \text { gram } \\
\text { of target at } 1 \mathrm{~cm}\end{array}$ \\
\hline
\end{tabular}

The importance of the source gamma rays will vary with source and shield thickness; therefore, source gamma ray dose rates have not been included in this presentation. They may, however, be estimated by using appropriate gamma energy attentuation coefficients for the shield material in the relation

$$
D=D_{\text {Source }} e^{-\mu t}
$$

where $\mu$ is the energy-dependent gamma ray attenuation coefficient $\left(\mathrm{cm}^{-1}\right)$ from Reference 6 and $t$ is shield thickness $(\mathrm{cm})$.

\section{EFFECT OF NEUTRON SOURCE SPECTRA}

Comparison of the slopes of the neutron dose rate attenuation curves in Figures 1 , 2 , and 3 reveal the obvious effect of the neutron source spectra. For higher energy neutron sources, attenuation for a given thickness is less, and therefore the neutron dose rate contribution to the total dose is greater.

The magnitude of the capture gamma ray dose for the different sources shown in Figures 4,5 , and 6 is not as sensitive a function of neutron spectra. The capture gamma ray dose rate contribution to the total dose can be expected to remain relatively constant. 
BORON OR LITHIUM ADDITION

TO REDUCE CAPTURE GAMMA RAYS

Neutron capture gamma ray dose rates from hydrogenous shields can be substantially reduced by adding $10_{\mathrm{B}}$ or ${ }^{6} \mathrm{Li}$ to the shielding material. The dose is reduced because a charged particle reaction is substituted for the hydrogen-radiative capture reaction in the shield. If the capture gamma dose is reduced, then the shield weight can be reduced or the source capacity of the shield can be increased.

The relative effect of ${ }^{6} \mathrm{Li}$ and ${ }^{10}{ }_{\mathrm{B}}$ addition to a spherical paraffin shield are shown in Figures 7 and 8 . The capture gamma ray reduction was found to be relatively insensitive to neutron source spectra. If ${ }^{6} \mathrm{Li}$ or $10_{\mathrm{B}}$ is added to a shield, the neutron dose is reduced very little because the neutron dose is primarily from fast neutrons.

\section{ACKNOWLEDGMENTS}

The authors wish to thank the Radiation Shielding Information Center at Oak Ridge for furnishing the necessary computer codes for this work.

\section{REFERENCES}

1. W. W. Engle, Ir. A Users Manual for ANISN. USAEC Report K-1693, Union Carbide Corporation, Nuclear Division, Oak Ridge Gaseous Diffusion Plant, Oak Ridge, Tenn. (1967).

2. J. R. Knight and F. R. Mynatt. MUG, A Program for Generalizing Multigroup Photon Cross Sections. USAEC Report CTC-17, Oak Ridge National Laboratory, Oak Ridge, Temn. (1970).

3. The National Bureau of Standards Handbook No. 63. U. S. Government Printing Office, Washington, D. C. (1957).

4. H. C. Claiborrie and D. K. Trubey. "Gamma Dose Rátes in a Slab Phantom." Trans. Am. Nue 1. Soc. 12, (1), 383 (1969).

5. R. G. Jaeger (Ed). Engineering Compendium on Radiation Shielding. Vol. 1, p. 31, 35, Springer-Verlag, New York (1968).

6. E. P. Blizard (Ed). Reactor Handbook. (2 ed), Vol. III, Part B, Shielding. Interscience Fublishers, New York (1962).

TABLE I

22 Group Source Distribution and Conversion Factors

\begin{tabular}{|c|c|c|c|c|c|c|c|}
\hline \multirow[b]{2}{*}{$\begin{array}{c}\text { Type } \\
\text { Radiation }\end{array}$} & \multirow[b]{2}{*}{ Group } & \multirow[b]{2}{*}{ Energy } & \multirow[b]{2}{*}{ Interval, Mev } & \multirow{2}{*}{$\begin{array}{l}\text { Dose Rate Conversion Factor, } \\
\left(\mathrm{mrem} \mathrm{hr}^{-1}\right) /\left(\text { particle } \mathrm{sec}^{-1} \mathrm{~cm}^{-2}\right)\end{array}$} & \multicolumn{3}{|c|}{ Source Distribution } \\
\hline & & & & & ${ }^{252} \mathrm{Cf}$ & $\frac{124}{\mathrm{Sb}-\mathrm{Be}}$ & ${ }^{239} \mathrm{Pu}-\mathrm{Be}$ \\
\hline Neutron & $\therefore 1$ & 10.0 & 14.92 & 0.147 & 0.0036 & & 0.00005 \\
\hline & 2 & 6.70 & 10.00 & 0.147 & 0.0279 & & 0.16240 \\
\hline & 3 & 5.49 & 6.70 & 0.142 & 0.0356 & & 0.09211 \\
\hline & 4 & .4 .49 & 5.49 & 0.139 & 0.0547 & & 0.15175 \\
\hline & 5 & 3.68 & 4.49 & 0.134 & 0.0730 & & 0.14217 \\
\hline & 6 & 3.01 & 3.68 & 0.130 & 0.0869 & & 0.10330 \\
\hline & 7 & 2.02 & 3.01 & 0.125 & 0.1894 & & 0.05431 \\
\hline & 8 & 0.91 & 2.02 & 0.130 & 0.3033 & & 0.16879 \\
\hline & 9 & 0.41 & 0.91 & 0.105 & 0.1462 & & 0.09797 \\
\hline & 10 & 0.11 & 0.41 & 0.056 & 0.0676 & & 0.02715 \\
\hline & 11 & 0.015 & $-\cdots \quad 0.11$ & 0.020 & 0.0119 & 1.0 & \\
\hline & 12 & 0.0000 & $0041-0.015$ & 0.0056 & & & \\
\hline & 13 & 0.0 & $\ldots 0.0000041$ & 0.00373 & & & \\
\hline Gamma & 14 & $6.0^{\circ}$ & 10.0 & 0.0081 & & & \\
\hline & 15 & 5.0 & 6.0 & 0.0061 & & & \\
\hline & 16 & 4.0 & 5.0 & 0.0054 & & & \\
\hline & 17 & 3.0 & 4.0 & 0.0045 & & & \\
\hline & 18 & 2.0 & 3.0 & 0.0036 & & & \\
\hline & 19 & 0.9 & 2.0 & 0.0031 & & & \\
\hline & 20 & 0.4 & 0.9 & 0.0014 & & & \\
\hline & 21 & 0.12 & 0.4 & 0.00065 & & & \\
\hline & 22 & 0.01 & 0.12 & 0.00015 & & & \\
\hline
\end{tabular}




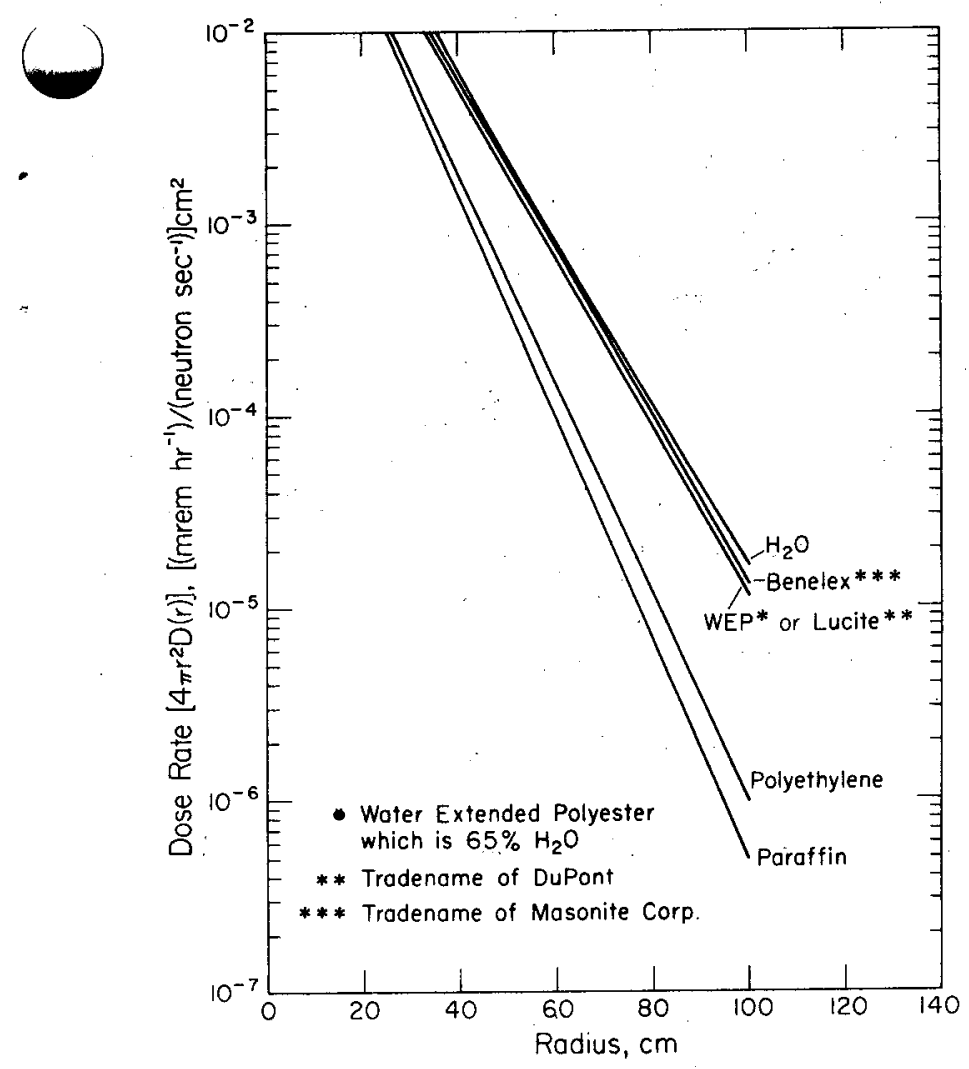

FIG. $1{ }^{239} \mathrm{PU}-\mathrm{Be}$ NEUTRON DOSE RATE

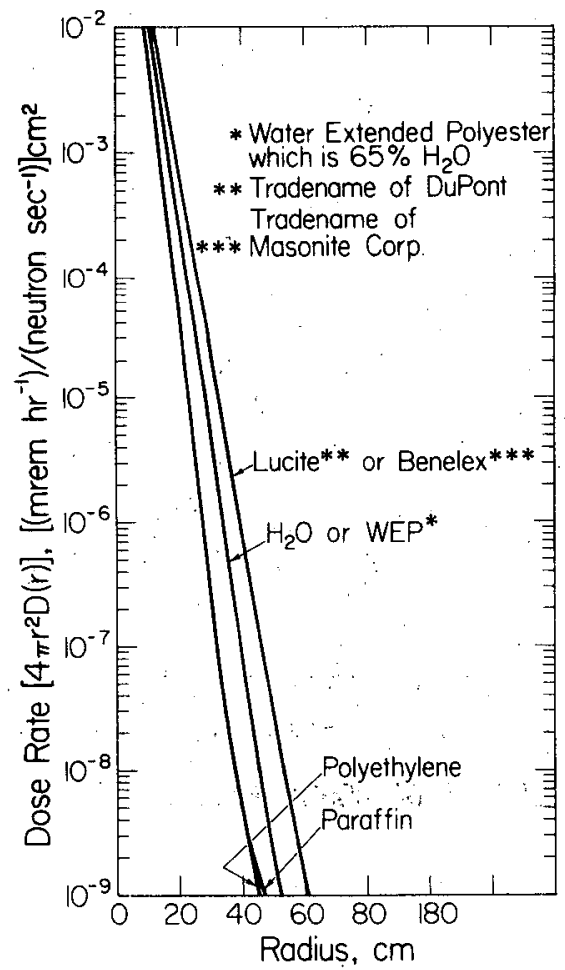

FIG. $3{ }^{124} \mathrm{Sb}-B \mathrm{e}$ NEUTRON DOSE RATE

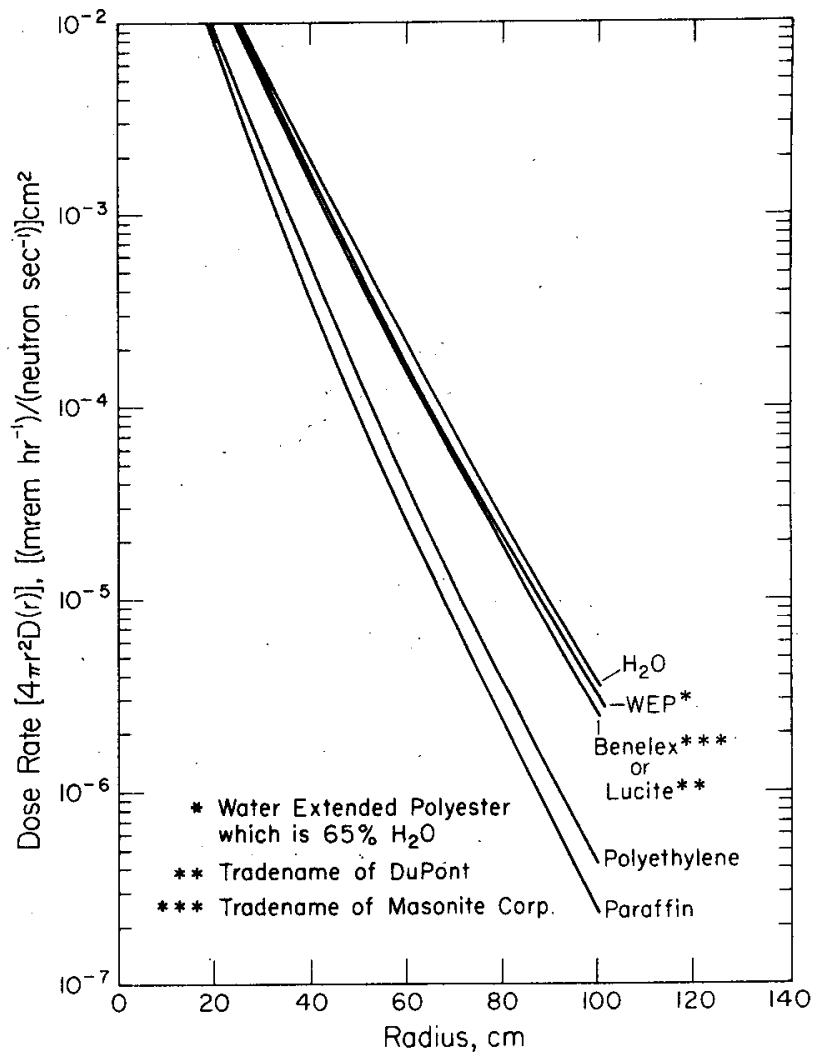

FIG. $2 \quad{ }^{252} \mathrm{Cf}$ NEUTRON DOSE RATE

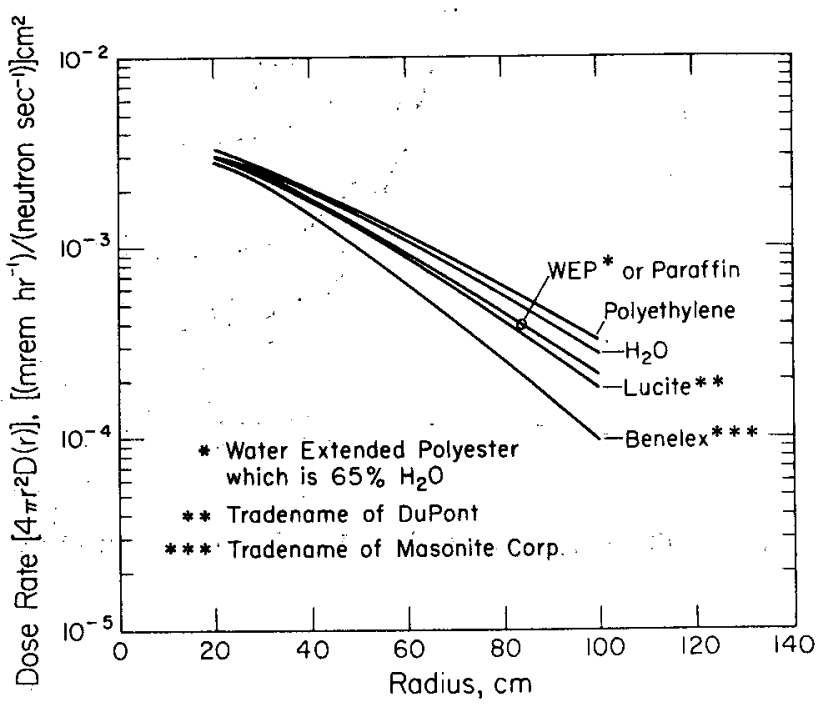

FIG. 4. ${ }^{239} \mathrm{Pu}$-Be CAPTURE GAMMA RAY DOSE RATE 


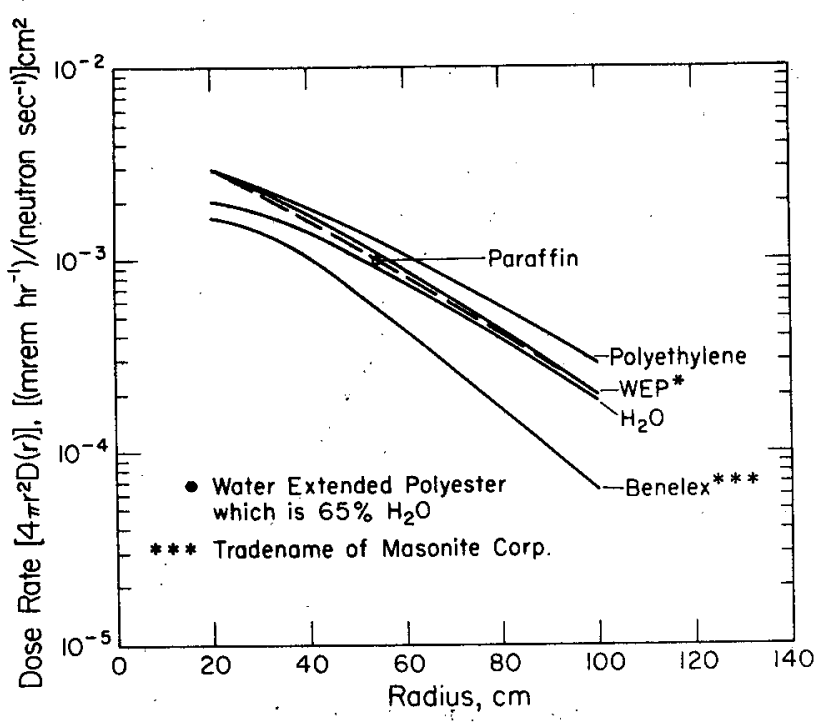

FIG. $5{ }^{252} \mathrm{Cf}$ CAPTURE GAMMA RAY DOSE RATE

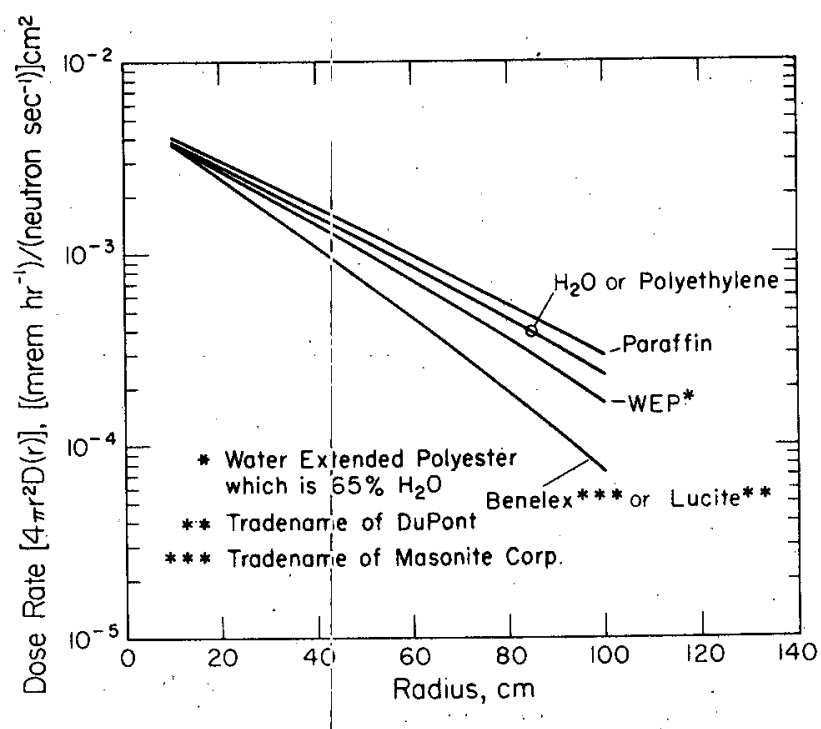

FIG. $6{ }^{124} \mathrm{Sb}$-Be (APTURE GAMMA RAY DOSE RATE

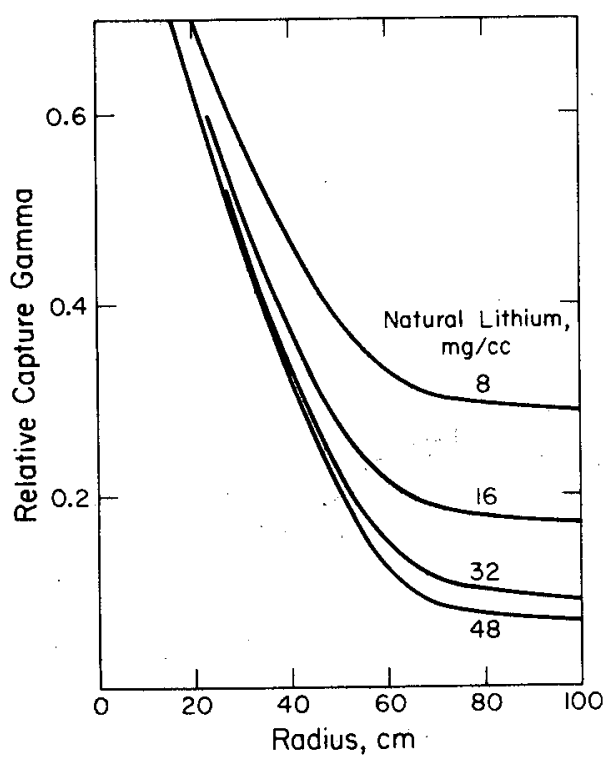

FIG. 7 EFFECT OF LITHIUM ADDITION TO PARAFFIN

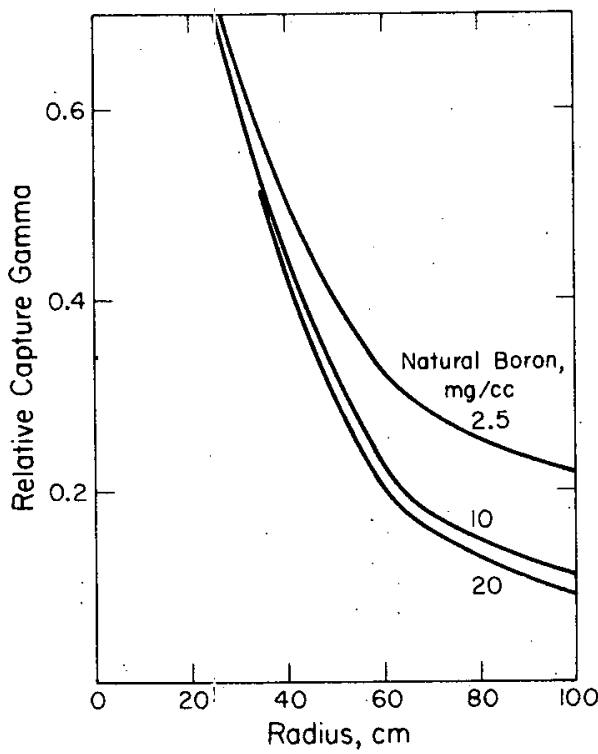

FIG. 8 EFFECT OF BORON ADDITION TO PARAFFIN 


\title{
CALCULATED AND MEASURED EFFECTIVENESS OF CALIFORNIUM-252 SOURCE SHIELDING
}

\author{
D. H. Stoddard \\ R. A. Moyer \\ Savannah River Laboratory \\ E. I. du Pont de Nemours and Company \\ Aiken, South Carolina
}

The Savannah River Laboratory has effectively used the discrete ordinates computer code ANISN to evaluate effectiveness of shields and casks for the shipment of ${ }^{252} \mathrm{Cf}$ sources. Gamma and neutron dose rate equivalents radiating from shields and casks containing ${ }^{252} \mathrm{Cf}$ sources were measured with radiological instruments and thermoluminescent dosimeters.

In most instances, the calculated data agree within a factor of two with the measured data. Differences generally indicate the calculations are conservative.

\section{INTRODUCTION}

${ }^{252} \mathrm{Cf}$ has radiation properties ( 1 ) that make it useful in applications where a compact source of neutrons is needed. The spontaneous fission properties of $252 \mathrm{Cf}$, however, present special shielding problems in handling, shipping, and transporting this material. The Savannah River Laboratory has been employing a discrete ordinates method of approximating the solution to the energydependent linear Boltzmann transport equation to determine shielding effectiveness of materials and to determine the shielding capability of storage vessels or casks.

Organizations contemplating $\cdot \mathrm{h}$ andling, shipping, or storing $252 \mathrm{Cf}$ sources are interested in comparing data acquired by calculations and that acquired by actual experiments and tests because such comparisons verify the calculation techniques.

The objective of this paper is to present ${ }^{252}$ Cf shielding data obtained by a specific calculation technique and compare these data with some actual measurements made with specific shields. The data are useful not only because of increased confidence in calculations but also because they demonstrate

The information contained in this article was developed during the course of work under Contract AT (07-2)-1 with the U. S. Atomic Energy Commission. a technique for measuring the capability of a cask.

The casks listed do not necessarily represent the most efficient shields and arrangements for californium. In some cases, the casks were used because a design was already available, and this represented the most expeditious method of cask design and approval.

For designing a new californium cask or shield for a specific use, see Reference 2, which gives shielding data on many materials. The data in this paper and the data in Reference 2 are expected to assist in the judicious selection of materials with which the reader may improve one of these already existing casks or design his own shielding. The primary purpose of this report is to demonstrate the validity: of the calculations for many of the materials available.

\section{GENERAL CALIFORNIUM RADIATION PROPERTIES}

Californium-252 decays $97^{\circ}$ by the emission of alpha particles and $3 \%$ by spontaneous fission. The gamma radiation associated with the alpha decay is primarily of low energy $(<0.25 \mathrm{Mev})$ and therefore can normally be neglected for cask shielding. The abundance of neutrons and gamma rays associated with the spontaneous fission process is the primary source of radiation that must be shielded. A more detailed description of the 
radiation properties used in the calculations are given in References. 1, 2, and 3 .

The source energy distribution used in the calculations is given in Table $\mathrm{I}$. Conversion factors used to convert calculated flux to dose rate are listed in Table II, and were based on the data reported in References 4 and 5 .

The dose rate for unshielded californium sources in air can be approximated by multiplying the unshielded neutron flux at the distance desired by the $4 \pi r^{2} D(r)$ dose rate factors listed in Table III.

\section{SHIELDING REQUIREMENTS}

Shielding necessary to meet a specific need will vary considerably; therefore, no attempt is made in this paper to specify shield materials or thicknesses. The designer interested in designing his californium container is referred to Reference 2. For shipping requirements, refer to the $U, S$. Code of Federal Regulations, Title 49, Chapter I, Part 173, "Regulations Applying to Shippers," paragraphs 173.389 through 173.399. For information on storage and general use, refer to the U.S. Code of Federal: Regulations, Title 10, Chapter. I, Part 20, "Standards for Protection Against Radiation."

\section{METHOD OF CALCULATION}

A11 shielding calculations in this report were performed using the ANISN (6) code.

ANISN is an acromym for the discrete ordinates method of approximating the solution for the energy-dependent linear Boltzmann transport equation with anisotropic scattering. Spherical geometry which closely approximated that of all casks was used with the source being small $\left(<1-\mathrm{cm}^{3}\right.$ sphere $)$.

The angular flux was approximated by a sixteenth order quadrature $\left(\mathrm{S}_{16}\right)$ and the anisotropy of elastically scattered neutrons and of Compton scattered photons was approximated by a third order. Legendre expansion $\left(\mathrm{P}_{3}\right)$. Multigroup theory of neutron and gamma ray transmission was used, dividing the 22 energy groups into 13 neutron and 9 gamma groups. The neutron groups are coupled with the gamma groups to calculate inelastic and capture gamma rays resulting from neutron scatter and absorption.

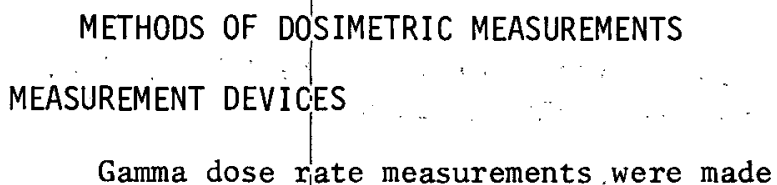

Gamma dose rate measurements, were made using a Victoreen* Mode1-440 instrument and with Harshaw TLD-700 ribbons** packaged in a small plastic vial (Figure 1). Both of these devices were calibrated with a radium gamma source.

Neutron dose rates were measured with a Trapelo*** "Snoopy" instrument and/or an SRL Fast Neutron Survey Meter (7), and with the timed exposure of a TLD neutron "badge" recently developed for personnel monitoring (8) (Figure 2). This TLD neutron "badge" consists of a 2-in.-polyethylene hemisphere that has been sliced $1 / 4-i n$. from the rounded end, which contains a Harshaw TLD-600 and Harshaw TLD-700 ribbon in the dome section, and another set of the two ribbons placed at the base section of the hemisphere. The curved surface of the polyethylene.hemisphere is covered with 1/32-in. cadmium and a shell of 20-gauge stainless steel. A stainless steel back plate holds the unit tcigether. The badge was attached to a 6-gal. water-filled polyethylene bottle used as a body phantom.

Neutron devices were calibrated with a 10-Ci Pu-Be'neution source or a $\mathrm{PuF}_{4}$ source.

\section{CONTAINERS AND DATA}

Figures 3-12i include cask descriptions, sketches of the sihielding and/or photographs of the casks, and the calculated and measured dose rate data. The dose rate data are listed in mrem/hr at the point of interest per $\mu \mathrm{g}$ of ${ }^{252} \mathrm{Cf}$ in the source. The points of interest included the dose rate at the surface of the container and at $36 \mathrm{in}$. in air from the container.

* Victoreen Insitrument Co.

* Harshaw Chemical Co. thermoluminescent dosimeters.

*** Trapelo Divi:sion of LFE Corp. Model $\mathrm{NP}-2$. 


\section{ACKNOWLEDGMENTS}

The authors acknowledge the Radiation Shielding Information Center for supplying the computer codes necessary for the calculations.

\section{REFERENCES}

1. D. H. Stoddard. Radiation Properties of Californium-252. USAEC Report DP-986, Savannah River Laboratory, E. I. du Pont de Nemours \& Co., Aiken, S. C. (1965).

2. D. H. Stoddard and H. E. Hootman. Californium-252 Shielding Guide. USAEC Report DP-1246, Savannah River Laboratory, E. I. du Pont de Nemours \& Co., Aiken, S. C. (to be issued in 1971).

3. J. J. Barker. Californium-252. Proceedings of a Symposium Sponsored by the New York Metropolitan Section of the American Nuclear Society, USAEC Report CONF-681032 (1969).

4. N.B.S. Handbook 63, U. S. Government Printing Office, Washington, D. C. (1957).

5. H. C. Claiborne and D. K. Trubey. "Gamma Dose Rates in a S1ab Phantom," Trans. Am. Nuc2. Soc. 12(1), 383 (1969).

6. W. W. Engle. ANISN Users Manual: A One Dimensional Discrete Ordinates Transport Code with Anisotropic Scattering. USAEC Report K-1693, Union Carbide Corp., Nuclear Div., Oak Ridge Gaseous Difension Plant, Oak Ridge, Tenn. (1967).

7. W. F. Splichal. A Portable MuZtipurpose Radiation Monitor. USAEC Report DP-993, Savannah River Laboratory, E. I. du Pont de Nemours \& Co., Aiken, S. C. (1966).

8. A. Korba and J. E. Hoy. "A Thermoluminescent Personne1 Neutron Dosimeter." Health Physics 18, 581 (1970).
TABLE I

Twenty-Two Group Source Distribution for ${ }^{252} \mathrm{Cf}$

\begin{tabular}{|c|c|c|c|}
\hline Group & $\begin{array}{c}\text { Energy } \\
\text { Interval, } \\
\text { Mev }\end{array}$ & $\begin{array}{c}\text { Type } \\
\text { Radiation } \\
\end{array}$ & $\begin{array}{c}\text { Source Distribution, } \\
\text { number/neutron }\end{array}$ \\
\hline 1 & $10-14.92$ & Neutron & 0.0036 \\
\hline 2 & $6.70-10.00$ & Neutron & 0.0279 \\
\hline 3 & $5.49-6.70$ & Neutron & 0.0356 \\
\hline 4 & $4.49-5.49$ & Neutron & 0.0547 \\
\hline 5 & $3.68-4.49$ & Neutron & 0.0730 \\
\hline 6 & $3.01-3.68$ & Neutron & 0.0869 \\
\hline 7 & $2.02-3.01$ & Neutron & 0.1894 \\
\hline 8 & $0.91-2.02$ & Neutron & 0.3033 \\
\hline 9 & $0.41-0.91$ & Neutron & 0.1462 \\
\hline 10 & $0.11-0.41$ & Neutron & 0.0676 \\
\hline 11 & $0.015-0.11$ & Neutron & 0.0119 \\
\hline 12 & $0.000004-0.015$ & Neutron & 0.0 \\
\hline 13 & $0.0-0.000004$ & Neutron & 0.0 \\
\hline 14 & $6.0-10.0$ & Gamma & 0.0012 \\
\hline 15 & $5.0-6.0$ & Gamma & 0.0056 \\
\hline 16 & $4.0-5.0$ & Gamma & 0.020 \\
\hline 17 & $3.0-4.0$ & Ganma & 0.071 \\
\hline 18 & $2.0-3.0$ & Gamna & 0.094 \\
\hline 19 & $0.9-2.0$ & Gamma & 1.06 \\
\hline 20 & $0.4-0.9$ & Gamma & 2.4 \\
\hline 21 & $0.12-0.4$ & Garma & 2.0 \\
\hline 22 & $0.01-0.12$ & Gamura & 0.0 \\
\hline
\end{tabular}

TABLE II

Dose Rate Conversion Factors $(\underline{4}, \underline{5})$

Group $\quad\left(\frac{\text { mrem-hr- }}{\text { neutron- } \mathrm{sec}^{-1}}\right) \mathrm{cm}^{2}$

0.147

0.147

0.142

0.139

0.134

0.130

0.125

0.130

0.105

0.056

0.0056

0.00373

0.0081

0.0061

0.0054

0.0045

0.0036

0.0014

0.00065

0.00015

TABLE III

Dose Rate Attenuation Factors

$$
4 \pi \mathrm{r}^{2} \mathrm{D}(\mathrm{r})
$$

Type

Radiation

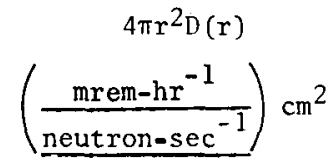

Neutron

$1.22 \times 10^{-1}$

Gamma

$8.58 \times 10^{-3}$

Tota1 


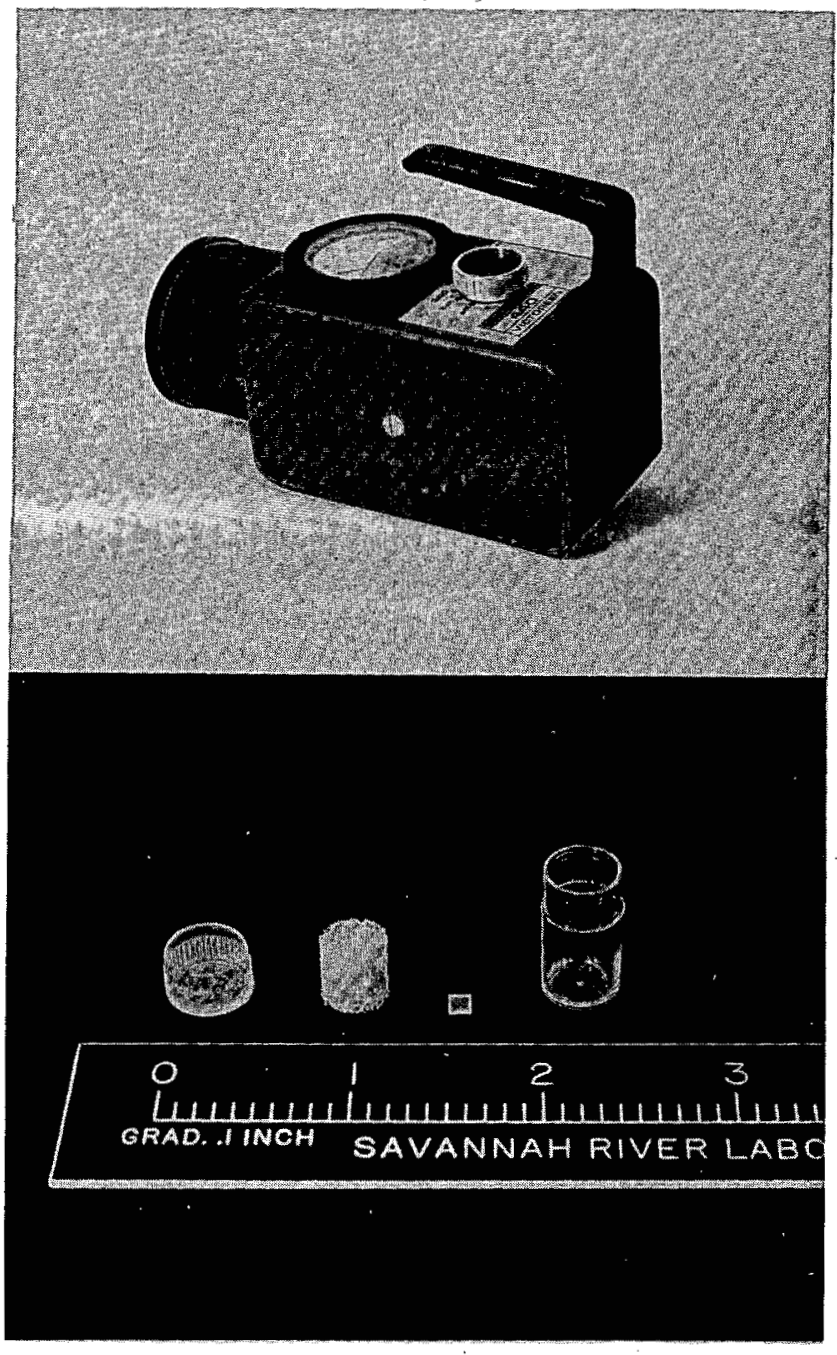

FIG. 1 GAMMA DOSE RATE MEASUREMENT DEVICES
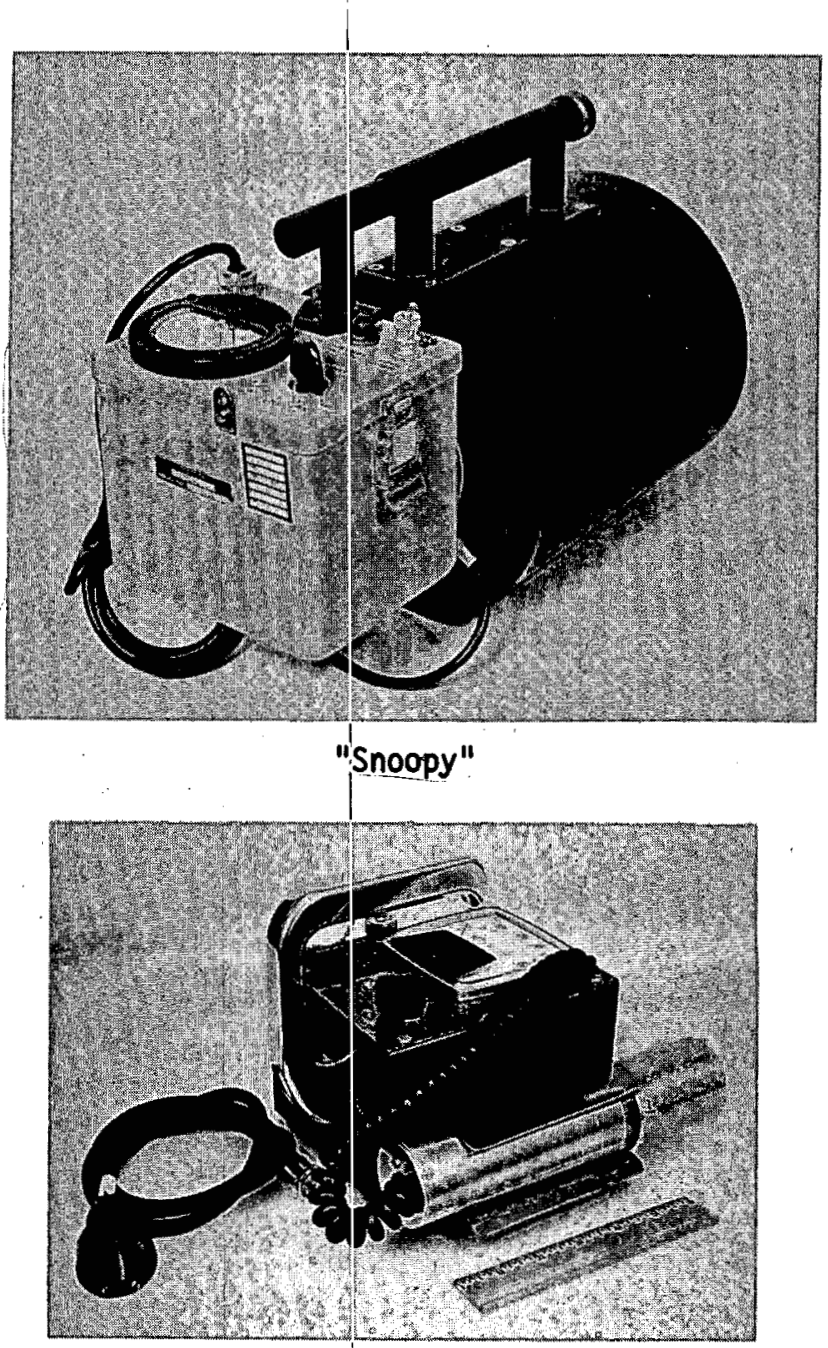

SRL Fast Neutron Survey Meter

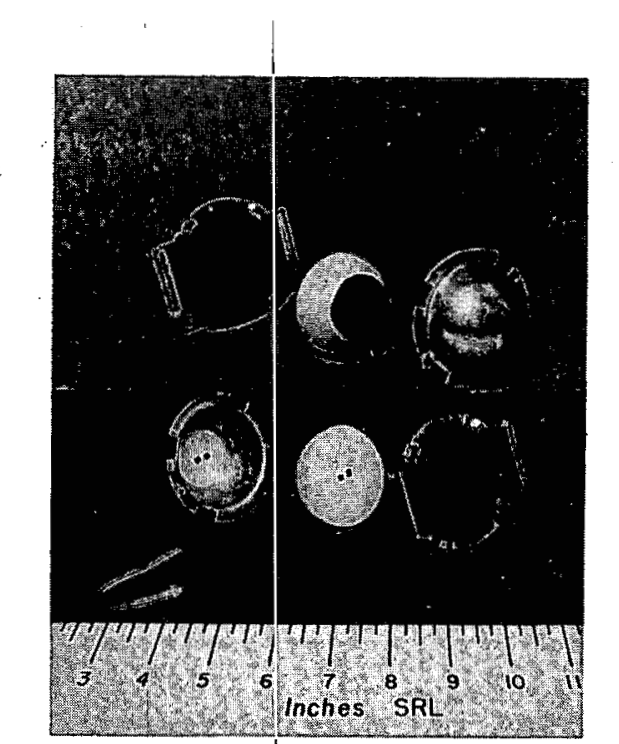

Thermoluminescent Neutron Dosimeter

FIG. 2 NEUTRON DOSE RATE MEASUREMENT DEVICES 

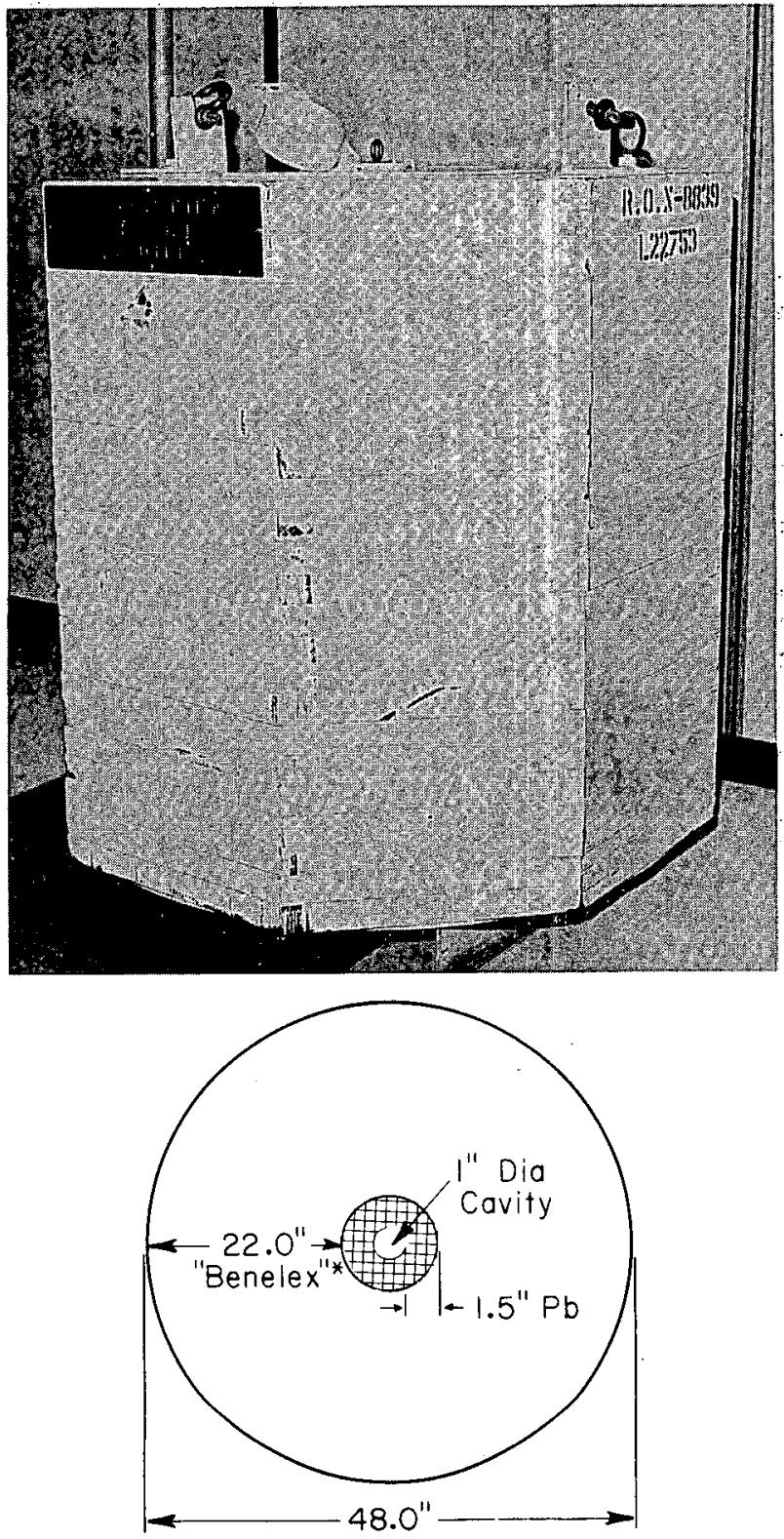

Dose Rate Data

Dose Rate per Microgram of Source,

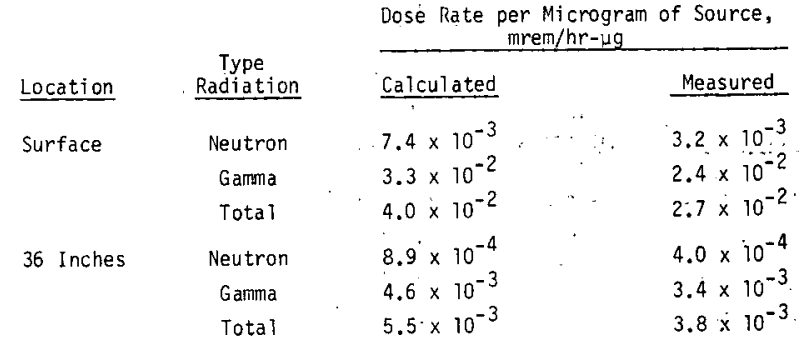

FIG. 3 DOSE RATE DATA FOR "BENELEX" CASK

* Registered trademark of Masonite Corp.
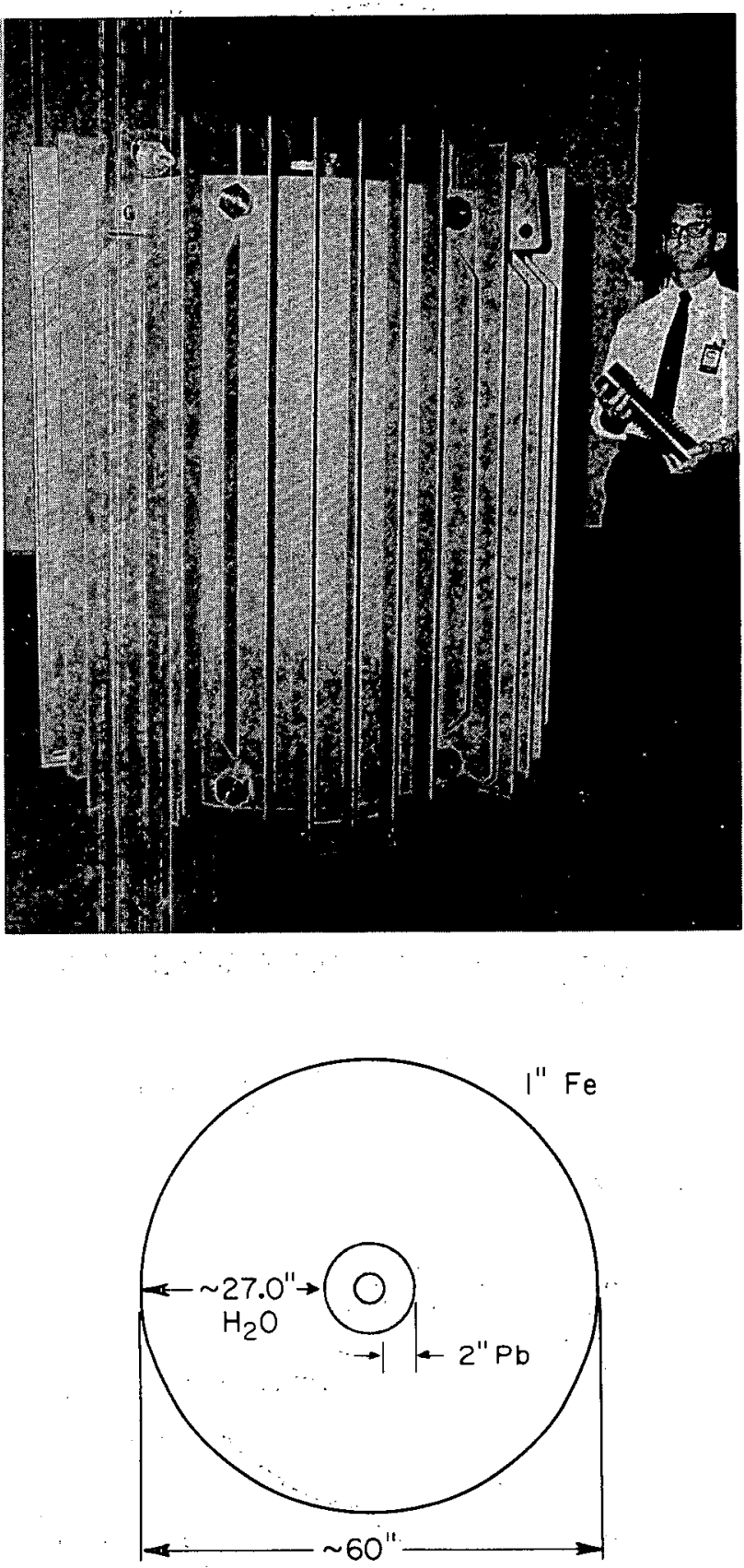

Dose Rate Data

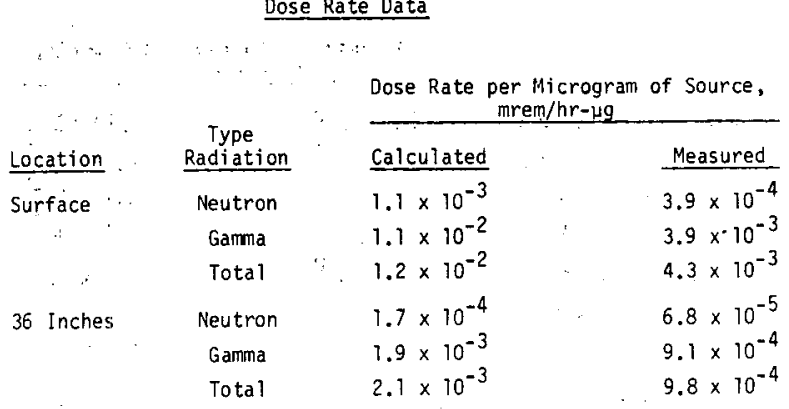

FIG. 4 DOSE RATE DATA FOR WATER-FILLED CASK 

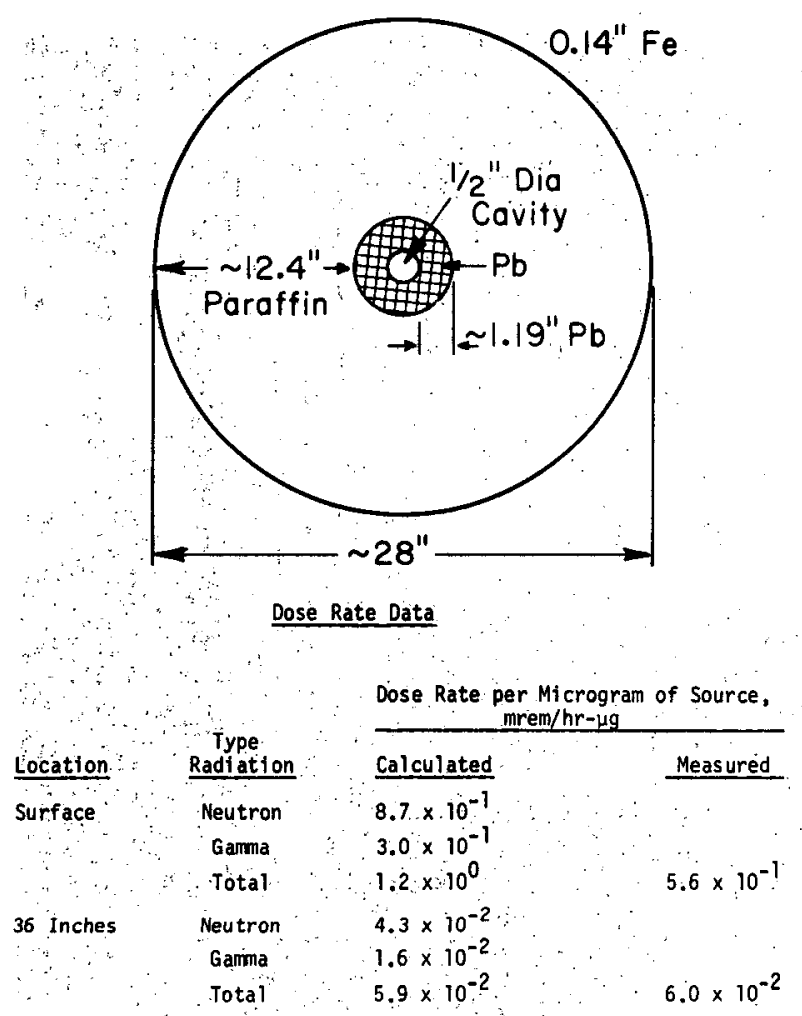

FIG. 5 DOSE RATE DATA FOR PARAFFIN-LEAD CASK

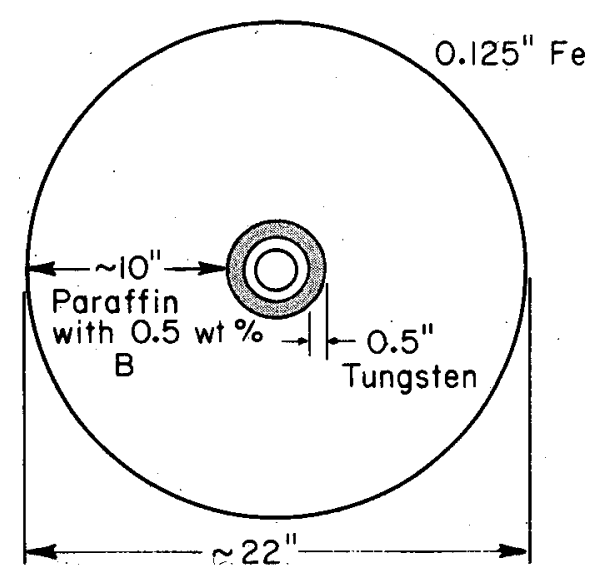

Dose Rate Data

\begin{tabular}{|c|c|c|c|}
\hline \multirow[b]{2}{*}{ Location } & \multirow[b]{2}{*}{$\begin{array}{c}\text { Type } \\
\text { Radiation } \\
\end{array}$} & \multicolumn{2}{|c|}{$\begin{array}{c}\text { Dose Rate per Microgram of Source, } \\
\text { mrem/hr- } \mu g\end{array}$} \\
\hline & & Calculated & Measured \\
\hline \multirow[t]{3}{*}{ Surface } & Neutron & $5.0 \times 10^{-1}$ & $8.8 \times 10^{-1}$ \\
\hline & Gamma & $6.6 \times 10^{-1}$ & $5.4 \times 10^{-1}$ \\
\hline & Total & $1.2 \times 10^{0}$ & $1.4 \times 10^{0}$ \\
\hline \multirow[t]{3}{*}{36 Inches } & Neutron & $2.5 \times 10^{-2}$ & $3.8 \times 10^{-2}$ \\
\hline & Gamma & $3.4 \times 10^{-2}$ & $2.1 \times 10^{-2}$ \\
\hline & Total & $5.9 \times 10^{-2}$ & $5.9 \times 10^{-2}$ \\
\hline
\end{tabular}

FIG. 6 DOSE RATE DATA FOR CASK OF TUNGSTEN AND PARAFFIN WITH 0.5 wt \% BORON
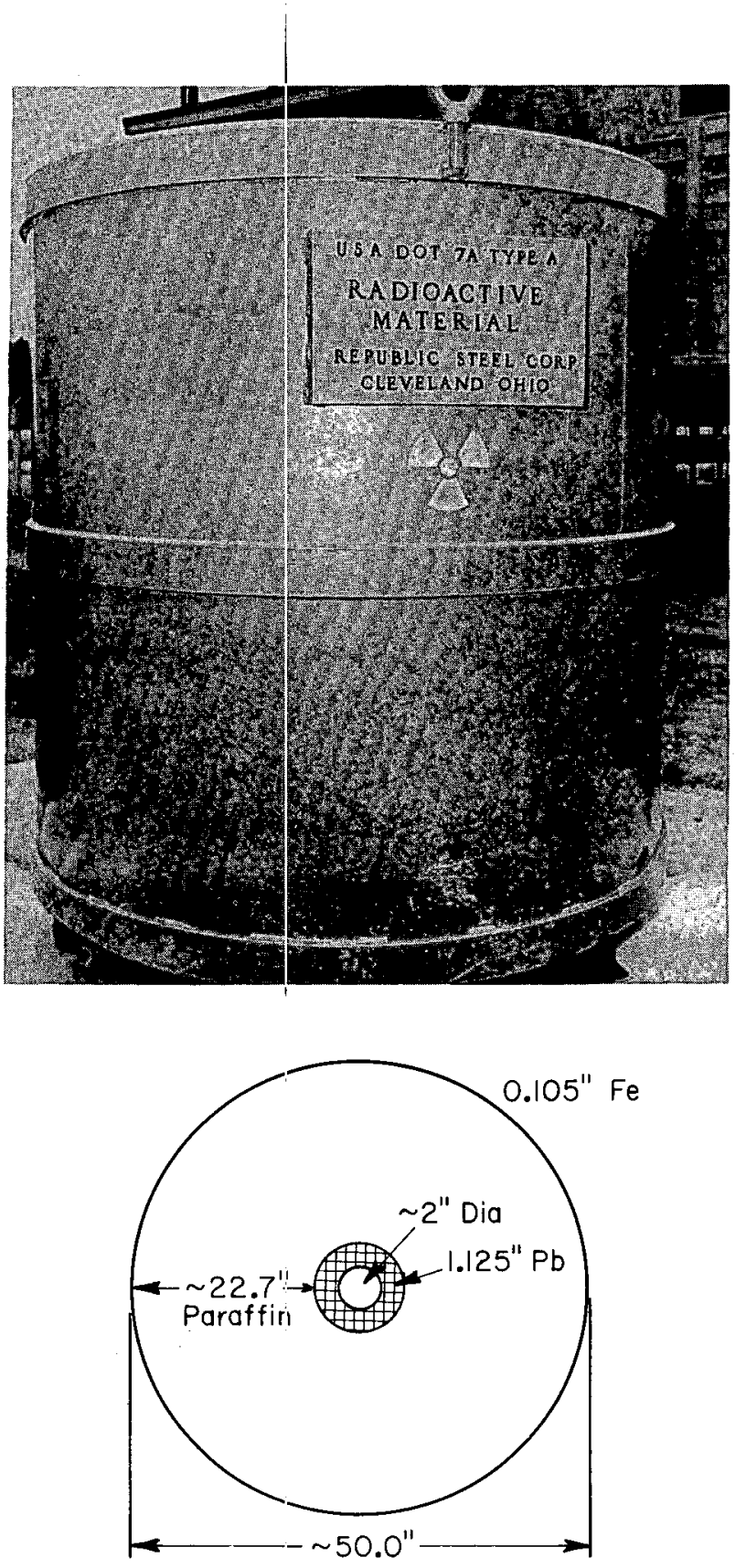

Dosi: Rate Data

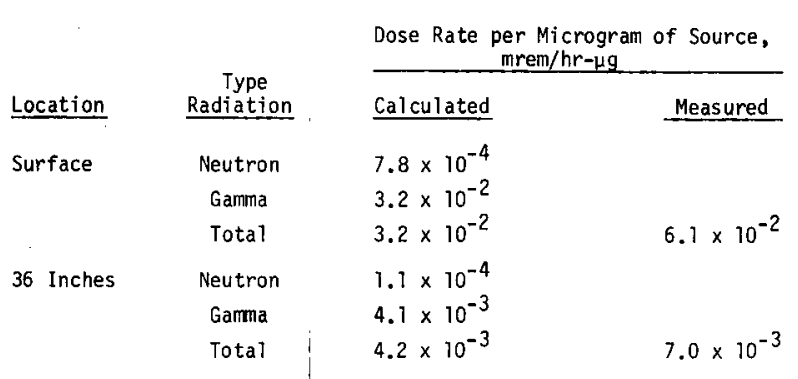

FIG. 7 DOSE RATE DATA FOR PARAFFIN-LEAD CASK 


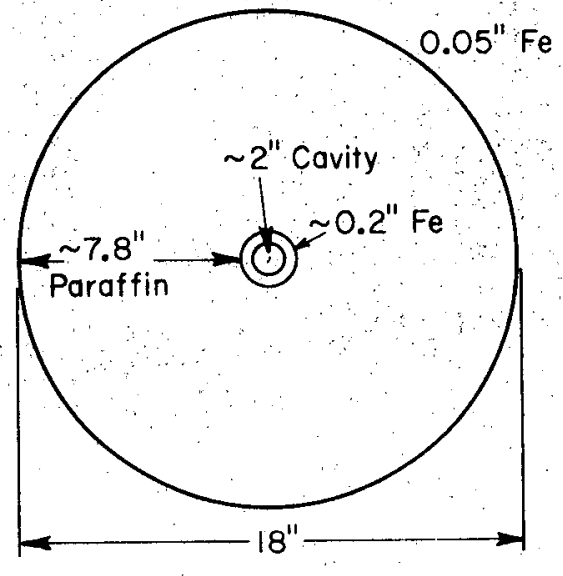

Dose Rate Data

\begin{tabular}{|c|c|c|c|}
\hline \multirow{2}{*}{ Location } & \multirow{2}{*}{$\begin{array}{c}\text { Type } \\
\text { Radiation } \\
\end{array}$} & \multicolumn{2}{|c|}{$\begin{array}{c}\text { Dose Rate per Microgram of Source, } \\
\text { mrem/hr- } \mu \mathrm{g}\end{array}$} \\
\hline & & Calculated & Measured \\
\hline \multirow[t]{3}{*}{ Surface } & Neutron & $1.3 \times 10^{0}$ & $2.1 \times 10^{0}$ \\
\hline & Gamma & $1.9 \times 10^{0}$ & $1.7 \times 10^{0}$ \\
\hline & Total & $3.2 \times 10^{0}$ & $3.8 \times 10^{0}$ \\
\hline \multirow[t]{3}{*}{36 Inches } & Neutron & $5.4 \times 10^{-2}$ & $1.2 \times 10^{-1}$ \\
\hline & Gamma & $7.1 \times 10^{-2}$ & $3.1 \times 10^{-2}$ \\
\hline & Total & $1.3 \times 10^{-1}$ & $1.5 \times 10^{-1}$ \\
\hline
\end{tabular}

FIG. 8 DOSE RATE DATA FOR PARAFFIN-IRON CASK

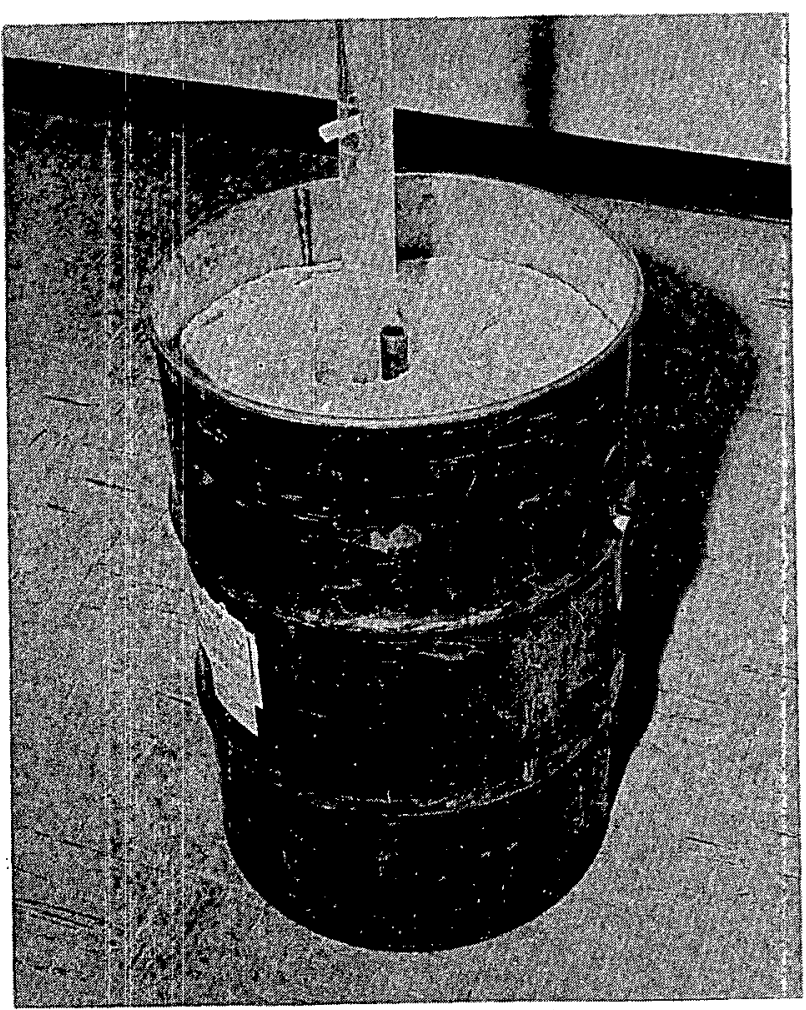

55. gal Cask Fabricated by Monsanto Research Corp.

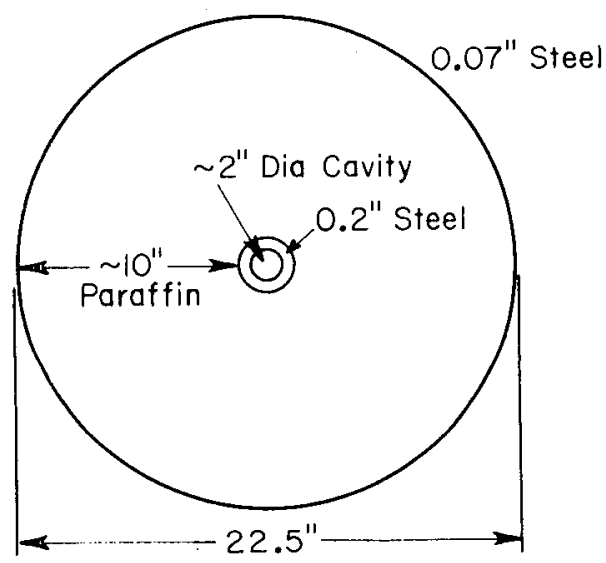

Dose Rate Data

\begin{tabular}{|c|c|c|c|}
\hline \multirow[b]{2}{*}{ Location } & \multirow{2}{*}{$\begin{array}{c}\text { Type } \\
\text { Radiation } \\
\end{array}$} & \multicolumn{2}{|c|}{$\begin{array}{c}\text { Dose Rate per Microgram of Source, } \\
\mathrm{mrem} / \mathrm{hr}-\mu \mathrm{g}\end{array}$} \\
\hline & & Calculated & Measured \\
\hline \multirow[t]{2}{*}{ Surface } & Neutron & $7.2 \times 10^{-1}$ & $4.1 \times 10^{-1}$ \\
\hline & Gamma & $1.4 \times 10^{0}$ & $8.3 \times 10^{-1}$ \\
\hline \multirow{4}{*}{36 Inches } & Total & $2.1 \times 10^{0}$ & $1.2 \times 10^{0}$ \\
\hline & Neutron & $3.4 \times 10^{-2}$ & $2.5 \times 10^{-2}$ \\
\hline & Gamma & $6.0 \times 10^{-2}$ & $5.0 \times 10^{-2}$ \\
\hline & Total & $9.4 \times 10^{-2}$ & $7.5 \times 10^{-2}$ \\
\hline
\end{tabular}

FIG. 9 DOSE RATE DATA FOR PARAFFIN-STEEL CASK 


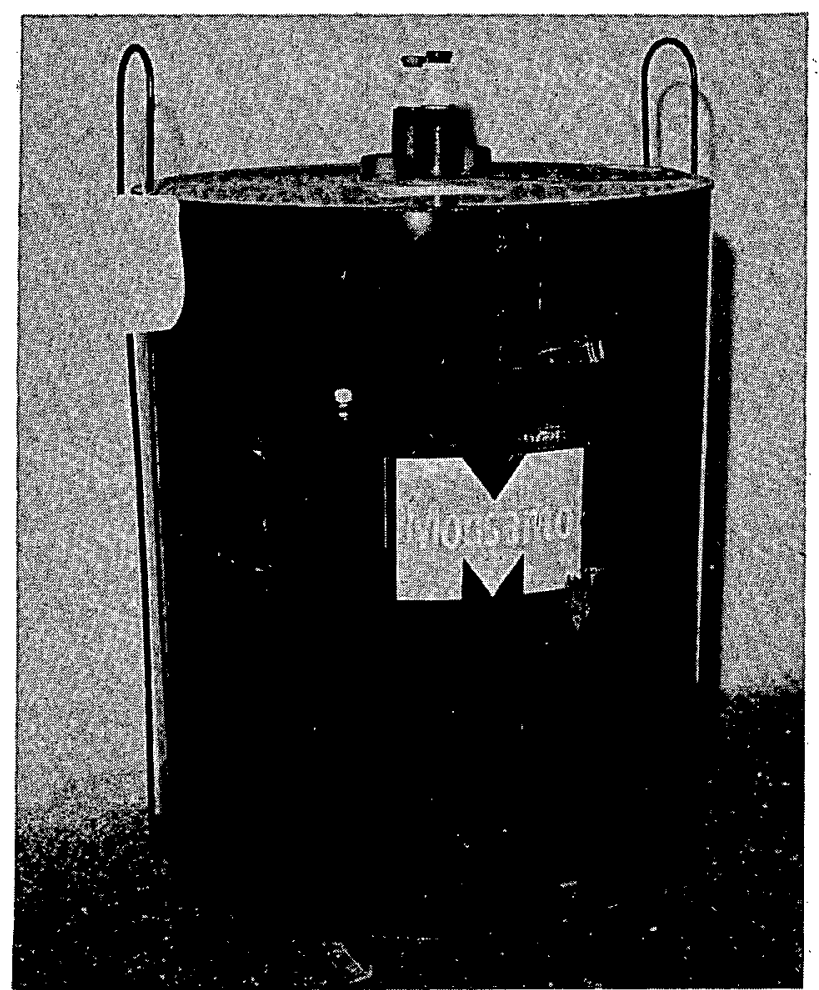

130 gal Cask Fabricated by Monsanto Research Corp.

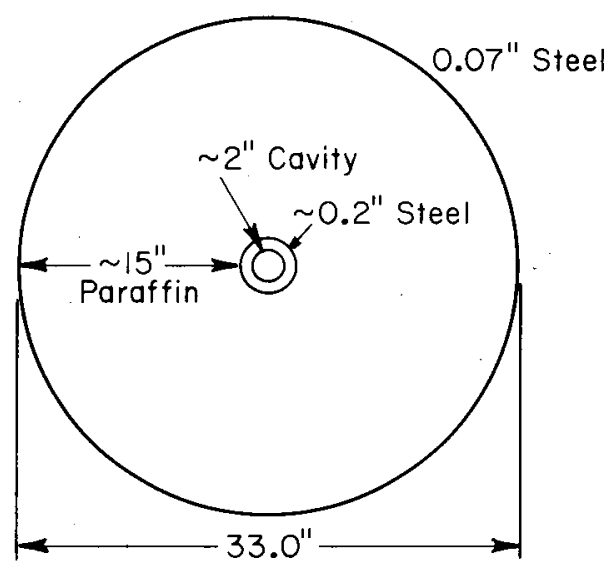

Dose Rate Data

\begin{tabular}{|c|c|c|c|}
\hline \multirow[b]{2}{*}{ Location } & \multirow{2}{*}{$\begin{array}{c}\text { Type } \\
\text { Radiation }\end{array}$} & \multicolumn{2}{|c|}{ 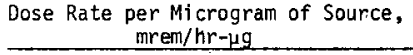 } \\
\hline & & Calculated & Measured \\
\hline \multirow[t]{3}{*}{ Surface } & Neutron & $3.8 \times 10^{-2}$ & $3.6 \times 10^{-2}$ \\
\hline & Gamma & $3.5 \times 10^{-1}$ & $3.4 \times 10^{-1}$ \\
\hline & Total & $3.9 \times 10^{-1}$ & $3.8 \times 10^{-1}$ \\
\hline \multirow[t]{3}{*}{36 Inches } & Neutron & $3.0 \times 10^{-3}$ & $3.8 \times 10^{-3}$ \\
\hline & Gamma & $2.8 \times 10^{-2}$ & $3.5 \times 10^{-2}$ \\
\hline & Total & $3.1 \times 10^{-2}$ & $3.9 \times 10^{-2}$ \\
\hline
\end{tabular}

FIG. 10 DOSE RATE DATA FOR PARAFFIN-STEEL CASK
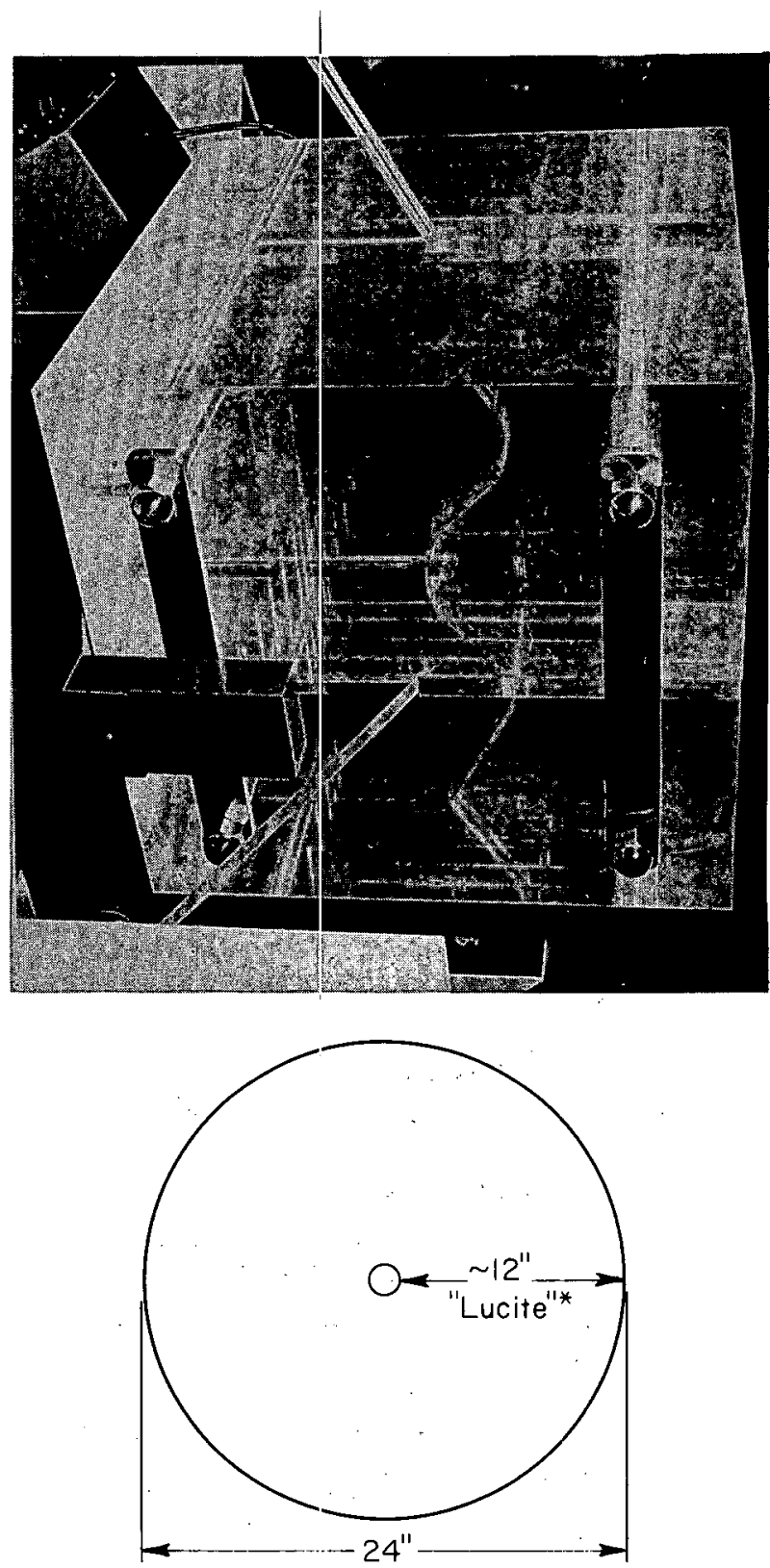

Dose Rate Data.

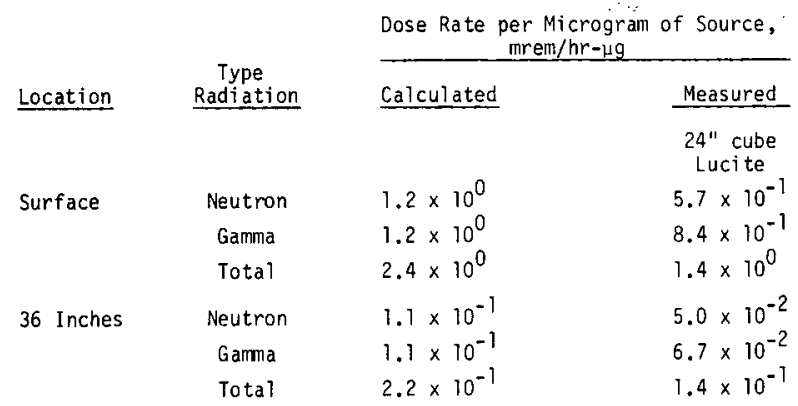

FIG. 11 DOSE RATE DATA FOR "LUCITE" SHIELD

* Registered trademark of DuPont. 

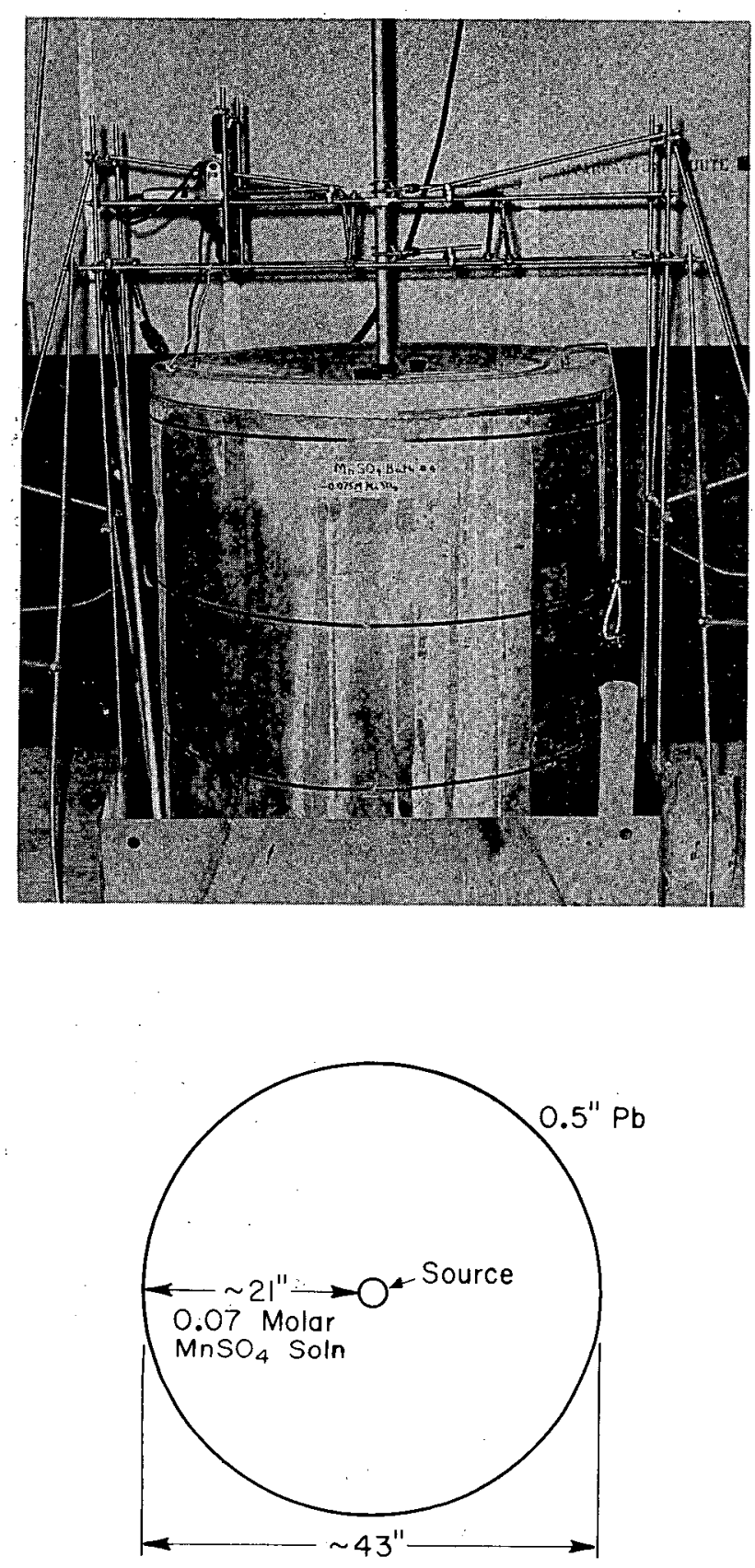

Dose Rate Data

\begin{tabular}{|c|c|c|c|}
\hline \multirow[b]{2}{*}{ Location } & \multirow{2}{*}{$\begin{array}{c}\text { Type } \\
\text { Radiation }\end{array}$} & \multicolumn{2}{|c|}{$\begin{array}{c}\text { Dose Rate per Microgram of Sources, } \\
\text { mrem } / \mathrm{hr}-\mu \mathrm{g}\end{array}$} \\
\hline & & Calculated & Measured \\
\hline \multirow[t]{3}{*}{ Surface } & Neutron & $1.2 \times 10^{-2}$ & $7.7 \times 10^{-3}$ \\
\hline & Gamma & $7.3 \times 10^{-2}$ & $3.2 \times 10^{-2}$ \\
\hline & Total & $8.5 \times 10^{-2}$ & $4.0 \times 10^{-2}$ \\
\hline \multirow[t]{3}{*}{36 Inches } & Neutron & $1.4 \times 10^{-3}$ & $1.2 \times 10^{-3}$ \\
\hline & Gamma & $1.0 \times 10^{-2}$ & $5.3 \times 10^{-3}$ \\
\hline & Total & $1.1 \times 10^{-2}$ & $6.5 \times 10^{-3}$ \\
\hline
\end{tabular}

FIG. 12 DOSE RATE DATA FOR $\mathrm{MnSO}_{4}$-Pb BATH 


\section{DESIGN AND CONSTRUCTION OF A VERSATILE ${ }^{252} \mathrm{Cf}$ NEUTRON SOURCE SHIELD AND EXPERIMENTAL FACILITY}

Stephen J. Gage

E. Linn Draper, Jr.

Gary D. Bouchey

Richard R. Day

Nuclear Reactor Laboratory

The University of Texas at Austin

Austin, Texas
The design and construction of a facility which combines the functions of a shipping container, experimental utilization assembly, and biological radiation shield for a 1000 microgram californium-252 neutron source is described in this paper. The facility is a right cylinder, $52^{\prime \prime}$ high and $48^{\prime \prime}$ in diameter, pierced at the midplane by four stepped beamports which converge to a central cavity. A water-extended polyester (WEP) riesin. with composition of $30 \%$ resin and $70 \%$ water was used to cast the bulk of the shield, with the internal beamport and central cavity structure forined of welded polyethylene and the exterior formed of rolled 16-gauge sheet steel. The fast-to-thermal flux ratio near the source can be maximized by lining the central cavity with boron or cadmium or the neutron energy spectrum can be degraded by inserting spectrum-tailoring materials into the cavity. The facility satisfied Department of Transportation regulations for a 7A Type A shipping container and is proving to be very useful for a variety of application experiments under the USAEC Market Evaluation Program.
INTRODUCTION

In December 1969, the staff of the Nuclear Reactor Laboratory at The University of Texas at Austin prepared and submitted a proposal for participation in the USAEC Californium-252 Market Evaluation Program. Negotations between the USAEC Savannah River Laboratory and the University of Texas at Austin were initiated in early 1970 and resulted in a contract covering the loan of a 1000 microgram Cf -252 neutron source to the University. The project outlined by the University included research and development activities in nuclear materials safeguards, forensic activation analysis, neutron radiography, and industrial process control with prompt capture gamna spectroscopy.

During Spring 1970, it was decided to design and fabricate a facility which combined the functions of a shipping container, experimental utilization assembly, and biological radiation shield for a 1000 microgram Cf-252 neutron source. This paper describes the design of the facility, its fabrication, and its overall performance both as a shipping container and as an experimental assembly.

\section{DESIGN CONSIDERATIONS}

In designing, the combined shipping/ experimental shield facility, it was recognized that a number of factors had to be considered and that trade-of $f s$ between the most important requirements would have to be made. The major factors considered were the experimental requirements, physical design considerations, radiation safety, and cost of materials and fabrication. The design consequently reflects compromises made to accommodate as many of the objectives of the UT-Austin project as possible consistent with good radiological health practices.

\section{FXPERIMENTAL REOQUIRERFNTS}

In the evaluation of several proposed industrial applications of Cf-252 neutron sources, the following experimental needs were recognized: (1) the ability to vary, or tailor, the neutron energy spectrum; (2) the ability to transfer samples in and out of the facility; and (3) the ability to extract beams of neutrons of variable energy from the facility.

Variable Energy Neutrons. It was desirable to have the capability to perform irradiations, both inside and outside the facility, with a variety of neutron spectra. 
Thus the design had to provide for (1) minimization of the thermal and intermediate neutron intensity in the irradiation positions when fast neutron irradiations are preferred, and (2) thermalization of source neutrons when thermal and intermediate neutron irradiations are preferred. To provide a maximum fast-to-thermal flux ratio near the source and to reduce the effect of thermal and intermediate neutrons a large central cavity was included in the design. This cavity can be lined with boron or cadmium, as can the beam tubes. The spectrum tailoring assemblies, which can be fabricated of hydrogenous materials such as water, paraffin, and polyethylene, and other spectrum-tailoring materials like graphite, iron, boron, lead, and cadinium, are inserted into the central cavity. The dimensions of the cavity (16" diameter $x 20$ " height) were established so that a polvethylene assembly and an enrichedurnaium, polyethylene-moderated subcritical reactor could be introduced into the facility. In Figures 1 and 2 are vertical and horizontal sections of the University of Texas at Austin Cf -252 shield facility. The major features of the facility will be discussed in the following. sections.

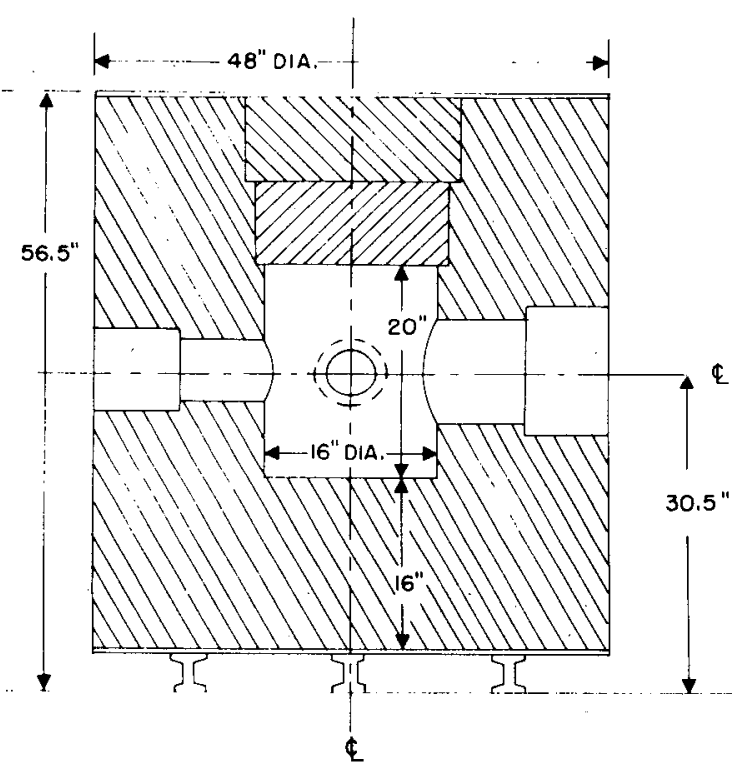

Fif. 1. Vertical Section of University of Texas at $\Lambda$ ustin $\mathrm{Cf}-252$ Shield Facility

Rapid Sample Transfer. A pneumatic sample transfer is an extremely valuable tool for the production and utilization of short-lived radioisotopes. The UT-

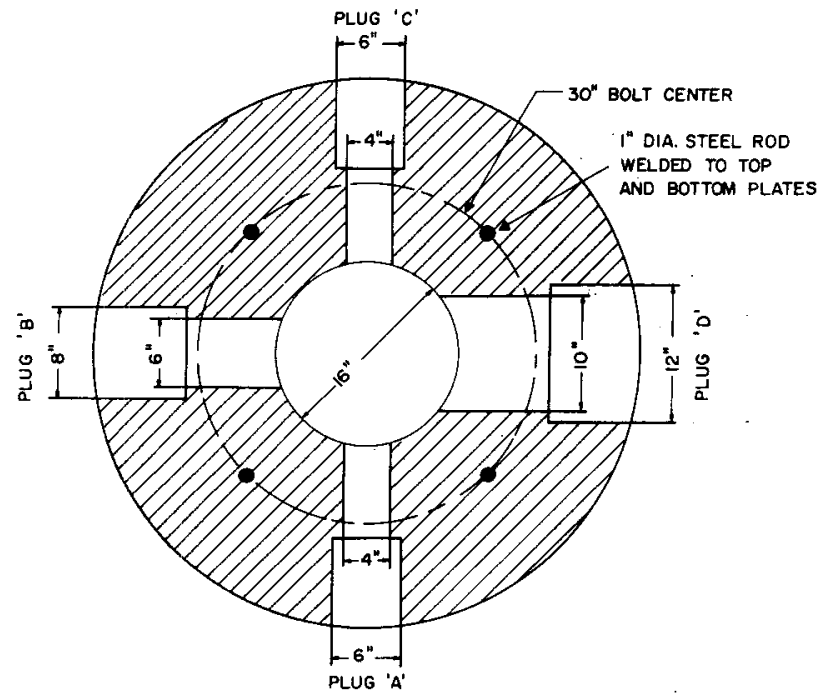

Figure 2. Horizontal Section of University of Texas at Austin CF-252 Shield Facility

Austin Cf -252 source evaluation project requires the use of such a system so provisions were made in the facility design. The transfer system designed and fabricated can be inserted through the 6" diameter so samples can be exposed to a variety of neutron spectra.

Neutron Beam Extraction. The Cf -252 source evaluation program also included experiments requiring neutron beam extraction. Two broadly classified experiments are neutron radiography and capture gamma ray analysis. Four beamports (two 4", one $6^{\prime \prime}$, and one 10" diameter ports) were necessary to accommodate the several beam experiments. These beamports are of stepped design to prevent - the possibility of neutron and gamma streaming. They are located 90 degrees apart at the horizontal midplane of the facility and converge to the central cavity. This arrangement provides maximum access to the facility and accommodates simultaneous experiments.

\section{PHYSICAL REOUIREMENTS}

Because the combined facility was to serve as a shipping container as well as an experimental shield, several physical limitations vere placed on the design. The facility had to be nurtable yet possess hirh shield integrity. An additional practical consideration was that the facility be 
relatively easy to construct.

Portability. The facility had to be shipped by truck from Austin, Texas to the Savannah River Laboratory to receive the 1000 microgram source and then make the return trip. The facility was designed to comply with the Department of Transportation shipping container regulations for radioactive materials in non-exclusive use vehicles. Preliminary calculations indicated that a combined factlity could be easily designed to meet these requirements (1).

Shield Integrity. In the design of such a combined facility, the shield integrity was of utmost importance. The facility had to be leakproof, fireproof, and strong enough to withstand abuse during shipping. For this reason, sheet steel was used to provide exterior protection and a solid neutron and gamma shielding material was used to form the bulk of the interior.

Several materials considered for the shielding material included paraffin; polyethylene, graphite, and water-extendedpolyester resin (WEP). The first three would have made excellent materials for the facility except that each is difficult to form and machine, reasonably expensive, and not fireproof. A new resin material, WEP, had been used successfully in the construction of a small portable neutron shield (2). This material is fireproof, leakproof, solid, hydrogenous, relatively inexpensive, and is relatively easy to handle and fabricate. WEP has physical properties that are four times stronger than wood but not as strong as concrete. In addition, WEP exhibits excellent neutron shielding properties that are about equal to those of paraffin, polyethylene, and water. For these reasons WEP was chosen as the neutron shielding material for the facility.

\section{RADIATION SAFETY REQUIREMENTS}

Radiation safety standards had to be carefully considered since the facility was to be used for shipping, storage, and experimental use. However, Department of Transportation shipping container regulations presented the most limiting case and therefore were the basis for shielding design.
A $1000 \cdot$ microgram $\mathrm{Cf}-252$ source yields about $2.5 \times 10^{9}$ neitrons per second and about $1.3 \times 10^{10}$ gamma photons per second. Preliminary calculations indicated that when used as a shipping container, approximately two feet of WEP surrounding the. source should provide acceptable neutron radiation levels. The overall diameter of the shield assembly was set then at $48^{\prime \prime}$ and the height|at 52". In addition, the gamma dose at the container surface could be reduced to acceptable levels by placing the source in a lead and steel chamber 4" in diameter. For shipping, a special WEP casting to fill the inner cavity was made and the lead and steel chamber was placed in the center of this casting. This shipping assembly is shown in Figure 3.

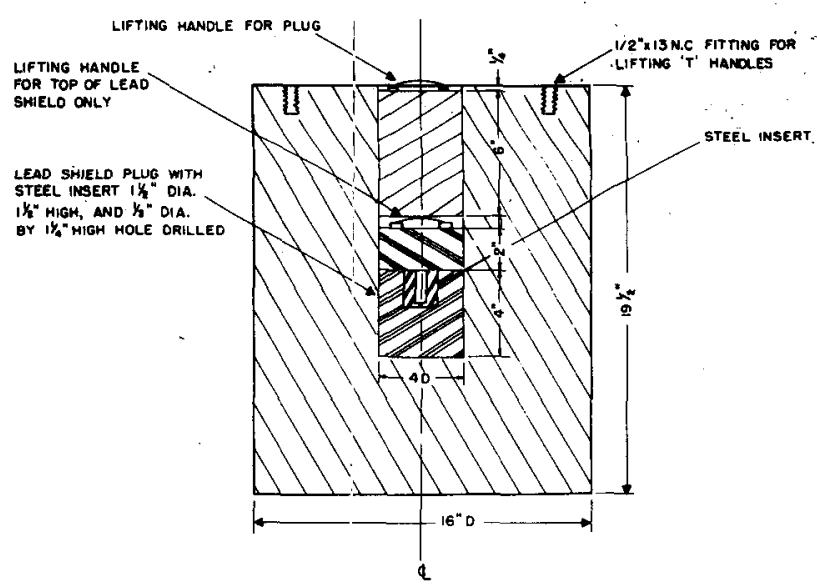

Fig. 3. Central Ciavity Shielding Assembly for use in Cf -252 Shield During Shipping,

\section{COST REQUIREMENTS}

It proved to be possible to keep the cost of the faciljty reasonable through the use of WEP for the neutron shielding material as compared to the cost if paraf$f$ in, polyethylene, and graphite had been used. Also, no expensive special purpose equipment had to be purchased to complete the project. The cost of the facility, excluding labor, vas about $\$ 840.00$. In Table $I$, an itemisation of these costs is presented. Total labor amounted to 325 hours, approximately eighty hours of which were spent in researching materials and learning to weld polyethylene and twenty hours were spent on a trip to $M$. D. Anderson Hospital and Tumol: Institute, in Houston, 
Itemization of Cf-252 Shipping/Experimental Shield Costs
Manhours Spent in Design and Fabrication of Cf-252 Source Facility

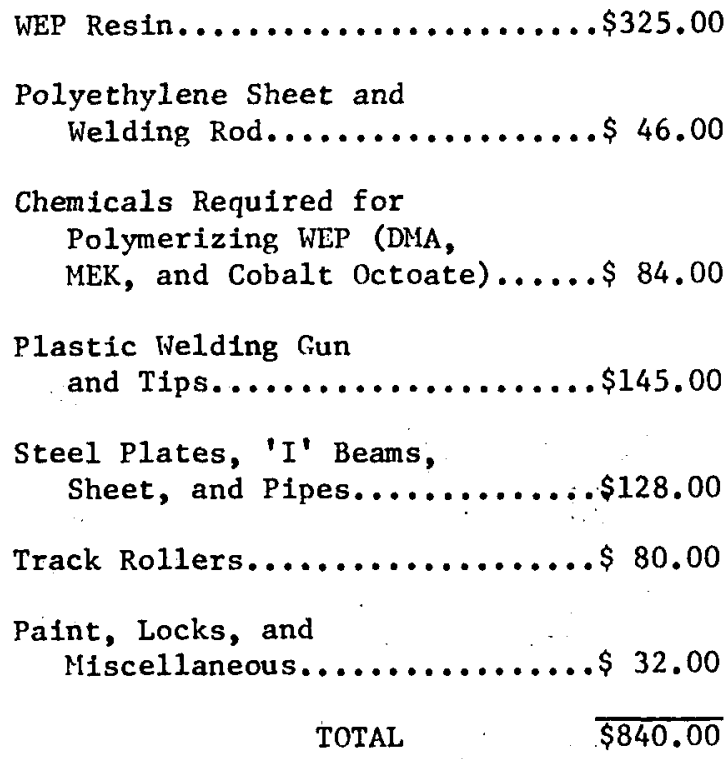

to consult with Dr. George Oliver on the mixing and casting of NEP. A breakdown of manhours spent on the project is given in Table II. (Numbers in brackets after each item are the number of men involved with that particular activity).

\section{FACILITY FABRICATION}

The Californium-252 shipping/experimental shield facility of The University of Texas at Austin was fabricated by Nuclear Reactor Laboratory and other university shop personnel. All the equipment used in fabrication, except for the polyethylene velding gun, were available in the university shops. Described in this section are the fabrication of the shield container and the internal structure and the mixing and casting, of the WLP shield assembly.

\section{SHIELD CONTAINER FABRICATION}

$\Lambda$ liobot circle.cutting machine was used to cut the $4^{\prime}$ dianeter upper and lower basseplates from 1/4" to $1 / 2$ " thick steel plate, respectively. The top plate also had a $20^{\prime \prime}$ diameter hole cut in its center to allow access to the central cavity. Three ' $I$ ' beams, 4" high, were welded to
Work Activity

Manhours

Design of Facility (5)........40

Material Acquisition (3).......40

Construction of Polyethylene Internal Form (1) .........660

Construction of Shield Container (3)..............20

Mixing and Casting

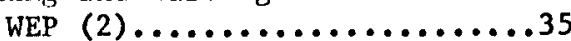

Painting (1)............... 5

Pouring and Fitting Beam

Port plugs and Central

Cavity PIug (2)...........20

Polyethylene Welding..........80

Consultation with Dr. George Oliver, Houston (1)..............20

TOTAL $325 \mathrm{Man}-$ hours

the lower baseplate so that a fork lift could be used to move the facility for shipping and handling. A $4^{\prime}$ high section of 16 gauge sheet steel was rolled in a $4^{\prime}$ diameter cylinder and welded to the upper and lower baseplates. Steel bands were used to align the cylinder for welding to the lower baseplates. Four holes were cut in the sides of the cylinder to provide access to the beam ports. Four vertical $1^{\prime \prime}$ diameter steel pipes were welded to the lower baseplate in a square lattice with a $30^{\prime \prime}$ spacing. Later, these pipes were welded to the upper baseplate and to the lifting fixture. Finally, the container was painted with a rust-inhibiting paint.

\section{INTERNAL STRUCTURE FABRICATION}

Because of the complicated internal structure, it was decided to use a casting form and to cast the bulk of the shield around this form. Materials considered for 
the form were wood, cardboard, lucite, and polyethylene. The first three materials would have been used only during casting and then removed. Removing the forms presented another problem since the inside surfaces of the casting would have to be coated with some impermeable material to prevent wear and prevent water from evaporating from the exposed surfaces of the WEP. Polyethylene, on the other hand, offered considerable advantage in that it could be: permanent, because it has excellent wear characteristics and would seal the WEP surfaces against evaporation. Polyethylene cylinders in the desired sizes were not commercially available stock itens from any plastic supplier and the costs of the material and fabrication precluded custom manufacture by a vendor. It was decided to use a hot gas welding gun to weld polyethylene sheeting into the desired shapes. This procedure was relatively: easy once sufficient experience was acquired in using the welding unit. To weld polyethylene, water-pumped nitrogen gas under pressure was used as the gas in the welding gun. Figure 4 is a picture of the completed form before insertion into the shield contalner.

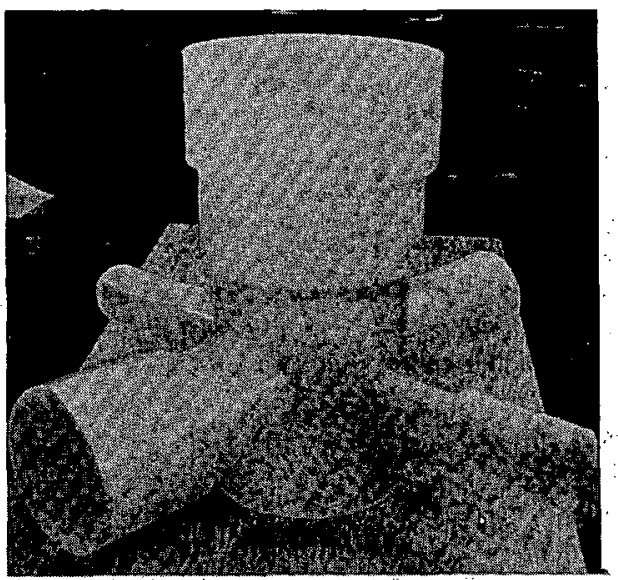

Fig. 4. Internal structure of $\mathrm{Cf}-252$ Shield Facility

Note the stepped beam ports, central cavity, and stepped top shield plugs. The completed form was held in place by a wooden base at the bottom and a wooden retaining ring around the top which also positioned the four vertical pipes. Figure 5 is a picture of the shield container with the internal form inserted and positioned by the retaining ring!

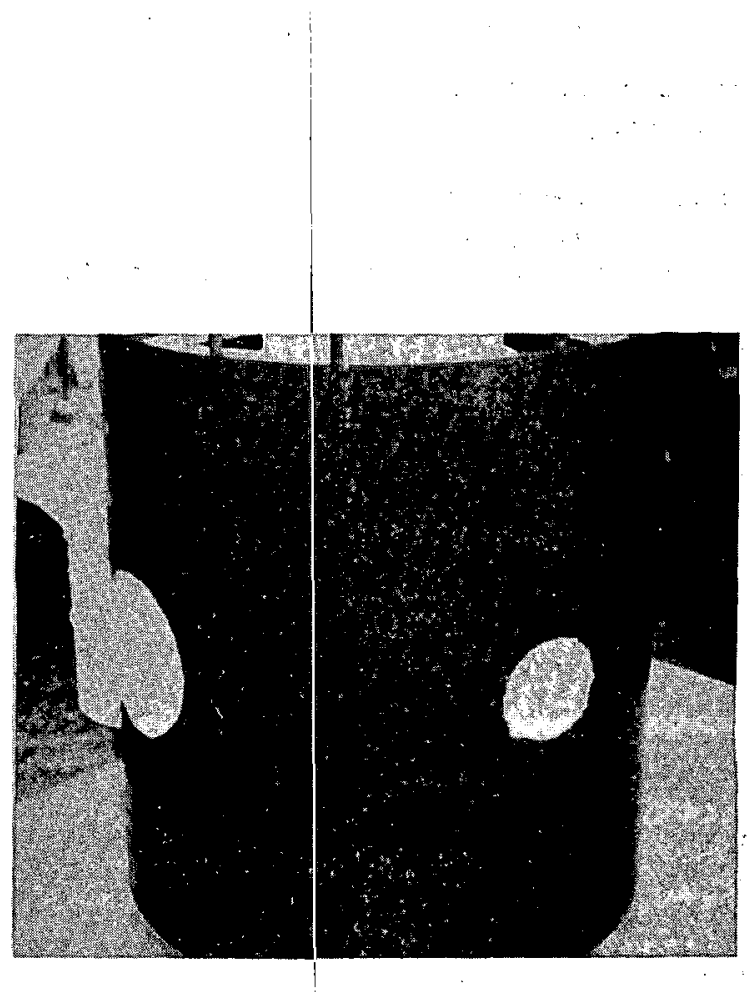

Fig. 5. Shield Container with Internal Forms Positioned for Casting WEP

\section{WEP PREPARATION}

The most critical part of the project was the preparation of the WEP. WEP can be prepared with water content between $50 \%$ and $90 \%$ by volume. However, the resin supplier (Ashland Chemical Co., Columbus, Ohio) suggested $70 \%$ as the highest water percent to be prepared because special mixing equipment, due to the increased.viscosity of the solution, is needed above this percentage. Hence, $70 \%$ water and $30 \%$ resin was chosen as the optimum mixture.

\section{Approximately 450 gallons of WEP} were added to cast the facility, beam plugs, shipping plug and top plugs. Because of this large quantity of mixture and the time required to $\mathrm{mix}$ the W'EP (about 30 minutes for 15 gallon batchi) it was decided to mix 15 gallon WEP. batches and store them in 55 gallon drums until enough had been accumilated so that the facility could be cast in a single batch operation. 
WEP is a mixture of WEP resin; promoters, water and catalyst. WEP resin is thick, sticky, goid-colored liquid. Two promoters, Dimethalanaline (DiA) and CobaltOctoate, are added to the resin before the water is mixed into the WEP. Approximately $3 / 4$ gallon of DII $\Lambda$ and $11 / 2$ gallons of $6 \%$ Cobalt-Octoate were required for entire shield casting. If properly mixed, WEP can be stored for three to five days in open drums provided the catalyst is not added. When the mixture is stored longer than 5 days, it will separate into WEP and water and will be ruined.

The actual mixing of the WEP was accomplished by using a 17 gallon drum and a specially designed mixing paddle. The small drum was used because it was necessary to mix the water into the WEP throughly to avoid eventual separation of the VEP and water. These small batches were transferred to the 55 gallon drums for storage. The special mixing paddle was a $12^{\prime \prime}$ diameter steel disk welded to a $3^{\prime}$ long shaft. Two radial cuts vere made in the disk and then a 2" pitch added to form screw type blades. This paddle, when used in a $12 \mathrm{hp}$, variable speed drill press, proved to be an excellent mixing device because it provided a smooth action to completely mix the resin and water without introducing air into the mixture.

\section{CASTINO}

The catalyst, methil-ethyl-ketone peroxide (MEK), was mixed directly into each 55 gallon drum and then the IEP poured into the shield container. Approximately $3 / 4$ gallon of ILK was required for the entire casting. The pitch of the mixing paddle vas increased to about 5 " to handle the greater volume in the 55 gallon drum. A special bridle sling that $f$ it around the 55 gallon drums vas used in conjunction with a small fork lift truck to lift and move the 400 pound drums into place for mixing the catalyst and pouring the WEP into the shield container. Figure 6 is a photorraph of nouring the WEP from the 55 gallon mixing drua into the container.

\section{COIPLETION OF FACILITY}

After the UFP solidified and cooled, the upper baseplate and lifting fixture vere velded into position. Removable steel doors were fitted to the beam port openings and padlocks attached. The facility vas spray

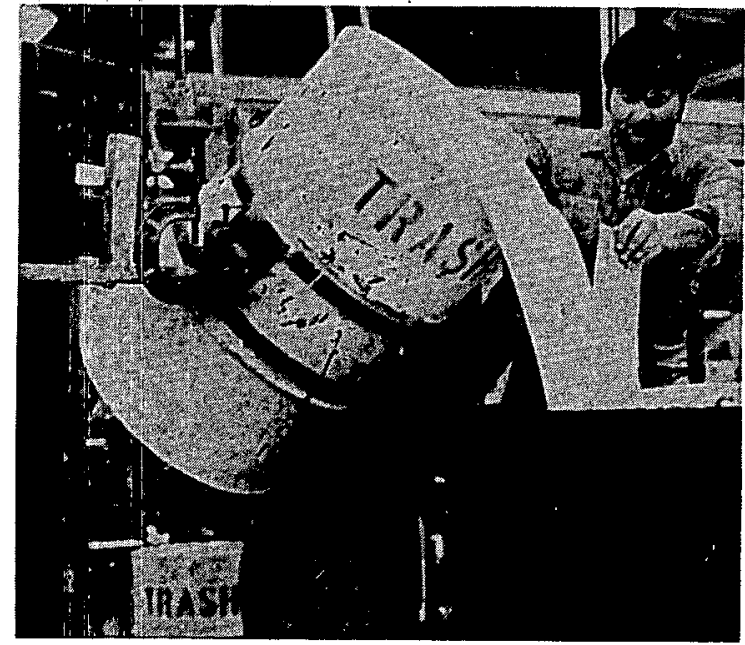

Fig. 6. Pouring of WEP From 55 Gallon ?fixing Drums Into Shield Container

painted and identification plates attached. one plate identified the shield assembly as a Department of Transportation $7 \Lambda$ Type A shipping container. Figure 7 is a photograph of the shipping container/experimental shield being moved by 6 ton fork lift truck prior to shipment to Savannah River Laboratory.

\section{FACILITY PERFOR:LANCE}

Since the true test of any design and fabrication is in the actual performance of the product, the performance of the facility as a shipping container and as an experimental assembly will be discussed in this section.

\section{PERFORIANCE AS A SHIPPING CONTAINER}

As a shipping container, the facility proved to be more than adequate with respect to portability, integrity, ruggedness, and radiological health standards. The facility can easily be moved by the use of a fork 


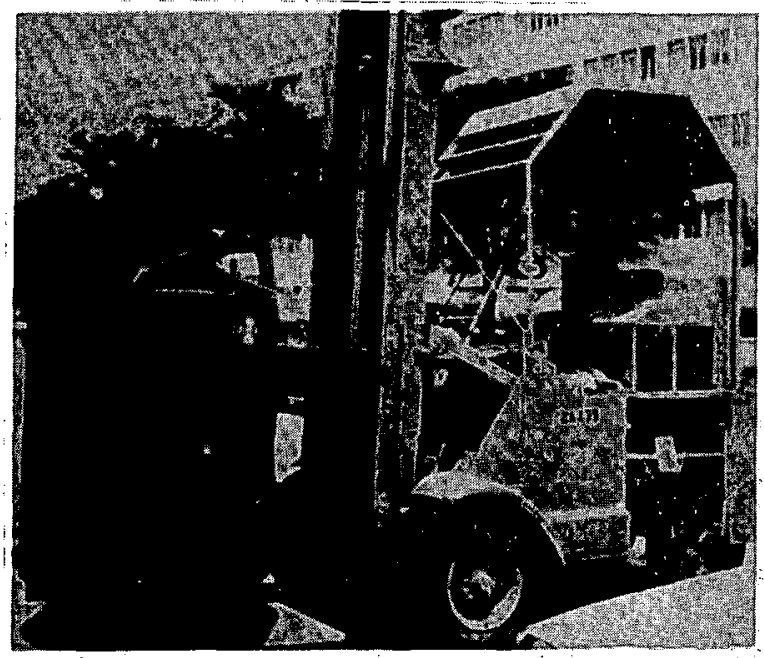

Fig. 7. Moving lhe Completed Shield Facility Prior to Shipping

lift truck or an overhead crane with a 5000 1b. capacity. In the Nuclear Reactor. Laboratory, the facility is mounted on "three track rollers (Stokvis-Multiton Corp., Port Washington, New York) that allow one man to easily reposition the shield. The only damage to the shield container during transit was some scuffing of the paint and a cracked nameplate.

Performance of the shipping container from the radiological health viewpoint can best be described by means of Table III. In this table, calculated and measured radiological dose rates are compared to the maximum allowable dose rates under the DOT regulations. It can be seen that total (neutron and gamma) dose rates measured at the surface of the shield are one-fourth of the allowable dose rate on contact and that the total dose rate measured at one meter from the surface is one-third the allowable dose rate at that point. From all indications then the shipping container provides adequate shielding for even a three milligram $\mathrm{Cf}-252$ neutron source.

\section{PERFORMANCE AS AN EXPERTMENTAL ASSEMBLY}

Performance of the facility as an experimental shield assembly has been better than anticipated. Several of the activities proposed for the USAEC Market Evaluation Program have been initiated and promising results can be reported.
In the evaluation of neutron radiography with a Cf: -252 source, fast, epithemal and thermal neutron beams have noi been used to obtain satisfactory radios raphs which allow much information to be gleened for the use of a portable neutron radiographic facility. All radiography has been perforned rith a 4" beamport and a special conical-sliaped collinator fauricated of boron-loaded UTP and lined with cadmium.

Nuclear naterials saferuards analysis with Cf -252 has also been initiated. Several items of special equinment liave been built for use in the facility to acconmodates these investigations. A boron liner 2" thick was built and inserted in the large central cavity to maximize the fast-tothermal neutron flux. In addition, a pneumatic transfer system was built and inserted through the $6^{\prime \prime}$ beamports. The transfer system is fully automated and can be used 24 hours a day for sample irradiations, without unduly interfering with other experiments, such as fast neutron radiography.

In summary, the overall performance of combined shipping container and experimental assembly has been satisfactory. The facility was used to ship a 1000 microgram Cf-252 source from Savannah River Laboratory to the Nuclear Reactor Laboratory of The University of Texas at Austin where it is now being utilized in investigations being conducted under the USAEC CE -252 Market Evaluation Program. The provision of a large central cavity and four beamports has greatly facilitated these studies, which include neutron radiography, activation analysis, and nuclear materials assay.

PEFEREINCES

1. Californium-252 Progress, 2,36 (January 1970).

2. Californium-252 Progress, 3, 15 (April 1970). 
TABLE III

Radiation Dose Rates Outside of Cf -252 Shield Pacility

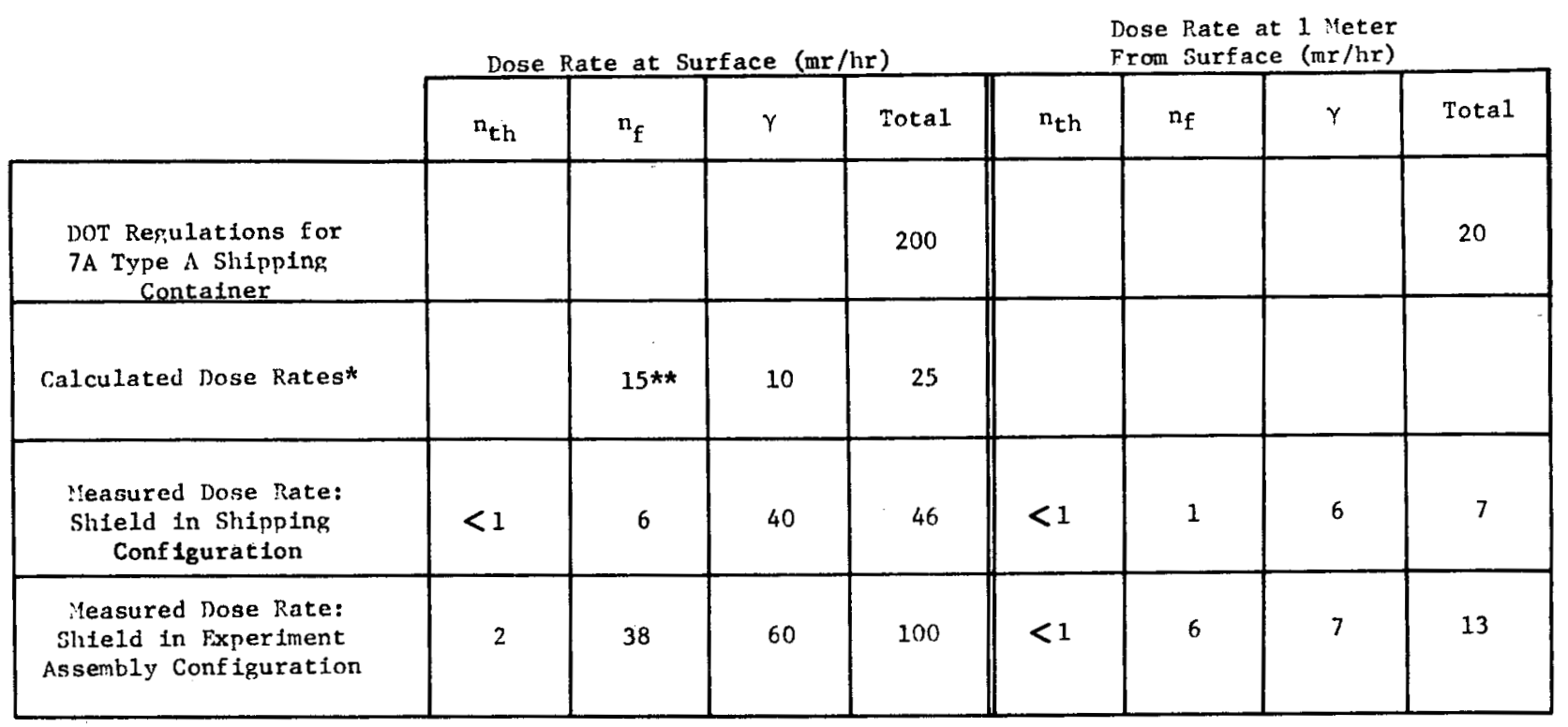

*Californium-252 dose rate attenuation curves presented in Californium-252 Progress, 2, 36-37 (January 1970), were supplemented by computer calculations based on fast neutron removal theory for composite shields.

**Total (thermal and fast) neutron dose rate. 
Session II

\section{MEDICAL AND FORENSIC USES OF NEUTRONS}




\section{NEUTRON RADIATION THERAPY}

Mary Catterall, R. H. Thomlinson, and S. B. Field

Medical Research Council

Hammersmith Hospital

London, England

\author{
Charles C. Rogers* \\ Virginia Commonwealth University \\ Richmond, Virginia
}

Before a controlled clinical trial of fast neutrons is started on the Medical Research Council's cyclotron at Hammersmith Hospital, London, a preliminary investigation is being conducted on patients with advanced malignant tumors. This paper describes the treatment and early observations on 72 such patients treated with fast neutrons since November 1969.

The dose rate is $\mathbf{4 0}$ rads per minute giving treatment times of $3-4$ minutes per field. This enables flexibility of the patient's position during treatment and overcomes the disadvantages of the fixed horizontal beam. Collimation is by borated wooden tubes; wedge filters can be used as in conventional radiotherapy. A dose of neutrons of about 1 rad per 1000 rads administered is received by the patient's eyes.

A total dose of 1440 rads given in 12 fractions over 25 days has been shown to be a practical therapeutic regime resulting in healing of skin reactions and regression of tumours. With this fractionation of 120 rads, the RBE for skin is about 2.9. Treatment times of $40-50$ days have been used in the most recently treated patients with doses of 1512 and 1576 rads given in 12 or 15 fractions.

A clinically significant skin sparing effect has been demonstrated.

* Special Fellow, National Cancer Institute

The present, investigation into the clinical effects of fast neutrons was started on the Medical Research Council's cyclotron at Hammersmith Hospital in November 1969, more than thirty years after Stone(1) had used them in Berkeley, California. Although he apparently cured some patients with very advanced cancers, he abandoned the method because he decided that the damage to normal tissues, which appeared months after treatment, was excessive and could not be related to the degree of the early reactions. Recently the records and photographs of his patients have been examined by Sheline, Field, Brennan, Raventos and Phillips (2) who noted that the physical characteristics of the neutron beam produced by the Cyclotron at Hammersmith are very similar to those used by Stone in 1940. The experimental data obtained by several workers relating $\mathrm{RBE}$ and dose per fraction for a variety of tissues tested on the Hammersmith cyclotron (reviewed by Field (3)) could therefore be applied to the treatments given in the. early trial. From this, the equivalent doses of gamma rays given to each patient were calculated in terms of the NSD of Ellis (4). Sheline et al (2) thereby showed that these doses were excessive in many instances because Stone had not known that the RBE varied with the size of each dose, nor had the exit doses been included in his calculations. In this retrospective survey, it seemed that both the early and late reactions were consistent with the doses given.

That a new trial of fast neutrons should be undertaken was suggested by the results of radiobiological research (5) which indicated that tumours containing hypoxic but clonogenic cells would be more effectively sterilised by radiations having a high linear energy transfer (IET). A controlled clinical trial will be necessary to determine whether this is the case in the treatment of cancer in humans but before such a trial could be started it was necessary to obtain data on the responses of the skin and tumours to various doses of neutrons and to compare these with those produced by $X$ rays. Details of the methods employed to do this have been described by Catterall, Rogers, Thomlinson and Field (6). The present paper describes treatment techniques and the calculations of doses given to 72 patients, together with the results noted in the first few months after treatment.

The physical properties of the beam have been described by Bewley and Parnell (7). Collimation is achieved by borated wooden tubes giving field sizes ranging from 27 to $440 \mathrm{sq}$. $\mathrm{cm}$., and although these are in the shape of squares or rectangles, paraffin wax filters of various shapes and sizes can be fitted to shield structures as required. An optical system shows the field size, the 
target skin distance, which is $120 \mathrm{cms}$. and the exit point of the beam. The output is 40 rads per minute, giving treatment times of less than 4 minutes per field. The depth doses for the fast neutron beam are intermediate between those of 60 Co and 300 KV $X$ rays and are nearly identical with those of ${ }^{137} \mathrm{Cs}$ at $50 \mathrm{~cm}$. FSD.

The beam is fixed and horizontal. Patients are treated either lying on a couch which can be tilted 15 degrees either side of the horizontal, or whilst sitting or kneeling in a chair, with arm rests, back, chest and head supports which can be adjusted to suit each patient. Both the chair and the couch can be rotated through 360 degrees and raised and lowered.

A mixture of paraffin wax and powdered carbon was found to give similar attenuation characteristics to soft tissues and is used to mould single units of bolus conforming to the site of treatment.

It was possible, using paraffin wax, to insert wedged filters into the neutron beam as in conventional radiation to treat tumours in certain sites and treatment plans were constructed in the usual way. The doses received on the skin beneath bolus and in cavities such as the mouth were checked by measuring the emission of gamma rays resulting from the activation of aluminium pellets placed at these sites. One pellet placed at the centre of the incident beam received a known dose of radiation and the doses received by the others were related to this.

\section{Dose, Number of Fractions and Overall Time}

To combine a limited availability of cyclotron time with a regime which could also be used with low IET radiation, it was decided to give all treatments three times weekly for 12 fractions over 25 deys. Sometimes; however, for clinical reasons 10 or 6 fractions were given in 21 or 11 days and in 3 early cases 13 and 14 fractions were given in 38 to 44 days. Fourteen of the latest cases have been treated over periods of 40 to 50 days in 12 - 16 fractions because it was thought that repopulation of normal tissues and re oxygenation of tumour cells might be enhanced by prolonging the overall time.

The Ellis formula (4) for gamma rays has been used to convert the low IET doses given in varying numbers of fractions and overall times to the equivalent in 12 fractions over
25 days, ie Total $\mid$ Dose $=$ N.S.D. $\mathrm{x} \mathrm{N}^{\cdot 24} \times \mathrm{T}^{\cdot 11}$ (when NSD $=$ a constant, $N=$ number of fractions, $T=$ overall treatinent time in days).

Bewley (unpublished data) has considered the data from the irradiation of pie skin with neutrons, relating dose as a function of the number of fractions (from 6 - 15) given in the same overall time. He gerived the relationship, Total Dose N*08. Since there is no evidence that the time factor is different for high IET radiation, a modified Ellis formula has been used for neutrons

$$
\text { ie Total Dose }=\text { N.S.D. } \times \mathrm{N}^{.08} \times \mathrm{T}^{\cdot 11}
$$

In order to choose the optimum dose of fast neutrons, it was desirable that the $\mathrm{RBE}$ of fast neutrons for skin should he determined. To do this, radiations of both high and low IET viere used and their effects were compared in the same patient whenever possib]e. This was achieved by selecting for treatment patients with multiple tumours or those with stage $\mathrm{T}_{4}$ breast cancer in whom more than one area had to be irradiated. In these cases one, or more, areas were irradiated with neutrons and the other, or others, with low LET radiations.

Treating in 12 fractions over 25 days, ranges of total rieutron doses from 1200 1760 rads were used and the effects compared with doses of $300 \%-4200$ rads of orthovoltage $X$ relys in the same fractionation and overall time. Finally a total dose of 1440 rads of neutrons was selected as causing a skin reaction within acceptable limits and leading to a satisfactory early response of the tumours. The effects of this dose were clinically the same as those produced by 4100 rads of orthovoltage $x$ rays and, using the Ej.Iis formula (4) this dose was equivalent to 6100 rads of gamma rays in 30 fractions ovej 40 days. When the overall treatment time was extended to 40 days, the dose given in 12 fractions was 1512 rads, while for 15 fractions in 50 days it was 1576 rads.

It was thought necessary to modify the dose given to veiny small or very large fields and a field of $90 \mathrm{sq}$. $\mathrm{cm}$. was taken as the standard. Factojs derived from a curve drawn through pulblished data on $X$ ray dose, field size and effect were applied to the standard neutron|dose to give biologically equivalent doses!

The doses of radiation received by the patients' eyes were measured by placing 
neutron film badges as near as possible to the eyes during each treatment. The total dose received during course of treatment was measured by the radiation protection service.

\section{Observations made on the Patients}

All patients had full physical examinations before, during and after treatment and the necessary radiological, haematological and special investigations were performed.

The skin reactions were observed clinically by the radiotherapists and, in an attempt to measure these more objectively, a system of numerical scorine was applied by four non-clinical scientists. This system was based on that of Fowler, Morgan, Silvester, Bewley \& Tumer (8) for pig skin and modified for use on patients. The scoring system is shown in Table $I$ and the method is fully described and its limitations discussed by Catterall et al (6).

TABLE I

The System of Scoring Skin Reactions

Developing Reaction

. O No visible reaction

1.0 Slight but definite erythema

2.0 Moderate erythema

3.0 Severe erythema (deep red or pink)

4.0 First sign of acute exudative response

5.0 Exudative response, over less than half the field

6.0 Exudative response, over more than half the field

7.0 Exudative response, over entire field

If the area is showing signs of scaling add

0.5 to erythema score.

+ or - can be added and count for \pm 0.33

Healing Reaction

4.0 Whole area covered by dry scab

3.0 More than half the area covered by dry scab

2.0 Less than half the area covered by dry scab

1.0 Small area of dry scab

If the area is stowing signs of scaling add

0.5 to erythema score

+ or - can be added and count for \pm 0.33

Bewley (9) calculated that a skin sparine effect, comparable with that of $137 \mathrm{Cs}$ would be found with neutrons. This possibility was examined in many of the areas irradiated by covering part of the field with $1 \mathrm{~mm}$. thickness of tissue equivalent plastic and comparing the skin reactions beneath the plastic and therefore with full proton build up, with the uncovered skin. Ninety six fields were scored in 35 patients, 67 after neutron and 29 after low IET radiation.

The tumours were observed by the clinicians and measured with engineer's calipers by technical officers having several years experience in measuring animal tumours. The average of measurements in two or where possible three dimensions was expressed as a fraction of that on the first day of treatment and plotted against time. A typical plot is shown in figure 1.

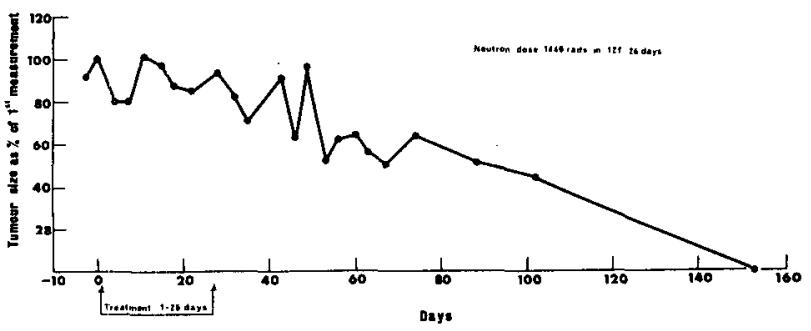

FIG . 1 TUMOUR MEASUREMENTS PLOTTED AGAINST TIME

All the patients were referred for neutron therapy because they had very advanced local or metastatic cancer. In all cases, hormone or cytotoxic therapy had been considered and been given and failed, or the growth was thought to be unlikely to respond satisfactorily to drugs or to low IET radiation. Treatment with fast neutrons was given in all cases to relieve symptoms or to cure a local lesion and no areas of skin were irradiated unless they were involved in tumour or were situated immediately adjacent to the tumour and so fell into the treatment zone.

A total of 72 patients has been treated. Sixteen died shortly after treatment and before a clinical assessment was possible. Three patients left Iondon and have been followed up by post only. 
This paper therefore describes 53 patients whose ages ranged from 41 to 84 years and? of whom were over 80 years of age. They were divided into 4 groups.

Group I. The eight patients in this group suffered from stage $\mathrm{T}_{4}$ breast cancer, 7 were also $\mathrm{N}_{3}$ and 4 were $\mathrm{M}_{1}$ or $\mathrm{M}_{2}$. Two areas at least were irradiated in each patient, for example, the breast by fast neutrons and the flandular areas by orthovoltage $X$ rays. The skin changes produced by the 2 types of radiation were observed and provided information on the comparability of the effects produced by the neutrns and the $X$ rays.

Group II. There were 15 patients in this group all of whom suffered from large tumours of more than $400 \mathrm{sq}$. $\mathrm{cm}$. or from multiple skin nodules. These tumours were divided into areas varying from 48 to $80 \mathrm{sq}$. $\mathrm{cm}$., some of which were treated with fast neutrons and some with orthovoltage $X$ rays or gamma rays from cobalt through $1 \mathrm{~cm}$. of wax. The purpose of using neutrons for these patients was to compare the tumour response and to ascertain that neutron therapy was not inferior to that of low IET radiation. Both the tumour and skin reactions were observed.

Group III. Twenty two patients suffering from advanced but superficial tumours which could easily be seen and palpated were treated with fast neutrons only. Six of these had metastatic elands in the neck, 8 had mouth tumours, 3 had advanced epidermal tumours, 3 had locally advanced $\left(\mathrm{T}_{4}\right)$ breast cancer and because of their age and general condition the glandular areas were not irradiated and 2 . other patients had large fibrosarcomas. The purpose of treating this group was to measure the tumour response and observe skin and mucous membrane reactions.

Group IV. In this group were three patients, with adenocarcinoma of the stomach, 2 with metastases from adenocarcinoma of the colon, one with recurrent bladder cancer, one with a grade 3 astrocytoma and one man with a well differentiated squamous carcinoma involving both his main bronchi.

Results

The systemic reactions of patients treated with neutrons: did not differ from those seen with other types of radiations. In the 14 months of observation there has been no significart effect on the peripheral blood. The average dose of neutrons to the eyes was one rad rier 1000 rads to the treated area, the range being from 0.4 to 2.7 rads.

\section{Skin Reactions}

A skin sparirig effect with fast neutrons was clearly demonstrated, when the total dose in 12 fractions over 25 days exceeded 1000 rads. A typical plot of the scores given to a patient in whom part of the field was covered with tissue equivalent plastic and the other part uncovered is shown in figure 2! the scores given to the covered part being.consistently higher than those to the uncovered part.

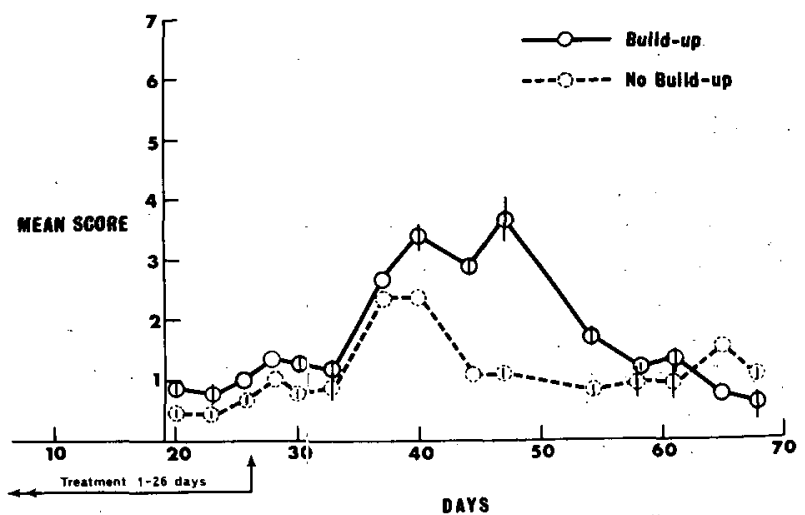

FTG. 2 SKINI SCORAS DEMONSTRATING
SKIN SPARIIG EFFECT OF NOUTRONS

Comparisons of skin reactions caused by neutron and low IET radiations were therefore only valid when full build up was achieved at the surface." The use of wax moulds produced maximum proton build up and resulted in exudative skin reactions scored 5,6 or 7 . However when the moulds were lined with $0.1 \mathrm{~mm}$. lead, these protons were stopped and full skin sparing was restored.

When treatments were given in 25 days, the peak of the skin reaction occurred $3-30$ days after the end of treatment, so that it was not possible to use the skin reaction seen during treatment as a guide to dosage and to have done so could have resulted in 
overdosage. When treatments were given over 40 - 50 days the reactions appeared at about the thirtieth day and increased for the last few treatments but not markedly after the end of treatment. In genera] the skin reactions were less marked in patients over 80 years of age and in those in poor general condition whilst infection tended to increase the reaction.

The reactions produced by 1440 rads of neutrons given in 12 . fractions of 120 rads each were thought by clinical observations to he equal to those nroduced by 4100 rads of orthovoltage $X$ rays, giving an $R B E$ for skin of 2.9. Using the skin scoring method and applying it to 1? fields of skin which were anatomically and physiologically alike, the ratio of the doses ranged from 2.8 to 3.0 . Five pairs of the fields indicated an RBE of Ereater than 2.8 and one pair an RBE of less than 2.8 .

\section{Tumour Response}

The early response of all the tumours treated with fast neutrons was favourable and not qualitatively different from that produced by low IET radiation.

At present, of the total of 72 patients treated, 21 are alive with no sign of cancer in the neutron treated area of whom 9 were treated more than 9 months ago, another 9 were treated $3-6$ months ago and 3 finished treatment less than 3 months ago. A further 22 patients are alive with palpable masses that are still regressing, of whom $?$ were treated more than 4 months ago and 1 , with a recurrence on the abdominal wall from an adenocarcinoma of the stomach, was treated ten months ago.

Nine patients died with no sicn of cancer in the treated area more than 3 months after treatment.

None in this short period of follow up has recurred within the treated area. One patient's tumour grew on the edge of the treated area, not all of the tumour having been included in the high dose region.

Of the sixteen patients who died before a clinical evaluation of the effects of treatment was possible, nine were in eroup II and had very large tumours or multiple metastases.

Graphic representations of the rates of regression were constmucted from the measurements of the tumours. The rates varied not only from one type of tumour to another in different patients but also when the same type of tumour was treated in the same patient, with neutrons or with low IET radiation. This variation may be concerned with the rate of destruction of the tumour parenchyma or with differences in the stromal reaction changing the rate of resorption.

The growth pattern shown in figure 1 illustrates the long period of time over which some of the tumours continued to regress. Fifty days after the end of treatment, this tumour was still about $60 \%$ of its original size and it was not for another 3 months that it became impalpable.

The composition of residual masses cannot be determined with certainty and in some cases improvement in general condition and gain in weight were associated with an jncrease in apperent size of the mass which could have been due to the deposition of fat. But if increase in size of a mass were due to proliferation of malignant cells, the timing of this regrowth would be recorded by the repeated measurements and would be a better guide to the effectiveness of therapy than the rate and degree of regression of tumours.

\section{Conclusions}

1. A fixed horizontal beam of fast neutrons was not a serious disadvantage providing a good dose rate was available.

2. It was quite possible to use wedges and moulds just as with conventional radiotherapy.

3. The neutrons produced by the cyclotron showed a clinically significant skin sparing. When moulds were used, this could be restored by lining the mould with a thin layer of lead.

4. The best estimate for the RBE of neutrons at 120 rads per fraction was 2.9 .

5. 1440 rads of fast neutrons given in 12 fractions over 25 days to a $90 . \mathbf{s q} . \mathrm{cm}$. area was a practical therapeutic regime. It caused a skin reaction, which was exudative in some cases, but which healed in all cases. The peak of the skin reaction occurred $3-30$ days after the end of treatment. It is not yet possible to comment on the patients whose treatment was extended over $40-50$ days. 
6. Early tumour responses to fast neutrons were favourable and were similar to those produced by low IET radiation.

\section{References}

1. R. S. Stone. "Neutron Therapy and Specific Ionization." Amer. J. Roentgenol. 59; 771 (1948).

2. G. E. Sheline, S. B. Field, J. T. Brennan, A. Raventos and T. L. Phillips. "Effects of Fast Neutrons on Human Skin." Amer. J. Roentgenol. In press.

3. S. B. Field. "The Relative Biological Effectiveness of Fast Neutrons for Mammalian Tissues." Radiology 93, 915 (1969).

4. F. Ellis. "Dose, Time and Fractionation: A Clinical Hypothesis." Clin. Radiol. 20, 1 (1969).

5. British Institute of Radiology Symposium "Pre-therapeutic Experiments with the Fast Neutron Beam from the Medical Research Council Cyclotron." Brit. J. Radiol. 36, 77 (1963).

6. M. Catteral1, C. Rogers, R. H. Thomlinson and S. B. Field. "An Investigation into the Clinical Effects of Fast Neutrons 1. Methods and Early Observations." Brit. J. Radiol. In press.

7. D. K. Bewley and C. J. Parnell. "The Fast Neutron Beam from the Medical Research Council Cyclotron." Brit. J. Radiol: 42, 281 (1969).

8. J. F. Fowler, R. I. Morgan, J. A. Silvester, D. K. Bewley and B. A. Turner. ivxperiments with Fractionated $X$ Ray Treatment of the Skin of Pigs." Brit. J. Radiol. 36, 188 (1963).

9. D. K. Bewley. "Pre-therapeutic Experiments with the Fast Neutron Beam from the Medical Research Council Cyclotron, II Physical Aspects of the Fast Neutron Beam." Brit. J. Radiol. 36,81 (1963). 


\section{FAST NEUTRON DEPTH DOSES AND SPECTRA OBTAINED BY BOMBARDING VARIOUS TARGETS WITH $16 \mathrm{MeV}$ DEUTERONS}

\section{J. Parnell}

M.R.C. Cyclotron Unit Hammersmith Hospital London, England

\begin{abstract}
The fast neutron beam from the Medical Research Council's cyclotron at Hammersmith Hospital, London is produced by bombarding a beryllium target with $16.7 \mathrm{MeV}$ deuterons. Whilst the dose rate is typically $40.50 \mathrm{rad} / \mathrm{min}$, the dose decreases to $50 \%$ at $7.5 \mathrm{~cm}-10 \mathrm{~cm}$ deep in tissue. Thus tumours which can be treated satisfactorily are limited mainly to those of the breast, head and neck and superficial sites. In an attempt to improve the depth dose characteristics experiments have been carried out using various thicknesses of beryllium and thick targets of other materials, i.e. heavy water, lithium and carbon. Using a $10 \mathrm{~cm} \times 10 \mathrm{~cm}$ field at a target-skin distance of $100 \mathrm{~cm}$ the greatest penetration was obtained with either heavy water or a thin beryllium target backed with gold, the thickness of the beryllium being about one quarter of the deuteron range. For thick targets of lithium, beryllium and carbon the greatest penetration was obtained with carbon, but this was inferior to that obtained with heavy water or the optimum thin beryllium target.
\end{abstract}

The fast neutron spectra obtained with some of these targets has been examined with a recoil proton spectrometer.

\section{INTRODUCTION}

The Medical Research Council's cyclotron at Hammersmith Hospital, London, is the only machine currently being used regularly for fast neutron radiotherapy. During the past $4 \frac{1}{2}$ years approximately 120 patients have been treated with this fast neutron beam, initially by Dr.R.L.Morgan and more recently by Dr.M. Catterall.

The fast neutron beam is produced by bombarding a beryllium target with $16.7 \mathrm{MeV}$ deuterons. The thickness of the beryllium is nominally $0.8 \mathrm{~mm}$. Thus the beryllium is not thick enough to stop the deuterons, but instead they are brought to rest in the copper backing on which the beryllium is mounted. The physical characteristics of this beam have been discussed previously $(1,2)$ whilst some of the recent clinical results have been reported elsewhere (3) and also at this Meeting $(\underline{4})$.

The basic physical properties which determine the suitability of a radiation beam for radiotherapy are the dose rate, the penetration of the beam into tissue and, to a lesser extent, the degree of skinsparing. with a fast neutron beam the gamma component should also be considered since if this is too high the possible improvement in the clinical response obtained by using neutrons might be reduced. For the fast neutron beam from the M.R.C. cyclotron the dose rate is typically $40-50 \mathrm{rad} / \mathrm{min}$. at $120 \mathrm{~cm}$ from the beryllium target, this being

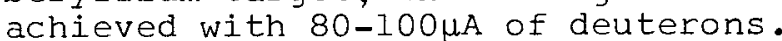
The penetration of the beam into tissue at this distance is such that the dose decreases to $50 \%$ of the maximum (which occurs at about $1.5 \mathrm{~mm}$ beneath the skin surface) at depths of $7.5 \mathrm{~cm}$ to $10 \mathrm{~cm}$ depending upon field size. In air, i.e. no scattering, the tissue kerma due to gamma rays is typically $4 \%-5 \%$ of the kerma in tissue due to neutrons. However this increases at depths within tissue to a maximum of about $20 \%$ ( 5 ).

Brennan has suggested that a neutron source suitable for radiotherapy should be able to deliver a dose rate of $10 \mathrm{rad} / \mathrm{min}$ incident upon the patient and that the dose should decrease to $50 \%$ at a depth of $10 \mathrm{~cm}$ for a $5 \mathrm{~cm} \times 5 \mathrm{~cm}$ field $(\underline{6})$. Fast neutron beams with a penetration satisfying this specification would allow treatment of deep sited tumours with dose distributions similar to those obtained with telecobalt units. However many tumours 
can be treated satisfactorily with radiations which have less penetration than this and thus, although. the specification should serve as a target at which to aim, it should not be regarded as a limit below which neutron beams should be considered inapplicable. The dose rate specification of $10 \mathrm{rad} / \mathrm{min}$ however should be" considered as the lower limit and there are good reasons for suggesting that it ought to be higher particularly for cyclotion produced fast neutron beams. "First, the physical size of the cyclotron means that treatments have to be carried out with a fixed beam direction unless the added complexity and expense of a steerable beam can be considered. Thus it is frequently necessary to support the patient: in relatively uncomfortable positions which he may not be able to maintain throughout a prolonged treatment. Second, many. tumours are treated more satisfactorily by using wedged fields. Using a $45^{\circ}$ wedge constructed from paraffin wax with the M.R.C. beam, Field and Parnell (unpublished) have obtained wedge factors in the range from $1.5-2.2$ depending upon field size. Third, accelerators such as cyclotrons have other important medical applications, e.g. radioisotope production, activation analysis etc. and these might restrict the time available for neutron radiotherapy. Thus, in order to treat a reasonable number of patients per session for a clinical trial a reasonably high. dose rate is required.

The dose rate obtained with the M.R.C. cyclotron is satisfactory but the penetration of the beam should be improved if it is to compete physically with modern $x$ - or gamma ray machines. However any improvement in the penetration should not be accompanied by a very large decrease in dose rate. Possible methods of improving the penetration of cyclotron produced fast neutron beams are (a) increased energy of the accelerated positive ion, (b) use of a reaction other than the bombardment of beryllium with deuterons; (c) increased target-skin distance (d) beam filtration (e) use of thin targets. For a fixed energy cyclotron such as the M.R.C. machine method (a). is inapplicable but this has been studied by Goodman, Marino and Brennan ( 7$)^{1}$. This paper will be concerned with methods (b) and (e).

\author{
The effect of varying the thick-
} ness of the tariget has been examined using the interaction of deuterons with berylitium! The rationale for using this approach to improve fast neutron penetration into tissue lies in the assumption that as the deuterons are slowed down within a thick target the neutrons produced witl have a gradually decreasing mean energy. rhus, if the lower energy deuterons are not allowed to interact in the target material the mean energy of the neutron beam should be higher than that obtained with a thick target. However, the deuterons must be brought to rest in a backing material and this should, ideally, produce no neutrons. Since this is not possible it is necessary to use a backing material for which the neutron yield is small compared with the yieldl from the primary target. Since the neutron yield per microamp decreases with increasing atomic number of the target ( 8 ) high atomic number backing materials are indicated. However the target currently used with the M.R.C. cyclotron does not stop the deuterons completely but instead they are brought to rest in the copper backing of the beryllium and thus the effect of target thickness was examined with two backing materials, copper and gold.

It was not practical to make frequent changies of the target with the existing neutron beam from the M.R.C. cyclotrion due to its regular use for other studies and hence these investigations were carried out using a second beam position. In this position the deuteron beam was focused as well as possible with the quadrupole focusing magnets of the beam transport system. The deuterons passed through a $0.025 \mathrm{~mm}$ thick titanium vacuum foil and about $5 \mathrm{~cm}$ of air before striking the target. The energy of the deuterons. incident upon the target was 


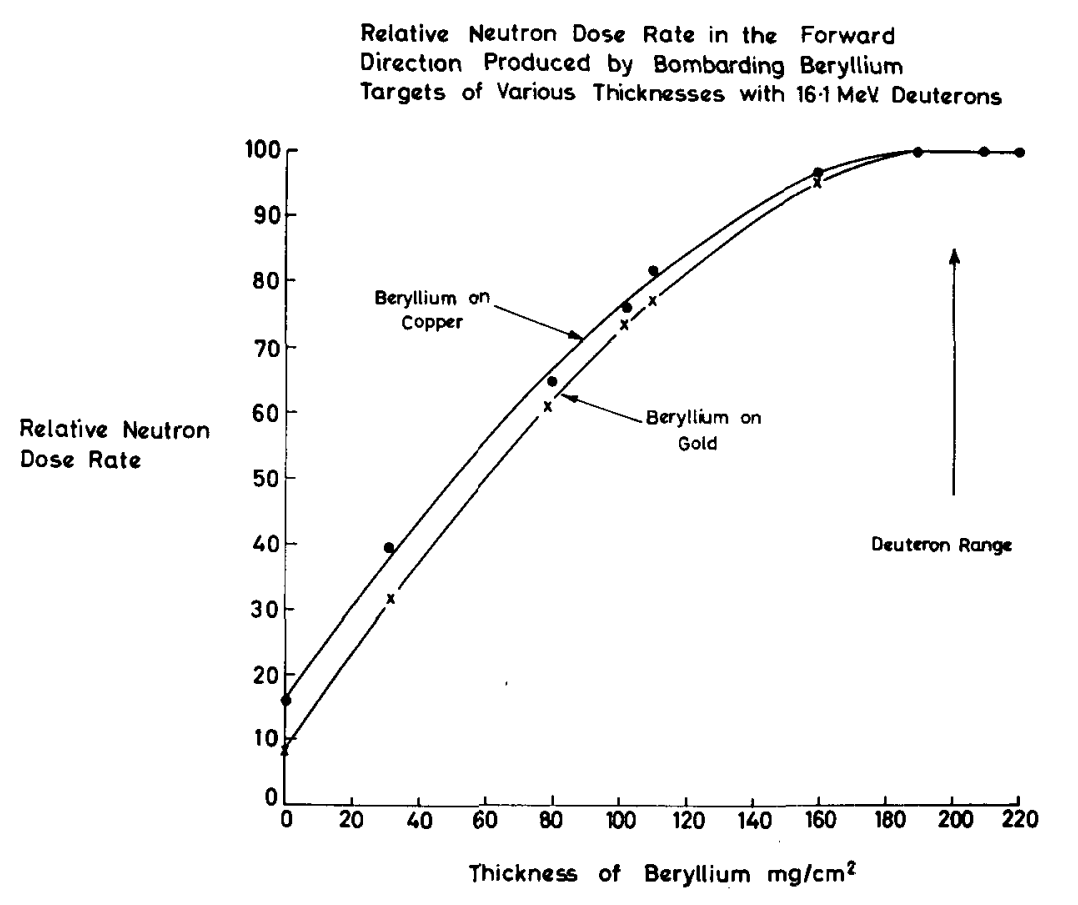

Fig.1

estimated to be $16.1 \mathrm{MeV}$. The beam incident upon the target had a diameter of $2 \mathrm{~cm}$, this being defined by a tantalum collimator placed in front of, but electrically insulated from, the target. Deuteron beam currents of $2-3 \mu \mathrm{A}$ were used throughout these investigations, and the charge collected from the target was used to normalise the observations.

The neutron beam was collimated with one of the standard borated wooden collimators which was surrounded by paraffin wax. Measurements were made at a target-skin distance of $100 \mathrm{~cm}$ where the field: size was $10 \mathrm{~cm} \times 10 \mathrm{~cm}$.

Depth dose measurements were made in a tissue equivalent liquid using a gamma compensated ionization chamber which was a modified version of that described by Bewley andParnell (1). Relative dose rate measurements for each target were also made with this chamber at $100 \mathrm{~cm}$ from the target but with the phantom removed.

Figure 1 shows the variation of fast neutron dose rate, expressed as a percentage of that obtained with a thick beryllium target, as a function of target thickness. Since copper produces more neutrons than gold the dose rates with copper backed targets are higher than for gold backed targets. The dose rates measured for pure copper and gold targets are higher than expected from the data of Allen et al ( 8 ) and this is probably due to neutron production in the cyclotron vacuum foil, the air gap between the vacuum foil and the target, and to a small fraction of the deuterons striking the tantalum collimator. There might also be an additional contribution relative to berylilum resulting from scattering from the collimators since for high atomic number materials the angular distribution of neutrons is not so sharply directed in the forward direction as it is with beryllium ().

The depth dose characteristics, represented by the depth at which the dose decreases to $50 \%$ in unit density tissue, as a function of target thickness is shown in Figure 2. The range of $16.1 \mathrm{MeV}$ deuterons in beryllium is estimated to be about $195 \mathrm{mg} / \mathrm{cm}^{2}$. Thus the measurements at $209 \mathrm{mg} / \mathrm{cm}^{2}$ are regarded as 


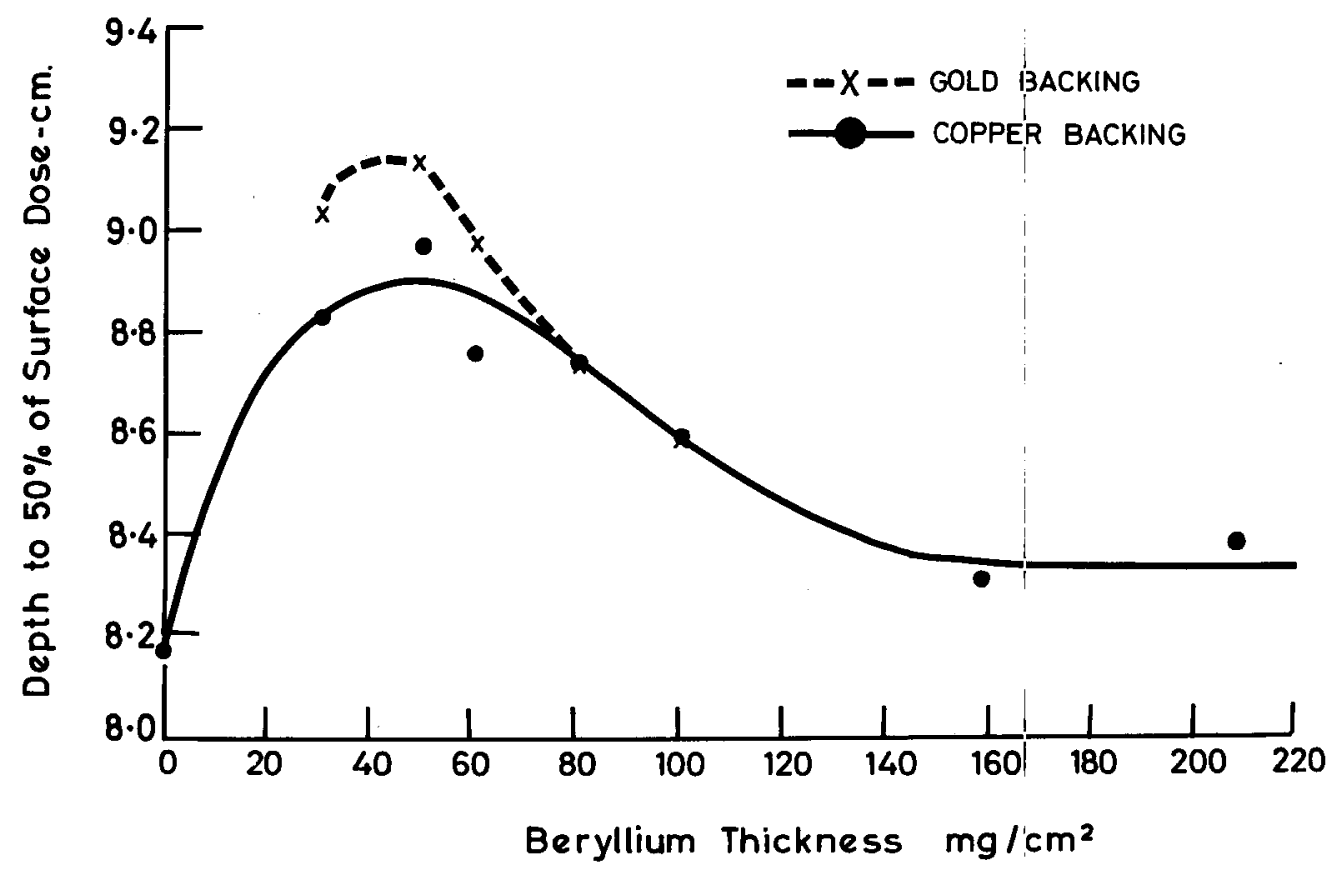

Fig. 2 Variation of depth at which the dose decreases to $50 \%$ of the maximum value as a function of target thickness

being characteristic of a thick target. The optimum thickness, with regard to neutron beam penetration, was about $50 \mathrm{mg} / \mathrm{cm}^{2}$ beryllium backed with gold, i.e. about one quarter of the range of the deuterons. On decreasing the thickness of beryllium from that which is sufficiently thick to ${ }_{2}$ stop the deuterons to about $80 \mathrm{mg} / \mathrm{cm}^{2}$ there is no significant effect of varying the backing material since in this region the yield of neutrons from the beryllium is considerably higher than that from the backing material. In the range from $80 \mathrm{mg} / \mathrm{cm}^{2}$ to $30 \mathrm{mg} / \mathrm{cm}^{2}$ the backing material gradually contributes a significant proportion of neutrons to the beam, gold contributing less than copper. Thus, with gold backed targets it is possible to obtain greater depth doses than with copper backed targets. For beryllium targets thinner than about $30 \mathrm{mg} / \mathrm{cm}^{2}$ the backing material contributes even more significantly to the neutron flux and the situation is approached where neutrons are produced only in the backing material.

\section{REACTIONS OTHER THAN THE BOMBARDMENT OF BERYLLIUM WITH DEUTERONS}

Three targets were considered as alternatives to beryllium for neutron production with deuterons. The first was lithium- 7 since the $Q$ value for the $\mathrm{Li}(\mathrm{d}, \mathrm{n}) \mathrm{Be}$ reaction is $115 \mathrm{MeV}$ whilst that for the $\mathrm{Be}(\mathrm{d}, \mathrm{n}) \mathrm{I}(\mathrm{B}$ reaction is $+4.4 \mathrm{MeV}$. Al though the $Q$ value for the ${ }^{12} \mathrm{C}(\mathrm{d}, \mathrm{n}){ }^{13} \mathrm{~N}$ réaction is $-0.3 \mathrm{MeV}$, carbon was considered as the second alteryative sjoce the $Q$ value for the ${ }^{12} \mathrm{C}(\mathrm{d}, 2 \mathrm{n}) \mathrm{i}_{\mathrm{N}}$ reaction is -20.6 $\mathrm{MeV}$ and hence this reaction will not occur at the cleuteron energy under consideration. Third, the use of deuterium as a target was also considered since there are no 3 low energy excited states in the ${ }^{3} \mathrm{H}_{3}$ produced by the reaction $\mathrm{D}(\mathrm{d}, \mathrm{n})^{3} \mathrm{He}$. This reaction/was examined using a heavy water target. A heavy water target was also examined using 
TABLE I

\begin{tabular}{|c|c|c|c|c|c|}
\hline \multirow[b]{2}{*}{ Reaction } & \multirow[b]{2}{*}{$\overline{\mathrm{E}}_{\mathrm{n}} \mathrm{MeV}$} & \multicolumn{2}{|c|}{$100 \mathrm{~cm}$ TSD } & \multicolumn{2}{|c|}{$120 \mathrm{~cm}$ TSD } \\
\hline & & $\begin{array}{c}\text { Dose rate } \\
\mathrm{rad} / \mathrm{min} / 100 \\
\mu A\end{array}$ & $\begin{array}{l}\% \text { Dose at } \\
\text { 10cm in } \\
\text { tissue }\end{array}$ & $\begin{array}{l}\text { Dose rate } \\
\text { rad/min/ } \\
100 \mu \mathrm{A}\end{array}$ & $\begin{array}{l}\% \text { dose } \\
\text { at locm } \\
\text { in } \\
\text { tissue }\end{array}$ \\
\hline $\begin{array}{l}\text { 16. } 7 \mathrm{MeV} \text { d on } 0.8 \mathrm{~mm} \\
\mathrm{Be} \text { on } \mathrm{Cu} \text { (existing } \\
\text { M.R.C. beam) * }\end{array}$ & 7.65 & 72 & 44.1 & 50 & 45.4 \\
\hline $\begin{array}{l}16.1 \mathrm{MeV} d \text { on thick } \\
\text { Be }\end{array}$ & 7.0 & 80 & 42.0 & 56 & 43.3 \\
\hline $\begin{array}{l}16,1 \mathrm{MeV} d \text { on } 101 \mathrm{mg} / \\
\mathrm{cm}^{1} \text { Be on } \mathrm{Cu}\end{array}$ & 7.7 & 60 & 43.4 & 42 & 44.7 \\
\hline $\begin{array}{l}16,1 \mathrm{MeV} d \text { on } 50 \mathrm{mg} / \\
\mathrm{cm}^{2} \mathrm{Be} \text { on Cu }\end{array}$ & 8.2 & 34 & 46.0 & 24 & 47.4 \\
\hline 16.1 MeV d on Li & - & 64 & 43.0 & 44 & 44.3 \\
\hline $16.1 \mathrm{MeV} \mathrm{d}$ on $\mathrm{C}$ & 7.1 & 58 & 43.8 & 40 & 45.2 \\
\hline Ió $\mathrm{MeV} d$ on $\mathrm{D}_{2} \mathrm{O}$ & 8.0 & 48 & 46.0 & 33 & 47.4 \\
\hline $10.5 \mathrm{MeV} d$ on $\mathrm{D}_{2} \mathrm{O}$ & 6.4 & 15 & 42.0 & 10 & 43.3 \\
\hline Il MeV d on thick Be & 5.7 & 30 & 38.5 & 21 & 39.7 \\
\hline $\mathrm{T}(\mathrm{d}, \mathrm{n})^{4} \mathrm{He}$ & 14 & - & 53 & - & 54.6 \\
\hline
\end{tabular}

* Low density berylium

approximately 10. MeV deuterons since it was suggested that this might form a suitable target for "compact" cyclotrons (9). In order to obtain a deuteron energy of about $10 \mathrm{MeV}$ the beam was passed through a gold degrading foil.

Table I summarises the dose rate and depth dose data of the various beams studied and also includes, for comparison purposes, data fgr $14 \mathrm{MeV}$ neutrons from the $\mathrm{T}(\mathrm{d}, \mathrm{n})^{4}$ He reaction (10). With the exception of the measurements for the existing neutron beam from the M.R.C. cyclotron (16.7 MeV deuterons on $0.8 \mathrm{~mm}$ beryllium backed with copper) the data for $120 \mathrm{~cm}$ targetskin distance have been estimated from measurements at $100 \mathrm{~cm}$ by applying an inverse square law correction only. Comparing the data for thick metallic targets (beryllium, lithium and carbon) it is seen that carbon gave better depth doses than lithium or beryllium. This is attributed to the fact that the ${ }^{12} \mathrm{C}(\mathrm{d}, 2 \mathrm{n}){ }^{\mathrm{I}} \mathrm{N}_{\mathrm{N}}$ reaction cannot occur at the deuteron energy under consideration, whilst the $Q$ values for the (d, 2n) reactions with lithium and beryllium are almost the same (about 
-4 MeV). The improvement obtained with a.lithium target in comparison with beryllium is attributed to the much larger $Q$ value for the $\mathrm{Li}(\mathrm{d}, \mathrm{n})$ Be reaction $(+15 \mathrm{MeV})$ in Gqmparison with that for the $\mathrm{Be}(\mathrm{d}, \mathrm{n}) \mathrm{IO}_{\mathrm{B}}$ reaction $(+4.4 \mathrm{MeV})$. A heavy water target bombarded with deuterons if about $16 \mathrm{MeV}$ gave depth doses which were identical with those obtained with the optimum thickness of beryllium ( $5 \mathrm{l} \mathrm{mg} / \mathrm{cm}^{2}$ backed with gold) and the dose rate obtained was higher than for this thin beryllium target but less than that obtained with thick beryliium. At a deuteron energy of about $10 \mathrm{MeV}$ the depth doses obtained with heavy water were very similar to that obtained by bombarding a thick beryllium target with $16 \mathrm{MeV}$ deuterons.

\section{NEUTRON SPECTRA}

A knowledge of the energy distribution of fast neutrons used for radiobiology and radiotherapy is necessary to improve the accuracy of dosimetry techniques and to assist in the interpretation of the biological response of the irradiated system. Since the keryllium target used routinely with the M.R.C. cyclotron is not thick enough to stop the deuteron beam it was not possible to estimate the neutron spectrum from published data and hence it was desirable to make a direct determination of the spectrum. In addition the energy spectra obtained with some of the targets discussed previously have also been determined.

The spectrometer used for this work was based on measurements of the energy, distribution of recoil protons emitted from a thin polythere radiator at a known angle (strictly, a distribution of angles) relative to the incident, well collimated, neutron beam. The radiator consisted of a $2 \mathrm{~cm} \times 2 \mathrm{~cm}$ square of polythene which was $10 \mu \mathrm{m}$ thick. Recoil protons were detected at an angle of $20^{\circ}$ by a counter telescope system consisting of a $12.5 \mu \mathrm{m}$ totally depleted silicon detector and a $2 \mathrm{~mm}$ thick lithium drifted silicon detector. The thick detector was situated $20 \mathrm{~cm}$ from the radiator and tie thin detector was about $3.5 \mathrm{~cm}$ in front of the thick one. The signal from the thin detector was alded electronically to that from the thick detector to give a pulse proportional to the energy of the recoil proton. The signal from the thin detector was also fed to the $Y$ converter of a pulse height analyser whilst the energy signal was fed to the $x$ converter; the analyser being operated in a bidimensional analysis mode. Background measurements were made by removing the polythene radiator and the energy calibration was obtained by using a standard alpha particle squbce ceqsisting gf a mixture of ${ }^{39} \mathrm{Pu}, 24 \mathrm{Am}$ and ${ }^{4} \mathrm{Cm}$, and a pulse generator.

Figure 3 shows the energy spectrum of the fast neutron beam produced by the M.R.C. cyclotron. The mean energy of the neutrons is estimated to kie $7.65 \mathrm{MeV}$ whilst the maximum eriergy is about $21 \mathrm{MeV}$ although there are very fow neutrons with energies greater than the energy of the incident deuterons.

Figure 4 compares the fast neutron spectra produced by bombarding three thicknesses of berylitum with 16.l MeV deuterons. In this figure the spectra have been normalised in such a manner that the area under the curve is proportional to the neutron dose rate obtained with that target. The spectra clearly show the reduction of the low energy component of the beam resulting from the use of thin targets. For example, a target thickness of $101 \mathrm{mg} / \mathrm{cm}^{2}$ reduces the energy of the incident deuterons from 16.1 $\mathrm{MeV}$ to $11.0 \mathrm{MeV}$ and consequently the high energy side of the spectrum is not significantly altered, the reduction in the neutron flux from this target, in comparison with the thick target, being due almost entirely to the reduction in the low energy component. For the thinnor target only about $2.5 \mathrm{MeV}$ of the deuteron energy is deplosited in the beryllium, 


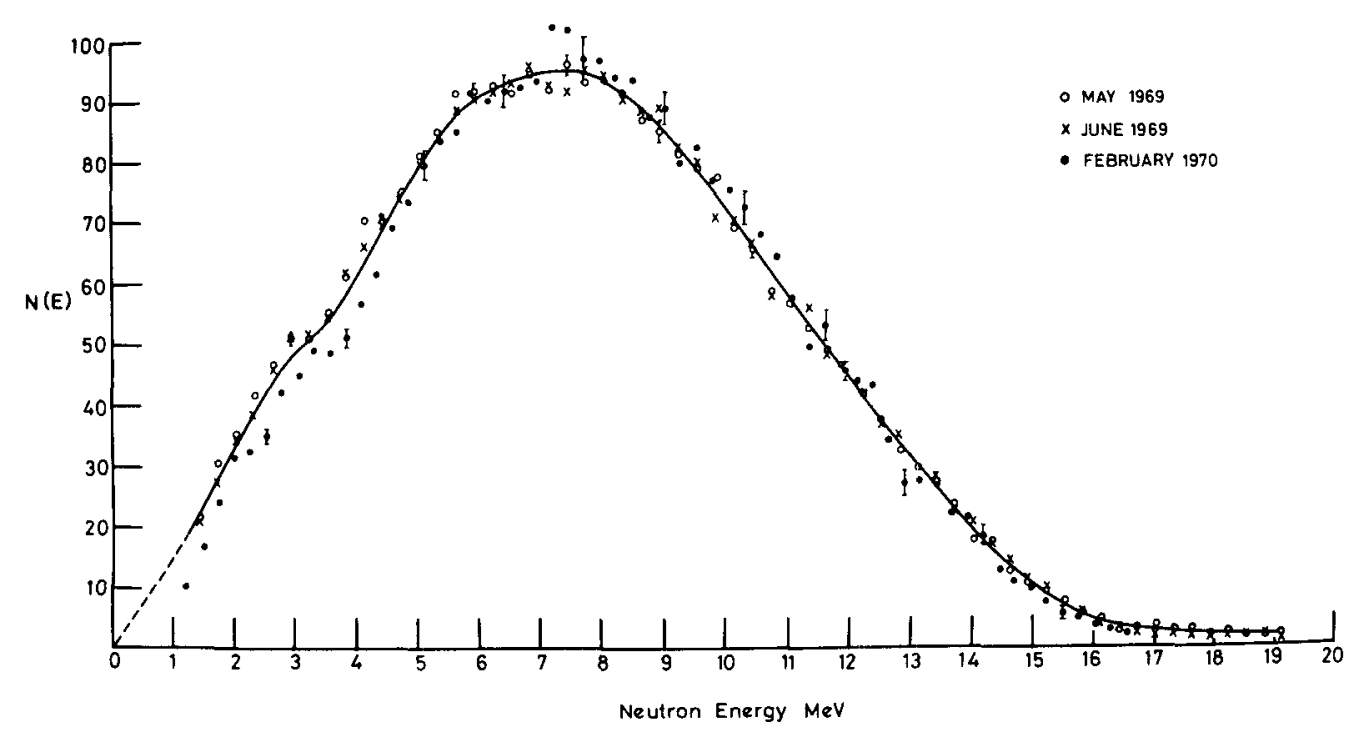

FIG. 3

the remainder of the energy being deposited in the gold backing. Consequently the spectrum obtained with this target diverges from that obtained with the thick target at a much higher energy. The mean energies of the three spectra shown in figure 4 are $7.0 \mathrm{MeV}$ for the thick target, $7.7 \mathrm{MeV}$ for the $101 \mathrm{mg} / \mathrm{cm}^{2}$ target and $8.2 \mathrm{MeV}$ for the $51 \mathrm{mg} / \mathrm{cm}^{2}$ target.

The mean energies of the other measured spectra are given in table 1 .

\section{DISCUSSION}

The fast neutron beam produced by the M.R.C. cyclotron has been developed to such an extent that it can be used routinely for radiotherapy. Whilst a cyclotron produced beam offers high dose rates the depth doses are inferior to those obtained with modern supervoltage or teleisotope units normally used for radiotherapy unless the cyclotron is capable of accelerating deuterons to energies of about $30 \mathrm{MeV}$. The existing fast neutron beam produced by the M.R.C. machine has depth doses which give $50 \%$ at $9 \mathrm{~cm}$. for a $10 \mathrm{~cm}$. $x$ $10 \mathrm{~cm}$. field at a target-skin distance of $120 \mathrm{~cm}$. and on this account the range of tumours which can be treated satisfactorily is limited. Methods have been examined which might be of value in imporoving the depth dose characteritics but the improvements obtained were not as great as was desired. The targets which produced the greatest penetration were either heavy water or $51 \mathrm{mg} / \mathrm{cm}^{2}$ beryliium backed with gold and these resulted in $50 \%$ depth doses at $9.1 \mathrm{~cm}$. for a $10 \mathrm{~cm}$. $x 10 \mathrm{~cm}$. field at $100 \mathrm{~cm}$. target-skin distance using a slightly lower energy deuteron beam than is routinely used for neutron production with the M.R.C. machine. The corresponding figures for a thick beryllium target and a target comparable with that used routinely in the cyclotron (101 mg/ $\mathrm{cm}^{2}$ beryllium on copper) are $8.4 \mathrm{~cm}$. and $8.6 \mathrm{~cm}$. respectively, these being at a target-skin distance of $1.00 \mathrm{~cm}$.

It was originally thought that the beryllium target in routine use was thick enough to stop the deuterons. 


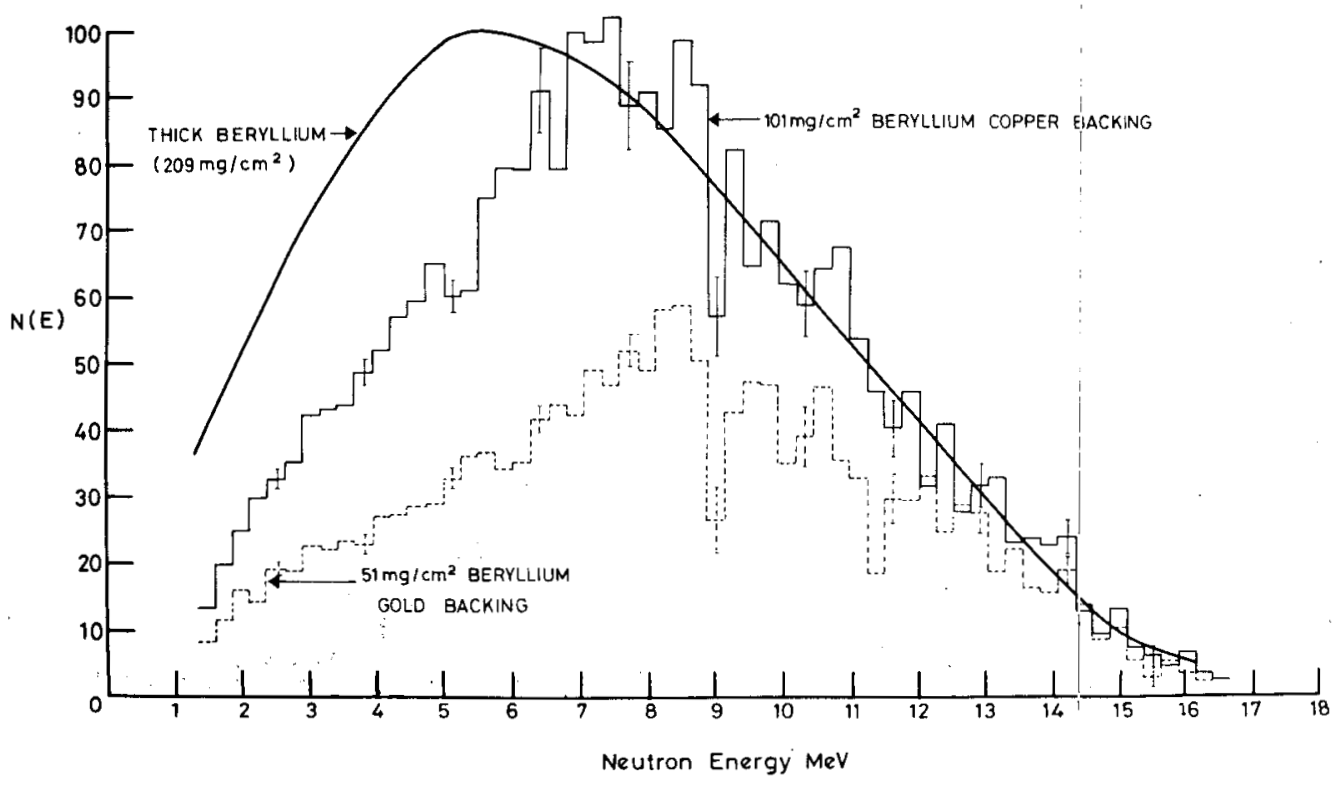

FIG. 4

Since this is not the case the use of the optimum thickness would not produce as great an improvement as it might otherwise have done. However an improvement could be gained by decreasing the thickness of the target to about half its present value but at the expense of dose rate. The use of such a target would shift the $50 \%$ contour by $5 \mathrm{~mm}$. but the dose rate would be decreased by a factor of about 1.8 .

The energy spectrum of the neutrons produced by the M.R.C. Cyclotron is continous, with very few neutrons having energies greater than that of the bombarding deuterons. The mean energy of this beam was estimated to be $7.65 \mathrm{MeV}$. In addition the maximum intensity of neutrons occurs at about the same energy.

The measurements of neutron spectra made with three different thicknesses of beryllium using 16.1 MeV deuterons clearly demonstrate the effect of reducing the target thickness. The mean energies of the beams obtained with these targets were estimated to be $8.2 \mathrm{MeV}$ (51 mg/ $\left.\mathrm{cm}^{2}\right) 7.7 \mathrm{MeV}\left(101 \mathrm{mg} / \mathrm{cm}^{2}\right)$ and 7.0 MeV (thick target).

The beryljium target currently used with the I.R.C. cyclotron has a nominal thickness of $0.8 \mathrm{~mm}$. which, on the assumption that the density of the beryllium is $1.8 \mathrm{gm} / \mathrm{cm}^{2}$ is equivalent to $0.144 \mathrm{mg} / \mathrm{cm}^{2}$. However, dose rate measurements with this target, arid the mean energy of the neutron beam produced with this target suggest that its equivalent thickness is only about $100 \mathrm{mg} / \mathrm{cm}$. This discrepancy is probably due to the method of preparation of. the target; the beryliium being formed by hot pressing sintered berylitum. In addition, the silver used to attach the berylitium to the copper backing could diffuse into the beryllium thereby reducing the effective thickness.

Spectra obtained with carbon and heavy water show the same 
general shape as that obtained with

thick berylium. However, the

carbon spectrum has no neutrons

greater than the energy of the

deuterons (due to the small negative

Q value of the ${ }^{12} \mathrm{C}(\mathrm{d}, \mathrm{n})^{13} \mathrm{~N}$ reaction.

\section{REFERENCES}

I. D.K.Bewley and C.J.Parnel.1.. "The Fast Neutron Beam from the M.R.C. Cyclotron." Brit J. Radiol. 42, 281 (1969).

2. D.K.Bewley. "Fast Neutron Beams for Therapy." in Current Topics in in Radiation Research. M. Ebert and Alma Howard. Eds. p.251. North Holland Publishing Company, Amsterdam (1970).

3. Mary Catterall, C. Rogers, R.H.Thomlinson and S.B.Field.

"An. Investigation into the Clinical Effects of Fast Neutrons

(i) Methods and Early Observations." Brit. J. Radiol. In press (197I).

4. Mary Catterall, C. Rogers, R.H.Thomlinson and S.B.Field.

"Neutron Radiation Therapy." ANS National Topical Meeting. Neutron Sources and Applications (1971).

5. N.J.MCNally and D.K.Bewley. "A Biological Dosimeter using Mammalian Cells in Tissue Culture and its use in obtaining Neutron Depth Dose Curves." Brit. J. Radiol. 42, 281 (1969).

6. J.T.Brennan. "Fast Neutrons for Radiation Therapy." Radiol. Clin. N. Amer. 7365 (1969).

7. L.J.Goodman, S.A. Marino and J.T.Brennan "Minimum Cyclotron Size for Radiation Therapy: A Study of Relevant Physical Parameters." In: Uses of Cyclotrons in Chemistry, Metallurgy and Biology. C.B. Amphlett Ed. p. 209. Butterworth \& Co. London (1970).

8. A.J.Allen, J.F. Nechaj, K-H Sun and B.Jennings. "Thick Target Fast Neutron Yield from $15 \mathrm{MeV}$ Deuteron and $30 \mathrm{MeV}$ Alpha Bombardment." Phys Rev. 81,536 (1951).

9. M.A.Chaudhri and G.J.Batra. "Production of Fast Neutrons by Cyclotrons." Presented at: Int. Canf. on the Use of Cyclotrons in Chemistry, Metallurgy and Biology, Oxford, U.K. (1969).

10. D.Greene and R.I.Thomas. "An Experimental Unit for Fast Neutron Radiotherapy." Brit. J. Radiol. 41, 455.(1968). 


\title{
EXPERIENCE IN TREATMENT DOSE CALCULATIONS FOR ${ }^{252}$ Cf PATIENTS
}

\author{
George D. Oliver, Jr., Peggy R. Wright, \\ and Peter R. Almond
}

The University of Texas

M. D. Anderson Hospital and Tumor Institute Houston, Texas

\begin{abstract}
An experimental program, designed to evaluate ${ }^{2 \leq 2} \mathrm{Cf}$ versus conventional interstitial therapy, has been in progress in the Departments of. Radiotherapy and Physics. Of significant importance in a treatment is the capability to calculate isodose distributions around the implant. A computer program has been developed which produces complete neutron and gamma-ray dose contours in selected planes through an implant. This program is based upon neutron transport theory calculations. Routinely hand calculations are performed at selected points of medical pertinence as a check. Gamna-ray dose calculations consistently show an average value of $75 \%$ of the neutron dose. Normally we use the calculated neutron physical dose rate times a selected RBE value of 7.5 and add the calculated gamma-ray physical dose times an RBE value of 1.0 to arrive at a biological dose rate in terms of $\mathrm{rem} / \mathrm{hr}$. Examples of patient dosimetry will be presented. Utilization. of this program for deep seated implant calculations yields more accurate numbers. Neutron doses to the patients' eye lens also have been calculated; some lip and cheek implants have been found to deliver excessive doses to the eye.
\end{abstract}

\section{INTRODUCTION}

For over 70 years gamma emitting radium has been used for treatment of neoplastic diseases; and more recently, iridium, cesium, gold, and cobalt have been employed. The latter treat by gamma-rays and produce a clinical result similar to radium. None of these materials were sought because of their basic capability for increasing the damage to malignant cells. Since a component of californium radiation consists of neutrons, high LET secondary radiation can be delivered to a target volume in the treatment of a malignant tumor. Qualitatively different results in the clinic may emerge because of possible advantages of californium neutrons: (1) the equalization of differential in sensitivity of cells due to their oxygenated state; (2) a higher relative biological effectiveness close to the sources with a decreasing effectiveness with distance from the source; (3) an apparent absence of dose rate effects at neutron dose rates $<50$ rads/ hr. In order to obtain any results, regardless of their basis or quality, a method is necessary for evaluation of the treatment dose from the radiation delivered by a californium implant.

\section{METHODS OF DOSE CALCULATION}

Much of the clinical experience with the use of radium can be applied to californium treatment and will be essential to the evaluation of californium. It is desirable that the full radiation distribution be known for the califiornium and the radium implants. which wili be compared. The method should be practical for the majority of hospitals and therepeutic institutions evaluating californium as a cancer tocl. Two processes are required to answer the therapist's questions about a particular implant. First, the source seometry in the implant must be determined Second, accurate and consistently reproducible assessments of dose must be possible. These processes need to be performed for the implant achieved in the patient rather than the one planned prior to implantation. The reconstruction of needles or afterloaded sources and dose evaluation methods for radium have received much attention. Both processes are feasible with hand or computer analysis. A computer method which presents complete dose distributions. in selectei planes through the source array is preferred over a manual method which presents doses at a few selected points.

This paper presents a computer method which originated from a parent method, used for radium, which has evolved in various forms over a 10 year period. It is sufficient that only a few such efforts be promoted; it also should be expected that these efforts become available for general use. With the expansion of the californium clinical program a calculative method should be useful in correlating patient data from different; institutions by providing a common dosimetry 
technique. In the event of expansion of the program this technique would be available upon request. The computerized capability provides for speed and flexibility of detailed information. It seems likely that this complete dosimetric picture provided by computer analysis will be beneficial to the radiotherapist in arriving at an optimum treatment plan of time and dose.

\section{MAIVUAL, CALCULATION}

Prior to the development of a computer program, a hand calculational technique was used for californium interstitial implants. In this method a cross sectional view of the array in the selected plane of calculation is obtained by geometric projections of each source as it intercepts the plane, using only AP and lateral films (1). Experimental data of isodose contours in a transverse plane through a $2.0 \mathrm{~cm}$ active length source were used to obtain concentric isodose contours in the plane perpendicular to the center of the source ( $\vec{z})$. Other concentric contours were developed from this data for sources having greater lengths. For a particular implant the appropriate concentric contour is selected which corresponds to the active length used in the actual implant. The doses contributed by each line source in the array to selected points of interest were calculated and summed by hand. The numerical technique for dose assessment is similar to the technique used at GustaveRoussy ( $\underline{3}$ ).

\section{CCMPUTER CALCUIATION (CALRAD)}

The computer code CALRAD (CAIifornium RAdiation Dosimetry) was designed to calculate the neutron and gamma-ray radiation distributions around individual implantations of californium-252 sources, The program described here derives from the program RADCOMP (4, 5) in that the input and output features as well as the general computational approach are the same; only those basic dosimetric parameters specific to californium have been substituted. Both the neutron and gamma-ray components of the program CAIRAD consider a line source having a constant linear activity $(\mathrm{\mu g} / \mathrm{cm})$ for the defined length ( $\mathrm{mm})$, surrounded by a cylindrical absorber of a stated thickness (mm) with an associated absorption coefficient, and lying within a medium whose absorption properties can be described. An interval method is used for computing the dose from a line source. Dividing the line source intc a large number of equal intervals, in this case twenty, simulates point sources. The dose to a point is obtained by numerically summing the dose contributed from each assumed point source. The method considers uniquely each line source in an array. Both types of medical californium sources (ALC, AT types) can be applied to this program.

Input data is derived from localization of the sources from a set of radiographs (either orthogonal or stereo) of the actual implant (5). The method for obtaining the implant geometry from films has been given in detail (6). A plane of calculation having multiple isodose contour lines, may have selected orientation around or passing through some or all of the sources in the implant. Output data may consist of a tabular listing of dose rates on a grid of points or automatically plotted and labeled isodose curves with the source positions projected onto the plane (Figures 1 - 4).

Gamma-ray Dose Calculation The gammaray dose contribution is calculated for a line source by using a solution of the Sievert integral in a manner similar to RADCOMP (7). The specific gamma-ray emission (I factor) for californium-produced gamma radiation has been taken as $1.2 \mathrm{R}-\mathrm{cm}^{2} / \mu g-\mathrm{hr}$ for a point source in air filtered by $0.5 \mathrm{~mm}$ of platinum. This number was determined in our laboratory by measurement with thermoluminescent materials and agrees to within $9 \%$ with a $\Gamma_{\text {Cfy }}$ of 1.33 calculated from stoddard's data (8).

The gamma-ray energy spectra of radium and californium are similar - both are multienergetic and cover the same energy range. Then the absorption. coefficients relating to radium gamma transmission in lead and platinum have been taken in the californium gammaray calculations. Effects on the dose from attenuation in the encapsulation wall (platinum-iridium) are considered for the appropriate source in the calculation.

The complex gamma-ray spectra of both radium and californium causes the transmission of gamma-rays through materials to decrease non-exponentially with the thickness of the absorber; this effect results in an absorption coefficient which varies as the gamma-rays penetrate the material. For penetration through tissue, which is taken to be the same as that through water for the gamma-rays only, an empirically derived $3 \mathrm{rd}$ crder polynomial expression describes the 
appropriate reduction in dose (7).

Neutron Dose Calculation the neutron dose contribution of a point source in tissue has been derived by Windham (9) from the solution of the monoenergetic neutron transport equation with a multi-energy grouping approximation. The dose is calculated in units of rads/hr for a finite line source by using a line integral of the point kernel solution. The solution assumes that an infinite homogeneous phantom surrounds the source, establishing full build-up effects. This assumption also establishes that any boundaries of media lie at a distance greater than several neutron mean free paths. A ninth order polynomial has been derived to fit the calculated data over the interval $0.15 \mathrm{~cm}$ to $11.5 \mathrm{~cm}$. This mathematical fit is directly applied in CALRAD with the interval method to describe neutron dose rates from any single or multiple line source arrangement in any selected plane of calculation. Although the polynomial accounts for tissue absorption, no consideration is given to attenuation in the Pt-Ir wall thickness. Fabrication of the ALC type sources is such that uniform specific activity is present and no accounting for celiular construction is required. Tandem combinations of ALC type sources do present cellular conditions, but are not considered as such.

\section{SPECIAL ASPECTS}

\section{BASIS OF TREATMENT DOSE DEFINITION}

The stated treatment doses in Table I of the preceding companion paper are the sum of the neutron and gamma-ray physical doses in rads taken at a distance from the source plane in the tumor which was selected by the radiotherapist as the distance for the meaningful therapeutic dose rate. In this form the doses bear little resemblance to the conventional doses of gamma-ray (radium) therapy. The individual components must be weighted according to their capability for biological damage in accordance with the dose rate variations (10). A value of 7.5 has been taken for the relative biological effectiveness of californium neutrons, based on a conservative compromise of the existing biological data comparing californium to radium at low dose rates $(11,12)$. An $R B E$ of 1.0 was selected for the gamma-ray component. Although these high values of $\mathrm{RBE}$ result in a low effective dose to a patient, they are in agreement with the philosophy of conservative treatment which prevails during these early stages of californium application to medicine. The ealifornium dose to be compared to conventional tumor doses is determined by $7.5 \frac{1}{x}$ neutron dose in rads plus $1.0 \mathrm{x}$ gamma-ray dose in rads; this yields the biological treatment dose in rem. This method attaches the weight to the dose component responsible for the most biological damage. It also accounts for the changing function of the neutron-gamma dose ratio with depth. Gamma-ray dose calculations for the treatment region have an average value of $75 \%$ of the neutron physical dose.

A less accurate method, which can be used as a handy reference system to convert from californium to equivalent radium dosage, is to sum the neuticon and gamma doses and multiply by an RBE of five. This approximation method usually gives biological doses which are 5-10\% lower than the preferred method, depending in the volume and geometry of the implant.

It is within the scope of the program to combine the doses for expression in units of REM. Weighting the two components of dose by preselected factor values of $\mathrm{RBE}$ and summing them can lead to an output of the data in terms of biological dose rates.

\section{BOUNDARY EFFECTS ON TUMOR DOSE}

All physics dosimetry and theoretical calculations of californium dose rates have been done for infinite, homogeneous phantoms of tissue composition. The numbers generated from these endeavcrs are accurate for application to clinical dose calculations of deep-seated implarts such as in the pelvis. When the source liles close to a boundary or near a tissue-air interface, such as for skin implants, a reduction of the tumor dose may occur due to the loss of back-scattered radiation. The megnitude of dose reduction has been estimated from 4 to $30 \%$ and may be larger for neutron than for gamma-ray interactions. Experiments and calculations are in progress to evelluate this problem.

\section{LENS DOSE FROM PR(NXIMAL IMPLANTS}

A criterion for accepting individuals for treatment having lesions in and around the head region will be the dose, especially the neutron dose, received by the eye lens during californium therapy. When the lens is expected to receive a dose of 6 - 10 rads, the patient may be rejected for californium 
therapy. This dose range approaches that extrapolated from animal data to produce an observable effect in humans, but not necessarily a vision-impairing defect (13). of the patients treated at this hospital only one, which is presented in this paper as patient case \#2, received an excessive eye lens dose ( 57 rads neutron and 50 rads gamma-ray). The implant was high on the cheek and had a source lying within a $\mathrm{cm}$ or so of the eye socket. Other cases, treating lesions of the lip, tongue, and mandible, had calculated neutron doses of $<1$ rad to the nearest eye lens.

\section{EXAMPLE PATIENTS AFTERIOADED WITH CAIIFORNIUM}

\section{EXAMPLE PATIENT \#-}

The first patient treated with californium had a superficial inguinal node implanted with $18 \mu \mathrm{gs}$ for 109 hours. Figures 1 and 2 are the neutron and gamma isodose distributions through a mid-implant plane of calculation, perpendicular to the direction of the sources. The $7.0 \mathrm{rad} / \mathrm{hr}$ neutron contour and $3.5 \mathrm{rad} / \mathrm{hr}$ gamma contour were selected as the treatment dose rates, representing about a 1 . cm wide treatment zone. The sources were approximately straight, as seen by the radiographs in the companion paper (Figure 1B). Projections on this plane of calculation have some distortion at the top of the implant.

\section{EXAMPIE PATIENI \#2}

The 7 th patient had a skin nodule in the cheek implanted with $13.5 \mu \mathrm{gs}$ for 67 . hours. Figures 3 and 4 describe the neutron and gamma isodose distributions in a mid-plane through the implant and perpendicular to the direction of the sources. Treatment dose rates of 8 . and $3.5 \mathrm{rads} / \mathrm{hr}$ were selected. The circled asterisk at the upper left is a projection of the eye lens position onto this plane. The excessive eye lens dose was caused by the crossing source in the implant, represented by the long dashed line. Reference should be made to Figure 5 of the companion paper.
REFERENCES

1. M. Def. Smith. "A Graphic Method of Reconstructing Radium Needle Implants for CaIculation Purposes." Am. J. Roentgenol. Rad. Therapy, Nuc1. Med. 79: (42) (1958).

2. G. D. Oliver, and C. N. Wright. "Dosimetry of an Implantable 252Cf Source." Radiology 92(1):143-147, January, 1969.

3. B. Pierquin, et al. "Technique de Dosimetrie en Curietherapie Interstitielle par Tomographie Transversale." Acta Radiol. 53, 314, (1960).

4. George W. Batten, Jr. "The M.D. Anderson Method for the Computation of Isodose Curves Around Interstitial and Intracavitary Radiation Sources. II. Mathematical and Computational Aspects." Am. J. Roentgenol., Rad. Therapy, Nucl. Med. 102:673-676 (1968).

5. Marilyn Stovall and Robert J. Shalek. "The M.D. Anderson Method for the Computation of Isodose Curves Around Interstitital and Intracavitary Radiation Sources, III. Roentgenograms for Input Data and the Relation of Isodose Calculations to the Paterson-Parker System." Am. J. Roentgenol., Rad. Therapy, Nucl. Med. 102:677-687 (1968).

6. R. J. Shalek, and M. Stovall. Radiation Dosimetry, Vol. III, pp. 774-801, Academic Press, New York (1969).

7. Robert J. Shalek and Marilyn Stovall. "The M.D. Anderson Method for the Computation of Isodose Curves Around Interstitial and Intracavitary Radiation Sources, I. Dose From Iinear Sources." Am. J. Roentgenol. Rad. Therapy, Nucl. Med. 102:662-672 (1968).

8. Deon H. Stoddard. Radiation Properties of Californium-252. USAEC Report DP-986, E.I. duPont de Nemours and Co., Savannah River Laboratory, Aiken, S.C. (1965).

9. J. P. Windham, et al. "Calculated Neutro Dose Rates for Implantable Californium252 Sources." Accepted for publication in Physics in Med. and Biol. (1971). 
10. National Bureau of Standards, Handbook 78, Report of the International

Commission on Radiological Units and Measurements (ICRU) pp. 2-3 (1959).

11. H. R. Withers, et al. "Response of Mouse Jejunal Crypt Cells to Low Dose Rate Irradiation with Californium Neutrons or Radium Gamma-Rays." To be published. (1971).

12. H. L. Atkins. "Comparison of Irradiation by Californium-252 and Radium on the Skin of Swine.". Radiology 96: $161-165$ (1970).

13. V. P. Bond. Iens Opacification in the Mouse - Implication for RBE and QF." Biophysical Aspects of Radiation Quality, IAEA Report No. 149 (1968). 
Patient No. ${ }^{252}$ CI IMPLANT-NEUTRON DOSE

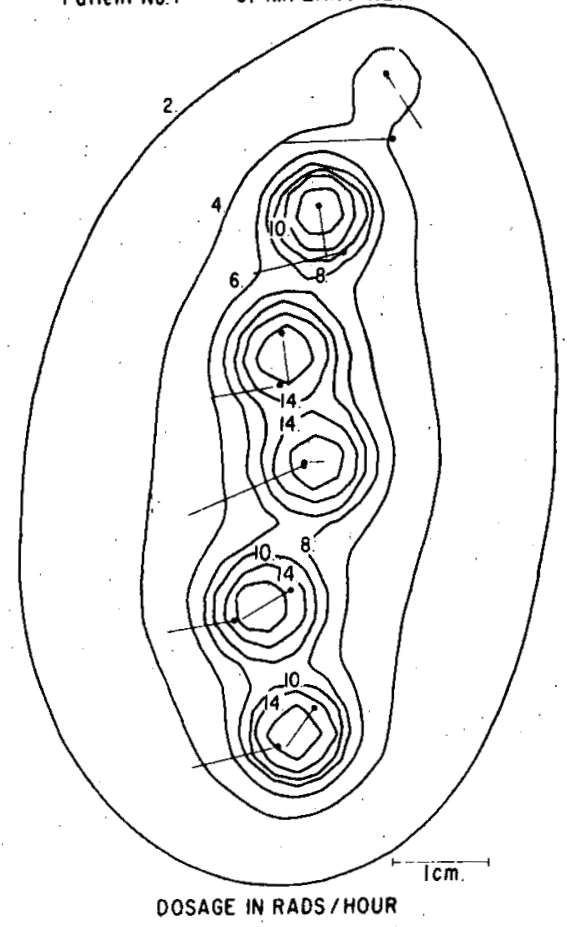

Figure 1. Neutron isodose contour pattern of example patient No. 1. The numbers represent dose rates in rads/hr.

Potient No.1 $252 \mathrm{CF}$ IMPLANT-GAMMA DOSE

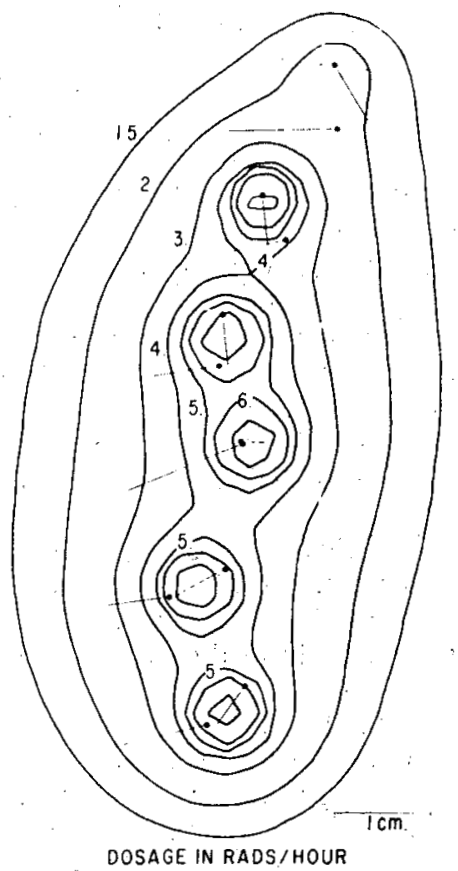

Figure 2. Gamma-ray isodose contour. pattern of example patient No. 1.

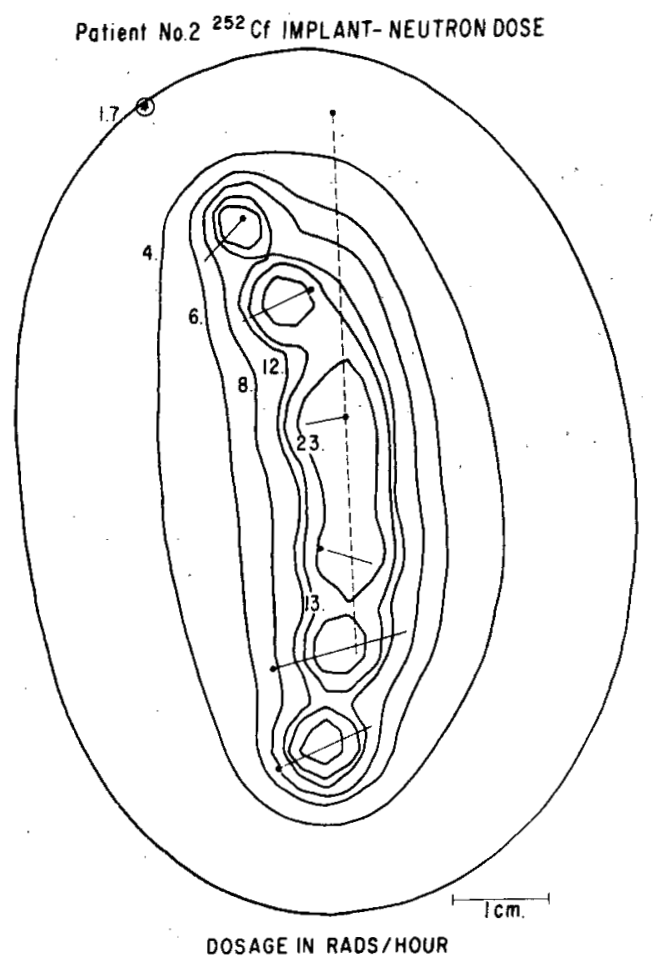

Figure 3. Neutron isodose contour pattern of example patient No. 2. The circled asterisk is a projection of the eye lens.

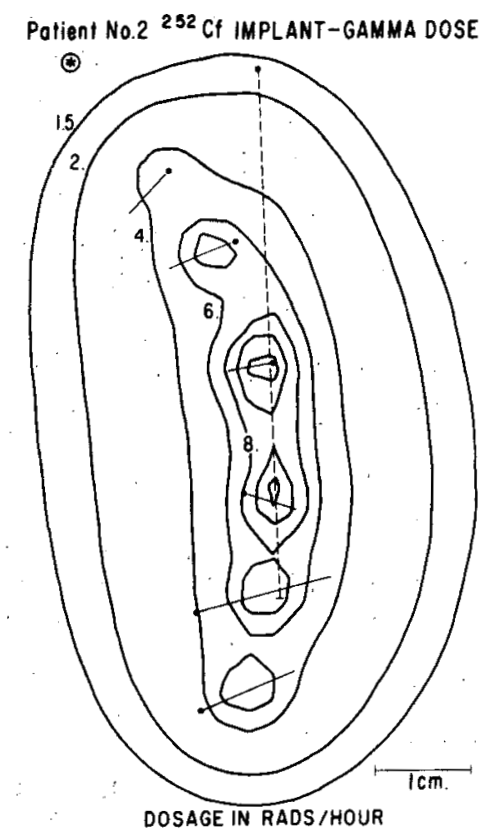

Figure 4. Gamma-ray isodose contour pattern of example patient No. 2. 


\title{
PRELIMINARY CLINICAL EXPERIENCE WITH
} CALIFORNIUM-252 INTERSTITIAL SOURCES

\author{
Joseph R. Castro \\ Department of Radiotherapy \\ The University of Texas \\ M. D. Anderson Hospital and Tumor Institute \\ Houston, Texas
}

\begin{abstract}
Californium-252 fast neutron emitting interstitial sources were utilized in the treatment of locally advanced or widely disseminated hum an squamous cancers. A clinical RBE factor of 5 was utilized to convert the doses fron rads (neutron plus gamma) to rem. A percutaneously inserted nylon tubing system was utilized with dummy sources placed for radiographic localization, and the radioactive sources afterloaded behind special shielding after the patient had returned to his room. Dosage ranged from 4,000 rem in 40 to 100 hours by californium-252 alone to 7,500 rem in si $x$ weeks when combined with external irradiation. Although the tumor dosage has been lower than desirable because of difficulties with manual and computer dose calculations, most of the patients have had local control of their tumors. A controlled clinical trial will be necessary to compare the effectiveness of californium-252 to radium-226 for interstitial and intracavitary usage.
\end{abstract}

The use of fast neutrons in the treatment of cancer has been. considered for some time and attempts have been undertaken at two institutions $(1,2,3)$. The underlying rationale for fast neutron therapy has been succinctly stated by Brennan (4), that is, a reduction of the radiation resistance of anoxic tumor cells by irradiation with high L. E. T. (linear energy transfer) particles such as fast neutrons. Any advantage of a fast neutron emitting source such as californium 252 for intracavitary and interstitial use over that of radium 226, thus depends directly on its presumed lower oxygen enhancement ratio (5). The O, E. R. for californium 252 has been measured in various test systems $(\underline{5}, \underline{6})$, and appears to be in the range of 1.6. to 1.1 as compared to about 2.0 (5) for radium 226 utilized at clinical dose rates of approximately 40 rads per hour. Therefore, a small but possibly important advantage may be gained in using californium 252 sources instead of radium 226 .

This investigation was supported in part by Public Health Service Research Grant No. CA-06294-09 from the National Cancer Institute.
The clinical use of californium 252 for interstitial use in a pilot group of patients was undertaken at the M. D: Anderson Hospital and Tumor Institute starting in November, 1969. Following the suggestion of Atkins (7), a c?inical RBE of 5 was selected for comparing californium implants to radium 226 implants. This report will summarize our experience to date.

\section{PATIENT MATERIAL AND METHODS}

Between November, 1969 and December, 1970 , fifteen patients had interstitial implants with californium 252. All patients had advanced disease, either with distant metastases or massive local tumors in which poor vascularization and anoxic tumor cells were likely. Californium 25:2 implantation was utilized as the sole treatment in six patients, and in combination with external irradiation in nine patients. All patients had squamous carcinoma with the exception of one with basal cel carcinoma of the skin. The implanted tumor sites ranged from meiastatic lymphadenopathy in the inguinal region or neck to advanced vaginal tumors (Table I). All patients were followed until death or local failure. Frequent 
follow-up visits were held and appropriate photographs taken to document: 1) tumor response and 2) radiation reaction in surrounding normal structures.

The usual treatment planning employed with interstitial radium therapy was utilized except that a clinical RBE of 5 was employed to convert the californium 252 dose from rads to rem. However, no attempt was made to systematically utilize the same dose rates for californium 252 as would be indicated in radium therapy. A RBE of 1.0 was taken for the rad dose from external irradiation. The doses ranged from 4,000 rem in 40 to 100 hours with californium 252 alone to 7,500 rem in five and one half weeks, when combined with external irradiation. In these instances the patient usually received 5,000 rads in five weeks with Cobalt 60 irradiation or the 22 Mev betatron prior to californium 252 implant.

The technique of implantation was modified from that suggested by Pierquin for iridium 192 implants (8). Nylon or teflon tubes were inserted percutaneously through the tumor, following which californium 252 tubes of 1.5 or $3.0 \mathrm{~cm}$. length were afterloaded into the tubing after radiographic control films. Dummy sources were used for radiographic localization. The use of afterloading techniques provided ample time for leisurely and accurate placement of the nylon tubes throughout the tumor. In the case of vaginal implants hollow stainless steel needles were utilized instead of nylon tubes. General anesthesia was required for the placement of the tubes or needles. Loading of the californium 252 sources was ordinarily done in the patients room where specially constructed shields were available. In most instances the loading could be rapidly accomplished particularly if the implant was superficially located. In a few instances loading was difficult because of curvature of the nylon tubes and the lack of flexibility of the californium sources. This was particularly evident in intraoral implants, and further modifications of the afterloading system or source fabrication may be needed to obviate this difficulty. The possible use of californium 252 needles for direct implantation in the tongue and floor of the mouth has been suggested. This has the obvious disadvantage of working with the radioactive material while the implant is being performed.

Dosimetric data was obtained both by hand calculation, and by utilization of a special computer program. The selection of the minimum tumor volume was facilitated by the availability of computer drawn isodose curves, giving dose rates in rads per hour for the neutron component of the californium 252 dose. The gamma component could be calculated separately. Similar tumor volumes to that employed in radium 226 interstitial therapy were employed. Generally, absorbed dose rates were somewhat higher in californium therapy than the usual radium therapy rates.

Because of an error in dosimetry recalculation of several of the implants showed the dose to the target volume to be considerably ( 20\%) less than expected. This has been corrected but may explain some of the failures (Table I, patients $3,6,7$ ).

The types of implants included planar (single and double) and volume implants. Anatomic coverage of the tumor was paramount in placement of the sources. No special attempt was made to follow any distribution. rules or to increase the amount of californium on the periphery of the tumor.

Examples of the implants performed are shown in the following case illustrations:

Case 1: (MQ) This fifty-six year old female presented in October, 1969, with Stage IV squamous cell carcinoma of the cervix. Clinically involved nodes were palpable in the left inguinal area. The patient received palliative radiation to the pelvis. A biopsy of the left inguinal nodes was positive for metastatic squamous cell carcinoma. A single plane californium 252 implant (Figure 1A, B) of the left inguinal 
nodes was performed on November 13 , 1969, delivering an estimated 1,145 rads (neutron plus gamma) in 109 hours at $0.5 \mathrm{~cm}$. distance from the plane of sources by manual calculation. This corresponded to 5,720 rem using an RBE of 5. One month post implant there was dry desquamating epidermitis with several small areas of moist reaction (Figure 2 ). These healed within two months (Figure 3). The palpable nodes gradually receded and remained under control until the patient expired of distant metastases eight and one half months post implantation.

Case 2: (FM) This eighty-eight year old white male presented with a basal cell carcinoma of the right nasal labial fold. A central ulceration of $2.5 \mathrm{~cm}$. in diameter was present with palpable infiltration into the surrounding soft tissues of the cheek, nose and commissure of the mouth for a distance of $3 \mathrm{~cm}$.

(Figure 4). The patient was deemed in poor medical condition for an extensive surgical resection or a prolonged course of external irradiation. On July 8, 1970, a californium 252 single plane implant was performed delivering an estimated 1,340 rads (neutron plus gamma) in 67 hours (Figure 5). This corresponded to 6,700 rem to a slab of tissue of approximately $1.5 \mathrm{~cm}$. in thickness. Over the next two months the patient developed a confluent moist epidermitis which slowly cleared (Figure 6). The ulcer healed, and was replaced by thin atopic skin (Figure 7). However, five months post implantation several small areas of recurrent tumor were evident at the margin of the previously ulcerated area. Recalculation of the dosimetry showed an error in the original computer output. The neutron plus gamma dose to the tumor volume was only 770 rads $(\sim 4,000$ rem), which may account for the persistent tumor.

\section{DISCUSSION}

Table I summarizes the results in the initial fifteen patients treated with californium 252.

Several comments can be made on the basis of the glinical experience:

1. With careful afterloading techniques it is possible to perform reasonably geometric implants in most tumor sites while maintaining c. low exposure dose to the physician and other health personnel.

2. Due to the rigidity of the californium sources, techriques for intraoral implants providing geometric coverage comparable to radium needles have not yet been developed. A possible solution other than the use of radioactive californium needles would be the production of more flexible californium afterloading tubes or $0.5 \mathrm{~cm}$. length sources which could be linked in more flexible chairis.

3. Using a clinical RBE of 5 the local reactions have been roughly comparable to those obtained with radium 226 interstitial implantations.

4. Over the relatively short time of follow-up, no untoward late reactions have been detected.

5. At least five of fifteen patients have persistent or recurrent disease within the implanted area indicating that californium 252 is not a panacea. However, errors in dosimetry with underdosage undoubtedly contributed to three of the failures.

6. A sufficient number of patients have had local control within the implanted area despite the relatively low doses delivered, indicating that californium 252 can be an effective interstitial source for irradiating human tumors. 
7. A controlled clinical trial will be necessary to prove or disprove the greater effectiveness of californium 252 over that of gamma emitting sources such as radium 226 or iridium 192.

Further investigation into clinical dosimetry is needed to develop more accurate computer calculation of isodose curves around arrays of californium sources. This should take into account both the neutron and gamma component of the dose. In particular further investigation may be needed into the dosimetry about sources placed close to a hollow cavity or the surface of the body.

Further studies are also needed to verify the clinical RBE to be utilized. With the selected RBE of 5 utilized in the patients in this study, the doses given may have been somewhat lower than clinically desired.

The dose to the lens may be prohibitively high in implants near the eye. For example, in Case 2, an implant of the right cheek and nasolabial area delivered approximately 600 rem (RBE:10) to the right lens. Intraoral or neck implants deliver a much lower dose to the lens.

The possible use of californium 252 for intracavitary treatment such as for carcinoma of the uterine cervix and endometrium has been contemplated. Initial dosimetry studies have been performed around representative californium source distributions in standard intrauterine and vaginal applicators. A pilot study in which these sources would be used in lieu of radium as part of the treatment for advanced uterine cervical or endometrial tumors should be undertaken.

\section{CONCLUSIONS}

1. A controlled clinical trial will be needed to demonstrate. (or disprove) any advantage of californium 252 over radium 226.
2. Further attempts to improve afterloading techniques are needed to facilitate implantation.

3. Further investigation into dosimetry with computer generated isodose curves is needed.

\section{REFERENCES}

1. Andrews, J.R. "The potentialities for fast neutron beam radiotherapy." Amer. J. Rad. $93,190(1965)$

2. Bewley, D. K. "The fast neutron beam from the W. R. C. cyclotron." Brit. J.Rad. 42 , 281 (1969).

3. Stone, R. S., and Larkin, J.C., Jr. "Treatment of cancer with fast neutrons." Radiology. 39, 608 (1942).

4. Brennan, J. T. "Fast neutrons for radiation therapy."

Rad. Clinica North Amer. Vol. VII, No. 2, August (1969).

5. Hall, E. J., and Fairchild, R. G. "Radiobiological measurements with californium 252." Brit. J. Rad. 43, 263 (1970).

6. Bushong, S. C., Prasad, N. , Briney, S. A., and Oliver, G. D. "Radiocytogenetic determination of the oxygen enhancement ratio of californium 252." Radiology. $96,167(19,0)$.

7. Atkins, H. L., Fairchild, R. G., and Robertson, J. S. "Comparison of irradiation by $253 \mathrm{CF}$ and radium." Radiology. 96,161 (1970).

8. Pierquin, B. "Precis de Curietherapie Masson et Cie, Editeurs:" Paris (1964). 
TABLE I

Californium 252 Patients

\begin{tabular}{|c|c|c|c|c|c|}
\hline $\begin{array}{l}\mathrm{Pt} . \\
\text { No. }\end{array}$ & Tumor & $\begin{array}{l}\text { Type of } \\
\text { Implant }\end{array}$ & $\begin{array}{c}\text { External } \\
\text { XRT }\end{array}$ & $\begin{array}{c}\text { Calif. } 252 \\
\text { Dose }\end{array}$ & Results \\
\hline 1 & $\begin{array}{l}\text { Squamous ca cervix } \\
\text { metastatic to } \\
\text { inguinal nodes }\end{array}$ & $\begin{array}{l}\text { Single } \\
\text { Plane } \\
11 / 69\end{array}$ & & $\begin{array}{c}1145 \text { rads } / 109 \\
\text { hours }\end{array}$ & $\begin{array}{c}\text { Local control until } \\
\text { death at } 8 \frac{1}{2} \text { mos. }\end{array}$ \\
\hline 2 & Squamous ca vagina & $\begin{array}{r}\text { Volume } \\
2 / 70\end{array}$ & $5000 \mathrm{R}$ & $\begin{array}{l}480 \text { rads } / 25 \\
\text { hours }\end{array}$ & $\begin{array}{l}\text { Local control in } \\
\text { pelvis - DM at } \\
5 \text { months }\end{array}$ \\
\hline 3 & $\begin{array}{l}\text { Squamous ca cervix, } \\
\text { Stage III meta to } \\
\text { inguinal nodes }\end{array}$ & $\begin{array}{l}\text { Single } \\
\text { Plane } \\
3 / 70\end{array}$ & & $\begin{array}{l}750 \text { rads } / 100 \\
\text { hours }\end{array}$ & $\begin{array}{l}\text { Recurrent disease } \\
\text { at } 9 \text { months }\end{array}$ \\
\hline 4 & $\begin{array}{l}\text { Squamous ca lung meta } \\
\text { to } \mathrm{rt} \text { submandibular } \\
\text { node }(4.5 \times 3.5 \mathrm{x} \\
2.0 \mathrm{~cm} .)\end{array}$ & $\begin{array}{l}\text { Double } \\
\text { Plane } \\
\quad 4 / 70\end{array}$ & & $\begin{array}{l}440 \text { rads } / 40 \\
\text { hours }\end{array}$ & $\begin{array}{l}\text { Expired } 12 \text { days } \\
\text { post implant of } \\
\text { CVA - ? DM (brain) }\end{array}$ \\
\hline 5 & $\begin{array}{l}\text { Squamous ca vagina } \\
\text { Stage III }\end{array}$ & $\begin{array}{r}\text { Volume } \\
5 / 70\end{array}$ & $5000 \mathrm{R}$ & $\begin{array}{l}187 \mathrm{rads} / 24 \\
\text { hours }\end{array}$ & NED at 7 months \\
\hline 6 & $\begin{array}{l}\text { Recurrent squamous } \\
\text { ca lower lip, post } \\
\text { surgery }\end{array}$ & $\begin{array}{l}\text { Single } \\
\text { Plane } \\
6 / 70\end{array}$ & $5000 \mathrm{R}$ & $\begin{array}{l}360 \text { rads/30 } \\
\text { hours }\end{array}$ & $\begin{array}{l}\text { Local failure at } \\
2 \text { months }\end{array}$ \\
\hline 7 & $\begin{array}{l}\text { Basal ca right } \\
\text { nasolabial fold }\end{array}$ & Planar & & $\begin{array}{l}770 \text { rads } / \zeta 7 \\
\text { hours }\end{array}$ & $\begin{array}{l}\text { Moist reaction - } \\
\text { healed at } 2 \text { mos. } \\
- \text { recurrence at } \\
5 \text { months }\end{array}$ \\
\hline 8 & $\begin{array}{l}\text { Squamous ca lung } \\
\text { with skin nodules }\end{array}$ & $\begin{array}{r}\text { Planar } \\
7 / 70\end{array}$ & & $\begin{array}{l}1410 \text { rads } / 70.5 \\
\text { hours }\end{array}$ & $\begin{array}{l}\text { Expired from DM at } \\
6 \text { weeks } \\
\text { Local lesions much } \\
\text { smaller - no } \\
\text { moist skin reaction }\end{array}$ \\
\hline 9 & $\begin{array}{l}\text { Squamous ca upper } \\
\text { lip }\end{array}$ & $\begin{array}{l}\text { Single } \\
\text { Plane } \\
7 / 70\end{array}$ & $5000 \mathrm{R}$ & $\begin{array}{l}377 \text { rads } / 26 \\
\text { hours }\end{array}$ & $\begin{array}{l}\text { Local control - } 5 \\
\text { months }\end{array}$ \\
\hline 10 & Squamous ca vagina & $\begin{array}{r}\text { Volume } \\
8 / 70\end{array}$ & $5000 R$ & $\begin{array}{l}255 \text { rads } / 51 \\
\text { hours }\end{array}$ & $\begin{array}{l}\text { Local control - } 2 \\
\text { months }\end{array}$ \\
\hline 11 & $\begin{array}{l}\text { Squamous ca vagina } \\
\text { Stage III }\end{array}$ & $\begin{array}{r}\text { Volume } \\
9 / 70\end{array}$ & $\begin{array}{l}5000 \mathrm{R}+ \\
1000 \text { para }\end{array}$ & $\begin{array}{l}300 \text { rads } / 25 \\
\text { hours }\end{array}$ & $\begin{array}{l}\text { Local control - } 2 \\
\text { months }\end{array}$ \\
\hline 12 & $\begin{array}{l}\text { Squamous ca cervix } \\
\text { meta to distal } \\
\text { urethra }\end{array}$ & $\begin{array}{r}\text { Volume } \\
9 / 20\end{array}$ & $\begin{array}{c}3000 \mathrm{R} \\
\text { (Trans-Vag) }\end{array}$ & $\begin{array}{l}550 \text { rads } / 69 \\
\text { hours }\end{array}$ & $\begin{array}{l}\text { Local control until } \\
\text { death CVA at } 2 \\
\text { months }\end{array}$ \\
\hline 13 & $\begin{array}{l}\text { Recurrent squamous } \\
\text { ca (lip) in neck - } \\
\text { post XRT }\end{array}$ & $\begin{array}{l}\text { Planar } \\
10 / 70\end{array}$ & & $\begin{array}{l}788 \text { rads } / 65.7 \\
\text { hours }\end{array}$ & $\begin{array}{l}\text { Partial tumor } \\
\text { regression - } 2 \\
\text { months }\end{array}$ \\
\hline 14 & $\begin{array}{l}\text { Squamous ca tongue } \\
\mathrm{T}_{3} \mathrm{~N}_{0} \text { (2nd primary) }\end{array}$ & $\begin{array}{r}\text { Volume } \\
10 / 70\end{array}$ & $\begin{array}{l}2500 \mathrm{R} / 3 \\
\text { Fractions }\end{array}$ & $\begin{array}{l}773 \mathrm{rads} / 70.3 \\
\text { hours }\end{array}$ & $\begin{array}{l}\text { Persistent tumor } \\
\text { (Dead) }\end{array}$ \\
\hline 15 & Squamous ca tongue & $\begin{array}{r}\text { Volume } \\
11 / 70\end{array}$ & $5500 \mathrm{R}$ & $\begin{array}{l}540 \mathrm{rads} / 67 \\
\text { hours }\end{array}$ & Presistent tumor \\
\hline
\end{tabular}




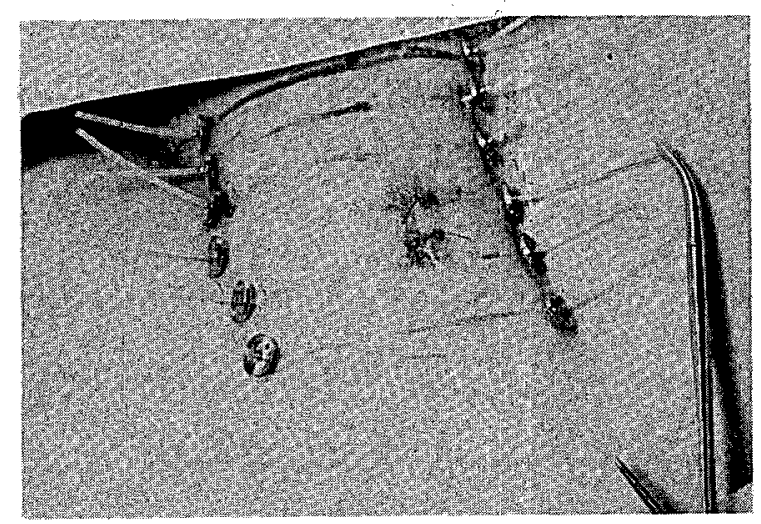

Figure 1A: Left inguinal node implant (Case 1).

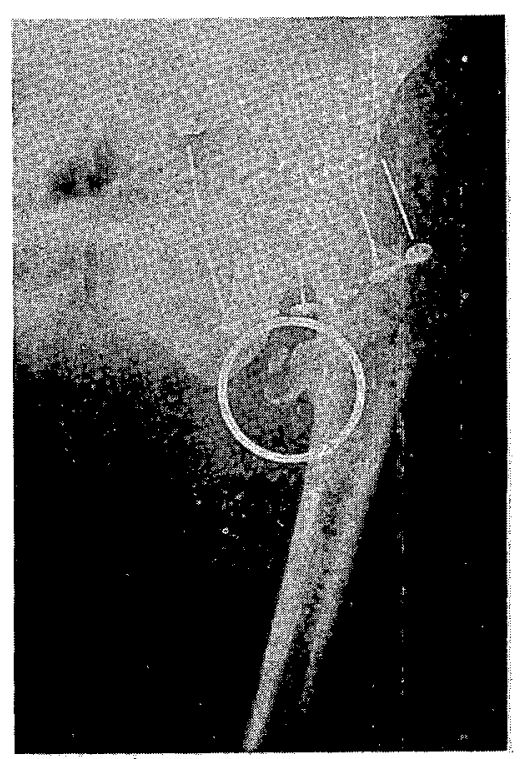

Figure 1B: Radiograph of implant.

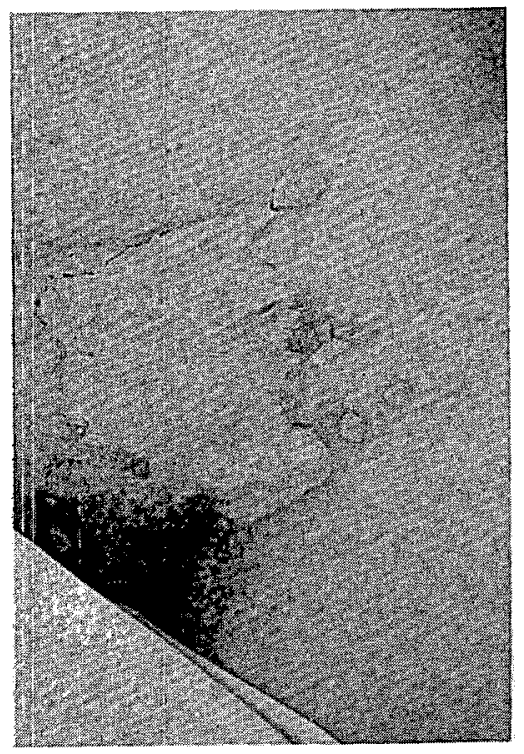

Figure 2: Dry desquamating epidermitis with some moist areas one month post implantation.

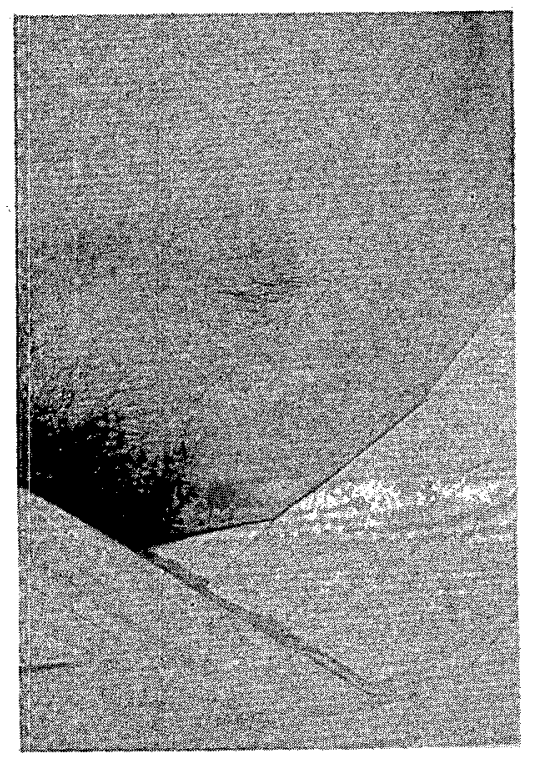

Figure 3: Healing of skin reaction about two months post implant. 


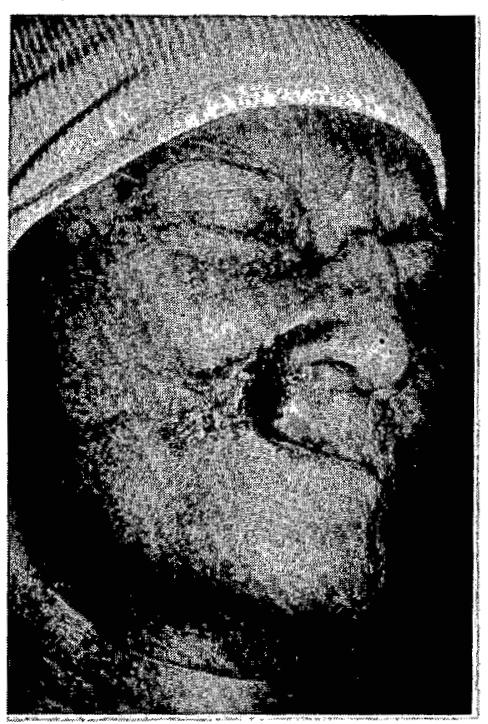

Figure 4: Basal cell carcinoma of nasolabial fold in an 88 year old man (Case 2).

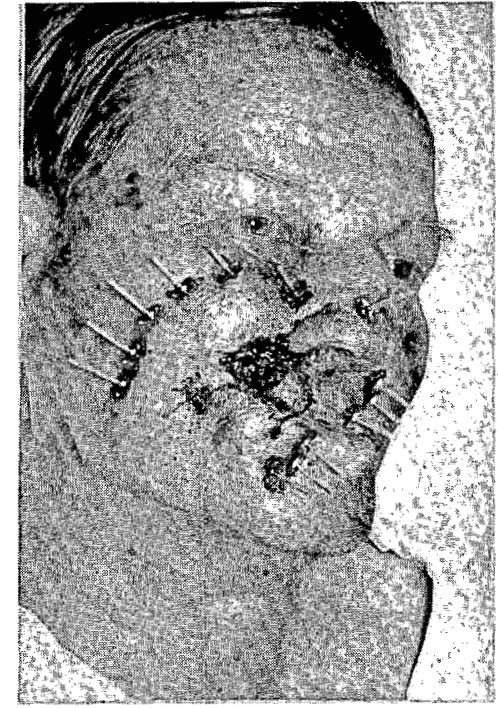

Figure 5: Planar implant in Case 2. Note close proximity of sources to lens of right eye. Bilateral cataracts were present prior to irradiation.

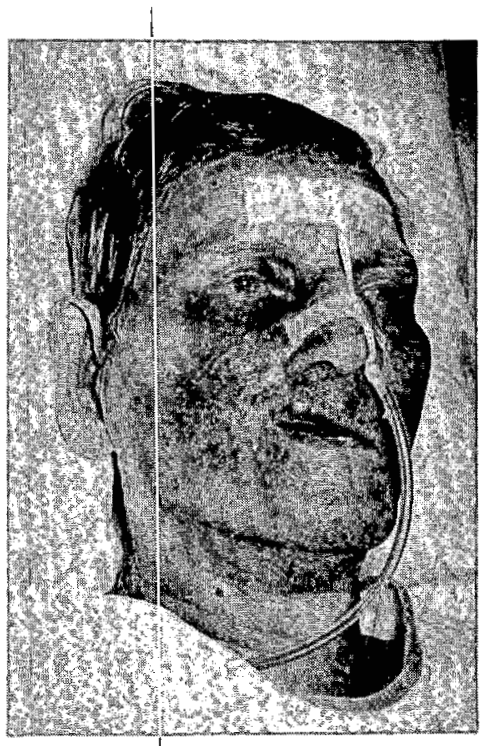

Figure 6: Moist epidermitis at 6 weeks post implant.

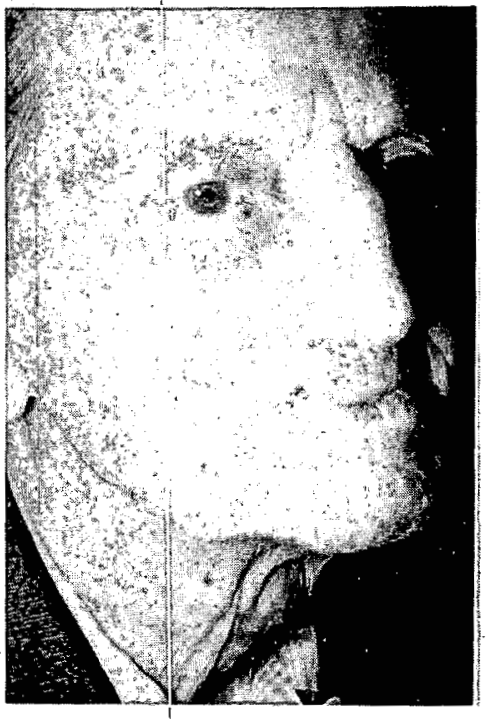

Figure 7: Healing of skin reaction and ulcer at 4 imonths. Recurrent nodule is present in upper lip at margin of implant. 


\title{
A CYTOGENETIC DETERMINATION OF THE OER AND RBE OF CALIFORNIUM-252
}

\author{
Stewart C. Bushong, Naresh Prasad, and Sharon A. Briney \\ Baylor College of Medicine \\ Houston, Texas \\ George Oliver
}

M. D. Anderson Hospital and Tumor. Institute

Houston, Texas

The RBE and OER for californium-252 have been determined on the basis of chromosome damage in Chinese hamster ovary cells. The cells were cultured in vitro and were suspended in a $12 \mathrm{~mm}$ diameter tube in McCoy's medium without serum for irradiation. The tube was positioned in a polystyrene irradiation jig with either californium or radium sources located concentrically at a distance of $9 \mathrm{~mm}$ from source center to cell suspension center. The californium source loading of $46 \mu \mathrm{g}$ resulted in a dose rate of $60.4 \mathrm{rads} / \mathrm{hr}$ with a neutron-to-gamma ratio of 1.41 . The radium dose rate of $57.8 \mathrm{rads} / \mathrm{hr}$ resulted from a source loading of $40 \mathrm{mg}$. During irradiation the cell suspension was aerated with either oxygen or nitrogen containing less than ten ppm oxygen contamination. Following irradiation the cells were placed in tissue culture flasks in fresh medium and incubated for ten hours. Colchicine was added to a concentration of $1 \mu \mathrm{g} / \mathrm{ml}$ and incubated for an additional two hours. The cells were harvested, cytogenetic slides were prepared, and the slides were scanned for chromosome aberrations. Statistical analysis of the single-hit chromosome aberration frequency resulted in linear regression equations. The ratio of the dose coefficients of these equations showed an RBE of 3.48 under aerobic conditions and 4.61 under anaerobic conditions. 'The OER for the radium exposures was 2.32 while that for the californium exposures was 1.75 . A therapeutic gain.factor. of 1.33 wäs demoristrated. Experiments are in progress at higher dose rates in or der to extend the total dose range of this study.

\section{INTRODUCTION}

The application of Californium -252 (Cf-252) as a substitute for radium in certain radiotherapy applications will depend in large measure upon its radiobiological properties. The physical properties of $\mathrm{Cf}-252$ are well known and have been adequately documented $(1-3)$. The radiobiological properties of $\mathrm{Cf}-252$ are just now being elucidated.

Two very important radiobiological characteristics are the relative biological effectiveness (RBE) and the oxygen enhancement ratio (OER). The RBE is the ratio of the dose of standard radiation to the dose of experimental radiation necessary to produce the same level of biological effect. Similarly, the OER is the ratio of the dose from a given radiation source administered under. hypoxic conditions to the dose administered under oxic conditions necessary to produce the same level of biological effect.

This paper reports the results of experiments designed to estimate the RBE and the OER for $\mathrm{Cf}-252$, the experimental radiation source. Radium is the standard radiation source. The estimates are based on chromosome damage in mammalian cells cultured and irradiated in vitro.

\section{METHODS AND MATERIALS}

Chinese hamster ovary cells were: used in all experiments. The cells were serially cultured in our laboratory according to standard methodology that has been reported elsewhere (4). Prior to irradiation, the cells were suspended in Puck's saline solution $A$, and the suspension was aerated for 15 minutes with either oxygen or pre-purified nitrogen. The pre-purified nitrogen contained less than 10 ppm of oxygen contamination.

During irradiation, the tube containing the cell suspension was placed in a cubical polystyrene phantom $30 \mathrm{~cm}$ on a side. The radiation sources were in the form of intracavitary tubes and were positioned concentrically around the cell suspension. The Cf-252 irradiations were limited by the quantity of material available, $40 \mu \mathrm{gms}$. The radium was loaded in an effort to match the total Cf -252 dose rate and 50 mgms were required for this purpose.

Gamma radiation dosimetry was performed with lithium flouride thermoluminescence dosimeters, and these 
measurements resulted in dose rates of $57.8 \mathrm{rads} / \mathrm{hr}$ from the radium and 25.1 rads/hr from the Cf -252 loading. Neutron dosimetry was performed with solid-state devices that have been previously described (5), and these measurements gave a neutron do se component of the Cf- 252 of 35.3 rads/hr. The resulting total dose rate during Cf- 252 irradiation was $60.1 \mathrm{rads} / \mathrm{hr}$, and the neutron to gamma ratio was approximately 1.41 to 1.0 .

Following irradiation, the cells were removed from suspension and resuspended in fresh McCoy's medium with $20 \%$ fetal calf serum added. At ten hours postirradiation colchicine was added to a final concentration of $1.0 \mu \mathrm{gm} / \mathrm{ml}$, and the cells were incubated for an additional two hours. This time insured that only first division, post-irradiation mitoses would be observed. Cytogenetic preparations were made using a modification of standard techniques and this methodology has been reported (6).

Microscopic evaluation of the prepared chromosome slides was performed in order to determine the numbers of chromosomes per cell and the numbers and types of chromosome aberrations from each wellspread mitotic igure. Isochromatid breaks and terminal deletions were scored as single-hit type chromosome aberrations while rings, dicentrics, and polycentric chromosomes were identified as multi-hit type aberrations. Chromatid gaps were not considered to be true aberrations unless the portion of the chromatid distal from the centromere was clearly out of place.

\section{RESULTS}

Tables 1 and 2 show the results of the cell irradiations from radium and $C f-252$, respectively. fin attempt was made to analyze at least 100 cells per radiation dose point. The data shown on Table $l$ is the result of three repetitive experiments while that for Table 2 resulted from four separate experiments. The chromosome aberration frequency as employed in this text and in the Tiables is determined by

Table 1

Chromosome Aberrations Induced in Chinese Hamster Ovary Cells by Radium Radiation (57.8 rads!/hour)

\begin{tabular}{|c|c|c|c|c|c|c|}
\hline \multirow[b]{2}{*}{$\begin{array}{l}\text { Dose } \\
\text { (rads) } \\
\end{array}$} & \multicolumn{3}{|c|}{ Aerobic } & \multicolumn{3}{|c|}{ Anaerobic } \\
\hline & $\begin{array}{c}\text { Cells } \\
\text { Examined }\end{array}$ & $\begin{array}{c}\text { Single-Hit } \\
\text { Aberrations } \\
\text { Per Cell } \\
\end{array}$ & $\begin{array}{c}\text { Multi-Hit } \\
\text { Aberrations } \\
\text { Per Cell } \\
\end{array}$ & $\begin{array}{c}\text { Cells } \\
\text { Examined } \\
\end{array}$ & $\begin{array}{c}\text { Single-Hit } \\
\text { Aberrations } \\
\text { Per Cell } \\
\end{array}$ & $\begin{array}{c}\text { Multi-Fit } \\
\text { Aberrations } \\
\text { Per Cell }\end{array}$ \\
\hline 0 & 200 & 0.23 & 0.04 & 200 & 0.18 & 0.02 \\
\hline 29 & 85 & $0: 18$ & 0.00 & 100 & 0.27 & 0.03 \\
\hline 43 & -- & --- & $\ldots$ & 100 & 0.22 & 0.02 \\
\hline 58 & 100 & 0.32 & 0.03 & 100 & 0.31 & 0.01 \\
\hline 87 & 100 & 0.60 & 0.05 & 100 & 0.29 & 0.02 \\
\hline 101 & 100 & 0.56 & 0.08 & 100 & 0.31 & 0.03 \\
\hline 116 & 100 & 0.65 & 0.12 & 100 & 0.43 & 0.04 \\
\hline
\end{tabular}


Table 2

Chromosome Aberrations Induced in Chinese Hamster Ovary Cells by Cf -252 Radiation (60. 4 rads/hour)

\begin{tabular}{|c|c|c|c|c|c|c|}
\hline \multirow[b]{2}{*}{$\begin{array}{l}\text { Dose } \\
\text { (rads) }\end{array}$} & \multicolumn{3}{|c|}{ Aerobic } & \multicolumn{3}{|c|}{ Anaerobic } \\
\hline & $\begin{array}{c}\text { Cells } \\
\text { Examined } \\
\end{array}$ & $\begin{array}{c}\text { Single-Hit } \\
\text { Aberrations } \\
\text { Per Cell } \\
\end{array}$ & $\begin{array}{c}\text { Multi-Hit } \\
\text { Aberrations } \\
\text { Per Cell } \\
\end{array}$ & $\begin{array}{c}\text { Cells } \\
\text { Examined } \\
\end{array}$ & $\begin{array}{c}\text { Single-Hit } \\
\text { Aberrations } \\
\text { Per Cell } \\
\end{array}$ & $\begin{array}{l}\text { Multi-Hit } \\
\text { Aberrations } \\
\text { Per Cell } \\
\end{array}$ \\
\hline 0 & 200 & 0.07 & 0.01 & 200 & 0.07 & 0.01 \\
\hline 15 & 100 & 0.31 & 0.16 & 100 & 0.29 & 0.05 \\
\hline 22 & -- & $\ldots$ & ---- & 100 & 0.42 & 0.09 \\
\hline 24 & 100 & 0.46 & 0.14 & $-\ldots$ & $-\ldots$ & $\ldots$ \\
\hline 30 & 67 & 0.55 & 0.12 & $-\cdots$ & --- & --- \\
\hline 35 & -- & -- & --- & 100 & 0.46 & 0.21 \\
\hline 45 & 100 & 0.71 & 0.16 & 100 & 0.64 & 0.17 \\
\hline 61 & 100 & 0.98 & 0.27 & 100 & 0.57 & 0.09 \\
\hline 90 & 100 & 1.26 & 0.50 & 100 & 0.77 & 0.22 \\
\hline
\end{tabular}

dividing the total number of chromosome aberrations observed at a given radiation dose by the total number of cells analyzed and therefore, it has units of aberrations per cell.

The single-hit type aberration data is plotted in Figures 1 and 2 for the radium and the Cf- 252 irradiations, respectively. The points on the graph are the average aberration frequency values reported in Tables 1 and 2 , and the lines are the result of a least squares regression analysis on each set of data. The actual aberration frequencies observed with the control cells were given equal weight to those observed with the irradiated cells, and therefore the regression line does not always intersect the ordinate at precisely the observed aberration frequency level.

The resulting regression equations from the single-hit type data are listed in Table 3. Analysis of the multi-hit

Table 3

Regression Equations from the Single-Hit Chromosome Aberration Data of Tables 1 and 2

\begin{tabular}{l|cc|c} 
& $\begin{array}{c}\text { Radium } \\
(57.8 \mathrm{rads} / \text { hour })\end{array}$ & $\begin{array}{c}\text { Californium } \\
(60.4 \mathrm{rads} / \mathrm{hour})\end{array}$ & $\mathrm{RBE}$ \\
\hline & $\mathrm{Y}=0.181+0.00388 \mathrm{D}$ & $\mathrm{Y}=0.103+0.0135 \mathrm{D}$ & 3.48 \\
Aerobic & $Y=0.183+0.00167 \mathrm{D}$ & $\mathrm{Y}=0.153+0.0077 \mathrm{D}$ & 4.61 \\
\hline Anaerobic & $Y=1.75$ & $\mathrm{TGF}=1.33$
\end{tabular}




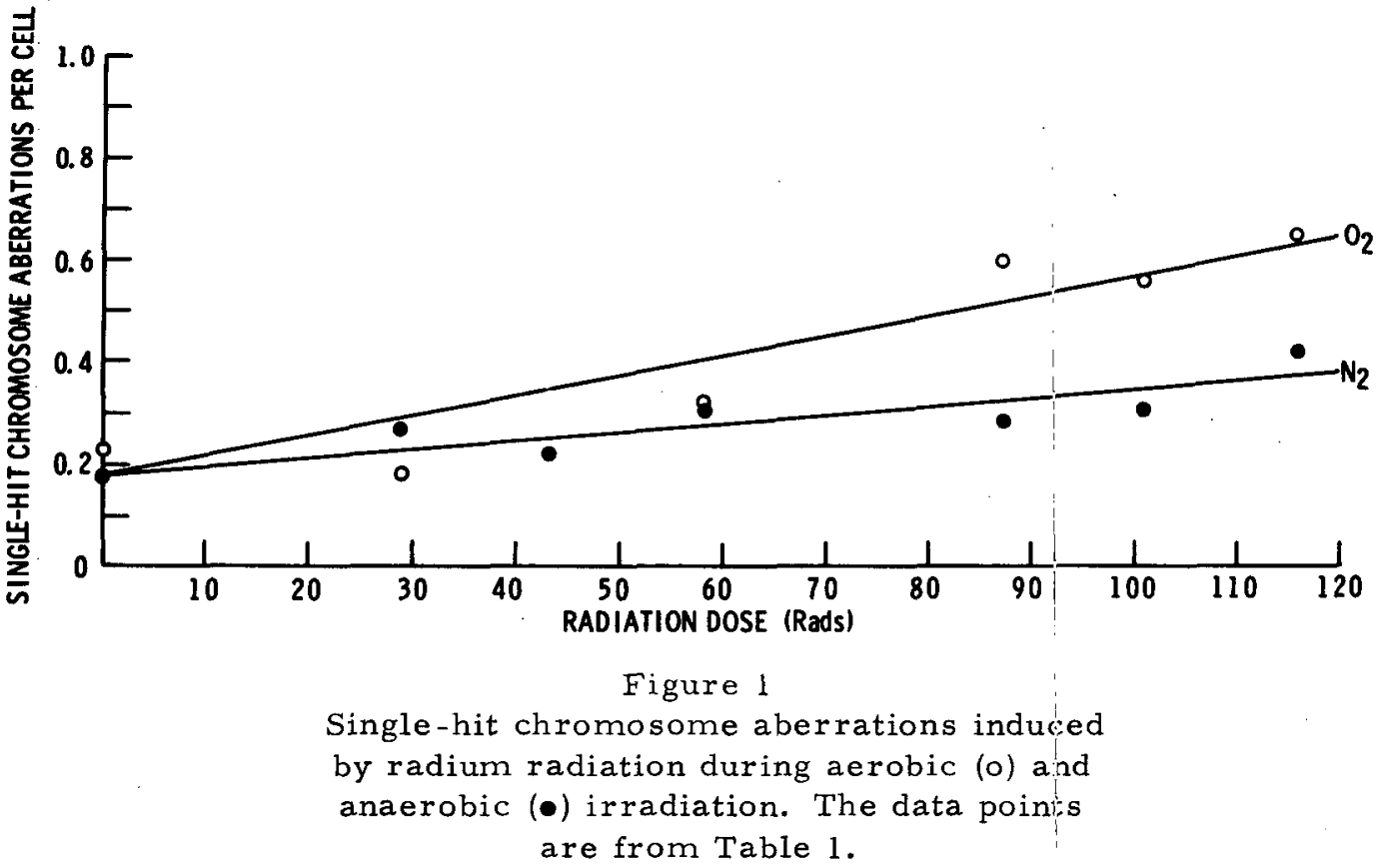

aberrations was not possible because of the low frequency of aberrations observed which in turn was apparently due to the small quantity of Cf- 252 that was available for these experiments.

Since the dose-response relationships exhibited in Table 3 are linear, the RBE and OER are simply the ratio of the dose coefficients selected appropriately. The results of this type of analysis are also shown in Table 3 . As noted, the RBE for Cf-252 varies from 3.48 to 4.61 depending upon the degree of oxygenation. The OER for Californium is shown to be 1.75 while that for radium was 2.32 .

\section{DISC USSION}

The RBE observed in these experiments for the Cf -252 irradiations was 3.48 and 4. 61 for the oxygenated and anoxic conditions, respectively. RBE as determined for other neutron sources at various dose rates and with different biological endpoints have been shown to lie in the range of approximately 2 to 10 . Examples of some relevant determinations of neutron RBE are shown in Table 4.

That the RBE is higher for an hypoxic system than for the oxygenated system is well established. The degree of increase is not, however, and the results reported here show a substantial increase in RBE for hypoxic cells irradiated with $\mathrm{Cf}-252$.

The OER reported for most photon radiation is in the range of 2 to 4 . Our finding of a value of 2.32 from radium gamma rays delivered at a rate of 57.8 $\mathrm{rads} / \mathrm{hr}$ is somevhat lower than most but in general agreernent with other data. We found an OER of 1.75 from our Cf- 252 irradiation system, and this value, is in line with what has been reported for other neutron sources. Values of OER ranging from approximately 1.2 to 2.0 have been reported for fast neutron irradiations, and some of these experiments are referred to in Table 5. It should be noted, however, that our finding of 1.75 reflects both the neutron and photon contribution from Cf-252.

The therapeutic gain factor (TGF) has been described as the anticipated increase 


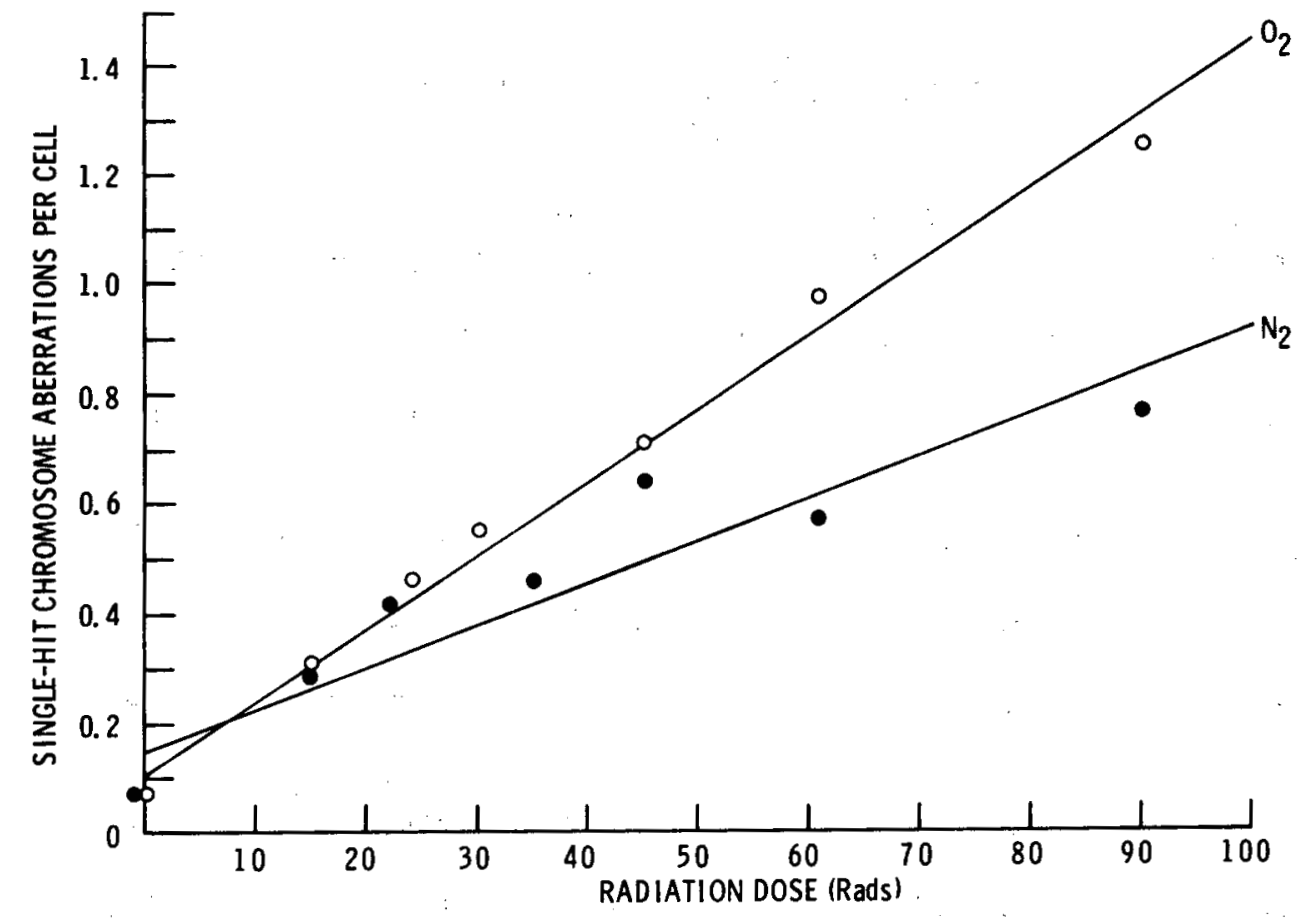

Figure 2

Single-hit chromosome aberrations induced by $\mathrm{Cf}-252$ radiation during aerobic (o) and anaerobic ( ) irradiation. The data points are from Table 2 .

Table 4

The Relative Biological Effectiveness of Californium-252 Radiation Compared to Radium

Dose Rate $(\mathrm{rads} / \mathrm{hr})$

16

16

12.5

43.5
Biological Response

He La cell survival Vicia faba root growth pig skin damage crypt cell survival

\section{$\underline{\mathrm{RBE}}$}

2. 86

5.3 to 6.5

4. 9

4. 9
Investigator

Fairchild

Hall

Atkins

Withers
Reference

7

8

9

10




\section{Table 5}

\section{THE OXYGEN ENHANCEMENT RATIC OF FAST NEUTRONS}

Neutron Source/

Average Energy

\section{$3 \mathrm{MeV}$ \\ $6 \mathrm{MeV}$}

$6 \mathrm{MeV}$

$6 \mathrm{MeV}$

$\operatorname{Be}(d, n)$

$14 \mathrm{MeV}$

$15 \mathrm{MeV}$

$6 \mathrm{MeV}$

Cf -252

\author{
Biological \\ Response
}

Vicia Faba root growth

Ascites tumor cell survival

RIB5 tumor growth

E Coli survival

human kidney cell survival

barley root growth

human kidney cell survival

Ascites tumor cell survival

Vicia Faba root growth
Neutron OER

1. 4

1. 8

1.3

Thornlinson

2.03

1.6

Alper

Barendsen

1. 92

Cercek

1.6

Broerse

1. 80 McNally

1.66

Hall
15 16

17

18

11

12

13

4

5

19 in efficacy of the experimental radiation due to the differential response of normal oxygenated tissue to hypoxic tumor tissue. The TGF can be computed from the ratio of the OERs or the RBEs as shown in Table 3. Our finding of a TGF of 1.33 indicates that Cf -252 may be $33 \%$ more effective than radium in the treatment of hypoxic tumor tissue. This gain is marginal and not as encouraging as one would like.

The justification for employing the dose coefficients from the linear dose-response relations in the computation of $\mathrm{RBE}$ and OER is based upon the fact that the intersection between the regression line and the ordinate is essentially constant. Were this intersection widely different, then the RBE and the OER would vary in magnitude according to the radiation dose delivered. This phenomenon is evident in many cell survival studies| where the extrapolation numbers are greatly different and it generally result's in a higher RBE and a higher OER with increasing radiation dose. Such was not the case in our experiments.

The RBE as defined for these studies is the dose ratio of both the standard and experimental radiation delivered at the same physical dose rate. This experimental design was all that could be accomplished with the radioactive material available. Further studies are planned such that the radiation dose required to produce a specified effect is delivered in the same period of time with both types of radiation. In other words, the estimate of 
$\mathrm{RBE}$ reported here will be incorporated into future experiments.

\section{REFERENCES}

1. S. G. Thompson, K. Street, Jr., A. Shiorso, et al. "The New Element Californium (Atomic Number 98)." Phys. Rev. 80, 790 (1950).

2. D. H. Stoddard. Radiation Properties of Californium-252. USAEC Report DP-986, E.I. du Pont de Nemours and Co., Savannah River Laboratory, Aiken, S. D. (1965).

3. P. R. Fields, H. Diamond. Californium-252, A Primary Standard for Neutrons. Neutron Dosimetry Symposium Proceedings, Harwell, Dec. 10-14, IAEA, Vienna, 581 (1963).

4. S. C. Bushong, N. Prasad, S. A. Briney, G. D. Oliver. "Radiocytogenetic Determination of the Oxygen Enhancement Ratio of Californium-252." Radiology 96:l, 167 (1970).

5. G. D. Oliver, Jr., C. N. Wright. "Dosimetry of an Implantable $252 \mathrm{Cf}$ Source." Radiology 92, 143 (1969).

6. S. C. Bushong, J. A. Watson, N. Wald. "The Oxygen Effect Based on Chromosome Damage in HeLa Cells." Int. J. Radiat. Biol. 13, 249 (1967).

7. R. G. Fairchild, R. M. Drew and H. I. Atkins. "The Relative Biological Effect of Cf-252 on HeLa Cells in Culture." Radiology 93, 1187 (1969)

8. Eric J. Hall and Ralph G. Fairchild. "Radiobiological Measurements with Californium-252." Brit. J. Radiol. 43, 263 (1970).
9. H. L. Atkins, R. G. Fairchild, and J. S. Robertson. "Comparison of Irradiation by Californium-252 and Radium on the Skin of Swine." Radiology 96, 161 (1970).

10. M. R. Withers, G. D, Oliver and D. W. Glenn. "Response of Mouse Jejunal Crypt Cells to Low Dose Rate Ir radiation with Californium Neutrons or Radium Gamma-rays." (to be published).

11. G. J. Neary, S. M. Tonkinson, and F. S. Williamson. "The Relative Biological Efficiency of Single Doses of Fast Neutrons and Gamma Rays on Vicia Faba Roots and the Effect of Oxygen." Int. J. Rad. Biol. 1, 201 (1959).

12. S. Hornsey and G. Silini. "Studies on Cell - Survival of Irradiated Ehrlich Ascites Tumour. "Int. Journ. Rad. Biol. 4: 2, 135 (1961).

13. R. Thomlinson. "A Comparison of Fast Neutrons and $X$-rays in the Relation to the Oxygen Effect in Experimental Tumours in Rats" Brit. J. Rad. 36, 89 (1963).

14. T. Alper. "Comparison Between the Oxygen Enhancement Ratios for Neutrons and $X$-rays as Observed with $\mathrm{E}$ coli B." ${ }^{11}$ Brit. J. Rad. 36, 97 (1963).

15. G. W. Barendsen, C. J. Koot, G. R. Van Kersen. "The Effect of Oxygen on Impairment of the Proliferative Capacity of Human Cells in Culture b Ionizing Radiations of Different LET." Int. J. Rad. Biol. 10, 317 (1966).

16. L. Cercek, M. Ebert, and D. Green. "RBE, OER, and Dose-rate Effects with $14 \mathrm{MeV}$ Neutrons Relative to $300 \mathrm{kVp} \mathrm{X}$-rays in Barley Roots." Int. J. Radiat. Biol. , 14:5, 453 (1968). 
17. J. J. Broerse, G. W. Barendsen. "Survival of Cultured Human Cells After Irradiation with Fast Neutrons of Different Energies in Hypoxic and Oxygenated Condition." Int. J. Rad. Biol. 13, 559. (1968).

18. N. J. McNally and D. K. Brewley. "A
Biological Dosimeter Using Mammalian Cells in Tissue Culture and Its Use in Obtaining Neutron Depth Dose Curves." Bt. J. Radio1. 42, 239 (1969).

19. E. J. Hall. "Cell Killing at very Low Dose Rates!' BNL-50203 (C57) $308 \cdot(1970)$. 


\title{
INTRODUCTION TO MEDICAL NEUTROGRAPHY
}

\section{Roland Buchet}

Professeur Au College De Medecine

Radiologiste Des Hopitaux De Paris

\begin{abstract}
This paper is a state of the art report on the medical applications of neutron radiography. It reviews the physical principles involved, the neutron sources suitable for radiography, and the techniques used for detecting and recording neutron images. It considers the distribution of elements within tissues, the role of hydrogen, and the constraints imposed by radiation hazards, patient tolerance, and conventional pathological specimen preparation techniques. Finally, it suggests a research approach for determining and expanding the limits and possibilities of medical neutron radiography.
\end{abstract}

The paper reviews the pertinent literature and concludes that medical neutron radiography will probably find a place among other medical graphic techniques which improve and enlarge the framework of conventional radiography.

This report concerns a process for obtaining an image of an object from a neutron flux (figures 1, 2, and 3). We will not discuss what could be called. "neutrometry," the process which would give numeric results by measuring the neutron flux using a detector at the end of the object examined.

$$
* * * * * * *
$$

Before considering the means of recording such an image, for a better understanding of the problem we should review briefly the details of neutronmatter interaction; this interaction determining the difference between neutron radiography and conventional radiography (X or ganma).

The particle used, the neutron, is one of the particles making up the nucleus of the atom. Electrically neutral, its mass is almost that of a proton (1850 times that

We wish to express our appreciation to: M. Jean Debiesse, Director of Centre d'Etudes Atomiques of Saclay, who has so kindly opened the doors of the Center for us; MM. Boutaine and Farny of the Department of experimental piles of the C.E.A., who have communicated to us information on industrial neutron radiography; Pr Dutreix and Dr. Vallée, our two colleague-friends who have helped us by their authoritative information. of an electron). Its interaction with traversed tissues is not the same as that of $X$ or gamma photons with the same tissues. --The latter takes place within the electronic cloud around the atom. The photon can either be absorbed by removing an electron-photo-electric effect or by undergoing a change of direction and loss of energy by collision with a projected electron (the "Compton Effect").

-For a neutron traversing the substance or tissue, interaction takes place in the nucleus of the atom and can undergo:

a) Either capture, that is true absorption of the neutron by the encountered nucleus. The neutron which does not possess an electric charge is not stopped by the "potentia 1 barrier" of the atomic nucleus. The new nucleus thus produced can be stable or unstable. In the latter case, where the nucleus is "excited," it can emit either a proton $(n, p)$, or an alpha particle $(n, \alpha)$, or more often a simple gamma photon (this $n, y$ capture leads to an isotope of the collision nucleus).* of course, the chance of such capture depends on the nature of the target nuclei which has more or less affinity for neutrons. There are, for example, 60,000 more chances of such capture

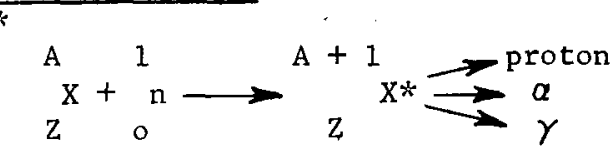

TRANSLATED FROM THE JOURNAL DE II-3RADIOLOGIE D`ELECTROLGIE ET DE MEDECINE NUCLEAIRE $51.269(1970)$. 

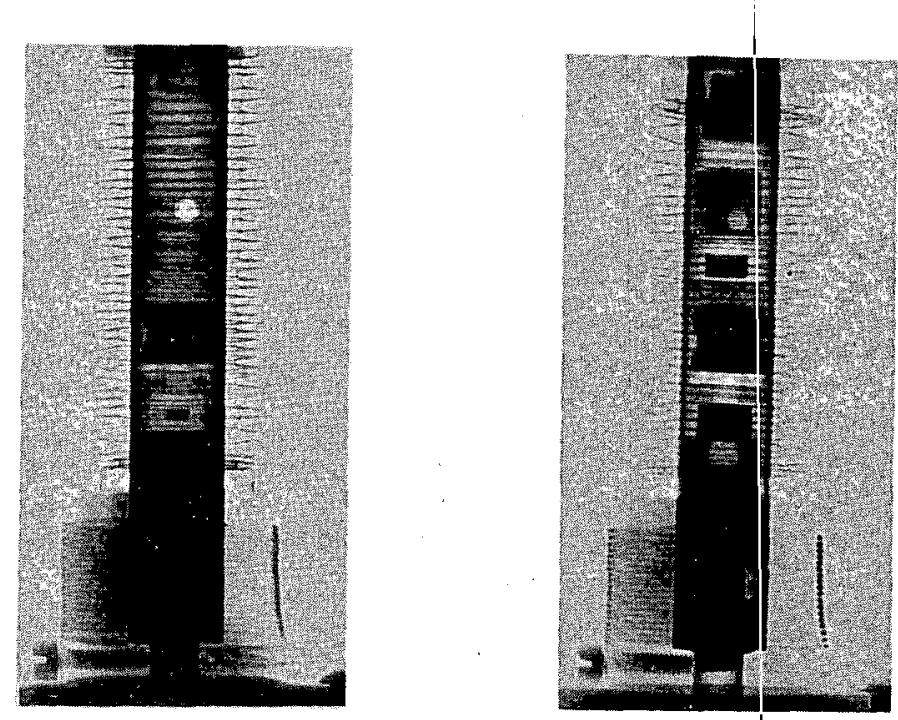

Fig. 1--Example of industrial neutron radiography. Comparison of an assembly of different parts by radiography (left) and neutron radiography (right).

Note the clarity of information on the neutron radiographic plate as compared with conventional radiography: visibility of the glycerine cup and at the lower part of the assembly, the vinyl tubes (courtesy of Frany and LaPorte, Experimental Reactor Dept., C.E.A.).

by a cadmium nucleus than by a light hydrogen nucleus and 700 times more for a light hydrogen nucleus than a heavy hydrogen nucleus.

b) Or elastic collision: neutron and nucleus act as two elastic balls which bounce off each other. While there is not a "nuclear reaction," this collision nevertheless produces two routine effects: one on the path of the neutron which is deflected and the other, a loss of energy.

At energies less than $20 \mathrm{MeV}$, interactions are caused essentially by elastic collisions on the nuclei of atoms.

When this energy goes below $20 \mathrm{KeV}$, the neutron is said to be in the "epithermal" region. Elastic collision remains then the dominant elementary interaction, but the other phenomenon of capture by the nuclei is also present.

This phenomenon is of practical importance only in the so-called "thermal" region (neutrons whose kinetic energy corresponds to that of thermal excitation) $(0.025 \mathrm{eV})$ (Table I).
TABLE I

Different Neutron Flux

\begin{tabular}{l|c|c}
\hline \multirow{2}{*}{$\begin{array}{c}\text { Neutron } \\
\text { Classification }\end{array}$} & \multicolumn{2}{|c}{ Energy Field } \\
\cline { 2 - 3 } Cold neutrons & & $<0.01$ \\
\hline Thermal neutroris & 0.01 & 0.5 \\
\hline Epithermal neutrons & 0.5 & 10 \\
\hline Slow neutrons & 10 & 1000 \\
\hline Intermediate neutrons & 1000 & $5 \times 10^{5}$ \\
\hline Fast neutrons & $5 \times 10^{5}$ & \\
\hline
\end{tabular}

The length of the path of the neutron until its energy is reduced to the thermal region varies from one neutron to another and is a function of the mean atomic mass. Eighteen collisions can "thermalize" a fast neutron when it strikes hydrogen nuclei --110 being necessary for carbon. The 
Comparison between $\mathrm{x}$-ray $(15 \mathrm{KeV})$ and neutron $(0.025$ eV) radiography of an osteomuscular part $1 \mathrm{~cm}$ thick. With neutrons the bone is radiotransparent, and there is little increace in fatmuscle contrast (Brown \& Parks).
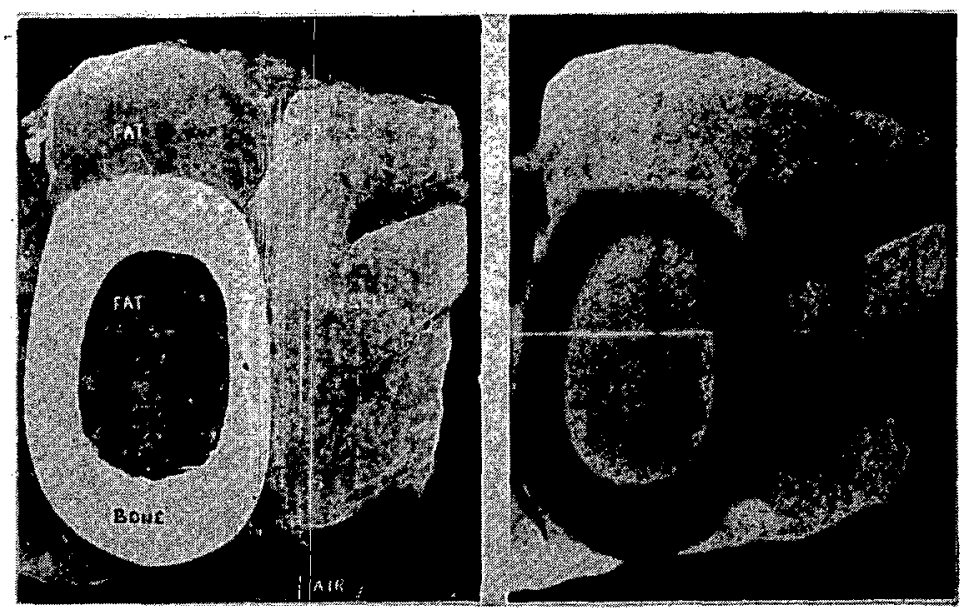

intensity of the neutron beam diminishes quite rapidly because of its scattering and absorption. Thus in the thermal region a tissue $5 \mathrm{~cm}$ thick reduces the intensity of a wide beam by $3 / 10$ and a narrow beam by $1 / 10$.

Each of these processes (capture, elastic collision) occurs with a certain probability:

--on the microscopic scale, given by the effective $\sigma$ section usually measured in barns ( 1 barn $=10^{-24} \mathrm{~cm}^{2}$ );

--on the macroscopic scale, given by the attenuation coefficient $\mu$ (measured in $\mathrm{cm}^{-1}$ ). The mass attenuation coefficient $\frac{\mu}{\rho}\left(\mathrm{cm}^{2} / \mathrm{g}\right)$ is also used.

Thus the laws that govern the attenuation of photon and neutron beams in traversing material of a given thickness (X) are of the we1l-known exponential form, taking into account their respective attenuation coefficients:

$$
I=I_{0} e^{-\mu^{x}}
$$

More precisely, for a neutron flux:

$$
\mathrm{I}=\mathrm{I}_{\mathrm{o}} \mathrm{e}^{-\left(\mu^{\dot{c}}+\mu^{\mathrm{s}}\right)} \mathrm{x}
$$

where $\mu^{c}$ and $\mu^{s}$ represent the attenuation coefficients relative to neutron capture and scattering.

It is wel1 to point out that these formulas are strictly true only for a narrow beam of neutrons with paralle 1 paths and that the phenomenon of "build-up," which is a disadvantage of using a large beam is not inconvenient in $X$ or gamma radiography (where the scattered Compton photons have a high probability of being absorbed close to their interaction site). This last case is not negligible in neutron radiography if the effective scattering cross section, or above all the thickness of the object, becomes large.

In fact, even before completing the traversal of the object, the neutrons assume the energy level of thermal excitation of the surrounding molecules and undergo hundreds of scattering collisions before being absorbed. Often more numerous than the direct neutrons which have not been scattered or absorbed, the scattered neutrons create a significant blur in the image.

These preliminaries established, the table of mass attenuation coefficients (fig. 4), allows one to easily ascertain different areas of application for neutron radiography and for conventional radiography.

Thus if the mass attenuation coefficient for photons increases slowly and steadily, it varies in an apparently uncertain manner. for neutrons. Other nuclear parameters than the atomic number intervene. They vary in a discontinuous manner from one substance to another (fig. 5) and even, for a given substance, from one isotope to another.

Two similar substances in the atomic tables can have very different effective cross sections (for example: difference of 1000 between the effective absorption cross sections of gadolinium and terbium).

As for the isotopes, an example of cross section variation is the ratio of 22,000 that exists between cross sections of $113 \mathrm{Cd}$ and ${ }^{114} \mathrm{Cd}$. 


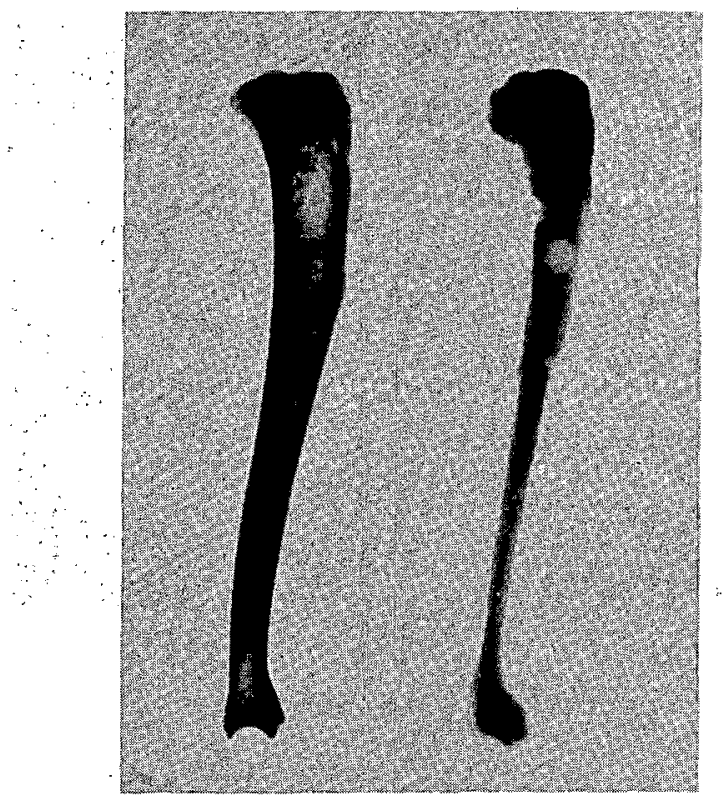

Fig. 3--Comparison between a radiogram (left) and a neutron radiographic image ( $r$ Ight). of a calf bone.

In the upper region of the tibia are porous images which is seen only at the back in conventional radiography. (Plate due to the courtesy of Boutaine and Farny, Experimenta1 Reactór Dept., C.E.A.).

In decreasing: order, elements having large mass attenuation coefficients are gadolinium, hydrogen (of understandable biological importance), samarium, boron, and cadmium (this will be discussed later) (fig. 6).

If a thermal neutron beam is compared with photons of $100 \mathrm{KeV}$, one observes hydrogen attenuates 150 times more neutron intensity than photon intensity. Lead, on the other hand, will attenuate 140 times fewer neutrons than photons: The ratio of mass attenuation coefficients for boron and iron are respectively 170 for neutrons and 0.4 for photons of $100 \mathrm{KeV}$.

\section{SOURCES}

Almost all the studies on neutron radiography have been conducted using nuclear reactors as the neutron source. It is in this industry that the technique has progressed and the basic theories have been studied.

The advantages of such sources is in the large, usable neutron flux (a paralle1 thermal flux of $10^{8} \mathrm{n} / \mathrm{cm}^{2} / \mathrm{sec}$ can be obtained over a useful surface of a few $\mathrm{dm}^{2}$ ). On the other hand, these sources have numerous inherent disadvantages such as the stability of the equipment, significant $Y$ "background noise," and of particular concern to us, cannot be used for medical purposes.

It is necessary to have portable sources of irradiation if this technique is to reach its $f_{11} 11$ value and become more commonly used like radiography or gamma radiography.

For any kind of source, even in the case of mobile sources, it is important to take into consideration certain requirements :

- The sources should be sufficiently strong, since it is necessary to integrate from $10^{5}$ to $10^{9} \mathrm{n} / \mathrm{cm}^{2}$ on detector screens (we will come back to this) placed about $1 \mathrm{~m}$ from the sourice:

--The sources hould also have small enough hazard wheln manipulated without requiring excessive protective shielding.

--Fina11y, any source emitting fast neutrons should be. moderated by "thermalization." Although the fast neutron source can be almost a point source, the volume of moderator in which the thermalization of these neutrons is carried out is about one $\mathrm{dm}^{3}$. Also, the strength per. surface unit of that source is much weaker than the initial point source of fast neutrons, and the mobile source of thermal neutrons would have to have about $10 \mathrm{~cm}^{2}$ of surface area to be strong enough.

Taking these requirements into account, two types of fast neutron sources can be considered furthei:

--particle accelerators used as neutron generators;

--radioisotopic neutron sources.

\section{ACCELERATORS}

Accelerators are analogous in neutron radiography to $X-1$ ay equipment in radiography. To obtain neutrons, they use the nuclear reactions following the collision of accelerated particles in electrostatic fields in suitable nuclear targets.

Fluxes on the order of $10^{4} \mathrm{n} / \mathrm{cm}^{2} / \mathrm{s}$ to $10^{6}$ can be obtained. These sources are. less expensive than formerly, but they must be supplied with $€$ lectric current at high voltage and require significant maintenance, such as frequent changing of the targets.

More than thirty reactions would permit production of neutrons, each characterized by the energy of the incident particle, the reaction efficiency, and the energy of the 


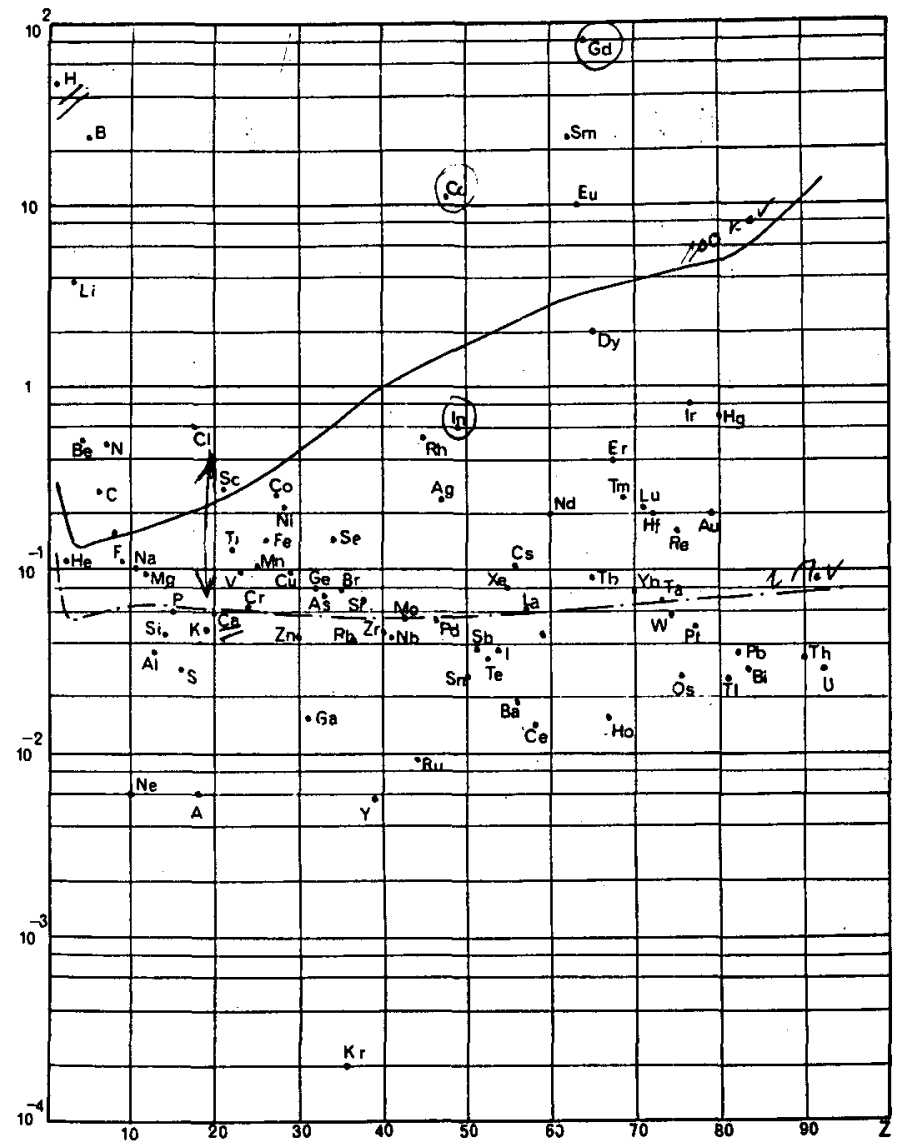

Fig. 4--Comparison of values for the mass attenuation coefficients $\left(\mathrm{cm}^{2} / \mathrm{g}\right.$ ) (thermal neutrons: - : photons of $100 \mathrm{KeV}:-$ : photons of $1 \mathrm{MeV}:-\ldots)$.

emitted neutrons. There are also two requirements limiting the choice of reactions available:

--the incident particles should be of low energy to limit the volume, weight, and cost of the generator producing the accelerating potential;

--the yield from the selected reactions should be sufficient to obtain a satisfactory number of neutrons per second.

Thus, due to these considerations

three reactions are usable:

deuterium-tritium

deuterium-deterium

deuterium-beryl1ium

The first doesn't have a threshold but has sufficient yield only for deutrons of energy greater than $100 \mathrm{KeV}$. Moreover, the energy released in the target in the form of heat is such that tritiated compounds are rapidly decomposed in spite of intensive cooling. This requires frequent renewal of targets (sometimes every twenty hours in

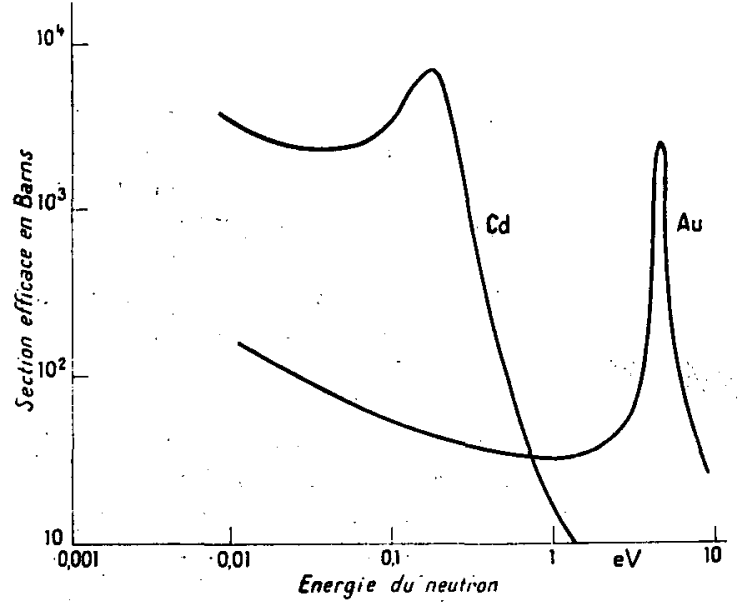

Fig. 5--The effective cross section passes through either a maximum of one or several values of neutron energy.

In the curve above, cadmium is an excellent absorbent of thermal neutrons (according to Tubiana, Dutreix and Jockey). 


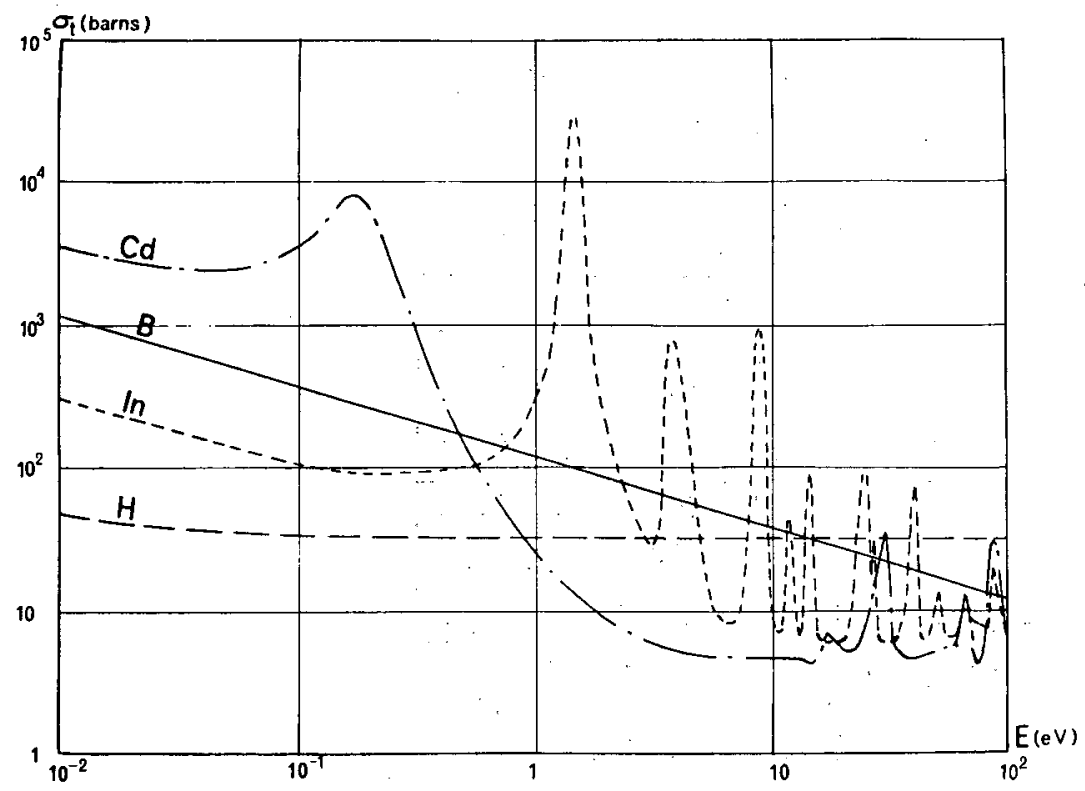

Fig. 6

Comparison of various tota 1 effective cross sections of hydrogen, boron, cadmium and indium as a function of energy.

certain accelerators having:high intensity deutron beams).

The D-D reaction is also without threshhold, but is impractical because its output is significant only for deutrons. with energy of at least $600 \mathrm{KeV}$. As for the $\mathrm{D}-\mathrm{Be}$ reaction, the yield becomes satisfactory only towards $800 \mathrm{KeV}$. Its great advantage, on the other hand, 1 ies in the fact that the target is not destroyed because of the high temperature tolerance of beryllium.

\section{RADIOISOTOPIC SOURCES}

They are analogous in neutron radiography to the use of sealed Co- 60 sources in gamma radiography. The usable flux is of the order of $10^{4} \mathrm{n} / \mathrm{cm}^{2} / \mathrm{s}$. This figure may be a little low; however, certain of them ( $\alpha$, n-reaction, $\alpha-B e$ ) have the advantages that no maintenance is required and the $Y$ background noise is practically nonexistent. On the other hand, the necessity of using an intermediate reaction to produce neutrons considerably limits the neutron yield of these sources.*

\footnotetext{
\% This intermediate reaction will not be necessary for isotopes decaying by spontaneous fission, such as californium252 , when they are available. Thus while one $\mathrm{Ci}$ of $\mathrm{Co}-60$ supplies $3.7 \times 10^{10}$ disintegrations per second, giving from each d'is integration two $\gamma$ photons, in an isotopic' neutron source, the intermediate reaction only produces from $10^{4}$ to $10^{5} \mathrm{n} / \mathrm{s}$.
}

Beginning with one of these sources, it is necessary when working with thermal neutrons to assure thermalization and collimation of the neutrons emitted by these sources. Various solutions have been applied to these problems. Without expanding on each of these, the principle is to associate:

--a certain moderator thickness (water, paraffin, graphite, polyethylene, etc.). The thickness varies with the energy of the neutrons emitted by the source;

$--a$ collimator of absorbent material (boron, cadmium) in the form of a cylinder or cone;

a11 of this is to obtain an approximately parallel flux of neutrons with energy near that of the thermal energy spectrum.

\section{DETECTION OF THE TMAGE}

Not carrying: an electric charge, neutrons cannot ionize matter directly." Consequently, they react very weakly: with a photographic emul'sion. An auxiliary reaction is therefore necessary to detect them. This is done by means of a converter system which converts the neutron flux into a charged particle flux or electromagnetic rays which act on the photographic emulsion.

Thus the so-called "direct" method or the "indirect" method of "image trans fer" can be used, depending on whether the film is subjected directly to neutron bombardment or to electromagnetic radiation. 


\section{DIRECT METHOD}

a) Photographic process using a converter of metallic sheets which are equivalent to intensifier screens used in radiography or gamma radiography.

The principle is as follows: neutrons which have not been attenuated by the examined object are absorbed by the nucleus of the metal, from whence $\beta$ particles and $\gamma$ photons are emitted in the following disintegration (when the nuclei formed by capture are radioactive).

A metallic element with a high cross section should be selected, such as gadolinium and rhodium.

Practically all films are naturally very sensitive to electrons emitted after the capture reaction, whether directly from the nuclear disintegration or from slowing down and capture of disintegration gammas. For a given film, the speed and definition obtained depend on the nature of the screen used, the energy of the neutron flux, and the $Y$ background noise.

b) Photographic processes using fluorescent screens.

c) Fluoroscopic processes, etc.

A disadvantage of al1 of these methods is the lack of discrimination against the gamma background noise.

\section{IMAGE TRANSEER METHOD}

The image transfer methods are almost insensitive to gamma.

It can be a question of:

a) Activation of metallic screens (gold, indium, dysprosium); then only the screen is irradiated. The materials with the strong activation cross sections give a $\beta$ emitter for a relatively short period. The neutron image is obtained secondarily by autoradiography--that is, by placing the irradiated screen on a radiographic film. A significant flux is necessary, but the resolution is excellent.

b) Using a thermoluminescent material (1ithium fluoride in particular).

Such are the principal processes for detection of the neutron radiographic image. With respect to medical radiography, speed and quality of information vary in inverse ratio. Thus in order of decreasing speed, come the fluorescent processes, then the metallic intensifier screens, and finally the image transfer method. This order corresponds approximately to the order of increasing image quality (fig. 7). It is desired that the image be clear, have good contrast and good resolu-
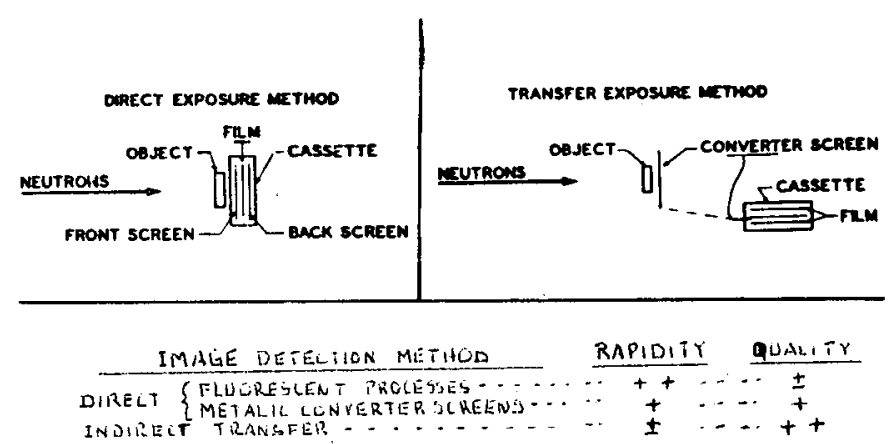

Fig. 7--Two detection methods for the neutron image and the quality of their response.

tion.

This quality is therefore related: to the nature of the film used, to the fineness of the grain and to that of the converter (radiation emitted, surface state, thickness).

The type of collimator used is also important. One wishes to obtain a minimal geometric image compatible with the acceptable time of exposure.

Having stated generally the principle and technique of neutron radiographic methods, we consider now the medical "spin-off" eventually possible from the process.

\section{MEDICAL APPLICATIONS}

A.--It is important at first to examine the "biological neutron-tissue" interactions.

One çan schematically, for a density of $1 \mathrm{~g} / \mathrm{cm}^{3}$, represent the tissues by the chemical formula $\mathrm{C}_{5} \mathrm{H}_{40} \mathrm{O}_{18} \mathrm{~N}$. The other constituents can be, according to the part of the body examined, sulfur, phosphorus, and especially sodium and calcium (Table II)

Where capture and especially elastic collisions (100 times more probable) can be observed, according to classical mechanics, light neutron projectiles undergo (in tissue components) an appreciable loss of energy only. in the frontal elastic collisions with particle-targets whose mass is approximately the same. Consequently, we have observed that slowing up of thermal neutrons will affect essentially the light nucle $i$ and therefore the hydrogen nucleus (the neutron loss of energy averages about half for a collision with a proton).

This fundamental phenomenon conditions and justifies at first approach any research on neutron radiography of human tissues. 
Linear Attenuation Coefficients by Thermal Neutrons

(according to J. P. Barton)

\begin{tabular}{l|c|c|c|c|c|c|c|c|c|c}
\hline \multirow{2}{*}{ Composition } & \multicolumn{4}{|c|}{ Tissue } & \multicolumn{1}{c}{ Bensity $1.0 \mathrm{~g} / \mathrm{cm}^{3}$ ) } \\
\cline { 2 - 11 } & $\mathrm{H}$ & $\mathrm{C}$ & $\mathrm{N}$ & 0 & $\mathrm{H}$ & $\mathrm{C}$ & $\mathrm{N}$ & $\mathrm{O}$ & $\mathrm{P}$ & $\mathrm{Ca}$ \\
\hline Weight \% & 10.1 & 12.1 & 4.1 & 73.6 & 3.39 & 15.5 & 3.97 & 44.1 & 1.02 & 22.2 \\
Scattering & 3.01 & 0.03 & 0.017 & 0.115 & 1.96 & 0.071 & 0.032 & 0.134 & 0.018 & 0.019 \\
Absorption & 0.02 & - & 0.003 & - & 0.013 & - & 0.006 & - & - & 0.003 \\
\hline
\end{tabular}

In fact, it is even more important in eventual medical applications. Since hydrogen represents approximately $10 \%$ of the body weight, there are more hydrogen atoms than atoms of other elements in biological tissues. The effective cross section of hydrogen is quite high, on the order of barns. It is only millibarns for oxygen. It's the same for other constituents: calcium, phosphorus, sulfur, which do not have an effective cross section high enough to compensate for their smaller concentrations. Only sodium, because of its importance in the organism, will have a relatively important cross section. To indicate the importance of this phenomenon, elements such as lead, calcium and iodine, which are opaque to $\mathrm{X}$-rays, are much less opaque than the hydrogen nuclei when subjected to thermal neutrons. Substances rich in hydrogen, such as lipides, which have weak opacity to X-rays, will be more visible because of their high neutron attenuation coefficients. This hydrogen content in lipides varies according to the tissues but is usually high in certain cerebral regions.

Thus, it is only in making a sharp contrast between bone and tissue that neutron radiography is not superior to X-rays. Micro-radiography offers more by way of optimum contrast. On the other hand, because neutrons penetrate bone more than tissue, bone can be analyzed according to its thickness and especially the soft part covered by the bone cortex. It also increases gaseous contrasts. At any rate, neutron radiography appears to be a graphic method affording values reverse of those of conventional radiography, in addition to more useful, detailed information.
However, contrary to the above mentioned advantages, the amount of hydrogen in living substances is so high that scattering constitutes a serious problem involving deterioration of contrast in samples more than two centimeters thick. According to our present knowledge, this would limit neutron radiography to investigations of digits or of soft, thin tissues.

Also, even though the first results obtained were relatively satisfactory on the industrial level, they leave much to be desired for biological applications on a large scale. The techniques are still in the experimental stage. For now, neutron radiography can only be conducted on the in vitro analysis of thin, normal biological or pathological samples. These could be treated with formaldehyde in order to preserve them before being submitted to the neutron flux. Formaldehyde is a hydrogen compound, and thus one risks altering the hydrogen content of the sample. Freezing would seem preferiable.

B.--Two essential conditions control the problem of medical applications:

--Exposure time should be short, as opposed to indust:rial neutron radiography where the length of exposure is left to the operator.

--Biological radiation tolerance should not be exceeded.

This neutron risk, well studied by Snyder (1957), Morgan (1958, and more recently by Barton (1963), is a function of the biological|damage which could result from capture in hydrogen and nitrogen. The capture cross section of hydrogen is 0.33 
barns at $0.025 \mathrm{eV}$ and involves gamma radiation (of $2.2 \mathrm{MeV}$ ) and a low energy deuteron nucleus $(1.3 \mathrm{KeV}) . *$

The capture cross section of nitrogen is 1.88 barns and results in the emission of a highly ionized proton $(660 \mathrm{KeV})$ and the production of radioactive $\mathrm{C}^{14}$ (ha1f-1ife 5,700 years). The neutron does not cause direct ionization. The elastic collisions with hydrogen and other nuclei do not transfer enough energy to involve active secondary ionization if the incident neutron flux has energy less than $10 \mathrm{KeV}$.

A first estimate of this risk would show that a sample the size of the whole human body subjected to a total dose of $10^{\mathrm{n} / \mathrm{cm}^{2}}$ and energy less than $10 \mathrm{KeV}$, would cause damage similar to that caused by one roentgen of X-rays.

Intensities higher than $10^{9} \mathrm{n} / \mathrm{cm}^{2}$

would appear acceptable for irradiation of local members (ankle, wrist for example) if narrow radiation beams are used. Experiments on animals by Brown and Parks give dosimetric figures from 0.8 to 5 rads, as opposed to 0.2 to 3.7 for the same specimen examined by $\mathrm{X}$-rays.

We recal1 that the dose tolerance (varying as a function of energy with factors from 20 to 30 times) would be 2.5 $\mathrm{mrem} / \mathrm{hr}$ for 700 thermal N/ $\mathrm{cm}^{2} / \mathrm{sec}$. (Jammet).

$$
\begin{gathered}
* \\
* \\
*
\end{gathered}
$$

The first approach in our research program on neutron radiography for medical purposes will be to proceed with the "Triton" reactor, according to four directions:

1. To try in vitro to improve the quality of the image. "Certainly the large size of the neutron emitting surface wil1 never reach the fine' focus of roentgen tubes, endangering the image definition obtained, but progress can be anticipated:

--in emitting: source material with radioisotopic generators of neutrons, - in collimation material, --in antiscatter grid material to suppress--for example with boron plates-the scattered neutron contaminants, --in detection material: a zinc sulfide scintillator charged with a material sensitive to neutrons ( 6 Li-fluoride) previously shown to give good information with a flux of $10^{3} \mathrm{n} / \mathrm{cm}^{2}$ but it seems desirable to carry this to $105 \mathrm{n} / \mathrm{cm}^{2}$ to

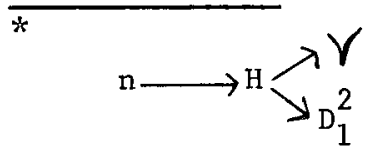

obtain, good contrast and good resolution. In addition, such screens are quite useful for neutron radiography of biological specimens because of short exposure time and very weak radiation dose which the screens permit (100 times less than that of the system using gadolinium).

One can also consider changing the modulated neutron flux after interaction with the object by reflecting light investigation. These latter are absorbed, amplified and analyzed by a television system.

We should expect however, with neutroscopy thus considered, to have definition of lesser quality than that of film. (Farny and $\mathrm{La}$ Porte)

In any event, one can be content with the images which will provide interesting information, even though they do not reach the clarity of conventional radiography, where the resolution is excellent. Doesn't thyroid autoradiography, for example, give fundamental radiodiagnostic information in spite of its imprecision?

2. Always in vitro, it will be suitable to consider neutron radiography of greater thicknesses. One can increase the possibilities of neutron radiography by having recourse to neutrons of high energy. A flux $10^{9} \mathrm{n} / \mathrm{cm}^{2} / \mathrm{sec}$. of high energy neutrons would seem to give the maximum exposure limit and an intensity of $10^{5} \mathrm{n} /$ $\mathrm{cm}^{2}$ the condition required at the detector level.

For this, in the limit of biological tolerance, it will be necessary to form very fast neutrons ( 1 neutron of $6 \mathrm{MeV}$ would be equivalent to radiation of $200 \mathrm{kV}$ ), at least intermediary, in order to penetrate most of the regions of the human body.

The use of grids will increase the contrast and details. Methods of direct exposure to fast neutrons will be settled by checks, resonance detectors; transfer techniques with indium foils would be preferable. Californium 252 sources, when available (besides their portable characteristics and reasonable price), would be, from this point of view, interesting for fast neutron research (fig. 8).

The neutron $\mathrm{risk}$ for an epithermal neutron would be similar to that of thermal neutrons and their greater penetration would allow a smaller radiation dose. It should be emphasized that the adjusted higher dose tolerance concerns only a single professional exposure and, consequently, there is not a precise limit for an occasional examination. 


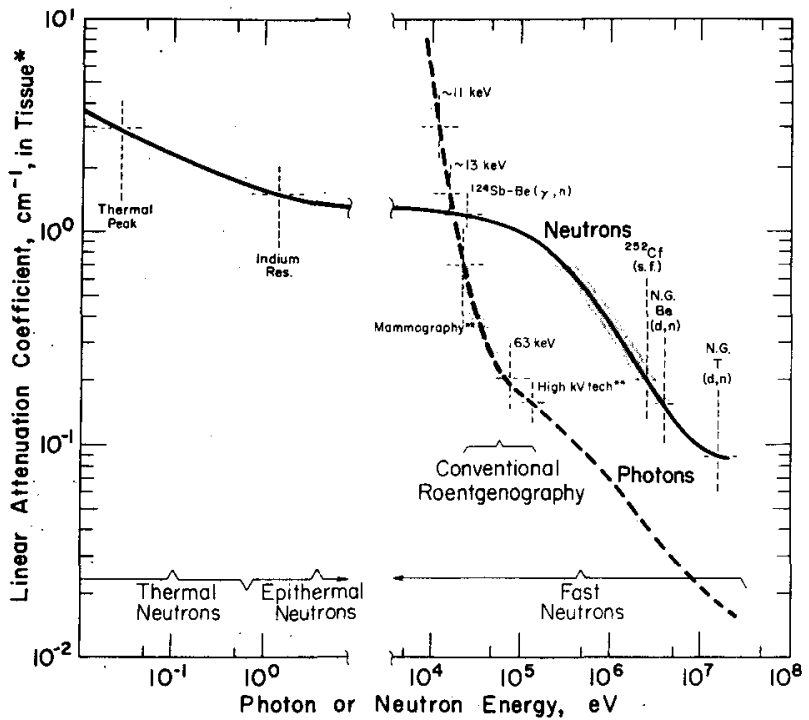

Fig. 8--Comparison of the attenuation differences between $\%$ photons or gamma and neutrons in the tissue.

The linear attenuation coefficients identical with conventional radiography are principally in the fast neutron region.

Californium sources would be equivalent to $63 \mathrm{KeV}$, whereas thermal neutrons would be equivalent to $11 \mathrm{KeV} \mathrm{X}$-rays and epithermal neutrons equivalent to $13 \mathrm{KeV}$ $\mathrm{X}$-rays (Brown and Parks).

Moreover, the use of epithermal neutrons permits those neutrons which have been scattered and slowed up to be absorbed by cadmium or some other absorbent placed in front of the detector screen. In fact, if the incident radiation consists almost entirely of monoenergetic neutrons, energy corresponding to ' a resonance peak in the detector material (indium, dysprosium) there is a significant discrimination in scattered neutrons.

3. Analysis of pathological specimens, in comparison with eikonography of the same sections obtained by radiograms (fig. 9), can also be a profitable method to estimate the possibilities of neutron radiography in medicine. If, as in the Frigerio statement cited by Barton, nany tumors have higher hydrogen content than normal tissue, one can easily conceive the possibilities of the method.

4. Finally, in addition to this simple neutron radiography, "without preparation" of any sort, an interesting medium of research can be neutron radiography with contrast agents (positive or negative). In fact, the opaque range of these contrast agents is greater for neutron radiography than for $\mathrm{X}$-rays, because the absorption coefficients--principally with low energy neutrons-have a variation scale covering several orders of magnitude.

The comparative experiments of Brown and Parks with air, heavy water, and gadolinium in a methacrylate phanton (fig. 10) and with gadolinium oxide, indium oxide, heavy wate: and air in animals (fig. 11) are in this respect very significant. However, before we consider the in vivo use of these substances, we must assure their lack of toxicity for the organism.

Fig. 9
dy of tumorous
ecimens: neutron
n the upper row;
elow $20 \mathrm{kV}$ in the
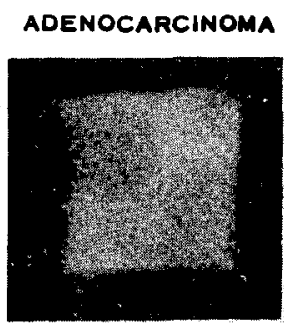

radiography in the upper row; radiography below $20 \mathrm{kV}$ in the

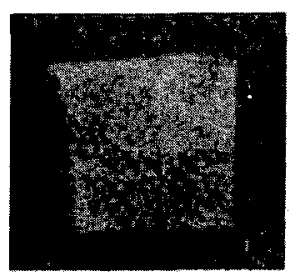

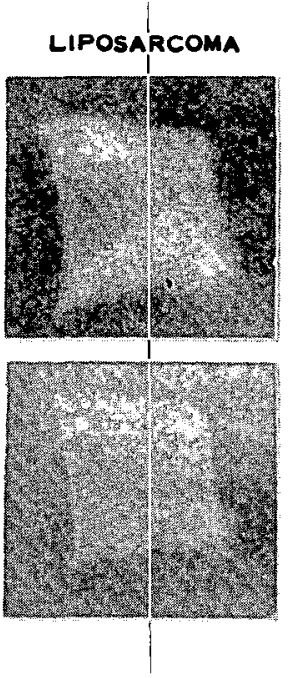

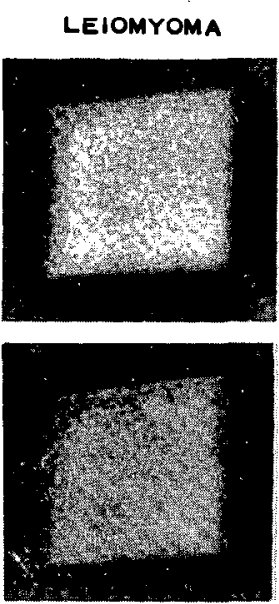




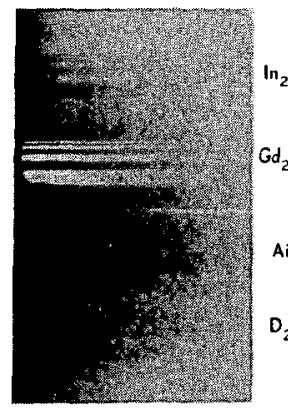

A

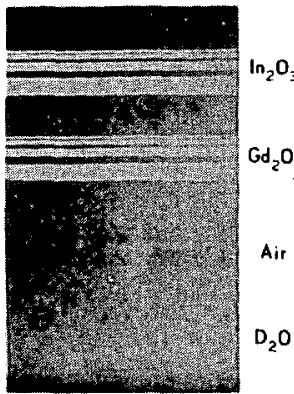

c
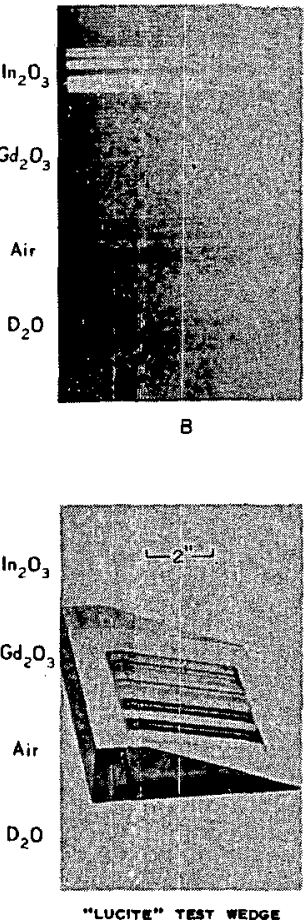

Fig. 10--Wedge-test evaluation of contrast agents by neutron radiography.

A-Thermal neutrons

B-Epithermal neutrons

C-X-rays of $45 \mathrm{KeV}$

Note the good visibility of air and heavy water in the neutron radiographic exposures and the distinct differences between thermal and epithermal neutrons with gadolinium oxide (Brown and Parks)。

\section{CONCLUSION}

Such are the limits and possibilities of neutron radiography. As to its application to medicine, it will probably find its place among other graphic techniques (such as subiraction, logetronics, scintography, thermography, and echography) which perfect and enlarge the framework of conventional radiography. Needless to say, initially, it must be investigated cautiously under pure experimental conditions. It is important to seek improvement which will perfect the information that can be provided by this technique. In particular, the ability to penetrate greater thicknesses must be developed.
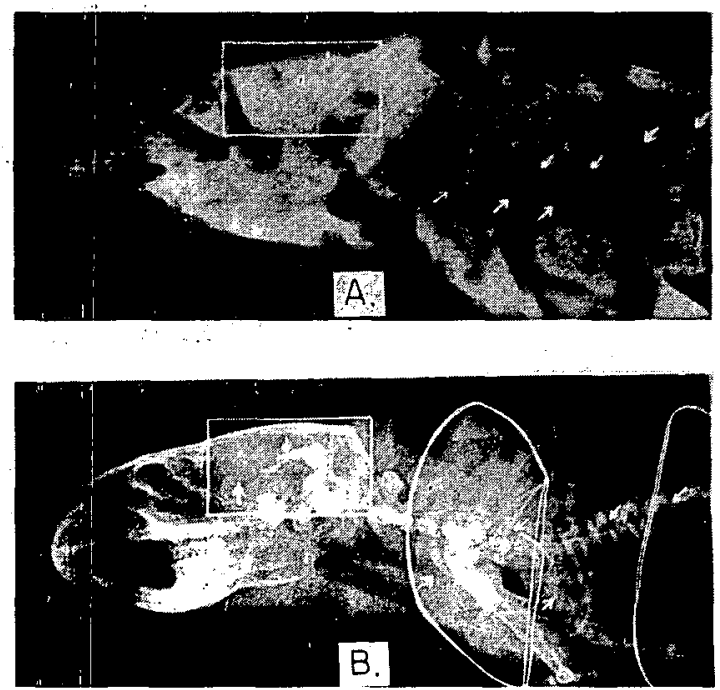

Fig. 11--Neutron ventriculography of a rat. A-Thermal neutron radiograph B-Conventional nonscreen radiography

Air and gadolinium oxide have been introduced by cisternal puncture. The neutron radiograph shows we 11 air in the ventricle (verticle arrow), trachea and the spinal canal (slanting arrow) (Brown and Parks).

\section{BIBLIOGRAPHY}

1. H. L. Atkins. "Biological Application of Neutron Radiography, 24th Nat. Conv. of S.N.T. Philadelphia (Oct. 1964). Report BNL-8165, CONF778-7 and Materia1s Eva1., 23, 453 (1965).

2. J. Anderson. "Neutron Radiography in Man." Brit. J. Radiol. 37, 957-958 (1964).

3. J. P. Barton. "Some Possibilities of Neutrón Radiography." Phys. Med. Biol. $8,33-42$ (1964).

4. J. P. Barton.: "Radiography with Resonance Energy Neutrons." Phys. Med. Biol.. 10, 209-212 (1965).

5. J. P. Barton. "Neutron Radiography Biological Aspects," Neutron Radiography Newsletter, №. 7, Nov. 1967.

6. J. P. Barton. "Divergent Beam Collimator for Neutron Radiography." Materials Evaluation, 45A-46A, 25 (1967). 
7. H. Berger. Neutron Radiography, Methods, Capabilities and Applications. p. 146, Elsevier Pub1. Co., Amsterdam (1965).

8. H. Berger "A Sumary Report on Neutron Radiography." Report A.N.L., July (1964).

9. M. Blankes and col1. "Improved Resolution Neutron Radiography." 5th Congrès International Sur Les Esisais Non Destructeurs, Montréa 1, May (1967).

10. J. L. Boutaine. "La Neutrographie: Son Principe; ses Possibilités. d'Emploi." La Neutrographie, Possibilities et Avenir. Saclay, C.E.A. (1966).

11. M. Brown and P. B. Parks. "Neutron Radiography in Biologic Media." Am. J. Roentgen. 106, 472-485 (1969).

12. C.E.A. "La Neutrographie: Possibilités et Avenir." Compte Rendu de la Réunion d'Information Organisé à Sac lay le 5 0ctobre 1966 (INSTN).

13. Farny and Laporte. Installation d'un Faisceau Sorti a Triton. Application Industrielle de la Neutrographie, C.E.A., Section de Physique et d'Experimentation, Saclay (1969).

14. E. Tachilin. "Photographic Detection of Fast Neutrons: Application to Neutron Radiography ." Phys. Med. and Bio1. 10, 477-490 (1965).

15. M. Tubiana, J. Dutreix, A. Dutreix, and $P$. Jockey. Bases Physiques de la Radiothérapie et de 1 a Radiobiologie. Masson et C ${ }^{\text {te }}$, ét., Paris (1963).

16. H. V. Watts. "Research on Neutron Interaction in Matter as Related to Image Formation." Report ARF, 1164-1227, August (1962). 


\title{
NEUTROGRAPHY IN MEDICAL RESEARCH AND PATHOLOGY
}

\author{
Michael J. Flynn \\ Glenn F. Knoll \\ Andrew K. Poznanski \\ University of Michigan \\ Ann Arbor, Michigan
}

The results of research in three areas of bio-medical applications of neutron radiography are reported. Neutrographs from thick bone sections are presented and analyzed with respect to their tissue types and extent of tumor involvement. The contrast provided by various high cross section elements in biological material is analyzed and the results for boron- 10 contrast are compared with experiment. A concept for micro-neutrography is presented and its application to biological studies is briefly discussed. The necessity of using reaction rate average cross sections in predicting the image response of thick organic objects is stressed, and the results presented are supplemented by several tables of microscopic and macroscopic cross sections along with contrast parameters calculated by a reaction rate analysis.

\section{INTRODUCTION}

The potential advantage of using neutrons to generate radiographs of biological objects has been discussed frequently over the last five years. In general, the image of a normal biological object yields a twodimensional representation of the total hydrogen atom thickness (atoms/unit area) normal to the image plane $(1,2)$. This frequently implies that a bone image, vividly apparent in a roentgenograph, does not appear in a neutrograph $(1,2,3)$. With these properties in mind, and the fact that exceptionally good contrast agents exist in certain high cross section elements, we have recently attempted to delineate specific applications of thermal neutron radiography in medical research and pathology. While no specific procedures may be recommended for routine applications, the following report presents specific results for three of the areas which we have considered.

It must be recognized at the outset that thermal neutron radiography is practically limited to samples no thicker than several neutron mean free paths. For most organic materials this implies a maximum sample thickness of 1 to $2 \mathrm{~cm}$ (about 3 to 6 mean free paths for thermal neutrons). At thicknesses greater than this the fraction of neutrons transmitted uncollided through the object is so small that it is extremely difficult to separate this image from background and object scattered neutrons. Being thuswise restricted, there are several catagories of applications which become of interest; the in vitro examination of tissue specimens (2) and the in vivo examination of small animals (1). Specifically considered in this paper are (a) the examination of gross pathological specimens (.5 cm thick) obtained from neoplastically involved bone regions, (b) the feasibility of high resolution micro-neutrography for the examination of thin specimens, and (c) the in vivo detection of high cross section isotopes in small animals.

$$
\text { DETECTION FOIL }
$$

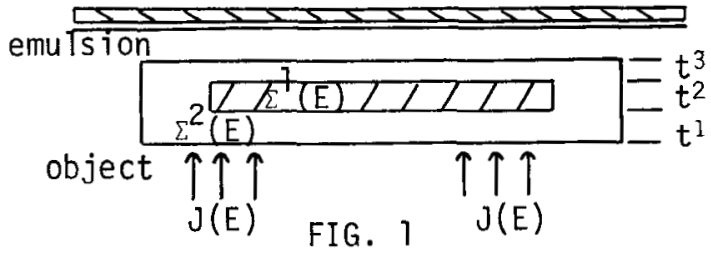

In each of these cases the film density and contrast response has been predicted by a simple mathematical description. The film density, D, for a direct exposure is assumed directly proportional to the reaction rate per unit area in the foil times the exposure time, i.e. $D=\mathrm{kRt}$. This assumption is in general valid for industrial $x$-ray films and a linear response has been measured in this laboratory for type-M $\mathrm{X}$-ray film exposed by 25 micron thick gadolinium up to a film density of 3.0. Referring to Fig. 1, the total reaction rate may be written as the integral over the differential reaction rate in energy,

$$
\mathrm{R}=\int_{0}^{\infty} \mathrm{R}(\mathrm{E}) \mathrm{dE}
$$


where,

$R(E)=J(E) e^{-\mu(E)} A(E)$

$J(E)=$ incident neutron current

$\mu(E)=\Sigma^{1}(E) t^{1}+\Sigma^{2}(E) t^{2}+\ldots \Sigma^{n}(E) t^{n}$

$A(E)=$ foil absorption $=$

$$
\left(1-e^{-\Sigma^{f}(E) t f}\right)
$$

The film density change (contrast) produced by a thickness $t^{\text {n }}$ of some material described by $\Sigma^{\mathrm{n}}(\mathrm{E})$ may be derived by differentiating $\mathrm{R}$ by $\mathrm{dt}^{\mathrm{n}}$,

$$
d R / d t^{n}=0^{f^{\infty}-\Sigma^{n}(E) R(E) d E}
$$

If we then define an average cross section as,

$$
\vec{\Sigma}^{n}=\int_{0}^{f^{\infty} \Sigma^{n}(E) R(E) d E / \int^{f^{\infty}} R(E) d E}
$$

and then recall that $D=k R t$, then the contrast may be described as

$$
\mathrm{dD} / \mathrm{d} t^{\mathrm{n}}=-\vec{\Sigma}^{\mathrm{n}} \mathrm{D}
$$

The authors are careful to point out that this expression depends on a reaction rate averaged cross section and hence on the type of foil used and on the object in consideration. The use of the commonly tabulated 'thermal averaged' or ' $2200 \mathrm{met} / \mathrm{sec}^{\prime}$ cross section in calculations of this type is likely to result in serious error particularly for the case of thick objects.

The average cross section for effectively thin objects may be computed using a reaction rate equal to the incident flux times the foil absorption, i.e. $J(E) \times A(E)$. In Table 1 are listed the average cross section for standard tissue with a composition of $\mathrm{C}_{7} \mathrm{H}_{70} \mathrm{O}_{32} \mathrm{~N}_{2}(\underline{4}, \underline{5})$ for two common foil materials along with the thermal energy $(.025 \mathrm{eV})$ cross section for the same composition. These cross sections and the contrast parameters subsequently reported in this paper were computed by numerical integration of the preceding integrals on a digital computer. The cross sections were specified by a 61 point mesh over the energy region from .001 to $10 \mathrm{eV}(\underline{6})$. The cross sections for organic materials were calculated using a bound atom value for hydrogen (7) ( $\mathrm{H}-\mathrm{C}$ bonded in cetane). The incident neutron current was specified by the Westcott relation (8) with a Maxwellian neutron temperature of $300.0^{\circ} \mathrm{K}$ and a Westcott epithermal index of $r=.01$. The numerical integration was based on the use of a Lagrange interpolating polynomial between mesh points.

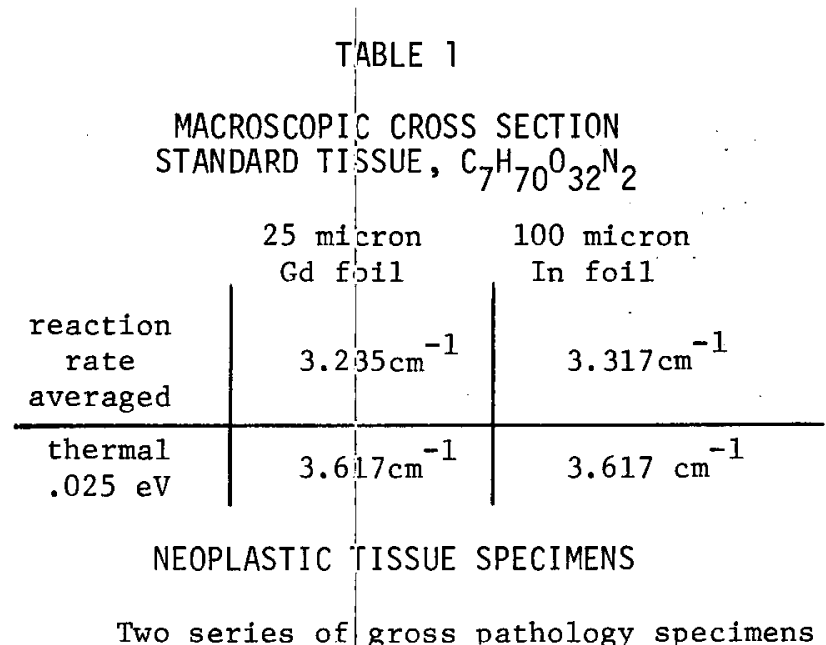
measuring approximately $.5 \times 2 \times 2 \mathrm{~cm}$ and containing a variety of neoplastic lesions have been examined for potentially useful patterns or region: of differentiation. It has been reported by Frigerio that tumorous regions have a higher hydrogen content than their surrounding issue( $(9)$ and the previous results of Brown and Parks indicate that this difference may be observable on a neutron radiograph (2). Attention was therefore focused in thits study on confirming this result and det-ermining if one can establish the extent of neoplastic involvement in the early stages of tumor formation.

The densitometric response of several detection systems to a neutron current transmitted through dif:Eerent thicknesses of standard tissue has been examined and the results are shown in Table 2 . The relative exposure time required to obtain the same film density without an object and with an object was calculated by taking the ratio of their total reaction rates. The tabulated reaction rate averaged cross sections indicate the sensitivity to small changes in thickness for a given thickness object with a particular detector; thus a $1 \mid \mathrm{mm}$ void in a $1 \mathrm{~cm}$ thick object will yield a density change of .275 when radiographed with a 25 micron thick gadolinium detector. The decrease in sensitivity for increasing thicknesses of tissue is due to the increasing hydrogen cross section at low energies which tends to shift the average energy of the transmitted neutrons to a higher energy/ where the tissue cross section is reduced The difference in averaged cross section for gadolinium and indium detectors is due to the non $1 / v$ absorption cross sections for the two metals (Gd resonance energy $=.031 \mathrm{eV}$, In resonance energy $=1.46 \mathrm{eV})$. The indium resonance activation is relatively smal the except when the thermal 
TABLE 2

EXPOSURE FACTORS AND REACTION RATE AVERAGED CROSS SECTIONS

\section{THICK 'STANDARD TISSUE' OBJECTS}

\begin{tabular}{|c|c|c|c|c|c|c|}
\hline \multirow[b]{2}{*}{$\begin{array}{c}\text { Object } \\
\text { Thickness }\end{array}$} & \multicolumn{2}{|c|}{25 Micron Gd Detector } & \multicolumn{2}{|c|}{250 Micron In Detector } & \multicolumn{2}{|c|}{250 Micron In Detector } \\
\hline & $\begin{array}{c}\text { Exposure } \\
\text { Factor }\end{array}$ & $\begin{array}{l}\text { Cross } \\
\text { Section }\end{array}$ & $\begin{array}{c}\text { Exposure } \\
\text { Factor }\end{array}$ & $\begin{array}{l}\text { Cross } \\
\text { Section }\end{array}$ & $\begin{array}{c}\text { Exposure } \\
\text { Factor }\end{array}$ & $\begin{array}{l}\text { Cross } \\
\text { Section }\end{array}$ \\
\hline 0.00 & 1.00 & $3.235 \mathrm{~cm}^{-1}$ & 1.00 & $3.314 \mathrm{~cm}^{-1}$ & 1.00 & $2.284 \mathrm{~cm}^{-1}$ \\
\hline 0.25 & 2.20 & 3.087 & 2.22 & 3.077 & 1.72 & 2.063 \\
\hline 0.50 & 4.69 & 2.958 & 4.67 & 2.866 & 2.81 & 1.885 \\
\hline 0.75 & 9.68 & 2.845 & 9.33 & 2.677 & 4.43 & 1.752 \\
\hline 1.00 & 19.50 & 2.746 & 17.80 & 2.507 & 6.77 & 1.655 \\
\hline 1.50 & 73.60 & 2.582 & 57.90 & 2.220 & 14.90 & 1.537 \\
\hline 2.00 & 258.00 & 2.450 & 165.00 & 1.996 & 31.40 & 1.477 \\
\hline
\end{tabular}

region of the spectra has been reduced, either by a thick tissue sample or by a cadmium filter as shown by the averaged cross section for a cadmium filtered indium detector. The radiographs presented below were prepared to a thickness of $.5 \mathrm{~cm}$ and detected with a 25 micron thick gadolinium foil and type $M x$-ray film. This was chosen because of the reasonable exposure factor, high hydrogen sensitivity, and good resolution associated with this system.

An initial survey of tissue sample image properties was made using cancerous specimens obtained from the brain, lung, esophagus, and bone regions. Each specimen was fixed in formaldehyde and stored in ethyl alcohol prior to being radiographed by a $20 \mathrm{KVP} x$-ray beam and by a thermal neutron beam. In general the images formed by the brain, lung, and esophagus were similar and it was concluded that both the roentgenograph and the neutron radiograph were yielding an image of the density variations. The results of most interest were obtained from the bone specimens, an example of which is shown in Figure 2. The dense regions in the positive roentgenograph clearly define the bone structures which appear in the positive neutrograph as regions of low density adjacent to the high density regions produced by the fibrous connecting tissue. The neutrograph further exhibits a lack of the dense calcium cross-structure apparent in the roentgenograph, thus allowing visualization of the regions of low neutron penetration in the lower right and the patterns of high neutron penetration in the central region. It was suspected that some of these patterns might be due to surface fluctuations and interior void regions.

A series containing 14 tumor entailed bone specimens was then examined to determine whether patterns of decreased neutron penetration in the neutrograph correspond to regions of neoplastic involvement. Each specimen was fixed in formaldehyde and stored in ethyl alcohol prior to examination with the neutron beam. To eliminate patterns resulting from voids and surface defects the specimens were placed in an aluminum sample holder designed to contain $.5 \mathrm{~cm}$ thick objects and the sample volume was filled with alcohol and sealed. The neutron beam in the area surrounding the sample was filtered by cadmium to reduce the neutron background and to prevent areas of extremely high film density from acting as a source for grain diffusion. Standard $\mathrm{H} \& \mathrm{E}$ sections were then prepared from each specimen*, which involves the decalcification, setting in wax, sectioning, and staining of the specimen. This procedure allowed the types of tissue present to be identified and compared to the patterns in the corresponding neutrograph.

Representative examples of this study are presented in Figures 3 through 8 in order of increasing tumor involvement. The dark globular shapes in the central portions of the $H \&$ E sections represent osteoid tissue, a protein around which the calcium had

${ }^{*} \mathrm{H} \& \mathrm{E}$ sections were prepared by $\mathrm{D}$. Weaver, M.D., Dept. of Pathology, University Hospital, Ann Arbor, Michigan. 


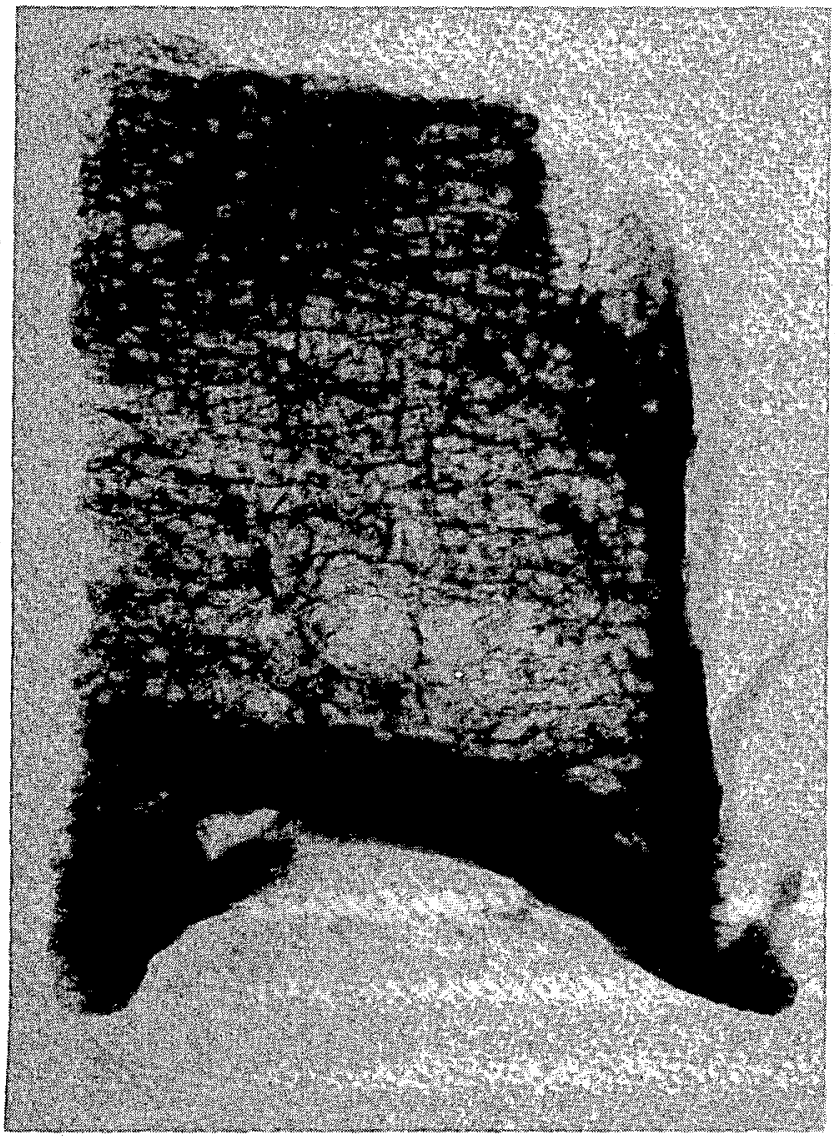

THERMAL NEUTRON RADIOGRAPH

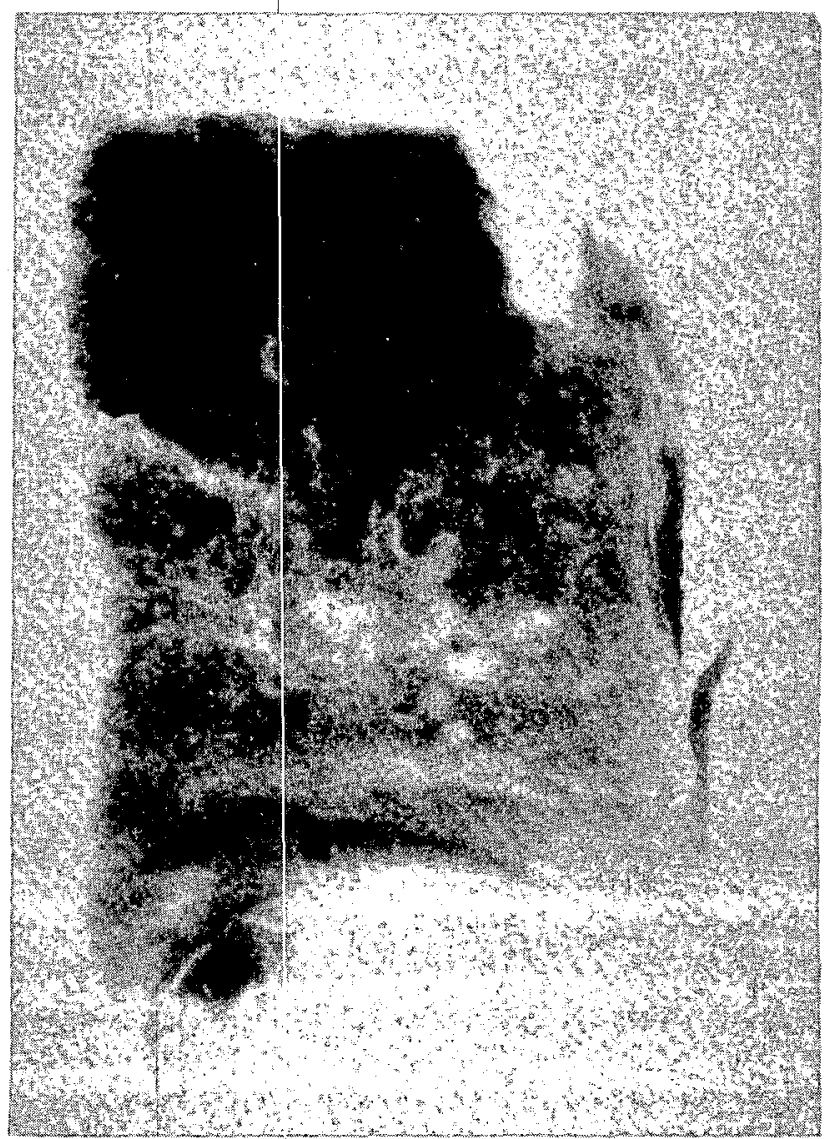

20 KVP ROENTGENOGRAPH

FIGURE 2

Bone specimen from the spinal region: Diagnostic micro-analysis indicates plasma cell myeloma involvement, a malignant tumor of marrow origin ( $4 \mathrm{X}$ enlargement).

been deposited. The speckled inter-osteoid regions correspond to healthy bone marrow tissue (see Figure 3) while the more homogeneous grey-toned inter-osteoid areas represent regions of neoplastic growth (see the central region of Figure 4). The grey even-toned areas on the outer boundaries are areas of fibrous connective tissue while the interosteoid regions of similar appearance represent fibroid tissue resulting from tumor involvement (see Figure 5). The specimen shown in Figure 8 is totally necrotic as evidenced by the dark inter-osteoid material. In the neutrograph the dark areas correspond to regions of decreased neutron penetration and hence increased hydrogen concentration. The light elongated patterns are readily seen to correspond to the osteoid regions. The fibrous connecting tissue appears equal in density to the surrounding alcohol and hence is not defined, as is the case with the necrotic tissue in Figure 8 . The healthy bone marrow tissue appears with a significantly lighter density as with the fibroid tissue and the regions of early tumor formation. only in the specimens which are totally involved and are exhibiting the onset of necrosis does there appear some contrast that might be attributed to increased hydrogen concentration in regions of advanced neoplasia (see the urper left region of Figure 6 and the bulk of Figure 7). Densitometric measurements on the original neutrographs indicate that the fibrous connecting tissue and necrotic tissue areas have a $4 \pm 1 \%$ greater hydrogen concentration than in the healthy marrow regions.

The resultis of this study establishes the radiographil $\left.\right|_{C}$ image properties of osseous material, marrow tissue, and fibrous connecting tissue in thick specimens from the spinal region. The neutrographs provide a detailed picture of the distribution of bone material throughout the specimen by virtue of the hydrogenous tissue displacement. There is no evidence of increased hydrogen concentration in the regions where the presence of 

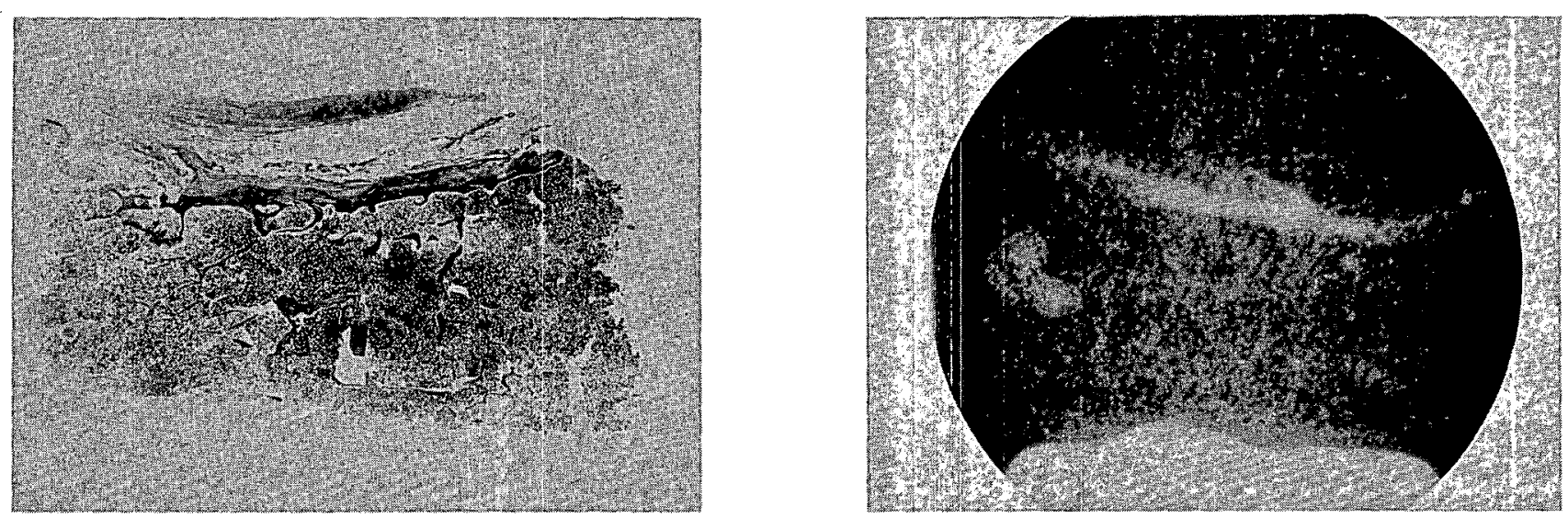

FIGURE 3

$H$ \& E section (left) and neutron radiograph (right) of bone section with slight tumor involvement (Oat cell carcinoma)
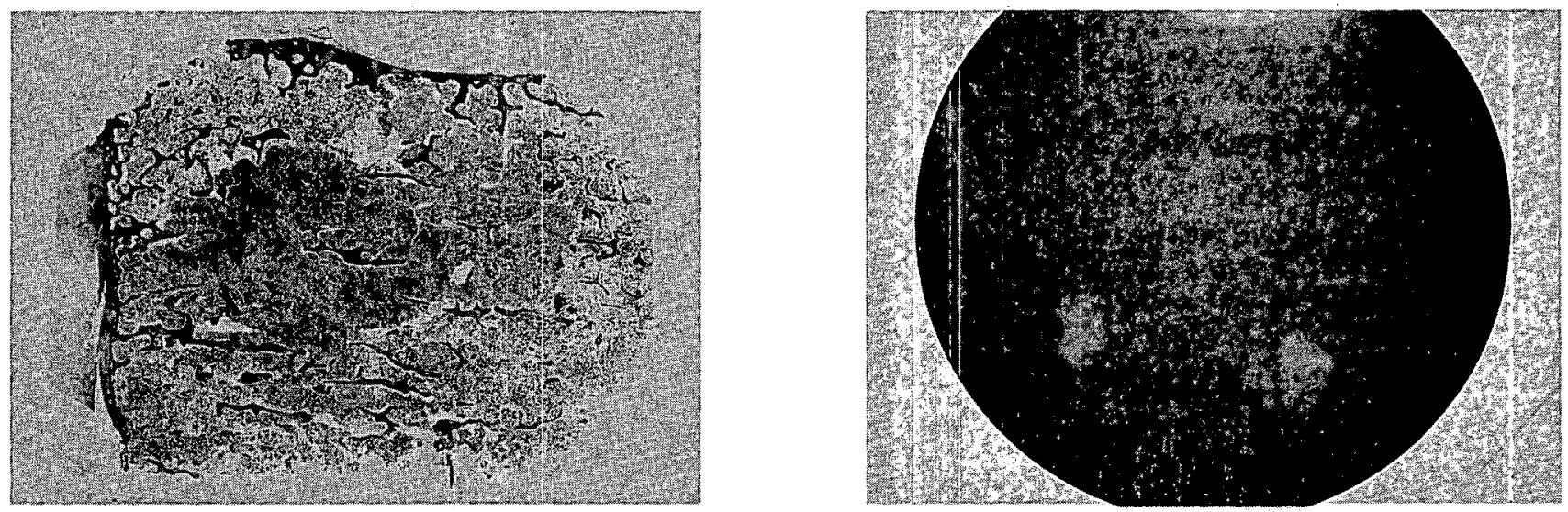

FIGURE 4

$H$ \& E section (left) and neutron radiograph (right) of bone section with large central tumor (0at cel1 carcinoma)
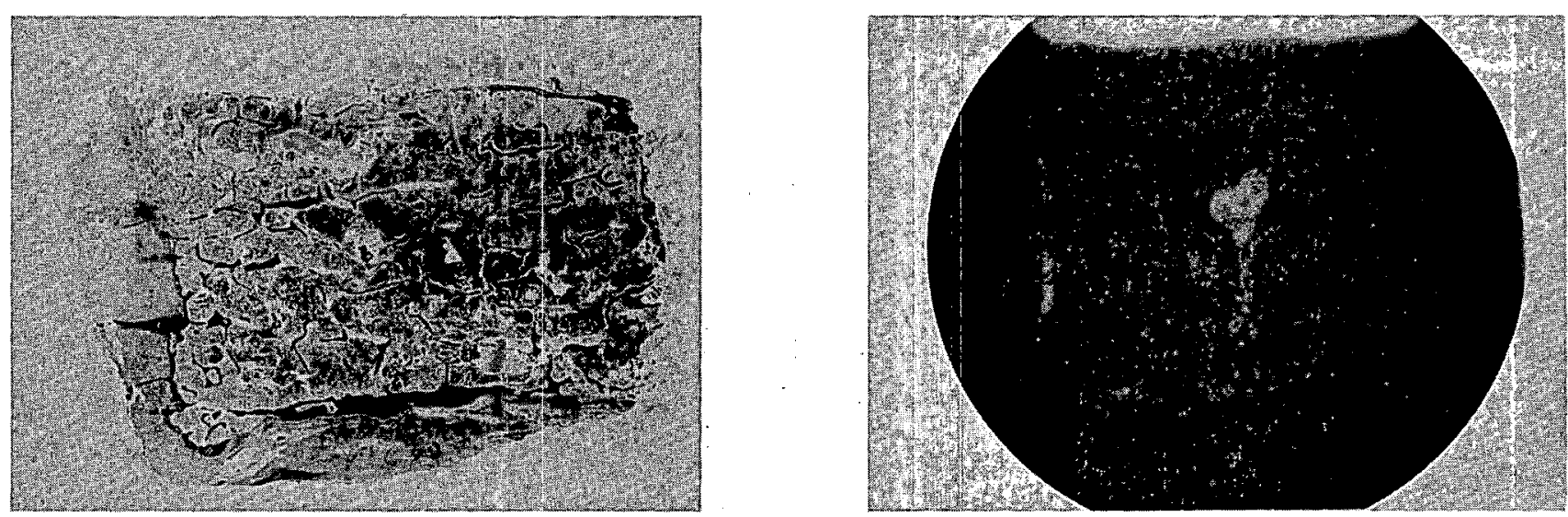

FIGURE 5

$H \& E$ section (left) and neutron radiograph (right) of bone section with extensive tumor involvement and scar formation (Hodgkin's disease) 

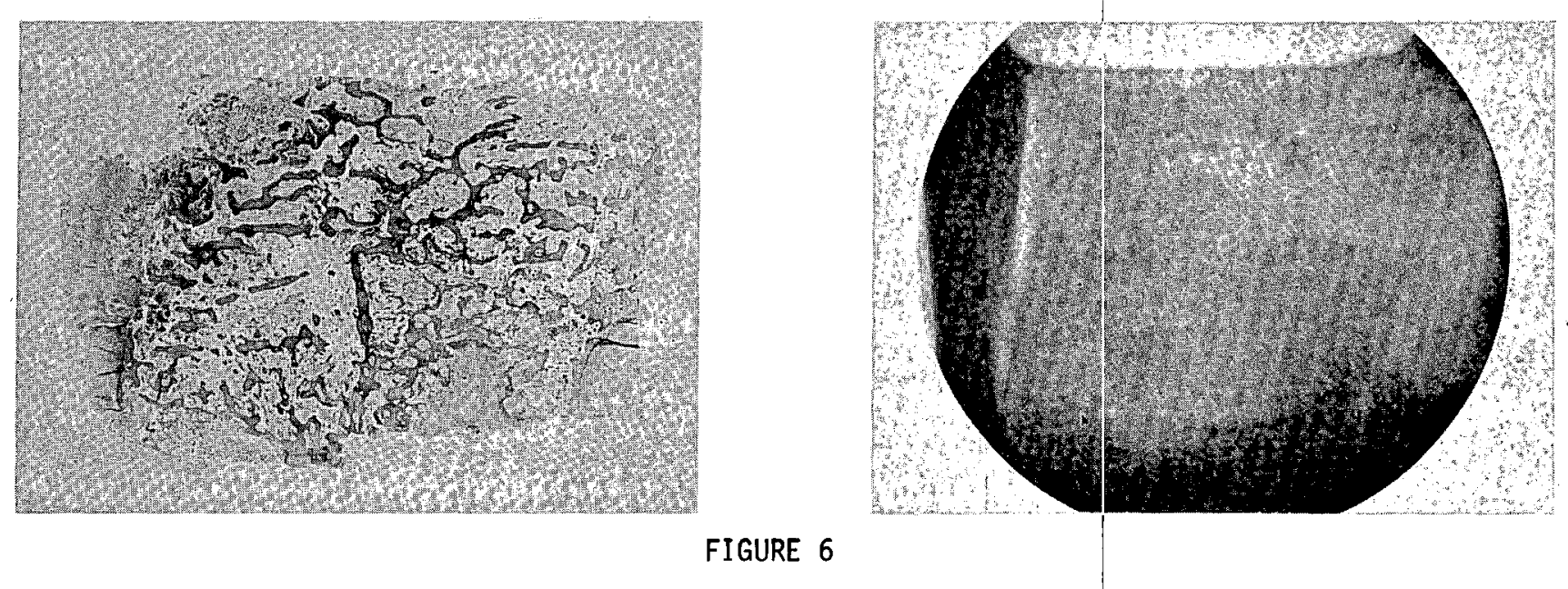

FIGURE 6

$H \& E$ section (left) and neutron radiograph (right) of bone section with severe tumor involvement (Prostate tumor metastasized to vertebrae)
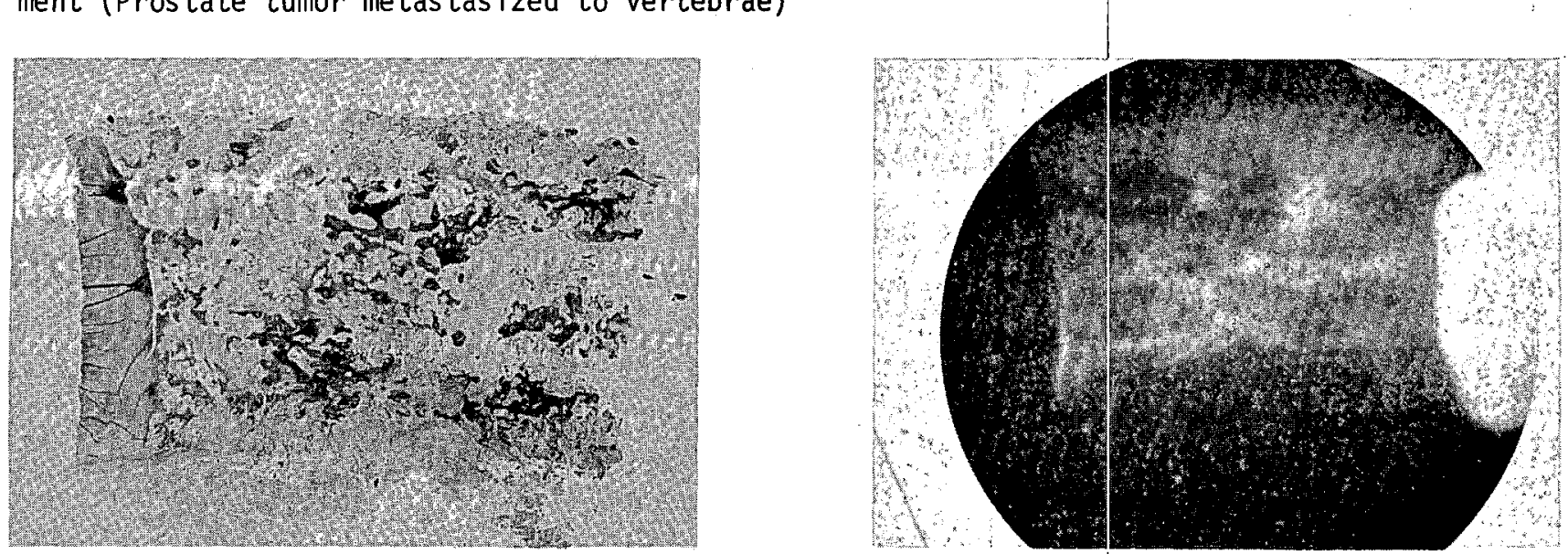

FIGURE 7

$H$ \& $E$ section (left) and neutron radiograph ( $r i g h t$ ) of bone section with severe tumor involvement and partial necrosis (adenocarcinoma of the breast metastasized to vertebrae)
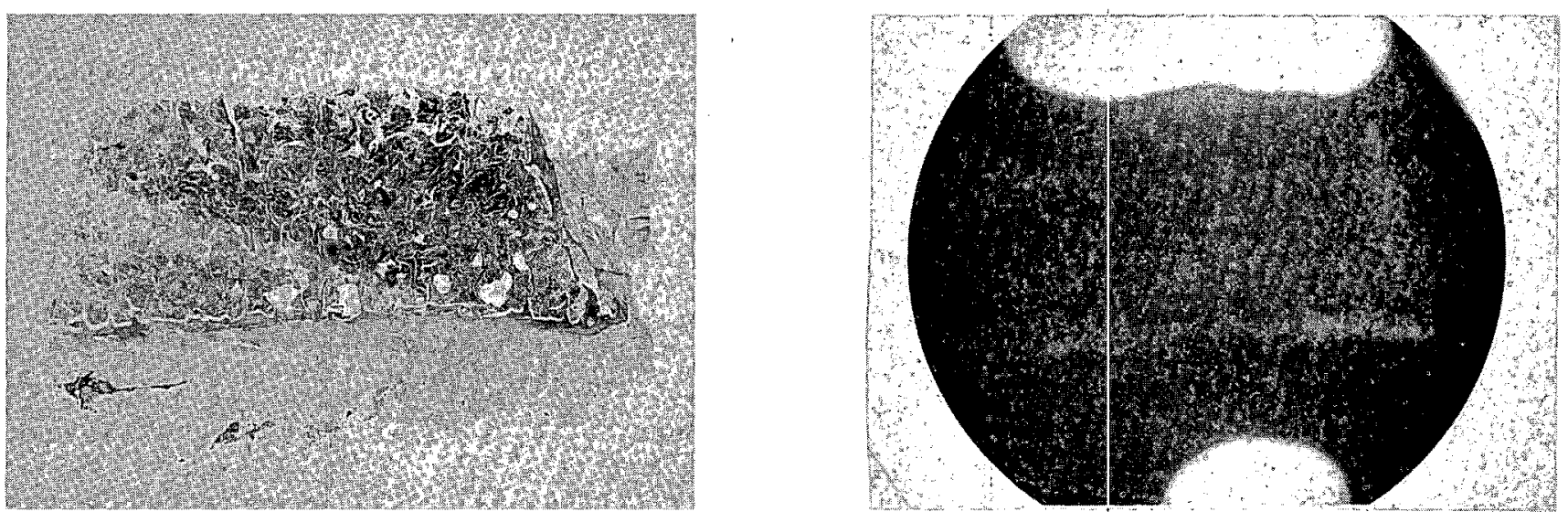

FIGURE 8

$H$ \& E section (left) and neutron radiograph (right) of a totally necrotic bone section (0at ce 11 carcinoma) 
TABLE 3

\section{CONTRAST SENSITIVITY AND MICROSCOPIC REACTION RATE AVERAGED CROSS SECTIONS}

\begin{tabular}{|c|c|c|c|c|c|c|c|}
\hline & & $\begin{array}{r}\text { FOR TH } \\
25 \text { Micr } \\
\text { Detec }\end{array}$ & $\begin{array}{l}\text { CK 'STAND } \\
\text { or }\end{array}$ & $\begin{array}{r}\text { TISSUE' }^{\prime} \mathrm{OB} \\
250 \mathrm{Mi} \\
\mathrm{Det}\end{array}$ & $\begin{array}{l}\text { ETS } \\
\text { gon In } \\
\text { ctor }\end{array}$ & $\begin{array}{l}250 \text { Micron } \\
250 \text { Micron }\end{array}$ & $\begin{array}{l}\text { In Det. } \\
\text { Cd Filt. }\end{array}$ \\
\hline $\begin{array}{l}\text { Contrast } \\
\text { Isotope }\end{array}$ & $\begin{array}{l}0.00 \\
0.50 \\
1.00 \\
2.00\end{array}$ & $\begin{array}{l}\text { Contrast } \\
\frac{\Delta \mathrm{D} / \mathrm{\mu g} / \mathrm{cm}^{2}}{\mathrm{D}} \\
2.12 \times 10^{-4} \\
1.82 \\
1.62 \\
1.37\end{array}$ & $\begin{array}{c}\text { Cross } \\
\text { Section } \\
\text { barns } \\
3527 \\
3025 \\
2691 \\
2274\end{array}$ & $\begin{array}{l}\text { Contrast } \\
\frac{\Delta \mathrm{D} / \mu \mathrm{g} / \mathrm{cm}^{2}}{\mathrm{D}} \\
2.27 \times 10^{-4} \\
1.76 \\
1.39 \\
.91\end{array}$ & $\begin{array}{c}\text { Cross } \\
\text { Section } \\
\text { barns } \\
3781 \\
2919 \\
2317 \\
1508\end{array}$ & $\begin{array}{l}\begin{array}{c}\text { Contrast } \\
\Delta \mathrm{D} / \mu \mathrm{g} / \mathrm{cm}^{2} \\
\mathrm{D}\end{array} \\
11.30 \times 10^{-5} \\
7.46 \\
5.25 \\
3.54\end{array}$ & $\begin{array}{c}\text { Cross } \\
\text { Section } \\
\text { barns } \\
1879 \\
1240 \\
873 \\
588\end{array}$ \\
\hline INDIUM & $\begin{array}{l}0.00 \\
0.50 \\
1.00 \\
2.00\end{array}$ & $\begin{array}{l}9.59 \times 10^{7} \\
8.34 \\
7.53 \\
6.58\end{array}$ & $\begin{array}{l}183 \\
159 \\
144 \\
125\end{array}$ & $\begin{array}{l}1.28 \times 10^{-6} \\
1.39 \\
1.75 \\
2.90\end{array}$ & $\begin{array}{l}244 \\
265 \\
334 \\
552\end{array}$ & $\begin{array}{l}3.67 \times 10^{-6} \\
4.70 \\
5.39 \\
5.92\end{array}$ & $\begin{array}{r}700 \\
897 \\
1027 \\
1128\end{array}$ \\
\hline Gd & $\begin{array}{l}0.00 \\
0.50 \\
1.00 \\
2.00\end{array}$ & $\begin{array}{l}1.12 \times 10^{-5} \\
1.13 \\
1.18 \\
1.34\end{array}$ & $\begin{array}{l}2913 \\
2945 \\
3084 \\
3504\end{array}$ & $\begin{array}{l}1.13 \times 10^{-5} \\
1.05 \\
.99 \\
.82\end{array}$ & $\begin{array}{l}2940 \\
2741 \\
2592 \\
2129\end{array}$ & $\begin{array}{l}4.88 \times 10^{-6} \\
2.89 \\
1.64 \\
.56\end{array}$ & $\begin{array}{r}1275 \\
754 \\
428 \\
147\end{array}$ \\
\hline
\end{tabular}

tumors has been verified, with the exception of the contrast produced by necrotic and highly advanced neoplastic involvement. It may be inferred from this that the previously suspected increase in hydrogen concentration is of a smaller magnitude than previously thought $(4 \%)$ and that this increase is as a result of the tumor damage and not a phenomena inherent in the tumor cells. It is speculated that this may be a result only of increased density in the necrotic core of the tumor. It should be carefully pointed out that the hydrogen concentration in the in vivo specimen may have been perturbed by the fixation and storing procedures; hence, these results must be interpreted in view of the specific procedures adopted.

\section{DETECTION OF LARGE CROSS SECTION ISOTOPES IN BIOLOGICAL MATERIAL}

The neutrographic contrast produced by small concentrations of large cross section isotopes has been examined from the perspective of determining the bound atom uptake in various regions of small animals. Of particular interest in this regard is the uptake in tumors of boron-10 compounds considered for application in neutron capture therapy; thus, the primary attention was focused on the determination of the minimum concen- tration of boron-10 which is visible on a neutron radiograph. The following presents contrast parameters for a number of isotopes calculated by a reaction rate analysis along with specific experimental results for the contrast produced by boron-10 in an organic test object.

Since most absorption cross sections are highly energy dependent, their effectiveness as contrast agents in thick hydrogenous objects will depend on the amount of spectral shift induced in the transmitted neutron curtent. The contrast produced per microgram/cc of an isotope may be calculated by adding a concentration dependent cross section to equation 1.2 ,

$$
\Sigma(E)=\left(C \frac{N o}{W} \times 10^{-6}\right) \sigma(E)
$$

where:

$$
\begin{aligned}
C & =\text { concentration in micrograms } / c c \\
N o & =.602 \times 10^{24} \\
\sigma(E) & =\text { microscopic cross section, barns } \\
W & =\text { formula weight of isotope }
\end{aligned}
$$

and differentiating the reaction rate, $R$, with respect to $\mathrm{Ct}$ (micrograms $/ \mathrm{cm}^{2}$ ):

$$
\frac{\mathrm{dR}}{\mathrm{dCt}}=-\left(\frac{\mathrm{N}_{0}}{\mathrm{~W}} \times 10^{-6}\right) \int_{0}^{\infty} \sigma(E) \mathrm{R}(\mathrm{E}) \mathrm{dE}
$$

or, 


$$
\begin{aligned}
\frac{\mathrm{dD}}{\mathrm{dCt}} & =-\left(\frac{\mathrm{No}}{\mathrm{W}} \times 10^{-6}\right) \bar{\sigma} \mathrm{D} \\
\bar{\sigma} & =\int_{0}^{\infty} \sigma(E) \mathrm{R}(\mathrm{E}) \mathrm{dE} / \int_{0}^{\infty} \mathrm{R}(\mathrm{E}) \mathrm{dE}
\end{aligned}
$$

The contrast sensitivity in units of inverse micrograms $/ \mathrm{cm}^{2}$ and the reaction rate averaged microscopic cross sections have been calculated for gadolinium, boron-10, and indium in various thicknesses of 'standard tissue' and for three detection systems. These results are presented for reference in Table 3 . The reduction in effectiveness of these contrast agents in thick objects is readily apparent; for example, the boron cross section is reduced by a factor of .76 when radiographed in a $1 \mathrm{~cm}$ thick biologic object with a 25 micron thick gadolinium detector. The contrast sensitivity for the cadmium filtered indium detector is seen to vary greatly for different objects and may increase or decrease with thickness depending on the isotope in question, pointing out the necessity of using reaction rate averaged cross sections for evaluating images in neutron radiography:

The contrast produced by various concentration of boron-10 has been experimentally examined using an acrylic plastic test object with an area of $2.5 \mathrm{~cm} \mathrm{X} 7.4 \mathrm{~cm}$ and a thickness of $1.15 \mathrm{~cm}$. The test piece had a $.6 \mathrm{~cm}$ diameter filter of cadmium and indium imbedded in the center so that the gamma fog and neutron scatter fog density could be recorded. Four void regions measuring $.6 \mathrm{~cm}$ thick by $.6 \mathrm{~cm}$ diameter were placed in the middle of the object and could be filled with different solutions by a syringe. Solutions with known concentrations of boron-10 were made from sodium metaborate and the film density produced on the neutrograph from these regions was recorded with an optical densitometer. These results are shown in Figure 9 along with a theoretical relation predicted by a reaction rate analysis. The experimental results validate the analytical techniques employed in the preceding sections and establish the contrast sensitivity for boron-10 when imaged by a 25 micron gadolinium foil.

The preceding results indicate that neutron radiography is not sufficiently sensitive to observe the in vivo uptake of boron-10 in small animals. For example, consider a $1 \mathrm{~cm}$ thick animal with a $.25 \mathrm{~cm}$ thick region subject to boron uptake, then a concentration of 247 ppm by weight would be required to achieve a contrast of $\mathrm{D} / \mathrm{D}=.01$ when using a 25 micron thick gadolinium detector. This concentration is of the same order of magnitude as
F.IGURE 9

B-10 CONTRAST

$1.15 \mathrm{~cm}$ THICK HYDROGENOUS TEST OBJECT

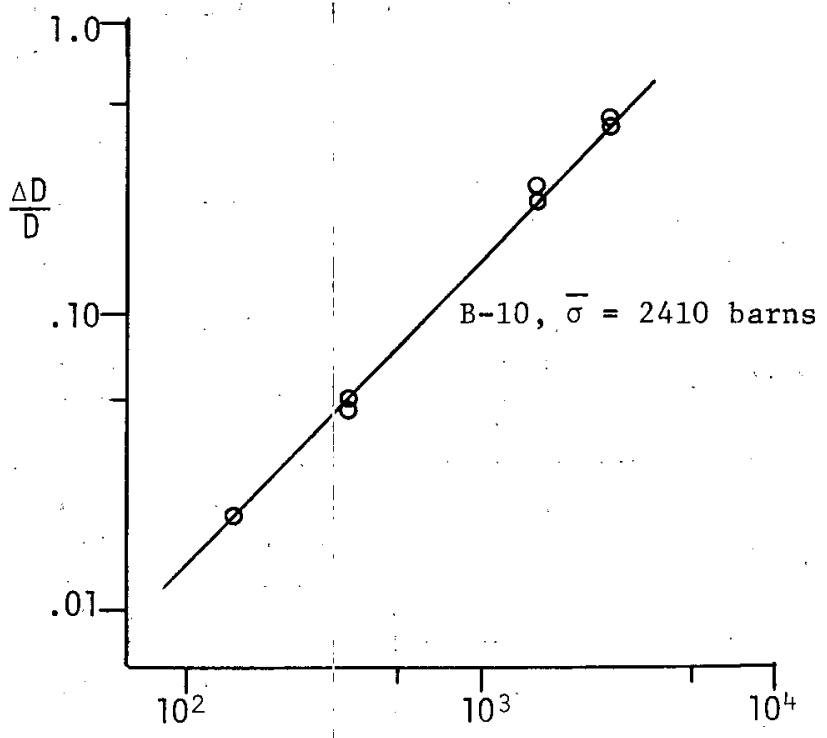

Boron-10 Concentration, $\mu \mathrm{g} / \mathrm{cm}^{2}$

might be expected throughout various regions of the animal Immediately following near lethal injection of: typical boron compounds, $(10,11)$ but is a factor of $5-10$ greater than static, bound concentrations presently being achieved in neutron capture therapy research $(9,12)$. Since there is no evidence that such a technicue would be adequate for any other isotopes, a program to investigate in vivo uptake has not been pursued. It is anticipated that the contrast parameters presented above will tie of use in other areas of bio-medical neutron radiography where the isotopes are considered in an unbound form, such as in arteriography.

\section{MICRO-NEUTROGRAPHY}

The techniques of micro-neutrography in the examination of biological material are considered below, albeit quite briefly. The success of this scheme in the examination of thin specimens depends first on whether sufficiently small resolution may be obtained to yield new patterns of differentiation and second on whether the differentiation or contrast sensitivity of these patterns is sufficient for a radiographic imaging system.

While these questions have not been answered. experimentally, several statements may be made based on analytical investigations.

The resolution characteristics of a neutron radiography facility may be examined 
by considering two contributions to the total image response:

I. $N\left(r^{f}-r\right) d r^{f}$, the neutron current distribution on the foil due to a neutron penetrating the object a $r$, i.e. a line spread function due to the finite size of the neutron source.

II. E(re-rf) dre, the energy deposition distribution in the emulsion for neutrons incident on the foil at $\mathrm{r}^{\mathrm{f}}$. This must detail the interaction mechanism of the neutrons in the foil, the decay spectra of activated nuclei, and the interaction of emitted radiations with the emulsion.

If the assumption is made that for all radiations emitted the specific energy loss per track length (Bragg relation) is constant throughout the foil and emulsion material and that energy is deposited in the emulsion as if all emitted particles have linear tracks (isotropic energy distribution) then a closed form analytical expression for the energy distribution function, similar to those describing autoradiographic resolution (13), may be derived for the case of a 'thin' detector. This function, while complex in nature, has a full width at half maximum of 2.15 microns for a 1 micron thick foil used in conjunction with a 1 micron thick emulsion. When this function is integrated with a neutron current distribution function having a width of $1 \mathrm{mi-}$ cron the resulting image density distribution for a line object has a width of 2.94 microns (assuming a linear density response). This approximate analysis points out the necessity for having a thin foil and a thin emulsion in addition to a small beam divergence, which has been confirmed by a more rigorous description of the resolution characteristics. It may be concluded that resolution on the order of several microns down to perhaps one micron may be obtained by using thin high resolution films such as Eastman Kodak High Resolution Film (14) in conjunction with thin vapor deposited foils (15). If the neutron current distribution is limited to a width of a 1 micron then the mean distance from the foil to the object material is limited to about 330 microns for a system whose beam has a half angle divergence of $1.5 \times 10^{-3}$ radians $(\mathrm{L} / \mathrm{D}=400)$. Thus, the micro-neutrography techniques should be of interest in the examination of specimens several hundred microns thick and placed in near contact with the detection system.
The contrast sensitivity provides a more severe limitation on the usefulness of microneutrography techniques than that imposed by resolution. Consider a 500 micron thick specimen and assume a reaction rate averaged cross section for the system of $3.0 \times 10^{-4}$ microns ${ }^{-1}$, then the contrast produced by the total specimen is $\Delta D / D=0.15$. The tissue cross section may be increased to about $7.3 \times 10^{-4}$ microns $^{-1}$ by using a cold neutron source with an average energy of $.001 \mathrm{eV}$ (neutron wavelength $=9.1$ angstroms) which would increase the contrast to $\Delta D / D=0.36$. This improved contrast would yield a system with greater ability to differentiate changes in hydrogen concentration while also increasing the foil absorption probability. Additional patterns of differentiation might be obtained by 'staining' with solutions containing large cross section isotopes. On the basis of the preceding it would appear that a micro-neutrography technique would be adequate to delineate regions containing relatively high hydrogen concentrations. Whether the technique is sufficiently sensitive to determine variations in hydrogen concentration or regions of contrast media for a thermal beam remains unanswered as with the sensitivity improvement provided by the use of a cold neutron source.

Contrary to the negative implications apparent in the first two sections of this paper, the application of micro-neutrography techniques in the examination of bio-medical specimens affords a positive ray of hope and it is for this reason that the concept has been presented at this time. It should be carefully noted, however, that the feasibility has not been proven and that its practical value will not be known until the examination of actual specimens has been accomplished. The authors are currently pursuing research in this area in an attempt to answer these questions and a more detailed analysis of the concept will be published at a latter date.

\section{SUMMARY}

In reviewing the results of the research presented in the preceding three sections it is apparent that no routine procedures or applications can be recommended at this time nor has the technique of neutron radiography proven valuable in the medical research applications considered. It is hoped that the procedures described here and the biological parameters derived will be of general use in. the evaluation of other possible bio-medical applications of neutron radiography. The 
section on bone specimens delineates the image properties of several different types of tissue of which neoplastic tissue is considered of prime interest. The tissue cross sections presented should be of value in determining the attenuation of objects other than those considered here. The behavior of contrast agents in biological material has been covered with some detail and the contrast sensitivity parameters should be useful as new applications are considered. The concept of reaction rate averaging has been stressed throughout this report as essential in predicting image response characteristics of thick objects. The averaging analysis is simple to perform and should be valuable in many bio-medical as well as industrial inspections of thick organic material.

\section{REFERENCES}

1. Barton; J. P., "Neutron Radiography Biological Aspects", Neutron Radiography Newsletter, No. 7, Nov. 1967.

2. M. Brown and P. B. Parks, "Neutron Radiography in Biological Media, Techniques, Observations, and Implications," USAEC Report No. DP-MS-68-2, E. I. du Pont de Nemours and Co., Savannah River Laboratory, Aiken, S. C. (1968).

3. M. Brown, J. J. Allen, P. B. Parks, "Slow Neutron Imaging of Fatty Tissues Through Deuteration with Heavy Water," USAEC Report No. DP-MS-68112, E. I. du Pont de Nemours \& Co., Savannah River Laboratory, Aiken, S.C. (1968).

4. H. Goldstein, Fundamental Aspects of Reactor Shielding, Chap. 2 (Reading, Mass.: Addison-Wesley Pub1. Co., Inc., 1959).

5. W. S. Snyder, J. Neufeld, "On the Passage of Heavy Particles Through Tissue", Radiation Research 6, 67-78 (1957).

6. Goldberg, Mughabghad, Purohit, Magurno, and May, Neutron Cross Sections, USAEC Report No. BNL-325 Supp1. No. 2, Second Edition, Brookhaven National Laboratory, 1966.

7. E. Melkonian, "Slow Neutron Velocity Spectrometer Studies of $\mathrm{O}_{2}, \mathrm{~N}_{2}, \mathrm{~A}, \mathrm{H}_{2}$, $\mathrm{H}_{2} \mathrm{O}$, and Seven Hydrocarbons", Physical Review, Vol. 76, No, 12, (1949).

8. C. H. Westcott, "The Specification of Neutron Flux and Neutron Cross Sections in Reactor Calculations", Journal of Nuclear Energy, 2, 59 (1955).

9. N. Frigerio, Private Communication, Argonne National Laboratories, Aug. 1970.

10. Fairchild, Tcinna, Seibold, and Straub, "Neutron Autcradiographic Determination of Boron-10 Concentration and Distribution in Mammalian Tissue", Radiation Research 36, 87-97 (1968).

11. A. H. Soloway, "Boron Compounds in Cancer Therapy" in Progress in Boron Chemistry, Vo1. 1 (New York: The Macmillan Company, 1964).

12. W. H. Sweet, "The Use of Thermal and Epithermal Neutrons in the Treatment of Neoplasms", Massachusetts General Hospital, Mass., Report TID 20856 (1964).

13. R. H. Herz, The Photographic Action of Ionizing Radiations (New York: WileyInterscience, 1969).

14. "Kodak High Resolution Film (Estar Thick Base), SO-343|' Kodak Pamphlet No. P-183, Eastman Kodak Co., Rochester, New York.

15. J. A. Dresser, Private Communication, Research Chemical, P. O. Box 14588 , Phoenix, Arizona 85031. 


\title{
NEUTRON RADIOGRAPHY IN DENTAL DIAGNOSIS
}

\author{
Manuel I. Weisman \\ Veterans Administration Hospital \\ Augusta, Georgia
}

Mark Brown

Medical College of Georgia

Augusta, Georgia

Neutron radiography has been felt to have a potentially useful role in the diagnosis of dental disease processes, particularly those involving the pulp of the tooth. Experiments were carried out to determine the applicability of the technique for use in a clinical situation. Radiographs comparing $X$-ray and neutron dental radiographs were obtained. Although additional information could be obtained using neutron radiography, the image quality was inferior. The technique was not thought to be clinically applicable because of a combination of physical, biological dose, and fiscal factors.

The internal structure of the tooth is very inaccesible to in vivo examination. It is surrounded by very hard tissues, the dentin and ename1. These are composed primarily of an inorganic calcium phosphate complex and are therefore relatively opaque to conventional x-rays. Ordinary radiographs of teeth are of little value in determining pulpal abnormalities unless the condition has deteriorated to such an extent to reveal a periapical osseous lesion. Pulpal abnormalities, such as chronic and acute pulpitis, early pulpal necrosis, hyperemia, and other degeneratory pulpal conditions that are confined to the tooth must be diagnosed by indirect crude tests and subjective observations.

Neutron radiography has been suggested as a method of directly visualizing some of these structures ( 1 ). In biological media, this phenomenon shows radiographic contrasts that are dependent primarily on the distribution of hydrogen atoms, and overlying calcified structures do not interfere $(\underline{2,3})$.

A study was devised to determine the applicability of neutron radiography to the study of the dental pulp. Conventional

The information contained in this abstract was developed during course of work under Contract AT (07-2)-1 with the U.S. Atomic Energy Commission. roentgenograms and thermal neutron radiographs of a fresh section of the mandible from a recently sacrificed dog ( $(24$ hrs.) were made. A premolar tooth was included in which the pulp had been removed. The Standard Pile Reactor of the Savannah River Laboratory was used as the neutron source. Dysprosium transfer techniques were used.

The neutron radiograph revealed the cuspid pulp ( $c p$ ) to be densely radiopaque: The dentin (d) was radiolucent, and the enamel (e) was still more radiolucent. The air filled pulp cavity (afpc) was clearly visualized.

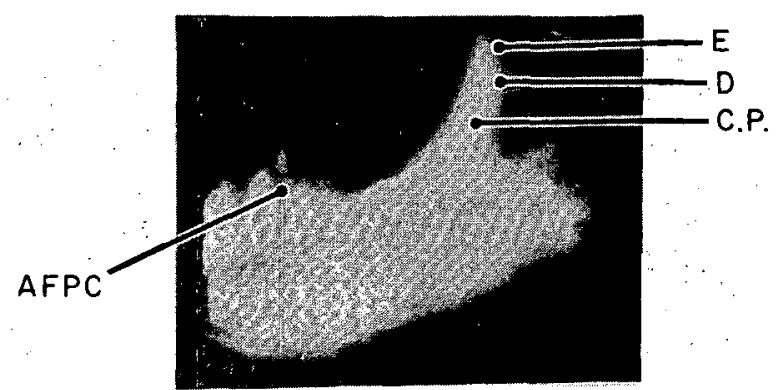

Fig. 1 Neutron radiograph $(0.025 \mathrm{eV})$

On the conventional $x$-ray studies, the radio-capacity of the enamel and dentin, as expected, precluded visualization of the 
normal pulp. However, the pulp chamber from which the pulp had been surgically removed (and replaced with air) was clearly seen. The radiographs taken with conventional $x$-ray techniques were significantly clearer and more readily interpreted.

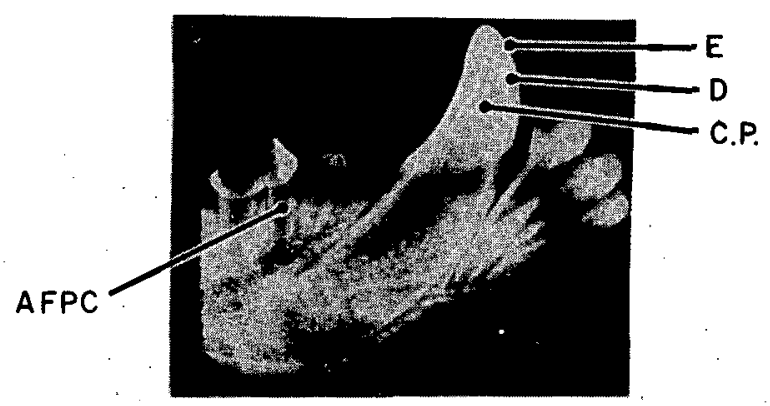

Fig. 2 Conventiona1 $\mathrm{x}$-ray study (60 kVp)

The neutron radiographs taken with the lip overlying the tooth were totally unsatisfactory due to interference from this hydrogen rich structure.

It is felt that the problems relating to providing dentists with safe, relatively inexpensive easy to use neutron sources probably precludes the use of neutron radiography as a practical clinical tool. In addition, scattered radiation to the very radio-sensitive lens of the eye (maximum permissible dose - 15 rem) poses a serious constraint. Finally, fast neutrons will probably be required to overcome interference from the overlying lips and cheeks, and an efficient fast neutron imaging system suitable for use in a clinical environment has not yet been developed.

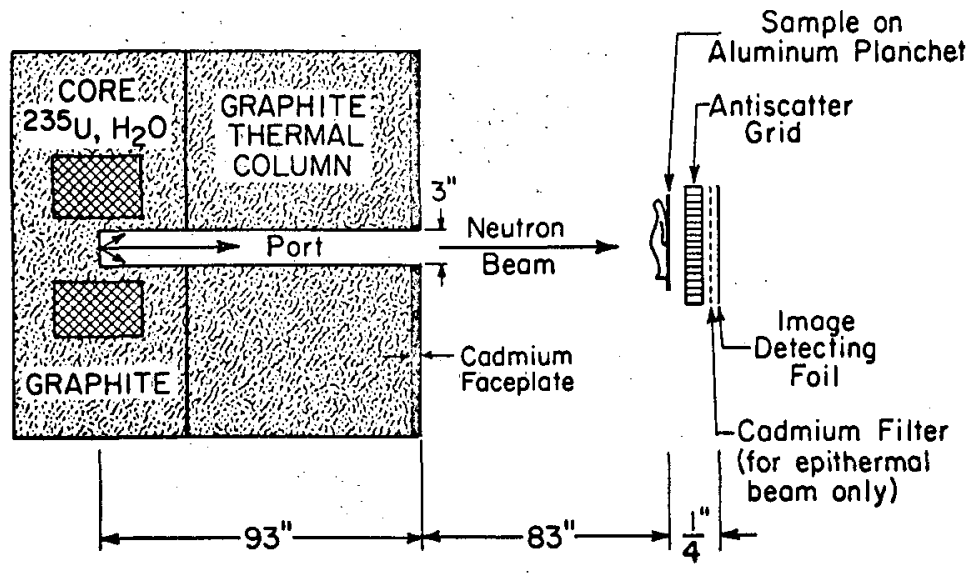

FIG: 3 THE EXPERIMENTAL ARRANGEMENT FOR LOW ENERGY NEUTRON RADIOGRAPHY USING THE STANDARD PILE REACTOR
Fig. 3 The Experimental Arrangement For Low Energy Neutron Radiography Using The Standard Pile Reactor

\section{REFERENCES}

1. M. Welsman and $M$. Brown, Neutron Radiography of the Dental Pulp. Proc. International Conf. on Pulpal Biol: Memphis, Ten:a., Sept., 1970. (In Press).

2. M. Brown and P. B. Parks. "Neutron Radiography in Biological Media: Techniques, Dbservations, and Implications.: Ain. J. Roent. 106, 472-485 (1969).

3. P. B. Parks, S. M. Reichard, and M. Brown. Deutel:ation in slow Neutron Radiography of Blological Media. USAEC Report - DP - 1229, E. I. du Pont de Nemours and Co., Savannah River Laboratory, Aiken, S. C. (1970).

4. M. Brown, J. J. Allen, and P. B. Parks. "Slow Neutron Imaging of Fatty Tissues Through Deuteration with Heavy Water" in Imaigery In Medicine, Biomedical Sciences Instrumentation, Vol. 6, p. 98-103: Thomas, D. F. and Sellers, E. E., Eds. Instrument Society of America, Pitt:sburg, Pa., (1969). 


\section{A ${ }^{252} \mathrm{Cf}$ FOCUSED NEUTRON GUN FOR ACTIVATION OF SKIN- TISSUE TO DETECT ABOVE-NORMAL AMOUNTS OF SODIUM}

Zahoor M. Alvi

Kaiser-Permanente Medical Center Los Angeles, California
A portable californium-252 neutron gun is designed to activate sodium atoms contained in the skin and subcutaneous tissue. Two sealed sources 10 microgram $(0.54 \times 10 \mathrm{mCi})$ each are positioned inside the probe assembly. Two cadmium lined collimators converge the neutron beams from the two point sources. The entrance field is just $1 \mathrm{~cm} \times 1 \mathrm{~cm}$ at the skin surface. The exposure time is of the order of 15 minutes. The thermal neutrons and fast neutrons with a maximum at $1 \mathrm{Mev}$, activate the sodium atoms and also other elements contained in a tissue volume about $2 \mathrm{ml}$ located few $\mathrm{mm}$ below the outer skin surface. The estimated induced activity is of the order of $2000 \mathrm{dpm}$. The Na-24 atoms that are formed decay with the emission of 1.37 Mev gamma photons. This activity is picked up by a solid-state lithium-drifted silicon detector mounted above the probe at the end of the central channel which provides direct pathway from the activated tissue. The $\mathrm{Li}(\mathrm{Si})$ detector provides good scatter discrimination. The detector output goes through a pulse-height analyzer and is related to the concentration of the sodium content below the skin near the sweat glands. Likewise potassium concentration can be determined. The $\mathrm{Na} / \mathrm{K}$ ratio can be used as a detection index for cystic fibrosis. The ratio is $2 \frac{1}{2}$ times higher for children with cystic fibrosis compared with children considered normal. The probe is intended to be compact and portable enhancing the application of neutron activation analysis in clinical medicine and research. The probe obviates the necessity of collecting any biological samples.

\section{INTRODUCTION}

The technique of Neutron Activation Analysis has been applied by various investigators in recent years to measure significant differences in the concentrations of some inorganic elements in biological samples taken from patients with cystic fibrosis. Sweat, Urine, Plasma, Duodenal fluid, teeth, finger \& toe Nails and Hair from the $\mathrm{CF}$ patients and from the Controls were subjected to neutron radiation inside a nuclear reactor. The irradiated samples were removed from the reactor and after waiting for some 24 hours for the short-lived activity to die-off, were counted in a well-type scintillation counter. In this fashion sodium, potassium and to a lesser degree magnesium, silicon, phosphorus, manganese, calcium and to a still lesser degree copper, iron, aluminium, titanium and boron were studied.

In the main their results show that sodium in the toe-nail is twice in concentration for the $C F$ compared to the normal. Eccrine sweat specimens show increased levels of sodium (2-5 times norma1) and Potassium (1-3 times norma1). There have been two major handicaps in the effective application of the very sensitive method of NAA for the diagnosis of cystic fibrosis.
One is that so far it has required the availability of nuclear reactor. The second and the most important is the fact that collection of the required biological samples is not an easy task and there are too many almost uncontrollable variables that enter into the process of sample collecting and subsequent handling or processing of the collected samples.

The paper describes a focussed neutron gun which is designed to obviate the necessity of collecting any biological samples. The activation is performed in-vivo. The design has been developed with utmost consideration to the clinical situation and the ease and reliability of operation so vitally needed in medical applications .

\section{Cf-252 FOCUSSED PROBE}

Figure 1 shows the details of a portable neutron probe using two loug Cf-252 sealed sources. Hoffer et.al. (1) have shown the feasibility of imaging iodine in the thyroid and in the cerebral blood flow measuring $\mathrm{K}$-shell fluorescence of the stable iodine using Am-241 in their probe. The neutron gun is designed with 10 microgram sources of Californium- 252 although it is conceivable that smaller neutron sources may be sufficient. 


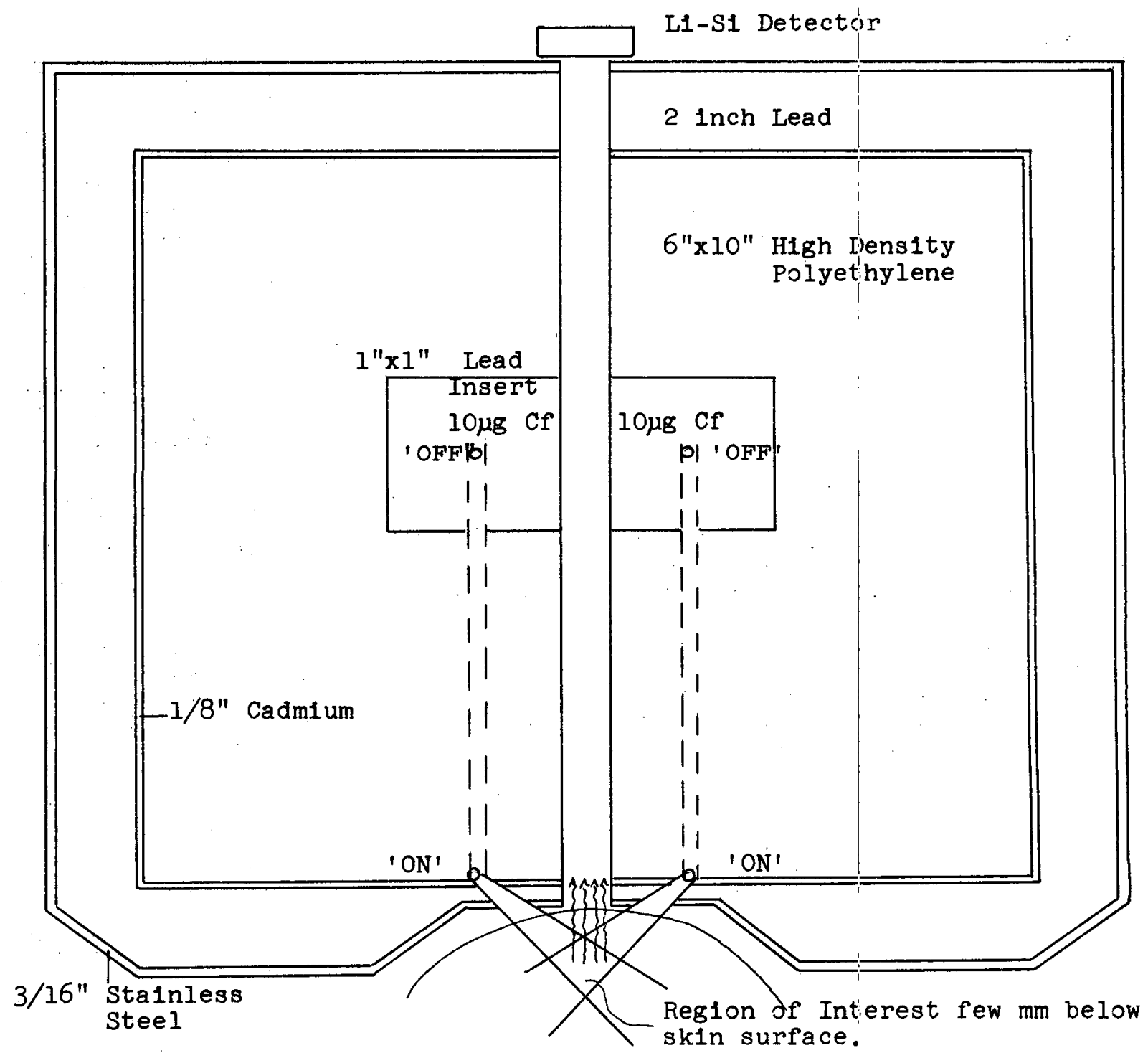

F1g. 1 Portable Neutron Probe using two $10 \mu \mathrm{g} C f-252$ sealed sources for neutron activation analysis of the dermal tissue for CF.

The two divergent collimating cones are cadmium lined.

Lithium-drifted Silicon Detector permits good scatter discrimination. (Nuclear Equipment Corporation) 
The probe incorporates multiple shielding design such that the surface dose remains within the permissible levels when the two sources are in the 'OFF' position as shown in Fig.1. There is 1 cubic inch lead insert which immediately surrounds the source proper in the 'OFF' position. Two blocks of high density polyethylene, each measuring $6 "$ wide, $10^{\prime \prime}$ high and $10 "$ arc length as part of the circumference of the cylindrical probe, surround the two lead inserts as shown. The high density polyethylene is enriched in natural Lithium to reduce capture gamma dose rates. The reduction results from the $6_{\mathrm{Li}}(n, \alpha) \mathrm{T}$ reaction which has a 945-barn cross section for thermal neutrons and produces no gamma radiation. The lithium absorption over rides the $H(n, r) D$ reaction (2) in the hydrogenous material, which is the predominant source of the secondary gamma activity. The passage of the sources from the 'OFF' to the 'ON' positions is through two narrow vertical slits in the two polyethylene blocks. The two blocks are lined with 1/8" Cadmium to further moderate high energy neutrons from the two sources. Following this there is two inch lead shield all around which is narrowed near the ' $O N^{\prime}$ positions. The lead shield is encased with $3 / 16$ " Stainless Steel. Along the central axis of the probe there is an open channel $\frac{1}{2} "$ " wide which provides a direct pathway for the gamma photons emerging from the activated nuclii contained within the irradiated tissue volume. The channel $i$ tself is not lined with any high $Z$ material which may contribute to scatter photons. In the 'ON' position the two sources sit behind the two divergent collimating cones which are also cadmium lined. The cones bring the two neutron beams to converge few $\mathrm{mm}$ below the skin surface. The entrance field at the skin is $1 \mathrm{~cm} x 1 \mathrm{~cm}$ for each neutron beam. The subcutaneous tissue volume enclosed is about $2 \mathrm{ml}$. The sodium in the form of $\mathrm{NaCl}$ contained in $2 \mathrm{~m} 1$ of the irradiated volume is estimated to be about $0.00446 \mathrm{gm}$. Therma1 neutrons from $\mathrm{Cf}-252$ cause the following reaction with natural sodium:

$$
\begin{aligned}
& 23 \mathrm{Na}+\mathrm{n}={ }_{11}^{24} \mathrm{Na}+\gamma(1.37 \mathrm{Mev}) \\
& \mathrm{Ha} 1 \mathrm{f}-1 \text { ife of } \mathrm{Na}-24,15 \mathrm{hrs} .
\end{aligned}
$$

The gamma photons from $\mathrm{Na}-24$ are detected by a solid-state lithiumdrifted Silicon detector (Nuclear Equipment Corp.) which is mounted above the central channel. The selection of Li-Si detector was prompted by the fact that it permits good scatter discrimination (1). The proper use of $\mathrm{Ge}(\mathrm{Li})$ detector requires the equipment that goes with the use of liquid nitrogen. Ge(Li) detectors are known to resolve the peaks of $\mathrm{Na}$ and $\mathrm{K}$ very well, a fact which NaI(T1) crystal detectors can not do well. With superior electronic subtraction techniques, NaI(T1) crystals may also be appropriate for the detection system.

\section{ESTIMATION OF SODIUM CONCENTRATION FEW MM BELOW SKIN:}

$$
\begin{aligned}
& \text { Sodium in human body }= \begin{array}{l}
0.15 \% \text { of total } \\
\text { body weight }
\end{array} \\
& \text { "Standard" man weighs } \begin{aligned}
70 \mathrm{~kg} \text { or } \\
70,000 \text { grams }
\end{aligned}
\end{aligned}
$$

\section{Skin weighs 2000 grams}

Subcutaneous Tissue weighs $4100 \mathrm{gms}$

$$
\begin{aligned}
\text { Total Sodium } & =0.15 \% \text { of } 70,000 \mathrm{gms} \\
& =105 \mathrm{gms} .
\end{aligned}
$$

$\mathrm{Na}$ in skin+subcutaneous tissue

$$
\begin{aligned}
=(6100 / 70,000) \times 105= & 9.15 \mathrm{gm} \\
& \text { in } 6100 \mathrm{~m} 1
\end{aligned}
$$

$\mathrm{Na}$ Conc. $=9150 \mathrm{mg} / 6100 \mathrm{ml}=1.5 \mathrm{mg} / \mathrm{ml}$

$\mathrm{Na}$ Conc. in Subcutaneous tissue alone $=9150 \mathrm{mg} / 4100 \mathrm{ml}=2.23 \mathrm{mg} / \mathrm{ml}$

Assuming all $9150 \mathrm{mg} \mathrm{Na}$ to be in subcutaneous tissue.

Checking above figure against another approach we note that from sweat test results for cystic fibrosis,

$\mathrm{Na}$ has been reported to be in the range $70 \mathrm{mEq} / \ell$.

$$
\begin{aligned}
\text { Since } & 23 \mathrm{mg}=1 \mathrm{mEq} \\
& 70 \mathrm{mEq} / \ell=1.61 \mathrm{mg} / \mathrm{m} 1
\end{aligned}
$$

The tissue is expected to contain somewhat greater amount of $\mathrm{Na}$ than 
the sweat it produces. Thus the 2.23 $\mathrm{mg} / \mathrm{ml}$ concentration appears to be reasonable.

$\mathrm{Na}$ in the subcutaneous tissue volume of $2 \mathrm{cc}$.

$=2.23 \mathrm{mg} / \mathrm{m} 1 \times 2 \mathrm{~m} 1$

$=4.46 \mathrm{mg}$

$=0.00446$ grams .

ESंTIMATION OF INDUCED ACTIVITY

Neutron activation analysis requires that we atleast have good estimate of the expected activity within the bounds of the equipment and the factors applied. The activity induced can be calculated from the following equation:

$A=N \emptyset \sigma_{\text {th }}\left[1-e^{-\frac{0.693}{T_{\frac{1}{2}}} \cdot t}\right]$

where

$\mathrm{A}=$ dps of activated $\mathrm{Na}-24$ atoms

$\mathrm{N}$ = Sodium nuclii in the tissue vol.

$=$ (Avogadro's No./Mass No.) $\mathrm{X}$ gm. of sodium in the irradiated tissue vol. in vivo.

$\emptyset_{\text {th }}=$ thermal neutron flux $2.34 \times 10^{6} \mathrm{n} / \mathrm{sec}$ per $\mu \mathrm{g}$ of $\mathrm{Cf}-252$

$\sigma_{\bar{x}}=\underset{\operatorname{barns}\left(0.53 \times 10^{-24} \mathrm{~cm}^{2}\right)}{\text { Crosection in }}$

$\mathrm{T}_{\frac{1}{2}}=$ Half-1ife of induced radionuclide. For Na-24; 15 hrs.

$t=$ Duration of irradiation 15 minutes.

Using two $10 \mu \mathrm{gg} C-252$ sources and for the irradiation time of $15 \mathrm{~min}$.

$$
\begin{aligned}
& \begin{aligned}
A= & \left(6.02 \times 10^{23} / 23\right) \times 0.00446 \\
& \left(20 \times 2.34 \times 10^{6}\right)\left(0.53 \times 10^{-24}\right)\left(1-e^{-0 / 155}\right)
\end{aligned} \\
& =\left(1.167 \times 10^{20}\right)\left(46.8 \times 10^{6}\right)\left(0.53 \times 10^{-24}\right) \\
& =33.288 \mathrm{dps} \\
& A \cong 2000 \mathrm{dpm}
\end{aligned}
$$

EXPOSURE DOSE ESTIMATION

Source: Two 10 microgram Cf-252 sealed sources.

SSD: 1 to 2 crn source to skin distance.

Entrance Field: $1 \mathrm{cmxlcm}$ for each neutron beam.

Tissue Volume: about $2 \mathrm{~m} 1$ located 1 to $2 \mathrm{~cm}$ below skin where the two convergent neutron beams intersect.

Established Skin Tolerance Dose:

$1570 *$ RADS at $15 \mathrm{rads} / \mathrm{hr}$.

(Increase in lose rate, understood to have little effect on the tolerance level)

Gamma Emission Rate: $1.3 \times 10^{8} \mathrm{r} / \mathrm{sec}$ at 0.043 and

0.1 Mev from $10 \mu g$

Neutron Emissiton Rate:

$2.34 \times 10^{7} \mathrm{n} / \mathrm{sec}$ with max. at $1 \mathrm{Mev}$ from $10 \mu \mathrm{g} \mathrm{Cf}-252$

Now from p.52?, Ref.(ㄹ)

$7.1 \times 10^{4} / \mathrm{Exp}_{\mathrm{e}} \mathrm{photon} \mathrm{s} / \mathrm{cm}^{2} / \mathrm{sec}$

$=1 \mathrm{Rem} / \mathrm{sec}$

where $E_{r}$ is Mev per photon which for Cf-252 is 0. LMev.

$\mu$ is the energy absorption co-efficient of ai:.

From Fig.9.1, p.522, Ref.(3) $\mu_{e}=\begin{aligned} & 3 \times 10^{-5} \mathrm{~cm}^{-1} \text { at } 0.1 \mathrm{Mev} \text {. The } \\ & \text { curve is near flat for energ- }\end{aligned}$ ies above $0.1 \mathrm{Mev}$.

Therefore for $1.3 \times 10^{8}$ photons/sec we have

$$
\begin{array}{ll}
\left(0.1 \times 3 \times 10^{-5}\right) /\left(7.1 \times 10^{4}\right) & \times 1.3 \times 10^{8} \times 1 \\
& \times 60 \times 15
\end{array}
$$

$=5$ Rem for 15 min. Exposure.

*Californium-?52 Progress Report非4, p. 8, Aug. 1970. 
Neutron Dose

From Fig.9.4, p.528, Ref. (3)

$8 \mathrm{n} / \mathrm{sec}$ at $1 \mathrm{Mev}$ deliver $1 \mathrm{mRem} / \mathrm{hr}$

Therefore $2.34 \times 10^{7} \mathrm{n} / \mathrm{sec}$ will

deliver $1 / 8 \times 2.34 \times 10^{7}=2900 \mathrm{Rem} / \mathrm{hr}$

and for $15 \mathrm{~min}$. exposure, neutron dose is 725 Rem.

Total $r+n$ dose at the entrance

field $1 \mathrm{~cm} x 1 \mathrm{~cm}$

$$
=5+725=730 \text { Rem }
$$

This is less than $50 \%$ of the more or less established dose for skin tolerance which is 1570 rads over $6 \mathrm{~cm} \times 6 \mathrm{~cm}$ skin area.

In the interest of the medical diagnostic test information, the calculated dose is considered here to be acceptable. It may turn out that smaller strength sources and less exposure time are adequate for sufficient amount of activity induced. In that event, the entrance dose will be greatly reduced. The design does require optimization from this point of view

\section{CONCLUSION}

The portable Cf-252 focussed neutron gun has design features which make it immediately applicable in clinical medicine and in research. In-vivo neutron activation analysis is possible with reasonable exposure periods. The exposure period will be optimized according to the strength of activity induced. Additional activation information with Cf-252 is available from (4) and (5). The multiple shield design, (6), has been carried out with the intent that surface doses are within permissible limits and the overall size of the probe is compact enough for easy adjustment over the limb area to be irradiated. The output of the detector goes through a pulse-height analyzer and is related to the concentration of the sodium content in the tissue. Likewise Potassium concentration can also be established. The $\mathrm{Na} / \mathrm{K}$ ratio can be used as a detection index for cystic fibrosis. It has been established by various investigators that this ratio is some $2 \frac{1}{2}$ times higher for children with cystic fibrosis compared with children considered normal. The probe has the greatest advantage in that it obviates the necessity of collecting any biological samples. As far as the use of $\mathrm{Cf}-252$ sources in radium-type meta11ic applicators is concerned, dosimetric considerations have been worked out in (7). It will be necessary to calibrâte the neutron probe using a especially prepared phantom which simulates the geometry of the in-vivo test and has comparable concentrations of sodium and other elements. In addition to the proposed application, it is anticipated that the neutron probe will be equally useful in the external measurement of elements such as calcium in peripheral bone structure etc.

\section{REFERENCES}

1. P. B. Hoffer, R. E. Polcyn, R. Moody, H. J. Lowe and A. Gottschalk. "Fluorescence detection: Application to the study of cerebra1 blood flow". J. Nuc1. Med. 10, 651 (1969).

2. Californium-252 Progress, 非, Nov.1970, Savannah River Lab', Aiken, S. Carolina.

3. S. Glasstone and A. Sesonske. Nuclear Reactor Engineering. p.522 and p.528, D. Van Nostrand Co., Inc., Princeton, N.J. (1967)

4. E. Ricci and T. H. Handley. "Activation analysis with Cf-252" Anal. Chem., 42, 378 (1970)

5. R. G. McAndrew, J. B. Smathers, R. E. Wainerdi, G. M. Harrison and R. Doggett. "Application of NAA to the sweat test diagnosis of cystic fibrosis". Nuc1. Apn1. Tech. 8,290 (1970)

6. H. E. Hootman. Estimation of Cf-252 Shielding Requirements. USAEC Report DP-1232, E.I. du Pont de Nemours \& Co., Savannah 
River Laboratory, Aiken, S.C. (1970).

7. Z. M. Alvi. "Cf-252 versus

Ra-226 Dosimetry." Trans. Am. Nuc1. Soc. 13, 435 (1970). 


\section{PRACTICAL APPLICATIONS AND LIMITATIONS OF FORENSIC ACTIVATION ANALYSIS}

\section{Michael Hoffman Maynard J. Pro}

U.S. Treasury Department

National Office Laboratory

Alcohol, Tobacco and Firearms Division

Washington, D. C.
FAA is an accepted and valuable tool for examining many types of physical evidence and it is currently used on a routine basis to supplement and extend other types of analyses. Soil, hair, paint, and gunshot residue are some of the materials commonly encountered in the crime laboratory to which FAA may be appropriately applied.

FAA can rarely be used alone, however, since other information about the crime scene samples and specimens from the suspect must be obtained by using multiple methods of analysis. Other factors such as analytical time and cost limit the use of FAA in the crime laboratory.

\section{TNTRODUCTION}

Neutron activation analysis used for the examination of evidentiary materials in criminal cases has been labeled forensic activation analysis (FAA). Many of the more common types of physical evidence amenable to chemical analysis have received at least some study by FAA to determine if this analytical method can be used to characterize these materials.

From the forensic viewpoint, the ultimate analytical achievement would be the ability to establish that a material with unique composition found at the crime scene completely matches a material with the same unique characteristics found, for example, on the suspect's clothing. This ability to establish, with a reasonable scientific certainty, that the two materials came from the same source and from no other would provide the basis for a positive identification. This complete characterization would be the chemical equivalent of a fingerprint identification with 12 points of comparison between the latent and inked prints.

Even though complete characterization of evidentiary materials is not possible through the use of FAA alone (or any other single analytical technique for that matter), much useful data about many types of physical evidence can be obtained. Often, when combined with information from other types of examinations, FAA findings may contribute consider- ably towards establishing the guilt or innocence of a suspect.

FAA has now developed to a point where it is an accepted tool for the examination of many evidentiary materials. It has not only been accepted in many trial courts, but its use has been upheld by superior courts as well.a,b It is the purpose of this paper to discuss some of the practical uses of this nuclear technique as well as its limitations when used on a routine basis in actual criminal cases.

\section{FAA APPLICABLE MATERIALS}

HAIR

Hair studies performed in Canada (I) and England (2) called attention to the value of FAA for examining this substance. Since that time, FAA has been further explored, and the similarity of the trace elemental composition of questioned and known hairs connected with criminal cases have been used to support and extend the microscopic examination of this material.

akelly v. U.S. United States Court of Appeals for the Second Circuit (10/28/69)

bStifel v. U.S. United States Court of Appeals for the Sixth Circuit (10/29/70) 
While FAA provides additional information for a conclusion that questioned hair could have come from a suspect, this conclution does not approach the degree of certainty of a fingerprint identification. In addition, there are analytical difficulties which arise because hair specimens from the scene are usually small or few in number, and may be contaminated with blood or other foreign substances.

In practice, only a small percentage of criminal cases justify the use of FAA for hair examinations. If the hairs are microscopically, indistinguishable, can be freed from possible contamination, and represent an adequate sample size, then FAA can be appropriately used.

\section{PAINT}

Tiny flecks of paint, visible only with a microscope are frequently encountered in

debris submitted for examination and comparison. If the questioned paint cannot be distinguished microscopically from that of the known source, the use of FAA is indicated. In cases of this type, FAA is not only valuable for examining small samples, but is also particularly suited for the analysis of black paint, which cannot be compared microscopically with respect to color and hue.

A study of black paints produced by 26 major U.S. manufacturers showed that the products of each manufacturer were distinguishable by FAA (3). It was further found that, while batch differences did exist, in about one-third of the black paints produced by these manufacturers no significant differences could be detected.

SOII

Encrustations of dried mud on a suspects clothing or possessions can be compared with soil from a specific location. Nomally, these soils are examined microscopically to determine their mineral compositions and to search for the presence of unusual foreign materials, such as paint chips or metal fragments. Density gradient measurements may also prove valuable in revealing the proportions of each soil fraction in the samples being compared.

FAA is useful in conjunction with these and other methods of instmmental analysis for determining the elemental composition of the questioned and know specimens. In practice, soils are examined by both non-destructive FAA and atomic absorption spectroscopy, because in the average criminal case involving soils, it has been found that a total of 20 or more elements can be detected and quantitatively measu'red when both methods are used (4).

\section{GUNSHOT RES IDUE}

FAA provides a sensitive and specific, although time consuming, method of detecting gunshot residues. Barium and antimony, when present in the cartridge primer, are deposited on the shooter's hand when the cartridge is detonated. The residue may be removed mechanically using the paraffin cast technique (5) in the crime lab or with cotton -tipped swabs (6) under field conditions.

It is necessary to examine an appropriate blank to be certain $\mathrm{Ba}$ and $\mathrm{Sb}$ are not part of the testing materials. It is also desirable to sample the residue from the interior of the spent cartridge case, if it is available, to determine the presence or absence of $\mathrm{Ba}$ and $\mathrm{Sb}$ in that particular brand and caliber of ammunition.

The detection of $\mathrm{Ba}$ and $\mathrm{Sb}$ on the back of the suspect's shooting hand and its absence on his other hand is indicative of the presence of gunshot residue. Since the argument may be raised that these elements represent occupational contamination, all swabs or paraffin casts should be examined for the presence of foreign material such as paint or grease so that these substances can be removed if they are present.

\section{MISCELIANEOUS MATERIALS}

Glass. The color, refractive index, and density of glass are useful properties for comparison purposises, but give little information about the composition of this amorphous material. FAA has been used to obtain information about the trace elemental composition of randomily collected window glass and the compositional variations within a single sheet ( 7$)(\underline{8})$.

Biillet Lead! Bullets are often badly mutilated or framented when they strike bone or other haind substances. If a ballistics comparison is not possible due to muti- 
lation of the bullet, FAA examination of the bullet leads removed from the victim and from the cartridges in the suspect's possession may be of some value.

Antimony, and occassionally tin, are added to bullet leads as hardening agents. Antimony may be determined non-destructively by FAA, and other elements such as As, Cu, and $\mathrm{Ag}$ may be measured after radiochemical separations or by atomic absorption spectroscopy.

Preliminary work on bullet leads by FAA and atomic absorption has indicated that the three major U.S. manufacturers use different alloys in their bullet leads (9) (10), and that batch differences do exis $\bar{t}$. $\overline{\mathrm{At}}$ present, the conclusions drawn from this type of comparison must be limited, because a single batch of lead normally represents a large number of bullets.

\section{PRACTICAL LIMITATIONS}

Forensic laboratories, which examine physical evidence from several hundred criminal cases each year, must operate under certain limitations which affect the extent to which FAA can be used.

\section{$\operatorname{cost}$}

The analysis is expensive and requires a high flux nuclear reactor and gamma-scintillation spectrometers with appropriate read-out equipment. While a neutron generator may be suitable for certain types of laboratory analyses, it cannot be used for volume work of the type required in comparative forensic analyses because of its low flux and limited sample capacity.

\section{TIME}

Unless the forensic laboratory is fortunate enough to have an on-site reactor, transportation time both to and from the reactor represents a considerable portion of the analytical time, and limits the analysis to the measurement of the Ionger lived radionuclides. Counting time can represent 2 hours or more per sample during the final stages of non-destructive analysis, and although more elements can be determined by performing radiochemical separations, this technique is time consuming and may not be feasible, especially with materials of unknown trace composition.

\section{NONDETECTABLE ELEMENTS}

Many. elements cannot be detected by FAA in some types of material Decause certain elements have small nuclear cross sections or have short half-lives. For example, even though soils contain approximately $1 \%$ aluminum, the 2.3 minute $28 \mathrm{Al}$ has decayed before it can be counted if the sample is not analyzed at the reactor site. Even elements with good sensitivities, if present in low concentrations, cannot be detected in samples with complex inorganic matrices without radio -chemical separations.

\section{NONDESTRUCTIVE FAA}

With the exception of $\mathrm{Ba}$ and $\mathrm{Sb}$ determinations in gunshot residues, it is usually not feasible to analyze physical evidence other than nondestructively because most materials cannot be characterized solely by FAA. For this reason, FAA is supplemented by other techniques such as microscopy, atomic absorption, x-ray diffraction, and GLC which are used to identify the substance and to obtain additional information for an adequate comparison of the evidentiary materials.

\section{FAA AND THE EXPERT WITNESS}

The role of the expert witness is solely to assist the court in reaching a decision. He brings to the court a knowledge beyond that possessed by the average lay juror and demonstrable observations he has made in the course of his examination of the evidence.

When FAA results are presented, the glamorization of the technique must be avoided not only because of the witnesses ethical responsibilities to the jury, but because this "window dressing" constitutes reversible error which is the basis for a new trial. The expert witness presents facts derived from his examinations and the court accords him an opportunity not given other types of witnesses..... the permission to express an opinion based on his experience, tests, and observations. This expert opinion however, is not binding on the jury, who can accept or reject it either totally or in-part. 
SUMMARY

As shown by research performed during the past decade, forensic activation analysis is a useful tool for examining many types of physical evidence. It is a șensitive, specific method of elemental analysis which can be performed non-destructively on extremely small specimens. The non-destructive capabilities of FAA permit the use of other analytical methods for obtaining additional information about the significant properties of the evidentiary material.

The experience gained in examining thousands of evidentiary specimens by FAA clearly shows that FAA is a valuable adjunct to other types of examinations. The lengthy analytical time and high cost of the analysis is more than justified when the only evidence available in a criminal case is to small to be examined by other instrumental methods. Even when enough material is present for multiple instrumental analyses, the use of

FAA is desirable since it permits a comparison of the trace elemental composition of the questioned and known specimens.

\section{REFERENCES}

1. A. K. Perkons and R. E. Jervis. "Trace Elements in Human Head Hair." J. of Forensic Sciences 11 (1), 50-63 (1966).

2. R. F. Coleman, F. H. Cripps, A. Stimson, and H. D. Scott. The Determination of Trace Elements in Human Hair by Neutron Activation and the Application to Forensic Science. AWRE Report \#0-86/66, United Kingdom Atomic Energy Authority, Aldermaston, England (1967).

3. K. B. Snow, C. M. Hoffman, R. L. Brunelle, and M. J. Pro. "Comparison of Black Paints by Neutron Activation Analysis." International Criminal Police Review, (231) 221-225 (1969).

4. C. M. Hoffman, R. I. Brunelle, and K. B. Snow. "Forensic Comparisons of Soil by Neutron Activation and Atomic Absorption Analysis." J. of Criminal Law and Police Science 60 (3), 395-401 (1969).

5. R. R. Ruch, V. P. Guinn, and R. H. Pinker. "Detection of Gunpowder Residues by Neutron Activation Analysis." Nucl. Sci. and Eng. 20, 381-383 (1964).
6. C. M. Hoffman. "A Simplified Method of Collecting Gurishot Residue for Examination by NAA." Identification News 18 (10), 7-8 (1968).

7. G. C. Goode, G. A. Wood, and R. F. Coleman. Interim Report on the Multi-Element Analysis of Gliass Fragments by Neutron Activation and the Application to Forensic Scierice. AWRE Report $\# 054 / 69$, United Kingdom Atomic Energy Authority, Aldermaston, Eingland (1969).

8. R. A. Schmitt and V. Smith. "Identification of Origin of Glass by Neutron Activation Analysils in a Forensic Case." $\mathrm{J}$. of Forensic Science 15 (2), 252-260 $(1970)$.

9. H. R. Lukens end V. P. Guinn. "Comparison of Bullet Lead Specimens by NonDestructive Neutron Activation Analysis." (Paper presentied at the 20th Annual Meeting of The American Academy of Forensic

Sciences, Chicago, П11. Feb. 22, 1968).

10. R. L. Brunelle and C. M. Hoffman. "Comparison of Bullet Leads." Identification News 19 (9), 5i-14 (1969). 
THE ROLE OF ENVIRONMENTAL CONTAMINATION IN CRIMINALISTICS: A Case History

K. K. S. Pillay, C. C. Thomas, Jr., and G. F. Mahoney*

Western New York Nuclear Research Center

Power Drive, Buffalo, New York

$$
877^{2^{\circ}}
$$

The case history of a unique application of neutron activation analysis to criminalistics is presented. Neutron activation analysis of trace levels of tungsten, cobalt, and tantalum present in a variety of evidence materials were employed to establish the probable presence of the suspect at the scene of a murder. The evidence materials included the blouse of the victim, bed sheets, a pair of pantyhose used in strangulation and head and pubic hair of the suspect. Stained areas of the fabrics were found to contain the elements tungsten, cobalt, and tantalum, which are not normally found in fabrics or in household environment. The occupation of the suspect exposed him to fine dust particles containing these elements. In addition, eyewitness accounts indicated that the suspect was in the neighborhood where the murder took place near the time of the murder. There was, however, no evidence other than the neutron activation analysis results to indicate that he may have been at the scene of the crime. The court apcepted the neutron activation analysis results and the jury trial resulted in a conviction.

* Present address: Federal Bureau of Investigation Laboratory Washington, D. C.

The effects of environmental contamination on human hair has been recognized for a number of years. Lima, et a1 (1) have reported that workers in an arsenic plant had adnormally high concentrations of both arsenic and antimony in their hair. Perkons has reported high concentrations of selenium in the hair of a group of selenium factory workers (2). We have found an abnormal level of mercury in the hair of a researcher working with elemental mercury and mercury compounds. Perkons states that his results indicate "that certain elements can be and are deposited in hair in increased amounts through occupational contact or environmental conditions by exposure to industrial dusts and vapors".

This phenomenon, thus, can be used in the characterization of individuals on the basis of the trace elements deposited on their hair, clothing and/or bodies. The presence of abnormally high concentrations of certain elements could play an important role in analysis of physical evidence. The present paper discusses a homicide-criminal assault case in which environmental conditions played a major role.

The victim was criminally assaulted and strangled. Strangulation was accomplished with a pair of pantyhose. Physical evidence obtained at the scene included a blouse, a blanket, the top bed sheet, pantyhose and two turkish towels. One towel had been used to bind the victims legs while the second towel was found around her throat. Dark smear areas were noted on the blouse, blanket and bed sheet. No hair samples were obtained, primarily as a result of the fact that large quantities of dog hair were present, making separation of non-animal hair from animal hair essentially impossible. Spectrographic analysis of stained areas of the blouse indicated the possible presence of tungsten, titanium, antimony, magnesium and iron. Several of these elements, in particular, titanium and antimony, might be expected to be present in synthetic fibers since they are common catalysts.

Investigation of the crime brought to light a possible suspect who was acquainted with the victim, knew the schedule of the people involved on the day of the crime and was known to be in the area. This stage of the investigation was reached some time after the crime partially as a result of an incorrect pronunciation of the suspect's name. The suspect had been in jail for approximately a week on another charge.

The suspect had been employed at the time of the crime by a firm manufacturing tungsten carbide and tungsten carbidegraphite products. The environmental conditions are such that the workers become heavily contaminated with dust. The material is deposited on their hair and clothing and becomes embedded in the exposed areas of the body. The dust typically contains tungsten, cobalt, tantalum and possibly titanium. The 
latter, as indicated previously, is frequently present in synthetic fibers and, therefore, was not condidered useful in our work.

Additional physical evidence obtained after identification of the suspect included samples of his head and pubic hair and specimens of materials produced by his employer. The latter included tungsten carbide and tungsten carbide graphite mixtures.

Neutron activation analysis for tungsten, cobalt and tantalum was performed on a number of the physical evidence samples in our laboratory. The following analyses were performed:

1. Tungsten Carbide: Less than $50 \mathrm{mg}$ of each sample was taken for analysis. After irradiation, each sample was smeared with Whatman $\# 1$ filter paper, and the composition of the material transferred to the smear as well as that of the bulk material was determined.

2. Hair: Head hair samples of about $10 \mathrm{mg}$ each were taken. One sample was analyzed with no further treatment. The second sample.was washed with diethyl ether for two hours in a sam11 soxhlet extractor. The third sample was washed for fifteen minutes with deionized water. The pubic hair sample $(\sim 200 \mu g)$ was washed with diethyl ether.

3. Blouse: Two, two-inch square samples were cut from stained areas. One of these samples was cut in half and one half was washed with soap and water prior to analysis. Two, two-inch square areas were also cut from areas with no visible stains. One of these was also divided in half and one half subjected to washing.

4\&5. Blanket and Top Bed Sheet: Twoinch square samples were cut from both the stained and unstained areas.

6. Pantyhose: The evidence submitted had been reconstructed into the knot shape it had at the crime scene. One side of the knot was torn, probably due to excessive strain. About $20 \mathrm{mg}$ of material was cut from this section. Another similar sample was cut from the opposite side of the knot. Both samples were divided into two sections. One half of each sample was washed with soap and water prior to analysis.

The samples and appropriate tungsten, cobalt and tantalum standards were sealed in separate polyethylene vials. The samples were irradiated for one hour at a thermal neutron flux of $5: \mathrm{x} 10^{12} \mathrm{n} \cdot \mathrm{Cm}^{-2} \mathrm{Sec}^{-1}$ and were allowed to decay for about four to five hours. Gamma-ray spectra of the samples and standards were taken at various times for two days. After completion of this phase of the analysis, the samples and standards were re-encapsulated in quartz vials and irradiated for ten hours at a thermal flux of $3 \mathrm{x}$ $10^{13} \mathrm{n} \cdot \mathrm{Cm}^{-2} \mathrm{Sec} .^{-1}$. After a decay period of three days, the gamma-ray spectra of the samples and standairds were taken. The decay pattern of the radioisotopes of interest were followed for three weeks to confirm the presence of ${ }^{18} 7_{\mathrm{W}},{ }^{150} \mathrm{Co}$ and ${ }^{182} \mathrm{Ta}$.

All gamma-ray spectra were obtained with a high resolution $20 \mathrm{~cm}^{3}$ lithiumdrifted germanium detector and a 1024 channel analyzer. Quantititive analytical data was obtained using the peak area integration and comparison standari technique.

The results o:E the analysis are shown in Tables 1 through 4 .

The following conclusions may be drawn from the results:

1. The identification of the presence of ${ }^{187} \mathrm{~W},{ }^{60} \mathrm{Co}$ and ${ }^{182} \mathrm{Ta}$ radioisotopes in the various irradiated aamples is conclusive as determined by thei:e unique gamma energies and half-lives. The primary nuclear transmutations responsible for the formation of these isotopes are:

$$
\begin{gathered}
{ }^{186_{\mathrm{W}}(\mathrm{n}, \gamma)}{ }^{187_{\mathrm{W}}} \\
{ }^{59} \mathrm{Co}(\mathrm{n}, \gamma){ }^{60} \mathrm{Co} \\
\text { and }{ }^{181_{\mathrm{Ta}}(\mathrm{n}, \gamma)}{ }^{182_{\mathrm{T}} \mathrm{a}}
\end{gathered}
$$

The possible interferences are:

$$
\begin{aligned}
& { }^{187} \operatorname{Re}(\mathrm{n}, \mathrm{p}){ }^{187} \mathrm{~W} \\
& 190 \text { os }(\mathrm{n}, \alpha){ }^{187} \mathrm{~W} \\
& 60_{\mathrm{Ni}}(\mathrm{n}, \mathrm{p}){ }^{60} \mathrm{Co} \\
& { }^{63} \mathrm{Cu}(\mathrm{n}, \alpha){ }^{60} \mathrm{Co} \\
& { }^{182} \mathrm{~W}(\mathrm{n}, \mathrm{p}){ }^{182} \mathrm{Ta} \\
& \text { and }{ }^{185} \operatorname{Re}(n, \alpha){ }^{182} \mathrm{j}^{\prime} \mathrm{a}
\end{aligned}
$$


have shown up in the gamma spectra. Since we did not identify any ${ }^{64} \mathrm{Cu},{ }^{58} \mathrm{Co}$ (from $\left.{ }^{58} \mathrm{Ni}(\mathrm{n}, \mathrm{p}){ }^{58} \mathrm{Co}\right),{ }^{186} \mathrm{Re},{ }^{188} \mathrm{Re},{ }^{185} \mathrm{Os}$ and ${ }^{193} \mathrm{Os}$, it is reasonable to assume that the observed ${ }^{187} \mathrm{~W},{ }^{60} \mathrm{Co}$ and ${ }^{182} \mathrm{Ta}$ radioactivitiés have resulted from neutron induced nuclear reactions on tungsten, cobalt and tantalum present in the materials analyzed.

2. The blouse and the pantyhose positively show the presence of tungsten, cobalt and tantalum.

3. The stained areas of the white blanket and the top bed sheet positively show the presence of cobalt and tantalum. The analysis for tungsten was inconclusive due to interference radioactivities from dirt on these materials.

4. The analysis of the washed samples of blouse and pantyhose indicate that tungsten, cobalt and tantalum found on these materials were from external contamination.

5. The analysis of the head hair samples of the suspect indicate the presence of tungsten, cobalt and tantalum. These analyses further show that in spite of water and ether washings, some amount of these elements are retained in the hair, probably due to exchange processes.

6. The analysis of the pubic hair when compared with the head hair indicated that the contamination of the head hair is probably from external sources.

7. The calculation of the ratios of tungsten, cobalt and tantalum in the tungsten carbide graphite and smears taken from these samples demonstrated that the smeared materials are not representative of the original samples with regard to their elementa1 ratios.

8. Since tungsten', 'cobalt and tantalum are highly unlikely to be present in smearable and particulate form in normal surroundings, it is probable that the tungsten, cobalt and tantalum in the form found on the evidence materials may have a common source of origin.

The above findings were presented at the jury trial in Onandaga County, New York in Apri1 1970. The neutron activation analyses results were accepted by the court. The trail resulted in a conviction.
In summary, the data from a case in which environmental contamination played a major role is presented. This case, in our opinion, is an example of a unique application of neutron activation analysis to criminalistics.

\section{REFERENCES}

BOOK 1. F. W. Lima, H. Slubata, and L. T. Atatla. "Activation Analysis Applied to Forensic Investigation: Some Observations on the Problem of Human Hair Individualization", Radiochemical Methods of Analysis. p. 119-132, Internationa1 Atomic Energy Agency, Vienna (1965).

DOCUMENT 2. A. K. Perkons Hair Individualization Study

by Neutron Activation. PhD. Thesis, University of Toronto (1965). 
TABLE 1

ANALYSIS OF WASHED AND UNWASHED FABRIC SAMF'LES

\section{Sample Identification}

\#3, Blouse Stained Area

\#3B (1), Blouse Unstained Area

\#3S, Blouse Stained Area

\#3SW, Blouse. Stained Area Washed

$\# 3 B(2), B$ louse Unstained Area

\#3BW, Blouse

Unstained Area Washed

\#4, White Blanket Stained Area

\#4B, White Blanket Unstained Area

\#5, Top Bed Sheet, Stained Area

\#5B， Top Bed Sheet Unstained Area

\#66T, Pantyhose, Top Part of Knot, Strained Area

0.088

\#6TW, Pantyhose, Top Part of Washed

0.094

1.2

3.9

1.7

97.1

28.8

29.5

2.0

2.6

0.8

5.4

\#6L, Pantyhose, Lower

Part of Knot

\#6LW, Pantyhose, Lower

Part of Knot, Washed Knot, Strained Area,
0.136

0.074

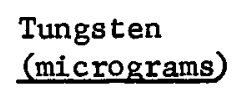

N.D.

0.03

N.D.

0.07

0.02

Not Conclusive

Due to Interferences

0.24

0.10

N. D. N. D.$$
0.074
$$

N.D. N.D.

$0.16 \quad 0.05$
N.D. N.D.

*Not Detectable 
TABLE 2

ANALYSIS OF HAIR SAMPLES

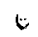

\begin{tabular}{|c|c|c|c|c|}
\hline $\begin{array}{c}\text { Sample } \\
\text { Identification* }\end{array}$ & $\begin{array}{c}\text { Sample } \\
\text { Weight (mg) }\end{array}$ & Tungsten (PPM) & Coba1t (PPM) & Tantalum (PPM) \\
\hline HHUW & 9.46 & 2244 & 39 & 15 \\
\hline HHWW & 10.66 & 1884 & 50 & 13 \\
\hline HHEW & 9.50 & 650 & 19 & N. D. *** \\
\hline PHEW & $<0.2$ & N. D. & N.D. & N. D. \\
\hline
\end{tabular}

\footnotetext{
*HHUW - Head Hair Unwashed

HHWW - Head Hair Water Washed

HHEW - Head Hair Ether Washed

PHEW - Publc Hair Ether Washed

w*Not Detectable
}

TABLE 3

$\ell$

ANALYSIS OF TUNGSTEN CARBIDE GRAPHITE SAMPLES

Sample

Identification

1A

$1 \mathrm{~B}$

$1 \mathrm{C}$
Tungsten (PPM)

$3.5 \times 10^{5}$

$5.8 \times 10^{5}$

$2.5 \times 10^{3}$
Cobalt (PPM)

$3.2 \times 10^{4}$

$5.2 \times 10^{4}$

$3.1 \times 10^{2}$
Tantalum (PPM)

$2.0 \times 10^{4}$

$4.1 \times 10^{4}$

$3.0 \times 10^{1}$ 
TABLE 4

RATIOS OF TUNGSTEN, COBALT AND TANTALUM IN SOME OF THE EVIDENCE SAMPLES

Sample Identification

1A, Tungsten Carbide Graphite (Small Piece)

1A, Tungsten Carbide Graphite (Smeared on Filter Paper)

1B, Tungsten Carbide Graphite (Sma11 Piece)

1B, Tungsten Carbide Graphite (Smeared on Filter Paper)

1C, Tungsten Carbide Graphite (Sma11 Piece)

1C, Tungsten Carbide Graphite (Fine Particles on Filter Paper)

3S, Blouse

Stained Area, Unwashed

6T, Pantyhose, Top Part of the Knot, Unwashed

6L, Pantyhose, Lower Part of the Knot, Unwashed

HHUW, Head Hair of suspect Unwashed

HHWW, Head Hair of suspect Water Washed

\begin{tabular}{|c|c|c|c|c|}
\hline Tungsten & $:$ & Cobalt & $:$ & Tantalum \\
\hline 17.5 & $:$ & 1.6 & $:$ & 1 \\
\hline 2.9 & $:$ & 3.6 & : & 1 \\
\hline 14.2 & $:$ & 1.3 & : & 1 \\
\hline 2.4 & : & 2.8 & $:$ & 1 \\
\hline 83.3 & $:$ & 10.3 & $:$ & 1 \\
\hline 74.2 & $:$ & 1.8 & $\vdots$ & 1 \\
\hline 31.3 & : & 0.9 & : & 1 \\
\hline 52.1 & $:$ & 2.3 & : & 1 \\
\hline 72.8 & : & 3.0 & : & 1 \\
\hline 147.6 & ; & 2.6 & : & 1 \\
\hline 144.9 & : & 3.8 & : & 1 \\
\hline
\end{tabular}




\title{
STATE-WIDE TRAINING AND SERVICE PROGRAM IN FORENSIC NEUTRON ACTIVATION ANALYSIS
}

\author{
James R. Vogt \\ Matthew E. Eichor \\ Ronald E. Mason
}

University of Missouri

Columbia, Missouri

\begin{abstract}
During the past year the University of Missouri has begun a state-wide program in forensic neutron activation analysis. This effort is intended to provide a sophisticated program in the characterization of physical evidence through the prudent application of neutron activation combined with the training of investigative officers in proper sample collection and handling procedures as well as in the capabilities and limitations of this analytical technique. A training program for police laboratory personnel is included to enhance their overall scientific background in practical applications of modern analytical chemistry. This part of the program is not limited to activation analysis. The training portion of the program is organized by the University's. Law Enforcement Extension Education staff who regularly conduct training programs throughout the State of Missouri. The overall program emphasizes close cooperation between investigating officers and the scientific staff in order to provide a coordinated approach.
\end{abstract}

INTRODUCTION

In recent years, the chemical characterization of physical evidence specimens has gained increasing importance in the investigative and prosecutive aspects of law enforcement. However, many state and local law enforcement agencies have neither the funds, the time nor the specialized personnel for the utilization of sophisticated analytical techniques on a routine basis. One solution is to expand the use of other state supported agencies - - such as state universities -- which have both the expensive specialized equipment needed and highly qualified professional personnel.

One such technique for which equipment is usually too expensive for law enforcement agencies to acquire, is neutron activation analysis. Since 1964, this analytical tool has seen increasing use in the characterization of physical evidence in court. Unfortunately, the technique has all too often been passed off as a panacea and has been, subject to "oversell" in a number of instances. A more prudent approach than has often been realized is needed in the application of this technique to law en forcement.
The University of Missouri has, on request, provided neutron activation analysis services to Missouri law enforcement agencies for several years. The majority of these analyses involved possible arsenic poisoning, although samples of hair, fiber and paint were also received occasionally. During this period no concerted effort was made to encourage the submission of samples because of limitations of personnel and funds. During the past year, however, funds became available to establish an extensive state-wide program in forensic activation analysis. This paper describes the objectives and activities of that program.

\section{PROGRAM AREAS}

It is the intent of this program to provide a sophisticated on-going capability in the characterization of physical evidence -- on a state wide basis. - through the prudent application of the technique of neutron activation analysis. In order to meet this objective there has been a heavy emphasis on developing a coordinated approach beginning with the crime scene search and ending with the final presentation of the evidence in court. Therefore, a significant portion of this program is devoted to 
the training of investigative law enforcement personnel in crime scene search techniques and proper sampling procedures as well as in the advantages and limitations of activation analysis. The three main phases of the program -- training, research and development, and service are discussed below.

\section{TRAINING}

A major portion of this program is devoted to training and orientation. It is believed from previous experience that an effective program requires close cooperation between law enforcement and scientific personnel. Equally essential is an understanding on the part of law enforcement personnel of the capabilities and limitations of neutron activation analysis as well as their role in correct sample collection, hand1ing and storage techniques. It is quite likely that one of the major impacts of this program in Missouri will be an increased awareness on the part of field investigators of the proper scientific techniques for the collection and preservation of physical evidence specimens.

The training program has been organized by the University's Law Enforcement Extension Education staff who regularly conduct training programs throughout the state of Missouri. The contacts made previously by the Law Enforcement Extension staff have been quite important in gaining acceptance for the activation analysis program on the part of local law enforcement officials.

The training portion of the program has been organized to provide instruction at three levels of intensity. The first area is a "popular" level presentation designed as an orientation for a broad cross-section of law enforcement and prosecution personnel. This series describes the basic concepts of neutron activation analysis, its application to forensic science, and the role of law enforcement agencies and personnel in the successful conduct of the project. Both the patrolman and prosecuting attorney are considered to be import- ant to this program. The patrolman is often the Eirst person at the crime scene and is in a position: to preserve the integrity of any physical evidence antil the arrival of investigative and laboratory personnel. Training of a patrclman can be quite important in the arrest of a person suspected of a shooting. Where practical; the patrolman can take steps to avoid contamination of the suspects hands si that a defense attorney's claim of possible contamination will carry less weight. Education of prosecuting attorneys is of particular importance because they often decide what laboratory tests will be run as well a's what evidence will be used and how the data will be presented in court.

The second level of the training program is a lecture and demonstration series aimed at experienced investigative personnel. The content of this section is dependent on the background of the particular class. In some areasi of the state there are some highly trained investigators. In other areas the investigative personnel can benefit from a more detailed presentation on crime scene search technigues and the preservation of physical evidence. These lectures involve more detailed instruction in activation analysis capabilities and limitations, as well as practice in scientific sample collection, handling and storage techniques. This phase of the training provides instruction and experience that are applicable to mary other physical evidence characterization techniques in addition to activation analysis.

The third level is a lecture, demonstration and laboratory program involving experienced police laboratory personnel. These sessions are conducted at the Columbia campus of the University of Missouri and consist of seminars in a number of modern analytical techniques conducted by faculty members who are specialists in a particular analytical area. This portion of the program is of particular interest to police laboratory directors who are receiving grants to modernize and expand their facilities. 
The training sessions involving investigative and laboratory personnel place particular emphasis on which analytical techniques may be most appropriate for a particular problem. The class is advised that for many types of evidence materials their existing methods are as good as or better than activation aralysis. The areas where activation anaIysis may be of importance to them. are discussed as is the current status of developmert of the service program in Missouri with respect to specific types of evidence material.

\section{RESEARCH AND DEVELOPMENT}

This area of effort might better be termed implementation and development. A concerted effort has been. made to implemert procedures developed by others for those forensic applications with which the Reactor Facility staff has not previously had extensive experience. It is necessary that the staff be intimately familiar with any technique used as well as with the expected variations to be found in a particular type of specimen to insure proper interpretation of the data. Extensive use of the literature has been made to avoid unnecessary duplication of effort.

In some cases a certain amount of development work is necessary to adapt a tectinique to the particular facilities available. For example, optimum irradiation, decay and counting times will vary with the irradiation and pulse height analysis facilities of the group doing the analysis. The high neutron flux and high gamma-ray heating at the Research Reactor Eacility makes it necessary to seal all samples in quartz tubing for irradiations of more than four hours. This has created some problems in sample preparation as well as problems in sample contamination from impurities in the quartz vials.

A certain amount of effort has been expendea in the development of new applications ana the improvement or extensicn of existing techniques. A series of äiscussions with the Springfjeld, Missouri Police Depart- mert indicated a reed for and interest ir a technique to characterize safe insulation. A pilot study is now in progress to determine the feasibility of using activation analysis for this purpose. Samples are being irradiated for 100 hours at a flux of $4.5 \times 10^{14} \mathrm{r}_{\mathrm{Cm}}^{-2} \mathrm{~s} \in \mathrm{c}^{-1}$. and counted using a $45 \mathrm{~cm}^{3} \mathrm{Ge}(\mathrm{Li})$ detector and a 8192 channel pulse height analyzer. An extensive survey of fiber samples is being conducted to determine the usefullness of activation analysis for the characterization of this type of physical evidence. These samples are irradiated for one minute and one hour in the pneumatic tube system at a flux of $4.5 \times 10^{13} \mathrm{n} \mathrm{cm}^{-2} \mathrm{sec}^{-1}$.

One application of forensic neutron activation analysis which usually arouses considerable interest is the characterization of hair. Since the high neutron flux of the Research Reactor provides a good capability for the analysis of small samples, this is an area of some interest to us. A statistical analysis of published analytical data on hair is being conducted as well as an analytical survey of selected specimens collected in the state of Missouri. We are particularly interested in determining the feasibility of reliable characterization of single hairs. This portion of the program will be enhanced when the Research Reactor power level is increased from 5 megawatts to 10 megawatts. This will provide a maximum flux of $9 \times 10^{14} \mathrm{n}$ $\mathrm{cm}^{-2} \mathrm{sec}^{-1}$ and $a$ flux in the pneumatic tubes of $9 \times 10^{13} \mathrm{n} \mathrm{cm}^{-2} \mathrm{sec}^{-1}$.

Hair samples are currently irradiated for one hour at a flux of 4.5

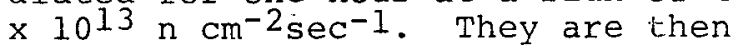
washed for one minute in diethyl ether and counted on a $45 \mathrm{~cm}^{3} \mathrm{Ge}(\mathrm{Li})$ detector after decay times of 6 minutes $2 \frac{1}{2}$ hours and 48 hours. Data are read out on 9 track 800 bpi magnetic tape and processed on an IBM System 360/65 computer using program BARFF. BARFF is used to process all spectra taken with $\mathrm{Ge}(\mathrm{Li})$ detectors. The program will analyze multiplets up to triplets, and beyond that will give data on the three most intense peaks of a multiplet along with a warning message to check the data carefully. Table I lists those elements deter- 
mined instrumentally following a one hour irradiation of a single hair four inches in length. The error. estimate is for the peak analysis from program BARFF.

TABLE I

Radionuclides Determined

In a single Hair

\begin{tabular}{|c|c|c|c|c|}
\hline $\begin{array}{l}\text { Energy } \\
\text { (Kev) } \\
\end{array}$ & Nucl & Lide & Decay & $\begin{array}{c}\text { Error } \\
\left(\frac{\text { 응 })}{}\right.\end{array}$ \\
\hline 77 & 197 & $\mathrm{Hg}$ & $48 \mathrm{hr}$ & $<5$ \\
\hline 389 & $87 \mathrm{~m}$ & $\mathrm{Sr}$ & $2.5 \mathrm{hr}$ & $<5$ \\
\hline 412 & 198 & $\mathrm{Au}$ & $2.5 \mathrm{hr}$ & $<5$ \\
\hline 439 & $69 \mathrm{~m}$ & $\mathrm{Zn}$ & $24 \mathrm{hr}$ & $<5$ \\
\hline 443 & 128 & $I$ & $6 \mathrm{~min}$ & $<5$ \\
\hline 776 & 82 & $\mathrm{Br}$ & $2.5 \mathrm{hr}$ & $<5$ \\
\hline 1014 & 27 & $\mathrm{Mg}$ & $6 \min$ & $<10$ \\
\hline 1039 & 66 & $\mathrm{Cu}$ & $6 \mathrm{~min}$ & $<10$ \\
\hline 1368 & 24 & $\mathrm{Na}$ & $6 \min$ & $<5$ \\
\hline 1525 & 42 & K & $2.5 \mathrm{hr}$. & $<5$ \\
\hline 1642 & 38 & $\mathrm{Cl}$ & $6 \min$ & $<5$ \\
\hline 1779 & 28 & Al & $6 \min$ & $<10$ \\
\hline 1811 & 56 & $\mathrm{Mn}$ & $2.5 \mathrm{hr}$ & $<5$ \\
\hline 3083 & 49 & $\mathrm{Ca}$ & $6 \min$ & $<10$ \\
\hline 3102 & 37 & $S$ & $6 \mathrm{~min}$ & $<10$ \\
\hline
\end{tabular}

of the fifteen elements determined, several are of minor importance for characterization purposes.

\section{SERVICE}

The major objective of this program is to provide a routine physical evidence characterization service to Missouri law enforcement agencies. Expert witness testimony is also provided as needed. As this paper is written this portion of the program is just getting under way. However, those cases which have been referred to us tend to support our belief that close contact between the investigat- ing officers and the scientific staff is very important in obtaining the maximum amount of reliable data from a particular sample. One must, of course, maintain an objective approach and avoid becoming emotionally involved in a particular case. However, the investigating officers can often provide iseful information which may aid in interpretation of the data obtained.

The training portion of this program should aid the service portion to a great extent. The proper collection of appropriate samples is obviously crucial to the success of the program. In addition, the training sessions provide investigative and laboratory personnel with enough background information to identify those samples which are appropriate for neutron activation analysis. Hopefully, this will avoid a siege of samples which are of little value or which could best be analyzed by other means.

Acknowledgement -- This work has been supported by the U..S. Department of Justice, the Missouri Law Enforcement Assistance Council and the Mid-Missouri Regional Law Enforcement Planning Council. 


\section{STATISTICAL INTERPRETATION OF TRACE ELEMENT PATTERNS IN PAPER}

\section{H. Richard Lukens \\ Howard L. Schlesinger}

Gulf Radiation Technology

San Diego, California
The qualitative and quantitative trace element patterns, as determined by neutron activation analysis (NAA), in a suite of 40 different bond papers was subjected to statisti$\mathrm{cal}$ analysis. The eight most frequently observed elements, $\mathrm{Ti}, \mathrm{Al}, \mathrm{Ca}, \mathrm{Na}, \mathrm{Mn}, \mathrm{Cl}, \mathrm{Cr}$, and $\mathrm{Sb}$ were selected for this purpose. With few exceptions the distribution functions of these elements could be described by neither Gaussian nor linear forms. However, the observed densities were found to be satisfactorily self-defining. That is, the frequence with which given combinations of concentrations of each pair of elements occurred (the $\mathrm{Na}-\mathrm{Cl}$ pair was the sole exception) could be accurately predicted by multiplication of their individual densities. Thus, in addition to the fact that different trace element patterns indicate different papers, it is now possible to compute the probability that a given trace element pattern of one bond paper will be matched by that of a different name brand of paper. The average, greatest, and least probabilities of such an accidental match, based on 7 elements, are on the order of $10^{-5}, 10^{-3}$, and $10^{-10}$, respectively.

\section{INTRODUCTION}

The examination of 120 samples of bond paper, representing one to several manufacturing runs of 40 different kinds of paper, by instrumental neutron activation analysis (NAA) has been previously reported. (1) The present paper describes the statistical analysis of the NAA results with respect to the concentrations of $\mathrm{Ti}, \mathrm{Al}, \mathrm{Ca}, \mathrm{Na}, \mathrm{Mn}$, $\mathrm{Cl}, \mathrm{Cr}$, and $\mathrm{Sb}$ in the samples. These elements were selected on the basis of their relatively high frequency of observation and their lack of correlation. A number of other elements were determined, also, but with comparatively lower frequency.

An important aspect of the sampling procedure was that the different runs of a given kind of paper in the sample were not separated by more than a few weeks. Hence, the practice of using papers from two or three consecutive runs in assembling packages of paper was reflected in the sampling procedure, and the variation of a given element among the different runs was similar to that within a package of paper. Furthermore, samples were taken at various times throughout each of several rather long manufacturing runs, which enabled the intra-run variation of the elements to be examined.

\section{ST ATISTICAL, PROCEDURE}

The wide range of concentrations exhibited for most of the elements suggested that the logarithm of each concentration value, rather than the values themselves, should be subjected to a distribution sutdy. Nevertheless it was first ascertained that the original values did not fit a standard distribution function. The logarithms were then taken, and are given in Table I.

The intra-and inter-run variations of the elements in a given kind of paper are given in Table II. It can be seen that in most cases the latter are greater than the. former. Note b), of Table II, refers to the standard deviation of mean values of the elements under discussion: and the relative standard deviations of the means were used to decide the widths of concentration increments to be employed in the analysis of concentration distributions.

It was decided to use 3 equal logarithmic increments per decade to study the distributions of $\mathrm{Ti}, \mathrm{Al}, \mathrm{Ca}, \mathrm{Na}, \mathrm{Mn}$, and $\mathrm{Cl}$, while 2 equal logarithmic increments per decade were used in the case of $\mathrm{Cr}$ and $\mathrm{Sb}$. The corresponding widths, in $\overline{0}$ mean 
i

the increments were as follows: $\mathrm{Ti}-3.7$, $\mathrm{Al}$ - 4.6, $\mathrm{Ca}$ - 5.6, $\mathrm{Na}-3.5, \mathrm{Mn}-3.6$, $\mathrm{Cl}-3.0, \mathrm{Cr}-2.6$, and $\mathrm{Sb}-2.6$. The increments and their numerical designations are given in Table III.

The logarithms of the concentrations, which are listed in Table I, were converted to the increment numbers pecified in $\mathrm{T}$ able III, and the resulting array of enumerated elemental concentration increments are given in Table IV. The frequency of observation of each code increment for each given element was tabulated as shown in Table $V$, and the resulting distributions were tested, by the chi-square test, against the hypotheses that they were log-normal (Gaussian) or log-linear (linear) derisities. The results of these tests a re given in Iable VI. It was clear from these results that the data could not be uniformly treated by either of these two, common distribution forms; and the results cast strong doubt that any other well-understood distribution form could be uniformly applied to the data set. Therefore, the observed distribution frequencies were treated as self-defining and tested for independence by combinatorial means.

Each possible combination of expected pair frequencies was computed by multiplying the individual element densities, including the null class (Table V). The hypothesis that the observed frequencies were not significantly different from these products was subjected to the chi-square test. The results of the test for each pair of elements, which are given in Table VII, indicate that the frequency distributions of all pairs, except $\mathrm{Na}-\mathrm{Cl}$, are suitably predicted by multiplication of their individual observed densities. Since $\mathrm{Na}$ is more frequently observed than $\mathrm{Cl}$, the former will usually be of more use as a comparison point, and $\mathrm{Cl}$ should not be used unless $\mathrm{Na}$ is not observed. In any event, only one of these two elements whould be used in the comparison of two samples of bond paper.

The foregoing tests indicate that, given the trace element qualitative and quantitative analysis (by the described method( 1 ) of a given paper, it is possible to compute the probability that a different paper, selected at random from the subject population, will have the same triace element pattern. The probability defined by multiplying the most frequent analyticial results for individual elements ( $T$ able $\mathrm{V}$, excluding chlorine) is $2.77 \times 10^{-3}$. All other combinations are less probably - the least probability is $9.8 \times 10^{-11}$, and the average probability is $3.7 \times 10^{-5}$.

Therefore, it has been shown that, in addition to the fact that trace element patterns of papers from a given batch of paper will match, the chance that matching patterns will be obtained from different papers is very small. Given the pattern of one bond paper, the probability of obtaining a matching pattern from a truly different bond paper can be estimated.

In addition to their immediate value, these results suggest the examination of an enlarged population sample to improve the accuracy and precision of probability estimates.

(1) H. L. Schlesinger and D.M. Settle, "A Large-Scale Study of Paper by Neutron Activation Analysis', Am. Acad. of Forensic Sci., 20th Annual Meeting, Chicago, Feb. $22-24,1968$. 
TABI,E I

Average Concentrations of the Elements in 40 Kinds of Bond Paper,

Log of Value in Units Indicated

Element and Concentration Unit

\begin{tabular}{|c|c|c|c|c|c|c|c|c|}
\hline $\begin{array}{l}\text { Kind of } \\
\text { Paper }\end{array}$ & $\begin{array}{l}\mathrm{Ti} \\
0.1 \% \\
\end{array}$ & $\begin{array}{c}\text { Al, } \\
10 \mathrm{ppm} \\
\end{array}$ & $\begin{array}{c}\mathrm{Ca}, \\
100 \mathrm{ppm} \\
\end{array}$ & $\begin{array}{c}\mathrm{Na}, \\
10 \mathrm{ppm} \\
\end{array}$ & $\begin{array}{c}\mathrm{Mn}, \\
0.1 \mathrm{ppm} \\
\end{array}$ & $\begin{array}{c}\mathrm{Cl}, \\
10 \mathrm{ppm} \\
\end{array}$ & $\begin{array}{c}\mathrm{Cr}, \\
0.1 \mathrm{ppm} \\
\end{array}$ & $\begin{aligned} & \mathrm{Sb}, \\
& 0.1 \mathrm{ppm} \\
&\end{aligned}$ \\
\hline 1 & $\overline{0.49}$ & $\overline{0.48}$ & 1.48 & 0.99 & 1.51 & 1.18 & & \\
\hline 2 & 0.34 & 0.23 & 1. 34 & 1.32 & 1.45 & 1. 48 & 0.85 & \\
\hline 3 & 0.52 & 1.22 & 1. 40 & 1.46 & 1.04 & 1.83 & & 0.77 \\
\hline 4 & 0.32 & 1. 24 & 1.48 & 1.37 & 0.85 & 1.75 & & 0.36 \\
\hline 5 & 1. 11 & 0.77 & 1.29 & 1.32 & 1.00 & 1.71 & & 1.26 \\
\hline 6 & 0.46 & 1.13 & 1.54 & 1.32 & 1.04 & 1.78 & & \\
\hline 7 & 0.52 & 1.67 & 0.91 & 1. 16 & 1.28 & 1.04 & 1.43 & 0.98 \\
\hline 8 & 0.90 & 2.13 & 0.68 & 1. 48 & 1.32 & 1.00 & & \\
\hline 9 & & 3. 10 & & 1.51 & 1.00 & 1.56 & 2.28 & 0.04 \\
\hline 10 & 1. 10 & 2.98 & & 1.61 & 0.88 & 1.40 & & 2.53 \\
\hline 11 & 1.03 & 2,58 & 1.13 & 1.71 & 1.04 & & & \\
\hline 12 & & 3.28 & & 1.76 & 1.04 & & 2.08 & \\
\hline 13 & 0.84 & 2.34 & & 1.71 & 1.85 & & & \\
\hline 14 & 0.91 & 2.32 & & 1.70 & 1.96 & & 1.20 & 0.54 \\
\hline 15 & 0.76 & 2.27 & & 1.95 & 2.23 & 1.62 & 1.04 & \\
\hline 16 & 1.10 & 2.20 & & 1.96 & 2.38 & 1.72 & & 0.84 \\
\hline 17 & 0.89 & 2. 24 & & 2.26 & 2.54 & & & 1.23 \\
\hline 18 & 0.42 & 2.66 & & 2. 18 & 2. 18 & & 1.86 & \\
\hline 19 & 1.08 & 3.13. & & 1.82 & 2. 18 & & & \\
\hline 20 & 0.75 & 2.28 & & 2.03 & 1.72 & & & \\
\hline 21 & 0.86 & 2.28 & & 1.88 & 1.36 & & & 1.20 \\
\hline 22 & 1.16 & 2. 43 & & $1: 76$ & 1.30 & & & 1.51 \\
\hline 23 & & 2.43 & 1.56 & 1.40 & 1.96 & 1.71 & & 1.61 \\
\hline 24 & & 2. 74 & & 1.44 & 1.08 & 1. 19 & 1.83 & \\
\hline 25 & & 3.11 & & 1.49 & 1.28 & 1.55 & 2.32 & \\
\hline 26 & & 3.15 & & 1.51 & 1.30 & 1.67 & 2.26 & \\
\hline 27 & & 2.99 & & 1.69 & 1.15 & 1.66 & 2.15 & \\
\hline 28 & & 2.80 & & 1.34 & 1.18 & & 1.95 & \\
\hline 29 & 0.19 & 1.95 & & 1.59 & 1.41 & 1.56 & 1.63 & 1.26 \\
\hline 30 & 0.54 & 2. 20 & & 1.45 & 1.23 & 1.36 & & 1.67 \\
\hline 31 & 0.94 & 2. 51 & & 1.87 & 1.41 & & & 2.11 \\
\hline 32 & & 2.28 & & 1.20 & 0.69 & 0.94 & 1.23 & 0.46 \\
\hline 33 & & 2.31 & & 1.20 & 0.69 & 1.35 & 1.36 & 0.77 \\
\hline 34 & 0.83 & 2.26 & 0.97 & & & & & \\
\hline 35 & 0.60 & 2.13 & 0.74 & & & & & \\
\hline 36 & 0.53 & 2. 48 & & 1.45 & 0.85 & 2.06 & & 1.11 \\
\hline 37 & 1.00 & 1.99 & & 1.63 & 0.92 & 2.08 & & 1.23 \\
\hline 38 & 0.83 & 2.28 & & 1. 55 & 0.98 & 2.08 & & 0.85 \\
\hline 39 & 0.95 & 1.96 & & 1.56 & 0.90 & 2. 10 & & 1. 18 \\
\hline 40 & 0.49 & 2. 48 & & 1. 49 & 0.88 & 2.08 & & 0.80 \\
\hline
\end{tabular}

j 
TABLE II

Intra-Run and Inter-Run Variations

\begin{tabular}{|c|c|c|c|c|c|}
\hline \multirow[b]{2}{*}{ Element } & \multicolumn{2}{|c|}{$\begin{array}{c}\text { Average Relative } \\
\text { Standard Deviation, } \% \\
\end{array}$} & \multirow[b]{2}{*}{ Element } & \multicolumn{2}{|c|}{$\begin{array}{l}\text { Average Relative } \\
\text { Stalidard Deviation, } \%\end{array}$} \\
\hline & Intra-Runa) & Inter-Run b) & & Intra-Runa) & Inter-Run b) \\
\hline $\mathrm{Ti}$ & 8 & 33 & $M n$ & 11 & 33 \\
\hline $\mathrm{Al}$ & 23 & 27 & $\mathrm{Cl}$ & 51 & 41 \\
\hline $\mathrm{Ca}$ & 19 & 22 & $\mathrm{Cr}$ & 22 & 70 \\
\hline $\mathrm{Na}$ & 39 & 35 & $\mathrm{Sb}$ & 12 & 68 \\
\hline
\end{tabular}

a) 24-hour production run

b) Average among different runs of 35 different kinds of papers! The average standard deviations of the means are $\sim 60 \%$ of the values listed.

TABLE III

Log-Concentration Increments

A. Ti, Al, Ca, $\mathrm{Na}, \mathrm{Mn}$, and $\mathrm{Cl}$

Increment

Number

1

2

3

4

5

6

7

8

9

10

$0.67-1.0$
B. $\mathrm{Cr}$ and $\mathrm{Sb}$

Increment

Number

Log-Concentration Increment

$-0.33$

$0.33-0.67$

$1.00-1.33$

$1.33-1.67$

$1.67-2.00$

$2.00-2.33$

$2.33-2.67$

$2.67-3.00$

$3.00-3.33$
$0-0.5$

$0.5-1.0$

$1.0-1.5$

$1.5-2.0$

$2.0-2.5$

$2.5-3.0$ 
TABL،E IV

Enumeration of Elemental Concentrations in Increments

Element and

Kind of Concentration Increment Paper $\mathrm{Ti} \mathrm{Al} \mathrm{Ca} \mathrm{Na} \mathrm{Mn} \mathrm{Cl} \mathrm{Cr} \mathrm{Sb}$
Element and

Kind of Concentration Increment

$\frac{\text { Paper }}{21} \frac{\mathrm{Ti}}{3} \frac{\mathrm{Al}}{7} \frac{\mathrm{Ca}}{\mathrm{Na}} \frac{\mathrm{Mn}}{6} \stackrel{\mathrm{Cl}}{\frac{\mathrm{Cr}}{6}} \frac{\mathrm{Sb}}{3}$

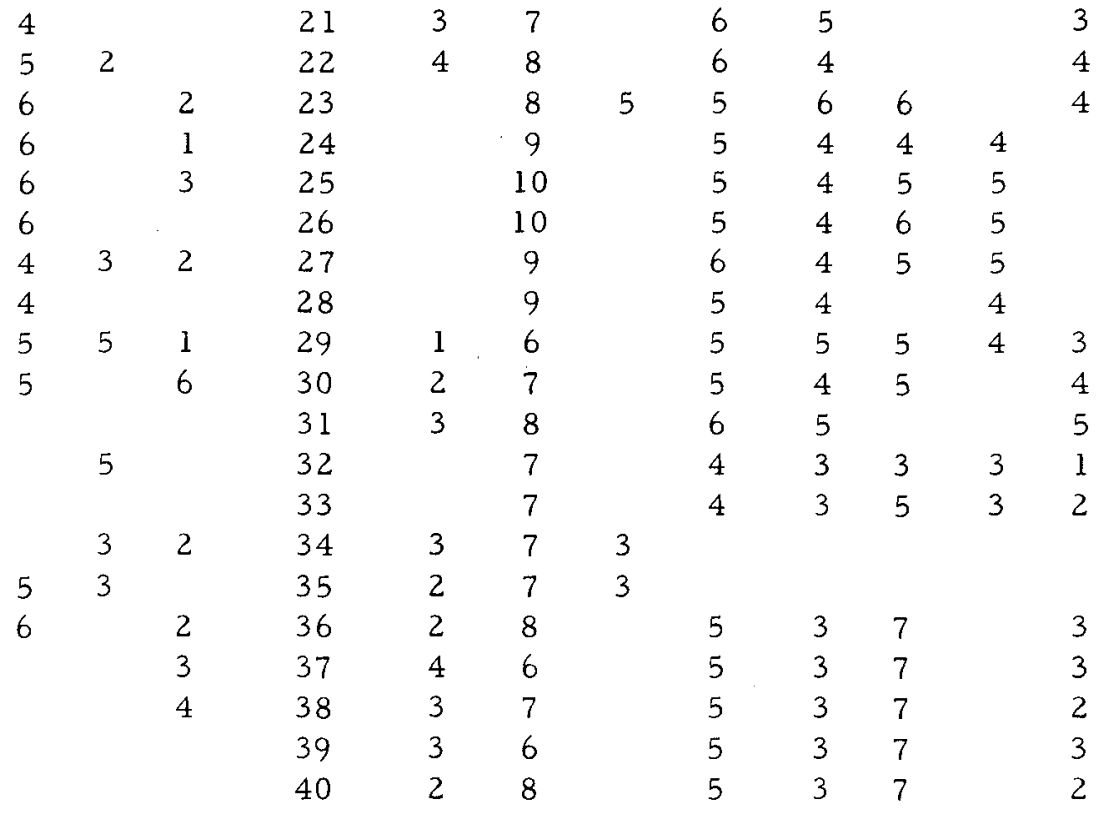

TABLE V

Distribution of Elemental Values, Incremental
Concentration Increment Number

1
2
3
4
5
6
7
8
9
10
Nul1 b)

\begin{tabular}{|c|c|c|c|c|c|c|c|}
\hline & & Obse & ation & uency & $7_{0}^{\text {a) }}$ & & \\
\hline$\overline{\mathrm{Ti}}$ & $\mathrm{A} 1$ & $\overrightarrow{\mathrm{Ca}}$ & $\mathrm{Na}$ & $\mathrm{Mn}$ & $\mathrm{Cl}$ & $\mathrm{Cr}$ & $\mathrm{Sb}$ \\
\hline 5.0 & 2.5 & & 2.5 & & & & 7.5 \\
\hline 25.0 & 2.5 & & & & & 2.5 & 17.5 \\
\hline 27.5 & 2.5 & 10.0 & 2.5 & 22.5 & 2.5 & 12.5 & 17.5 \\
\hline 17.5 & 7.5 & 5.0 & 15.0 & 37.5 & 10.0 & 7.5 & 10.0 \\
\hline & & 15.0 & 42.5 & 12.5 & 22.5 & 12.5 & 2.5 \\
\hline & 10.0 & & 27.5 & 10.0 & 17.5 & & 2.5 \\
\hline & 32.5 & & 7.5 & 7.5 & 12.5 & & \\
\hline & 20.0 & & & 5.0 & & & \\
\hline & 10.0 & & & & & & \\
\hline & 12.5 & & & & & & \\
\hline 25.0 & & 70.0 & 5.0 & 5.0 & 35.0 & 65.0 & 42.5 \\
\hline
\end{tabular}

a) Zero observations not specifically cited.

b) Percent of kinds of papers in which the element was not observed.

(1) 
TABLE VI

Distribution Function (Density) Analysis

Total

Frequency of Obser-

Element

$\mathrm{Ti}$

Al

$\mathrm{Ca}$

$\mathrm{Na}$

$\mathrm{Mn}$

Cl

Cr

$\mathrm{Sb}$ vation, $\%$

75

100

30

95

95

65

35.

57.5
Value of Chi-Square For Hypothesized Density Gaussian . Linear

13.5

6.4

112.0

14.5

3. 8 .

124.5

30. 1

20.7

23.2
35. 7

2. 0

24.2

19.1

6.9

3.2

9.6
Acceptability of

Degrees of Freedon?

Hypothesized Density Gaussian Iinear No No No: No

No Yes

Yes No

No . No

No Yes

No Yes

No No

TABLE VII

Acceptability of Hypothe sis that Observed Frequency of Element Concentration Pairs is not Significantly Different than the Product of Their Individual Densities

Hypothesis Accepted? (At 2.05 Leirel)

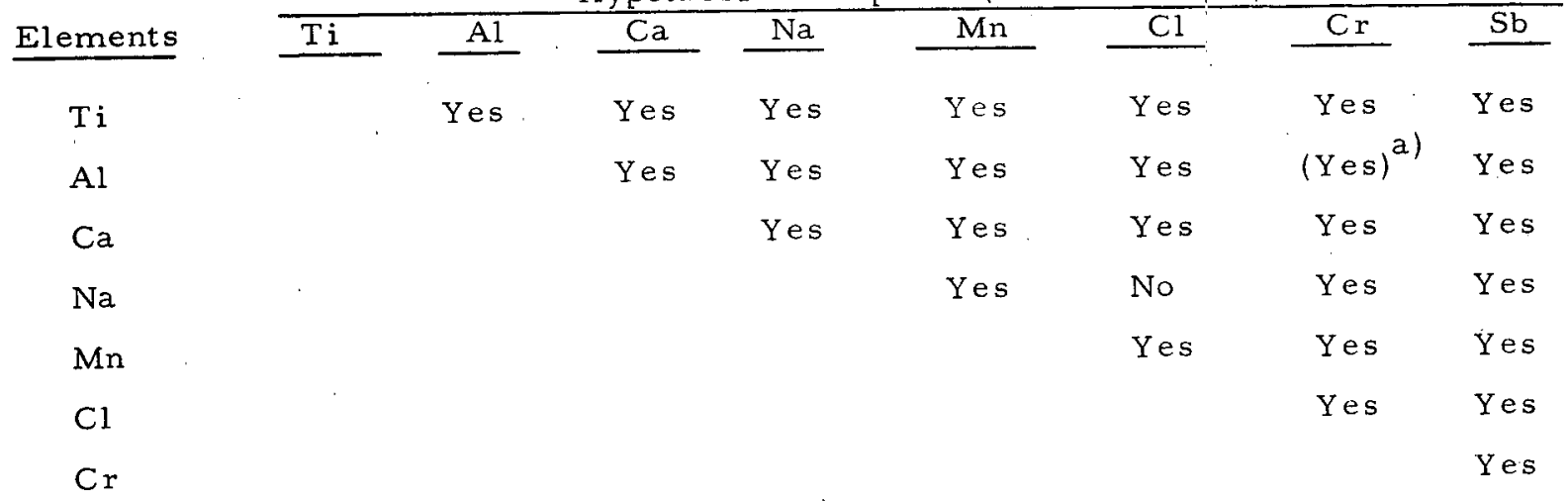

a) Not rejected at .01 level. Would be accepted at higher levels ( $>$. 10) with rejection of one observation where the expectation was 0.025 . 